\title{
Fully kinetic PiC simulations of current sheet instabilities for the Solar corona
}

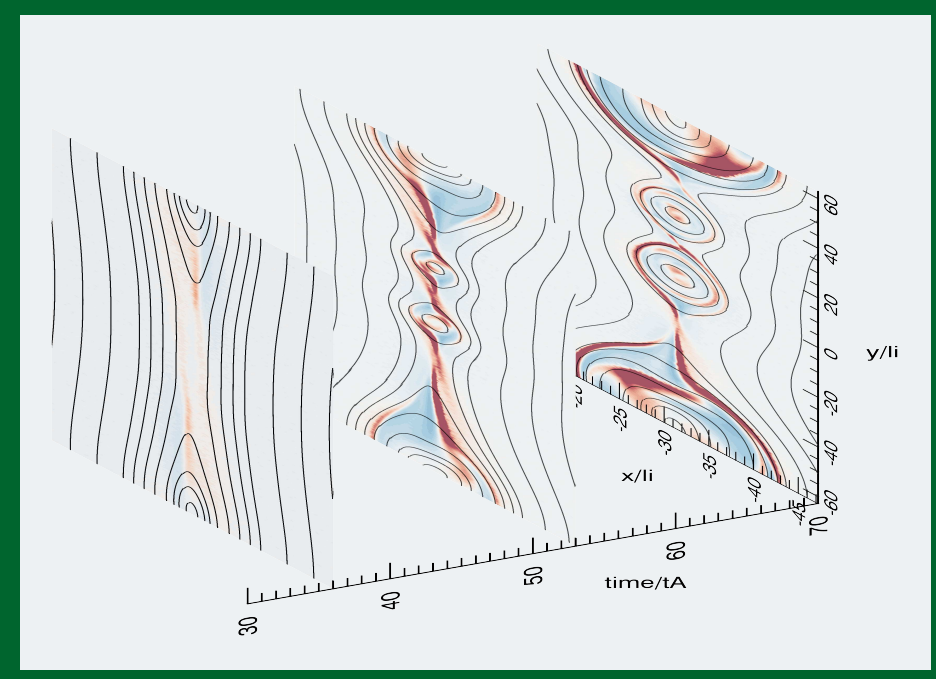

\section{Patricio A. Muñoz Sepúlveda}

International Max Planck Research School for Solar System Science at the University of Göttingen 


\title{
Fully kinetic PiC simulations of current sheet instabilities for the Solar corona
}

\author{
Dissertation \\ zur Erlangung des mathematisch-naturwissenschaftlichen Doktorgrades \\ "Doctor rerum naturalium" \\ der Georg-August-Universität Göttingen \\ im Promotionsprogramm PROPHYS \\ der Georg-August University School of Science (GAUSS)
}

vorgelegt von

\section{Patricio A. Muñoz Sepúlveda \\ aus Concepción, Chile}

Göttingen, 2015 
Betreuungsausschuss

Prof. Dr. Jens Niemeyer

Institut für Astrophysik, Universität Göttingen, Germany

Prof. Dr. Jörg Büchner

Max-Planck-Institut für Sonnensystemforschung, Göttingen, Germany

Prof. Dr. Hardi Peter

Max-Planck-Institut für Sonnensystemforschung, Göttingen, Germany

Mitglieder der Prüfungskommision

Referent: Prof. Dr. Jens Niemeyer

Institut für Astrophysik, Universität Göttingen, Germany

Korreferent: Prof. Dr. Jörg Büchner

Max-Planck-Institut für Sonnensystemforschung, Göttingen, Germany

2. Korreferent: Prof. Dr. Uwe Motschmann

Institut für Theoretische Physik, Technische Universität Braunschweig, Germany

Weitere Mitglieder der Prüfungskommission:

Prof. Dr. Wolfgang Glatzel

Institut für Astrophysik, Universität Göttingen, Germany

PD Dr. Wolfram Schmidt

Hamburger Sternwarte, Fachbereich Physik, Universität Hamburg, Germany

Prof. Dr. Laurent Gizon

Institut für Astrophysik, Universität Göttingen, Germany

Max-Planck-Institut für Sonnensystemforschung, Göttingen, Germany

Prof. Dr. Ansgar Reiners

Institut für Astrophysik, Universität Göttingen, Germany

Tag der mündlichen Prüfung: 25.06.2015 


\section{Bibliografische Information der Deutschen Nationalbibliothek}

Die Deutsche Nationalbibliothek verzeichnet diese Publikation in der Deutschen Nationalbibliografie; detaillierte bibliografische Daten sind im Internet über http: //dnb .d-nb. de abrufbar.

ISBN 978-3-944072-15-9

uni-edition GmbH 2015

http://www.uni-edition.de

(C) Patricio A. Muñoz Sepúlveda

(c) (i) This work is distributed under a

Creative Commons Attribution 3.0 License

Printed in Germany 



\section{Contents}

Summary 11

1 Why current sheet instabilities? an introduction 13

1.1 Properties of the solar corona and magnetic reconnection . . . . . . . . 13

1.2 The role of current sheets in magnetic reconnection . . . . . . . . . . . . 16

1.3 Current sheets and magnetic reconnection in other environments . . . . . 17

1.4 General concept of instability . . . . . . . . . . . . . . . . . . 18

1.5 The importance of kinetic instabilities . . . . . . . . . . . . . . . 19

1.6 Outline of this thesis . . . . . . . . . . . . . 20

2 Plasma physics background: an overview of plasma models (used in this thesis) 25

2.1 Kinetic theory . . . . . . . . . . . . . . . . . 25

2.1 .1 Momenta of the VDF . . . . . . . . . . . . . 26

2.1.2 Maxwell equations and single particle description . . . . . . . 27

2.1.3 What is a collisional in a plasma? The kinetic Boltzmann equation 27

2.1 .4 Collisionless approach and closure: Vlasov-Maxwell system . . . 29

2.2 Multi-fluid models . . . . . . . . . . . . . . . . 30

2.2 .1 Two-fluid theory and Generalized Ohm's law . . . . . . . . . . 31

2.2 .2 One fluid variables . . . . . . . . . . . . . . . . . . . . . . . . . 31

2.2 .3 One-fluid Generalized Ohm's law . . . . . . . . . . . . . . . . . . . . . . 33

2.3 Hall-MHD . . . . . . . . . . . . . . . . . . . . 34

2.4 Resistive MHD . . . . . . . . . . . . . . . . . . . . 34

2.5 Ideal MHD and Alfvén theorem . . . . . . . . . . . . . . . . . . . . . . . . 35

2.6 Double adiabatic approximation (CGL $\ldots \ldots \ldots$. . . . . . . . 37

3 Current sheet equilibria 39

3.1 MHD equilibria and magnetohydrostatics . . . . . . . . . . . . 39

3.1 .1 Force free fields . . . . . . . . . . . . . . . . . . . . 40

3.2 Kinetic equilibria of current sheets . . . . . . . . . . . . . . . . . . . . . . . . 40

$3.2 .1 \quad$ Harris type equilibria $\ldots \ldots \ldots \ldots$. . . . . . . . . . . . . . . . . . . . . . . .

3.2 .2 Force free equilibria . . . . . . . . . . . . . . . . 43

3.2.2.1 Linear kinetic force free equilibria . . . . . . . . . 44

3.2.2.2 Non-linear kinetic force-free equilibria . . . . . . . . 45

3.2.2.3 Force free configuration used in this work . . . . . . 46 
4 Magnetic Reconnection 49

4.1 MHD reconnection . . . . . . . . . . . . . . . . 50

4.1.1 Sweet-Parker reconnection model . . . . . . . . . . . . . 50

4.1 .2 Petschek reconnection . . . . . . . . . . . . . . . . 52

4.1.3 Non-stationary reconnection model: Plasmoid instability . . . . . 52

4.2 Collisionless magnetic reconnection . . . . . . . . . . . . . . 53

$4.2 .1 \quad$ Hall effects . . . . . . . . . . . . . . . . 53

4.2.2 Role of dispersive waves in collisionless reconnection . . . . . 55

4.2.2.1 Traditional explanation . . . . . . . . . . . . . 55

4.2.2.2 Recent works showing opposite evidence . . . . . . . 58

4.2 .3 Non-gyrotropic pressure effects . . . . . . . . . . . . 60

4.2 .4 Effects of electron inertia . . . . . . . . . . . . . . 62

4.2 .5 Anomalous dissipation effects .............. . . 62

4.3 Influence of guide field on Hall-MHD reconnection . . . . . . . . 63

4.3.1 Effects on the symmetries of steady-state magnetic reconnection . 64

4.3.2 Consequence: Cavities and electron acceleration . . . . . . . . 66

4.3 .3 Effects on reconnection rate . . . . . . . . . . . . . 67

5 Instabilities in CS: Theoretical background 69

5.1 Tearing mode instability of CS . . . . . . . . . . . . . . 69

5.1 Physical mechanism and fluid approach . . . . . . . . . . . . . 70

5.1 .2 Kinetic approach . . . . . . . . . . . . . . 72

5.1.2.1 Linear instability . . . . . . . . . . . . 73

5.1.2.2 Non-linear evolution and saturation of tearing . . . . 75

5.1 .3 Influence of a guide field . . . . . . . . . . . . . 78

5.1.3.1 Fluid approach in the low beta limit . . . . . . . 80

5.1.3.2 Tearing growth rates in a linear force free configuration 80

5.1.3.3 Tearing growth rates in non-linear force free configuration 81

$\begin{array}{lll}5.1 .3 .4 & \text { Saturation mechanism in the presence of a guide field . } 82\end{array}$

5.1 .4 Influence of anisotropy . . . . . . . . . . . . . . . 82

5.1.4.1 Relation with CS bifurcation . . . . . . . . 86

5.2 Temperature anisotropy driven instabilities . . . . . . . . . . . . . . 87

5.2 .1 Unmagnetized Weibel instability . . . . . . . . . . . . . 88

5.2.1.1 Weibel instability in CS . . . . . . . . . . . . . . 90

5.2 .2 Magnetized: Mirror instability . . . . . . . . . . . . . . . 90

5.2.2.1 Relation between mirror and Weibel instabilities . . . . 93

5.2 .3 Magnetized electron whistler instability . . . . . . . . . . . . 99 93

5.2 .4 Magnetized: Firehose instability . . . . . . . . . . . . . . . . . . . . . . 94

5.3 Shear flow driven instabilities . . . . . . . . . . . . . . . . . . . . . . . 94

$5.3 .1 \quad$ MHD/fluid Kelvin-Helmholtz instability . . . . . . . . . . . . . . 94

5.3 .2 Kinetic scale K-H instability . . . . . . . . . . . . . . . . 96

5.3.2.1 Shear flow gradient kinetic instabilities . . . . . . . . 98

$5.3 .3 \quad$ Magnetic field generation via kinetic K-H instability . . . . . . . 99

5.3 .4 K-H interaction with magnetic reconnection . . . . . . . . . . 101

5.3.5 Shear flow interaction with magnetic reconnection . . . . . . 103

5.4 Streaming instabilities . . . . . . . . . . . . . . . . . . . 104 
$5.4 .1 \quad$ Field aligned instabilies with oblique propagating . . . . . . . . . 104

5.4.2 Cross-field instabilies with perpendicular propagating . . . . . . 104

5.4.2.1 Electron cyclotron drift instability (ECDI) . . . . . . 105

$5.4 .2 .2 \quad$ MTSI/KCSI instabilities . . . . . . . . . . . . 107

5.4.2.3 Cross field streaming instabilities and reconnection . . 112

5.4.2.4 Lower Hybrid Drift Instability (LHDI) $\ldots . . . . . .113$

5.4 .2 .5 LHDI in M.R. . . . . . . . . . . . . 116

6 Kinetic PIC code simulations $\quad 119$

6.1 General scheme . . . . . . . . . . . . . . . . . . . . . . . . 119

6.2 Coarse-graining of phase space and shape function . . . . . . . . 121

6.2 .1 Most common shape functions . . . . . . . . . . . . . . . 123

6.3 Discrete Vlasov-Maxwell system for particles . . . . . . . . . . . . . 125

6.4 Particle mover . . . . . . . . . . . . . . . . . . . 126

6.5 Discrete Vlasov-Maxwell system for fields: FDTD schemes and Yee lattice 128

6.6 Coupling particles with fields: current assignment . . . . . . . . . . . 129

6.6 .1 Current smoothing . . . . . . . . . . . . . . . . . . . . 131

6.6 .2 Energy conserving PIC codes . . . . . . . . . . . . . 132

6.7 Maxwell solven . . . . . . . . . . . . . . . . . . . . . . 132

6.7.1 Approximation of the Maxwell equations . . . . . . . . . . 134

6.8 Numerical consequences of the coarse-graining, grid and time step . . . 135

6.8 .1 Stability conditions . . . . . . . . . . . . . . . . . 135

6.8.1.1 Practical consequences of the stability conditions . . . 137

6.8 .1 .2 Implicit codes . . . . . . . . . . . . . . . . 137

$6.8 .2 \quad$ Numerical heating . . . . . . . . . . . . . . . . . . 138

6.8.2.1 Grid heating and "aliasing" instability . . . . . . . . 138

6.8.2.2 "Scattering" heating . . . . . . . . . . . . . . . . . 139

6.8 .3 Consequence of coarse-graining: numerical collisions . . . . . 141

6.8.4 Side effect of collisions: Entropy in Vlasov plasmas . . . . . . 142

6.8.5 Side effect of collisions: Enhanced stopping of high energy particles 144

6.8.6 Consequences of coarse-graining: numerical noise . . . . . . . 144

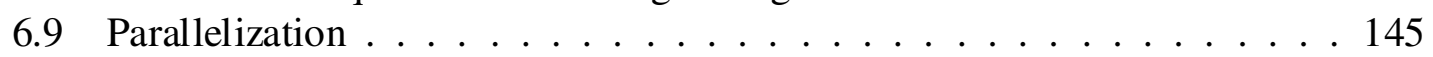

6.10 The ACRONYM code . . . . . . . . . . . . . . . . . . . . . . 147

$7 \quad$ Instabilities of antiparallel Harris CS and anisotropic heating 151

7.1 Simulation setup . . . . . . . . . . . . . . . . . . 152

7.1 .1 General considerations . . . . . . . . . . . . . . . . 152

7.1 .2 Parameters . . . . . . . . . . . . . . . . 152

7.1 .3 Justification for the size along $\mathrm{d} \ldots \ldots . . \ldots \ldots$

7.1.4 Justification for the size along $\mathrm{x}$ : boundary conditions . . . . . 155

7.2 Numerically developed anisotropic heating . . . . . . . . . . . 155

7.2 .1 Numerical heating and shape function . . . . . . . . . . . . 155

7.2 .2 Anisotropic numerical heating and stability of CS . . . . . . 158

7.2 .3 Numerical CS bifurcation and entropy . . . . . . . . . . . . . 163

7.2.3.1 Collisions and location of bifurcation . . . . . . . 165

7.2.3.2 Collisions in 2D geometry . . . . . . . . . . . . . 165 
7.2.4 Numerical heating and background plasma effects . . . . . . . . 165

7.2 .5 Restriction to an antiparallel magnetic field configuration . . . . . 168

7.3 Imposed temperature anisotropy and instabilities . . . . . . . . . . . . 168

7.3 .1 Initial temperature anisotropy relaxation . . . . . . . . . . 168

7.3.1.1 Enhancement of tearing mode with the opposite ani sotropy 170

7.3.1.2 Macroparticles per cell and anisotropy relaxation . . . . 170

7.3.2 Temperature anisotropy relaxation via Weibel instability . . . . . 171

7.3.2.1 Spatial structure of Weibel instability . . . . . . . . . . 173

7.3.2.2 Weibel threshold condition ... . . . . . . . 175

7.3 .3 Mirror instability produced via Weibel instability . . . . . . . . 175

7.3.3.1 Field aligned temperature anisotropy . . . . . . . . . 175

7.3.3.2 Mirror threshold condition . . . . . . . . 176

7.3.3.3 Size of mirror mode structures and propagation direction 178

7.3.4 Growth rates of the initial Weibel/mirror instabilities . . . . . . . 179

7.3.4.1 Other possible temperature driven instabilities . . . . . 181

7.3.5 Consequence of Weibel/Mirror instabilities: bifurcation . . . . . 181

7.3.6 Macroparticles per cell and Weibel/mirror instabilities . . . . . 183

7.3.7 Influence of ions in the temperature anisotropy driven instabilities 186

7.4 Numerical parameters and tearing mode . . . . . . . . . . . . . . 187

7.4 .1 Influence of the shape function . . . . . . . . . . . . . . . 187

7.4.1.1 Time evolution of power in tearing islands . . . . . 187

7.4.1.2 Identification of linear/non linear stage . . . . . . . 188

7.4.1.3 Comparison with linear growth rates . . . . . . . . . 190

7.4.2 Influence of the number of macroparticles . . . . . . . . . . . . 191

7.4 .3 Influence of the mass ratio . . . . . . . . . . . . . . . . 192

7.5 Conclusions . . . . . . . . . . . . . . . . . . . 193

$8 \quad$ Instabilities of Harris CS in the presence of small guide fields 197

8.1 Simulation setup . . . . . . . . . . . . . . . . . . 198

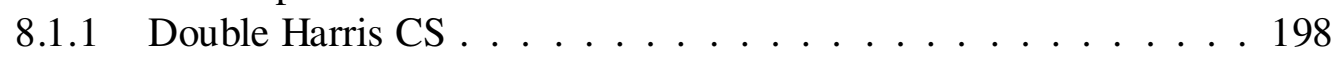

8.1 .2 Parameters ........................ 199

8.2 Onset of guide field reconnection: Tearing instability . . . . . . . . . . 201

8.2.1 Numerical considerations with guide field . . . . . . . . . . 201

8.2.1.1 Effects of guide field on the accuracy of particle moven 201

8.2.1.2 Resolution of electron gyration and bifurcation . . . . . 203

8.2.2 Evolution of tearing instability on dependence on guide field . . . 205

8.3 Mechanism of guide field reconnection . . . . . . . . . . . . . . . 207

8.3.1 Calculation methods for averages and derivatives . . . . . . . . 207

8.3.1.1 Time average and derivatives . . . . . . . . . . . 208

8.3.1.2 Spatial average and smoothing . . . . . . . . . . 209

8.3.1.3 Mean field generalized Ohm's law . . . . . . . . . 210

8.3 .2 Dissipative mechanism in the antiparallel limit . . . . . . . . 211

8.3 .3 Guide field dependence . . . . . . . . . . . . . . . . . . . . . 214

8.4 Deviations in the generalized Ohm's law and cross-field instabilities . . . 217

8.4.1 Electron bulk acceleration and cross-field instabilities . . . . . . 2 218

8.4.2 Thermal and non-thermal effects on the instabilities and Ohm's law 220 
8.5 Conclusions . . . . . . . . . . . . . . . . . . . 222

9 Instabilities of force free CS in large guide fields and comparison with GK 227

9.1 Gyrokinetic approach . . . . . . . . . . . . . . . . . . . . . . . . . . . 229

9.1 .1 Gyrokinetic theory . . . . . . . . . . . . . . . . . . . 229

9.1.2 Gyrokinetic simulations of tearing instability/magnetic reconnection 231

9.2 Simulation setup . . . . . . . . . . . . . . . . . . . . . . . 234

9.2 .1 Force free CS . . . . . . . . . . . . . . . . . . . 234

9.2.1.1 Justification for the use of a force free CS . . . . . . 234

9.2.1.2 Double force free CS to be used . . . . . . . . . . . 235

9.2 .2 Perturbation . . . . . . . . . . . . . . . . . . . . . 236

9.2 .3 Parameters . . . . . . . . . . . . . . . . . . . . . . 237

9.3 Global evolution: reproduction of previous work . . . . . . . . . 242

9.3.1 Reconnection rates and fast magnetic reconnection . . . . . . . 242

9.3.2 Parity/symmetry of reconnection quantities and linear scaling . . 244

9.4 Core magnetic field and pressure equilibrium condition . . . . . . . 246

9.4.1 Numerical reason for core magnetic field and pressure equilibrium 247

9.4.2 Time evolution of deviations in the pressure equilibrium condition 250

9.5 Core magnetic field and shear flow . . . . . . . . . . . . . . 253

9.5 .1 Initial shear flow . . . . . . . . . . . . . . . . 253

9.5.2 Current/flows in secondary magnetic islands . . . . . . . . . 255

9.5.3 Electron/ion flow in later times . . . . . . . . . . . . . . 257

9.5.4 Time evolution of electron/ion flows: influence of initial shear flow 258

9.5 .5 Boundary effects in core magnetic field generation . . . . . . . 260

9.5.6 Influence of shear flow in reconnection . . . . . . . . . . 261

9.5.7 Other related studies about magnetic field generation by shear flows 262

9.6 (Thermal) heating mechanisms . . . . . . . . . . . . . . . . 262

9.7 Non-thermal and dissipative mechanisms _ . . . . . . . . . 265

9.7 .1 Deviations from thermal equilibrium . . . . . . . . . . . . 265

9.7.2 Charge separation and in-plane electric fields . . . . . . . . . 269

9.7 .3 Dissipation measures . . . . . . . . . . . . . . . . . . . 271

9.8 Evidence for cross-field streaming instabilities . . . . . . . . . . . . 272

9.8 .1 Criteria for instabilities ruled out . . . . . . . . . . . . 272

9.8.2 Modified two stream/Kinetic cross-field streaming instabilities . . 273

9.8 .3 Gradients driven instabilities . . . . . . . . . . . . . . . 276

9.8.3.1 Lower hybrid drift instability (LHDI) . . . . . . . . . 276

9.8.3.2 Comparison of ExB and diamagnetic drifts . . . . . . 277

9.8.3.3 Electron-ion hybrid/Kinetic Kelvin-Helmholtz instabilities 278

9.8.3.4 Thermal effects due to secondary instabilities . . . . . . 279

9.9 Finite plasma beta effects . . . . . . . . . . . . . . . . . . 280

9.9.1 Differences in the pressure equilibrium condition . . . . . . . 280

9.9.2 Flow pattern structure in magnetic islands and separatrices . . . . 283

9.9.3 Summarv: critical parameters for reliable PIC/GK comparison . . 285

9.10 Role of numerical noise . . . . . . . . . . . . . . . . . . . . 285

9.11 Conclusions . . . . . . . . . . . . . . . . . . . . . . . . . . . 287 
10 Conclusions and Outlook 293

10.1 Conclusions . . . . . . . . . . . . . . . . . . . . . . 293

10.2 Outlook . . . . . . . . . . . . . . . . . . 296

\begin{tabular}{|lr}
\hline A Terminology, definitions and relations & 297
\end{tabular}

A.1 Plasma parameters and abbreviations . . . . . . . . . . . . . . . . . . . 297

A.1.1 Abbreviations . . . . . . . . . . . . . . . . . . . 297

A.1.2 List of symbols and definitions . . . . . . . . . . . . . . 297

A.2 Geometry . . . . . . . . . . . . . . . . . . . . . . . . . . 299

A.3 Relation between quantities and parameter setup . . . . . . . . . . . . . 300

A.3.1 General relations between plasma parameters . . . . . . . . . . . 300

A.3.2 Independent input parameters for a Force Free CS . . . . . . . . 301

A.3.3 Independent input parameters for a Harris CS . . . . . . . . . . 302

\begin{tabular}{lr}
\hline Numerical tools & $\mathbf{3 0 5}$
\end{tabular}

B.1 Digital filters . . . . . . . . . . . . . . . . . . . 305

B.1.1 Frequency response . . . . . . . . . . . . . . . . 306

B.1.2 Examples of commonly used filters . . . . . . . . . . . . 308

B.1.2.1 Moving average . . . . . . . . . . . . . . . . . 309

B.1.2.2 Binomial . . . . . . . . . . . . . . . 310

B.1.2.3 Gaussian filten . . . . . . . . . . . . . . . . . 311

B.1.2.4 Sharp-cutoff . . . . . . . . . . . . . 312

B.1.2.5 Savitzky-Golay filters . . . . . . . . . . . . . 312

B.1.3 Numerical derivatives . . . . . . . . . . . . . . . . . . . . 317

B.1.3.1 Extended finite differences . . . . . . . . . . . 317

B.1.3.2 Smooth derivatives with S-G filters . . . . . . . . 318

B.1.3.3 Combining smoothing formulas and derivatives . . . . 320

B.2 Some calculation methods . . . . . . . . . . . . . . . 321

B.2.1 Higher order momenta of the distribution function . . . . . . . 321

B.2.2 Reconnection rate . . . . . . . . . . . . . . . . . 323

B.2.3 Tearing mode growth rates . . . . . . . . . . . . . . . . . . 324

B.2.4 Discrete coarse-grained entropy . . . . . . . . . . . . . 325

B.2.5 Rotation of pressure tensor . . . . . . . . . . . . . . 325

B.2.6 Non-Gyrotropy of the pressure tenson . . . . . . . . . . . . 326

\begin{tabular}{|l|l}
\hline Bibliography & 327
\end{tabular}

\begin{tabular}{|l|l|}
\hline Scientific Contributions & 363
\end{tabular}

\begin{tabular}{|ll}
\hline Acknowledgements & 365
\end{tabular}

\begin{tabular}{|l|l}
\hline Curriculum vitae & 367
\end{tabular} 


\section{Summary}

The plasma of the Solar corona is practically collisionless. This imposes an important role of waves and micro-turbulent interactions with particles for plasma transport, dissipation, stability, etc, since binary particles collisions are inefficient. As a result the Solar corona is also a natural laboratory for testing basic plasma turbulent phenomena. Current sheets (CS), through which magnetic energy can be released, are ubiquitous in this environment. Different from many other plasmas, the presence of large guide magnetic fields plays a crucial role in the corona. In general, CS are prone to a large number of macro and micro-instabilities, the more the thinner they become. CS can lead to or are formed as consequence of magnetic reconnection. This is a fundamental physical process in the Universe that converts magnetic into other forms of energy which goes along with change of the magnetic connectivity.

In this context, we aim towards an appropriate characterization of the influence of the guide magnetic field on the instabilities of and magnetic reconnection through CS, for which in coronal plasmas small scale kinetic effects are essential. In order to investigate the essential nonlinear properties, we use fully kinetic Particle-in-Cell (PIC) numerical simulations to adequately describe the collisionless solar coronal plasma. The kinetic approach is necessary to properly describe coupling of scales in collisionless magnetic reconnection and to provide, in the end, macroscopic transport properties appropriately describing the coronal plasma. In order to validate our methods, we also analyze the limits cases of zero (antiparallel configuration) and infinite guide fields (gyrokinetic theory) for comparison.

In the case of antiparallel Harris CS, we find several instabilities driven by temperature anisotropy which might be numerically induced when more realistic parameters (high mass ratios) are used in PIC simulations. We reveal that they may mimic real collisional physical processes, and we show how they can be efficiently avoided by choosing appropriate numerical parameters, such as the shape functions (interpolation schemes).

Next, we analyze the instabilities of Harris CS in the presence of small guide fields. We develop methods to calculate spatial and temporal derivatives, as well as averages, for a proper calculation of the mechanisms supporting the reconnected electric field. Our methods, more accurate than previously used ones, reveals the appearance of additional terms in the mean field generalized Ohm's law at the edge of the magnetic islands, arising from the interaction with electromagnetic fluctuations. Our findings reveal cross-field streaming and pressure gradient driven instabilities, causing plasma heating, particle acceleration and turbulence.

In the third and last part, we analyze instabilities of force free CS in the presence of large guide fields, which we compare with the results of gyrokinetic simulations. For $\beta_{i}=0.01$, we find that gyrokinetic simulations model sufficiently well the regions close to 
the reconnection $\mathrm{X}$ point for guide fields $b_{g} \gtrsim 5$, and practically everywhere for $b_{g} \gtrsim 30$. But only a fully kinetic PIC simulation can reveal, e.g., the physics of secondary magnetic islands for moderate guide fields $b_{g} \gtrsim 5$, where macro and micro instabilities driven by shear flow and streamings generate magnetic fields, particle heating and acceleration besides of high frequency electromagnetic fluctuations. For $\beta_{i} \sim 1$, the applicability of gyrokinetic simulation is much less restricted, in the sense that the convergence to the PIC simulations results requires even lower guide fields.

Our results have important implications for understanding the role of current sheet instabilities in the solar corona and their macroscopic consequences for the overall dynamics and energy conversion processes. This also applies for astrophysical collisionless plasmas, as well as laboratory plasmas and nuclear fusion facilities on Earth. 


\section{Why current sheet instabilities? an introduction}

\subsection{Properties of the solar corona and magnetic reconnec- tion}

The solar corona is the outer layer of the Solar atmosphere, directly visible from the Earth only during eclipses. It is composed by a practically collisionless (see definition of "collision" in Sec. 2.1.3) and low beta ( $\beta$, to be defined in Eq. (3.4)) plasma, permeated by magnetic fields, electromagnetic turbulence and waves (see some characteristic parameters in Table 1.1). One of its most striking features is its very high temperatures above $10^{6} \mathrm{~K}$. This is about three orders of magnitude hotter that the photosphere $\left(T \sim 6 \cdot 10^{3} \mathrm{~K}\right)$, the Sun's visible surface located below. One of the most long standing open questions in the solar corona research is trying to explain how the solar corona is heated to such high temperatures.

Many explanations for the coronal heating have been proposed, which can be divided basically in two groups (see Aschwanden 2005, Chap. 9). The first one are AC mechanisms, based in the dissipation of the energy of electromagnetic waves. The second ones are DC mechanisms, based in the dissipation of electric currents, e.g. in the so called nanoflares. Basically, it proposes that current sheets (CS, to be discussed in Sec. 1.2) in small flares, distributed everywhere in the solar corona (in both active and quiet regions), are continuously releasing energy and contributing to the steady heating of the corona (Cassak and Shay 2011). This is is in opposition to the much larger, but infrequent, release of energy by flares and coronal mass ejections (CME's). In all these scenarios, magnetic energy is converted to heat and plasma acceleration via a sudden change in the topology of the magnetic field (see Somov 2006, and references therein), a process called magnetic reconnection (to be discussed in Chapter 4). Since reconnection usually takes place through CS, they are the main focus of this thesis.

There are many models of magnetic reconnection in solar flares, relying on a host of different mechanisms from the pure magnetohydrodynamics (MHD, see definition of this plasma model in Sec. 2.2) to fully kinetic ones (see Parker 1983, 1994, Priest et al. 2005, Somov 2006, Cassak and Shay 2011, and references therein). There is no unique explanation: some of these models seem suitable to explain some events particularly in collisional regimes, but there is a lack of models for collisionless cases. As pointed out by Cassak and Shay (2011), the main problem for validation of these models is the huge gap between the length scales of a typical solar flare (on the order of $10^{7} \mathrm{~m}$ ) and the diffusion region of collisionless reconnection where magnetic field lines reconnect (on the order 

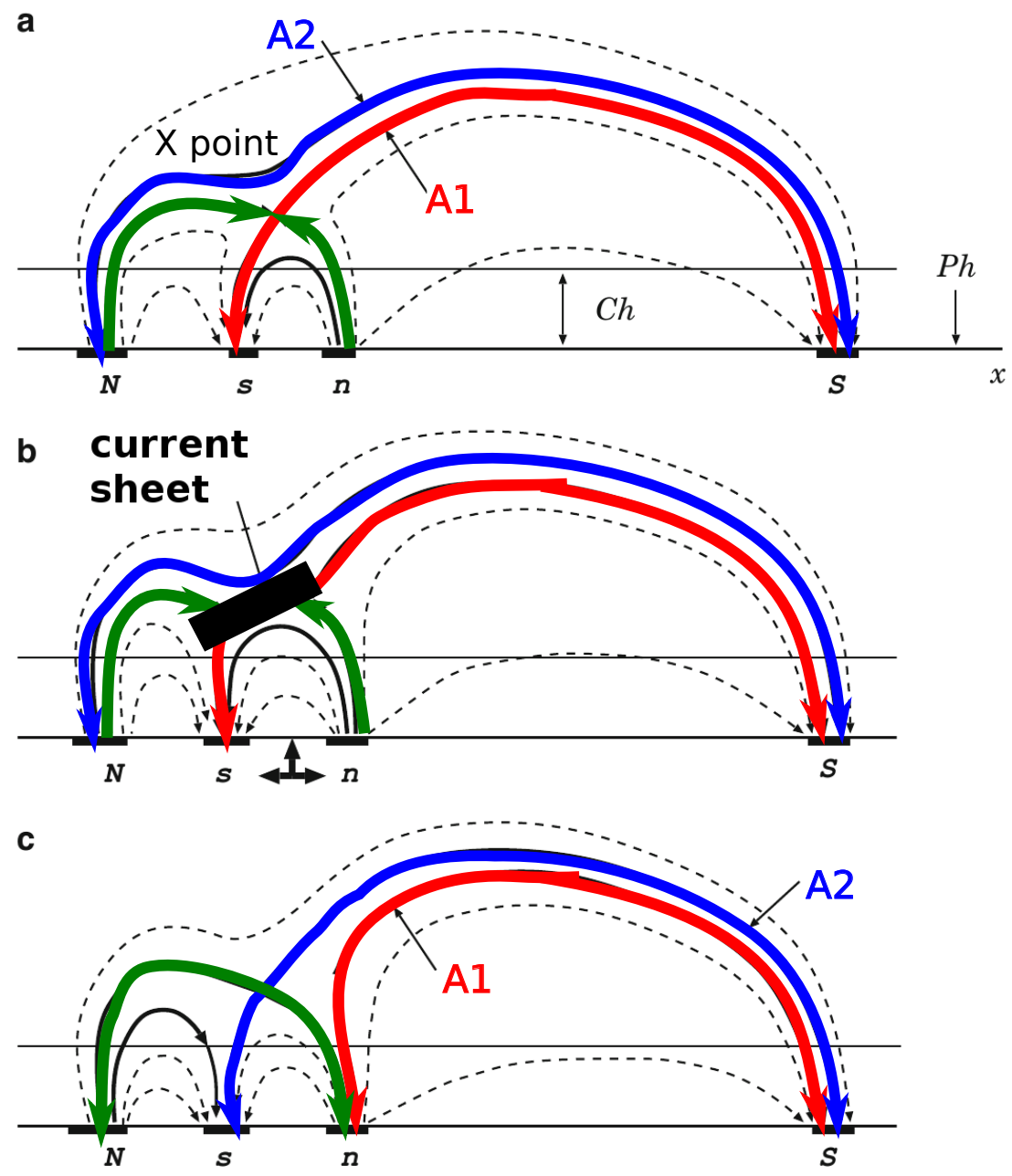

Figure 1.1: Scheme showing a possible scenario of current sheet and magnetic reconnection in a solar flare. In stage a), the magnetic field line Al has end-points in "s" and " $S$ ", while A2 in " $N$ " and " $S$ ". In stage b) a current sheet is formed in the indicated place. After the reconnection process takes place in the current sheet, the change in topology alters the end point of the line Al to the other side of the X point: from " $s$ " to " $n$ ", and similarly for the line A2: from " $N$ " to " $s$ ". Adapted from Fig. 4.1 of Somov (2006).

of $d_{e} \sim 2 \mathrm{~cm}$. See Table 1.1) 1. A realistic numerical simulation that could test these models would have to resolve a separation of 9 orders of magnitude in length scales, something totally impractical nowadays. Moreover, as we will describe in Chapter 4, magnetic reconnection also involves a very slow build-up phase (up to weeks in the case of flares), but a very sudden release of energy during its onset (on the order of minutes). This wide time-scale separation impose an additional severe constraint on any kind of numerical validation of realistic models of solar flares.

But how do we know that magnetic reconnection is operating in the solar corona? The CS where this process takes place cannot be resolved by observations due to their small size, on the order of the diffusion region $d_{i}$ in the collisionless coronal plasma (Büchner

\footnotetext{
${ }^{1}$ All the symbols to be shown in this thesis are defined and described in Appendix A.1.2.
} 


\begin{tabular}{ccccc}
\hline Plasma & $n_{e}\left[\mathrm{~cm}^{-3}\right]$ & $B[T]$ & $T_{e}[\mathrm{eV}]$ & $\omega_{p e}\left[\mathrm{~s}^{-1}\right]$ \\
\hline Solar corona (flares) & $10^{9}$ & $2 \cdot 10^{-2}$ & 200 & $1.4 \cdot 10^{9}$ \\
Solar wind & 7 & $7 \cdot 10^{-9}$ & 10 & $2.5 \cdot 10^{5}$ \\
Magnetotail & 0.3 & $2 \cdot 10^{-8}$ & 600 & $4 \cdot 10^{4}$ \\
Magnetopause & 10 & $5 \cdot 10^{-8}$ & 300 & $1.1 \cdot 10^{5}$ \\
\hline Magnetar flares & $10^{35}$ & $2 \cdot 10^{11}$ & $5 \cdot 10^{5}$ & \\
\hline MRX & $10^{13}$ & 0.1 & 10 & \\
ITER & $10^{14}$ & 5.3 & $2 \cdot 10^{4}$ & \\
\hline Plasma & $S$ & $\omega_{p e} / \Omega_{c e}$ & $c / v_{t h, e}$ & $d_{e}[\mathrm{~m}]$ \\
\hline Solar corona (flares) & $1 \cdot 10^{13}$ & $1-2$ & 50 & 0.2 \\
Solar wind & $3 \cdot 10^{12}$ & $100-150$ & 200 & $1.2 \cdot 10^{3}$ \\
Magnetotail & $4 \cdot 10^{15}$ & $10-30$ & $20-70$ & $8.0 \cdot 10^{3}$ \\
Magnetopause & $6 \cdot 10^{13}$ & $10-70$ & $40-70$ & $2.7 \cdot 10^{3}$ \\
\hline Magnetar flares & $6 \cdot 10^{16}$ & - & - & \\
\hline MRX & $3 \cdot 10^{3}$ & $50-200$ & $220-320$ \\
ITER & $6 \cdot 10^{8}$ & - & - & \\
\hline \hline
\end{tabular}

Table 1.1: Some important parameters of plasmas in different environments where magnetic reconnection can develop. The symbols are described in Appendix A.1.2. Top: Measured parameters. Bottom: Derived quantities. Adapted from Ji and Daughton (2011), Kivelson and Russell (1995), Boyd and Sanderson (2003). $d_{e}$ is the typical spatial dimension of the diffusion region. In the magnetopause and magnetotail, $T_{i} \sim 10 T_{e}$.

2007). But by means of indirect methods and numerical simulations (relating the motion of photospheric magnetic field with the plasma bulk flows) is possible to estimate, e.g, the reconnected electric field $E_{\text {rec }}$. This quantity, closely related to the reconnection rate, (to be defined precisely in Chapter 4, Eq. (4.2)) quantifies the release of energy via magnetic reconnection. By means of the previously mentioned methods, this (properly normalized) electric field gives estimations of $E_{\text {rec }} /\left(B_{\infty y} V_{A}\right) \sim 0.001-0.2$ (with $V_{A}$ the Alfvén speed calculated on the asymptotic magnetic field $B_{\infty y}$ sustaining the CS. See definition in Appendix A.1.2) in the solar flares (see Cassak and Shay 2011, and references therein). Therefore, a successful model of magnetic reconnection should predict rates on this range (the upper limit is commonly called fast magnetic reconnection). On the other hand, there are also observations of the flows resulting from reconnection in EUV and visible range, as well as the radio and $\mathrm{X}$-ray emission resulting from electron acceleration (Karlický 2014). 


\subsection{The role of current sheets in magnetic reconnection}

As we already mentioned without a proper definition (see Fig. 1.1), current sheets (CS) constitute a basic ingredient for magnetic reconnection observed in the solar corona. Indeed, most of the theories of reconnection in the solar corona predict that it should take place and dissipate energy in these regions with enhanced current density and (by Ampère's law Eq. (2.10)) an associated strong magnetic field shear. CS form when two highly conductive plasmas (see discussion in Eq. (2.49)) with different orientations in their internal magnetic field come together due to an external driver. In the solar corona, this is realized due to the motion of magnetic flux tubes as a result of the moving photospheric plasma underneath (Parker 1994, Büchner 2006).

Magnetic reconnection in CS starts when steeper gradients are formed due to the aforementioned external driver. This produce higher currents, being therefore concentrated in smaller spatial regions (a thin CS) with a small typical length scale $L_{B}$. This length will be reduced more and more making CS thinner and thinner (Schindler 2007) as long as the external driver is active, with a corresponding increase in the total energy stored in this configuration. If in addition there is some other process enhancing the resistivity $\eta$ in that region, the magnetic Reynolds number Eq. (2.46) will be increased (Koskinen 2011). 2 Therefore, due to the resistive Ohm's law Eq. (2.44) or induction equation, the diffusive term in the Ohm's law $\eta \vec{J}$ will gain importance in comparison with the convective one $\vec{V} \times \vec{B}$, leading to a breaking of the frozen-in condition Eq. (2.49) and allowing thus a rearrangement of magnetic field lines. Or in other words, the plasma loses its highly conductive nature: the bulk plasma flow can move independently from the magnetic field lines. As we are going to explain in Chapter 4, this global change in the magnetic field topology transforms the magnetic into particle energy, in the form of bulk flow motions, heating and particle acceleration. For this reason CS are the preferred locations where magnetic reconnection can take place, at least in the context of resistive MHD (and sometimes is taken as part of its definition, according to Koskinen (2011)).

The physical process that can interrupt the continuous thinning of the CS (with the corresponding increase in the total energy stored in the magnetic configuration) is known as tearing instability, leading to CS with typical length scales $L_{B} \sim d_{i}$ at the end of this saturation stage. This instability, to be discussed in 5.1, prepares the onset for the fast release of energy during magnetic reconnection.

In addition, the associated gradients in magnetic field, density, temperature currents, and the electron-ion streaming (necessary to produce the currents), act as additional sources of free energy to drive a large number of macro- and micro- instabilities (see Silin et al. 2002, Silin and Büchner 2003, Silin 2004, and references therein). And because these effects will be enhanced when current sheets are thinner (especially around ion length scales $d_{i}$ ), it is expected that kinetic effects, such as resonant wave-particle interactions, play an important role as physical mechanisms leading to generalized forms of dissipation in collisionless plasmas (see Büchner and Daughton 2007, Galeev and Sudan 1984, and also Sec. 4.2.5). A few of the most important instabilities taking place in thin current sheets will be discussed in Chapter 5 .

\footnotetext{
${ }^{2}$ All the Plasma Phy sics concepts discussed here are discussed in detail in Chapter 2.
} 
a)

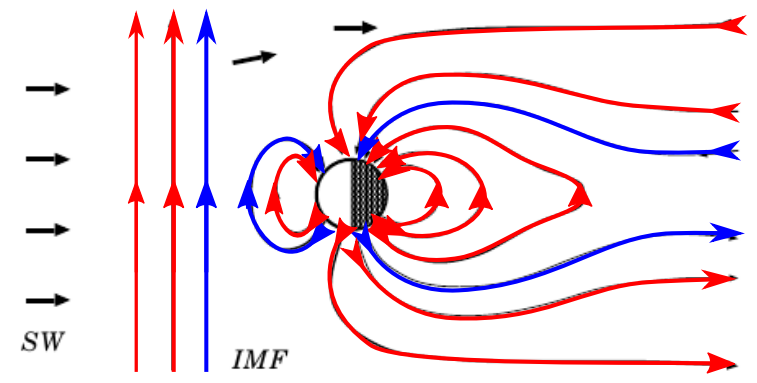

b)

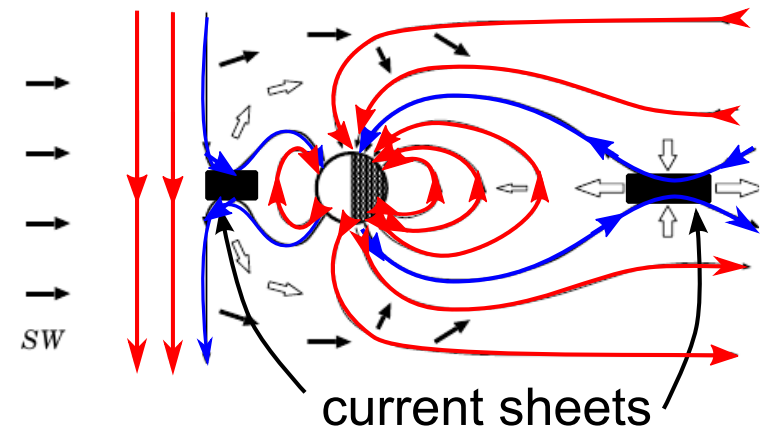

Figure 1.2: Scheme of current sheet formation and reconnection in two possible configurations of the Earth's magnetopause. Top: "Closed" magnetosphere (Northward IMF,Interplanetary Magnetic Field), no magnetic dayside reconnection. $S W$ is the direction of the incoming Solar Wind. Bottom: "open" magnetosphere, dayside and nightside magnetic reconnection is allowed through the formation of current sheets in the indicated locations. Red lines are magnetic field lines not involved in reconnection, while blue lines are the ones forming the current sheets. Adapted from Fig. 8.2 of (Somov 2006)

\subsection{Current sheets and magnetic reconnection in other environments}

Current sheets and magnetic reconnection are also ubiquitous and important in many other space, astrophysical and laboratory plasmas, such as in Earth's magnetosphere (see, e.g., Dungey 1961, Schindler and Birn 1978, Kivelson and Russell 1995, Hesse et al. 2014, and references therein). It can take place through current sheets formed in both magnetopause and magnetotail (see some characteristic parameters in Table 1.1), opening the magnetosphere and leading to the development of magnetic substorms, as can be seen in Fig. 1.2. While in the magnetotail reconnection is usually symmetric, in magnetopause is remarkably asymmetric: different conditions are present in each side of the current sheet. And different from the solar corona, this setting provides an unique opportunity for insitu measurements of magnetic reconnection, such those carried out by, e.g., the Cluster, Themis and the just launched MMS space missions.

Magnetic reconnection has also been proposed to explain the acceleration of the solar wind (see, e.g., Fisk et al. 1999, Büchner and Nikutowski 2005, and references therein). It has been detected recently in the solar wind (Gosling 2011). It is interesting to mention that although the data for its detection was available for many decades ago, there 
were not too many attempts to look at it for signatures of reconnection, because it was thought that should not play an important role in the overall solar wind physics. Now there have been observed even kinetic effects (Xu et al. 2015), and associated turbulence in this environment (Osman et al. 2014, Vörös et al. 2014).

Magnetic reconnection also plays an essential role to explain the huge release of magnetic energy in "extreme" astrophysical environments, such as flares in pulsars' magnetospheres (magnetars), gamma ray bursts (GRB) and accretion disks of AGNs (see Uzdensky and Rightley 2014, Kagan et al. 2015, and references therein). Those environments are called extreme because their (usually pair) plasmas are embedded in intense magnetic fields, besides of being very hot $k_{B} T_{e} \gg m_{e} c^{2}$ and dense (see some characteristic parameters in Table 1.1). This is in the realm of relativistic quantum plasma physics, a theory that only recently started to be developed.

CS instabilities and magnetic reconnection are also of essential importance in laboratory plasmas. This is the case of MRX, "Magnetic Reconnection Experiment", a laboratory at PPPL with the purpose of studying the basic physics of magnetic reconnection, or VINETA II, another (linear) magnetic reconnection experiment in Germany (Bohlin et al. 2014). It is also relevant for fusion plasmas, such as in ITER: "International Thermonuclear Experimental Reactor" a tokamak reactor at France studying the feasibility of practical fusion reaction. See additional details in Yamada et al. (2010), Zweibel and Yamada (2009) and references therein. In fusion devices, magnetic reconnection has to be avoided in order not to destroy the magnetic topology that keeps the hot plasma confined inside of tokamaks, etc.

\subsection{General concept of instability}

This thesis is devoted to the study of CS instabilities. Several specific cases will be analyzed in Chapter 5. For that reason, now we will briefly describe the general concept of plasma instability, in addition to introduce their related terminology.

Physically, an instability is due to the loss of equilibrium of the forces acting in a plasma. Small perturbations will remove the system away from this unstable state. This is in contrast to a stable equilibrium state, where these effects will only make the system oscillate around its equilibrium configuration. Theoretically, linear instabilities are described by a linear analysis of a given plasma model, determining if the plasma is stable against small perturbations. The standard Ansatz is assuming that the solution for a given quantity $A=A(t)$ can be expressed in the frequency domain $\widetilde{A}=A(\omega)$ by means of the Fourier transform 3 in the following way:

$$
\widetilde{A}(\omega)=\frac{1}{\sqrt{2 \pi}} \int_{-\infty}^{\infty} A(t) e^{i \omega t} d t \quad \Rightarrow \quad A(t)=\frac{1}{\sqrt{2 \pi}} \int_{-\infty}^{\infty} \widetilde{A}(\omega) e^{-i \omega t} d \omega
$$

\footnotetext{
${ }^{3}$ This is suitable if the problem can be considered as one of normal modes. But in kinetic theory, when analyzing any effect related with Landau damping (Landau 1946), it is more convenient to analyze the problem as one of initial values. In this case, the Fourier transform has to be replaced by a Fourier-Laplace transform (see, e.g., Baumjohann and Treumann 1997, Sec. 10).
} 
It is often the case that in the solution to a problem the frequency cannot be considered as purely real, but in general a complex number:

$$
\omega=\omega_{r}+i \gamma \quad \text { with } \quad \omega_{r}=\mathfrak{R e}(\omega) \text { and } \quad \gamma=\mathfrak{I m}(\omega)
$$

Plugging this into the solution for $A(t)$ in Eq. 1.1), each normal mode will have the following dependence

$$
\exp (-i \omega t)=\exp (\gamma t) \exp \left(-i \omega_{r} t\right)
$$

This means harmonic oscillations modulated (or with an envelope) by $\exp (\gamma t)$. If $|\gamma| \ll$ $\left|\omega_{r}\right|$, the oscillations will be weakly affected by that term, and we have a normal mode or eigenmode. If $\gamma<0$, the oscillations will decrease with time: the mode is called damped or stable. If $\gamma>0$, the oscillations will grow with time, indicating a linear instability with the associated wave mode is said to be unstable. For this reason $\gamma$ is called growth rate. Of course, an instability cannot grow forever in nature, there is always some mechanism that prevents that, leading to a different configuration of the plasma. This process is known as the saturation of an instability, in many cases bringing the system to a quasi-steady state.

Plasma instabilities can be of different origin: they are always fed by a source of free energy which can be, e.g, relative streaming, inhomogeneities in the density (drift instabilities), magnetic or electric fields, inhomogeneities in the velocities (shear), magnetic tension, wave-wave interactions (parametric instabilities), etc. These cases are called macroinstabilities, since they depend on macroscopic quantities. Instabilities can, however, also arise from a "deformation" of a distribution function $f$ (deviations from the equilibrium Maxwellian $f$ ). In this case, they are called microinstabilities. The physical mechanisms of all instabilities requires free energy. If it is not resupplied (in a closed system), they will deplete it (i.e. reducing the gradients, streamings or thermalizing the distribution function) bringing the system back towards an stable state equilibrium. For further details about plasma instabilities, see the list of textbooks listed at the beginning of Chapter 2, and in particular the textbook by Gary (1993).

\subsection{The importance of kinetic instabilities}

Although some consequences of magnetic reconnection in the solar corona, are visible at very large scales (rearrangement of magnetic fields), the physics behind takes place at very small scales (diffusive or non-ideal process. See Sec. 2.5). Indeed, as we mentioned before, reconnection requires the existence of CS over extended periods of time (e.g., in MHD models of solar flares, Cassak and Shay 2011). In collisionless plasmas they can be properly described only with kinetic theories, beyond fluid models as MHD (see Sec.2.2). Basically, in these plasmas it is necessary to take into account the microphysics of the particle interacting in the plasma (see details in Sec. 2.1). Thus, the aforementioned micro-scale physics is the first main justification for the use of kinetic theories for the analysis of these processes.

The second reason has to do with the fact that the most of the space plasmas are practically collisionless, i.e.: they have such low density and high temperatures that a particle can travel very large distances without experience significant interactions with other ones over long time (see a more detailed discussion in Sec.2.1.3). This can be seen in the high 
value of the Lundquist number $S$ given in Table 1.1, a measure of this behavior. In these environments, the particles can be very far away from the thermodynamic equilibrium, and therefore collective plasma phenomena dominate the global evolution of the system. On the other hand, at the macroscopic level the transport processes governing the evolution of some key quantities as resistivity, diffusion, heat conduction, equation of state, etc , rely on the assumption of thermodynamic equilibrium, equivalent to a local dependence. Then, in the collisionless coronal plasma, these transport processes are controlled mostly by the non-local, long range collective wave-particle interactions, very different from the collision-dominated fluid picture. This has dramatic consequences in many of the phenomena that can be observed, in the Solar corona. As stated in Schwartz et al. (2013): "If we lived in a fluid Universe, there would be no solar flares, no ultra-relativistic cosmic rays, and no Aurora Borealis". For further details about the importance of the kinetic effects in space and astrophysical plasmas, see, e.g., the review of Schwartz et al. (2013) and the book by Balogh et al. (2014).

\subsection{Outline of this thesis}

In the previous sections of this chapter we have motivated the importance of the study of collisionless current sheet instabilities and magnetic reconnection in the solar corona, where a external guide field is an essential ingredient. The aim of this thesis is precisely a proper description of these kinetic processes in this physical system via fully-kinetic Particle-in-Cell (PiC) codes, capable to model accurately this kind of phenomena. We gradually approach to this goal in three result Chapters 7 to 9 .

First, in the result Chapter 7 , we analyze spontaneous instabilities in Harris CS in the limit case of zero guide field. We will see that a proper modeling of the physical CS instabilities require a careful tuning of numerical parameters in a PIC code, in order to distinguish them from instabilities driven by numerical heating that previous works were not taking properly into account. Then, in our second result Chapter $\mathbf{8}$, we switch to more realistic CS configurations by adding a small guide field. Here we explore the dissipative mechanisms allowing the fully developed stage of magnetic reconnection on dependence on guide field, via the analysis of the Generalized Ohm's law, properly averaged in time and space via the techniques explained in Appendix B.1. This is particularly important because many previous studies have not taking into account these averaging processes and their physical implications. Finally, in our last result Chapter 9 we address magnetic reconnection and instabilities in the regime of moderate and large guide field, suitable for the Solar corona. Since PIC simulations in this parameter range can be computationally very expensive, we change our equilibrium to force free CS in addition to compare our results with a gyrokinetic code, valid in the limit of infinite guide field. Our purpose here is trying to clarify to what extent the gyrokinetic simulations can be used to replace the PIC simulation in the realistic limit of finite guide fields. For that is necessary a careful benchmark considering all the assumptions in the gyrokinetic plasma model, and a detailed description of the instabilities and physical processes that can be modeled only by a fully-kinetic PIC code.

However, it is necessary to pass through a long road full of possible pitfalls coming from an inadequate understanding of the physical and/or numerical assumptions of the 
models and algorithms in order to understand properly our results. That is why we are going to explain next the somehow unconventional format of this thesis in the sense of having a very long introduction divided in five additional chapters (including this one, from Chapters 1 to 6 ).

The theoretical study of Plasma Physics relies heavily in a clever set of assumptions and approximations. There are many plasma models suitable to describe some phenomena but they are only valid under some parameter regimes. We are going to briefly describe the ones applied to the processes to be observed in our results in Chapter 2, with special emphasis in the assumptions and approximation where they are valid. Many times these models are applied for cases beyond their validity regime, rendering any conclusion or result based on them invalid. For that reason we start in Sec. 2.1 introducing the velocity distribution function VDF $f$, basis of all kinetic treatments, and then showing how the macroscopic quantities used in fluid models can be derived from it in Sec.2.1.1. Next, we present the basic equation of collisionless plasmas, called Vlasov equation, in Sec. 2.1.4. The assumptions under which it is valid, absence of collisions, are reviewed just before, in Sec. 2.1.3. Then, we go down into the hierarchy of plasma models by introducing the multifluid approaches, based on a proper averaging of the Vlasov equation, and its closures in Sec. 2.2. In particular, the equations and assumptions of two-fluid models are described in Sec. 2.2.1. Then, we eliminate partially distinctions between plasma species by combining them in one-single variable to arrive to Hall-MHD model in Sec. 2.3. This model is essential to understand the physics of collisionless magnetic reconnection that we are going to present in our results. When the Hall term, arising from a decoupling between electrons and ions is neglected, we arrive to resistive MHD in Sec. 2.4. It is often the case that resistivity can be neglected (especially in the collisionless solar corona plasma), in which case we get a reduced system of equations known as ideal MHD, to be described in Sec. 2.5. In that section we also present the Alfvén's theorem (also known as frozenin condition), stating that any change of topology in the magnetic field configuration, a necessary condition for magnetic reconnection, require a mechanism beyond ideal MHD. This condition is nicely summarized in the Generalized Ohm's law, presented for first time in Sec. 2.2.1. Any plasma description beyond ideal MHD contributes with terms in that equation responsible for the violation of Alfvén's theorem, allowing the dissipation necessary for magnetic reconnection. Therefore, it is of essential importance to clearly understand any of the approximations used to get to this point, since when going down into the hierarchy of plasma models we are neglecting effects that can be important to explain the phenomena to be shown in this thesis (starting from the same definition of the of single-particle distribution function, collisions, two fluid effects, etc). In resistive MHD all the possible mechanisms for breaking the frozen-in condition are collapsed into one single parameter, the resistivity, but that turns out to be an oversimplification in many cases. That is the main justification for presenting all these models. Finally, in the last section of this chapter, Sec. 2.6, we present the CGL approximation, an approach useful to model low $\beta$ plasmas with strong guide field such as the one to be shown in our results of Chapter 9 .

The study of CS instabilities requires the specification of initial equilibria. The ones to be used in this thesis will be described in Chapter 3. As explained in Sec. 3.1, there are many possible MHD equilibria but very few kinetic ones (see Sec. 3.2). The most famous is the Harris equilibrium (Sec. 3.2.1) which is also an MHD equilibrium. We are going 
to use this one in our setup to be shown in Chapter 7. The Harris equilibrium also turns out to be unaltered with the addition of a guide field, and that is why we are also going to use for the guide field cases in Chapter 8 . However, in the large guide field limit this equilibrium turns out to be inconvenient for some numerical reasons.Due to this, in our last result Chapter 9 , we switch to a force free equilibrium to be described in Sec. 3.2.2.3. However, this is an MHD equilibrium, approximation to the Harris equilibrium in the strong guide field limit. Since in our results of Chapter 9 we focus in the non-linear or quasy-steady stage of magnetic reconnection, the distinction is not so important. There is an exact kinetic force free equilibrium described Sec. 3.2.2.2, but it does not include the guide field dependence.

In Chapter 4, we review the literature about the fully-developed stage of magnetic reconnection to put in context our work about guide field dependence in Chapter 8 and Chapter 9 . First, we review the generalities of the MHD models of magnetic reconnection in Sec.4.1, and how they are inappropriate to describe this physical process for the parameter regimes used in our studies. Then, in Sec. 4.2, we switch to collisionless magnetic reconnection, more suitable in our case. In particular, we emphasize the importance of Hall effects (Sec.4.2.1) in the sense of that, although not breaking the frozen-in condition of ideal MHD, they allow dispersive waves (Sec. 4.2.2) that can explain the fast reconnection rates observed in our studies in Chapter 9. Then, we explain three collisionless dissipative mechanisms (terms in the Generalized Ohm's law) that can violate the Alfvén theorem in Secs. 4.2.3 to 4.2.5. We are going to identify all of them in our results in Sec. 8.3 and Sec. 8.4 , but only after developing a proper way to calculate time and spatial averages resorting to the techniques explained in Appendix B.1. Finally, in Sec. 4.3 we address what it is known about the guide field effects on magnetic reconnection, in order to compare our results with the theoretical and numerical expectations of two-fluid theory. In particular, in Sec.4.3.1, we describe the effects of a guide field on the symmetries and scaling of some key quantities on the steady-state stage of magnetic reconnection. This is the theoretical framework in which we are going to compare our results in the large guide field limit of Chapter 9 . As we are going to show, any deviation from this two fluid behavior can be attributed to some specific kinetic effects to be found in our results.

Next, in Chapter 5, we briefly describe and analyze the most important collisionless instabilities in CS relevant for our results. First, we review the spontaneous instability leading to magnetic reconnection, the tearing instability, in Sec.5.1. We show its physical MHD mechanism in Sec. 5.1.1 in order to emphasize how different is its kinetic description in Sec.5.1.2. While in the first case its growth rates are controlled by the resistivity, in the kinetic case they depends on the behavior of the particle orbits, and thus on how thin is the CS halfwidth in comparison with the typical length scale $\rho_{i}$. We explain all the approximations used to derive its growth rates (without guide field in Sec. 5.1.2.1 and with guide field in Sec.5.1.3) against which our results will be compared in Chapter 7 and Chapter 8. This will allow to identify precisely the physical origin of the discrepancies. We also describe the saturation mechanisms (without guide field in Sec. 5.1.2.2 and with guide field in Sec. 5.1.3.4), in order to explain what physical process control the coalescence and merging of magnetic islands in our results of Chapter 7 and Chapter 8 . Finally, in Sec. 5.1.4, we review the tearing mode theory extended for temperature anisotropies, since this will appear due to purely numerical reasons in our simulations of Chapter 7 , allowing us thus to explain its consequences in our system. This is especially relevant for 
the bifurcation induced in CS (indirectly) due to this effect (see Sec. 5.1.4.1).

In Chapter 5 we also reviewed some other instabilities consequence of magnetic reconnection to be found in our results. In Sec.5.2 we explain the theoretical expectations about the instabilities driven by temperature ani sotropy. The most important features of Weibel and mirror instabilities are explained in Sec. 5.2.1 and Sec. 5.2.2, allowing us to justify its appearance in our simulations of Harris CS when an initial temperature anisotropy is imposed (see Sec. 7.3). We emphasize the conditions and approximations under which they are expected, since these instabilities are not commonly seen in Harris CS. The theory about Weibel instability will also be useful to understand its behavior when a guide field is present (see Sec. 8.3). In Sec. 5.2.3 and Sec. 5.2.4 we also show the reasons because other two related instabilities driven by temperature anisotropy are ruled out in our simulations.

In the last part of Chapter 5 we discuss two family of instabilities driven by bulk flows that will appear in our simulations in the large guide field limit in Chapter 9 . The first ones explained in Sec. 5.3 are driven by the shear flow, initially present in the our simulation setup. They are very related with the classical MHD Kelvin-Helmholtz instability to be explained in Sec. 5.3.1. The kinetic version of this instability is explained in Sec. 5.3.2 in order to have the arguments to claim its presence under the conditions present in our simulations. We also see a magnetic field generation in our simulations with large guide field and initial shear flow, and that is why we describe in Sec. 5.3.3 a similar mechanism observed in simulations for a different parameter range (in order to gain physical insight). The consequences of the shear flow in reconnection are reviewed in Sec.5.3.4 and Sec.5.3.5, allowing to understand quantitatively its effects in our results for the specified parameter range. Finally, in Sec. 5.4 we describe instabilities driven by relative streaming between electrons and ions. The ones relevant for the results shown in Chapter 9 are cross-field, i.e: with propagation perpendicular to the dominant out-of-plane guide magnetic field and driven by in-plane streamings. They are analyzed in Sec. 5.4.2. We explained the high frequency cross-field instability in Sec. 5.4.2.1 that will be ruled out, and another similar low frequency instability (modified-two stream instability MTSI) in Sec.5.4.2.2. In that section, as well as in Sec. 5.4.2.3, we describe its most important features that will support our conclusions in identifying the behavior seen in our simulations as due to that instability. In Sec.5.4.2.4 we describe the lower hybrid drift instability (LHDI). Although it is technically a instability driven by pressure gradients, it can also be interpreted as driven by streaming originated from diamagnetic drifts. All the evidence to be found in our results as due to that instability is explained in the mentioned section as well as in Sec.5.4.2.5. All these cross-field instabilities turn out to be important to explain the differences between our PIC simulations results shown in Chapter 9 and the results given by gyrokinetic simulations, since these last ones have restrictions on the magnitude on the allowed cross-field streaming speeds.

Finally, the last introductory Chapter $\mathbf{6}$ is devoted to the main numerical tool used to obtain the results shown in this thesis: fully kinetic PiC codes, with an emphasis on the methods used by the ACRONYM code. Starting from some generalities of the PIC method in Sec. 6.1, we then explain in Sec. 6.2 the consequences of coarse-graining the phase space via the introduction of the shape function, used in the interpolation scheme between the grid and the macroparticles. This often overlooked numerical parameter will turn out to be of essential importance in avoiding numerical artifacts in the PIC sim- 
ulations shown in Chapter 7. Then, we describe different parts of the PIC algorithms in Secs. 6.4 to 6.7 to finally discuss the most important numerical effects that we will be facing in Sec. 6.8. First, we analyze the stability conditions constraining our choice of parameters in the PIC simulations to grid cell sizes and time steps on order of electron scales, implying the additional requirement of artificial reduced mass ratio and other similar numerical parameters, in order to make the simulations computationally less demanding. Then, in Sec. 6.8.2 we describe two mechanisms of numerical heating, one of them related with the non-fulfillment of the stability condition (Sec.6.8.2.1) and the other one due to numerical scattering (Sec.6.8.2.2). The latter one will show up spontaneously and unexpectedly in our simulations of Chapter $\square$, since so far there were no enough studies about this numerical effect and parameter regimes where it should be operating. This mechanism of numerical heating is closely related with the numerical collisions inherent to all PIC codes, a side-effect of the coarse-graining the phase space. That is why we discuss this process in Sec. 6.8.3, in particular because it can mimic real collisional phenomena in a PIC plasma, deviating from the predictions of a real collisionless Vlasov plasma. These collisions can be measured through the entropy to be discussed in Sec.6.8.4, a concept from statistical mechanics that requires a careful adaptation to be applied to the non equilibrium phenomena driven by both numerical collisional and physical collisionless processes taking place in a PIC plasma, characterized by a coarsegrained distribution function. Finally, in Sec.6.10 we describe briefly all the PIC methods implemented specifically in the ACRONYM code.

Only after all these introductory chapters we can get a deeper understanding of the meaning of our results, shown in Chapters 7 to 9 , in the more general context of kinetic plasma physics. Finally, we summarize our findings in the conclusion Chapter 10 , also with a brief outlook about possible extensions of our work. 


\section{Plasma physics background: an overview of plasma models (used in this thesis)}

A plasma is sometimes called the fourth state of matter. It is basically an ionized gas whose particles exhibit a collective behaviour. Its basic interaction is through electromagnetic fields. Although not commonly seen on the Earth's surface, more than the $99 \%$ of the visible (baryonic) matter in the Universe it is in plasma state, in particular the space environment in our solar system (the Sun, solar wind and the planetary magnetospheres). This implies the paramount importance of an adequate study of plasmas. In this section we will briefly describe some of the main plasma models used in this thesis, focusing on the kinetic approach and some of their approximations. A more complete discussion can be found in the general plasma physics textbooks (in decreasing order of use for the purpose of this thesis): Baumiohann and Treumann (1997), Treumann (2001), Krall and Trivelpiece (1973), Bellan (2006), Somov (2013), Parks (2004), Koskinen (2011), Cravens (1997), Boyd and Sanderson (2003), Bittencourt (2004).

Note that all the symbols and definitions to be used from now on are listed in Appendix A.1.2.

\subsection{Kinetic theory}

A basic model of a collisionless plasma is the one-particle kinetic theory. The foundations of kinetic theory are based in the idea of an efficient approach to describe the position $\vec{x}$ and velocity $\vec{v}$ of the large number $N$ of particles (electrons, protons, and possibly other heavy ions) that compose a plasma, without having to follow the details of the motion of each one of them. In the standard kinetic theory, the solution is to introduce the phase space distribution function $f_{\alpha}=f_{\alpha}(\vec{x}, \vec{v}, t)$ for each plasma specie $\alpha$, defined as:

$$
f_{\alpha}(\vec{x}, \vec{v}, t)=\frac{d N_{\alpha}}{d x^{3} d v^{3}}
$$

i.e.: the number of particles $d N_{\alpha}$ per infinitesimal element of the $6 \mathrm{D}$ phase space: the probability of finding a particle in $d v^{3} d x^{3}=d v_{x} d v_{y} d v_{z} d x d y d z$ around the point $(\vec{x}, \vec{v})$ at the time $t$. But instead of using the number of particles $N_{\alpha}$, it is more convenient to use the number density $d n_{\alpha}=d N_{\alpha} / d x^{3}$ and the commonly used velocity distribution function 
VDF, defined as:

$$
f_{\alpha}^{V}(\vec{x}, \vec{v}, t)=\frac{d n_{\alpha}}{d v^{3}}
$$

From now on, we will drop the superscript $V$ from this quantity.

\subsubsection{Momenta of the VDF}

By integrating Eq. 2.2) in the velocity space $\vec{v}$ with an appropriate weight, we get the momenta of the VDF (Baumjohann and Treumann 1997): the macroscopic quantities described by fluid models. The zero order moment is obtained by integrating directly:

$$
n_{\alpha}(\vec{x}, t)=\int_{-\infty}^{\infty} f_{\alpha}(\vec{x}, \vec{v}, t) d v^{3}
$$

The integral is taken over all the velocity space $d v^{3}$. The first order moment, the bulk (or mean) flow velocity, is obtained by weighting with the velocity

$$
V_{\alpha, i}(\vec{x}, t)=\frac{1}{n_{\alpha}(\vec{x}, t)} \int_{-\infty}^{\infty} \vec{v} f_{\alpha}(\vec{x}, \vec{v}, t) d v^{3}
$$

with the index $i=x, y, z$ indicates the spatial component. The second order moment can be identified as the pressure tensor

$$
P_{\alpha, i j}(\vec{x}, t)=m \int_{-\infty}^{\infty}\left(v_{i}-V_{\alpha, i}(\vec{x}, t)\right)\left(v_{j}-V_{\alpha, j}(\vec{x}, t)\right) f(\vec{x}, \vec{v}, t) d v^{3}
$$

where $i$ and $j$ are the spatial indices of this $3 \times 3$ tensor. This quantity measures the fluctuations of the velocities with respect to their mean value, being thus proportional to the standard deviation in the statistic terminology. We can recover some thermodynamics concepts by identifying the diagonal elements of this tensor pressure via the ideal gas relation $p_{i}=n_{i} k_{B} T_{i}$, where $T_{i}$ is the kinetic temperature:

$$
T_{i}(\vec{x}, t)=\frac{p_{i}(\vec{x}, t)}{k_{B} n(\vec{x}, t)}=\frac{m}{k_{B} n(\vec{x}, t)} \int_{-\infty}^{\infty}\left(v_{i}-V_{i}(\vec{x}, t)\right)^{2} f(\vec{x}, \vec{v}, t) d v^{3}
$$

This kinetic temperature is different from the thermodynamic one, since it can be applied for any kind of VDF, for situations very far away from the thermodynamic equilibrium. One can recover the usual (scalar) temperature $T$ and pressure $P$ by averaging the three components of the kinetic temperature: $T=\sum_{\alpha} T_{j} / 3$ and $P=n k_{B} T$. Both definitions are equal when the VDF is a Maxwellian one (see Eq. (2.17)), since it represents a state of thermodynamic equilibrium 


\subsubsection{Maxwell equations and single particle description}

Since a plasma is a gas of ionized particles, the basic force describing their interaction is from electromagnetic nature, with the electromagnetic fields $\vec{E}$ and $\vec{B}$ given by the (in vacuo) Maxwell equations:

$$
\begin{aligned}
\nabla \cdot \vec{E}(\vec{x}, t) & =\frac{\rho(\vec{x}, t)}{\epsilon_{0}} \\
\nabla \cdot \vec{B}(\vec{x}, t) & =0 \\
\nabla \times \vec{E}(\vec{x}, t) & =-\frac{\partial \vec{B}(\vec{x}, t)}{\partial t} \\
\nabla \times \vec{B}(\vec{x}, t) & =\mu_{0} \vec{J}(\vec{x}, t)+\mu_{0} \epsilon_{0} \frac{\partial \vec{E}(\vec{x}, t)}{\partial t}
\end{aligned}
$$

The Lorentz force on each single charged particle of the plasma is given by:

$$
\vec{F}_{L}=m_{\alpha} \frac{d \vec{v}}{d t}=q[\vec{E}(\vec{x}, t)+\vec{v} \times \vec{B}(\vec{x}, t)]
$$

However, as we mentioned previously, is more convenient to use the $\operatorname{VDF} f(\vec{x}, \vec{v}, t)$ instead of a single particle description of plasma, and thus Eq. (2.11) lose their importance for modeling its characteristic collective behaviour. In order to obtain an equation describing the evolution of $f(\vec{x}, \vec{v}, t)$, first is necessary to clarify the concept of collision between charged particles in a plasma.

\subsubsection{What is a collisional in a plasma? The kinetic Boltzmann equa- tion}

The precise definition of "collision" in a plasma is quite involved. This is because the plasma particles do not experience binary collision like in a neutral gas, but long range electromagnetic forces with an inverse dependence on the inter-particle distance. In order to understand better this key concept, first it is necessary to introduce some definitions.

The smallest characteristic and typical length scale of a plasma is called the Debye length, being defined by:

$$
\lambda_{D e}=\sqrt{\frac{\epsilon_{0} k_{B} T_{e}}{n_{e} e^{2}}}
$$

for length scales below $\lambda_{D e}$, the electric fields are effectively shielded and quasineutrality holds. Now, if there are enough particles in a sphere of radius $\lambda_{D e}$, i.e.:

$$
\Lambda=n_{e} \lambda_{D e}^{3} \gg 1
$$

where $\Lambda$ is called the plasma parameter, the plasma will be dominated by collective interactions instead of collisions. This is can be intuitively understood due to the fact that the more particles that are together, the smoother will be the deflections due to long range 
electromagnetic scattering that the particles will experience 1 . The plasmas where the collective behavior is dominant $\Lambda \gg 1$ are called collisionless, characterized by a very unique and fascinating behavior: they can display large deviations from thermodynamic equilibrium for long times, due to the long range and therefore non-local electromagnetic interactions. On the other hand, when the plasma parameter $\Lambda$ is small, the plasma is called collisional, and any deviation from thermodynamic equilibrium is quickly attenuated: the plasma behaves like a fluid, with exclusively local interactions. The MHD plasma model (to be described in Sec.2.2) is based on this assumption of thermodynamic equilibrium due to the dominant collisions. However, this behavior is far from being accurate to describe the mostly collisionless plasmas in the solar corona, as well as in other space environment, turning the foundations of its applicability invalid: a kinetic treatment is essential in these cases (although many times MHD can still give relatively good predictions for large scales).

On the other hand, it can be shown that the effective electron-ion collision frequency $v_{c}$, a measure of the classical cross-section of scattering as for neutral gases, it is proportional to the logarithm of the plasma parameter (called Coulomb logarithm):

$$
v_{c} \propto \frac{n_{i} \ln (\Lambda)}{T_{e}^{3 / 2}}
$$

and thus the electron mean free path $\lambda_{c}$, a measure of how long a particle can travel in the plasma without significant deviations in its trajectory by the surrounding electromagnetic field, is given by:

$$
\lambda_{c}=\frac{v_{t h, e}}{v_{c}} \propto \frac{T_{e}^{3 / 2}}{n_{i} \ln (\Lambda)}
$$

The mean free path is small in collision dominated plasmas, such as in MHD, while it can be very large in collisionless plasmas such as those of the solar corona, implying that a kinetic treatment is essential to understand their properties. For further details, see Boyd and Sanderson (2003, Sec. 1.4.1), Krall and Trivelpiece (1973, Chap. 6) and Somov (2013, Chap. 8).

Under very general conditions in the frame of one-particle kinetic theory, it is possible to show (see, e.g. Baumjohann and Treumann (1997, Chap. 7), Stix (1992, Sec. 8.7), or Bittencourt (2004, Chap. 21)) that the single-particle distribution function $f$ satisfies the Boltzmann or kinetic equation:

$$
\left[\frac{\partial}{\partial t}+\vec{v} \cdot \frac{\partial}{\partial \vec{x}}+\frac{q_{\alpha}}{m_{\alpha}}(\vec{E}(\vec{x}, t)+\vec{v} \times \vec{B}(\vec{x}, t)) \cdot \frac{\partial}{\partial \vec{v}}\right] f_{\alpha}(\vec{x}, \vec{v}, t)=\left.C \frac{\partial f_{\alpha}}{\partial t}\right|_{c}
$$

Note that the electromagnetic fields in the left hand side are macroscopic, in the sense of not including micro-scale fields due to particle collisions (Cravens 1997). The term in the right hand side is proportional to the inter-particle collisions, involving higher order

\footnotetext{
${ }^{1}$ This fact has a critical consequence when a plasma is simulated with Particle-in-Cell codes. Due to computational constraints, the amount of computational particles is always less than in a real plasma, implying that the collisional effects will be greatly enhanced. This will be described in Sec. 6.8.3 and one chapter of this thesis is devoted partially to study the numerical collisional effects on CS instabilities (Chapter 7$)$
} 
correlations of the distribution function. Usual explicit expressions for this term are the Krook or Fokker-Planck collision models. In summary, Eq. 2.16) states that the total derivative of $f$ evolves according to the time rate of its change due to the collisions.

Finally, it is interesting to mention that the asymptotic solution (for long times) of the Boltzmann equation Eq. (2.16) (together with the Maxwell equations) always converge to an unique distribution function: a drifting Maxwellian VDF:

$$
f_{\alpha}(\vec{v})=n_{0 j}\left(\frac{m}{2 \pi k_{B} T_{j}}\right)^{3 / 2} \exp \left(-\frac{m_{\alpha}\left(\vec{v}-\vec{U}_{j}\right)^{2}}{2 k_{B} T_{j}}\right)
$$

characterizing thus the thermodynamic equilibrium.

\subsubsection{Collisionless approach and closure: Vlasov-Maxwell system}

Under the assumption of a collisionless plasma, suitable for the low $\beta$ plasmas found, e.g., in the solar corona, the right hand side of the kinetic Boltzmann equation vanishes. Thus, the equation describing the evolution of $f$ on dependence on the electromagnetic fields is the so called Vlasov equation (Vlasov 1938): 2

$$
\left[\frac{\partial}{\partial t}+\vec{v} \cdot \frac{\partial}{\partial \vec{x}}+\frac{q_{\alpha}}{m_{\alpha}}(\vec{E}(\vec{x}, t)+\vec{v} \times \vec{B}(\vec{x}, t)) \cdot \frac{\partial}{\partial \vec{v}}\right] f_{\alpha}(\vec{x}, \vec{v}, t)=0
$$

When we introduced the distribution function instead of a single particle description, the sources of the Maxwell equation, the charge $\rho(\vec{x}, t)$ and current density $\vec{J}(\vec{x}, t)$ can be obtained from the momenta of order 0 Eq. (2.3) and 1 Eq. (2.4) of the VDF, adding them up over all the plasma species.

$$
\begin{aligned}
\rho_{c}(\vec{x}, t) & =\sum_{\alpha} q_{\alpha} \int d v^{3} f_{\alpha}(\vec{x}, \vec{v}, t) \\
\vec{J}(\vec{x}, t) & =\sum_{\alpha} q_{\alpha} \int d v^{3} \vec{v} f_{\alpha}(\vec{x}, \vec{v}, t)=\sum_{\alpha} q_{\alpha} n_{\alpha}(\vec{x}, t) V_{\alpha}(\vec{x}, t)
\end{aligned}
$$

The set of the previous Vlasov and Maxwell equations, plus the previous sources $\rho$ and $J$ as function of the VDF, constitute a complete set of non-linear integro-differential equations in the variables $\vec{E}, \vec{B}$ and $f$, with very few known analytical solutions.

${ }^{2}$ This equation states the conservation of $f$ along the orbit followed by an element in the phase space $(\vec{x}, \vec{v})$, or in short conservation of phase space volume :

$$
\frac{d f(t, \vec{x}, \vec{v})}{d t}=\frac{\partial f_{\alpha}}{\partial t}+\vec{v} \cdot \frac{\partial f_{\alpha}}{\partial \vec{x}}+\vec{a} \cdot \frac{\partial f_{\alpha}}{\partial \vec{v}}=0
$$

with $\vec{v}=d \vec{x} / d t, \vec{a}=d \vec{v} / d t$, and the acceleration $\vec{a}=\vec{F}_{L} / m$ given by the Lorentz force Eq. (2.11). As a consequence, we have the Liouville's theorem: any stationary $(\partial / \partial \rightarrow 0)$ exact solution of the Vlasov-Maxwell system $f_{\alpha}$ is a function of the constant of motions along the non-perturbed particle orbits. Then, solving Vlasov equation can be reduced to find these constants of motion via a Hamiltonian approach. Note that the same non-perturbed particle orbits are used to find linearized solutions of the Vlasov-Maxwell system via the methods of characteristics. For details, see e.g., Swanson (2003, Sec. 4.3) or Krall and Trivelpiece (1973, Sec. 8.10). 


\subsection{Multi-fluid models}

Often the solutions of the Vlasov equation provide more information than the necessary to describe reliably a plasma: it more than enough just to have a few macroscopic quantities. By integrating the Vlasov equation in the velocity space with an appropriate weight, it is possible to derive equations describing the evolution of macroscopic measurable quantities, such as density, velocity, temperatures, heat flux, kurtosis and so on. This standard procedure is well explained in most of the aforementioned textbooks (e.g.: Baumjohann and Treumann 1997, Chap. 8).

Thus, the moment of order zero is obtained integrating the Vlasov equation in the velocity space (symbolically: $\int f_{\alpha} d v^{3}$ Eq. (2.19)), resulting in

$$
\frac{\partial n_{\alpha}}{\partial t}+\frac{\partial\left(n_{\alpha} V_{\alpha, j}\right)}{\partial x_{j}}=0
$$

corresponding to the continuity equation (conservation of mass).

The moment of order one is obtained multiplying by $\vec{v}$ and then integrating the Vlasov equation in the velocity space (symbolically: $\int f_{\alpha} \vec{v} d v^{3}$ Eq. (2.19)), resulting in

$$
m_{\alpha} n_{\alpha} \frac{d V_{\alpha, i}}{d t}=m_{\alpha} n_{\alpha}\left(\frac{\partial V_{\alpha, i}}{\partial t}+V_{\alpha, j} \frac{V_{\alpha, i}}{\partial x_{j}}\right)=q_{\alpha} n_{\alpha} E_{i}+\varepsilon_{i j k} J_{\alpha, j} B_{k}-\frac{\partial P_{\alpha, i j}}{\partial x_{j}}
$$

where $J_{\alpha, j}=q_{\alpha} n_{\alpha} V_{\alpha, i}$ and $d / d t=\partial / \partial t+V_{\alpha, j} \partial / \partial x_{j}$ is the advective derivative (also known as convective, Lagrangian or comoving derivative). This is the momentum equation. Note that the moment of order zero has one unknown quantity: $V_{\alpha, j}$, with their evolution given by the next moment of order one. But in the latter, it appears a new unknown, the tensor pressure $P_{\alpha, i j}$. Their evolution is given by the moment of order two, obtained multiplying the Vlasov equation by the tensor product $v_{i} v_{j}$ and integrating in the velocity space (symbolically: $\int f_{\alpha} v_{i} v_{j} d v^{3}$ Eq. (2.19)):

$$
\frac{d P_{\alpha, i j}}{d t}+\frac{\partial Q_{\alpha, i j k}}{\partial x_{k}}+P_{i j} \frac{\partial V_{\alpha, k}}{\partial x_{k}}+P_{j k} \frac{\partial V_{\alpha, i}}{\partial x_{k}}+P_{k i} \frac{\partial V_{\alpha, j}}{\partial x_{k}}-\frac{q_{\alpha} B_{k}}{m_{\alpha}}\left[\varepsilon_{i l k} P_{j l}+\varepsilon_{j l m} P_{l i}\right]=0
$$

(written in the form by Walker (2005, Eq. 5.28) or Schindler (2007, Eq. 3.44)) In Eq. (2.24) 3 appears a new unknown, the heat tensor $Q_{i j k}$. We can already notice the trend: the next higher order moment is always necessary to have the full solution of the previous one. This is an infinity hierarchy, called BBGKY (after Bogoliubov, Born, Green, Kirkwood, Yvon. See Baumjohann and Treumann (1997), Krall and Trivelpiece (1973)). For having practical solutions, it is necessary to close the system by assuming a suitable Ansatz, such as an equation of state, on dependence on the physical properties of the system to be described. The ones that will be used in this thesis will be given next.

\footnotetext{
${ }^{3}$ This expression is not often used in this very general form, but instead as an energy evolution equation by assuming isotropic pressure. We will not give that equation here because it will not be used. See any of the textbooks listed at the beginning of this section for further details.
} 


\subsubsection{Two-fluid theory and Generalized Ohm's law}

In the plasmas to be analyzed in this thesis we consider two species: electrons $e$ and ions $i$. The corresponding set of equations for both species Eq. (2.22) and Eq. (2.23), with the Maxwell equations and a suitable closure for the pressure tensor will form the so called two-fluid equations. It is possible then to obtain a per species Ohm's law (Johnson 2011) by solving Eq. (2.23) for the electric field. For the case of electrons, we get:

$$
E_{i}+\underbrace{\varepsilon_{i j k} V_{e, j} B_{k}}_{\text {convective/ideal }}=-\underbrace{\frac{1}{e n_{e}} \frac{\partial P_{e, i j}}{\partial x_{j}}}_{\text {pressure/ambipolar/polarization }}-\underbrace{\frac{m_{e}}{e} \frac{d V_{e, i}}{d t}}_{\text {inertia }}
$$

Below each term is indicated their standard name by which they are called in the context of magnetic reconnection theories. In particular, the convective electric field,

$$
\vec{E}_{\text {convective }}=-\vec{V}_{e} \times \vec{B}
$$

represents the electric field produced by the electron flow motion: it vanishes in the electron frame of reference $\vec{E}^{\prime}=\vec{E}_{\text {convective }}+\vec{V}_{e} \times \vec{B}=0$. Note that this change of reference frame only affects the components of $\vec{E}$ perpendicular to the flow motion, while the components along $\vec{V}_{e}$ do not change. It is also interesting to mention that from the basic theory of electrodynamics, it is not possible to find a frame of reference where $\vec{E}=0$ if $\vec{E} \cdot \vec{B} \neq 0$ or $c^{2} B^{2}>E^{2}$ (a condition rarely satisfied in magnetized plasmas, where the magnetic energy is dominant).

The two fluid Ohm's law Eq. (2.25) reveals the electric field necessary to keep the plasma quasineutral (Cravens 1997). Note that changing to a reference frame comoving with electrons, the electric field will be equal to the pressure and inertia term.

For application to 2D magnetic reconnection, it is al so useful to give the explicit $z$ component (the out-of-plane direction in our standard geometry. See Appendix A.2) of the previous Eq. (2.25):

$$
E_{z}+V_{e, x} B_{y}-V_{e, y} B_{x}=-\frac{1}{e n_{e}}\left(\frac{\partial P_{e, x z}}{\partial x}-\frac{\partial P_{e, y z}}{\partial y}\right)-\frac{m_{e}}{e}\left(\frac{\partial V_{e, z}}{\partial t}+V_{e, x} \frac{\partial V_{e, z}}{\partial x}+V_{e, y} \frac{\partial V_{e, z}}{\partial y}\right)
$$

\subsubsection{One fluid variables}

MHD (Magnetohydrodynamics) is one of the most used plasma models of Plasma Physics, especially suitable for large scale models. It does not distinguish between plasma species: it assumes a single fluid component moving with the center of mass (very close to the motion of the heaviest specie, the ions for an electron-proton plasma). The single 
fluid variables (without subscript) are defined as follows:

$$
\begin{aligned}
n & =\frac{\sum_{\alpha} m_{\alpha} n_{\alpha}}{\sum_{\alpha} m_{\alpha}} \\
\rho_{m} & =\sum_{\alpha} n_{\alpha} m_{\alpha} \approx n_{e} m_{i} \\
\rho_{c} & =\sum_{\alpha} q_{\alpha} m_{\alpha} \\
\vec{V} & =\frac{\sum_{\alpha} m_{\alpha} n_{\alpha} \vec{V}_{\alpha}}{\sum_{\alpha} n_{\alpha} m_{\alpha}} \approx \vec{V}_{i} \\
\vec{J} & =\sum_{\alpha} n_{\alpha} q_{\alpha} J_{\alpha} \\
P & =\sum_{\alpha} p_{\alpha}, \quad \text { with } p_{\alpha}=P_{\alpha, j j} / 3, \quad \text { assuming } P_{\alpha, i j}=p_{\alpha} \delta_{i j}
\end{aligned}
$$

where the approximations in the right hand side of the equations have been done considering and electron-proton plasma and $m_{e} \ll m_{i}$. Now, assuming the following approximations (Boyd and Sanderson 2003):

- $m_{e} \ll m_{i}$ (relevant for simulations with artificially reduced mass ratio)

- Quasineutrality $\left|\omega \Omega_{c e} / \omega_{p e}\right|^{2} \ll 1$ or $n_{i} \approx n_{e}$ (from the Gauss law). Note that this implies strictly zero net charge, and therefore it is not possible to determine the electric field $\vec{E}$ from Eq. (2.7). That is why the electron momentum equation can be used to obtain the electric field, as discussed below in the context of the Ohm's law.

- Strong collisions: $v_{c}^{-1} \ll \sqrt{m_{e} / m_{i}} \tau$, with $v_{c}$ the collision frequency $\tau$ the typical time scales.

- Non relativistic: $\omega / k \sim L / \tau \sim V \ll c$. Neglect displacement current in Ampère's law Eq. (2.10).

- Large length scales $L \gg \rho_{i}, \lambda_{c}=v_{t h, e} v_{c}^{-1}$, with $\lambda_{c}$ the mean free path.

- Slow time scales $\omega<\Omega_{c i}$.

it is possible to combine the per-specie momenta of Vlasov equation (adding the perspecies contributions with a given weight) and get the MHD equations for continuity (conservation of mass), conservation of mass

$$
\begin{aligned}
& \frac{\partial n}{\partial t}+\frac{\partial\left(n V_{\alpha}\right)}{\partial x_{j}}=0 \\
& m n \frac{d V_{i}}{d t}=m n\left(\frac{\partial V_{i}}{\partial t}+V_{\alpha} \frac{V_{i}}{\partial x_{j}}\right)=\rho E_{i}^{\prime}+\underbrace{\varepsilon_{i j k} J_{\alpha} B_{k}}_{\text {Lorentz force }} \underbrace{-\frac{\partial P}{\partial x_{i}}}_{\text {Pressure force }}
\end{aligned}
$$

Neglecting the Lorentz force, the latter is nothing else than the Navier-Stokes of hydrodynamics (HD). Note that we also need an equation of state, where the most common 
approach is assuming an ideal gas law $P=n k_{B} T$ and then relating $P$ with either the density or temperature (in order to reduce the unknown variables from 3 to 2 ). Two commonly used are the adiabatic (negligible heat flux):

$$
P=P_{0}\left(\frac{n}{n_{0}}\right)^{\gamma}
$$

where $\gamma$ is the adiabatic or polytropic index (5/3 for a 3D plasma). This is valid for $v_{t h} \ll L / T$ (Bellan 2006), where $L$ and $T$ are typical length and time scales. We also have the isothermal for $\gamma=1$

$$
P=n k_{B} T_{0}
$$

valid for $v_{t h} \gg L / T$ (dominant heat flux). These equations can also be applied for the respective per-specie pressures in the framework of multifluid theory.

\subsubsection{One-fluid Generalized Ohm's law}

The multifluid momentum Eq. (2.23) was derived in the framework of collisionless Vlasov theory. But a single fluid theory assumes thermodynamic equilibrium (i.e.: Maxwellian distribution functions), and for that it is required a process capable to bring the plasma to that state: collisions. This adds a friction or drag term proportional to the current (and resistivity $\eta$ )

$$
\vec{R}=\eta n e \vec{J}
$$

to the right hand side of the equation, representing phenomenologically a momentum transfer between electrons and ions (and consequently, with opposite sign in each equation). Here, the resistivity is defined in terms of the collision frequency $v_{c}$ as follows:

$$
\eta=\frac{m_{e} v_{c}}{n e^{2}}
$$

As we will see, this term can couple the current $\vec{J}$ (an unknown in the system Eq. 2.34)Eq. (2.35)) with the electromagnetic field. In fact, subtracting the momentum equations for species Eq. (2.23) with a given weight, one can derive the following equation (Johnson 2011):

$$
\begin{aligned}
& E_{i}+\epsilon_{i j k}\left(V_{j}-\frac{m_{i}-m_{e}}{e n m} J_{j}\right) B_{k}=\eta J_{i}+\frac{1}{e n m} \frac{\partial\left(m_{e} P_{i, i j}-m_{i} P_{e, i j}\right)}{\partial x_{j}} \\
& +\frac{m_{i} m_{e}}{e^{2} n m}\left[\frac{\partial J_{i}}{\partial t}+\frac{\partial\left(v_{i} J_{j}+v_{j} J_{i}-\frac{m_{i}-m_{e}}{e n m} J_{i} J_{j}\right)}{\partial x_{j}}\right]
\end{aligned}
$$

Now, assuming the aforementioned MHD approximations (in particular quasineutrality), it is possible to neglect most of the terms in the right hand side (e.g. the derivatives of the second order terms in velocity) and get the equation known as one-fluid generalized Ohm's law:

$$
E_{i}+\underbrace{\epsilon_{i j k} V_{j} B_{k}}_{\text {ideal/convective }}=\underbrace{\eta J_{i}}_{\text {resistive }}+\underbrace{\frac{1}{e n} \epsilon_{i j k} J_{j} B_{k}}_{\text {Hall }}-\underbrace{\frac{1}{e n} \frac{\partial P_{e}}{\partial x_{i}}}_{\text {pressure/ambipolar }}-\underbrace{\frac{m_{e}}{e^{2} n} \frac{d J_{i}}{d t}}_{\text {inertia }}
$$

This (and in general, any other version of) generalized Ohm's law gives the evolution of the current $\vec{J}$ in terms of the electric field $\vec{E}$. 


\subsection{Hall-MHD}

Comparing Eq. (2.41) with the two-fluid (electron) Ohm's law Eq. (2.25), we can easily identify each term with their respective approximation, excepting two additional ones only appearing Eq. (2.41): the resistive and the Hall term. The first one can be understood since no collisions were assumed in Eq. (2.25). On the other hand, the Hall term appears if we replace the MHD approximation for velocity Eq. (2.31) into Eq. (2.32) for an electron-proton plasma, we get

$$
\vec{V}_{e}=\vec{V}-\frac{\vec{J}}{e n}
$$

relating thus the electron velocity $\vec{V}_{e}$ (a two-fluid variable) with the MHD variables $\vec{V}$ and $\vec{J}$. Assuming negligible electron mass, this is precisely the second term in the left hand side of Eq. (2.40). Thus, the Hall term $\vec{J} \times \vec{B}$, associated with the second term in Eq. (2.42), arises from a differential motion between the bulk velocity and electron flows. This Hall term is especially important for current sheets where there are transverse currents to the magnetic field. Now, we can neglect the remaining pressure and inertia terms in the right hand side of Eq. 2.41) (balancing the terms) under the following conditions (Baumjohann and Treumann 1997, Boyd and Sanderson 2003):

1. $\frac{\rho_{e}}{L} \ll \frac{V}{v_{\text {th }, e}}$ or $\frac{\rho_{i}}{L} \ll \frac{V}{c_{s}}$ (from the ratio between pressure and convective terms). $L$ and $V$ are typical length and velocity scales. This is equivalent to the small Larmor radius approximation. It allows to neglect the pressure term. It can be well satisfied for cold plasmas and also for $\beta \ll 1$.

2. $\omega \ll v_{c}$ (from the ratio of inertia to resistive terms) It allows to neglect the inertia term.

3. $\omega \ll \Omega_{c e}$ (from the ratio of inertia to Hall terms) It allows to neglect the inertia term. This is easily satisfied in most cases.

Thus, we get the Hall-MHD Ohm's law:

$$
\vec{E}+\underbrace{\vec{V} \times \vec{B}}_{\text {ideal/convective }}=\underbrace{\eta \vec{J}}_{\text {resistive }}+\underbrace{\frac{\vec{J} \times \vec{B}}{e n}}_{\text {Hall }}
$$

The Eq. (2.43) plus the MHD equations form the so called Hall-MHD model.

\subsection{Resistive MHD}

In order to neglect the Hall term in Eq. 2.43), we require

- $\Omega_{c e} \ll v_{c}$ (from the ratio between Hall and resistive term). This condition is not satisfied in many cases where MHD it is usually applied, since $v_{c}$ is usually large in collision dominated plasmas. It will be satisfied more easily in weak magnetic field with high collisionality, a scenario not so feasible to find in space plasmas. 
- $\frac{c}{V} \frac{d_{e}}{L_{B}} \Omega_{c e} \frac{1}{\Omega_{p e}} \frac{\rho_{i}}{L} \frac{c_{s}}{V} \ll 1$ (from the ratio between Hall and convective term). This is equivalent to the first condition for getting the Hall-MHD equations if $\beta \sim 1$ : the small Larmor approx. Otherwise, for $\beta \ll 1$, the small Larmor approx. becomes even more stringent: $\frac{\rho_{i}}{L} \ll \sqrt{\beta}$. Since in many space plasmas $\beta$ can be very low, to neglect the Hall term is not justified.

under such conditions, we have the usual MHD's Ohm law.

$$
\vec{E}+\vec{V} \times \vec{B}=\eta \vec{J}
$$

Sometimes, instead of an expression for the electric field, the Ohm's law is used implicitly to derive the induction equation, by replacing $\vec{E}$ in Faraday equation Eq. (2.9), using Ampère's law Eq. 2.10) neglecting displacement current plus some vectorial identities, obtaining thus an expression for the evolution of the magnetic field:

$$
\frac{\partial \vec{B}}{\partial t}=\underbrace{\nabla \times(\vec{V} \times \vec{B})}_{\text {convection }}+\underbrace{\frac{\eta}{\mu_{0}} \nabla^{2} \vec{B}}_{\text {diffusion }}
$$

The second term in the right hand side represents the diffusion of the magnetic field in a frame of reference comoving with the fluid $\left(\vec{E}^{\prime}=\vec{E}+\vec{V} \times \vec{B}\right)$, while the first one represents the convection of magnetic field due to the fluid motion. The relative importance between both terms can be calculated by means of the ratio of the typical scaled between the convective to the diffusive term, resulting in the magnetic Reynolds number $R_{m}$ :

$$
R_{m}=\frac{\mu_{0}}{\eta} L_{B} V
$$

where $L_{B}$ is the length scale of variation of $\vec{B}$ and $V$ a characteristic fluid velocity. If $R_{m} \ll 1$, the diffusive term dominates and we get:

$$
\begin{aligned}
& \vec{E}^{\prime}=\vec{E}+\vec{V} \times \vec{B}=\eta \vec{J} \\
& \frac{\partial \vec{B}}{\partial t}=\frac{\eta}{\mu_{0}} \nabla^{2} \vec{B}
\end{aligned}
$$

The first equation indicates that the electric field in the velocity frame of reference is proportional to the current, while the second is a diffusion equation for the magnetic field. The typical timescale in which this process develops is $\tau_{d}=L^{2} / \eta$. See Chapter 4 for a comparison of these times in space plasma environments.

\subsection{Ideal MHD and Alfvén theorem}

In an ideally conducting fluid we can get a much more simplified set of equation by assuming:

- $R_{m} \gg 1$ (from the ratio between resistive and conductive terms). A condition satisfied in most of space plasmas, as can be seen in Table 1.1. 
- If $\beta \ll 1, R_{m} \gg 1 / \beta$. This more stringent condition over Eq. (2.46) comes from balancing the terms in the pressure evolution equation, obtained from the pressure evolution equation ( $\sim$ second moment of the Vlasov equation) not shown here.

In these cases, the resistive term in Eq. 2.44) and the magnetic field will evolve only due to the fluid velocity. Thus, we get the equations:

$$
\begin{aligned}
& \vec{E}+\vec{V} \times \vec{B}=0 \\
& \frac{\partial \vec{B}}{\partial t}=\nabla \times(\vec{V} \times \vec{B})
\end{aligned}
$$

The first equation indicates that the electric field is always zero in a reference frame comoving with the fluid 4 . Moreover, taking the dot product with the unitary vector in the magnetic field direction: $\hat{b}=\vec{B} / B$, we get:

$$
E_{\|}:=\vec{E} \cdot \hat{b}=0
$$

i.e.: an ideal MHD plasma cannot have parallel electric fields. Recall from the discussion following Eq. (2.26) that if the previous condition is not satisfied $\vec{E} \cdot \vec{b} \neq 0$ (a non-ideal plasma), it is not possible to find a reference frame where $\vec{E}=0$ or $\vec{B}=0$. On the other hand, the consequence of the (equivalent) second equation in Eq. (2.49) is the so called Alfvén or frozen-in flux theorem (Alfvén 1942): the magnetic field lines (more precisely: magnetic flux tubes) are "frozen" into the plasma, they always move together (more precisely, any transverse plasma motion). We also can say that the fluid (or more precisely the ions or any other specie involved) is magnetized. This implies that the magnetic field lines cannot change their topology: they cannot break. This result is of central importance for the purposes of our thesis: magnetic reconnection involves a change in the topology of the plasma, and thus a violation of the Alfvén theorem. Due to Eq. (2.52), this is equivalent to the existence of non-vanishing parallel electric fields $E_{\|} \neq 0$. When this happen, we say that the fluid or the corresponding specie (ions and/or electrons) are unmagnetized.

Therefore, the violation of Alfvén theorem with their associated consequences requires that any of the assumptions that led to that equation do not hold. In resistive MHD, the only way is that the resistive term cannot be neglected in going from Eq. (2.44) to Eq. 2.49) (high $R_{m}$ ). Reconnection driven by this term will be discussed in Sec. 4.1. In Hall-MHD, the Hall term can also be responsible for this in going from Eq. (2.44) to Eq. (2.49), which requires $\omega \gtrsim \Omega_{c i}$ among other assumptions. Reconnection driven by this

\footnotetext{
${ }^{4}$ Note that the previous condition requires neglecting the Hall term in Eq. (2.43). Actually, if it is keep (so, working in the Hall-MHD model) in addition to the same condition $R_{m} \gg 1$, we realize that is the electron fluid the quantity that is actually frozen into the plasma. Indeed, we can replace Eq. 4.5) into Eq. (2.41) (coming back to two fluid variables), yielding:

$$
\vec{E}+\vec{V}_{e} \times \vec{B}=0
$$

The same is automatically also seen from Eq. (2.27). Then, the electric field in the electron frame of reference vanishes. Only under the assumption of $\vec{V}_{i} \approx \vec{V}$ in Eq. 4.5) (i.e.: neglecting Hall term) we can get the condition of vanishing electric field in the bulk flow velocity frame of reference Eq. (2.49). Note that Eq. 2.51) do not imply that the plasma is frozen-into the magnetic field, since the bulk mass flow is still carried by the ions. It is often the case in space plasmas that the Hall term turns out to be more important than the resistive one, and that is why Eq. (2.51) is specially important in these scenarios.
} 
term will be discussed in Sec.4.2.1. Finally, MHD with the one-fluid generalized Ohm's law Eq. (2.41) opens another two possibilities: either the pressure/ambipolar term or the electron inertia. Their associated effects on reconnection will be discussed in Sec. 4.2.3 and Sec.4.2.4. We will see that especially the last two terms become critical in our studies of magnetic reconnection.

\subsection{Double adiabatic approximation (CGL)}

When a plasma is magnetically dominated, the assumption of isotropic pressure is not justified anymore, even in strongly collisional plasmas. In these cases, it is useful to assume that the pressure tensor is diagonal:

$$
P_{i j}=P_{\|} \hat{b}_{i} \hat{b}_{j}+P_{\perp}\left(\delta_{i j}-\hat{b}_{i} \hat{b}_{j}\right)=\left(\begin{array}{ccc}
P_{\perp} & 0 & 0 \\
0 & P_{\perp} & 0 \\
0 & 0 & P_{\|}
\end{array}\right)
$$

where $\hat{b}_{i}=\hat{z}$ in the last pressure tensor matrix (without loss of generality). Each diagonal term in the pressure tensor obey separately an equation of state of the kind Eq. (2.36). It is possible to prove that in this case, the adiabatic indices relating $P_{\{\|, \perp\}}$ with $T_{\{\|, \perp\}}$ are different for each direction and functions of the position for inhomogeneous plasma. For this reason is called "double-adiabatic theory". Then, we can derive the corresponding momentum equation by replacing the scalar pressure $P$ with the previous anisotropic pressure tensor in Eq. 2.35)

$$
\rho \frac{d V_{i}}{d t}=-\partial_{i}\left(P_{\perp}+\frac{B_{m}^{2}}{2 \mu_{0}}\right)+\frac{B_{l} \partial_{l}}{\mu_{0}}\left[\left(1+\frac{P_{\perp}-P_{\|}}{B_{m}^{2} / \mu_{0}}\right) B_{i}\right]
$$

with the perpendicular and parallel components:

$$
\begin{aligned}
\rho \frac{d V_{\perp}}{d t} & =-\partial_{\perp}\left(P_{\perp}+\frac{B_{m}^{2}}{2 \mu_{0}}\right)+\frac{B_{l} \partial_{l}}{\mu_{0}}\left[\left(1+\frac{P_{\perp}-P_{\|}}{B_{m}^{2} / \mu_{0}}\right) B_{\perp}\right] \\
\rho \frac{d V_{\|}}{d t} & =-\partial_{\|} P_{\|}-\left(P_{\perp}-P_{\|}\right) \frac{\partial_{\|} B_{\|}}{B}
\end{aligned}
$$

Under equilibrium conditions $d / d t \rightarrow 0$, the first previous equation generalizes the condition Eq. (3.3) to this case of anisotropic pressure (to be used for anisotropic initializations):

$$
\frac{\partial}{\partial x_{\perp}}\left(P_{\perp}+\frac{B_{m}^{2}}{2 \mu_{0}}\right)=\frac{B_{l}}{\mu_{0}} \frac{\partial}{\partial x_{l}}\left[\left(1+\frac{P_{\perp}-P_{\|}}{B_{m}^{2} / \mu_{0}}\right) B_{\perp}\right]
$$

The equations Eq. 2.55) are known as Chew-Goldberg-Low (CGL) (Chew et al. 1956). This plasma model, valid for strong guide field, assumes the constants

$$
\begin{aligned}
C_{1} & :=\frac{P_{e, \|} B^{2}}{\rho^{3}}, \\
C_{2} & :=\frac{P_{e, \perp}}{B \rho}
\end{aligned}
$$


for electrons and similarly for other components. These constants are derived from a general equation for the evolution of an anisotropic (but gyrotropic) pressure tensor, neglecting the heat flux (which contributes to the pressure transport along the magnetic field lines). The second constant has a relatively simple interpretation: the average energy per particle is proportional to the magnetic field strength. This is equivalent to say that the magnetic moment,the ratio of perpendicular particle kinetic energy to the magnetic field strength:

$$
\mu=\frac{K_{\perp}}{B}=\frac{m v_{\perp}^{2}}{2 B},
$$

of a particle in conserved, valid under the adiabatic approximation. This approach will prove to be useful to model some phenomena in the low $\beta$ regime in the Chapter 9 . 


\section{Current sheet equilibria}

\subsection{MHD equilibria and magnetohydrostatics}

It is possible to rewrite the Lorentz force in the MHD momentum equation by combining it with the Ampère's law (as used in MHD, without displacement current), obtaining:

$$
\vec{J} \times \vec{B}=-\nabla \underbrace{\left(\frac{B^{2}}{2 \mu_{0}}\right)}_{P_{b}=\text { magnetic pressure }}+\underbrace{\frac{1}{\mu_{0}}(\vec{B} \cdot \nabla) \vec{B}}_{\text {magnetic tension }}
$$

We have identified each term with their usual name. The right hand side is called magnetic tension, since it acts as an effective force when the magnetic field lines are curved. The term inside of the parenthesis in the left hand side with dimensions of pressure is usually called magnetic pressure, proportional to the magnetic field energy. It produces a force from the regions with high to low magnetic pressure.

Now, under stationary conditions $d / d t \rightarrow 0$, we can introduce Eq. 3.1) in the momentum equation Eq. (2.35), and obtain the equilibrium equation:

$$
\nabla\left(\frac{B^{2}}{2 \mu_{0}}+P\right)=\frac{1}{\mu_{0}}(\vec{B} \cdot \nabla) \vec{B}
$$

This is valid assuming an isotropic pressure tensor. If the magnetic field is mostly homogeneous, we can neglect the right hand side (magnetic field lines weakly curved) and obtain:

$$
\nabla\left(\frac{B^{2}}{2 \mu_{0}}+P\right)=0 \quad \Leftrightarrow \quad\left(\frac{B^{2}}{2 \mu_{0}}+P\right)=\text { const } .
$$

This is the pressure equilibrium condition in magnetohydrostatics. From the previous expression we can also define a very useful quantity, the total plasma beta $\beta$

$$
\beta=\frac{P_{t h}}{P_{B}}=\frac{2 \mu_{0} P}{B^{2}}=\frac{2 \mu_{0} n k_{B} T}{B^{2}}
$$

where it can also be specialized for each specie adding the corresponding subscript $e$ or $i$ in $n$ and $T$. This parameter tell us the relative importance of the magnetic to thermal effects in a plasma. Low beta plasmas $\beta \ll 1$ are magnetically dominated, while high beta plasmas $\beta \gtrsim 1$ are gas dominated. 


\subsubsection{Force free fields}

Under the same stationary conditions discussed before, sometimes it is useful to approximate the momentum equation Eq. 2.35) for cases where the pressure force can be neglected in comparison with the Lorentz one. The order of magnitude of this ratio turns out to be the same as beta. Therefore, pressure can be neglected for low $\beta$ plasmas, magnetically dominated, very well suited for the solar corona, for example. In this case, we obtain

$$
\vec{J} \times \vec{B}=0 \quad \Leftrightarrow \quad \mu_{0} \vec{J}=\alpha \vec{B}
$$

i.e.: the currents in a plasma always flow parallel to a magnetic field. $\alpha$ is a constant that can depend on the position. If $\alpha$ does not vary/varies from one magnetic field line to other, the force-free magnetic fields are called linear/non-linear. Linear force free fields are globally constant (Harrison and Neukirch 2009b). This relation also implies a coupling between the strength of the current density $J$ and the magnetic field $B$.

Note also that Eq. (3.5), by using Ampère's law, is equivalent to:

$$
\vec{B} \times(\nabla \times \vec{B})=0
$$

i.e.: the topology of the magnetic fields are governed by themselves.

\subsection{Kinetic equilibria of current sheets}

Any theoretical or numerical investigation of instabilities and other kinetic processes in plasmas should start from plasma kinetic equilibria: exact solutions of the VlasovMaxwell system in stationary state $\partial / \partial t \rightarrow 0$. It is interesting to notice that different from the Boltzmann equation that has an unique equilibrium for a given system for long timescales (due to the thermalizing effect of collisions), the Vlasov equation has an infinite number of solutions (see, e.g., Krall and Trivelpiece 1973, Sec. 7.7). But in practice, there are very few analytical known equilibria, because of the difficulty of solving exactly the nonlinear integro-differential system of equations. The standard methods are based on Hamiltonian approaches and constant of motion (see discussion in Sec. 2.1.4 for further details). Now, we show two families of solutions of the Vlasov-Maxwell system for CS, necessaries to initialize our simulations.

\subsubsection{Harris type equilibria}

By using the aforementioned method, Harris (1962) found the first exact kinetic equilibrium for an antiparallel magnetic field configuration, and since then it has been the most used equilibrium for magnetic reconnection studies. It assume quantities varying in the direction $x$ with a net current in $z$ direction, with the three constants of the motion for the system: the canonical momentum in the current direction $z, y$ and the Hamiltonian:

$$
\begin{aligned}
p_{\alpha, z} & =m_{\alpha} v_{z}+q A_{z}(x) \\
p_{\alpha, y} & =m_{\alpha} v_{y} \\
H_{\alpha} & =\frac{m_{\alpha}}{2} v^{2}+q_{\alpha} \phi
\end{aligned}
$$


Then, the solution $f=f\left(H, p_{\alpha, y}, p_{\alpha, z}\right)$ consists in drifting Maxwellian distribution function along $z$ with spatially constant drift speed $U_{z, \alpha}$ and temperatures $T_{\alpha}$ for each plasma specie:

$$
\begin{aligned}
f_{\alpha} & =n_{0 \alpha}\left(\frac{m_{\alpha}}{2 \pi k_{B} T_{\alpha}}\right)^{3 / 2} \exp \left(-\frac{H_{\alpha}-U_{z, \alpha} p_{z, \alpha}}{k_{B} T_{\alpha}}\right) \\
& =n_{\alpha}(x)\left(\frac{m_{\alpha}}{2 \pi k_{B} T_{\alpha}}\right)^{3 / 2} \exp \left[-\frac{m_{\alpha}}{2 k_{B} T_{\alpha}}\left(v_{x}^{2}+v_{y}^{2}+\left(v_{z}-U_{z, \alpha}\right)^{2}\right)\right]
\end{aligned}
$$

Note the spatially varying density $n_{\alpha}(x)$. Assuming a quasi-neutral electron-proton plasma with $n(x)=n_{i}(x)=n_{e}(x)$ (and $n_{0}=n_{0 \alpha}$ ), it can be shown that the density, pressure and current profiles (per specie and total) are respectively given as:

$$
\begin{aligned}
n(x) & =n_{0} \exp \left(\frac{e U_{z, \alpha}}{k_{B} T_{\alpha}} A_{z}(x)\right)=\frac{n_{0}}{\cosh ^{2}(x / L)} \\
P_{\alpha}(x) & =n(x) k_{B} T_{\alpha}=\frac{n_{0} k_{B} T_{\alpha}}{\cosh ^{2}(x / L)} \\
P(x) & =P_{i}(x)+P_{e}(x)=\frac{n_{0} k_{B}\left(T_{i}+T_{e}\right)}{\cosh ^{2}(x / L)} \\
\overrightarrow{J_{i}}(x) & =n_{0} e U_{z, \alpha} \exp \left(\frac{e U_{z, \alpha}}{k_{B} T \alpha} A_{z}\right) \hat{z}=\frac{2 e n_{0} U_{z, \alpha}}{\cosh ^{2}(x / L)} \hat{z} \\
\vec{J}(x) & =\vec{J}_{i}(x)+\vec{J}_{e}(x)=\frac{e n_{0}\left|U_{z, i}-U_{z, e}\right|}{\cosh ^{2}(x / L)} \hat{z}
\end{aligned}
$$

while the vector potential and magnetic field are:

$$
\begin{aligned}
& \vec{A}(x)=A_{z}(x) \hat{z}=L B_{\infty y} \log \cosh \left(\frac{x}{L}\right) \hat{z} \\
& \vec{B}(x)=B_{y}(x) \hat{y}=-\frac{d A_{z}}{d x} \hat{y}=B_{\infty y} \tanh \left(\frac{x}{L}\right) \hat{y}
\end{aligned}
$$

The respective magnetic and current density profiles are shown in Fig. 3.1.

\footnotetext{
${ }^{1}$ The reason for expressing these solutions in terms of the vector potential $A_{z}$ is the convenience when they are applied to solve the linearized Vlasov equation for instabilities such as the tearing mode (see Sec. 5.1).
} 


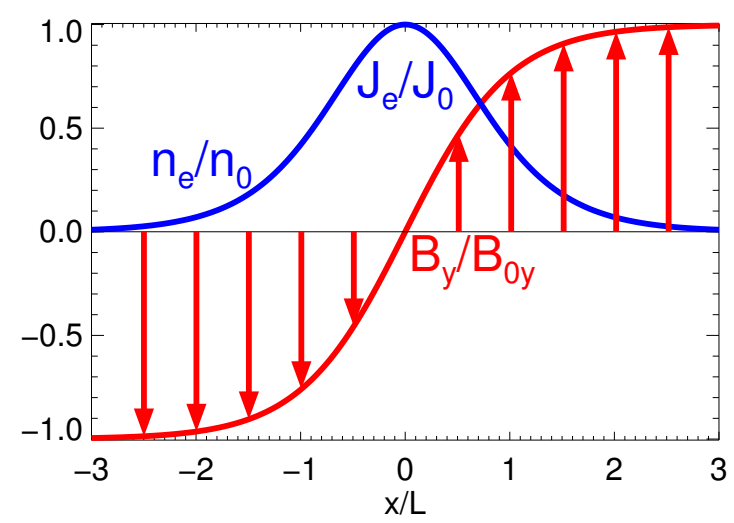

Figure 3.1: Magnetic field $\vec{B}$, density $n$ and current density $\vec{J}$ profiles for a Harris CS, given by Eq. (3.18) and Eq. (3.16), respectively. Note that $n_{0}$ is the peak central density, while $B_{\infty y}$ is the asymptotic Harris magnetic field. $n_{e} / n_{0}$ and $J_{z} / J_{0}$ have identical profiles due to the uniform drift speed assumption.

The Harris solution has also to satisfy the pressure equilibrium condition Eq. (2.57) (with the right hand side equal to zero), in the more general case of anisotropic distribution functions (different parallel and perpendicular temperatures). Then, the magnetic pressure at the infinity has to be balanced by the transverse thermal pressure at the center of the CS.

$$
\frac{\left|B_{\infty y}\right|^{2}}{2 \mu_{0}}=n_{0}\left(k_{B} T_{e, \perp}+k_{B} T_{i, \perp}\right) .
$$

In this more general case, all the previous expression where the temperature is involved have to be changed by considering only the perpendicular one. For details about the extension of Harris equilibrium for anisotropic distributions and their consequences, see Quest et al. (2010). By means of the previous expression, it is possible to prove that the halfwidth $L$ is related with the asymptotic magnetic field and drift speed via:

$$
L=\frac{B_{\infty y}}{\mu_{0} e n_{0}\left|U_{z, i}-U_{z, e}\right|}=\frac{2}{e B_{\infty y}} \frac{k_{B} T_{e}+k_{B} T_{i}}{\left|U_{z, i}-U_{z, e}\right|}, \quad \text { or } \quad \frac{\left|U_{i}\right|}{v_{t h, i}}=\frac{2 \rho_{i}}{L}
$$

Note the inverse proportionality between the halfwidth and drift speed: this expression implies that thinner CS $\left(\rho_{i} \sim L\right)$ will have larger drifts speed in terms of $v_{t h, i}$. This relation can be understood since the gradients in pressure will produce a diamagnetic drift, perpendicular to both magnetic field $(\hat{y})$ and gradient direction $(\hat{x})$, i.e.: in the out-of-plane direction $\hat{z}$ :

$$
\vec{U}_{z, \alpha}=-\frac{\nabla P_{e, \perp}(x) \times \vec{B}(x)}{n_{0} q_{\alpha} B(x)^{2}}
$$

For further details, see, e.g., the textbooks Bellan (2006, Sec. 4.4) or Baumjohann and Treumann (1997, Sec. 7.4). In obtaining these solution it has been used $\phi=0$, absence of initial electric fields. This gives a constraint between drift speeds and temperatures:

$$
\frac{U_{z, i}}{T_{i}}=-\frac{U_{z, e}}{T_{e}}
$$


i.e.: for equal temperatures the drift speeds of each specie sustaining the CS will be equal (but oppositely directed), as well as the currents and the pressures for both electrons and ions.

An important point to mention is that the addition of a constant magnetic guide field in the form:

$$
\vec{B}(x)=B_{y}(x) \hat{y}+B_{z} \hat{z}=B_{\infty y} \tanh \left(\frac{x}{L}\right) \hat{y}+B_{z} \hat{z}
$$

do not alter the Harris solution, it is still an exact solution of the Vlasov-Maxwell system and it does not change Eq. (3.19). It is also important to mention that the inclusion of a constant background pressure $P_{\text {back }}$ :

$$
P(x)=\frac{n_{0} k_{B}\left(T_{i}+T_{e}\right)}{\cosh ^{2}(x / L)}+P_{\text {back }}
$$

do not change the equilibrium Eq. (3.19) as well. This is especially useful in simulations using this kinetic equilibrium as initial equilibrium state.

For details about the specific choice of the minimum set of parameters to initialize a PIC simulation of Harris CS, in addition to other useful relation between parameters, see Appendix A.3.3.

After the original discovery of the Harris solution, Hoh (1966) generalized the Harris equilibrium for relativistic regimes using Maxwellian distribution functions. $\mathrm{Fu}$ and $\mathrm{Hau}$ (2005) showed that a kappa distribution function al so satisfies the same magnetic field and current profiles Eq. (3.18) and Eq. (3.16) as the classical Harris sheet solution. Balikhin and Gedalin (2008) generalized the work of Hoh (1966) by deriving fully relativistic non-Maxwellian distribution functions solutions of the Harris sheet profile. See also Treumann and Baumjohann (2013a) for other details.

\subsubsection{Force free equilibria}

There are also other kinetic equilibria in plasmas which directly address the CS feasible to find in the low $\beta(\ll 1)$ solar coronal plasma. In these strongly magnetized environments we can consider force free magnetic fields, discussed in Sec. 3.1.1. Note that force free magnetic fields do not require necessarily low beta plasmas (but the opposite is always true), instead, the plasma beta can be arbitrary (thermal and magnetic pressures can be chosen independently), as well as the ratio of drift to thermal speeds (Bobrova et al. 2001). This is opposite to the Harris equilibrium (Harris 1962), where magnetic and thermal pressures have to be of the same order, implying that the ratio of drift to thermal speeds has to be fixed. Another important consequence of the plasma equilibria satisfying the force-free condition is that they have constant density and pressure (Harrison and Neukirch 2009b).

It is important to notice that a Harris equilibrium with a strong enough guide field $\left(B_{g} \gg B_{0 y}\right)$ can be used to mimic a force free magnetic field. In a force free configuration, due to Eq. (3.5) $\vec{J} \| \vec{B}$. In the Harris case with guide field, since the direction of the total magnetic field $\vec{B}=B_{\infty y} \hat{y}+B_{g} \hat{z}$ will be mostly in the direction of the guide field $\hat{z}$, we have that $\vec{B}$ is approximately parallel to $\vec{J}=J \hat{z}$. But different from the latter, the strength of $J$ and $B$ are decoupled. Another difference is that in force free equilibria an increase 
in the shear of $B$ implies a higher free energy in the system, while in Harris equilibrium an increase in the constant guide field strength do not add any additional energy into the system (Neukirch et al. 2009).

\subsubsection{Linear kinetic force free equilibria}

In MHD, many force free equilibria can be found, both linear and non-linear. Recall that from Eq. (3.5) a linear force free field has a constant $\alpha$ in $\mu_{0} \vec{J}=\alpha \vec{B}$ that does not vary from one magnetic field line to another (globally constant, see Harrison and Neukirch $2009 \mathrm{~b}$ ). But a MHD equilibrium is not justified in collisionless plasmas often present in astrophysical scenarios. On the other hand, there are very few exact force-free kinetic equilibrium (Neukirch et al. 2009). The difficulty in finding solutions of the VlasovMaxwell system is because is an inverse problem: for a given magnetic field and current configuration, one has to find the distribution function as solution of the Vlasov equation. The first solutions to be discovered were 1D and linear, based on the following sinusoidal dependence on the magnetic field (Sestero 1967), sometimes called a "sheet pinch":

$$
\begin{aligned}
& B_{y}=-B_{0} \sin (k x) \\
& B_{z}=\mp B_{0} \cos (k x)
\end{aligned}
$$

Note that the total magnetic field is constant, with their components being rotated in a helix of constant angle between them of $\phi=2 \pi / k$. The density is also constant, identical for both electrons and ions. Because of this, the electric field vanishes, in the same way as the Harris equilibrium. This is one of the most simple examples of sheared force-free magnetic field configurations, and it has been a popular choice in many studies of magnetic reconnection (Bobrova et al. 2001), especially those with the gyrokinetic framework. In addition, it has also the computational advantage of being suitable without further modifications for periodic boundary conditions, widely available in simulation codes. Other linear force free equilibrium with sheared magnetic field were found by Channell (1976), although not with constant density, and Bobrova et al. (2001). The latter also found the following distribution function satisfying Eq. (3.25) (different from the found one by Sestero 1967).

$$
\begin{aligned}
f_{\alpha} & =\frac{n_{0 \alpha}}{\left(2 \pi m_{\alpha}\right)^{3 / 2} T_{\alpha, \|} T_{\alpha, \perp}^{1 / 2}} \exp \left\{-\frac{m_{\alpha}}{2}\left[\frac{\left(v_{y}-U_{y, \alpha}\right)^{2}+\left(v_{z}-U_{z, \alpha}\right)^{2}}{T_{\alpha, \|}}+\frac{v_{x}^{2}}{T_{\alpha, \perp}}\right]\right. \\
& \left.-\left[\frac{e_{s}}{T_{a \perp}} \phi(x)+\frac{1}{2} \frac{e_{a}^{2}}{m_{a} c^{2}} \frac{\Delta T_{\alpha}}{T_{\alpha, \perp}^{2}} \frac{B_{0}^{2}}{k^{2}}\right]\right\}
\end{aligned}
$$

with the drift speed $U_{y, \alpha}$ and inhomogeneity scale of the magnetic field $k$ given by

$$
\begin{aligned}
\vec{U}_{s}(x) & =\frac{q_{\alpha}}{m_{\alpha} c} \frac{\Delta T_{\alpha}}{T_{\alpha, \perp}} \frac{\vec{B}(x)}{k} \\
k & =\frac{\omega_{p e}}{c}\left(\frac{\Delta T_{e}}{T_{e, \perp}}+\frac{m_{e}}{m_{i}} \frac{\Delta T_{i}}{T_{i, \perp}}\right)^{1 / 2}
\end{aligned}
$$

Here, the equilibrium exists only if there is a non zero temperature anisotropy $\Delta T_{\alpha}=$ $T_{\alpha, \perp}-T_{\alpha, \|}$ between the parallel $T_{\alpha, \|}$ and perpendicular $T_{\alpha, \perp}$ components to the local magnetic field. A higher temperature anisotropy is associated with a smaller length scale 
for the inhomogeneity in the magnetic field. The big advantage of using the distribution function by Bobrova et al. (2001) is that they derived kinetic growth rate of the tearing instability in this configuration, finding that is only dependent on the shape of the magnetic field in the region away from the reversal point, and not on the details of the distribution function (see Sec.5.1.3.1). As a consequence, the same solution is valid in collisional regimes, suitable for some laboratory plasmas. This solution has been used to study streaming/Buneman micro-instabilities (Nishimura et al. 2003, Li et al. 2003), with the field aligned current as their source of free energy. Sakai et al. (2001) also studied these processes with the magnetic field given Eq. (3.25) but with Maxwellian distribution functions instead of the exact solution Eq. (3.27). Later, 3D PIC simulation for relativistic flows also used the same previous approach (Sakai and Matsuo 2004).

\subsubsection{Non-linear kinetic force free equilibria: the force free Harris sheet}

Only recently the first force-free Vlasov Maxwell equilibrium distribution function was found by Harrison and Neukirch (2009b) (see al so Neukirch et al. 2009) by extending the Fourier transform method first developed by Channell (1976). This method of solving the Vlasov-Maxwell equation is based in an analogy of following the trajectory of a pseudo-particle in a conservative potential. It was first applied by the same authors (Harrison and Neukirch 2009a) to derive the linear force-free equilibrium Eq. (3.25). Finally, the result for the (non-relativistic) distribution function for the specie $\alpha$ is:

$$
f_{\alpha}=\frac{n_{0 \alpha}}{v_{t h, \alpha}^{3}} \exp \left(-\frac{H_{\alpha}}{k_{B} T_{\alpha}}\right)\left[\exp \left(-\frac{U_{0 z, \alpha} p_{z \alpha}}{k_{B} T_{\alpha}}\right)+a_{\alpha} \cos \left(\frac{U_{0 y, \alpha} p_{y \alpha}}{k_{B} T_{\alpha}}\right)+b_{\alpha}\right]
$$

with $U_{0 z, \alpha}=U_{0 y, \alpha}, a_{\alpha}$ and $b_{\alpha}$ are constants with the constraint $0<a_{\alpha}<b_{\alpha}$. The magnetic field is given by:

$$
\begin{aligned}
& B_{y}=B_{0} \tanh (x / L) \\
& B_{z}=B_{0} \cosh ^{-1}(x / L)
\end{aligned}
$$

Similar to Eq. 3.25), the total magnetic field is constant $\left(B_{0}\right)$. Note that the $B_{y}$ component is the same as in the Harris sheet, but in this case the force balance is sustained by a magnetic shear instead of the plasma pressure. For that reason the authors called this equilibrium as "force free Harris sheet". The components of the current density are given by:

$$
\begin{aligned}
& J_{y}=\frac{B_{0}}{\mu_{0} L} \frac{\sinh (x / L)}{\cosh ^{2}(x / L)}=e n_{0}\left(U_{0 z, i}-U_{0 z, e}\right) \frac{\sinh (x / L)}{\cosh ^{2}(x / L)} \\
& J_{z}=\frac{B_{0}}{\mu_{0} L} \frac{1}{\cosh ^{2}(x / L)}=e n_{0}\left(U_{0 z, i}-U_{0 z, e}\right) \frac{1}{\cosh ^{2}(x / L)}
\end{aligned}
$$

while the drift speeds for each specie are given by

$$
\begin{aligned}
& U_{y, \alpha}(x)=\frac{U_{0 z, s} \sinh (x / L)}{\left(\frac{1}{2}+b\right) \cosh ^{2}(x / L)} \\
& U_{z, \alpha}(x)=\frac{U_{0 z, s}}{\left(\frac{1}{2}+b\right) \cosh ^{2}(x / L)}
\end{aligned}
$$


Different from the Harris case, we note that the density current is sustained for a spatially varying drift speed of electrons and ions, while the density is constant everywhere. The macroscopic and microscopic parameters are related by

$$
\begin{aligned}
\frac{B_{0}^{2}}{2 \mu_{0}} & =\frac{T_{e}+T_{i}}{T_{e} T_{i}} n_{0} \\
L & =\sqrt{\frac{2\left(T_{e}+T_{i}\right)}{\mu_{0} e^{2} T_{e} T_{i} n_{0}\left(U_{0 z, i}-U_{0 z, e}\right)^{2}}} \\
a & =\frac{1}{2} \\
b & =\frac{T_{e} T_{i}}{n_{0}\left(T_{e}+T_{i}\right)} P_{b}
\end{aligned}
$$

where $P_{b}$ is the background pressure. Note the similarity in the relation for the halfwidth in terms of other parameters in comparison with the Harris case. Harrison and Neukirch (2009b), Neukirch et al. (2009) also investigated a family of kinetic equilibrium with a smooth transition between the Harris equilibrium and the force free case previously described. Wilson and Neukirch (2011) generalized the Harrison's force free solution for a different dependence on the particle energy, but with the same dependence on the canonical momenta. Later, Stark and Neukirch (2012) generalized this force free solution for the relativistic regime. Finally, Abraham-Shrauner (2013) generalized the hyperbolic spatial dependence of the magnetic fields Eq. (3.32) to Jacobian elliptic functions, with the previous solutions as particular cases.

It is important to remark that none of the works about this new non-linear force free equilibrium have analyzed thoroughly their stability properties so far (no calculation of growth rates). Only some conditions about the number of maxima of these distributions functions have been established. Different from the single peaked Maxwellian distribution function, the force free solution Eq. (3.31) or its generalizations may display multiple maxima in $v_{x}$ or $v_{y}$ directions, which have implications for velocity space micro-instabilities such as two-stream or bump-on-tail (besides of macro-instabilities such as tearing mode. See Neukirch et al. (2009) for further details). A more complete stability analysis would be an interesting topic for future research.

Finally, it interesting to notice that is expected that the development of reconnection changes in force-free configuration in comparison with Harris sheet equilibrium. This is because many micro-instabilities found in density gradients of the Harris equilibrium will change radically in force-free configuration, affecting the reconnection process.

\subsubsection{Force free configuration used in this work}

The magnetic field configuration to be used in the force free results to be shown in this thesis, unless stated otherwise, is the following:

$$
\begin{aligned}
& B_{y}=B_{\infty y} \tanh \left(\frac{x}{L}\right), \\
& B_{z}=B_{\infty y} \sqrt{b_{g}^{2}+\cosh ^{-2}\left(\frac{x}{L}\right)}=\sqrt{B_{T}^{2}-B_{\infty y} \tanh ^{-2}\left(\frac{x}{L}\right)},
\end{aligned}
$$


where the relative guide field is $b_{g}=B_{G} / B_{\infty y}$, the total magnetic field has constant magnitude $B_{T}=B_{\infty y} \sqrt{1+b_{g}^{2}}$. Noticing that the magnetic field is given asymptotically by $\vec{B}= \pm B_{\infty y} \hat{y}+B_{g} \hat{z}$, we can calculate the total rotation shear angle with respect to the center of the current sheet

$$
\theta=\operatorname{acos}\left(\frac{b_{g}^{2}-1}{1+b_{g}^{2}}\right)
$$

The magnetic field Eq. (3.42) reduces to the kinetic equilibrium Eq. (3.32) for $b_{g}=0$. The force free magnetic field Eq. (3.42) is sustained by the following expression for the current density (calculated to fulfill the Ampère's law):

$$
\begin{aligned}
& J_{e, y}=-\frac{1}{\mu_{0}} \frac{\partial B_{z}}{\partial x}=\frac{B_{\infty y}}{\mu_{0} L} \frac{\tanh (x / L) \cosh ^{-2}(x / L)}{\sqrt{b_{g}^{2}+\cosh ^{-2}(x / L)}} \\
& J_{e, z}=\frac{1}{\mu_{0}} \frac{\partial B_{y}}{\partial x}=\frac{B_{\infty y}}{\mu_{0} L} \cosh ^{-2}(x / L)
\end{aligned}
$$

with $\vec{J}_{e}=\vec{J}$, assuming stationary ions. $J_{e, z}$ is the out-of-plane current with the same form as in the Harris equilibrium. But different from the Harris case, now we have in addition an in-plane current $J_{e, y}$, parallel to the reconnected magnetic field, that will be an important fact to compare with. The respective profiles are shown in Fig. 3.2.

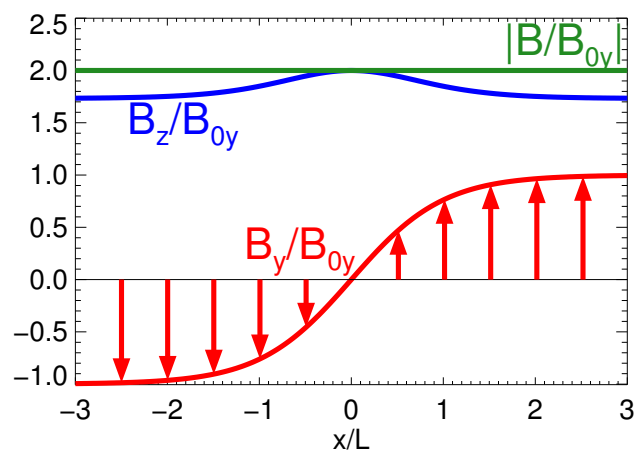

(a) $\vec{B}$

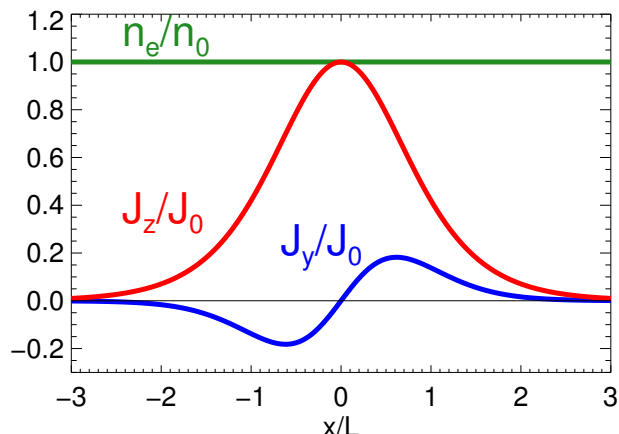

(b) $\vec{J}$

Figure 3.2: Magnetic $\vec{B}$ and current density $\vec{J}$ profiles for a force free current sheet with $b_{g}=2$, given by $E q .(3.45)$ and $E q .(3.45)$, respectively.

To the best of our knowledge, there is no known equilibrium distribution function for the configuration given by Eq. (3.42) and Eq. (3.45). Since we do not know the exact form of the VDF, we will be using the known one from the Harris equilibrium: drifting Maxwellians Eq. (3.11) but with constant density. This approach is valid assuming that the force free magnetic field Eq. (3.42) is close to the given by the exact Harris solution plus a guide field Eq. (3.23), which is true for strong enough guide field strength. Thus, we have the following VDF:

$$
f_{\mathrm{ff}}=\frac{n_{0}}{\left(\sqrt{2 \pi} v_{t h, e}\right)^{3}} \exp \left[-\frac{1}{2 v_{t h, e}^{2}}\left(v_{x}^{2}+\left(v_{y}-V_{y, e}(\vec{x})\right)^{2}+\left(v_{z}-V_{z, e}(\vec{x})\right)^{2}\right)\right]
$$


where, following the Ampère's law, $V_{\{y / z\}, e}=-J_{\{y / z\}, e} /\left(e n_{0}\right)$ are spatially varying drift speeds and $n_{0}=n_{e}=n_{i}$ is the constant background density. Also note the constant temperatures $v_{t h, e}$. Because of this, we will have a constant thermal pressure everywhere, in addition to the constant magnetic pressure due to the form of Eq. (3.42). This implies that the MHD pressure equilibrium condition is satisfied trivially initially (although with the magnetic pressure being much larger than the thermal pressure).

For details about the specific choice of the minimum set of parameters to initialize a PIC simulation of this force free CS, in addition to other useful relation between parameters, see Appendix A.3.2.

The force free magnetic field Eq. (3.42) has been used by Drake et al. (2003), Che et al. (2011) for studies of anomalous resistivity triggered by Buneman instability, in the context of magnetic reconnection with strong guide field. Hesse et al. (2005b) also used a similar configuration for 3D studies of the role of pressure term in sustaining the reconnected electric field. They used that setup in order to avoid the fast growth of LHDI and kink modes in comparison with tearing mode in $3 \mathrm{D}$ configurations, but with the trade-off of the appearance of some artifacts originating from the lack of initial exact kinetic equilibrium. Recently, it has also been used by Liu et al. (2013) for magnetic reconnection studies in $3 \mathrm{D}$ with a similar setup to the previous two references but much larger simulations boxes. They found that the streaming micro-instabilities do not play an important role in comparison with the macro-instability tearing mode. Two recent 2D PIC simulations using the same setup are Liu et al. (2014), TenBarge et al. (2014). 


\section{Magnetic Reconnection}

Magnetic Reconnection is one of the essential mechanisms in the universe for the conversion of magnetic energy into particle energy (heating, bulk flows and particle acceleration, etc). This takes places due to a rearrangement of magnetic field lines (they "reconnect"), something not allowed in ideal MHD due to the Alfvén theorem or frozenin condition (see Sec. 2.5). It is in some way the opposite of dynamo processes, in which the bulk flow energy can be converted to magnetic energy. The condition for its existence in solar flares was first pointed out by Giovanelli (1946), while its mechanism first proposed and explained by Dungey (1953). The term "reconnection" was first coined by Parker (1957). The latter work pointed out that diffusion of magnetic field lines can also convert magnetic energy into other forms of energy (the diffusion Eq. (2.47) for $R_{m} \ll 1$ ), and rearrange magnetic field lines, but at a much larger timescales (in magnetotail and solar flares, diffusive timescales are typically on the order of $\tau_{\text {diff }} \sim 10^{14} s \sim 10^{7}$ years, while the release of energy is on timescales of minutes). Thus, the key characteristic of magnetic reconnection that differentiates it from diffusive processes are their "fast" time scales (how fast will be defined later), although in principle many different mechanisms can lead to the same macroscopic result: the breaking of the frozen-in condition Eq. (2.49) with their associated parallel aligned electric fields Eq. (2.52).

Nowadays, the basic theory of magnetic reconnection is routinely explained in textbooks (Biskamp 2000, Priest and Forbes 2000, Birn and Hesse 2007), while some recent reviews indicating the modern challenges in the current understanding of this process can be found in Zweibel and Yamada (2009), Yamada et al. (2010), Treumann and Baumjohann (2013b) and Karimabadi et al. (2013).

Magnetic reconnection involves two separated time-scales (Cassak and Shay 2011). One is slow (weeks in solar active regions / 3 hours in between magnetospheric substorms), during which the magnetic energy is build-up and concentrated in curved magnetic field lines, with a very high magnetic tension. This process prepares the onset of magnetic reconnection, and it is associated with the tearing mode, to be discussed in Sec. 5.1. The second time-scale is the "fast" one ( 2 minutes in solar flares/ 10 minutes in substorms), during which all that stored energy is suddenly released and the effects of reconnection can be easily observable. In general, it has been found than this second fast phase is much simpler to describe theoretically than the first spontaneous one of slow build-up (known as tearing mode).

The key parameter that quantifies how fast develops the second (fast) phase of reconnection, and thus the rate of energy release (the parameters that it is actually observable), is the reconnection rate. It is the out-of-plane electric field $E_{z}$ in the upstream of the current sheet, being normalized to $V_{A} B$ (in S.I. units. In CGS, the normalization is with respect to $\left(V_{A} / c\right) B$ ), with $V_{A}$ the Alfvén speed calculated with the asymptotic magnetic 
field. By using Faraday's law and Stokes theorem, this is equivalent to the rate of change of magnetic flux $\Psi$ across the boundaries of the reconnection region. In a $2 \mathrm{D}$ configuration with no variations in the $z$ direction, it can be shown Somov (2013) that given a magnetic island with known $\mathrm{X}$ and $\mathrm{O}$ points (see scheme of tearing islands with $\mathrm{X}$ and $\mathrm{O}$ points in Fig. 5.1) will have the following reconnected flux density $\psi^{\prime}$ :

$$
\psi^{\prime}(x=\text { center }, t)=\frac{\psi}{\Delta z}=\frac{1}{\Delta z} \iint \vec{B} \cdot d \vec{S}=\frac{1}{\Delta z} \iint \vec{B} \cdot \vec{x} d z d y=\int_{O}^{X} B_{x}(x=\text { center }) d y
$$

where $S$ is a surface between the $X$ and $O$ points, which can be identified as the extrema of the vector potential (maximum and minimum). Its normal is pointing in the $x$ direction (it can be defined as $d \vec{S}=d z \hat{z} \times d y \hat{x}$ ). We have assumed that the size of this surface in the $z$ direction is $\Delta z \rightarrow 0$, and that $B_{x}$ does not change in this direction. Since now on, we will identify the reconnected flux density $\psi^{\prime}$ simply as the reconnected flux $\psi$ (omitting '). The previous expression is equivalent to:

$$
\frac{d \psi}{d t}=\frac{d}{d t}\left(\int_{O}^{X} B_{x}(x=\text { center }) d y\right)=\frac{1}{\Delta z} \oint \vec{E} \cdot d \vec{l}=E_{z}(X)-E_{z}(O)
$$

One key open problem in magnetic reconnection study, even nowadays (Cassak and Shay 2011, Karimabadi et al. 2013), it is to find what determines the reconnection rate Eq. (4.2). In this subsection we briefly describe the two first proposed mechanisms of steady magnetic reconnection that gives scaling relations for the reconnection rates, in the context of the MHD plasma model.

\subsection{MHD reconnection}

\subsubsection{Sweet-Parker reconnection model}

The first model of 2D steady state magnetic reconnection was proposed by Sweet (1958) and Parker (1957). It assumes antiparallel magnetic field lines supported by a current sheet, with a rectangular non-MHD region where the dissipation $(\eta \neq 0)$ and the rearrangement of magnetic field lines takes place, i.e: an $X$ point (the definition of $X$ and $\mathrm{O}$ points is given in Fig. 5.1). This non-ideal region has a length of $L$ in the outflow direction and a half-thickness of $\delta$ in the inflow direction, as shown in Fig. 4.1. 


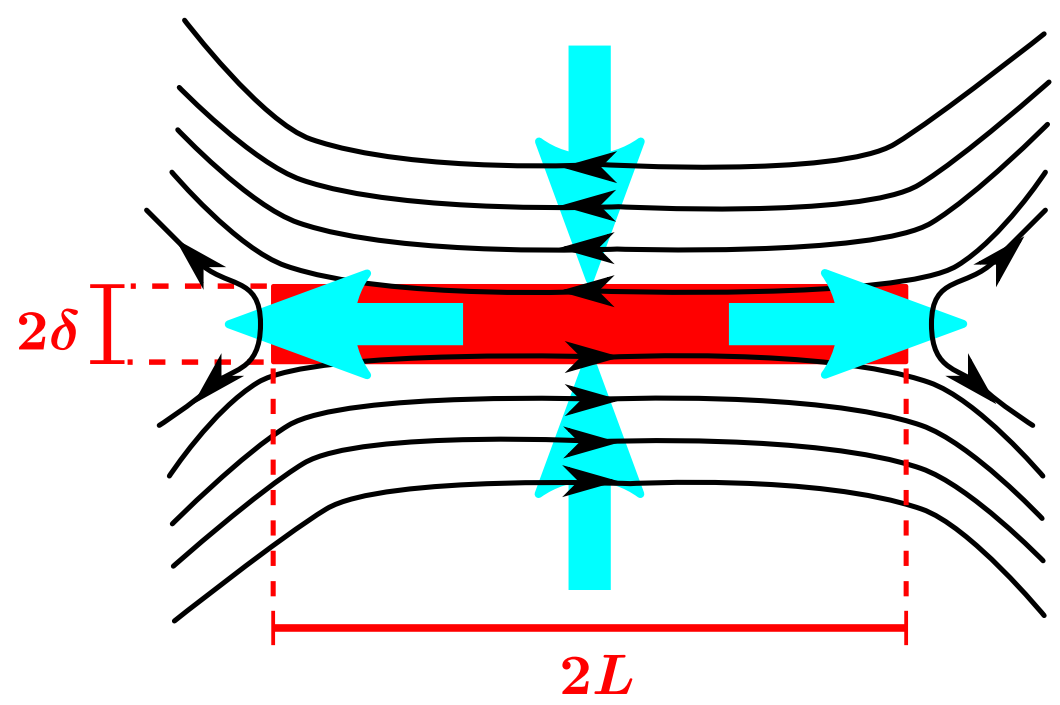

Figure 4.1: Sweet Parker reconnection. $\delta$ is the half-thickness of the diffusive region along the inflow direction, while L its length along the outflow region. Adapted from Fig. 1 of Zweibel and Yamada (2009)

In steady state, by simple 2D geometric arguments and conservation of mass (in addition to incompressibility), one can show (Zweibel and Yamada 2009) that the outflow plasma moves at the Alfvén speed $V_{A}$ and the electric field $\vec{E}$ points in the out-of-plane direction, being conductive outside of the reconnection region (second term in the left hand side of Eq. (2.44)), while is resistive inside of the non-ideal region. This has as a consequence that the reconnection rate is equal to the aspect ratio of the diffusion region (its width divided its length). The latter, in turn, is equal to the ratio between the inflow $V_{\text {in }}$ to outflow speed $V_{A}$, scaling with the Lundquist number as:

$$
\frac{d \psi}{d t}=E_{z}=\frac{\delta}{L}=\frac{V_{i n}}{V_{A}}=S^{-1 / 2} \propto R_{M}^{-1 / 2} \sim \tan \theta
$$

where $\tan \theta$ is the opening angle of the separatrices, the region around the $\mathrm{X}$ point with and $\mathrm{X}$ shape, separating magnetic domains. The second equality means that the inflow speed is the precisely the width $\delta$ of the diffusion region, a consequence from the fact all the incoming flow has to pass through that region (due to the conservation of mass). In addition, there is an equipartition of the magnetic energy in equal amounts for both bulk outflow plasma and electron heating (Zweibel and Yamada 2009). Then, faster reconnection rates imply a high aspect ratio (shorter layers), high inflow speeds and more open separatrices. As we will see, these three consequences will still be good approximation much beyond the MHD models, providing a good first order estimation about reconnection speed.

However, the scaling of these quantities with $S^{-1 / 2}$ would make reconnection too slow in many space environments where $S$ (or $R_{M}$ ) is very large. Indeed, it cannot explain the observed rates of energy release in many space environments (see Sec. 1.1). In order to match with the observational constrains, it is thus required that the dissipation region has to be much smaller than the system size $\delta \ll L$. This can be understood because only thin diffusive regions can provide the high currents (since $\nabla B \propto J$ by Ampère's law) necessary to dissipate the magnetic energy stored in this configuration. 


\subsubsection{Petschek reconnection}

The first improvement to solve the issue with the slow reconnection rates predicted by Sweet-Parker mechanism was proposed by Petschek (1964). The idea is to avoid the bottleneck that produce all the incoming flow passing through the width $\delta$ and thus limiting the reconnection rates given by Eq. (4.3). Instead of the full length $L$, Petschek proposed a shorter diffusive region $L *$, possible due to the deflection of the incoming flow by slow shock modes. This is in some sense equivalent to an increasing resistivity closer to the X point (not uniform like in Sweet-Parker model). By applying the MHD jump conditions at the shock fronts, it is possible to prove the existence of an additional acceleration and current due to the Lorentz force compared to the Sweet-Parker model, and thus the reconnection rates are given by

$$
E_{z}=\frac{V_{\text {in }}}{V_{A}} \approx \frac{\pi}{8 \log S}
$$

Thus, the reconnection rates decay much slower with the Lundquist number than in the Sweet Parker model, thanks to the inverse logarithmic dependence on $S$. Typical estimates provides reconnection rates of the order of $\left(E_{z} / B_{0}\right) \sim 0.01-0.1 V_{A}($ Koskinen 2011). Thus, the Petschek model is considered the first fast magnetic reconnection mechanism proposed, enough to explain the observations in space environments. It is interesting to mention that in this model the transfer of energy goes mostly to outflow bulk energy of ion, not producing an efficient electron heating like in the equally distributed energy of Sweet-Parker model (Zweibel and Yamada 2009).

However, although phenomenologically correct in this sense, there are many puzzling questions about the mechanism (and also about the numerical implementation) in the Petschek model that produces these enhanced reconnection rates. In particular, because the Petschek reconnection rate have a very weak dependence on $S$ and so $R_{m}$ and the resistivity $\eta$, it is practically independent on the physics of the diffusion region. But this is precisely the key problem in magnetic reconnection: determining what violates the frozen-in condition (see discussion in Sec. 2.5) allowing the breaking of magnetic field lines. Therefore, it is necessary to go beyond MHD to address this issue.

\subsubsection{Non-stationary reconnection model: Plasmoid instability}

Other factor that recently was found to be able to enhance and modulate reconnection rates in the resistive MHD approach are plasmoids, proposed by Loureiro et al. (2007). Also known as secondary magnetic islands, they are generated in very elongated (long and thin) current sheets, producing a time dependent and "bursty" reconnection. This is an alternative way of getting fast magnetic reconnection even for very high Lundquist numbers, and very different from the classical models such as Petschek reconnection. It has become a very active topic of research during the last years (see Loureiro et al. 2013a, and references therein). They have also been observed in full 3D PIC simulations with very large simulation boxes (see Daughton et al. 2011a, and references therein). 


\subsection{Collisionless magnetic reconnection}

In the present thesis we address collisionless magnetic reconnection in the solar corona, with negligible collisional resistivity $\eta \rightarrow 0$. Therefore, the aforementioned MHD models do not apply and specific collisionless plasma mechanisms are necessary to allow magnetic reconnection. They provide faster reconnection rates than Sweet-Parker model, matching better with the observations (see the review, e.g., Uzdensky 2006). As we discussed in Sec. 2.5, any term in the generalized Ohm's law in its two-fluid Eq. (2.25) or one-fluid Eq. (2.41) version provides the necessary ingredient to rearrange the magnetic field lines. Now, we will briefly describe the effects of each one of these terms: Hall, pressure and inertia, in the reconnection process. But keep in mind, however, that the Hall term or anisotropic pressure tensors (but otherwise gyrotropic) cannot by themselves break the frozen in condition (Egedal et al. 2013). Only the non-ideal terms in the right hand side of the generalized Ohm's law allow this kind of mechanism.

\subsubsection{Hall effects}

As discussed in Sec.2.3 and Sec. 2.4, the Hall term,

$$
\vec{E}_{\text {Hall }}=\frac{1}{e n} \vec{J} \times \vec{B}
$$

in the 1-fluid Ohm's law Eq. 2.41) 1 describes the consequences of the differential motion of electrons and ions. It is important for low $\beta$ plasmas, and CS sustained by transverse currents (to the magnetic field). It can be shown that is is especially relevant for CS on the order of $d_{i}$. Different from the aforementioned MHD models with only out-ofplane current, when the Hall term dominates over the resistive one, it introduces in-plane currents due to the decoupling between electrons and ions. As a result, the diffusion region is divided in two regions: an electron diffusion region of the order of $d_{e}$ embedded in a larger ion diffusion region of the order of $d_{i}$. In between the ions become demagnetized, while the electrons are still magnetized on the reconnecting magnetic field. The incoming ion flow is stopped and deflected at distances $d_{i}$ from the $\mathrm{X}$ point, while something similar for the electrons but a distances $d_{e}$. This was predicted theoretically and confirmed with simulations at the end of the 90s (see Shay et al. (1998, 2001) or Biskamp (2000) for a general review).

\footnotetext{
${ }^{1}$ note that it does not appears in the per-specie or two-fluid Ohm's law Eq. $(2.25)$ since it involves two species.
} 


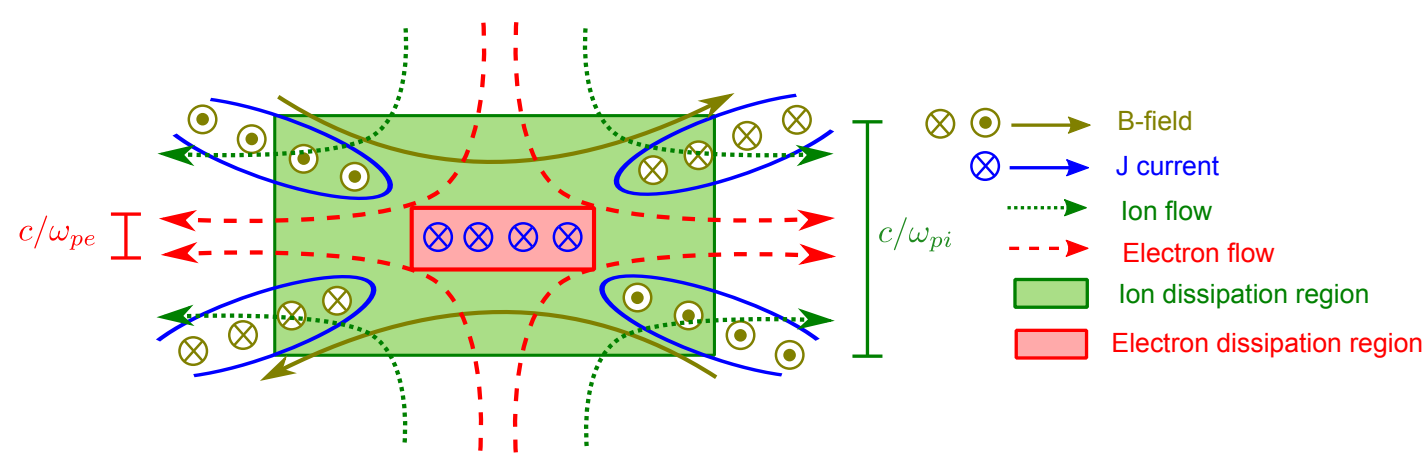

Figure 4.2: Hall-MHD reconnection. The typical length scale of the electron diffusion regions is $d_{e}$ (shown in red color), where the electron motion (shown in red dashed lines) decouple from ions (in red dashed lines). Outside of the red region, the electrons are magnetized. The ion diffusion region is much larger, on the order of $d_{i}$ and shown in green color. Outside of this regions, the ions are magnetized and frozen-into the plasma. In between these regions is where most of the Hall effects take place. This charge motion induces in-plane currents (shown in continuous blue lines), generating an out-of-plane magnetic field $B_{z}$ with opposite polarity at each other side of the $X$ point, forming a characteristic quadrupolar pattern (shown in olive color). Adapted from Fig. 4 of Zweibel and Yamada (2009).

In this reconnection model, the characteristic outflow speeds from the $X$ point are of the order of the in-plane Alfvén speed $V_{A}$ for ions and in-plane electron Alfvén speed $V_{A e, y}$ for electrons (Shay et al. 2001), in contrast to MHD models where the maximum outflow speed is always on the order of $V_{A}$. Only inside of the smaller electron diffusion region they become demagnetized (breaking the frozen-in condition).

A key feature of the Hall mediated reconnection is a characteristic quadrupolar shape of the out-of-plane magnetic field (already found in 1979 by B. Sonnerup). This can be seen by rewriting Eq. (4.5) using the decomposition in Eq. (2.11) and specializing to the z component:

$$
\frac{1}{e n}(\vec{J} \times \vec{B})_{z}=\frac{1}{e n \mu_{0}}(\vec{B} \cdot \nabla) B_{z}
$$

And due to the characteristic direction of the incoming and outcoming electron flows depicted in Fig.4.2, it is possible to prove that this magnetic field has a quadrupolar structure: $B_{z} \propto x y$ (see details in Zweibel and Yamada 2009, Uzdensky and Kulsrud 2006).

The importance of the Hall term facilitating fast magnetic reconnection was highlighted by the famous GEM (Geospace Environmental Modeling) challenge (Birn et al. 2001), which showed that all simulation models that incorporated this term (full PIC by Pritchett (2001), hybrid and two fluid) developed fast reconnection rates, in agreement with the observations 2 . Only the MHD simulation model, without Hall term, showed slow reconnection rates (Sweet-Parker like Eq. (4.3)). Then, they inferred that the reconnection rate should be practically independent on the physical mechanism breaking the frozen-in-condition, either fluid or kinetic. These results imply than the ion behaviour always determine the reconnection rates, but the inclusion of electron dynamics is essential

\footnotetext{
${ }^{2}$ Note that, fortunately for the simulation studies that are restricted to reduced mass ratios, it was shown that reconnection rates are more or less insensitive to the value of this parameter $m_{i} / m_{e}$ (Shay et al. 1998).
} 
for the mechanism (Treumann and Baumjohann 2013b). This result was also confirmed by another similar benchmark study of forced magnetic reconnection for different codes: the "Newton challenge" described in Birn (2005) (with fully PIC simulations by Pritchett (2005b)). However, many questions remained unanswered after the publication of these results. Especially what is the physical reason behind these enhanced reconnection rates. The most popular explanation is based on the waves that can be developed in this system, to be explained next.

\subsubsection{Role of dispersive waves in collisionless reconnection}

\subsubsection{Traditional explanation}

Rogers et al. (2001) proposed a model to predict the parameter regime in which a collisionless plasma can develop fast reconnection rates, based in the waves that the system can support. This has become the mainstream explanation since those years, although recently there have been some works that have challenged it, finding that fast magnetic reconnection can take place even in regimes where was predicted to be slow (TenBarge et al. 2014, Liu et al. 2014, Stanier et al. 2015). Let us first analyze the assumptions and predictions of the model by Rogers et al. (2001).

The Hall term that appears in two fluid plasma models brings the dynamics of whistler and kinetic Alfvén waves to magnetic reconnection. Both are dispersive waves, with frequencies that increases quadratically for smaller wavelengths $\omega \propto k^{2}$ (or phase speeds proportional to $\omega / k \propto k$ ). Before of a proper description of both waves, we need to define some characteristic speeds and length scales:

$$
\begin{gathered}
B_{k}=\vec{B} \cdot \hat{k}, \quad V_{A k}=\frac{B_{k}}{\sqrt{\mu_{0} n_{0} m_{i}}}=\frac{B_{k}}{B} V_{A}, \quad \beta_{j k}=\frac{2 \mu_{0} n_{0} m_{i} k_{B} T_{j}}{B_{k}^{2}}, \quad \beta_{k}=\frac{2 \mu_{0} n_{0} m_{i} k_{B}\left(T_{i}+T_{e}\right)}{B_{k}^{2}} \\
C_{s}^{2}=\frac{k_{B}\left(T_{i}+T_{e}\right)}{m_{i}}, \quad C_{M}=V_{A}^{2}+C_{s}^{2}, \quad C_{M k}^{2}=\frac{V_{A}^{2}}{1+\left(k d_{e}\right)^{2}}+C_{s}^{2} \\
\rho_{s}=\frac{C_{s}}{\Omega_{c i}}, \quad d_{k}=d_{i} \frac{V_{A k}}{C_{M}}=d_{i} \frac{1}{\sqrt{\frac{B^{2}}{B_{k}^{2}}+C_{s}^{2} / V_{A k}^{2}}}, \quad d_{s}=d_{i} \frac{C_{s}}{C_{M}}=d_{i} \sqrt{\frac{\beta / 2}{1+\beta / 2}}=\frac{\rho_{s}}{\sqrt{1+\beta / 2}}
\end{gathered}
$$

with $j=i, e$ for ions or electrons. $C_{S}$ and $C_{M}$ are the sound and magnetosonic speeds, respectively. The subscript $k$ indicates quantities calculated with the magnetic field in the propagation direction of the wave. If the waves propagates on the reconnection plane, then $B_{k} \sim B_{\infty y} . d_{k}$ can be interpreted as an "effective ion inertial length": $d_{i}$ reduced by a ratio of speeds that depends on the relative guide field and total plasma beta, while $d_{s}$ the ion inertial length reduced by the ratio between thermal to magnetosonic speeds. $\rho_{s}$ is the ion Larmor radius calculated on the sound speed based in both electron and ion contributions. Now, the properties of the aforementioned waves can be summarized as follows:

1. Whistler waves. $\omega=k^{2} d_{i} V_{A x}$. Amplitude $\propto k V_{A}$. 
They have short wavelengths and high phase speeds:

$$
d_{e}<k^{-1}<d_{k}, \quad \omega / k \gg C_{M}
$$

from where the condition for their existence can be deduced:

$$
\mu_{k}:=\frac{d_{e}^{2}}{d_{k}^{2}}=\frac{m_{e}}{m_{i}} \frac{C_{M}^{2}}{V_{A k}^{2}}=\frac{m_{e}}{m_{i}} \frac{B^{2}}{B_{k}^{2}}\left(1+\frac{\beta}{2}\right) \ll 1
$$

i.e: $\mu_{k}$ can be interpreted as an "effective mass ratio": $m_{i} / m_{e}$ reduced by a factor depending on the relative guide field and total plasma beta. Assuming the geometry $\vec{B}=B_{\infty y}\left(\hat{y}+b_{g} \hat{z}\right)$, with $b_{g}=B_{g} / B_{\infty y}$ the relative guide field and $\vec{k}=k \hat{y}$, the condition becomes:

$$
\mu_{k} \approx \frac{m_{e}}{m_{i}}\left(1+b_{g}^{2}\right)\left(1+\frac{\beta}{2}\right)=\frac{m_{e}}{m_{i}}\left(1+b_{g}^{2}+\frac{\beta_{k}}{2}\right) \ll 1
$$

Whistlers are mostly seen in antiparallel reconnection in high beta plasma regimes $(\beta \gtrsim$ 1. Note that $\beta_{i} \sim 1$ implies similar scales for the ion diffusion region compared to the ion Larmor radius $d_{i} \sim \rho_{i}$ ): they are suppressed for strong enough guide fields. Note that in the low beta plasma regime, $\mu_{k} \approx\left(m_{e} / m_{i}\right)\left(B / B_{\infty y}\right)^{2}$, which allow to rewrite the whistler condition as $B_{\infty y}^{2} \gg\left(m_{e} / m_{i}\right) B_{g}^{2}$ in the strong guide field case. One known theoretical prediction is that a quadrupolar structure in the out-of-plane magnetic field should be observed in regimes where whistler are present. This has been seen in many previous works (see, e.g., the 2D PIC simulations by Ricci et al. 2004).

2. Kinetic Alfvén waves. (KAWs) $\omega=k \rho_{s} k_{\|} V_{A}$ for $k \rho_{s} \gg 1$. Note the typical length scale of KAWs $\rho_{s}$ : the ion Larmor radius based on the electron temperature. When they operates, this quantity replace the typical length scale of the diffusion region $d_{i}$ (Ricci et al. 2004). They have larger wavelengths but lower phase speeds than whistlers

$$
\max \left(d_{e}, d_{k}\right)<k^{-1}<\rho_{s}, \quad V_{A k} \ll \omega / k \ll C_{S}
$$

Note that $\rho_{s} \sim d_{s}$ in the low beta regime $\beta \ll 1$ due to the relations in Eq. (4.7). Assuming additionally low frequencies $\omega<\Omega_{c i}$, they have to satisfy:

$$
\frac{\beta_{k}}{2}:=\frac{d_{s}^{2}}{d_{k}^{2}}=\frac{C_{s}^{2}}{V_{A k}^{2}}=\frac{2 \mu_{0} n_{0} k_{B}\left(T_{i}+T_{e}\right)}{B_{k}^{2}}=\frac{1}{2}\left(\beta_{i k}+\beta_{e k}\right)=\frac{\beta_{k}}{2}>1
$$

Assuming the same geometry as for whistlers before, we have the condition:

$$
\frac{\beta_{k}}{2}:=\frac{\beta_{\infty y}}{2}=\left(1+b_{g}^{2}\right) \frac{\beta}{2} \gg \max \left\{\mu_{k}, 1\right\}
$$

where $\beta_{\infty y}$ is the plasma beta calculated with the asymptotic magnetic field. Then, KAWs are expected in environments with strong enough guide field and/or high plasma betas. The first condition make feasible to describe them by means of reduced MHD models or EMHD. One prediction of these models is a quadrupolar electron density structure in regimes where KAWs should be present. This signature was first observed in 2D PIC simulations by Ricci et al. (2004). See Sec. 4.3.1 for more details. 
Therefore, the values of the parameters $\beta_{k}$ and $\mu_{k}$ determine which waves can be present. Note that this is equivalent to the relative value of the three characteristic scale lengths $d_{e}$, $d_{k}$ and $d_{s}$. There are four possible regimes shown schematically in Fig. 4.3.

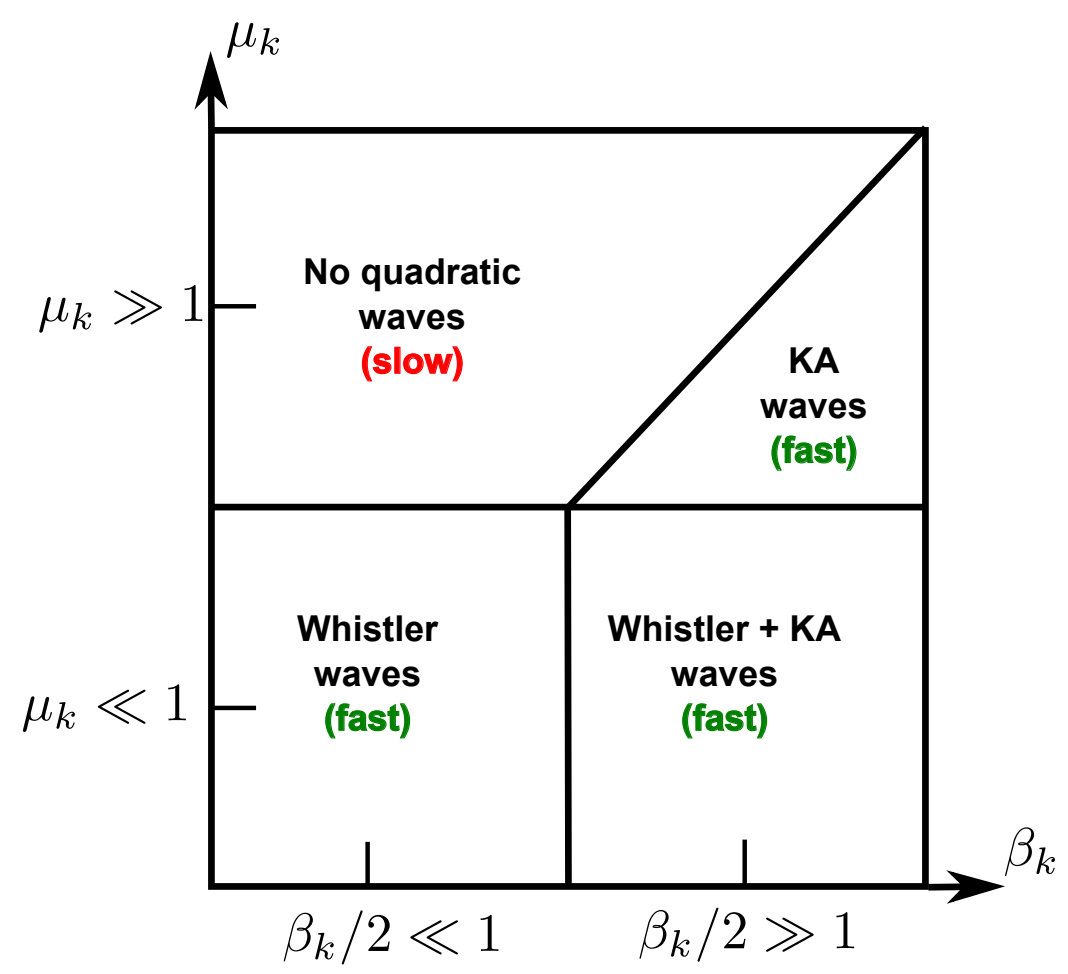

Figure 4.3: Parameter regime for waves expected in two fluid magnetic reconnection, as function of the parameter $\mu_{k}$ and $\beta_{k}$. Adapted from (Rogers et al. 2001).

These dispersive waves are expected to form quasi-steady structures in the electron outflow regions and along the separatrices (Treumann and Baumjohann 2013b). Now, in the regimes where these waves are present, it should be expected fast magnetic reconnection. Rogers et al. (2001) argued that this is because the dispersive nature of whistler/kinetic Alfvén waves can keep constant the acceleration in the outflow region if they behave as standing waves in that region, opening up the reconnection layer. The latter process takes place by assuming that the electron outflow speed is on the order of the phase wave speed $V_{e, \text { out }} \sim \omega / k \propto k$. Since typically $k \sim 1 / L$, this results in a constant mass flow $L V_{e, \text { out }}$ from the reconnecting layer even though $L$ decreases. As a result, when the length scales are compressed in the ion diffusion region, the electron outflow speed has to increase above $V_{A}$ in order to balance the higher whistler wave speed for these small scales. Thus, the reconnection rate, proportional to the inflow speed, is not limited anymore to the Alfvén speed $V_{A}$ as in Sweet-Parker model of resistive MHD, reaching the higher values observed in many space environments. This is equivalent to have a scale invariant reconnection rate (Ricci et al. 2004).

The previously described process is in opposition to models without Hall term where no dispersive waves are allowed, such one fluid MHD. Only the (ordinary) Alfvén waves can exist in these models. The dispersiveless character of these standing waves can produce the collapse of the open outflow layer away from the $\mathrm{X}$ point, leading to a reduction in the reconnection rates. This is the so called "slow" regime, characterized by $\mu_{k} \gtrsim 1$ 
and $\beta_{k} / 2 \lesssim \mu_{k}$, equivalent to $\frac{\beta}{2}\left(1-\frac{m_{e}}{m_{i}}\right) \lesssim \frac{m_{e}}{m_{i}}$. The second condition for non-existence of KAWs implies a very small plasma $\beta \ll m_{e} / m_{i}$ in such a way that the first condition for the non-existence of whistlers becomes $B_{\infty y}^{2} \lesssim\left(m_{e} / m_{i}\right) B_{g}^{2}$. One can understand intuitively the absence of fast magnetic reconnection in low plasma betas, since ions/electrons are tightly coupled in strongly magnetized environments, and do not allow the decoupling necessary to produce the Hall effect source of the dispersive waves (Ricci et al. 2004).

Rogers et al. (2001) tested this prediction by means of two fluid simulations. In regimes where was expected both whistler and KAW to be present (right bottom corner of Fig. 4.3), they found the highest reconnection rates $\left(\dot{\psi} / \dot{\psi}_{N} \sim 0.12\right)$. These rates were reduced when only one of these waves were present, but they were still substantial $\left(\dot{\psi} / \dot{\psi}_{N} \sim 0.06\right)$. Consequently, a reduction in the opening angle of the separatrices was seen. And finally, in the parameter regime where no dispersive waves were allowed (left top corner of Fig. 4.3$)$, reconnection was slow $\left(\dot{\psi} / \dot{\psi}_{N} \ll 0.05\right)$ and it was observed that the separatrices were barely open.

\subsubsection{Recent works showing opposite evidence}

Several recent works have challenged the traditional explanation of fast magnetic reconnection $\left((d \Psi / d t) / \dot{\Psi}_{N} \sim 0.1\right)$ based in the presence of dispersive waves. One reason is because the corresponding linear theory is rigorously valid only for homogeneous plasmas, while magnetic reconnection develops highly non-homogeneous structures. In any case, many of those studies agree that this process is possible in parameter regimes without the presence of fast dispersive waves. For example, in electron-positron (pair) plasmas (equivalent to the limit $m_{i}=m_{e}$ ) the Hall term is absent, then no dispersive waves such whistler or KAWs are allowed and thus reconnection should always be slow 3 . This also implies that there is no quadrupolar structure of the out-of-plane $B_{z}$. However, 2D PIC simulations of pair plasmas (antiparallel configuration) by Bessho and Bhattacharjee (2005) have shown fast reconnection rates, due to the still active off-diagonal terms of the pressure tensor (analogous to a localized resistivity). Similar results were seen in the large scale 2D PIC simulation carried out by Daughton and Karimabadi (2007), due to the production of plasmoids (related with secondary magnetic islands) in the large extension of their simulated current layer. It is interesting to notice at this point that secondary magnetic islands and plasmoids are also seen in electron-proton plasmas, modulating temporally the reconnection rates in large systems (see Daughton et al. 2011a, and references therein). Later, Chacón et al. (2008) developed an analytical model for explaining the fast reconnection rates in large guide field and low beta pair plasmas, but in the framework of a fluid model with a viscous closure for the pressure tensor. On the other hand, hybrid simulations by Karimabadi et al. (2004) where the Hall term is imposed to be zero also showed fast magnetic reconnection rates, although the interpretation of these results faced some criticism afterwards (Malakit et al. 2009).

Coming back to electron-proton plasmas, the recent work by TenBarge et al. (2014) also confirmed fast reconnection rates by means of gyrokinetic and PIC simulations of force free current sheets with guide fields up to $b_{g}=50$ (and low plasma beta $\beta_{i}=0.01$ ).

\footnotetext{
${ }^{3}$ In general, there is no other electromagnetic wave of dispersive nature in pair plasmas. This is valid for a wave-number regime of weak damping and even allowing arbitrary propagation angle. See Gary and Karimabadi (2009) for rigorous proof.
} 
Our results to be shown in Chapter 9 also support this conclusion. Liu et al. (2014) al so reported fast reconnection rates by means of 2D PIC simulations of force free current sheet with guide fields up to $b_{g}=80$. The latter study al so demonstrated that the dominant terms in the Ohm's law, which can support the reconnected electric field, do not change significantly between parameter regimes with or without the presence of these waves. There was no fully satisfactory explanation given there, but it was speculated that the reason for the persistence of fast magnetic reconnection rates is the invariance of the most unstable (collisionless) tearing mode at $k L \sim 0.5$. Although the fast waves can be suppressed by changing the guide field, the latter parameter does not. And $k L$ fixes the aspect ratio that can explain the fast reconnection rates.

Very recently, Stanier et al. (2015) showed that even two fluid Harris sheets simulations can display fast reconnection rates in low beta plasma regimes (not allowing dispersive waves). They proposed a mechanism and scaling laws with the same theoretical framework to explain the simulation results without resorting to the presence of dispersive waves. However, it is still unclear why the previous two-fluid simulations based in the work by Rogers et al. (2001) reported slow reconnection rates.

Another even more recent work by Cassak et al. (2015) proposed an alternative mechanism for fast magnetic reconnection in regimes that do not allow whistler or kinetic Alfvén waves, i.e.: without significant contribution of the Hall term. By means of a two fluid analysis using the CGL equations of state (discussed in Sec. 2.6, for anisotropic pressure tensor in the large guide field limit), these authors showed that another kind of dispersive waves can exist in magnetic reconnection scenarios only due to an electron temperature anisotropy (consistent with some former studies, somehow ignored, by Ambrosiano et al. 1986, Guo et al. 2003). These temperatures anisotropies are self-generated by the following process. Similarly to the Hall model plus guide field by Kleva et al. (1995), the model by Cassak et al. (2015) also predicts a quadrupolar structure of the out-of-plane $B_{z}$ but due to the magnetic flux convected by ions instead of electrons. As a result, $B_{z}$ has opposite polarity to the one predicted by the Hall model. Correspondingly, $P_{e, \perp}$ develops a quadrupolar structure of opposite polarity to preserve the pressure equilibrium condition, as well as the density $n_{e}$. Density gradients will produce parallel electric fields $\nabla_{\|} n_{e}=E_{\|}$ in the same way as these one explained in Sec. 4.3.1. Because they are directed from low to high density regions, this electric field has to sustained by a $P_{e, \|}$ with opposite polarity to $P_{e, \perp}$ and $n_{e}$, generating the required electron temperature anisotropy. The latter contributes to the reconnected electric field in a similar way to the pressure term in the standard generalized Ohm's law Eq. (2.25) or Eq. (2.41):

$$
E_{\text {anisotropy }, \mathrm{z}}:=-\frac{1}{e n_{e}} \frac{\partial P_{e}^{z j}}{\partial x_{j}}=-\frac{1}{e n_{e} \mu_{0}} \frac{\partial B_{l}}{\partial x_{l}}\left[\left(\frac{P_{e, \perp}-P_{e, \|}}{B^{2} / \mu_{0}}\right) B_{z}\right]
$$

Thus, this mechanism of fast magnetic reconnection is important in low plasma beta environments (strong guide field) or regimes with high in-plane plasma beta and high enough electron temperature anisotropy (but with an special form, otherwise reconnection turns out to be slow again). It is also dominant over the traditional Hall term in low beta plasmas with $T_{e}>T_{i}$ (instead of the KAW parameter regime according to Rogers et al. 2001). It is important to note that the guide field cannot be too high, otherwise $\rho_{i}<d_{e}$ preventing the separation of scales needed for fast reconnection. The predictions of the latter model might explain several recent simulation results of magnetic reconnection in the strong 
guide field regime, as those reported in the previous paragraphs and also our results to be shown in Chapter 9.

\subsubsection{Non-gyrotropic pressure effects}

The pressure or ambipolar term in Eq. (2.41) becomes important when its typical gradient length scale (the inhomogeneity scale) becomes comparable with $\rho_{i}$ (see Sec. 2.3), i.e.: when the finite Larmor radius (FLR) approximation is not valid anymore. This is the case of thin CS. The pressure term can be written for the out-of-plane direction (supporting thus the reconnected electric field) as

$$
E_{z, \text { pressure }}:=-\frac{1}{e n_{e}}\left(\frac{\partial P_{e, x z}}{\partial x}-\frac{\partial P_{e, y z}}{\partial y}\right)
$$

We can notice that only the off-diagonal terms of the electron pressure tensor contributes for breaking the frozen-in condition (a gyrotropic, even anisotropic, pressure tensor is not enough). This was first pointed out by Vasyliunas (1975). In an antiparallel configuration, a large number of fully-kinetic PIC simulation studies over the last two decades have confirmed that the pressure term is the dominant one to sustain the reconnected electric field (see Yamada et al. 2010, Hesse et al. 2014, Treumann and Baumiohann 2013b, and references therein). This is still true in 3D cases (see, e.g., Hesse et al. 2005a) and with the addition of a guide field (see, e.g., Ricci et al. 2004).

In the traditional HD Navier Stokes-equation, the non-gyrotropic terms in the pressure tensor are considered as a pseudo-viscosity. As pointed out by Treumann and Baumjohann (2013b, and references therein), they can be produced due to the bouncing or meandering motion of electrons around the neutral sheet line when they are trapped in the Speiser orbits (Büchner and Zelenyi 1987). A physical interpretation on how the electrons performing these orbits can generate the off-diagonal terms in the pressure term can be found in Yamada et al. (2010). The final result is that the electron become demagnetized, allowing the breaking of frozen-in condition, for distances less than their typical meandering length (bounce width) given by (Biskamp and Schwarz 2001, Kuznetsova et al. 2000, Ricci et al. 2004):

$$
\lambda_{x, e}:=\left[\frac{2 m_{e} k_{B} T_{e}}{e^{2}\left(\partial B_{y} / \partial x\right)^{2}}\right]^{1 / 4}
$$

and under these circumstances the electron pressure will become non-gyrotropic. For a Harris sheet in antiparallel configuration, this typical length scale can be approximated as (Hesse et al. 2014):

$$
\lambda_{x, e} \approx d_{e} \sqrt{\beta_{e}}
$$

where the quantities in the right hand side are calculated at the boundary of this region: the diffusion region. Note that this distance can becomes much smaller than $d_{e}$ for a low beta regime. It is interesting to notice that by replacing the derivatives in each term of Eq. (4.15) by the typical meandering length Eq. (4.16), it is possible to approximate both 
terms as derivatives of lower order momenta: only bulk velocities are required (see, e.g., Hesse et al. 2011, 2014, or references therein):

$$
\begin{aligned}
P_{y z, e} & \approx \frac{P_{e}}{\Omega_{c e}} \frac{\partial V_{e, y}}{\partial y} \\
P_{z x, e} & \approx-\frac{P_{e}}{\Omega_{c e}} \frac{\partial V_{e, x}}{\partial x}
\end{aligned}
$$

where $P_{\rho}=\sum_{i} P_{i i, e} / 3$ is the isotropic part of the tensor pressure. Then, plugging this back to Eq. (4.15), one can find an approximate expression for the reconnected electric field $E_{z}$, and so the reconnection rate, when magnetic reconnection is dominated by this term:

$$
E_{z} \approx \frac{1}{e} \frac{\partial V_{e, y}}{\partial y} \sqrt{2 m_{e} k_{B} T_{e}}
$$

This expression can be generalized, revealing a very interesting physics about the diffusion region, when it is written in the following form (see, e.g., Hesse et al. 2014):

$$
E_{z} \approx \frac{1}{2 e n_{e}} \Lambda^{2} \frac{\partial V_{e, y}}{\partial y} \nabla^{2}\left(m_{e} n_{e} V_{e, z}\right)
$$

where $\Lambda$ is the typical length scale of the diffusion region: $d_{e}$ for antiparallel reconnection or $\rho_{e}$ in case of guide field reconnection. The right hand side is a diffusion term related with the out-of-plane current density $J_{z}$, suggesting that the reconnected electric field is opposing to the decay of $J_{z}$ by accelerating particles inside of the diffusion region. .

On the other hand, as pointed out by Hesse et al. (2004) (see also Hesse et al. (2014)), the non-gyrotropy of the pressure tensor is closely related with the heat flux, which was usually neglected in many former two-fluid models. They could show, analytically and by means of 2D PIC simulations, that an appropriate description of that term require the inclusion of the heat tensor in the pressure evolution equation in the immediate vicinity of the X point, especially in the guide field case. Indeed, the generalization of Eq. (4.22) for guide field case is

$$
\begin{aligned}
& P_{y z, e} \approx-\frac{P_{x x, e}}{\Omega_{c e}} \frac{\partial V_{e, z}}{\partial x}+\left(P_{z z, e}-P_{x x, e}\right) \frac{B_{y}}{B_{z}}+\frac{1}{\Omega_{c e}} \frac{\partial Q_{x z y, e}}{\partial x} \\
& P_{z x, e} \approx \frac{P_{y y, e}}{\Omega_{c e}} \frac{\partial V_{e, z}}{\partial y}+\left(P_{z z, e}-P_{y y, e}\right) \frac{B_{x}}{B_{z}}+\frac{1}{\Omega_{c e}} \frac{\partial Q_{y z x, e}}{\partial x}
\end{aligned}
$$

In any case, the non-gyrotropy of the electron pressure tensor can be used as a proxy of the locations where magnetic reconnection is being driven by the pressure term (see Appendix B.2.6 for the method of calculation). This has been applied by Hesse et al. (2014) (and references therein) to PIC simulations of magnetic reconnection. They found that in many cases non-gyrotropic distributions cannot locate precisely neither the $\mathrm{X}$ points nor the separatrices. Instead, they indicate intrinsic kinetic features of the reconnection process that cannot be attributed to a fluid behavior. 


\subsubsection{Effects of electron inertia}

The electron inertia term in Eq. (2.41) can be written for a 2D configuration as:

$$
E_{z, \text { inertia }}:=-\frac{m_{e}}{e}\left(\frac{\partial V_{e, z}}{\partial t}+V_{e, x} \frac{\partial V_{e, z}}{\partial x}+V_{e, y} \frac{\partial V_{e, z}}{\partial y}\right)
$$

This term becomes important only inside of the small electron diffusion region $d_{e}$, due to the small electron mass. It has been shown (see Yamada et al. 2010, Treumann and Baumjohann $2013 \mathrm{~b}$, and references therein) that the most important parts are the non-linear terms (spatial gradients) in comparison with the time derivative. Overall, the electron inertia also contributes significantly to the reconnected electric field compared to the pressure term, at least in an antiparallel configuration. But the inclusion of a strong enough guide field magnetizes the electrons, making their pressure tensor more gyrotropic and leading to a loss of its importance in sustaining the reconnected electric field. This was first pointed out in 2D PIC simulations by Horiuchi and Sato (1997). More precisely, the guide field reduces the length scales for which the pressure tensor can be non-gyrotropic, while not affecting significantly the electron inertia term (Ricci et al. 2004, Pritchett 2005a). Therefore, in these cases, the electron inertia term becomes dominant over larger scales than the pressure term which is reduced to $\rho_{e}$ on the guide field. However, some recent studies (Horiuchi et al. 2014) have pointed out that this is not always the case, even for strong guide fields. This might be related with the choice of temperatures in former studies favoring $T_{i} \gg T_{e}$ (for magnetospheric applications), which makes the ratio $d_{e} / \rho_{e} \gg 1$ (for $\beta_{i}$ constant!) due to the relations in Appendix A.3.1. This reduces the importance of the pressure inertia term that should dominate on length scales $\rho_{e}$.

\subsubsection{Anomalous dissipation effects}

So far, all the previously mentioned mechanism for breaking the frozen in condition were derived in the framework of the collisionless Vlasov theory or MHD with constant scalar resistivity $\eta$. But it is also possible to take into account electromagnetic fluctuations over the mean fields in all the terms in the Vlasov equation. For example,

$$
\vec{E}=\langle\vec{E}\rangle+\delta \vec{E}
$$

where terms in brackets indicate an ensemble average and $\delta$ the fluctuations (with short time and spatial scales) with respect to the mean value $\langle\vec{E}\rangle$ (varying over long time and spatial scales). It is possible to show that the Vlasov equation Eq. (2.19) for the mean distribution function $\langle f\rangle$ has an non-zero right hand side $\Theta^{4}$, that can be interpreted as a form of collisions (Yoon and Lui 2006):

$$
\left[\frac{\partial}{\partial t}+\vec{v} \cdot \frac{\partial}{\partial \vec{x}}+\frac{q_{\alpha}}{m_{\alpha}}(\langle\vec{E}\rangle+\vec{v} \times\langle\vec{B}\rangle) \cdot \frac{\partial}{\partial \vec{v}}\right]\left\langle f_{\alpha}\right\rangle=-\frac{q_{\alpha}}{m_{\alpha}}\left\langle(\langle\vec{E}\rangle+\vec{v} \times\langle\vec{B}\rangle) \cdot \frac{\partial \delta f_{\alpha}}{\partial \vec{v}}\right\rangle
$$

The remaining Maxwell equations have the same standard form, but expressed in terms of the ensemble averaged quantities. It is also possible (see Yoon and Lui 2006) to obtain

\footnotetext{
${ }^{4}$ Note that this separation of scales introduced by the mean and fluctuating quantities in the electromagnetic fields is not unique, implying that the collision term in Eq. (2.19) do vary for different choices, affecting the macroscopic description. See also Schindler (2007, p. 32).
} 
analogous equations for the fluctuating distribution function $\langle f\rangle$ and Maxwell equations via a quasilinear analysis (neglecting second order terms). By taking the momenta of the previous equations, one can recover the usual resistive MHD equations for the ensemble average quantities. In this case, the resistive or collisional terms in the momentum and energy equations arise as result of a transfer of momentum and energy between a selfgenerated wave field (represented by the fluctuating fields). The waves can be generated by many different micro-instabilities. Finally, the net effect is seen macroscopically as an effective drag on the plasma particles, a kind of "anomalous" resistivity (in the sense of not based in collisions like the MHD resistivity). This can be better seen by combining the ensemble averaged two-fluid equations in a generalized one-fluid Ohm's law Eq. (2.41) for the ensemble averaged quantities:

$$
\left\langle E_{i}\right\rangle+\varepsilon_{i j k}\left\langle V_{j}\right\rangle\left\langle B_{k}\right\rangle=\underbrace{\eta_{i j} J_{j}}_{\text {anomalous }}+\frac{1}{e\langle n\rangle} \epsilon_{i j k}\left\langle\delta J_{e, j} \delta B_{k}\right\rangle-\frac{1}{e\langle n\rangle} \frac{\partial\left\langle P_{e}\right\rangle}{\partial x_{i}}-\frac{m_{e}}{e^{2}\langle n\rangle} \frac{d\left\langle J_{i}\right\rangle}{d t}
$$

where correlations higher than second order has been neglected. The additional "anomalous" resistive term 5 can be written as (Yoon and Lui 2006):

$$
\begin{aligned}
E_{i, \text { anomalous }} & :=-\eta_{i j} J_{j} \\
& =-\frac{1}{n_{e}} \int d v^{3}\left(\left\langle\delta E_{i} \delta f_{e}\right\rangle+\left\langle\varepsilon_{i j k} v_{j} \delta f_{e} \delta B_{k}\right\rangle\right) \\
& =-\frac{1}{\langle n\rangle}\left(\left\langle\delta n_{e} \delta E_{i}\right\rangle+\left\langle\varepsilon_{i j k} \delta\left(n_{e} V_{j}\right) \delta B_{k}\right\rangle\right)
\end{aligned}
$$

Note that the resistivity is now a tensor instead of a scalar $\eta_{i j}$ : the electric field does not have to be parallel to the current. Thus, this formalism can incorporate the effects of micro-turbulence in macroscopic MHD equations by means of "anomalous" transport coefficients, from which we have given only the example of anomalous resistivity. But in order to calculate theoretically the right hand side of Eq. 4.29), it is necessary to determine the saturation levels of the micro-instabilities driving the fluctuations. The most important ones are current aligned streaming instabilities such as Buneman (along $\hat{z}$ ), and also cross-field instabilities such as MTSI. For further details, see the early review about electrostatic fluctuations Davidson and Krall (1977), the textbooks Treumann (2001, Sec. 12.1), Biskamp (2000, Sec. 7.1.4), the articles Treumann (2001), Büchner and Elkina (2006), Yoon and Lui (2006), the recent review Treumann and Baumjohann (2013b) and all the references therein.

\subsection{Influence of guide field on Hall-MHD reconnection}

As we discussed in Chapter 4, the classical picture of 2D magnetic reconnection involves antiparallel magnetic fields in, e.g., a Harris equilibrium (see Sec. 3.2.1), in such a way that the direction of the total $\vec{B}$ reverses in $180^{\circ}$. However, it is often the case that

\footnotetext{
${ }^{5}$ It is interesting to mention that the contribution of this anomalous term to the balance of terms in the Ohm's has been measured applying explicitly this expression to observations of magnetic reconnection in the Earth's magnetotail by Lui et al. (2007).
} 
an out-of-plane magnetic field is present (and this does not alter the Harris equilibrium). This produces two main effects in the magnetic field configuration: 1) the center of the CS become magnetized: it is not a neutral line anymore and 2) the rotation or shear of the total $\vec{B}$ across the CS is by an angle $\theta$ smaller than $180^{\circ}$ (the larger the $b_{g}$, the smaller $\theta$ ). A specific example of the magnitude of this angle for a force free case is given in Eq. (3.44). Only one component of the magnetic field reverses sign at the center, and that is why guide field reconnection is sometimes called "component reconnection" (Lapenta et al. 2010).

There are many unique features consequences of the previous two effects. The first one is closely related with 1) and the build-up phase of magnetic reconnection. The tearing instability, the spontaneous instability leading to magnetic reconnection to be discussed extensively in Sec. 5.1, is severely quenched if $b_{g}$ is strong enough. That is because, at the kinetic level, it relies on resonant wave-particle interactions depending on the particle orbits close to the unmagnetized center of the CS (in an antiparallel configuration). These orbits are very sensitive to any perturbation, such as a guide field that can magnetize these particles, destroying the resonant condition driving the tearing mode. The analytical theory and mechanism of the tearing instability in this case are less clear theoretically, relying mostly in numerical solutions of the linearized dispersion relations (Karimabadi et al. 2005). For details, see discussion in Sec. 5.1.3.

At a more macro-level, the effect 2) of a guide field on the fully-developed or steady state magnetic reconnection (the second phase) can be analyzed with a two-fluid approach in terms of the symmetries of the system, based on the work started by Kleva et al. (1995) and then developed by Rogers et al. (2001). This is related with the properties of dispersive waves that the system allows, affecting the reconnection rates on dependence on the corresponding parameter regime. This is going to be discussed next.

\subsubsection{Effects on the symmetries of steady-state magnetic reconnec- tion}

For clarity in the terminology that will be used in the rest of this work, we will define the parity of all relevant quantities with respect to the $y$ axis along the current sheet. This means that a quantity with even/odd symmetry (or symmetric/antisymmetric) will $\mathrm{keep} /$ reverse sign under the transformation of coordinates $\left(x-L_{c}\right) \rightarrow-\left(x-L_{c}\right)$, where $L_{c}$ is the location of the left or right CS. This is valid for regions around the separatrices, away from both $\mathrm{X}$ and $\mathrm{O}$ points.

It has been known since some time ago that the fluctuations in the plasma pressure generated by magnetic reconnection are symmetric in absence of guide field (Rogers et al. 2003). This is the characteristic quadrupolar signature of the out-of-plane magnetic field $B_{z}$ in an antiparallel configuration (see discussion of Eq. (4.6)). This comes from the intrinsic symmetry in the 2D solutions of the two fluid equations (invariance under the transformation $x \rightarrow-x$ and $y \rightarrow-y$ ). The presence of a finite guide field breaks these symmetries. But the symmetries of some quantities (all except density $n$, thermal pressure $P_{\text {th }}$ and out-of-plane magnetic field $B_{z}$ ) reappear again in the strong guide field regime $B_{g} \gg B_{\infty v}$ (Rogers et al. 2003), according to the predictions of reduced MHD models (Kleva et al. 1995). These models predicts that the density $n$ and the total thermal pressure $P_{t h}$ should have odd symmetry in this regime, due to a compression of electrons along 
the magnetic field lines on the reconnection plane $\left(\frac{\vec{B}}{B_{g}} \cdot \nabla V_{e, z} \approx \nabla_{\|} V_{e, \|}\right)$. This generates parallel electron pressure gradients $\nabla_{\|} n_{e}$ via the 1-fluid generalized Ohm's law Eq. (2.41) neglecting the inertia and Hall terms (so, in between the two and 1 fluid model) in the isothermal limit:

$$
E_{\|}=\eta J_{\|}-\frac{1}{e n} \frac{d P_{e}}{\partial x_{\|}}=\eta J_{\|}-\frac{k_{B} T_{e}}{e n} \nabla_{\|} n_{e}
$$

where we have written the non ideal parallel component of the electric field as $E_{\|}$(i.e.: in the framework of reference of the moving fluid $\left.(\vec{V} \times \vec{B})_{\|}\right)$. This $E_{\|}$allows the decoupling of ion/electrons at the length scale $\rho_{s}$ (defined in Eq. (4.7)) when this length scale is larger than the resistive one, producing fast magnetic reconnection by breaking the frozen in condition Eq. (2.52). Note that this is mechanism is different from the standard picture of Hall-driven reconnection Sec. 4.2.1 (due to the Hall term $\vec{J} \times \vec{B}$ ) or the pressure-term driven reconnection Sec. 4.2.3 (due to the non-gyrotropy of the pressure tensor $P_{e}$ ).

The previous process can be understood in physical terms as follows (see Kleva et al. 1995, Ricci et al. 2004, for further details). By assuming quasineutrality, the compression of electrons has to be equal to the ion one $\nabla_{\|} V_{e, \|}=\nabla_{\|} V_{i, \|}$. Then, the main contribution to the gradients in $V_{i}$ has to come from polarization drifts $\vec{V}_{p}=\left(1 /\left(B \Omega_{c i}\right)\right) d \vec{E} / d t$, since the ion $E \times B$ drift do not allow variations in this simplified model and no initial gradients are assumed (that may contribute to a possible diamagnetic drift. Note also that the electron polarization drift is negligible in comparison to the ion one). The polarization drift makes $\nabla_{\|} n_{e}$ change of direction across the CS close to the X point, because the rotation of the asymptotic magnetic field along that direction. This results in a quadrupolar structure of $n_{e}$ and so in the total thermal pressure.

The previous model is valid under the assumption of small fluctuations on the order $O\left(V_{i, x} / V_{A}\right)^{2}$, i.e.: the ion inflow speed into the diffusion region should be much smaller than the Alfvén speed, or equivalently, the opening angle of the separatrices should be small. It is also required that the plasma beta calculated with respect to the asymptotic magnetic field be $\beta_{y}=2 \mu_{0} n_{0} k_{B} T_{i} / B_{\infty y} \gtrsim 1$ (although there are recent opposite evidence that this is not necessary, such as Hosseinpour and Mohammadi (2013). See more details at the end of this section). Thus, the magnitude of the pressure fluctuations under this ordering is given by (see Rogers et al. 2003, Eq. 17):

$$
\frac{\delta P_{t h}}{P_{t h, 0}} \sim \frac{d_{i}}{l_{x}} \frac{1}{b_{g}}
$$

where $P_{t h, 0}=P_{t h}(t=0)=2 n_{0} k_{B} T_{i}$ is the total initial thermal pressure (with contributions of both electrons and ions) and $l_{x}$ is the typical length scale of variation of these quantities across the current sheet, away from the $\mathrm{X}$ points, with an order of magnitude $l_{x} / d_{i} \sim$ $\sqrt{\beta_{i} / 2}$. The fluctuations are defined as $\delta P_{t h}=P_{t h}(t)-2 n_{0} k_{B} T_{i}$. Therefore, Eq. 4.33) predicts that the fluctuations in thermal pressure goes down with increasing guide field, if this is strong enough.

It is also possible to get a relation analogous to Eq. (4.33) for the magnetic pressure. Assuming that the pressure equilibrium condition (in the general anisotropic case Eq. (2.57), with the right hand side equal to zero) should hold even in the non-linear stage of magnetic reconnection (this assumption is valid under the previously mentioned ordering), we have in the strong guide field limit the following condition for the fluctuating 
quantities

$$
\delta P_{t h}+\frac{B_{g}}{2 \mu_{0}} \delta B_{z}+\frac{B_{\infty y}^{2}}{2 \mu_{0}}=\text { constant }
$$

with $P_{t h, \perp}:=n_{e} k_{B} T_{e, \perp}+n_{i} k_{B} T_{i, \perp}$ (note that it is required the perpendicular component of the thermal pressure). From Eq. (4.34) it follows that $\delta P_{t h}$ will necessarily have antisymmetric or odd parity: a quadrupolar structure, in agreement with the argument following Eq. (4.32). All the other terms should be balanced separately. Following Rogers et al. (2003), the dominance of regimes with different parity of $\delta B_{z}$ is given by the ratio of both terms:

$$
\frac{\delta B_{z}^{\text {odd }}}{\delta B_{z}^{\text {even }}} \sim \frac{\beta_{y}}{2} \frac{d_{i}}{l_{x}} \frac{B_{\infty y}}{B_{z}} \sim \sqrt{\frac{\beta_{y}}{2}} \frac{\rho_{s, e}}{l_{x}}
$$

where $\rho_{s, e}=\sqrt{k_{b} T_{e} / m_{i}} / \Omega_{c i}=\sqrt{\beta / 2} d_{i}$ is the ion gyroradius calculated with the electron temperature. Note that $\rho_{s}$ is the typical length scale of kinetic Alfvén waves (see Sec. 4.2.2), signatures of guide field reconnection. Eq. (4.35) means that the specific symmetry of $\delta B_{z}$ will depend on $\beta_{y}$. In the low beta case $\beta_{y} \gg 1$, and thus $\delta B_{z}$ will be predominantly antisymmetric, being given by the following estimation:

$$
\frac{\delta B_{z}}{B_{g}} \sim-\frac{\delta P_{t h}}{B_{g}^{2} / \mu_{0}} \sim \frac{d_{i}}{l_{x}} \frac{\beta_{i}}{b_{g}}
$$

Then, similar to $\delta P_{t h}, \delta B_{z}$ also scales linearly with the guide field, assuming $\beta_{i}$ constant. What it is more important is the prediction about the quadrupolar structure of the $\delta B_{z}$ in the limit of strong guide field. And due to the pressure equilibrium condition, $\delta P_{t h}$ will also have a quadrupolar structure but with opposite polarity. We have to remark that this mechanism is only possible in the strong guide field regime, being different from the classical quadrupolar structure of $\delta B_{z}$ due to the Hall term in an antiparallel magnetic reconnection configuration (recall discussion of Eq. (4.6)). The signatures of these predictions were found first in two fluid (Rogers et al. 2003) and later also in PIC code simulations (Ricci et al.2004, Pritchett|2005a).

It is important to mention that recently, it has been shown by Hosseinpour and Mohammadi (2013) that the assumption $\beta_{y} \gg 1$ is not necessary for the appearance of an odd-parity magnetic field structure. This can happen even in the opposite limit, providing that exists a shear flow parallel to the reconnected magnetic field. This study came up with that conclusion by taking into account the electron inertia in the dispersion relation for the collisionless tearing mode.

\subsubsection{Consequence: Cavities and electron acceleration}

Several simulation studies have confirmed the previous theoretical prediction: in presence of a guide field, the diffusion region becomes asymmetric or tilted. Basically, this is due to the fact that the $\vec{E} \times \vec{B}$ drifts change the symmetric pattern of electrons and ions flow motion compared with the antiparallel case (Ricci et al. 2004), where $\vec{B}$ only have inplane components. This results in the aforementioned quadrupolar structure, manifested in the region close to the $\mathrm{X}$ points as a pair of separatrices with alternate enhanced and depleted density (see Lapenta et al. 2010, and references therein). The dynamics in the low density pair of separatrices is quite interesting. Indeed, these regions also known as 
cavities, are sites of electron acceleration due to the generation of strong parallel electric field $E_{\|}$, make them prone to streaming instabilities such as Buneman when their speed exceed the $v_{t h, e}$. In the non-linear stage, they become electron holes and the electrons can be accelerated efficiently until relativistic speeds (Pritchett 2006). Note that his electron acceleration mechanism is only possible with a guide field, and specially efficient in 3D reconnection, a fact that it has been reported since Drake et al. (2003). This is because the guide field points in the same direction as the reconnected electric field. Although overall magnetic reconnection is globally less efficient with guide field (see below), it opens the opportunities for this kind of very energetic process leading to strong heating, turbulence and even radiation, although in very localized regions (Treumann and Baumjohann 2013b).

\subsubsection{Effects on reconnection rate}

The arguments related with the presence of dispersive waves by Rogers et al. (2001) (see Sec.4.2.2) can also explain a reduction in reconnection rates with increasing guide field. For example, a system initially without guide field (antiparallel configuration) and low $\beta<1$ will allow only whistler waves since $\mu_{k} \ll 1$ and $\beta_{k} / 2<1$. The dispersive properties of whistler waves in the outflows allow fast magnetic reconnection. As the guide field increases while $\beta_{k}$ is kept constant, the system increases $\mu_{k}$ towards values higher than 1 . This suppress whistler waves and so the mechanism that allows the opening of the reconnection layers. Therefore, reconnection turns out to be slow. Similarly, let a system with initially high plasma $\beta>1$ but no guide field. This will support both KAW and whistler, allowing even higher reconnection rates than in the previous case. As the guide field increases whistler can be suppressed in cases with $\mu_{k}>1$, but the mechanism that support KAWs will still be active. As a result, a reduction in reconnection rates will also be seen but their values will still be significant ("fast"), in the sense of $\left.\dot{\psi} / \dot{\psi}_{N} \lesssim 0.1\right)$. Finally, in a strongly magnetized regime where only KAWs are allowed, an increase in the guide field $b_{g}$ (with the associated increase in $\mu_{k}$ ) will reduce the electron gyroradius on the guide field $\rho_{e, b g}$. Since this is the typical length scale of KAWs and proportional to their phase speed, the mechanism allowing the opening of the separatrices and fast magnetic reconnection will be reduced, resulting in a decrease of reconnection rates for higher $b_{g}$ (see Sec. 4.2.2). This was confirmed by Rogers et al. (2001) via twofluid simulations.

The previous explanation can also be understood in more basic physical terms (Yamada et al. 2010) by considering that the inflows from the $\mathrm{X}$ point become slower (and so $d \psi / d t$ decreases according to Eq. (4.3)) because of the additional magnetic pressure exerted by the guide field (equivalent to the gradual suppression of whistler waves). In addition, a strong guide field make the plasma more incompressible, confining them in a smaller region than in the antiparallel case (equivalent to the appearance of KAWs dynamics at $\rho_{e}$ lengths scales).

This mechanism for explaining the reduction in reconnection rate was already reported by Biskamp et al. (1997) with two fluid simulations. However, they incorrectly predicted a strong drop in reconnection rates (practically suppression) in the strong guide field regime, switching to the "slow" regime. That was because they neglected the contribution of KAW s in sustaining reconnection rates, by not allowing parallel compression 
of the electron pressure $\nabla_{\|} P_{\rho}$. The reduction in reconnection rates was confirmed on that same year by Horiuchi and Sato (1997) via 2D PIC simulations of driven reconnection, which took into account that mechanism, although without explicit mention.

After the discovery of the KAWs allowing fast magnetic reconnection by Rogers et al. (2001), several 2D PIC simulations studies confirmed the reduction, but not suppression, of reconnection rates in presence of a guide field. This was already reported by Pritchett (2001) in the PIC version of the GEM challenge (Birn et al. 2001), including the initial perturbation. They showed that a slight reduction of $\Psi$ for guide fields up to $b_{g}=2$, concluding that for $b_{g}<1$ the antiparallel reconnection rate is very weakly affected. This almost negligible reduction in reconnection rates for the low guide field regime $b_{g}<1$ was also confirmed later by a 2D PIC simulation evolving only from initial noise (Karimabadi et al. 2005).

Ricci et al. (2004) carried out simulations (with both implicit and explicit codes) of Harris CS with initial perturbation (similar to the GEM challenge ), finding this reduction for several guide field cases ranging from $b_{g}=0 \rightarrow 5$. The onset of reconnection was similar for a similar time in all the cases, however.

Pritchett and Coroniti (2004) also found in 3D PIC simulation a monotonous decrease in reconnection rates with guide field up to $b_{g}=5$, but different from the previous study, they noticed a delay in the reconnection onset for higher $b_{g}$. They carried out these simulation in the "whistler only regime" $\beta_{k} / 2 \ll 1$ and $\mu_{k} \ll 1$. A recent study confirming the same finding is Horiuchi et al. (2014). 


\section{Instabilities in CS: Theoretical background}

Because we will present only 2D simulation results (in the reconnection plane $x-y$ ), we review only the CS instabilities that can grow in this reduced geometry (ruling out instabilities propagating along the out-of-plane current direction $\hat{z}$ ).

\subsection{Tearing mode instability of CS}

The most fundamental instability of CS is the tearing instability. As we already mentioned in Chapter 4, this instability characterizes the build-up phase of magnetic reconnection. It slowly changes the magnetic field topology by forming magnetic islands, growing initially from perturbations like noise (thermal in real environments or numerical in simulations). It is called "tearing" because its growth leads to "tear" the CS. The tearing growth prepares the global topology for the fast release of energy in magnetic reconnection. As pointed out by Schindler (2007): "The situation is similar to phase transitions in equilibrium thermodynamics, where quasi-static changes drive the system toward the point at which the phase transition takes places spontaneously"

It is important to mention that although the field topology in the X points of these magnetic islands may resemble steady state magnetic reconnection (like in the Sweet-Parker model), the tearing instability is essentially a different process (see discussion in Schindler 2007, Sec. 11.2.10). Steady state magnetic reconnection is always forced: it is necessary to have a finite electric field at the boundaries to drive it (e.g.: the conductive electric field $\vec{V} \times \vec{B}$ due to the inflow). On the other hand, tearing is a spontaneous instability: it does not require such electric fields: all the components of $\vec{E}$ should be zero at the boundaries.

However, a theory that can predict the observed timescales of this process in space plasmas is difficult. This is because, as mentioned by Cassak and Shay (2011), the tearing instability is strongly dependent on the specific initial conditions and parameters of the system, which are mostly unknown. These can be the initial perturbation, resistivity, the presence of normal or guide magnetic fields, the initial thickness of the current sheet, the size of the system, the boundary conditions, the specific kinetic equilibrium, pressure anisotropies (and even numerical parameters such as the mass ratio in kinetic simulations), etc. Indeed, as written by Priest and Forbes (2000): "The tearing-mode instability appears to be a rather delicate creature, which can easily be switched by other effects (such as a flow, a normal magnetic field component, or a pressure anisotropy)....". This has lead to a very rich field of study since it was first proposed in the 60s by Furth et al. (1963). 


\subsubsection{Physical mechanism and fluid approach}

Let us discuss now the physical mechanism behind the development of the tearing instability in CS, first with a fluid approach. The magnetic field configurations given by the Harris Eq. (3.18) or force free solutions Eq. (3.25) or Eq. (3.42) are stable in the ideal MHD limit (due to the frozen-in condition or Alfvén theorem. See Sec. 2.5). In collision dominated plasmas, this configuration can become unstable to small perturbations by adding finite resistivity (Furth et al. 1963) in the form of electron collisions. These perturbations will propagate along the antiparallel magnetic field $B_{y}(x)$ with a wavevector $k_{y}$. In presence of an out-of-plane magnetic guide field, the antiparallel magnetic configuration Eq. (3.18) will be sheared, and the tearing perturbations will propagate in the direction normal to the equilibrium magnetic field (Priest and Forbes 2000):

$$
F:=\vec{k} \cdot \vec{B}=0
$$

the so called resonant layer. The instability condition is that the length scale gradient of the current $J_{7}$ has to be smaller that the typical length scale of the perturbations $k^{-1}$ (Zweibel and Yamada 2009). This requirement is necessary to overcome the stabilizing effect of the magnetic tension. The development of tearing mode driven by the current shear reduces the stored magnetic energy transforming it into ion bulk motion and overall heating. This growth of tearing mode is stopped when the magnetic islands width get larger than the resistive layer, due to the restoring Lorentz force $\vec{J} \times \vec{B}$.

In order to carry out the analytical analysis of tearing mode, Furth et al. (1963) divided the geometry of the problem in two domains. The first correspond to the (internal) region inside of the resistive layer, where it is necessary to solve the linearized resistive MHD equations, while outside (external region) the solution can be obtained by using the ideal (non-resistive) MHD approach. This is very similar to the boundary layer method used in hydrodynamic 1 . One can solve the equations independently in each region. If $\widetilde{\psi}_{1}(x)$ is the (Fourier transform of) perturbed vector potential in 2D geometry satisfying $\vec{B}=\nabla \psi$ for the in-plane components of the magnetic field (in our notation, $A_{z}=\psi$ ), the equation to be solved is of a Grad-Shrafanov type:

$$
\frac{d^{2} \widetilde{\psi}_{1}}{d x^{2}}-k^{2} \widetilde{\psi}_{1}=-\mu_{0} \widetilde{J}_{z 1}
$$

where $k=\left|k_{y} \hat{y}+k_{z} \hat{z}\right|$. By assuming a simplified form of the perturbed current $\widetilde{J_{1}}$ expressed in terms of $\bar{\psi}_{1}$, it is possible to express to get a second order differential equation in $\widetilde{\psi}_{1}$ in terms of $F$ given by Eq. 5.1)

$$
\frac{d^{2} \widetilde{\psi}_{e, 1}}{d x^{2}}-\left[k^{2}+\frac{1}{F} \frac{d^{2} F}{d x^{2}}\right] \widetilde{\psi}_{e, 1}=0
$$

Specializing to the Harris equilibria where $F=k_{y} B_{\infty} \tanh (x)$, one can find the following equation in the external region:

$$
\frac{d^{2} \widetilde{\psi}_{e, 1}}{d x^{2}}-\left[k_{y}^{2}-\frac{2}{\cosh ^{2}(x)}\right] \widetilde{\psi}_{e, 1}=0
$$

\footnotetext{
${ }^{1}$ However, in resistive MHD and different from the kinetic approach, the width of the internal region can only be known a posteriori, depending on the solution of the problem, turning out to be $\epsilon \sim \gamma^{1 / 4} /\left(k^{1 / 2} S^{1 / 4}\right)$
} 
Note that a constant out-of-plane guide field in the Harris equilibrium will not change this equation (but it will affect the internal solution). This has the form of a Schrödinger equation in a Teller potential (Galeev and Sudan 1984). Its (eigenfunction) solution is known from quantum mechanics, and after applying the appropriate boundary conditions (e.g.: tearing solutions are always of even parity and $\widetilde{\psi}_{e, 1}(\infty)=0$ ), it can be expressed in the more general form of a gradient of the vector potential evaluated at the center of the CS $x=0$ (jump across the resistive layer), called delta stability parameter

$$
\Delta^{\prime}:=\left[\frac{\psi_{e}^{\prime}(x)}{\psi_{e}(x)}\right]_{0^{-}}^{0^{+}}=\frac{1}{\widetilde{\psi}_{e, 1}(x=0)} \lim _{\epsilon \rightarrow 0}\left[\frac{d \widetilde{\psi}_{e, 1}}{d x}(\epsilon)-\frac{d \widetilde{\psi}_{e, 1}}{d x}(-\epsilon)\right]
$$

with the index $e$ indicates the external solution and ' the spatial derivative $d / d x$. For this Harris geometry, the solution of Eq. (5.4) can be expressed $\Delta^{\prime}=\frac{1-k_{y}^{2}}{k_{y}}$. This Delta $\Delta^{\prime}$ stability parameter represents the driver of tearing perturbations, a measure of the free energy stored in the magnetic field configuration (independent of the plasma model, valid even with fully-kinetic approaches), since is proportional to the gradient of $\vec{B}$, and thus proportional to the current $J$ via the Ampère's law. Because of that, note that small/large $\Delta^{\prime}$ regime refers to large/small $k_{y}$. In the framework of MHD, Furth et al. (1963) proved that tearing is unstable if and only if $\Delta^{\prime}>0$. This implies that only modes with $k_{y}<1$ will be tearing unstable. Note that all these expressions are in units of the halfwidth $x \rightarrow x / L$, a convenient choice since (ideal) MHD is scale-free.

For the internal solution $\psi_{i}(x)$, it is necessary to use the resistive MHD model and thus the equation to solve is much more involved than Eq. (5.4). In order to simplify the problem, the usual method is recurring to the so called constant $\psi$ approximation: to consider $\psi$ constant throughout the internal region, equivalent to have a perturbed current density $J_{z}$ constant (valid assuming the internal region width much smaller than the resistive skin depth $\sqrt{\eta / \gamma}$, and therefore inappropriate for thin CS or short-wavelengths).

Now, in order to get a dispersion relation $\gamma(k)$, the usual method 2 is matching the logarithmic derivatives of the external solution Eq. (5.5) with the internal solution (not shown here) in the limit of a resistive layer of infinite width (analogous to the Born approximation in quantum mechanics considering the constant $\psi$ approximation), thus assuring a smooth transition across the boundary, i.e.:

$$
\Delta^{\prime}=\left[\frac{\psi_{e}^{\prime}(x)}{\psi_{e}(x)}\right]_{0^{-}}^{0^{+}}=\lim _{x \rightarrow \infty} \frac{\psi_{i}^{\prime}(x)}{\psi_{i}(x)}
$$

By means of this method, the results obtained by Furth et al. (1963) showed that the growth rate of the most unstable mode is $\sim \tau_{A} S^{-3 / 5}$, while the aspect ratio between the width of the resistive layer compared and the total length of the system is $\sim S^{-2 / 5}$. Both results are similar to the obtained for steady state Sweet-Parker reconnection: $\sim \tau_{A} S^{-1 / 2}$ for both parameters. It is also interesting to mention the dependence of the growth rates on the wavevector: $\gamma \propto\left(1-k^{2}\right)^{4 / 5}$

\footnotetext{
${ }^{2}$ First applied to this problem by Furth et al. (1963). See further details in the textbooks Schindler (2007, Sec 10.3) or Priest and Forbes (2000, Sec. 6.2).
} 


\subsubsection{Kinetic approach}

The theory of resistive tearing mode continued being developed after Furth et al. (1963), extending these results by taking into account a host of different effects and geometries. However, most of the space plasmas are collisionless, as evidenced by their large magnetic Reynolds numbers (see Table 1.1), in such a way that any theory based in an MHD approach turns out to be invalid for their application to these environments. In this context, the first study of tearing mode using a collisionless kinetic approach was carried out by Coppi et al. (1966), as a proposed mechanism for magnetic reconnection in the Earth's magnetotail during substorms. They proved that this instability is due to a coupling between a negative energy perturbation (associated with the tearing of the CS) and the electrons energized by Landau resonance in a small region close to the neutral point in a CS (the aforementioned resonant layer $\vec{k} \cdot \vec{B}=0$ ), with typical size of $d_{i}$. As explained by Li et al. (2003), in this diffusive region magnetic perturbations induce electric fields. They produce, in turn, induced localized currents $J$ in a region close enough to the resonant layer $|x|<\Delta_{N S}$, because only there the electrons have Doppler frequency shift smaller than the growth rate $\gamma$ (Landau damping or Cherenkov interaction). The internal region,

$$
\Delta_{N S}=\sqrt{\left.2 \frac{v_{t h, e} m_{e}}{e} \frac{d x}{d B_{y}}\right|_{0}}=\sqrt{2 \rho_{e} L}
$$

is usually called electron singular layer thickness, where most of the electron perform "exotic" orbits (more precisely: "meandering" across the center of the CS). The last equality is valid assuming a Harris equilibrium where the magnetic field length scale is $\left.\frac{d x}{d B_{y}}\right|_{0}=L / B_{\infty y}$

Later, other works were completing the kinetic theory of tearing mode (Schindler 1974, Drake and Lee 1977b, Galeev 1979, Lembege and Pellat 1982), especially dealing with the stabilizing effect of a normal magnetic field to the CS, important for explaining the onset of substorms in the Earth's magnetotail. The idea is that tearing requires a normal (in-plane) magnetic field $B_{x}$ to be driven unstable, but it cannot be too high in order to not be stabilized, in such a way that will exist if and only if the following condition is satisfied (Galeev 1979, Treumann and Baumjohann 2013b):

$$
\frac{(k L)^{3}}{\left(1-(k L)^{2}\right)\left(1+T_{i} / T_{e}\right)}<\frac{B_{x}}{B_{\infty y}}<\frac{\left(1-(k L)^{2}\right)\left(1+T_{i} / T_{e}\right)}{\sqrt{\pi T_{i} / T_{e}}}\left(\frac{\rho_{i}}{L}\right)^{3 / 2}
$$

assuming $(k L)^{2}<\frac{L}{\rho_{i}} \lesssim 1$. The lower bound is due to Landau damping: the growth rates (as given, e.g., in Eq. (5.13)) have to overcome the ion gyration on this normal magnetic field $\gamma>\Omega_{c i} B_{x} / B_{\infty y}$, tending to stabilize the tearing mode. The upper bound is due to the electron magnetization: tearing islands cannot grow beyond the halfwidth $k L \sim 1$ because the electrons become gyrotropic. This stabilization issue if of central importance, because at the beginning, Coppi et al. (1966) proposed that only electrons are the resonant particles driving the tearing mode. Later, Schindler (1974) realized the upper bound in Eq. (5.8), concluding that since the electrons can easily become magnetized suppressing tearing, the ions should be the resonant particles driving the instability, with electrons acting simply as a background. But Galeev (1979) found that both species play an essential role: if one 
of them is stabilized by the conditions in Eq. (5.8), tearing mode can still exist by the resonance effects of the another specie. The controversy remained, however, due to the difficulty of treating analytically the response of both species in a dispersion relation.

All these works used the same matching method of inner and external solutions as the MHD approach given by Eq. (5.5), but with the internal (kinetic) solution obtained by solving the linearized Vlasov equation inside of $\Delta_{N S}$ (Drake and Lee 1977b, see). The solution of Vlasov equation requires the integration of the unperturbed orbits of the particles in the equilibrium magnetic field, the so called "characteristics method" (see details in the discussion of Eq. (2.19)). The first approximation was just using straight lines (Dobrowolny 1968). But later it was realized than this approximation is too simplistic to describe the complicated and chaotic orbits of the particles around the center of the CS (Büchner 1986, Büchner and Zelenyi 1991): some of the particles cross the center of the CS, other do not, besides of the existence of gyrotropic and meandering populations.

\subsubsection{Linear instability}

Now, let us talk about the main results obtained by these studies regarding the predicted growth rates of the collisionless tearing mode (details about these calculations can be found, e.g, in Schindler 2007, Sec. 10.4). An important assumption for the analytical solutions is choosing the mass ratio $m_{i}=m_{e}$, since the difference in the electron/ion response disappears, with the corresponding electrostatic effects due to charge separation neglected. This is equivalent to consider only one resonant specie (e.g. the ions) while the other (electrons) are added just as neutralizing background. The solution of the kinetic equations in the external region $x>\Delta_{N S}$ is the same as in the resistive MHD case by Furth et al. (1963), being given (in the Harris case) by Eq. (5.5) replacing $k_{y} \rightarrow k_{y} L$ to translate from the dimensionless units of MHD (and adding an extra factor $1 / L$ in the right hand side to compensate for the units of $d / d x$. Explicitly,

$$
\Delta^{\prime}=2 \frac{1-\left(k_{y} L\right)^{2}}{k_{y} L^{2}}
$$

Note that in this region the solution is simple because all particles have gyrotropic orbits, and thus the form of $\widetilde{J}_{z 1}$ in Eq. (5.2) is the same as in MHD.

But for the internal region $x<\Delta_{N S}$, the idea is to write the distribution function as a function of the vector potential $f=f\left(A_{1 z}\right)$ and for that it is necessary an exact kinetic Vlasov equilibrium, like the Harris one in the form Eq. 3.17). Then, one can get the perturbed current by integrating $f\left(A_{1 z}\right)$ via Eq. (2.21) to get $\widetilde{J}_{1 z}$. Thus it is possible to obtain a second order differential equation for the eigenfunctions $\widetilde{A_{1 z}}$, with solutions that can be found by assuming appropriate boundary conditions and the same constant $\psi$ approximation as in MHD. Finally, one matches with the previous external solution $\Delta^{\prime}$ via Eq. (5.6). By neglecting diamagnetic drifts (equivalent to thick CS $\Delta_{N S} \ll \rho_{i}, L$ ) and assuming $k L \lesssim 1$, this procedure followed by Laval et al. (1966), Drake and Lee (1977b), Quest and Coroniti (1981a) and Galeev and Sudan (1984) gives growth rates:

$$
\frac{\gamma}{k_{y} v_{t h, e}}=\frac{1}{2 \sqrt{\pi}} \frac{\Delta^{\prime}}{\Delta_{N S}} d_{e}^{2}
$$


This expression is geometrically general, in the sense that it is not only for Harris equilibria, but it can also be applied to any other geometry by knowing the specific form of the external solution encoded in $\Delta^{\prime}$, besides of the electron singular layer thickness $\Delta_{N S}$. It is also possible to derive a expression identical except by numerical factors just by a heuristic argument highlighting the physical mechanism of the tearing mode via Landau resonance (see Drake and Lee 1977b, for further details). Specializing to the Harris case with the external solution $\Delta^{\prime}$ given by Eq. (5.9), the full growth rates can be written in the following normalized way (Karimabadi et al. 2005):

$$
\frac{\gamma}{\Omega_{c i}}=\left(\frac{m_{e}}{m_{i}}\right)^{1 / 4}\left(\frac{T_{e}}{T_{i}}\right)^{5 / 4}\left(1+\frac{T_{i}}{T_{e}}\right) \frac{1-\left(k_{y} L\right)^{2}}{\sqrt{8}}\left(\frac{\rho_{i}}{L}\right)^{5 / 2}
$$

Note that this expression is not valid close to $k L=0$ or $k L=1$ (marginal stability) and the maximum growth rate is reached at $k L=0.5$. These properties are very similar to the ones obtained with the resistive MHD solution by Furth et al. (1963). In this context it is interesting to mention that the first fully-kinetic PIC simulations of collisionless tearing mode trying to test the prediction Eq. (5.11) was carried out by Katanuma and Kamimura (1980).

However, we would like to have a more accurate expression, valid also for thin CS, the purpose of this thesis. In this context, Pritchett et al. (1991) found an equation for $\widetilde{A_{1 z}}$ in the inner region $x<\Delta_{N S}$ including diamagnetic drift effects on the particle orbits, being thus suitable for thin CS (since they are related by Eq. (3.20) or Eq. A.4)):

$$
\frac{d^{2} \widetilde{A}_{1 z}}{d x^{2}}-\left[k_{y}^{2}-\frac{2-2 \gamma M\left(1+4 \rho_{i}^{2} / L^{2}\right)}{L^{2} \cosh ^{2}(x / L)}\right] \widetilde{A}_{1 z}=0
$$

with $M=\sqrt{\pi} L^{2} /\left(4 \rho_{i}^{2} v_{t h, e} k_{y}\right)$ and assuming $T_{i}=T_{e}$. Analytical expressions can be found in the limit of thick $C S$, with $\Delta_{N S} / L \ll 1$ (equivalent to $L / \rho_{i} \gg 1$ ). If one additionally assumes $\gamma M \sim \sqrt{L / \Delta_{N S}},(k L)^{2}<1$, and the same constant $\psi$ approximation as in MHD, it is possible to perform an expansion in terms of $\sqrt{\Delta_{N S} / L}$. Then, matching with the known external solution via Eq. (5.6), it is possible to obtain (to a first order) the following dispersion relation:

$$
\frac{\gamma}{\Omega_{c i}}=\sqrt{\frac{\pi}{8}}\left(\frac{\sqrt{2} \rho_{i}}{L}\right)^{5 / 2}\left(1-\left(k_{y} L\right)^{2}\right) .
$$

This expression is very similar to Eq. 5.11), except by some numerical factors. On the other hand, for the more interesting case of thin $C S \Delta_{N S} / L \approx 1$, a simple expansion in $\sqrt{\Delta_{N S} / L}$ is not possible. But by making the change of variable $\zeta=\tanh (x / L)$ in Eq. 5.12), this expression becomes the Legendre equation, with known solutions. In this case, the final result for the dispersion relation can be written as (Pritchett et al. 1991):

$$
\frac{\gamma}{\Omega_{c i}}=\frac{2 \sqrt{2}}{\sqrt{\pi}}\left(\frac{\rho_{i}}{L}\right)^{3} \frac{k_{y} L\left(2+k_{y} L\right)\left(1-k_{y} L\right)}{1+4\left(\frac{\rho_{i}}{L}\right)^{2}}
$$

Note that for $\Delta_{N S} / L \approx 1$, the maximum growth rates given by this expression, as can be expected, are much larger than for thicker CS Eq. (5.13). Also, the maximum growth 
rate is shifted towards values a little bit higher than these of thick CS: $k L \approx 0.55$. Some years after this expression was obtained, Brittnacher et al. (1995) extended the solution of Eq. (5.12) for arbitrary thickness of the $C S \Delta_{N S} / L$, although in the following implicit form:

$$
\frac{\Delta_{N s}}{L} Z\left(\frac{\gamma}{k_{y} \sqrt{2} v_{t h, i}}\right)=\frac{1}{\sqrt{\pi}}\left(\frac{d_{i}}{L}\right)^{2} \frac{\left(2+k_{y} L\right)\left(1-k_{y} L\right)}{1+4\left(\frac{\rho_{i}}{L}\right)^{2}}
$$

where $Z$ is the plasma zeta function (Fried and Conte 1961):

$$
Z\left(\xi_{\alpha}\right)=\frac{1}{\sqrt{\pi}} \int_{-\infty}^{\infty} \frac{\exp \left(-x^{2}\right)}{x-\xi_{\alpha}} d x, \quad \operatorname{Im}\left(\xi_{\alpha}\right)>0
$$

with argument:

$$
\xi_{\alpha}=\frac{\omega}{k \sqrt{2} v_{t h, \alpha}}=\frac{\omega_{r}+i \gamma}{k \sqrt{2} v_{t h, \alpha}}
$$

All the previous results so far were based on the assumption of $m_{i}=m_{e}$, equivalent to one resonant specie. When this assumption is lifted, the perturbations cannot be described any longer by a single function $A_{3}$. since it is also necessary to consider the electrostatic perturbations $\phi$. This also introduces additional complications, such as in-plane electric fields due to charge separation. In this case one has to rely on numerical solutions of the linearized Vlasov equation to find the particle orbits, besides of solving the eigenfunction problem given by the integro-differential Maxwell equations Eq. (5.2) for $A_{z 1}$ (same as in the limit $m_{i}=m_{e}$ ) and the Poisson equation for $\phi$ and $\rho_{c}$ (electric charge). By means of a Hermite expansion of the eigenfunctions, Daughton (1999) (see also Daughton 2003) implemented a solver of this kind, finding that for realistic mass ratios $m_{i}=1836 m_{e}$, the maximum growth rates predicted by Eq. (5.15) are reduced by a factor of 1.5 to 2 . This agrees with earlier studies about the stabilizing electrostatic effects on the tearing mode in an antiparallel configuration (Hoshino 1987). It is interesting to mention that the previous study also found that for strong guide fields, the electrostatic effects enhance tearing mode growth rates. Daughton (1999) also showed that tearing mode growth rates are practically unchanged when in a Harris CS is superimposed a stationary background population. Silin et al.(2002) also investigated the tearing mode, among other CS instabilities, for $m_{i} \neq m_{e}$ including both electromagnetic and electrostatic perturbations, although in the large wavelength limit and assuming straight-line particle orbits, confirming the finding of Daughton (1999).

\subsubsection{Non-linear evolution: coalescence, size and saturation of tearing islands}

The coalescence of neighboring magnetic tearing islands can be understood intuitively due to the attraction of parallel currents in their $\mathrm{O}$ points. However, a theoretical description is much more involved, because it is part of the non-linear development of tearing

\footnotetext{
${ }^{3}$ Note that this $A_{z}$ component of the vector potential is the only one relevant for the tearing mode instability analysis. It has been shown, by means of kinetic simulations by Daughton (1999) that the in-plane components $A_{x}$ and $A_{y}$ are usually negligible, even in $3 \mathrm{D}$ geometries.
} 
mode, depending on many other factors (see further details in Priest and Forbes (2000, Sec. 6.5.3) for the MHD case and Karimabadi et al. (2005) for a discussion about this issue in kinetic PIC simulations). Something similar happens with their saturation level: even much before of reaching this stage, tearing mode growth rates are severely affected by a host of non-linear effects (see Biskamp 2000, Sec. 4.1.2). Both processes are closely related with the growth and limit size of these magnetic islands when they reach the linear marginal stability condition $k L \sim 1$ (see, e.g., Eq. (5.13)). Nevertheless, some simple arguments allows to understand the width of these magnetic islands and their relation with the reconnected flux. Indeed, following Karimabadi (2005) (based on Drake and Lee (1977a). See also the textbook Bellan (2006, Sec. 12.6)), during the linear stage where the islands are small enough, one can assume a sinusoidal perturbation due to the tearing mode in the normal direction to the asymptotic magnetic field: $\delta B_{x} \exp \left(-i\left(\omega t+k_{y} y\right)\right)$, while the vector potential due to a Harris reversal magnetic field can be approximated by a quadratic dependence on $x / L$ if the islands are small enough, in such a way that:

$$
A_{z}(x, y)=A_{z, \text { harris }}(x)+\delta A_{z}(y)=-\frac{B_{\infty y}}{2 L} x^{2}-\frac{\delta B_{x}}{k_{y}} \cos \left(k_{y} y\right)
$$

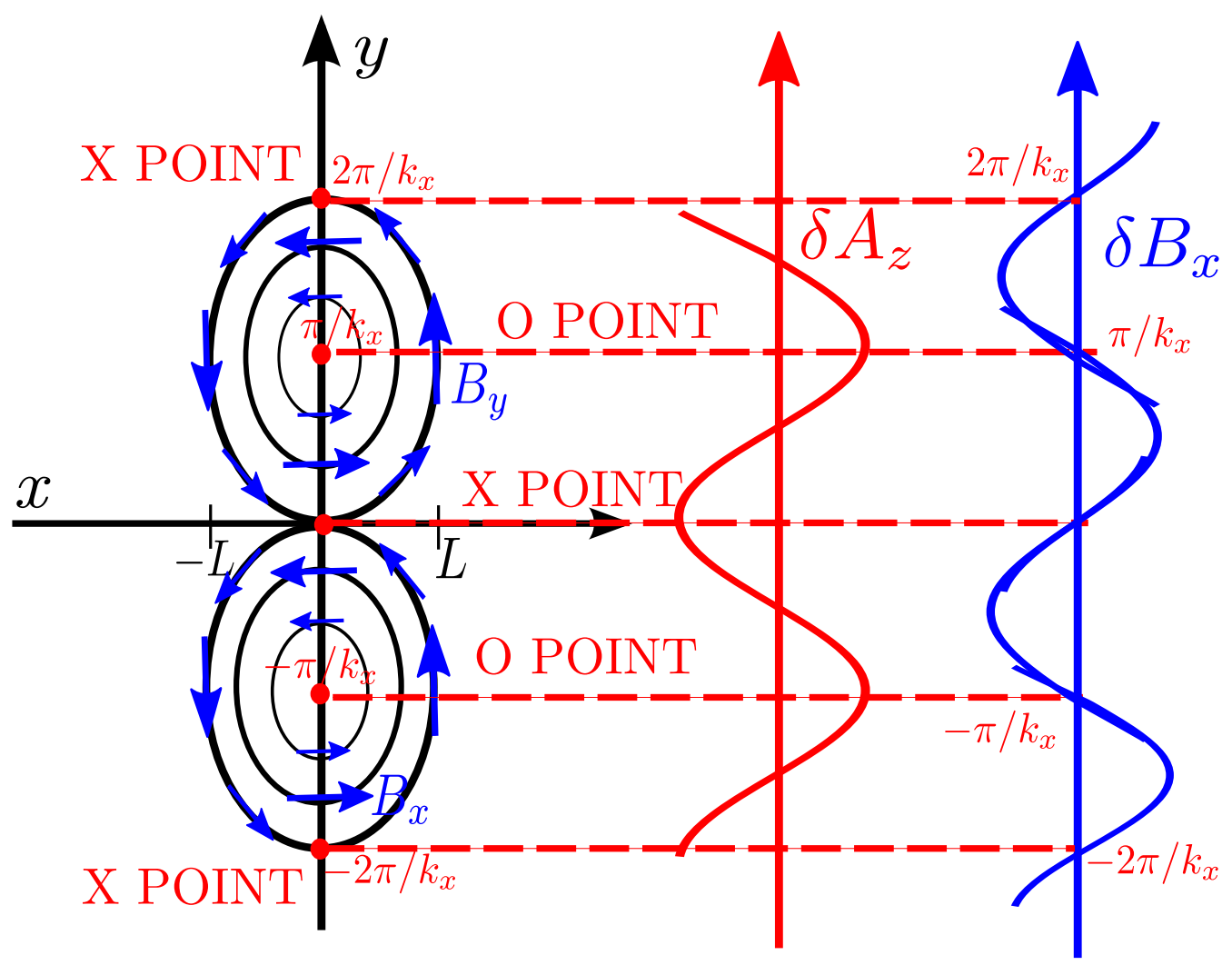

Figure 5.1: Scheme of tearing islands with the vector potential Eq. (5.18). Note that both $X$ and $O$ points satisfy $\delta B_{x}=B_{y}=0$, but in the $X$ points the perturbed potential $\delta A_{z}=0$ is a local minimum, while in the $O$ points is a local maximum.

With this simplified model, the $\mathrm{X}$ point will be located at the origin $(x, y)=(0,0)$ and thus $A_{z}(x)=-\delta B_{x} / k_{y}$, while the nearest $\mathrm{O}$ point will be located at $(x, y)=\left(0, k_{y} \pi\right)$ and 
thus $A_{z}(O)=\delta B_{x} / k_{y}$. Then, the reconnected flux will be given by $\psi=2 \frac{\delta B_{x}}{k_{y}}$. Since in 2D the magnetic field lines will be given by $A_{z}=$ constant, we have the equation for the magnetic field lines which pass through the $\mathrm{X}$ points:

$$
\frac{2}{k_{y} L} \frac{\delta B_{x}}{B_{\infty y}}\left(1-\cos \left(k_{y} y\right)\right)=\left(\frac{x}{L}\right)^{2}
$$

Therefore, the halfwidth $W_{s}$ (across $\mathrm{x}$ ) of the magnetic islands is given by the distance along $x$ for which $y=\pi / k_{y}$. Replacing in the previous equation, we get:

$$
\frac{W_{s}}{L}=2 \sqrt{\frac{\delta B_{x} / B_{\infty y}}{k_{y} L}}=\sqrt{\frac{2 \Psi}{B_{\infty y} L}}
$$

Thus, during the course of tearing evolution, the island halfwidth will increase as the square root of the normal magnetic field $\delta B_{x}$ or the reconnected flux $\psi$. Since the most unstable tearing mode will have $k_{y} L \approx 0.5$, an island of this size will have a width $\left|\frac{W_{s}}{L}\right|_{\max }=$ $2 \sqrt{2 \delta B_{x} / B_{\infty y}}$. For completeness (although straightforward from the definition) the height of the tearing islands $H_{\alpha}$ (along $y$ ) will be given by the distance between neighboring $\mathrm{X}$ points:

$$
\frac{H_{\alpha}}{L}=\frac{2 \pi}{k_{y} L}=\frac{\lambda_{y}}{k_{y}}
$$

For the most unstable tearing mode, an island of this size will have a height $\left|\frac{H_{\alpha}}{L}\right|_{\max }=$ $4 \pi \approx 12.6$. Note that the tearing islands will be much more elongated along $y$ than $x$, since $\delta B_{x} / B_{\infty y} \ll 1$. From this simple model we can find a physical explanation for the electron singular layer width $\Delta_{N_{\mathrm{c}}}$ and the stabilization of tearing mode due to trapping of electrons (as first proposed by Drake and Lee 1977a). Indeed, in order for an electron to be trapped in closed orbits inside of the magnetic islands, we require that its gyroradius on the perturbed normal magnetic field,

$$
\delta \rho_{e}=\frac{m_{e} v_{t h e}}{e \delta B_{x}}=\frac{\Delta_{N s}^{2}}{2 L \delta B_{x} / B_{\infty y}},
$$

has to be less than the half-height of the island $\delta \rho_{e} \lesssim H_{\alpha} / 2=\pi / k_{y}$. Note that during the tearing evolution, $\rho_{e}$ decreases due to the increase of $\psi$ and so $\delta B_{x}$. Therefore, the minimum island halfwidth required to keep the electrons trapped inside can be obtained by using Eq. (5.20) and the previous requirement, turning out to be on the order of the electron singular layer width (Karimabadi 2005):

$$
\frac{\left|W_{s}\right|_{\text {trapping }}}{L}=\sqrt{\frac{2}{\pi}} \Delta_{N s} \sim 1.13 \sqrt{\frac{\rho_{e}}{L}}
$$

Tearing islands larger than $\left|W_{s}\right|_{\text {trapping }}$ will contain mostly gyrotropic electrons, and thus tearing mode will get suppressed: $\Delta_{N s}$ represents the maximum allowed size. This electron trapping is the most known saturation mechanism for the tearing instability (see further details in Drake and Lee (1977a) and in the textbook Biskamp (2000, Sec. 7.5.2)). 
On the other hand, Eq. (5.23) also allows to estimate, by combining with Eq. 5.20), the maximum amount of reconnected flux before tearing gets saturated:

$$
\frac{\psi}{B_{\infty y} d_{i}}=\frac{1}{2} \frac{\Delta_{N s}^{2}}{L d_{i}}=\frac{2}{\pi} \frac{\rho_{e}}{d_{i}}=\frac{2}{\pi} \frac{v_{t h, e}}{c} \frac{\omega_{p e}}{\Omega_{c e}}
$$

for $T_{i}=T_{e}$. In a Harris CS, due to the constraint Eq. A.7), the last equality becomes $0.5 \sqrt{m_{e} / m_{i}}$. This also imposes a severe constraint in any simulation of tearing mode: the initial numerical noise has to be lower than this quantity, a stringent requirement if more realistic (higher) mass ratios are used. However, the evolution of reconnection can proceed beyond this limit without too much trouble, as it has been demonstrated by means of PIC simulations (Pritchett2005a).

Another recent proposed saturation mechanism for the tearing instability is due to temperature ani sotropies. The non-linear evolution of the tearing mode leads to a parallel $\left(k_{y}\right)$ heating of electrons, due to the Landau resonance (transfer of energy from the tearing wave field to the particles). It can be shown than this effects lead to a maximum island halfwidth of (Treumann and Baumjohann 2013b):

$$
\frac{\left|W_{s}\right|_{\text {anisotropy }}}{L} \sim 3 \frac{\rho_{e}}{L}
$$

It has been shown by Karimabadi (2005) that this process will dominate over electron trapping for islands of size around $\Delta_{N s}$ for most of the parameter range found in realistic plasmas, allowing a larger saturation amplitude (compare with Eq. (5.23)). In general, temperature anisotropy (due to preferential electron parallel heating) will be the dominant saturation mechanism for thin CS and large mass ratios. They found that electron trapping becomes important for saturation of tearing only when the temperature anisotropy is suppressed. This can be caused, e.g., by excitation of Weibel instability producing turbulence and the consequent pitch angle scattering isotropizing the distribution function.

\subsubsection{Influence of a guide field}

The presence of an out-of-plane magnetic guide field alters dramatically the particle orbits in a current sheet: they become gyrotropic. Consequently, the dispersion relation and associated growth rates describing this instability will change accordingly (since they will modify the particle response in the linearized Vlasov equation). This depends, however, on the guide field strength $B_{g}$. The first studies about guide field tearing by Drake and Lee (1977b), Quest and Coroniti (1981b) argued that if $\rho_{e}$ based on $B_{g}$ is smaller than $\Delta_{N S}$, the electrons inside of the singular layer will still be unmagnetized, with their orbits determined by $B_{\infty y}$ like in the antiparallel case. Therefore, a thermal electron will be affected significantly by the guide field when $\rho_{e, B_{c}}=m_{e} v_{t h, e} /\left(e B_{c}\right)=\Delta_{N S}$, with the critical guide field $B_{c}$ expressed as (Daughton 2005):

$$
\frac{B_{c}}{B_{\infty y}}=\frac{1}{\sqrt{2}}\left(\frac{\rho_{i}}{L}\right)^{1 / 2}\left(\frac{T_{e} m_{e}}{T_{i} m_{i}}\right)^{1 / 4}
$$

This quantity, measuring how much the electron orbits will be modified by the guide field, allows to identify different three guide field regimes and their respective tearing growth rates dependence on $B_{g}$. 
- Weak: $B_{g}<B_{c}$.

- Intermediate: $B_{c}<B_{g}<3 B_{c}$. The fastest growing modes will have a component in the current direction $k_{z}$ : a drift-tearing mode (Daughton 2005). In the other two limits (weak and strong), the fastest tearing mode always is in the reconnection plane $\left(k_{z}=0\right)$

- Strong: $B_{g}>3 B_{c}$.

The " 3 " in the last relation is in order to magnetize practically all the electrons (since $B_{c}$ will magnetize $v \lesssim v_{t h, e}$, roughly $68 \%$ of the total electron population, while $3 B_{c}$ will magnetize $v \lesssim 3 v_{t h, e}$, roughly 99.7\%). The last expression for the growth rates also indicates the stabilizing effect of a strong enough guide field. However, the dependence is weak, and tearing instability can still exist for a large range of guide fields. For weak guide fields, Daughton (2005) demonstrated via 2D PIC simulations that a change from $B_{g}=0$ to $B_{g}=B_{\infty y}$ can reduce growth rates by a factor of 3.75 .

In the strong guide field limit, Drake and Lee (1977b) derived an expression analogous to Eq. 5.10) for the strong guide field case, assuming additionally $\Delta_{G F} \gg \rho_{e}$ (with $\Delta_{G F}$ to be defined in Eq. (5.39)), equivalent to low beta plasmas $\beta_{e} \ll \rho_{e} / L$. Later, Quest and Coroniti (1981b) lifted that assumption, allowing finite $\beta$ plasmas $\beta_{e} \sim 1$ finding the expression

$$
\frac{\gamma}{k_{y} v_{t h, e}}=\frac{1}{2 \sqrt{\pi}} \frac{\Delta^{\prime}}{l_{s}} d_{e}^{2}
$$

with $l_{s}$ the shear scale of the magnetic field:

$$
l_{s}=\left(\left.\frac{1}{B_{z}} \frac{d B_{y}}{d x}\right|_{0}\right)^{-1}
$$

For the Harris case, $l_{s}=L B_{g} / B_{\infty y}$, and thus:

$$
\frac{\gamma}{k_{y} v_{t h, e}}=\frac{1}{2 \sqrt{\pi}} \frac{\Delta^{\prime}}{L} \frac{B_{\infty y}}{B_{g}} d_{e}^{2}
$$

It is interesting to mention that the growth rates in antiparallel reconnection Eq. 5.10) will become equal to these on the guide field Eq. (5.27) when $l_{s}=\Delta_{N S}$, which for a Harris equilibrium becomes

$$
\frac{B_{g}}{B_{\infty}}=\sqrt{\frac{2 \rho_{e}}{L}} .
$$

Eq. (5.29) can be specialized to the Harris case by using the external solution for $\Delta^{\prime}$ in Eq. (5.9), being possible to write it in the form (Karimabadi et al. 2005):

$$
\frac{\gamma_{G F}}{\Omega_{c i}}=\left(\frac{m_{e}}{m_{i}}\right)^{1 / 2}\left(\frac{T_{e}}{T_{i}}\right)^{3 / 2}\left(1+\frac{T_{i}}{T_{e}}\right) \frac{1-\left(k_{y} L\right)^{2}}{\sqrt{\pi}} \frac{B_{\infty y}}{B_{g}}\left(\frac{\rho_{i}}{L}\right)^{3}
$$

Thi s inverse dependence on the growth rates of the tearing mode vs the guide field strength is theoretically expected, as it has been shown in many previous simulation studies, such 
as Pritchett et al. (1991). Note that all the previous discussions and analytical formulas in presence of a guide field have been about the electron tearing mode. Büchner et al. (1991) showed that an ion tearing mode can become unstable for large values of $B_{g}$. Much later, numerical solutions of the linearized Vlasov equation by Karimabadi et al. (2005) have shown a slightly weaker dependence of the growth rates on the guide field than the predicted by Eq. 5.31) $: \gamma \propto\left(B_{g} / B_{\infty y}\right)^{-2 / 3}$. But even recently, the approximation Eq. (5.31) has been used as an analytical benchmark for guide field tearing mode (see, e.g. Lin et al. 2011, Wang et al. 2011).

\subsubsection{Fluid approach in the low beta limit}

A kinetic (Vlasov) approach is not the only way of obtaining tearing mode growth rates for collisionless plasmas, where only electron inertia provides the mechanism for the violation of frozen-in condition. Fluid models can also be used, especially in the limit of strong guide field. In this sense, Porcelli (1991) derived tearing growth rates under the assumption of a semi-kinetic approach for ions (first order finite Larmor radius effects) and a fluid treatment (or drift-kinetic) for electrons by using the generalized Ohm's law (see Sec. 2.2.1). This requires $\rho_{s} \gg d_{e}$, with $\left.\rho_{s}=\left(v_{t h, i}+v_{t h, e}\right) / \sqrt{m_{i} / m_{e}}\right) / \Omega_{c i}$ the ion sound Larmor radius (by Eq. (4.7) and the relations in Appendix A.3.1, this is equivalent to $\beta \gg 2 m_{e} / m_{i}$ ). In this region, the electrons behave as an isothermal, non-adiabatic fluid. Besides of that, it is also necessary to assume $\gamma \gg k_{\|} c_{s}$ (no sound-wave coupling), equivalent to $\beta \ll 2\left(m_{e} / m_{i}\right)^{1 / 4}$ (Pueschel et al. 2011). These authors obtained approximations for both small and large $\Delta^{\prime}$ parameter regimes. The last one corresponds to the mode $m=1$ in the terminology used in magnetically confined plasmas. Both expressions are particular cases of the (implicit) dispersion relation derived later by Rogers et al. (2007) (with the purpose of studying gyrokinetic simulations of magnetic reconnection. See Sec. 9.1.2 for further details):

$$
\frac{\gamma \tau_{A}}{k_{y} \rho_{s}}=\left[\frac{2}{\pi} \frac{d_{e}}{\rho_{s}}\left(1-\frac{\pi}{d_{e} \Delta^{\prime}} \frac{\gamma \tau_{A}}{k_{y} \rho_{s}}\right)\right]^{1 / 3}
$$

In the small $\Delta^{\prime}$ parameter regime (wide magnetic shear length scale or large normalized wavenumber $k_{y} \rho_{s}$ ), it can be shown that this relation is identical to the fully-kinetic dispersion relation for thick CS on guide field Eq. (5.27), except by numerical factors and $T_{i}=T_{e}$, confirming the suitability of the fluid model in this regime. On the other hand, in the large $\Delta^{\prime}$ parameter regime (narrow magnetic shear length scale or small normalized wavenumber $k_{y} \rho_{s}$ ), the growth rates can be written as (Rogers et al. 2007, Pueschel et al. 2011):

$$
\frac{\gamma}{k_{y} v_{t h, e}}=\left(\frac{2}{\pi}\right)^{1 / 3} \frac{d_{e}}{l_{s}}\left(\frac{\beta_{e} m_{i}}{2 m_{e}}\right)^{-1 / 6}\left(1+\frac{T_{i}}{T_{e}}\right)^{1 / 3}
$$

\subsubsection{Tearing growth rates in a linear force free configuration}

Bobrova et al. (2001) worked out the linear tearing theory for the sheet pinch force free equilibrium given by the (sinusoidal) magnetic field Eq. (3.25). The $\Delta^{\prime}$ parameter 
Eq. (5.5) for this geometry in the external region is:

$$
\Delta^{\prime}=-2 \sqrt{1-\kappa^{2}} \frac{\cos \left(\sqrt{1-\kappa^{2}} \pi\right)}{\sin \left(\sqrt{1-\kappa^{2}} \pi\right)}
$$

with $\kappa=k_{y} / \alpha$. For the internal region, it is necessary to consider the corresponding (anisotropic) distribution function Eq. (3.27), since the particle orbits will be greatly modified compared to the Harris case with guide field. Finally, matching external and internal solution via Eq. (5.6), the dispersion relation is:

$$
\gamma=2 \frac{1-\cos \left(\sqrt{1-\kappa^{2}} \pi\right)}{\sin \left(\sqrt{1-\kappa^{2}} \pi\right)} \kappa \sqrt{1-\kappa^{2}}\left(d_{e} \alpha\right)^{2} \alpha v_{t h, e}
$$

Later, Li et al. (2003) corrected the previous result, finding a smaller $\gamma$ by a factor $2 \sqrt{\pi}$. This expression implies that only modes with wavelength greater than $2 \pi / \alpha$ (shear magnetic field scale) are unstable. But there is no upper limit. This unphysical results indicates additional process not taken into account for large wavelength (or small $k$ ), that should keep finite growth rates.

\subsubsection{Tearing growth rates in non-linear force free configuration}

A two fluid treatment of the tearing instabilities in force free magnetic configuration Eq. (3.42) was worked out by Mirnov et al. (2004), in the limit of strong guide field $B_{z}=B_{G}=$ constant. However, in these case the mechanism that breaks the frozen in condition is both the electron inertia (a collisionless mechanism) and the resistivity. Ahedo and Ramos (2009) extended that work by including finite guide fields, but also considering massless electrons. They identified six different parameter regimes giving explicitly the corresponding dispersion relations and growth rates, although in the limit of small $\Delta^{\prime}$. Recently, Liu et al. (2013) analyzed the stability properties for the force free magnetic configuration Eq. (3.42) and Maxwellian distribution function with a fully kinetic treatment in a 3D geometry. By solving Eq. (5.3) for this geometry 4 , they found the following $\Delta^{\prime}$ parameter:

$$
\Delta^{\prime} \approx 2 \frac{1+b_{g}^{2} \tan ^{2} \theta-(k L)^{2}}{k L^{2}}
$$

where the tearing mode is assumed to have a wavevector $\vec{k}=k_{y} \hat{y}+k_{z} \hat{z}$ with the angle $\theta=\tan ^{-1}\left(k_{z} / k_{y}\right)$. The resonance layer is located at $x_{0} / L=-\tanh ^{-1}\left[\sqrt{1+b_{g}^{2}} \sin \theta\right]$. This expression becomes identical to the Harris case Eq. (5.9) if no out-of-plane tearing propagation is allowed $\left(k_{z}=0\right)$. The growth rate is then found by the matching of internal to external solutions in this guide field case: the Eq. (5.27) obtained by Drake and Lee (1977b):

$$
\frac{\gamma}{k v_{t h, e}} \approx \frac{d_{e}^{2} \Delta^{\prime}}{2 \sqrt{\pi} l_{s}\left[1+\sqrt{m_{e} T_{e} /\left(m_{i} T_{i}\right)}\right]}
$$

\footnotetext{
${ }^{4}$ Note that this is an approximation: it is necessary to match asymptotic solutions in the limit of large and small $k$. See Baalrud et al. (2012) for details. For the case of Harris sheet with guide field and allowing obliquely propagating tearing modes, see Daughton et al. (2011b).
} 
with $l_{s}$ is the shear magnetic field strength, generalization of Eq. $(5.28)$ to this $3 \mathrm{D}$ geometry:

$$
\frac{1}{l_{s}}=\frac{1}{k}\left(\frac{d k_{\|}}{d x}\right)_{x=x_{0}}=\frac{\cos ^{2} \theta-b_{g}^{2} \sin ^{2} \theta}{L \cos \theta \sqrt{1+b_{g}^{2}}}
$$

with $k_{\|}=\vec{k} \cdot \vec{B} / B$. Again, we recover the result Eq. (5.29) in case of an in-plane propagating tearing mode $\vec{k}=k_{y} \hat{y}$.

\subsubsection{Saturation mechanism in the presence of a guide field}

Now, let us talk about the saturation mechanism in presence of a guide field, in general much less understood than in the antiparallel case. If it is strong enough, electrons become highly gyrotropic, inhibiting any saturation mechanism related to temperature anisotropies. Then, only electron trapping can be responsible for tearing mode saturation. Drake and Lee (1977b) and Quest and Coroniti (1981b) showed that the region where this effect can take place, different from the antiparallel case $\Delta_{N S}$, becomes significantly modified in presence of a guide field, depending not only on the geometry, but also on the growth rates and wave vector number:

$$
\Delta_{G F}=\frac{\gamma_{G F} l_{s}}{k_{y} v_{t h, e}}=\frac{\gamma_{G F} L}{k_{y} v_{t h, e}} \frac{B_{g}}{B_{\infty y}}
$$

where the last equality is valid assuming Harris equilibria by using Eq. 5.28).

Later, Karimabadi (2005) showed that in the strong guide field regime, this mechanism leads to a maximum tearing island amplitude (halfwidth) comparable to the predicted in the antiparallel case due to temperature anisotropy (Eq. (5.25), unless the guide field is too strong $B_{g} \gg B_{\infty y}$ ), and larger than the one due to electron trapping Eq. (5.23)

$$
\frac{\left|W_{s}\right|_{\text {trapping,GF }}}{L} \sim 1.8 \frac{\rho_{e}}{L}
$$

This value is higher than the calculated by previous works (Drake and Lee 1977b), due to the inclusion of finite Larmor radius effects. But it is still too low to explain the observed saturation amplitudes of tearing mode in the magnetosphere (Karimabadi et al. 2004, Karimabadi 2005, Karimabadi et al. 2005). These authors proposed another mechanism: multimode tearing, allowing the magnetic islands to grow to much larger amplitudes to these predicted by single mode tearing.

\subsubsection{Influence of anisotropy}

Different effects can affect the evolution of the tearing mode. In our simulations of Harris CS (to be shown in Chapter 7), one of these effects, the pressure anisotropy, will turn out to be essential to explain the stabilization of tearing mode. That is why we are going to describe its theoretical framework in this subsection.

The first studies of the temperature ani sotropy effects on the tearing mode by Laval and Pellat (1967) and Forslund (1968) found that the instability is stabilized if the electron parallel 
temperature (along the tearing direction $y$ ) is greater than the perpendicular ones, with the following threshold:

$$
A_{e}=\frac{T_{e, \|}}{T_{e, \perp}} \gtrsim\left(1-\frac{\sqrt{2} \rho_{e}}{L}\right)^{-1} .
$$

Note that this threshold is more restrictive (higher) for thin CS (small $L / \rho_{e}$ ). The reason is because an electron anisotropy will drive unstable a Weibel instability (see discussion in Sec. 5.2.1) in a CS with a fast growth rate $\gamma / \Omega_{c e} \sim\left(1-1 / A_{e}\right)^{5 / 4}$ when the following conditions is satisfied:

$$
A_{e}=\frac{T_{e, \|}}{T_{e, \perp}} \gtrsim\left(1-\left(\frac{\sqrt{2} \rho_{e}}{L}\right)^{2}\right)^{-1} .
$$

This implies that in thin CS $\rho_{e} / L \ll 1$, Weibel becomes unstable always for lower anisotropy levels than the ones required for tearing stabilization. Providing $A_{e}>1$, or $T_{e, \|}>T_{e, \perp}$, this mode will propagate perpendicular to the magnetic field ("colder" direction $\perp$, across the CS in $x$ ) and it is damped and does not propagate along the warmer direction $y=\|$. Following Karimabadi et al. (2004) and Karimabadi et al. (2005), this direction is the same as the propagation of tearing mode $k_{y}$. Then, they can couple 5 and the resulting "hybrid" mode gets damped as well, besides of a reduction in the range of unstable wavenumbers $k_{y} L$. On the other hand, under opposite conditions of anisotropy, $A_{e}<1$, Forslund (1968) found that tearing growth rates will be greatly enhanced, besides of extending the range of unstable wavenumber $k_{y} L$ (usually between 0 and 1 in the standard isotropic case), and thus allowing the interaction of multiple tearing modes, increasing the saturation level (Karimabadi et al.2005). In this case, both Weibel and tearing instabilities have wavevectors along $k_{y}$ (since now the "colder" temperature is $T_{e, \|}$ ). In addition, this enhanced tearing mode will shift its fastest growing mode towards shorter wavelengths (higher $k_{y}$ ). This anisotropy enhanced tearing mode has been observed in 2D PIC simulations with an initially imposed ani sotropy by Karimabadi et al. (2004) and Haijima et al. (2008). The latter work also noticed a critical halfwidth $L_{c}$, below which this boosted tearing instability can transfer energy towards large wavelengths mode during the non-linear evolution.

Note that the evolution of tearing mode in a CS leads naturally to a preferential electron heating along the parallel direction, developing anisotropies $A_{e}>1$. This is because the tearing mode develops an electric field along its propagation direction: $E_{y}$, allowing free streaming of electrons parallel to the asymptotic magnetic field $\left(B_{\infty v}\right)$, resulting in an overall increase in the electron heating $T_{e, \|}$ (Karimabadi et al. 2004). Hirabayashi and Hoshino (2013) argued this preferential heating should also take place in a CS as a consequence of the CGL equations Eq. (2.58): the reduction in the magnetic field strength as the reconnection proceeds (due to conversion into other forms of energy) produces a drop in $T_{e, \perp}$ because of the second adiabatic invariant (magnetic moment), while an increase in $T_{e, \|}$ due to the conservation of the first one, with a final result of $T_{e, \|}>T_{e, \perp}$. Therefore, if these anisotropies get larger than the threshold Eq. 5.41), the tearing mode will tend to suppress itself during the course of its evolution. This is in someway opposite to the

\footnotetext{
${ }^{5}$ note that this is valid only in an antiparallel geometry. A guide field will suppress these effects, because it make electrons gyrotropic inhibiting the Weibel instability. Then, guide field tearing mode it is expected to be weakly affected by the presence of anisotropies (Karimabadi et al. 2005).
} 
conclusion about enhancing of tearing growth rates by Vainshtein and Mazur (1982), being thus one of the mechanisms of saturation of tearing instability (see Sec. 5.1.2.2). If Weibel instability is triggered as cause of this anisotropy, it can produce pitch angle scattering tending to isotropize again the electron distribution function, counteracting tearing and allowing saturation via electron trapping, at much lower amplitudes.

However, the destabilizing effect of electron temperature anisotropies $A_{e}<1$ remained for a long time not actively investigated, because it was thought (see, e.g., Burkhart and Chen 1989, and references therein) that any electron temperature anisotropy should be quickly isotropized in realistic plasmas due to the several temperature driven instabilities, with time scales much shorter than those typically found in magnetic reconnection (see Sec. 5.2). In this context, the attention changed to the effects of ion anisotropies. Chen et al. (1984) carried out an study of tearing mode focusing mostly in anisotropic ions and weakly anisotropic electrons. By using a straight orbit approximation for both species and the usual matching between internal and external solution Eq. (5.6), they found an expression analogous to Eq. 5.10) (and thus only valid for very thick CS) with the following additional terms due to anisotropies (in a Harris sheet geometry):

$$
\begin{aligned}
\frac{\gamma}{k_{y} v_{t h, e}} & =\frac{1}{\sqrt{2 \pi}}\left(\frac{\rho_{e}}{L}\right)^{3 / 2}\left(1+\frac{T_{i, \perp}}{T_{e, \perp}}\right)\left[\frac{1-\left(k_{y} l\right)^{2}}{k_{y} L}\right. \\
& \left.-\sqrt{2} \alpha_{i}\left(1+\frac{T_{i, \perp}}{T_{e, \perp}}\right)^{-1} \frac{m_{e}}{m_{i}}\left(\frac{L}{\rho_{i}}\right)^{3 / 2}-\sqrt{2} \alpha_{e}\left(1+\frac{T_{e, \perp}}{T_{i, \perp}}\right)^{-1}\left(\frac{L}{\rho_{e}}\right)^{3 / 2}\right]
\end{aligned}
$$

with $\alpha_{j}=1-1 / A_{j}$. From this expression one can infer that linear tearing growth rates will be increased for anisotropies $A_{i}=T_{i, \|} / T_{i, \perp}<1$ (in agreement with Forslund 1968), while in the opposite case they will have a stabilizing effect (reducing $\gamma$ ). The same enhancement in the tearing growth rates takes place for electron temperature anisotropies $A_{e}<1$, being dominant over the ion contribution for similar levels of anisotropy. This is due to their smaller gyroradius compared to the electron one (by a factor equal to the mass ratio $m_{e} / m_{i}$ ). Note also that this effect will be stronger for thicker CS $L / \rho_{i}>1$.

Interestingly, Chen et al. (1984) realized that the usual method of finding solutions in the two internal and external regions (as applied in Furth et al. (1963) and Drake and Lee $(1977 b))$ does not give accurate results when the species have anisotropic temperatures. It is necessary to use additionally an intermediate region $\left(\Delta_{N S}=\sqrt{2 \rho_{e} L}<x<\sqrt{2 \rho_{i} L}\right)$, where the ions orbits make the most significant contribution to the dispersion relation. Then, by solving numerically the resulting dispersion relation, Chen et al. (1984) found that the ion contribution to $\gamma$ is much larger than the predicted by Eq. (5.43): very weak ion anisotropies are sufficient to destabilize tearing mode. The difference between the analytical numerical solutions were found to be as high as one order of magnitude. Part of this disagreement is due to the assumption of very thick CS. Later, Burkhart and Chen (1989) confirmed this fact by improving this three-region treatment using numerically exact particle orbits, founding that for $A_{e}<1$, the anisotropic tearing growth rates increases even more (factor of 5).

Later, there were other works that questioned the lack of importance in magnetic reconnection of the anisotropic tearing when $A_{e}<1$. 2D PIC simulations carried out by Ricci et al. (2004), Daughton et al. (2004) found that LHDI (see Sec. 5.4.2.4) can generate precisely this condition at the edges of a CS (perpendicular electron heating), possibly 
enhancing tearing growth rates and helping thus to the reconnection onset. This also has another interesting effect, to be discussed in Sec.5.1.4.1. However, due to 2D constraints, these simulations could not address simultaneously the development of tearing mode due to this effect (LHDI develops in the out-of-plane direction).

Recently, there have been studies improving the analytical calculation about the ani sotropic tearing by Chen et al. (1984) as expressed in Eq. (5.43), such as Karimabadi et al. (2004). By means of these improved relations, the latter work found that, for $A_{e}<1$, the hybrid mode generated by the coupling between tearing and Weibel instabilities (anisotropic tearing) will always have growth rates in between the "slow" tearing (ion timescales) and the fast Weibel (electron timescales), besides of allowing modes with $k_{y} L>1$. Quest et al. (2010) carried out a detailed analytical study of all the modes driven by both electron and ion anisotropy in a Harris CS. They used a semi-analytical framework called "exact drift", calculating analytically the particle orbits to obtain some relevant quantities (bounce period and mean drifts), and then solving numerically the dispersion relation. Assuming $A_{i}=0$ and weakly electron anisotropies $A_{e}$ ("classical tearing limit"), they derived the following anisotropic tearing relation in the approximation of straight-line orbits for thin CS, improving thus Eq. (5.43):

$$
\frac{\gamma}{k_{y} v_{t h, e}}=0.6\left(\frac{\rho_{e}}{L}\right)^{3 / 2}\left(1+\frac{T_{i, \perp}}{T_{e, \perp}}\right) \frac{1-k_{y} L\left(k_{y} L+\sqrt{2 \rho_{e} / L}\right)}{k_{y} L+\sqrt{2 \rho_{e} / L}}-\frac{1}{\sqrt{\pi}}\left(1-\frac{1}{A_{e}}\right)
$$

Many interesting properties can be derived from this expression. First, note that the second term in the right hand side, associated with the anisotropy, does not depend on the CS thickness like the first one, associated with tearing. Thus, if the growth rate is dominated by the electron anisotropy term, it becomes more or less independent on the thickness. On the other hand, maximizing Eq. (5.44), one can get the maximum growth rates and corresponding unstable wavenumber (Quest et al. 2010):

$$
\begin{aligned}
\frac{\gamma_{\max }}{\Omega_{c e}} & \approx 0.6\left(\frac{\rho_{e}}{L}\right)^{5 / 2}\left(1+\frac{T_{i, \perp}}{T_{e, \perp}}\right)+0.27 \frac{\left(1-\frac{1}{A_{e}}\right)^{2}}{\sqrt{\rho_{e} / L}\left(1+\frac{T_{i, \perp}}{T_{e, \perp}}\right)} \\
k_{\text {max }} L & \approx \frac{1}{1.2 \sqrt{\pi}} \frac{\left|1-\frac{1}{A_{e}}\right|}{\left(\frac{\rho_{e}}{L}\right)^{3 / 2}\left(1+\frac{T_{i, \perp}}{T_{e, \perp}}\right)}
\end{aligned}
$$

From Eq. (5.45), it is possible to estimate the minimum anisotropy level required for which this effect (second term) dominates over the isotropic tearing (first term):

$$
A_{e}>\left[1-\sqrt{2}\left(\frac{\rho_{e}}{L}\right)^{3 / 2}\left(1+\frac{T_{i, \perp}}{T_{e, \perp}}\right)\right]^{-1}
$$

with a value usually small in most of the cases of interest. Note that electron anisotropy effects are more important for thick CS, but only weakly. In the regime of anisotropic tearing $A_{e}<1$ with enhanced growth rates, Eq. (5.44) predicts a wider width of unstable wave numbers between $0<k_{y} L<k_{\max } L \approx 2 k_{\max } L$. In the opposite case, $A_{e}>1$, an improved version of the tearing stabilization threshold Eq. (5.41) can be obtained by finding the value of $A_{e}$ in Eq. (5.44) that makes to vanish the width of unstable wavenumbers $k_{y} L$ :

$$
A_{e}=\frac{T_{e, \|}}{T_{e, \perp}}>\left[1-0.6 \sqrt{\pi} \frac{\rho_{e}}{L}\left(1+\frac{T_{i, \perp}}{T_{e, \perp}}\right)\right]^{-1} \text {. }
$$


Quest et al. (2010) also derived a analogous expression to Eq. 5.44) but only for ion anisotropies, founding that this effect is more important for thicker CS than an equivalent electron anisotropy. This is because, in this case, it is not possible to separate the free energy source terms related with the current and anisotropy like in the case of pure electron anisotropy: both are coupled, and therefore the growth rates will increase for thicker CS for the anisotropy dominated regime (not being independent like in the case of pure electron anisotropy). In addition, by means of an expression similar to Eq. (5.47), they showed that the ions anisotropy effects will dominate over the electron anisotropy if:

$$
\frac{1-1 / A_{i}}{1-1 / A_{e}}>\left(\frac{T_{e, \perp}}{T_{i, \perp}}\right)^{1 / 4}\left(\frac{m_{i}}{m_{e}}\right)^{3 / 4}
$$

which is a requirement difficult to fulfill. Then, ion anisotropy effects will be significant only if $A_{i}$ is very high and the electron anisotropy is negligible.

\subsubsection{Relation with CS bifurcation}

There are some observational evidence in the Earth's magnetosphere of current sheets with bifurcated structure (Runov 2003), i.e.: double peak in $J_{z}$ : a depletion of current density at the center of the CS. In these cases, the peak in the current does not coincide with the minimum in the magnetic field strength. For example, Greco and Taktakishvili (2002) suggested that chaotic electron scattering (Büchner and Zelenyi 1987) can lead to the formation of bifurcated CS. Several models have been built on this idea, generalizing Harris sheet equilibria (see Sitnov 2003, Zelenyi et al. 2004, and references therein). The last two works emphasize the key role of temperature anisotropy (in the electrons or ions) for the construction of these kind of double peaked solutions. Mok et al. (2006) and Janaki et al. (2012) also found the same relation for a two-fluid model of a bifurcated CS (extended to the kinetic regime by Yoon et al. 2014), demonstrating the requirement of an anisotropic and non-gyrotropic tensor pressure for the construction of such solutions. On the other hand, temperature anisotropies generated by instabilities have also been associated with bifurcation. Indeed, as we already mentioned, Ricci et al. (2004), Daughton et al. (2004) found that LHDI (in the current aligned plane) can produce $A_{e}<1$, leading to a bifurcation of the CS. This effect is caused by the generation of an electrostatic potential and their associated resonant scattering, being more efficient for thin CS $L<2 \rho_{i}$ (and therefore, bifurcation caused by LHDI would not be seen for thicker CS). They noticed that this bifurcation becomes weaker when lower mass ratios are used.

The previous mechanisms were all specific for thin CS, where the electron dynamics is essential. In the opposite case, Schindler and Hesse (2008) showed that an initially thick enough CS $\left(L \gg \rho_{i}\right)$ can bifurcate via quasi-steady compression (driving boundary conditions: a temporally varying plasma inflow, equivalent to a conductive electric field applied to the boundaries). This was demonstrated via 1D PIC simulations. They noticed bifurcation in both electron and ion current densities. The electron bifurcation was explained as a result of an electric shielding (of the convective incoming Hall electric field in a region close to the center of the CS), while the ion bifurcation due to momentum conservation (considering the incoming driving plasma flow). The resulting bifurcated CS has both ion and electron temperature anisotropies. Schindler and Hesse (2008) correlated this with 
the simple fact, as derived from the momentum equations, that both quantities are proportional to the gradients in the out-of-plane bulk flows $d V_{e z} / d x$ (see Eq. (7.2)), and thus to the density current gradients $d J_{e, z} / d x$. Therefore, bifurcated CS necessarily must have temperature anisotropies.

Coming back to thin CS, Camporeale and Lapenta (2005) noticed that a bifurcated CS becomes stable against the tearing instability. They studied bifurcated CS equilibria (also relying on anisotropic temperatures) analytically and with 1D and 2D implicit PIC simulations, and even for cases initialized with an initial perturbation, a bifurcated CS did not evolve towards the formation and merging of magnetic island nor reach any stage of explosive magnetic reconnection. This process showed almost no dependence on the mass ratio. They proposed an heuristic explanation for that: a bifurcated CS can be seen as the non-linear saturation of tearing mode, based on the fact that these structures arise naturally in the late stages of the evolution of this instability. Indeed, it has been observed that bifurcation can take place in the reconnection outflow, in magnetotail observations (Hoshino et al. 1996) and both 2D hybrid (Hesse et al. 1998, Shay et al. 1998) and fullykinetic 2D PIC simulations (Karimabadi et al. 2005). The last work identified that only multimode tearing allow this process.

Later, Matsui (2008) (see also Matsui and Daughton 2008, for a more extended discussion) complemented the previous investigations by developing the linear stability analysis of the tearing mode in bifurcated current sheets. They constructed generalizations of the Harris equilibrium, allowing both bifurcated $J_{z}$ and arbitrary temperature anisotropy. They found that both growth rates and range of unstable $k_{y} L$ of the tearing instability are strongly reduced (short wavelength mode are stabilized) in presence of an initially bifurcated $J_{z}$ (compared to the pure Harris case) as consequence of a reduction in the delta stability parameter $\Delta^{\prime}$ Eq. (5.5). This parameter operates as a positive driver for tearing when the slope of the current profile $J_{z}$ increases in the direction to the center of the CS, but it has an opposite (stabilizing) effect on tearing when the slope has opposite sign, like in the case of bifurcated $J_{z}$. The class of kinetic equilibria developed by Matsui (2008) shows a bifurcated $J_{z}$ due to the effects of a non-zero electrostatic potential $\phi$, producing an $\vec{E} \times \vec{B}$ drift in the out-of-plane electron bulk velocities (the same reason of the observed bifurcation in the reconnection outflows). They also investigated the effects of an electron temperature anisotropy $A_{e}<1$ in one of the populations for the considered equilibrium, with parameters fitting the late stages evolution of the LHDI, as seen in the simulations by Daughton et al. (2004). They confirmed the strong destabilizing influence of $A_{e}<1$ on the tearing mode, counteracting the stabilization due to bifurcation when both are present.

\subsection{Temperature anisotropy driven instabilities}

A temperature anisotropy is a important source of free energy for instabilities. Consequently, there are many kinetic instabilities driven by temperature anisotropies, such as the whistler, mirror, firehose, etc. (see, e.g., Gary 1993, Sironi and Narayan 2015). Under some circumstances, our simulations of Harris CS without guide field to be presented in Chapter 7 can become unstable to several of these instabilities. That is why in the rest of this section, we are going to describe briefly their most important properties in the same framework. 


\subsubsection{Unmagnetized Weibel instability}

The Weibel instability is an electromagnetic instability first discovered for unmagnetized plasmas by Weibel (1959). It is driven just by an anisotropy in the distribution functions, leading to the growth of the (electromagnetic) ordinary O mode (Treumann and Baumjohann 2001). Note that an anisotropic distribution function is stable against electrostatic perturbations. The linear theory for a homogeneous and unmagnetized plasma (Krall and Trivelpiece 1973 ) predicts a propagation in the "colder" direction (or more correctly, perpendicular to the "warmer" temperature), with the associated electric field in the "warmer" direction. Assuming propagation direction in $k_{x}$ and a temperature anisotropy $T_{e, y}>T_{e, x}$ in a bimaxwellian distribution function, we have the following dispersion relation for electromagnetic waves (Krall and Trivelpiece 1973):

$$
k_{x}^{2} c^{2}-\omega^{2}+\sum_{\alpha} \omega_{p \alpha}^{2}\left(1-\frac{T_{\alpha, y}}{T_{\alpha, x}}\right)=\sum_{\alpha} \omega_{p \alpha}^{2} \frac{T_{\alpha, y}}{T_{\alpha, x}} \xi_{\alpha} Z\left(\xi_{\alpha}\right)
$$

with thermal speed $v_{t h, e}=\sqrt{k_{B} T_{e, x} / m_{e}}$, indices $\alpha=i, e$ representing ions and electrons, and $Z\left(\xi_{\alpha}\right)$ is the plasma zeta function (see Eq. (5.16)). Now, in the low frequency regime $\omega /\left(k_{x} v_{t h, e}\right) \ll 1$ and weak anisotropy $\gamma<k_{x} v_{t h, e}$, it is possible to derive the following estimate for the growth rates:

$$
\gamma=\sqrt{\frac{2}{\pi}} k_{x} v_{t h, e} \frac{T_{e, x}}{T_{e, y}}\left(\frac{T_{e, y}}{T_{e, x}}-1-\left(\frac{k_{x} c}{\omega_{p e}}\right)^{2}\right) .
$$

The results for strong anisotropy $\omega /\left(k_{x} v_{t h, e}\right) \gg 1$ can be found, e.g., in Krall and Trivelpiece (1973). In the expression Eq. (5.51), the ion contribution has been neglected because it is smaller by a factor $m_{i} / m_{e}$. Thus, Weibel instability is mostly driven by electrons. It can also exist due to an anisotropy in the ion distribution function, but it has to be very large with a negligible electron temperature anisotropy to be noticed, something unrealistic in most of the plasmas in nature or simulations (Baumjohann et al. 2010). Now, from Eq. (5.51) we can derive the maximum growth rate

$$
\gamma_{\max }=\sqrt{\frac{8}{27 \pi}} \omega_{p e} \frac{v_{t h, e}}{c} \frac{T_{e, x}}{T_{e, y}}\left(\frac{T_{e, y}}{T_{e, x}}-1\right)^{3 / 2} .
$$

with the corresponding wave number

$$
k_{x, \max }=\frac{\omega_{p e}}{c} \sqrt{\frac{1}{3}\left(\frac{T_{e, y}}{T_{e, x}}-1\right)},
$$

This implies that the associated wavelength $\lambda_{x, \max }=2 \pi / k_{x, \max }$ gets larger for smaller anisotropies. Note that the growth rate is zero in the limit of large wavelengths $k_{x}=0$ and also at $k_{x}=\sqrt{3} k_{x, \text { max }}$. From Eq. 5.51) we can al so determine the minimum temperature anisotropy for the triggering of this instability, its threshold:

$$
\left(\frac{T_{e, y}}{T_{e, x}}-1\right)>\left(\frac{k_{x} c}{\omega_{p e}}\right)^{2} \text {. }
$$


One characteristic signature of Weibel instability is its ability to produce quasy-stationary and propagating magnetic fields perpendicular to the direction of the "warmer" temperature. These structures have a short length scale on the order of $\lambda=2 \pi d_{e}$ (Baumjohann et al. 2010), being located in adjacent cells with alternate (antiparallel) polarities of $\vec{B}$ (in "filamented" structures) due to the requirement of zero divergence of the magnetic field, leading potentially to small scale reconnection (see Treumann and Baumjohann 2012, and references therein) They are generated via a (turbulent) non-dynamo mechanism described in Fried (1959), Medvedev and Loeb (1999), due to the release of free energy in form of waves, reducing the anisotropy of the distribution function via pitch angle scattering (see also Tautz and Shalchi 2008, Medvedev and Loeb 1999). However, the theoretical description of the mechanism behind this $\vec{B}$ generation (potentially amplified to very high values due to the aperiodic nature of Weibel $\omega \sim 0$ ) is not so straightforward, since it requires to deal with the non-linear evolution of the instability and a proper calculation of the thermal fluctuation levels (Treumann et al. 2010). It is also interesting to mention that these magnetic fields generated by Weibel instability might provide the conditions necessary for the triggering of other magnetized temperature driven instabilities, such as mirror and/or firehose ones (Treumann and Baumjohann 2014). Even more: these Weibelgenerated magnetic fields (especially in the relativistic regime) have been proposed to be the mechanism behind the magnetization present in the early universe, as well as responsible for the formation of collisionless shocks in active galaxy nuclei (AGN), gamma ray bursts (GRB) and pulsar winds (see Stockem et al. 2006, and references therein). And only very recently it was possible to get experimental evidence confirming this mechanism in a laboratory plasma (Huntington et al. 2015).

Weibel instability is non-resonant (it does not rely on wave-particle resonance), depending only on the macro or bulk properties of the distribution function. This implies that most of the plasma particles can participate of the instability, with a very efficient transfer of kinetic (stored in the anisotropy of the distribution function) to magnetic energy. Therefore, it is very robust and can be active under a wide range of conditions (Tautz and Schlickeiser 2006), the reason why it is sometimes called an "universal" instability (Baumjohann et al. 2010). This fact has a consequence an interesting property: most of the previous results, such as growth rates, do not depend on the specific bimaxwellian form of the distribution function, as shown by Davidson (1972). A plasma with an effective temperature due to, e.g.: two counterstreaming populations with (cold) temperature $T_{e}$ at speed $U_{e, 0}$, can give origin to a instability with identical properties as Weibel by identifying $T_{e}=T_{e, x}$ and $k_{B} T_{e, y}=0.5 m_{e} U_{e, 0}^{2}$ (Krall and Trivelpiece 1973). This fact and the previous discussed generation of filamented magnetic fields is the reason because Weibel is also called "filamentation" instability, especially under the presence of a background magnetic field. However, the latter name is sometimes reserved when there is only relative streaming of species (see a discussion about the terminology in Bret et al. (2005) and Bret (2009)).

Weibel instability can also operate under the presence of magnetic fields, as long as they are not too strong $\Omega_{c e} \ll \omega_{p e}$. A magnetic field tends to suppress this instability because the particle gyromotion produce an isotropization of the temperature perpendicular to it, inhibiting the magnetic field generation responsible for the isotropization of the distribution function via pitch angle scattering. Nevertheless, under the previous assumption of weak magnetic field, the growth rates given are not reduced too much. The main 
effect of the magnetic field is manifested in the fact that Weibel gets a finite oscillation frequency, on the order of $\Omega_{c e}$. See Hededal and Nishikawa (2005), Stockem et al. (2006), Tautz and Schlickeiser (2007) and Tautz and Shalchi (2008) for further details.

\subsubsection{Weibel instability in CS}

Since Weibel instability develops preferentially in unmagnetized environments, its presence in magnetic reconnection (and CS in general) has been far away from being a focus in the research of this process. However, under some circumstances can be observed, especially in Harris CS without guide field, since there is a neutral line with vanishing magnetic field. Indeed, a number of studies have found signatures of its presence in studies of magnetic reconnection of pair (electron-positron) plasmas (Swisdak et al. 2008, Zenitani and Hesse 2008, Liu et al. 2009), as well as in the classic electron-proton plasmas (Lu et al. 2011, Schoeffler et al. 2013). In these plasmas, Weibel instability has been found operative inside of the magnetic tearing islands, as a result of the temperature anisotropy developed there. This, in turn, is produced by the first order Fermi mechanism: bouncing and acceleration of particles inside of these structures. In pair plasmas it can even provide a mechanism for fast magnetic reconnection, since the turbulence generated by Weibel instability can broaden the current layer (Liu et al. 2009). On the other hand, Weibel instability has also been proposed to be generated due to the counterstreaming electron inflows close to the $\mathrm{X}$ point, at length scales smaller than $d_{e}$ (Baumjohann et al. 2010, Treumann et al. 2010).

\subsubsection{Magnetized: Mirror instability}

The mirror instability is one of the magnetized instabilities that can be excited in a plasma when there is a field aligned anisotropy $T_{\alpha, \perp}>T_{\alpha, \|}$ (Hasegawa 1969, Gary 1993) $(\alpha=e, i) 6$. It is interesting to mention that this condition is more feasible to find in space plasmas than the opposite $T_{\alpha, \perp}<T_{\alpha, \|}$, even though there is no intrinsic bias towards this kind of heating. According to Gary (1993), this is because parallel heating will produce acceleration of particles along the magnetic field lines. They will escape quickly from the region where they are energized, leaving behind particles with a higher $T_{j, \perp}$ and producing thus the mentioned anisotropy. As we will see in the theoretical expressions for growth rates and thresholds, mirror develops preferentially in high $\beta$ plasmas with relatively low anisotropies (Génot et al. 2001), such as those found in the solar wind (Klein 2013) 7 .

The mirror instability was first discovered and described with a fluid description, where the specie responsible for the anisotropy are only ions. The linear theory predicts a practically zero real frequency, oblique propagation with respect to the magnetic field

\footnotetext{
${ }^{6}$ There is another instability driven by a similar condition, called ion-cyclotron instability. For high $\beta$ plasmas, the mirror instability requires lower thresholds in the temperature anisotropy than ion-cyclotron, and that is why the latter will not be analyzed here. See Gary (1993) for further details.

${ }^{7}$ In general, all the electromagnetic instabilities driven by temperatures anisotropies are severely quenched in low $\beta$ plasmas. This can be understood due to the fact that these instabilities require perturbations in $\vec{B}$, which become more difficult in plasmas dominated by magnetic pressure and very low plasma pressure: they cannot change so easily the structure of the background magnetic field (Krall and Trivelpiece 1973).
} 
(maximum growth rate nearly in the perpendicular direction) and preferentially longitudinal over perpendicular fluctuations $\delta B_{\|} \gg \delta B_{\perp}$ (Treumann and Baumjohann 2001, Gary 1993). A fluid approach can predict quite accurately the right threshold condition. Assuming $T_{i}=T_{e}$, very low frequencies $\omega \ll \Omega_{c i}$ and very long wavelengths $\lambda \gg \rho_{i}$, the anisotropic MHD equations (Hasegawa 1975) can predict a instability associated with the compressional Alfvén wave (or magnetosonic) mode whenever that the following condition is satisfied:

$$
\frac{P_{\perp}}{P_{\|}}>1+\frac{1}{\beta_{\perp}}
$$

where the pressures correspond to the scalar fluid ones, being proportional to the respective kinetic temperature.

After the initial fluid studies, a kinetic approach started by Tajiri (1967) and other workers revealed that, different from Weibel, mirror instability is essentially a resonant instability, being driven by a wave-particle interaction (gyro-cyclotron resonance) of the anisotropic specie, and thus a kinetic description is essential to understand its mechanism (see also Klein 2013). These kind of calculations demonstrated that both ions and electrons contributes equally to the instability condition (Hasegawa 1975), as can be seen from the expression for the threshold taking into account these effects (Hall 1979, Pokhotelov et al. 2000, Hellinger 2007):

$$
\sum_{\alpha} \beta_{j, \perp}\left(\frac{T_{j, \perp}}{T_{j, \|}}-1\right)>1+\frac{\left(\sum_{\alpha} \frac{T_{j, \perp}}{T_{j, \|}}\right)^{2}}{2 \sum_{\alpha} \beta_{j, \|}^{-1}}
$$

In this same framework, it is possible to derive, by assuming cold electrons and an ion anisotropy, the maximum growth rate of the mirror mode Hasegawa 1975, Treumann and Baumjohann 2001):

$$
\gamma_{\max }=\sqrt{\frac{2}{\pi}} \frac{T_{i, \|}}{T_{i, \perp} \beta_{i, \perp}}\left[\beta_{i, \perp} D+\frac{k_{\|}^{2}}{k_{\perp}^{2}} \sum_{\alpha}\left(\frac{\beta_{j, \|}-\beta_{j, \perp}}{2}-1\right)\right] k_{\|} v_{t h, i, \|}
$$

Since the mirror mode has practically perpendicular propagation, the second term is neglected 8 .

The essential feature of mirror instability (in both MHD and kinetic models), usually used as signature of its presence in in-situ measurements of solar wind plasmas, is the out of phase correlation between the density and magnetic field fluctuations (Hasegawa 1975, Southwood and Kivelson 1993):

$$
\frac{\delta n_{e}}{n_{e}} \sim\left(1-\frac{\beta_{\perp}}{\beta_{\|}}\right) \frac{\delta B}{B_{0}} .
$$

This implies, whenever the mirror threshold condition Eq. 5.55) is satisfied, that the plasma density will be higher in regions with small magnetic field strength, and vice versa. This can be understood due to the mechanism behind this instability (Treumann and Baumjohann

\footnotetext{
${ }^{8}$ This term gives the growth rate of the complementary instability: the so called firehose instability driven by $T_{j, \|}>T_{j, \perp}$ and with parallel propagation. See next Sec.5.2.4 for further details
} 
2001): particles become trapped in magnetic mirror structures whenever their perpendicular pressure is much larger than the parallel one according to Eq. (5.56). In that case, the free energy stored in the perpendicular gyromotion of the particles is transferred to their bouncing back and forth between the ends of the mirror structures, producing the oscillations described by Eq. (5.58) due to a mirror force $\nabla(\mu B)$. Most of the particles in the bulk of the plasma will preserve their energy during this process, as a consequence of the conservation of the conservation of the first adiabatic invariant (magnetic moment), but a few particles with small parallel velocity will interact via gyro-resonances, generating cyclotron waves. And only these few resonant particles are the responsible for the plasma response of the mirror instability. See Southwood and Kivelson (1993) for an expanded discussion.

For many years, the mirror instability was studied with the purpose of applications to solar wind, where the ion anisotropy is the most important one. Only recently (Pokhotelov et al. 2000, Pokhotelov 2002, Gary and Karimabadi 2006) the attention has changed to the study of its properties when the main driver is an electron temperature anisotropy. In particular, Pokhotelov et al. (2000) derived an improved threshold condition (compared to Eq. (5.56)), taking into account kinetic effects with a finite temperature electron response, finding a correction due to a stabilizing effect of the field aligned electric field arising from the differential motion of ions and electrons:

$$
D=\left(\frac{T_{i, \perp}}{T_{i, \|}}-1\right)+\frac{T_{e, \perp}}{T_{i, \perp}}\left(\frac{T_{e, \perp}}{T_{e, \|}}-1\right)-\frac{1}{\beta_{i, \perp}}-\frac{T_{e, \|} T_{i, \|}}{2 T_{i, \perp}\left(T_{e, \|}+T_{i, \|}\right)}\left(\frac{T_{i, \perp}}{T_{i, \|}}-\frac{T_{e, \perp}}{T_{e, \|}}\right)^{2}>0
$$

The last term is the aforementioned correction, saturating at high enough electron temperatures. Under the same assumptions, it is possible to derive (Pokhotelov et al. 2000) an expression for the maximum growth rates (improved version of Eq. 5.57)):

$$
\gamma_{\max }=\frac{k_{\perp} v_{t h i, \|} T_{i, \|}}{\sqrt{\pi} T_{i, \perp} \beta_{i, \perp}} \frac{4 A^{3 / 2}\left(1+\frac{T_{e, \|}}{T_{i, \|}}\right)^{2}\left[1+\left(\beta_{i, \perp}-\beta_{i, \|}\right) / 2\right]}{\left(1+\frac{T_{e, \|}}{T_{i, \|}}\right)^{2}+\left(1+\frac{T_{e, \perp}}{T_{i, \perp}}\right)^{2}},
$$

with

$$
A=\frac{D}{3 \beta_{i, \perp}^{-1}\left(1+\frac{\beta_{i, \perp}-\beta_{i, \|}}{2}\right)} .
$$

where $D$ is the left hand side of the mirror threshold condition given by Eq. (5.59). Although this expression increases with $k_{\perp}$, it is only valid neglecting finite Larmor radius effects: $k_{\perp} \rho_{i} \ll 1$. Therefore, the maximum growth rates will have wavelengths around the ion gyroradius $k_{\perp}^{-1} \sim \rho_{i}$, since FLR effects are expected to reduce growth rate in the short wavelength limit (Pokhotelov et al. 2000). Later there have been works analyzing with more detail these finite electron Larmor radius effects (Hellinger 2007, Kuznetsov et al. 2012), showing that they can indeed stabilize short wavelengths modes, but not affecting significantly the mirror threshold condition.

Eq. 5.60) also demonstrates that the mirror mode propagates practically in the perpendicular $y$, satisfying $k_{\perp}=k_{y} \gg k_{\|}=k_{x}$. The propagation angle $\theta$ can be then estimated by:

$$
\tan (\theta)=\frac{k_{\perp}}{k_{\|}}=\frac{1}{\sqrt{A}}
$$




\subsubsection{Relation between mirror and Weibel instabilities}

There have been some previous investigations pointing out a very close relationship between Weibel and mirror instabilities (Hasegawa 1969, Chen et al. 1984, Shi et al. 1987, Pokhotelov et al. 2010). For example, the latter work identified that the linear responses of both have mathematical identical form, by identifying quantities related with $\rho_{e}$ (in the mirror case) to $d_{e}$ (in the Weibel case). It is also necessary to identify the parallel and perpendicular direction from these with respect to the magnetic field (mirror) to these related purely with the temperature (Weibel). Due to this fact, both linear growth rate and non-linear evolution have very similar properties.

On the other hand, Treumann and Baumjohann (2014) proposed a very interesting mechanism relating even more both instabilities. The idea is based in the fact that a Weibel unstable (anisotropic) plasma will generate magnetic fields. These magnetic fields can act as an initial seed for the triggering of magnetized temperature anisotropy driven instabilities, in particular mirror instability. The effect is the appearance, on top of the filamented Weibel cells, of a chain of bubble or holes mirror-like structures, generating additional levels of turbulence due to the simultaneous existence of these instabilities. As we will see in Chapter 7 , a very similar situation can take place in the initially unmagnetized center of an anisotropic Harris CS.

\subsubsection{Magnetized electron whistler instability}

Similar to the previously discussed mirror, the (electron) whistler instability is other instability driven by the same condition $T_{e . t}>T_{e . \|}$. Under such condition, according to Gary and Cairns (1999), Gary and Li (2000) and Gary and Karimabadi (2006), the electron whistler instability may be more important, because it has larger growth rates and lower thresholds than the electron mirror instability, at least for a large range of parameters with $\beta_{e, \|} \gtrsim 0.1$. More specifically, its threshold (marginal stability condition) calculated from linear theory is given by:

$$
\frac{T_{e, \perp}}{T_{e, \|}}-1=A \beta_{e, \|}^{-\alpha}
$$

with the two fitting parameter that can be approximated as $\alpha=0.5$ and $A \approx 0.55$ according to Sironi and Narayan (2015) (they depend on the choice of the maximum growth rate). This instability can generate right-handed transverse electromagnetic waves of (mostly) parallel propagation. Therefore, in a $2 \mathrm{D}$ guide field magnetic reconnection setup, like the one to be analyzed in chapter 9 , this instability is not allowed since it would propagate in the out-of-plane $\hat{z}$ direction. Nevertheless, it has been known (Gary and Cairns 1999, Gary and $\mathrm{Li}$ 2000) that the electron whistler instability becomes more electrostatic and with a more oblique propagation angle (at maximum growth rate) as the local plasma beta $\beta_{e \|}$ decreases, allowing the possibility in a $2 \mathrm{D}$ setup. However, we can check that Eq. (5.63) predicts a too high threshold of the electron temperature anisotropies in low plasma beta with strong magnetization $\omega_{p e} \sim \Omega_{c e}$ (like the one to be analyzed in Chapter 9). 


\subsubsection{Magnetized: Firehose instability}

Although we did not find evidence of the firehose instability (Parker 1958) in the simulations to be shown in this thesis (Chapter 7), for reasons of completeness we are going to describe very briefly its properties (in order to compare it with the mirror instability). This is a (practically) parallel propagating electromagnetic instability driven by the opposite condition to the mirror instability $T_{\alpha, \|}>T_{\alpha, \perp}$ (Krall and Trivelpiece 1973, Gary 1993, Camporeale and Burgess 2008), and associated with Alfvén waves. The threshold condition can be expressed in an analog way to Eq. 5.56) (Treumann and Baumjohann 2001):

$$
\sum_{\alpha} \beta_{\alpha, \|}-\beta_{\alpha, \perp}>2
$$

Thus, we can see that this instability requires (very) high beta plasmas for their existence (such as in the solar wind). Its growth rate is also given by the same expression as for the mirror instability Eq. (5.57), but with a dominant second term instead of the first one, in such a way that can be rewritten in the following way (Treumann and Baumjohann 2001):

$$
\gamma_{\max }=\frac{k_{\|} V_{A}}{2} \sqrt{\beta_{j, \|}-\beta_{j, \perp}-2}
$$

Thus, we can see the much slower growth of this instability (Alfvén time scales) compared to the other anisotropy driven ones, such as mirror or Weibel. The mechanism (and the name) behind this instability is because of the nature of the driving force: the parallel pressure along the magnetic field lines operates by increasing the amplitude of the unstable (Alfvén) waves, similarly to water flowing in a (loose) hose (Krall and Trivelpiece 1973). There are different kinetic effects (resonance) important in some parameter regimes of this instability, especially in the (less investigated) case when electrons are the specie carrying the temperature anisotropy. For a review, see Michno (2014).

Evidence of the firehose instability has been found in 2D PIC simulations of Harris CS initialized with a temperature anisotropy by Schoeffler et al. (2011), Matteini et al. (2013). These works detected some signatures of their presence at the edges of the CS: a low frequency kinking of magnetic field lines, with the corresponding particle scattering and subsequent reduction of the temperature ani sotropy in these locations. These authors also observed that when the CS is firehose unstable, the growing of magnetic tearing islands in inhibited (they just oscillate together with the firehose fluctuations), allowing the tearing mode growth only after the anisotropy is depleted. This suppression of tearing mode is a very similar scenario to the one to be seen in Chapter 7 .

\subsection{Shear flow driven instabilities}

A shear flow as source of free energy can drive a MHD Kelvin-Helmholtz instability at macroscopic level as well as kinetic instabilities at smaller scales, with several different names.

\subsubsection{MHD/fluid Kelvin-Helmholtz instability}

Kelvin-Helmholtz $(\mathrm{K}-\mathrm{H})$ is a macro instability very well known in hydrodynamics since the XIX century. Its basic mechanism can be understood as follows: any pertur- 
bation in the streamlines lines of counterstreaming shear flows leads to unbalanced pressure, which is amplified as a consequence of the conservation of energy in incompressible fluid dynamics (Bernoulli principle). It transforms the bulk kinetic energy of flows with relative streaming into kinetic energy of vortices and magnetic energy, being able to generate anomalous viscosity and drag via turbulence (Miura and Pritchett 1982). It has been observed in many space and astrophysical environments, such as planetary magnetospheres, solar wind, solar coronae, etc. (see Miura and Pritchett 1982, and references therein). Especially in the latter scenarios a magnetic field plays an essential role, and that is why a correct description requires at least an MHD plasma description. In this context, Chandrasekhar (1961) performed a linear MHD stability analysis of this instability, assuming a velocity shear layer of zero thickness (tangential discontinuity) and incompressibility. He found a growth rate on dependence on the wave number $\vec{k}$ (perpendicular to the shear velocity gradient, with its main component along the interface between the flows) and the velocity bulk flow in each side of the shear layer $\vec{V}_{1}$ and $\vec{V}_{2}$ (see also Ma 2012, for an extended discussion):

$$
\gamma=\sqrt{\alpha_{1} \alpha_{2}\left[\left(\vec{V}_{1}-\vec{V}_{2}\right) \cdot \vec{k}\right]^{2}-\alpha_{1}\left(\vec{V}_{A, 1} \cdot \vec{k}\right)^{2}-\alpha_{2}\left(\vec{V}_{A, 2} \cdot \vec{k}\right)^{2}}
$$

where $\alpha_{i}=\rho_{i} /\left(\rho_{1}+\rho_{2}\right)$ are the relative densities of the fluids in each side of the shear layer and $\vec{B}$ is the background static magnetic field. In the limit of zero magnetic field, one recovers the hydrodynamics results $\left.\gamma=k \mid \vec{V}_{1}-\vec{V}_{2}\right) \mid$ (for constant density). This means that tangential discontinuities are always unstable (no bottom velocity threshold), with a growth rate proportional to the shear flow relative speed. In addition, they are stronger for smaller wavelengths (for $k \rightarrow \infty$ ). The magnetic field strength diminishes the growth rates and can even stabilize this instability if it is high enough (Alfvén speed has to exceed twice the relative flow speed) and has a component along the $\vec{k}$ direction (perpendicular component has no effect). More precisely, a system with shear flows will be K-H unstable if it can overcome the stabilizing effect of the field line tension (Miura and Pritchett 1982):

$$
\left|\vec{V}_{1}-\vec{V}_{2}\right|>2 V_{A}(\vec{k} \cdot \vec{b}) / k_{y}
$$

which states that only super-Alfvénic flows can be K-H unstable (result valid in the incompressible limit).

Miura and Pritchett (1982) studied the K-H instability for more general conditions than in previous works. By lifting the incompressibility assumption in the MHD analysis (essential for moderate to low values of plasma beta), they found an upper limit in the relative speed between the flows, beyond which K-H can be stabilized. This threshold arises as a consequence of the finite sound speed required to transmit the information necessary for the formation of vortices, constraining the regime where this instability can operate to subsonic flows (due to this, the growth rates given by the compressible MHD formalism have as an upper bound the incompressible MHD growth rates, for an infinite sound speed). More precisely, let $m_{\alpha}=\left|\vec{V}_{1}-\vec{V}_{2}\right| / c_{s}, M_{A}=\left|\vec{V}_{1}-\vec{V}_{2}\right| / V_{A}$ the sound and Alfvén Mach speeds, $k_{y}$ the wavenumber along the flows and $k_{z}$ the wavenumber perpendicular to both the flow and the shear velocity gradient. Then, under some additional simplifying assumptions, we have the instability condition:

$$
M_{f}^{2}=\frac{M_{A}^{2} m_{\alpha}^{2}}{m_{\alpha}^{2}+M_{A}^{2}}<4 \frac{k_{y}^{2}+k_{z}^{2}}{k_{y}^{2}}\left[1-\frac{4(\vec{k} \cdot b)^{2}}{k_{y}^{2}\left(M_{A}^{2}+m_{\alpha}^{2}\right)}\right]
$$


which reduces to the subsonic condition $m_{\alpha}<2$ for the parallel case ( $b=\hat{y}$ and $k_{z}=0$ ) and to $M_{f}<2$ for the transverse case $\left(b=\hat{z}\right.$ and $\left.k_{z}=0\right)$. $M_{f}$ is the fast Mach number. For obliquely propagating modes with finite $k_{z}$, the instability range is larger. Furthermore, by combining Eq. (5.67) and Eq. (5.68), it is possible to find the following condition for $\mathrm{K}-\mathrm{H}$ instability relating the three characteristic speeds for both parallel and perpendicular case:

$$
\begin{aligned}
2 V_{A} & <V_{0}<2 C_{s} \\
2 V_{A} \frac{k_{z}}{k_{y}} & <V_{0}<2 \sqrt{V_{A}^{2}+C_{s}^{2}}
\end{aligned}
$$

Note that the latter equation indicates that K-H instability grows more easier in the transverse than in the parallel case. And the appearance of the magnetosonic speed on the right hand side of Eq. (5.69) has to do with the additional contribution of the magnetic pressure to the total pressure, which increases the upper stability limit. This extends the speed range in which a flow can be unstable.

It is interesting to mention a remark on the previous result for $2 \mathrm{D}$ configurations with no variations in the $\hat{z}$ direction, but arbitrary direction of $\vec{B}$. The right hand side of Eq. (5.70) uses the Alfvén speed with respect to the total magnetic field, since it comes from an effect associated with the magnetic pressure. On the other hand, the bottom threshold depends on the in-plane Alfvén speed, since it comes from an effect related with the magnetic field tension that cannot be curved in the out-of-plane $(\hat{z})$ direction.

We can also find a relation between the relative flow speed and the plasma beta:

$$
2 \sqrt{\frac{1.2}{\beta}} \cos \theta<m_{\alpha}<2
$$

where $\theta$ is the angle between $\vec{V}_{1}-\vec{V}_{2}$ and $\vec{B}$. This condition indicate than in low beta plasmas, $\mathrm{K}-\mathrm{H}$ can exist only if $\theta \sim 90$, a feasible condition in force free magnetic reconnection configuration with strong guide field (see Chapter 9). It is also important to mention that the growth rates decreases from transverse $(\theta=90)$ to parallel configurations $(\theta=0)$.

Miura and Pritchett (1982) also studied a finite shear layer thickness, finding that the fastest growing modes do not have the smallest wavelengths as in the zero thickness case, but a size comparable to the width of the shear flow layer $(k \Delta x=0.5)$. All the modes with wavelengths smaller than this threshold will be K-H stable $(k \Delta x>2$, with $\Delta x$ the shear layer thickness). In addition, wider shear layers make the K-H growth rate smaller. Both restrictions also recover known hydrodynamics results.

Finally, another important feature of the compressible MHD K-H instability pointed out by the same authors is the generation of alternating (AC) field aligned currents inside of the velocity shear layer, via a dynamo process. It can also produce several Alfvénic waves (slow, magnetosonic, fast), confined or not to the shear layer (depending on $\theta$ ).

\subsubsection{Kinetic scale K-H instability}

$\mathrm{K}-\mathrm{H}$ instability not only takes place at MHD scales. It can also be generated at much smaller scales, which requires other formalism for their correct description. For example, 
Hall-MHD effect have been taken into account in Huba (1994) and Yoon et al. (1996) (see also references therein), with a magnetic field perpendicular to the shear flow direction. Electron MHD (EMHD) also predicts a Kelvin-Helmholtz instability at electron scales (see, e.g., Sundar and Das 2010), propagating in a direction perpendicular to both shear flow (same as the current in EMHD) and in the magnetic field direction (it has also been associated with a sausage mode Das and Kaw 2001). But there are many other effects that can only be captured with a kinetic treatment. In this context, kinetic linear theory, electrostatic PIC and electromagnetic hybrid simulations have been used to study instabilities driven by shear flows (K-H in the fluid limit) since many years ago, under different approximations. For example, Ganguli et al. (1988), Lemons et al. (1992), Cai et al. (1990), Pritchett (1993) and Cai et al. (1993) studied, under the electrostatic approximation, the transverse K-H instability with the shear flow provided by the $\vec{E} \times \vec{B}$ drift. They found two related instabilities in this configuration: one for large wavelengths $k \rho_{i} \ll 1$ with similar growth rates to the MHD fluid one (although its growth rates decreases when approaching to the kinetic limit: for wavenumbers between $0.1 \lesssim k \rho \lesssim 1$ ), and another one for short wavelengths $k \rho_{i}>1$ with features of an ion-cyclotron wave (which depend on the ratio $\left.\rho_{i} / \Delta x\right)$.

An early kinetic study particularly interesting for the purposes of this thesis is Wang et al. (1992), focused on the effects of a magnetic field parallel to the shear flow on the (electromagnetic) macroscopic K-H modes $k \Delta x<1$. They showed that the MHD bottom and upper thresholds for the triggering of K-H instability Eq. (5.69) are not affected significantly by kinetic effects (Landau damping). But the growth rates and range of unstable wave numbers are modified on dependence on the ratio between thermal, Alfvén and flow speed. This is because there is no Bernoulli principle in a kinetic plasma description, the basic mechanism of the fluid K-H instability. As a consequence, there is no upper bound on the perturbation of the pressure given by the sound speed or their kinetic equivalent thermal speed (as in MHD), even though this approaches to infinity. Therefore, for thermal speeds much larger than the flow speed, the maximum kinetic growth rates can be larger than the MHD ones, providing that the flow speed is larger than the in-plane Alfvén speed. In addition, the range of unstable wave numbers is wider for this case than in MHD (smaller wavelengths are allowed: $k \Delta x>1$ ). On the other hand, in the opposite case of small thermal speeds, the kinetic growth rate can be smaller than the MHD one, due to a phase difference between the magnetic and pressure perturbations different from the obtained with the fluid approach (anti-phase, $180^{\circ}$ ).

Later, one of the earliest attempts to study the collisionless K-H instability via an PIC code simulation was undertaken by Cai et al. (1993). They performed electrostatic 2D simulations of this instability for systems with ion gyroradius $\rho_{i}$ comparable to the shear layer width $\Delta x$, and with a magnetic field perpendicular to the velocity flow. The initialization was based on previous works by the same authors finding suitable distribution functions and methods for loading particles. They found good agreement with previous MHD estimations and simulations of the K-H instability, in the sense that the maximum growth rates are independent on the kinetic scale $\rho_{i} / \Delta x$. But their simulations also showed additional purely kinetic instabilities on dependence on the ratio $\rho_{i} / \Delta x$ : one with short wavelength for small values of that parameter and Bernstein waves when that parameter is larger.

More recently, simulations of K-H instability with more realistic parameters and large 
domains have also been performed with hybrid codes (Filippychev 2002), (4D) Vlasov codes (Umeda et al. 2010) and fully kinetic PIC simulations (Nakamura et al. 2010, 2011, Huebl et al. 2014). An interesting recent study is the one carried out by Henri et al. (2013). They benchmarked the magnetized K-H instability with a variety of different codes, from MHD to fully kinetic ones 9 . They confirmed that in hybrid and PIC codes, the linear growth rate of K-H instability is different depending on the relative orientation of the magnetic field with respect to the vorticity $\nabla \times \vec{V}$, as expected from previous theoretical works and simulations (Cai et al. 1990, Nakamura et al. 2010, Cerri et al. 2013). This is an effect that arises as a result of the particle gyration: in all the codes that do not they take into account finite Larmor radius corrections, this effect is absent (a similar asymmetric effect comes from a Hall-MHD effect. See details in Huba (1994)). They also pointed out the key role played by the compressibility in the development of the K-H instability, since it depends critically on the model used (related with the closure in the equation of state for fluid codes).

\subsubsection{Shear flow gradient kinetic instabilities}

Shear flows can also be seen as gradients in the flow speed, being related thus with the pressure gradient instabilities to be analyzed later. When shear flows reach velocity gradients (see Chen et al. 2015, and references therein) on the order of kinetic scales $\rho_{i}$ or $\rho_{e}$, instabilities closely related with the kinetic electron Kelvin-Helmholtz can take place. One important for our purposes is the so called electron-ion hybrid (EIH) instability (Ganguli et al. 1988, Romero et al. 1992), since it can generates waves typically in the lower hybrid-range $\Omega_{l h}$ range. Furthermore, their effects are always added to the ones produced by other streaming or pressure gradient driven instabilities. Let us describe in more precise terms this instability. EIH is an electrostatic instability that takes place when there are cross-field (perpendicular to the magnetic field) velocity shear gradients (or second derivative of the electron $\vec{E} \times \vec{B}$ drift) with typical scales $L_{e}$ in between ion and electron scales $\rho_{e}<L_{E}<\rho_{i}$. It is in a high electrostatic frequency branch of the familiar Kelvin-Helmholtz instability. Both frequencies and growth rates are on the order of $\Omega_{l h}$ (Romero et al. 1992). Typical perpendicular wavelengths are much smaller than the ion Larmor radius $k_{\perp} \rho_{i} \gg 1$ but larger than electron Larmor radius $k_{\perp} \rho_{e}<1$. This has an interesting implication: the non-linear evolution can provide a mechanism for perpendicular ion heating, where the threshold condition is for shear frequencies larger

\footnotetext{
${ }^{9}$ It is interesting to notice that the auth or of this study identified several spurious phenomena in the PIC simulations of K-H instability, caused by the fact that they initialized the setup with an MHD and not a Vlasov equilibrium. Indeed, one of the main problems of simulating K-H with a PIC code is finding a suitable equilibrium. Unfortunately, very few exact kinetic equilibria are known for shear flows configurations. One of the first ones was discovered by Cai et al. (1990), with several simplifying assumptions $\left(\rho_{i} \gtrsim \Delta x\right.$ : "strong shear case", uniform magnetic field unaffected by plasma dynamics). Mahajan and Hazeltine (2000) found a generalization of the Harris equilibrium for shear flows, assuming that the magnetic field is selfgenerated. Both equilibria rely on the agyrotropy of the pressure tensor. Later, Cerri et al. (2013) (see also Cerri et al. 2014) implemented some kinetic effects (finite ion Larmor radius) into a sheared MHD equilibrium. The justification for this is that in collisionless systems, the approximation of an isotropic pressure as in MHD is not valid, since the tensor pressure and shear flow are strongly coupled. In this way, they found a quasi-kinetic equilibrium ("extended fluid model") efficient in reducing several of the consequences of starting with a non-equilibrium configuration (via hybrid Vlasov simulations). This approach was also applied successfully later by Kemel et al. (2014) in PIC simulations.
} 
than $\Omega_{l h}$ :

$$
\frac{1}{\Omega_{l h}} \frac{d V}{d x} \gtrsim 1
$$

assuming gradient in $x$ direction and flows in the $y$ direction.

Similarly to the MTSI and LHDI (see Sec.5.4.2), instabilities with frequencies in this intermediate range between $\Omega_{c i}>\omega>\Omega_{c e}$ can drive particles out of their $\vec{E} \times \vec{B}$ drifts, a result especially relevant in magnetically dominated plasmas (Chen et al. 2015).

Finally, it is interesting to mention that the EIH instability (and other related to the kinetic scale K-H instability) has similar growth rates as the classical (MHD) KelvinHelmholtz instability, in spite of their very different mechanisms. This has led to the conjecture that it should be a very deep physical connection between those apparently different instabilities (Bret 2015).

\subsubsection{Magnetic field generation via kinetic K-H instability}

Very recently, it has been shown that kinetic electron scale K-H instability can generate large scale stationary (DC) magnetic fields via a dynamo-like process (Alves et al. 2012, Grismayer et al. 2013a,b, Alves et al. 2014). This is different from other collisionless mechanisms that can generate magnetic fields at small scales, in particular the Weibel instability (e.g., in collisionless shocks). Alves et al. (2012) demonstrated this process via 3D PIC simulations of (mostly relativistic) cold shear flows $\left(V_{0} \gg v_{t h, e}\right)$ in unmagnetized electron-proton plasmas. Their two fluid linear theory was based on the relativistic cold dispersion relation of K-H instability by Gruzinov (2008), which gives, for a tangential discontinuity (zero shear layer thickness), a growth rate:

$$
\gamma^{2}=\frac{\omega_{p e}^{2}}{2}\left(\sqrt{1+8 \frac{\left(k V_{0}\right)^{2}}{\omega_{p e}^{2}}}-1-2 \frac{\left(k V_{0}\right)^{2}}{\omega_{p e}^{2}}\right)
$$

with $k$ the wavenumber parallel to the shear flow direction. From this expression we can infer that the unstable wave number range is $k d_{e}<V_{0}$, different from the MHD result Eq. (5.66) in absence of magnetic field. The maximum growth rate is $\gamma_{\max }=\omega_{p e} / \sqrt{8}$ at the wavenumber $k=\sqrt{3} \omega_{\text {pe }} /\left(\sqrt{8} V_{0}\right)$. The real frequency is zero for all the unstable cases.

Note that this instability is in the opposite regime of the MHD K-H instability, where the shear flow speed is much less than the thermal one (in order to satisfy the MHD upper threshold Eq. (5.69), absent for these regimes). Therefore, the nature of this instability is very different from the MHD K-H, in which the mechanism is the amplification of the perturbed pressure due to Bernoulli principle. In this cold case, there is no pressure that can play that role and because the system is collisionless, there is no Bernoulli principle (see Sec.5.3.2). As explained by Grismayer et al. (2013b), this instability is produced due to the coupling of longitudinal modes (associated with the permittivity) at each side of the shear layer in the interface. This process is similar to the two-stream micro-instability.. Therefore, this kind of shear flow instabilities at electron scales can only takes place for fast flows (in comparison with the thermal speed).

Alves et al. (2012) generalized the previous expression by including density asymmetry, finding that the growth rates are reduced and the frequency gets a real oscillating part. 
Their 3D simulations showed the generation of an unexpected DC $(k=0)$ magnetic field in the interface between the counterpropagating flows, with direction perpendicular to the plane formed by the shear flow direction and the shear gradient. This is in addition to the expected fluctuating (AC) component of magnetic field on the plane of $\mathrm{K}-\mathrm{H}$ instability, necessary to roll-up the electrons forming the $\mathrm{K}-\mathrm{H}$ vortices. The $\mathrm{AC}$ component is the dominant one in the non-relativistic regime, while the DC component dominates scenarios with relativistic flows (because AC fluctuations are a decreasing function of the relativistic gamma factor). The physical mechanism for generation of this DC magnetic field is based in a current imbalance (DC current sheet) resulting from the mixing of electrons crossing the deformed shear interface during the non-linear evolution (ions cannot follow them due to their inertia), amplifying any initial magnetic field perturbation in a way similar to the Weibel instability. This is a purely kinetic effect that cannot be taken into account in fluid model, such as the fluid dispersion relation Eq. (5.73) that does not predict instability for $k=0$. The reason is that in these linearized calculations the dynamic deformation of the interface between the electron flows and the corresponding mixing is not considered (to zeroth order is fixed).

The DC magnetic field can be generated via two different mechanisms: either due to initial thermal effects in cases of warm shear flows, or due to the cold kinetic K-H instability in the opposite case (Grismayer et al. 2013a). In the first one, a simplified 1D kinetic (warm) model is used where an initial temperature drives the mixing of electrons across the shear layer. This model is valid as long as the self-generated electromagnetic field do not affect the free motion of the particles. In this way, they estimated that the generated DC magnetic field grows linearly on time. At saturation time, it has a typical thickness $L_{s a t}$ of the order of the ion Larmor radius on this magnetic field, approximately equal to $L_{s a t} \sim \gamma_{0} d_{e}$ ( $\gamma_{0}$ is the relativistic gamma factor). The strength of this DC magnetic field has a maximum value of

$$
B_{D C}^{s a t} \sim \frac{\gamma_{0} V_{0}}{c} \frac{m_{e} \omega_{p e}}{e}
$$

For the second (cold) case, the pure cold 2D K-H instability is the mechanism that drives the generation of DC magnetic field. The spread in the particles (mean) velocities produced by K-H play the role of an effective temperature driving the mixing of electrons across the shear layer. The calculations show that the linear growth on time and maximum value of the DC magnetic field have similar values to the warm scenario. So, it is seems to be a quite general process in these configurations, persisting for very long (ion) times scales. Grismayer et al. (2013a) and Alves et al. (2014) also estimated that a finite shear layer thickness $\Delta X$ reduces the maximum value of the DC magnetic field according to $B_{D C}^{s a t}(\Delta X) \sim B_{D C}^{s a t}(0) /\left(\Delta X / d_{e}\right)$, where $B_{D C}^{s a t}(0)$ is given by Eq. (5.74).

This mechanism of magnetic field generation has also been observed in $2 \mathrm{D}$ (Liang et al. 2013a,b) and 3D (Nishikawa et al. 2013, 2014) PIC simulations in different configurations, indicating the robustness of this process. In particular, some of these works analyzed pure electron-positron plasmas, where the Hall term is absent, hinting the kinetic nature of this process has nothing to do with the decoupling of electron/ions as in other fluid studies. Those studies were applied to explain the magnetic fields of gamma-ray bursts, AGNs and blazars.

It is worth mentioning that magnetic field generation has also been observed in MHD simulations of K-H instability, but with different mechanisms for the amplification: a tur- 
bulent dynamo (see, e.g., Zhang et al. 2008) or a Biermann battery (see, e.g., Modestov et al. 2014).

\subsubsection{K-H interaction with magnetic reconnection}

K-H instabilities can form in magnetic reconnection configurations in basically two different ways (see Nakamura et al. (2008) or Ma (2012) for an extended discussion). The first one is when the shear flow direction is parallel (or at least a component) to the reconnecting antiparallel magnetic fields. In this case, magnetic reconnection (tearing mode) is the dominant instability and the $\mathrm{K}-\mathrm{H}$ vortices play a secondary role, leading to a plasma mixing across the shear layer. The second possible configuration happens when the shear flow is so strong that the $\mathrm{KH}$ vortices can grow large enough to stretch magnetic field lines and produce localized magnetic reconnection events. Both configurations have been extensively observed and investigated in the context of magnetospheric reconnection. In the rest of this thesis we will focus in the first configuration.

With an MHD approach, the simultaneous existence of both $\mathrm{K}-\mathrm{H}$ and tearing modes is not allowed in 2D configurations (Ma 2012). Indeed, the only possible configuration in 2D is for wavevectors $\vec{k}$ of both tearing and K-H modes parallel to both the antiparallel magnetic field and the shear flow (the K-H $\vec{k}$ cannot have a component across the shear layer $x$, and no variations in $z$ are allowed in this setup). If the flow is sub-Alfvénic, by condition Eq. (5.67) K-H modes will be stable and the system will be tearing mode dominated. In this case, the effects of the flow is modifying the magnetic reconnection configuration by producing a density asymmetry in the outflow region, and decreasing the tearing mode growth rates. On the other hand, for super-Alfvénic flows K-H unstable, it was found that tearing modes will be stable (Chen et al. 1997). Then, the only way of having both instabilities is by allowing an oblique component of $\vec{k}$ in the out-of-plane direction, that necessarily requires a $3 \mathrm{D}$ configuration.

But there are also non-MHD mechanisms that allow 2D configurations with both K-H and tearing modes. Califano et al. (1999) introduced electron inertia effects in this system by using an EMHD model (where $d_{i} \rightarrow \infty$ ), allowing the study of the interaction between $\mathrm{K}-\mathrm{H}$ and tearing instabilities.

Later, by using an incompressible Hall-MHD linear theory model and corresponding 2D simulations, Chacón et al. (2003) showed that K-H instability and tearing mode can be simultaneously unstable for some parameter regimes. They studied configurations with flows parallel to the magnetic field and identical shear profiles. This is due to the decoupling between electron and ion flows inside of the sheared layer, when the latter is of the order of the relevant kinetic scales $\left(d_{i}\right.$ or $\left.\rho_{i}\right)$. In that case, the electron layer can be tearing unstable while the ion layer can be prone to K-H instability. They showed that Hall effects allows the formation of tearing mode islands and ion flow vortices in both subAlfvénic and super-Alfvénic regimes on dependence on the ratio $d_{i} / L$, in contrast with the aforementioned MHD predictions. More precisely, in the EMHD limit of large $d_{i}$ (ions with infinite inertia in comparison with electrons), the separation of electron to ion scales is large enough to have simultaneously and completely independent both $\mathrm{K}-\mathrm{H}$ and tearing instabilities. When $d_{i}$ is large but still comparable to $d_{e}$ (the so called Hall-MHD regime), both instabilities are not only simultaneously unstable, but also can be strongly coupled to each other. The dominant mode depends on the Alfvénic Mach number $M_{A}$, with the 
strongest coupling for values in a narrow range just above $M_{A}=1$. In general, the growth rates of the K-H instability were found to decrease when $\Delta x<d_{i}$, due to the Hall term. Note that these results are, however, restricted for cold ions and incompressible plasmas, assumption not valid in strongly magnetized environments.

2D two fluid simulations of the coupling between $\mathrm{K}-\mathrm{H}$ and tearing instability have been carried out by Nakamura et al. (2008) (with MHD scale K-H vortices, without guide field). They found that although K-H can start to grow for flows satisfying $M_{A}>2$ (the same MHD condition as Eq. (5.67)), highly rolled vortices that can stretch magnetic field lines requires much higher super-Alfvénic flow speeds $\left(M_{A}>5\right)$. For scenarios weakly K$\mathrm{H}$ unstable $\left(M_{A} \sim 2\right)$, reconnection mostly helps the growth of $\mathrm{K}-\mathrm{H}$ vortices by stretching the magnetic field lines. They pointed out that tearing mode has lower growth rates when the system is $\mathrm{K}-\mathrm{H}$ unstable, due to the magnetic field associated with the growth of this instability generated via the Hall term (additional magnetic pressure inhibits the thinning of the CS).

Full electromagnetic PIC simulations of the coupling between K-H instability and magnetic reconnection have also become available recently, in the context of magnetospheric environments, both in 2D (Nakamura et al. 2011) and 3D (Nakamura et al. 2013). These works investigated strong shear scenarios, where magnetic reconnection is a secondary instability generated inside of the K-H vortices.

$\mathrm{K}-\mathrm{H}$ instability can also interact with magnetic reconnection in a more subtle way. For example, K-H vortices have been reported in 2D PIC simulations of force free current sheets with guide field up to $b_{g}=80$ by Liu et al. (2014). They were located in the reconnection outflows at electron scales, but not related directly with secondary magnetic islands (no wrapping of magnetic field lines around them). As a result, they produce only small modulations of the reconnection rates, but not affecting their mean value. On the other hand, Fermo et al. (2012) reported in 2D PIC simulations of Harris sheets with guide field the formation of secondary magnetic islands (with wrapping of magnetic field lines around them) generated via this instability and not due to the tearing one. Those magnetic islands started as K-H vortices, were generated in the downstream region, and did not have the typical flow pattern expected from a tearing island, but vortical flows. Since those vortices had typical length scales of $k^{-1} \sim d_{e}$, they pointed out that the onset of $\mathrm{K}-\mathrm{H}$ instability requires, in addition to super-Alfvénic flows as in MHD, the additional requirement of growth rates $\gamma$ higher than the whistler wave frequency $\omega_{W}$. By assuming a typical growth rate linearly dependent on the shear flow speed and the wavenumber (similar to the MHD result), this condition reads:

$$
\gamma \sim \Delta V_{e, y} k \sim \frac{\Delta V_{e, y}}{d_{e}} \gtrsim \omega_{W} \sim \Omega_{c e, y} \frac{\left(k d_{e}\right)^{2}}{1+\left(k d_{e}\right)^{2}}
$$

where $\Omega_{c e, y}$ is the electron cyclotron frequency calculated on the magnetic field parallel to the interface between the counterstreaming flows. This condition can be rewritten as:

$$
\Delta V_{e, y}>\frac{V_{A e, y}}{2}
$$

with $V_{A e, y}$ the electron Alfvén speed based calculated with the $y$ component of the magnetic field (same as $\Omega_{c e, y}$ ). 


\subsubsection{Shear flow interaction with magnetic reconnection}

Other works have studied the role of the shear flows on the development of the tearing mode, in systems K-H stable. 2D MHD and Hall-MHD simulations (see Shi et al. 2005, and references therein) have shown that shear flows can tilt the magnetic islands and produce concentric vortical flows inside of them, as long as the shear flow speed is in a given sub-Alfvénic range. Later, Cassak (2011a) found the scaling laws that establish how reconnection rates decrease with increasing symmetric (same speed at each side of the interface) shear flows on a current sheet. This was derived under a Hall-MHD model without guide field, and tested with corresponding 2D simulations of Harris sheets (also by Cassak 2011b). The reason is because the outflows from the X point become slower. They are generated by the magnetic tension in the reconnected magnetic field lines, which is released by the shear flow. Let the reconnection rate be $(d \psi / d t)_{0}$ in absence on shear flow, while for finite values the reconnection rate is given by:

$$
\frac{d \psi}{d t_{0}}=\frac{d \psi}{d t} \sqrt{1-\frac{V_{0}^{2}}{V_{A}^{2}}}
$$

They also calculated the expected tilt angle $\theta_{\text {tilt }}$ of the dissipation region (with respect to the direction parallel to the current sheet) due to dynamic pressure of the shear flow:

$$
\tan \left(\theta_{\text {tilt }}\right) \sim \frac{V_{0}^{2}}{2 V_{A}^{2}}
$$

Roytershteyn and Daughton (2008) analyzed the stability of collisionless tearing mode in thin current sheets with this configuration by incorporating flows with symmetric profiles in the Harris equilibrium (not counterstreaming flows). They considered both magnetic and shear flows on ion kinetic length scales. For thick current sheets $L \gtrsim \rho_{i}$, the shear flow speed is the dominant parameter in the evolution of the CS, being able to either stabilize or destabilize the tearing mode in some parameter regimes. The latter case is opposite to the Hall-MHD predictions where a shear flow always stabilize the tearing mode. For thin current sheets $L \lesssim \rho_{i}$, the shear flow always stabilizes the reconnection process, in addition to produce strong non thermal features in the distribution function. An important finding is that the shear flows never stabilize completely the tearing instability in their analyzed parameter range, even with super-Alfvénic flows. All this was confirmed via solutions of the full linearized Vlasov-Maxwell systems and 2D PIC simulations.

Later, Hosseinpour and Mohammadi (2013) carried out a two fluid analysis of collisionless tearing mode under the influence of a shear flow. By taking into account electron inertia, they showed that the out-of-plane Hall magnetic field can exhibit a significant symmetric structure for low $\beta_{y}<1$, something not predicted by the standard two fluid model developed by Rogers et al. (2001) who did not consider the effects of the shear flow, relevant for force free CS. But the work by Hosseinpour and Mohammadi (2013) also predicts antisymmetric or quadrupolar structure of $\delta B_{z}$ for the regime $\beta_{y} \gg 1$, even with strong flow speeds, in agreement with Rogers et al. (2001). 


\subsection{Streaming instabilities}

\subsubsection{Instabilities of field aligned currents with oblique or perpendic- ular propagation}

Field aligned currents $\vec{V}_{0} \| \vec{B}$ can produce instabilities although the drift speed between ions and electrons is below $v_{t h, e}$. This condition necessarily requires obliquely propagating waves with $\left|\vec{k} \cdot \vec{V}_{0}\right| \ll V_{0}$ and thus a phase speed $\omega / k \ll v_{t h, e}$. The most known example is the so called ion cyclotron harmonic wave instability (ICHWI, see Drummond and Rosenbluth (1962)). It drives waves at the harmonics of $\Omega_{c i}$, with propagation almost perpendicular to $\vec{B}$ and wavelengths comparable to $\rho_{i}$ (Brown et al. 2013). They are the low frequency relatives of the electron cyclotron harmonic waves, also known as Bernstein waves (Bernstein 1958). Different from Buneman or ion acoustic instability that involves a coupling with Langmuir waves, in this case the coupling is with the slow extraordinary mode or lower hybrid mode. These waves are strongly damped by electron/ion cyclotron resonance if they have a parallel propagating component (not perpendicular propagation $\vec{k} \perp \vec{B}$ ). On the other hand, they are not affected significantly damped when $T_{i} \sim T_{e}$ as the ion-acoustic or Buneman waves. Kindel and Kennel (1971) showed that this critical threshold for the electrostatic ion-cyclotron instability is reduced to values $V_{0} / v_{\text {th }, e}>0.2$ for $T_{i} \sim T_{e}$, facilitating its excitation over ion-acoustic or Buneman in scenarios with weak currents or when the direction $\vec{k} \| \vec{B}$ is neglected for numerical reasons (as in 2D simulations). Therefore, it might be triggered more easily in reconnection, although their slow growth rates comparable to $\Omega_{c i}$ can make it more difficult to see since other faster processes can take over. Higher currents will suppress this instability by destroying the ion gyromotion, leading to a demagnetization of them and preparing the conditions for the possible excitation of ion-acoustic instability (Hasegawa 1975). On the other hand, the final non-linear saturate stage of this instability in a spatial modulation in the ion-density at scales of $\rho_{i}$ due to ion phase space holes, moving across the magnetic field at speed comparable with $v_{t h, i}$. These ion structures occurs because the low phase speed of the ICH waves allow a strong interaction with ions, instead of electrons as with the high frequency waves generated by the Buneman instability.

An important characteristic of these waves is that a reduced mass ratio accelerate their growth, an important fact to keep in mind for PIC simulations (Brown et al. 2013). However, and because this instability has frequencies on the order of $\Omega_{c i}$, which correspond to the typical time scales of magnetic reconnection, if it is developed will be overcomed by the main process, being unlikely to play any important role.

\subsubsection{Instabilities of cross-field currents with nearly perpendicular propagation}

A relative drift between electrons and ions perpendicular to the magnetic field: $V_{0} \perp \vec{B}$ can also generate electrostatic or electromagnetic instabilities. In this configuration a magnetic field has a destabilizing effect, making a plasma more prone to additional instabilities than unmagnetized environments. This is especially important when the plasma is inhomogeneous and/or the characteristic electron frequencies are comparable $\omega_{p e} \sim \Omega_{c e}$. 
And different from many other streaming instabilities, they can exist even for $T_{e} \sim T_{i}$.

It is important to remark that all the instabilities to be described, because of their typical frequencies and propagation direction, share an important feature: they can diffuse particles across the magnetic field 10 . This means that if the waves generated by them are strong enough, wave-particle interaction can move electrons and/or ions out of their $\vec{E} \times \vec{B}$ drifts across the magnetic field (Chen et al. 2015). As we will see in our simulations to be shown in Chapter 9, this fact has an important practical consequence when comparing with reduced plasma models, such as the gyrokinetic approximation. Indeed, since the gyrokinetic approach assumes that the motion of particle across the magnetic field is uniquely described by these drifts, it is understandable that these instabilities will have effects that can only be captured by means of a full kinetic approach.

\subsubsection{Electron cyclotron drift instability (ECDI)}

The electrostatic instabilities with relative bulk flow between electrons and ions as their source of free energy are known generically as cross-field streaming or beam-cyclotron drift instabilities. We are going to focus in the particular case of drifting electrons, where the corresponding instability is called electron-cyclotron drift instability (ECDI). ECDI has a minimum drift speed threshold much lower than $v_{t h, e}$, with typical frequencies relatively high: on the order of the electron cyclotron harmonics $n \Omega_{c e}$ (Hasegawa 1975).

The cold dispersion relation for this instability was first studied by Buneman (1962). Later, Wong (1970), Gary (1970) and Forslund et al. (1970) studied the thermal effects on the ECDI, finding the same hot dispersion relation as for (electron) Bernstein waves but with an additional term due to the ions

$$
\left(k \lambda_{D e}\right)^{2}=-1+e^{-\lambda} I_{0}(\lambda)+2 \omega^{2} \sum_{n=1}^{\infty} \frac{e^{-\lambda} I_{n}(\lambda)}{\omega^{2}-\left(n \Omega_{c e}\right)^{2}}+\frac{T_{e}}{2 T_{i}} Z^{\prime}\left(\frac{\omega-k V_{0}}{\sqrt{2} k v_{t h, i}}\right)
$$

with $\lambda=\left(k \rho_{e}\right)^{2} / 2$ and $I_{0}$ is the modified Bessel function of order 0 . Here we have assumed strictly perpendicular propagation $\vec{k} \| \vec{V}_{0} \perp \vec{B}$ (see derivation, e.g., in Gary 1970), unmagnetized ions and neglecting all kind of gradients. As an electrostatic dispersion relation describing longitudinal waves, it is valid for low beta plasmas. From the analysis of this dispersion relation, Forslund et al. (1970) found that the source of this instability is the resonance of electron cyclotron waves with the tail of the ion VDF. In other words, it is produced due to the coupling of (Doppler-shifted) slow electron Bernstein with ion acoustic waves (Lashmore-Davies and Martin 1973). Thus, in the terminology previously mentioned, it is a reactive instability. As a result, it produces perpendicular ion heating during this linear development, besides of the expected electron heating due to the electron cyclo-resonance. The unstable roots are asymptotic to the electron cyclotron harmonics $\omega=n \Omega_{c e}$ for large $k \rho_{e}$ (or $\left.\lambda\right)$. An approximate expression for the growth rates when $\gamma \ll \omega_{r}$ can be written as:

$$
\frac{\gamma}{\Omega_{c e}} \approx \frac{n}{2 \sqrt{\pi} k \rho_{e}} \frac{T_{e}}{T_{i}} \frac{\operatorname{Im} Z^{\prime}\left(\left(\omega_{r}-k V_{d}\right) / k v_{t h, i}\right)}{\left[1+\left(k \lambda_{D e}\right)^{2}-\left(T_{e} / 2 T_{i}\right) \operatorname{Re} Z^{\prime}\left(\left(\omega_{r}-k V_{d}\right) / k v_{t h, i}\right)\right]^{2}}
$$

\footnotetext{
${ }^{10}$ The diffusion across magnetic fields can be characterized by the coefficient $D_{r}\left\langle(\Delta r)^{2}\right\rangle$ depending on the wave electric field amplitude, as well as the diffusion in velocity space along the magnetic field $D_{v}\left\langle(\Delta v)^{2}\right\rangle$. See details in Drummond and Rosenbluth (1962).
} 
From this we can infer that this instability is very weakly dependent on both temperature and mass ratio. It has a cutoff for wavelengths smaller than the Debye length $k \lambda_{D e}>1$, which occurs for smaller values of the drift speed $V_{0} / v_{t h, e}$ (for larger frequency ratios $\left.\omega_{p e} / \Omega_{c e}\right)$. Therefore, their typical wavelengths are of the order $k \lambda_{D e}<1$. Since the frequency ratio is proportional to the Alfvén speed due to the relations in Appendix A.3.1, this means that the instability is more efficient in environments with high density and low magnetic field strength. More precisely, an approximate instability criterion can be written as (Forslund et al. 1972):

$$
\frac{V_{0}}{v_{t h, e}} \gtrsim n \frac{\Omega_{c e}}{\omega_{p e}}
$$

and it also requires $V_{0}>v_{t h, i}$. From here we can see that is more difficult destabilize higher harmonics (characterized by $n$ ), i.e.: they require higher values of the drift speed. Furthermore, high values of the frequency ratio $\omega_{p e} / \Omega_{c e}$ allow a lower threshold in the drift speed 11. For $V_{0}>v_{t h, i}$ and large wavelengths $k \lambda_{D e}<1$, this instability has a maximum growth rate of the order (Forslund et al. 1972):

$$
\gamma_{\max } \sim \frac{\Omega_{c e}}{\sqrt{\pi}} \frac{V_{0}}{v_{t h, e}} \frac{T_{e}}{T_{i}}
$$

Note the dependence on the temperature ratio in the previous expression. This instability is more efficient for large electron temperatures. For $T_{i} \sim T_{e}$, the nature of this instability changes from reactive to dissipative (only the branch related with electron Bernstein waves are unstable), implying an easier quenching (in comparison to other reactive instabilities) due to quasilinear effects that breaks the resonance condition (see Lashmore-Davies and Martin 1973). It is also interesting to see the cold limit $V_{0}, \omega / k \gg$ $v_{\text {the }}$ of the dispersion relation Eq. 5.79)(see Forslund et al. (1970) and also the textbook Birdsall and Langdon (1991)):

$$
1-\frac{\omega_{p e}^{2}}{\omega^{2}-\Omega_{c e}^{2}}-\frac{\omega_{p i}^{2}}{\left(\omega-k V_{0}\right)^{2}}=0
$$

For $\Omega_{c e} \ll \omega_{p e}$, this becomes identical to the cold Buneman dispersion relation (see, e.g., Treumann 2001, Sec 2.3) although the geometry involved and mechanism for the wave interaction are quite different. For this instability, the coupling is not with the ion-acoustic waves, but between the upper-hybrid mode with the Doppler shifted lower hybrid mode.

Later, Forslund et al. (1972) extended the previous analysis by allowing propagating with arbitrary angle and including collisional effects. They found the turbulence spectrum generated by this instability covers a wide range of angles centered in the drift speed direction, in the plane perpendicular to $\vec{B}$. As expected, they also confirmed the narrow range of angles of unstable waves for non perpendicular propagation $(\vec{k} \cdot \vec{B} \neq 0)$, due to

\footnotetext{
${ }^{11}$ This fact impose a severe restriction on PIC simulations of these instabilities. In fact, due to numerical constraints, these simulations require a lower value of the frequency ratio $\omega_{p e} / \Omega_{c e}$ than the usually found in nature. This leads to artificially large charge separation and correspondingly large electric fields, making the system more unstable (Winske et al. 1987, Chen et al. 2012). In addition, the threshold for these streaming instabilities will be higher than in real physical environments, making more difficult the excitation of the processes associated with them.
} 
the cyclotron damping. On the other hand, they also established that an electron collision frequency of the order of the growth rate is required for stabilization, making those effects negligible in our case. Forslund et al. (1972) also carried out PIC simulations of this instability for the non-linear evolution and saturation of this instability, although in a high beta parameter range. For wavelengths satisfying $1<k \rho_{e}<2 \pi$, they observed strong perpendicular electron heating, being attributed to a resonance in the main part of the electron VDF and the corresponding trapping. This contributes, as expected, to rise the threshold Eq. (5.81) and thus to self-suppress this instability.

During the early years of the study of the ECDI other works reached slightly different conclusions. Among them it is worth to mention the one carried out by Lampe et al. (1971), applied especially for low beta plasmas. They found that the fast growth rates of the ECDI transform it in a ion-acoustic instability in the course of its evolution, avoiding the electron trapping seen in the simulations by Forslund et al. (1972). The fast exponential electron heating slows down very quickly, due to the relatively low level of saturation turbulence, generating ion trapping. And since the ion-acoustic instability requires $T_{e} \gg T_{i}$, in many cases the final state will be a stable one. Another work to be analyzed with more detail later is Fujimoto and Machida (2003), with results also supporting the conclusion that the electron heating by this instability is not too efficient for relatively low drift speeds $V_{0}<v_{t h, e}$. Indeed, they estimated the electron temperature at saturation to be:

$$
T_{e, s a t} / T_{e, 0} \sim\left(V_{0} / v_{t h, e}\right)^{2}
$$

Zhou et al. (1984) analyzed the plasma beta effects on this instability, founding a reduction in the growth rates as this parameter increases. In addition, magnetic gradient will al so contribute to stabilize the ECDI.

\subsubsection{Modified two stream instability (MTSI) / Kinetic cross-field streaming in- stability KCSI}

Krall and Liewer (1971) (see also Krall and Liewer 1972) discovered a cross-field streaming instability not only more or less independent on the temperature ratio and with threshold much lower than $v_{t h, e}$ (both properties similar to ECDI), but also with an intermediate range of frequencies $\Omega_{c i}<\omega<\Omega_{c e}$. They called modified two-stream instability or MTSI. It is usually applied for a parameter regime where the ions are unmagnetized while the electrons are strongly magnetized, implying wavelengths $\rho_{e} \lesssim \lambda \ll \rho_{i}$. Its typical frequency and growth rates are close to the lower hybrid frequency $\Omega_{l h}$ (less than the typical ones from the ECDI) and it has almost perpendicular propagation (same as ECDI). This and many other properties can be derived from the electrostatic dispersion relation (McBride 1972):

$$
1-\frac{1}{2 k^{2} \lambda_{D e}^{2}} \frac{T_{e}}{T_{i}} Z^{\prime}\left(\frac{\omega-\vec{k} \cdot \vec{U}}{\sqrt{2} k v_{t h, i}}\right)-\frac{e^{-\lambda} I_{0}(\lambda)}{2 k^{2} \lambda_{D e}^{2}} Z^{\prime}\left(\frac{\omega}{\sqrt{2} k v_{t h, e}}\right)+\frac{1-e^{-\lambda} I_{0}(\lambda)}{\left(k \lambda_{D e}\right)^{2}}=0
$$

with $\lambda=\left(k \rho_{e}\right)^{2}$ and $I_{0}$ is the modified Bessel function of zeroth order. As usual, this is calculated in the electron frame of reference with ions drifting with speed $V_{0}$. It is also assumed $\Omega_{c i} \ll \omega \ll \Omega_{c e}$ and $k \rho_{e}<1$. Compare with the dispersion relation for its highfrequency relative ECDI Eq. (5.79). Due to the assumption of low frequencies $\omega \ll \Omega_{c e}$, 
only the term $n=1$ in the infinite sum Eq. (5.79) is kept. McBride (1972) pointed out and tested a very interesting property of this dispersion relation: it is independent on the mass ratio if the angle $\sin \theta=k_{z} / k$ is rescaled to $\theta^{\prime}=\theta \sqrt{m_{i} / m_{e}}$. If it is additionally assumed small $\theta$ and $\lambda<1$, the dispersion relation can be expressed independently of $\omega_{p e} / \Omega_{c e}$ rescaling the frequency and wavenumber as:

$$
\begin{aligned}
& \omega=\frac{\omega}{\omega_{p i}} \sqrt{1+\omega_{p e}^{2} / \Omega_{c e}^{2}} \\
& k^{\prime}=k \frac{\Omega_{c e}}{\omega_{p e}} \sqrt{1+\omega_{p e}^{2} / \Omega_{c e}^{2}}
\end{aligned}
$$

So, Eq. (5.85) has only two real free parameters: $T_{e} / T_{i}$ and $V_{0} / v_{t h, i}$. Numerical solution of the unstable waves predicted by this dispersion relation show that MTSI always have a negative group speed $\partial \omega_{r} / \partial k \lesssim 0$, similar to the generic property of the ion-acoustic wave (Gary et al. 1987), in addition to a phase speed smaller than the drift speed: $\left|\omega_{r} / k\right|<V_{0}$. The latter means that the positive slope of the ion VDF drives the instability.

Now, using the appropriate asymptotic expansion of the plasma zeta function in the cold limit $k \rho_{e} \ll 1, k v_{t h, i}<|\omega-\vec{k} \cdot \vec{U}|$ and $k v_{t h, e}<|\omega|$, we have (McBride 1972):

$$
1+\frac{k_{\perp}^{2}}{k^{2}} \frac{\omega_{p e}^{2}}{\Omega_{c e}^{2}}-\frac{k_{\|}^{2}}{k^{2}} \frac{\omega_{p e}^{2}}{\omega^{2}}-\frac{\omega_{p i}^{2}}{\left(\omega-k_{\perp} V_{0}\right)^{2}}=0
$$

From here we can see its similarity with the dispersion relation of the Buneman instability (see, e.g., Treumann 2001, Sec 2.3), and hence the origin of the name MTSI. This fact also implies that is a non-resonant or fluid-like instability. The second term is due to the polarization drift of electrons across the magnetic field $\vec{v}_{P}=-\left(d E_{\perp} / d t\right) /\left(B \Omega_{c e}\right)$, while the second gives the free streaming of electron along the magnetic field lines. This has a very interesting interpretation by comparing with the Buneman dispersion relation: the electrons behave as if they had an "effective mass" $m_{e, \text { eff }}=\frac{k^{2}}{k_{1}^{2}} m_{e}$, which is larger for propagation angles nearly perpendicular $\frac{k}{k_{\|}} \gg 1$ (McBride 1972). We can have a deeper insight about the range of frequencies involved in this MTSI by simplifying Eq. (5.88) and rewrite it as (Treumann and Baumjohann 2001):

$$
1-\frac{\Omega_{l h}^{2}}{\left(\omega-k_{\perp} V_{0}\right)^{2}}-\frac{m_{i} k_{\|}^{2}}{m_{e} k^{2}} \frac{\Omega_{l h}^{2}}{\omega^{2}}=0
$$

with $\Omega_{l h}$ is the lower hybrid frequency (see Appendix A.1.2). This expression allow us easily to make a correspondence with the Buneman instability dispersion relations can be obtained by replacing $\omega_{p e}$ with $\Omega_{l h}$ and $\omega_{p i}^{2}$ with $\left(m_{i} k_{\|}^{2} / m_{e} k^{2}\right) \Omega_{l h}^{2}$, respectively. Therefore, this reactive instability involves the coupling (Lashmore-Davies and Martin 1973) of a lower hybrid mode $\left(\omega \approx \Omega_{l h}\right)$ with a Doppler shifted electron Langmuir mode $(\omega \approx$ $\left.k_{\perp} V_{0}-\left(k_{\|} / k\right) \sqrt{m_{i} / m_{e}} \Omega_{l h}\right)$. From the cold dispersion relation Eq. (5.89) we can derive the following condition for the existence of MTSI (Treumann and Baumjohann 2001):

$$
\frac{k_{\perp}^{2} V_{0}^{2}}{\Omega_{l h}^{2}}<\left(\frac{m_{e} k^{2}}{m_{i} k_{\|}^{2}}\right)^{1 / 3}\left[1+\left(\frac{m_{i} k_{\|}^{2}}{m_{e} k^{2}}\right)^{1 / 3}\right]^{3}
$$


By comparing with the respective condition on the threshold speed of the Buneman instability $\left(k V_{0}\right)^{2}<\omega_{p e}^{2}\left[1+\left(\frac{m_{e}}{m_{i}}\right)^{1 / 3}\right]^{3}$ (see, e.g., Treumann 2001, Sec. 2.3), we can infer that MTSI requires a much lower threshold than the latter to be driven unstable, because $\Omega_{l h} \ll \omega_{p e}$. From Eq. (5.90), we can also derive the following relation that gives the propagation direction of the MTSI waves with maximum growth rate (more unstable roots of Eq. (5.85)):

$$
\left(\frac{k_{\|}^{2}}{k_{\perp}^{2}}\right) \sim \frac{m_{e}}{m_{i}} \ll 1
$$

i.e., close to perpendicular propagation. However, a necessary condition for the existence of this instability is a finite $k_{\|}$, or not strictly a perpendicular propagation. Note that this condition is relaxed, in the sense of more oblique waves are allowed, when a reduced mass ratio is used. This also implies that the "effective electron mass" for these waves is similar to the ion mass: $m_{e, e f f} \sim m_{i}$. Finally, for nearly perpendicular propagation direction, the maximum growth rate, wave number and real frequency are given by:

$$
\begin{aligned}
\gamma_{\max } & =\Omega_{l h} / 2 \\
k_{\perp, \max } V_{0} & \sim \Omega_{l h} / 2 \\
\omega_{r, \max } & =k_{\perp, \max } V_{0} / 2 \sim \Omega_{l h}
\end{aligned}
$$

Note that through $\Omega_{l h}$, it can be inferred a weak dependence of this instability on the frequency ratio $\omega_{p e} / \Omega_{c e}$ (e.g.: a decrement of 3 orders of magnitude in the frequency ratio will decrease growth rates only in a factor of two. See further details in Gary et al. (1987) or Lashmore-Davies and Martin (1973)). The estimation Eq. (5.94) allow us to rewrite the condition to overcome ion Landau damping, $\left|\omega-\vec{k} \cdot \vec{V}_{0}\right|>k v_{t h, i}$, as an instability threshold on the drift speed (McBride 1972):

$$
V_{0}>v_{t h, i}
$$

In a similar way, by using both estimations Eq. (5.93) and Eq. 5.94), we can find the following condition to avoid electron Landau damping given by $\omega>k_{\|} v_{t h, e}$ :

$$
\frac{k_{\|}}{k_{\perp}} \lesssim \frac{V_{0}}{v_{t h, e}} \quad \Leftrightarrow \quad \tan \theta \lesssim \frac{m_{e}}{m_{i}} \frac{V_{0}}{v_{t h, e} m_{e} / m_{i}}
$$

which is a restriction on the propagation direction (McBride 1972): waves with propagation far from strictly perpendicular will experience more electron Landau damping. From here and Eq. (5.91) we can understand the decrease in the Buneman instability threshold from $V_{0}>v_{t h, e}$ to the MTSI threshold

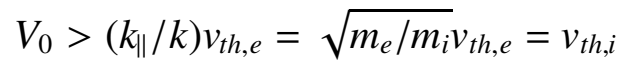

Other properties can be obtained just through a numerical solution of the hot dispersion relation Eq. (5.85). One of these properties is the dependence of the growth rates on the drift speed $V_{0} / v_{t h, i}$ (note that the cold dispersion relation Eq. (5.88) is independent on this parameter). Following this method, McBride (1972) found that the maximum value 
of the growth rates is a decreasing function of the drift speed, as well as the propagation angles of this maximum and the cut-off angles in which MTSI ceases to exist. On the other hand, it was also shown that $\gamma_{\max }$ is a weakly decreasing function of the temperature ratio. The propagation angle in which this $\gamma_{\text {max }}$ takes place also decreases the angle $\theta$ (it is not constant as the approximation Eq. (5.91) may suggest). However, for large ratios $T_{e} / T_{i}$ the unstable range of wave numbers is enhanced because the ion-acoustic instability, with parallel propagation, takes over. Indeed, it can be shown that both instabilities are closely related, especially in the case of $T_{e}=T_{i}$ when the propagation shift from perpendicular to parallel (Lashmore-Davies and Martin 1973). This implies the change of nature from a reactive (MTSI) to dissipative instability (ion-acoustic), being more easily stabilized.

In contrast to most of the other streaming instabilities analyzed so far that only heat electrons, MTSI can also heat ions in the perpendicular direction to the magnetic field, along the direction of the unstable waves. This is in addition to the expected parallel electron heating due to their free streaming along $\vec{B}$ (and different from the perpendicular electron heating due to ECDI because the cyclotron resonances). It is possible to relate the magnitude of the relative heating by noticing first that the effective electron and ion mass are equal, $m_{e \text {, ff }}=m_{i}$, for a propagation direction of $\sin \theta=k_{\|} / k=\sqrt{m_{e} / m_{i}}$ (which correspond to the most unstable waves). Then, it is expected than the perpendicular ion heating can be comparable with the parallel electron heating for this propagation direction. By using some quasilinear estimates at saturation time, and validates afterwards with $2 \mathrm{D}$ simulations, McBride (1972) found the relation:

$$
\sqrt{\frac{k_{B} T_{i \perp}}{m_{i}}}=\sqrt{\frac{k_{B} T_{e \|}}{m_{i}}} \sim \frac{V_{0}}{2}
$$

i.e.: the streaming free energy is equipartitioned between the thermal energies of ions and electrons.

So far, all the previous analysis and conclusions were done assuming only electrostatic fluctuations and small plasma beta. McBride (1972) and McBride and Ott (1972) al so analyzed the electromagnetic effects in MTSI. Many of the conclusions can be understood from the cold dispersion relation considering both effects (but still $k_{\|} \gg k_{\perp}$ and $\omega \ll \Omega_{c e}$ ), analogous to Eq. (5.89):

$$
1+\frac{k_{\perp}^{2}}{k^{2}} \frac{\omega_{p e}^{2}}{\Omega_{c e}^{2}}\left(1+\frac{1}{1+\beta_{e}} \frac{\Omega_{c e}^{2}}{(c k)^{2}}\right)-\frac{k_{\|}^{2}}{k^{2}} \frac{\omega_{p e}^{2}}{\omega^{2}} \frac{1}{1+\frac{\Omega_{c e}^{2}}{(c k)^{2}}}-\frac{\omega_{p i}^{2}}{\left(\omega-k_{\perp} V_{0}\right)^{2}}=0
$$

These authors established that the extra terms due to electromagnetic effects have a stabilizing effect, imposing an upper limit in the value of drift speed and restricting the instability to

$$
\frac{V_{0}}{V_{A}} \lesssim \sqrt{1+\beta_{e}}
$$

implying and extra condition on the lower threshold $V_{0}>v_{t h, i}$ to $v_{t h, i}<V_{A} \sqrt{1+\beta_{e}}$. Combining both conditions:

$$
1<\frac{V}{v_{t h, i}} \lesssim \sqrt{2 \frac{1+\beta_{e}}{\beta_{i}}}
$$


Furthermore, the electrostatic instability is recovered in the limit of small speeds $V_{0}$ in comparison with $V_{A} \sqrt{1+\beta_{e}}$.

After the initial studies in the early 70s summarized in McBride (1972), other works analyzed the electromagnetic effects on this instability in the high beta range of parameters. The reason is because the usual electrostatic approximation is valid for small wavelengths, assumption not necessarily valid for the expected wavelengths of MTSI in a wider set of parameter regimes. Lemons and Gary (1977) found that these effects reduce the growths rate of MTSI, but at the same time make wider the unstable range of wavenumbers (with important consequences for its overall contribution to the turbulence level). The electromagnetic contributions to the dispersion relation are also more dominant, in comparison with the electrostatic ones, for non-perpendicular propagation. The relative importance of the electromagnetic corrections is also an increasing function on the plasma beta. More precisely, they are dominant for values greater than one of the coupling parameter (Davidson et al. 1977):

$$
\delta_{E M}=\frac{\omega_{p e}}{c^{2} k^{2}}=\frac{\beta_{e}}{\left(k \rho_{e}\right)^{2}} \gtrsim 1
$$

Finally, Lemons and Gary (1977) also found that MTSI has a comparable electrostatic and magnetic fluctuation level for low plasma betas, even though it is mostly an electrostatic instability in this regime. Later, Wu et al. (1983) analyzed the large drift regime of this instability, finding that the nature of this instability is essentially kinetic (electrons become more resonant producing enhanced Landau damping) and electromagnetic when $V_{0} \gg V_{A}$ in high beta plasmas, in the transition to the ECDI. This means that the propagation angle at maximum growth rate decreases significantly (shift from perpendicular) and the unstable modes are modified whistler waves. On the other hand, in low beta plasmas, electromagnetic effects can suppress the instability for $V_{0}>V_{A}$, but they are unimportant in the opposite regime, in agreement with the findings of McBride (1972). They also found that in low plasma betas, the levels of electrostatic and magnetic fluctuations will be similar (Wu et al. 1983). Because the different nature of MTSI in the high beta regime, it is often called kinetic cross-field streaming instability or KCSI. However, other works prefer to call both MTSI and KCSI with the generic name electron-ion modified two stream instability (Gary et al. 1987).

Another later work by Winske et al. (1985) investigated the ion and electron heating for this instability in both low beta (MTSI) and high beta regimes (KCSI), with both quasilinear theory and 2D PIC simulations. In general, the fluctuations levels and both electron and ion heating rates decrease with an increasing plasma beta (it has low saturation levels in the high beta regime). They showed that for an increasing plasma beta, the heating becomes more isotropic for ions. This reflects the fact that higher beta implies a more oblique propagation of the unstable waves, and therefore a more comparable heating between both directions. Regarding the electrons heating, this becomes more field aligned, i.e.: more anisotropic with an increasing plasma beta. On the other hand, the relative ratio between parallel electron heating and perpendicular ion heating is an increasing function of the plasma beta. Another important finding of Winske et al. (1985) is that for low beta the saturation mechanism is comparable between electron and ion trapping, while for high beta it becomes easier for the unstable waves to trap electrons due to the more oblique propagation. 


\subsubsection{Cross field streaming instabilities and magnetic reconnection}

A long time after the pioneer studies of cross-field streaming instabilities in the 70s, Fujimoto and Machida (2003) applied these old findings to a scenario likely to be developed during magnetic reconnection. In particular, they proposed that due to the decoupling of ions and electrons in the diffusion region, large cross-field Hall currents can be generated, which can interact with the out-of-plane Hall magnetic field via the electrostatic cross-field streaming instabilities previously discussed. Of course, this effect might be additionally enhanced by imposing a magnetic guide field. Note that the relative streaming of the electrons and ions arises because the outflow speeds from the $\mathrm{X}$ point are of the order of the in-plane Alfvén speed $V_{A}$ for ions and in-plane electron Alfvén speed $V_{A e, y}$ for electrons (Shay et al. 2001). By performing a linear analysis and 2D PIC simulations with much higher resolution than the original works from the 70s, they found a very interesting interplay between the two cross-field streaming instabilities KCSI and ECDI. It is important to mention than in the linear analysis, they considered frequencies higher than the ion-cyclotron frequency, since modes with lower frequencies will not have time to grow in the diffusion region. More precisely, $\Omega_{u h} \gtrsim \omega>\Omega_{l h} \gg \Omega_{c i}$, with $\omega_{u h}=\sqrt{\omega_{p e}^{2}+\Omega_{c e}^{2}}$ the upper hybrid frequency and $\Omega_{l h}$ the lower hybrid frequency (see Appendix A.1.2). They also allowed arbitrary propagation direction and electromagnetic fluctuations in addition to the electrostatic ones. In this way, they could study both KCSI and ECDI with an unified approach.

The mechanism proposed by Fujimoto and Machida (2003) can be understood as follows. The kinetic cross-field streaming instability KCSI requires much lower threshold $V_{d} / v_{t h, e}$ on the relative drift speed than ECDI, being more likely to be developed first in the diffusion region of magnetic reconnection as the current sheet thins. This instability has relatively low growth rates but can produce a weak parallel heating of electrons. Then, when the Hall current or relative speed between electron-ions overcomes a critical threshold (a significant fraction of $v_{t h, e}$ according to Eq. (5.81)), the fastest growing ECDI can be triggered. This threshold was about $V_{d} / v_{t h, e} \sim 0.6$ for the parameter regime analyzed. As we discussed, ECDI heats the electrons in the perpendicular direction very quickly.

One of the findings of the linear dispersion analysis of Fujimoto and Machida (2003) is that the critical threshold in which ECDI is dominant in comparison with KCSI is a decreasing function on the mass ratio, important for numerical simulations.

There have been previous investigations about the role of streaming instabilities in force free configurations of magnetic reconnection, but in the relativistic regime with pair plasmas (see Haruki and Sakai 2001, Sakai and Matsuo 2004). They also noticed the generation of quasi-steady magnetic fields (similar to the previously described and reported by Alves et al. (2014), caused by the Kelvin-Helmholtz (macro) instability in unmagnetized plasmas). Nevertheless, they are strongly dissipated in a short time because of the electrostatic fluctuations caused by the streaming instability. In Liu et al. (2014) it was shown that reduction of reconnection rates may happen when intense electric fields, due to secondary streaming instabilities, are generated in magnetic reconnection configurations for a strong guide field. 


\subsubsection{Lower Hybrid Drift Instability (LHDI)}

The modified two stream instability MTSI have frequencies near the lower hybrid frequency $\Omega_{l h}$, with propagation oblique to $\vec{B}$. Their generalization for inhomogeneous plasmas (in density, temperature or magnetic field) is called Lower Hybrid Drift instability (LHDI), being first found by Krall and Liewer (1971). Basically, assuming inhomogeneity only in density and/or pressure, the diamagnetic drifts generated by the pressure gradients produce cross-field currents that behaves analogously to the ones required for the triggering of MTSI (for a more detailed proof of their equivalence, see Lashmore-Davies and Martin 1973). As a result, the range of conditions in which MTSI can exist is extended due to the gradients (destabilization becomes easier).

Now, in order to estimate how inhomogeneous a plasma should be to trigger LHDI, we need to define a characteristic quantity. In the frame of reference of stationary ions, the electrons will be drifting with the diamagnetic drift given by Eq. (3.21), or, assuming gradients in $\hat{x}$ direction and magnetic field in the $\hat{z}$ direction,

$$
\begin{aligned}
\vec{V}_{D, \perp} & =\frac{v_{t h, e}^{2}}{\Omega_{c e}}\left(\frac{1}{n_{e}} \frac{d n_{e}}{d x}+\frac{1}{T_{e}} \frac{d T_{e, \perp}}{d x}\right) \hat{y} \\
\Leftrightarrow \quad \frac{\vec{V}_{D, \perp}}{v_{t h, i}} & =\rho_{i}\left(\frac{1}{n_{e}} \frac{d n_{e}}{d x}+\frac{1}{T_{e}} \frac{d T_{e, \perp}}{d x}\right) \hat{y}
\end{aligned}
$$

It is interesting to note that if we denote the approximate length scale of variations of the gradients as $\left(1 / n_{e}\right) d n_{e} / d x \sim\left(1 / T_{e}\right) d T_{e} / d x \sim 1 / L_{n}$, then we have an inverse relation between the magnitude of the diamagnetic drift and the scale of inhomogeneity in units of $\rho_{i}$ :

$$
\frac{V_{D, \perp}}{v_{t h, i}}=\frac{1}{L_{n} / \rho_{i}}
$$

In the so called drift-approximation, the full motion of electrons will also have to incorporate at least the $\vec{E} \times \vec{B}$ drift (dominant over gradient drifts especially in low beta plasmas. See Gary (1970) and Lashmore-Davies and Martin (1973)) for a frame of reference noncomoving with them. Note that a more general expression should also include magnetic field gradients, but they usually are negligible in low beta plasmas. Then, an approximate threshold condition of this instability, assuming $(\omega / k) \gg v_{t h, i}$ and neglecting electron Larmor radius effects $k_{\perp} \rho_{e}>1$ can be written as (Krall and Liewer 1971):

$$
V_{0} V_{D, \perp} \gtrsim \frac{T_{i}+T_{e}}{m_{i}}
$$

with $V_{0}$ is the same cross-field electron-ion drift speed discussed for the streaming instabilities (possibly arising as a result of the $\vec{E} \times \vec{B}$ in a frame of reference with electrons non-stationary). Note that the right hand side is just the square of the sound speed. Combining with Eq. (5.104), we have the alternate expression:

$$
\left|\frac{1}{P_{e, \perp}} \frac{d P_{e, \perp}}{d x}\right|^{-1} \lesssim \rho_{i}
$$


with $P_{e, \perp}=n_{e} k_{B} T_{e, \perp}$. This simply means that the length scale of the pressure gradient should be smaller than $\rho_{i}$ in order to drive LHDI unstable. The inclusion of the associated gradient drifts and its relatively long wavelengths makes necessary the addition of electromagnetic effects (Lemons and Gary 1977, Lashmore-Davies and Martin 1973) for their linear analysis (contrary to KCSI or ECDI for which an electrostatic approach is enough due to their short wavelengths). However, a first order approximation can be obtained in the electrostatic approximation. We assume the same conditions as for MTSI: unmagnetized ions, magnetized electrons (wavelengths $\rho_{e}<\lambda<\rho_{i}$ ). Then, the real part of the dispersion relation becomes for phase speeds much larger than the ion thermal speed $(\omega / k) \gg v_{t h, i}$ (Treumann and Baumjohann 2001):

$$
1+\frac{\omega_{p e}^{2}}{\Omega_{c e}^{2}}+\frac{1}{k^{2} \lambda_{D e}^{2}} \frac{\Omega_{d e}}{\left(\omega-\vec{k} \cdot \vec{V}_{D, \perp}\right)}+\frac{1}{k^{2} \lambda_{D i}^{2}}=0
$$

Note the similarity of this expression with the corresponding dispersion relation for the Buneman or MTSI instabilities Eq. (5.88). Therefore, in a similar way, it will have solutions for $\omega / k<V_{D, \perp}$. Assuming additionally $T_{e} \ll T_{i}$, it is possible to find analytical approximations of the maximum growth rate, frequency and wavenumber (Davidson and Gladd 1975):

$$
\begin{aligned}
\frac{\gamma}{\Omega_{l h}} & =\frac{1}{4} \sqrt{\frac{\pi}{2}}\left(\frac{V_{D, \perp}}{v_{t h, i}}\right)^{2} \\
\frac{\omega}{\Omega_{l h}} & =\frac{1}{\sqrt{2}}\left(\frac{V_{D, \perp}}{v_{t h, i}}\right) \\
k \rho_{e} & =\sqrt{2} \sqrt{\frac{T_{i}}{T_{e}}}
\end{aligned}
$$

which implies a phase speed at maximum growth rate $\omega / k \approx V_{D, \perp} / 2$. Now, let us discuss some of the properties that only a numerical solution of the full electrostatic dispersion relation can provide. As it was found first by Gladd (1976), the propagation direction of the most unstable waves is perpendicular to both magnetic field: it propagates much less obliquely than MTSI, with maximum growth rates in the regime $k_{\|} / k_{\perp} \ll 1$. In addition, it is wavevector is also orthogonal to the pressure gradients (Yoon and Lui 2008). Similar to MTSI, it is not too dependent on the temperature ratio, something especially relevant in a plasma with equal temperature for electrons and ions where other instabilities, such ion-acoustic, should be damped (according to Krall and Liewer (1971): "both [MTSI and LHDI] are quite happy growing in a $T_{e} \sim T_{i}$ plasma)". By analyzing an intermediate range of drift speeds $V_{D, \perp} \sim V_{0} \sim v_{t h, i}$, Davidson and Gladd (1975) found that for $T_{e} \sim T_{i}$ its typical wavenumbers at maximum growth rates satisfy $k_{\perp} \rho_{e} \sim 1$ over a wide range of values of $V_{0}$ and $V_{D, \perp}$ (compare with MTSI that have larger wavelengths $k_{\perp} \rho_{e}<1$ ). Therefore, finite electron Larmor radius effects are essential to describe properly this instability in this parameter range. This is something to keep in mind when comparing with gyrokinetic simulations in which these effects are ordered out. It was also shown that the instability is not completely shut-down when $V_{D, \perp}, V_{0}<v_{t h, i}$ as the threshold condition Eq. (5.106) may suggest. Instead, when taking into account finite electron Larmor radius effects by solving the full dispersion relation, growth rates are still a significant 
fraction of $\gamma \sim \Omega_{l h}$ when the streaming or diamagnetic drift are below the thermal speed. Davidson and Gladd (1975) also analyzed the non-linear evolution of this instability and its contribution to the anomalous transport, founding that the fastest electron and ion heating is reached when $V_{D, \perp}, V_{0} \sim v_{t h, i}$.

All the previous conclusions and analysis were done under the assumption of unmagnetized ions: $\omega \gg \Omega_{c i}$ and $k_{\perp} \rho_{i} \gg 1$. For diamagnetic drift speeds low enough, this assumption breaks down. As pointed out by Davidson et al. (1977), taking into account these finite ion Larmor radius effects implies another different bottom constraint on the minimum value of $V_{D, \perp}$, or equivalently, the minimum inhomogeneity required to trigger LHDI (since both are related by Eq. (5.104)). This cannot be taken into account by means of the previously discussed linear analysis and electrostatic dispersion relation, and therefore Eq. (5.106) cannot be valid in this regime. The result is the instability threshold condition:

$$
\left|\rho_{i}\left(\frac{1}{n_{e}} \frac{d n_{e}}{d x}+\frac{1}{T_{e}} \frac{d T_{e, \perp}}{d x}\right)\right|>\frac{4}{\sqrt{2}} \frac{\Omega_{c i}}{\Omega_{l h}}=\frac{4}{\sqrt{2 m_{i} / m_{e}}} \sqrt{\frac{1+\omega_{p e}^{2} / \Omega_{c e}^{2}}{\omega_{p e}^{2} / \Omega_{c e}^{2}}}
$$

For realistic frequencies ratios in many astrophysical environments $\left(\omega_{p e} \gg \Omega_{c e}\right)$ and realistic mass ratios, the value is quite low $\left(4 /\left(\sqrt{2 m_{i} / m_{e}}\right) \sim 10^{-3}\right)$, indicating that even mild gradients in density (compared with the ion gyroradius) will make a plasma unstable to LHDI. This can be seen more clearly if we denote the typical length scale of the inhomogeneity as $L_{n}$, since the previous instability threshold in the "realistic limit" becomes approximately:

$$
\frac{L_{n}}{\rho_{i}} \lesssim \frac{\sqrt{2}}{4} \sqrt{\frac{m_{i}}{m_{e}}} \sim 15
$$

On the other hand, PIC simulations require much lower frequencies ratios as well as mass ratios, implying that the threshold required for LHDI is higher than in nature: gradient scale lengths should be much steeper, on the order of $L_{n} \lesssim \rho_{i}$. Therefore, PIC simulations makes more difficult the development of LHDI, allowing an artificial stable parameter regime that it should not exist in the reality.

Friedberg and Gerwin (1977) complemented the previous study of Davidson et al. (1977) by analyzing analytically the behaviour of LHDI in the limit of even lower diamagnetic drift speeds $V_{D, \perp}<0.5 v_{t h, i}$. They found that in this limit, LHDI becomes ion-cyclotron drift instability, with very different properties and much lower frequencies. This is consequence of the resonant (with the characteristic frequencies $n \Omega_{c i}$ ) character of the instability in this regime. If the diamagnetic drift speed decreases even more, both instabilities are completely stabilized for $V_{D, \perp} / v_{t h, i} \lesssim \sqrt{m_{i} / m_{e}}$, which is usually very small (except in PIC simulations with reduced mass ratios). This is an indication of the universal character of this instability: it is almost always present whenever there are gradients in the thermal pressure, although their growth rates may be small in comparison with other instabilities.

Electromagnetic or finite plasma beta effects on the electrostatic dispersion relation were studied by Gladd (1976). They concluded that these effects manifest through the induction of gradients in magnetic field, allowing the coupling between electrostatic and electromagnetic modes. Therefore, many of their consequences can be analyzed with the same dispersion relation, just extending the diamagnetic drift speed to include magnetic 
gradients. They found that in high beta plasma regimes LHDI can be very weak, because the gradients in magnetic field are more important and in the opposite direction to pressure gradients, and thus, a cancellation of the associated diamagnetic drift is expected. The dependence on beta of the magnetic gradient drift speed (using the same geometry as in Eq. (5.104)):

$$
\frac{\vec{V}_{B, \perp}}{v_{t h, i}}=-\rho_{i}\left(\frac{1}{B} \frac{d B}{d x}\right) \hat{y}
$$

can be understood by writing its ratio with respect to the diamagnetic drift speed Eq. (5.104), resulting in (Davidson et al. 1977):

$$
\frac{V_{B, \perp}}{V_{D, \perp}}=-\frac{1}{2} \frac{T_{e}}{T_{i}}\left(\beta_{e}+\beta_{i}\right)+\frac{\beta_{e}}{2} \frac{T_{e}}{T_{i}} \frac{d T_{e} / d x}{d n_{e} / d x}
$$

Note that in deriving this expression the Maxwell equations have been used to relate the different gradients:

$$
\frac{1}{B} \frac{d B}{d x}=-\frac{1}{2}\left(\beta_{i}+\beta_{e}\right) \frac{1}{n_{e}} \frac{d n_{e}}{d x}-\frac{1}{2} \beta_{e} \frac{1}{T_{e}} \frac{d T_{e}}{d x}
$$

Thus, $V_{B, \perp}$ is comparable to $V_{D, \perp}$ whenever $\beta_{\rho} \approx 1$. By performing numerical solutions of the full dispersion relation, Davidson et al. (1977) found a more precise critical threshold in $\beta_{e}$ beyond which LHDI is completely stabilized. This threshold increases with larger diamagnetic drift speeds and it does not exist for $T_{i} \gg T_{e}$. Furthermore, they also showed that the most significant decrease in the LHDI growth rates due to finite plasma beta effects is in the regime $V_{D} \lesssim v_{t h, i}$. Gladd (1976) also analyzed the effects of changing the frequency ratio $\omega_{p e} / \Omega_{c e}$. Similarly to MTSI, the growth rates and wave number at maximum growth rate decrease weakly as this parameters is reduced. And finally, although the wave number range is not affected significantly by variations in the temperature ratio $T_{e} / T_{i}$, the growth rates are enhanced as this parameter increase (although too high values may favor the ion-acoustic instability, which should be the dominant one over LHDI)

Davidson and Krall (1977) analyzed the non-linear evolution of the instability by means of a quasilinear approach, finding the relevant values of the saturation wave amplitudes and associated anomalous resistivity. Similar to MTSI, and for low diamagnetic drift speeds, LHDI saturates mostly via ion trapping, reducing the gradients and associated diamagnetic drifts. Note that all these saturation processes are not valid if the system is being driven externally and continuously replenishing the free energy of the gradients, as it would happen during the magnetic reconnection process. In this case, a macroinstability tearing mode would produce local gradients that can excite short-wavelengths LHDI modes.

\subsubsection{LHDI in M.R.}

Historically, it has been thought that LHDI should happen at the edges of a Harris current sheet, where the equilibrium density gradients provide a diamagnetic drift perpendicular to the reconnection plane (Huba et al. 1977). LHDI should take place in that region mostly for three reasons: the pressure gradients are higher there, the electrons are 
magnetized (not true at the unmagnetized center of a CS) and the stabilizing finite plasma beta effects are reduced in comparison with the center (the plasma beta decreases asymptotically from infinite at the center for a neutral sheet to low values at the infinity. How low it will depend on the background density). In the early years after the discovery of LHDI, there were many works analyzing the role of this instability in magnetic reconnection configurations, mainly because it can provide relatively high values of anomalous resistivity (Huba et al. 1977) that may explain the turbulence levels observed in magnetotail reconnection (Huba et al. 1978).

It is important to remark that most of the works about LHDI in magnetic reconnection have focused in $3 \mathrm{D}$ geometries. That is because LHDI should propagate perpendicular to both pressure gradient and magnetic field, which are located on the reconnection plane. Then, the propagation direction should be perpendicular to the reconnection plane. On recent example is the work by Divin et al. (2015). They investigated development of LHDI in the reconnection jet fronts (also known as dipolarization fronts), starting from a 2D PIC simulation, and then performing a localized 3D PIC simulation using the data provided by the first one in the later stages of the reconnection process. This was done to save computational resources, since a high resolution 3D PIC simulation developing magnetic reconnection self-consistently in not too practical.

However, very few works have analyzed the possibility of LHDI in a geometry where there is a strong guide magnetic field and the gradients are developed self-consistently during the reconnection process. In this case, the diamagnetic drifts are on the reconnection plane. 



\section{Kinetic PIC code simulations}

In this section we are going to discuss the main numerical tool used in the simulations of this thesis: the fully-kinetic Particle-in-Cell (PIC) codes. In order to narrow the scope of this discussion, we focus mostly in the algorithms implemented by the PIC code used to obtain the results to be shown in this thesis: ACRONYM (Kilian et al. 2012). Only tangentially other kind of codes and approaches will be mentioned. A brief description of the capabilities of this code is given at the end of this chapter (Sec. 6.10).

\subsection{General scheme}

This kind of codes were invented at the end of the 50s by Buneman (1959) and Dawson (1962) to study the electromagnetic interactions among a large amount of plasma particles. Therefore, they were designed for the simulation of fully-kinetic plasma processes 1 . They have become one of the most convenient tools for simulations of collisionless plasmas in a wide variety of physical environments. Nowadays, they are highly optimized and designed to run in the most powerful supercomputers.

The basic theory and applications of PIC codes are very well explained in the classical reference tex tbooks (the "PIC bibles"): Birdsall and Langdon (1991) and Hocknev and Eastwood (1988), besides of other nice reviews: Dawson (1983), Winske and Omidi (1996), Pritchett (2000), Verboncoeur (2005), Tskhakaya et al. (2007), Lapenta (2012) and sections of books: Büchner et al. (2003). A review about the early history of these plasma codes can be found in Birdsall and Fellow (1991). A pedagogical introduction including a PIC code is in the textbook by Matsumoto and Omura (1993). A short review about the latest standard methods used in the state-of-the-art relativistic electromagnetic PIC codes can be found in Vay and Godfrey (2014) (many of them implemented in ACRONYM). Note that most of these textbooks/reviews analyze electrostatid PIC codes models, in reduced geometries. Electromagnetic PIC codes are analyzed to much less extent, and that lack of

\footnotetext{
${ }^{1}$ In this thesis we use (abusing the terminology, but also for brevity) the word "PIC" as synonym for "fully-kinetic PIC" (code or simulation). However, it is necessary to remark that there are PIC codes modeling other physical models, and not only a fully-kinetic plasma described by the Vlasov (or Boltzmann) equation. Indeed, the PIC method was originally developed for compressible hydrodynamics flows (by F.H. Harlow in 1955), representing the continuum fluid as an Eulerian (fixed coordinate system) grid of cell plus a Lagrangian (coordinate system comoving with the fluid) set of marker particles. Note that the fully-kinetic PIC method does the same with the continuum phase-space of Vlasov plasmas (see Sec. 6.2). This kind of "hydrodynamic" PIC codes can simulate efficiently certain kind of problems (distorted flows), but its use is not widespread nowadays because has low accuracy and it is prone to some instabilities (in stagnating flows). For further details, see Harlow (1988) and Brackbill (2005).

${ }^{2}$ See Sec.6.7.1 for clarification about this term
} 
coverage is even more notorious for 3 $3 \mathrm{~V}$ 目 plasma models, the most required ones for realistic applications.

The basic idea of the PIC method can be summarized in Fig. 6.1. First of all, the simulation domain is divided in a grid with many cells, filled with computational particles that can be everywhere (from this fact comes the name "particle-in-cell"). The electromagnetic fields $\vec{E}$ and $\vec{B}$ are given and calculated only in these grid points. In order to have their values at the (arbitrary) particle position, it is necessary to use an interpolation scheme (step 1: "Weighting $(E, B)_{i} \rightarrow F_{i}$ " in Fig. 6.1). Then, the code can simulate the motion of these computational particles by solving the Lorentz equation (step 2: "Integration of eqs of motion, moving particles" in Fig.6.1 using Eq. (2.11)), under the action of the electromagnetic force due to $\vec{E}$ and $\vec{B}$. Then, the sources of the electromagnetic field, charge $\rho_{c}$ and current density $\vec{J}$, are calculated via weighting (step 3: "Weighting $(x, v)_{i} \rightarrow(\rho, J)_{i}$ " in Fig. 6.1, using Eq. (2.20)-Eq. (2.21)) from the (arbitrary) particle position back to the grid points, via an interpolation scheme (the inverse process as step 1). Finally, the Maxwell equations are solved (integrated) in the grid position from $e \rho$ and $\vec{J}$ to get $\vec{E}$ and $\vec{B}$ (step 4, "Integration of field eqs on grid" in Fig. 6.1, using Eq. 2.7)Eq. (2.10)). With the values of the electromagnetic field at the grid points, the loop repeats again back to the step 1 .

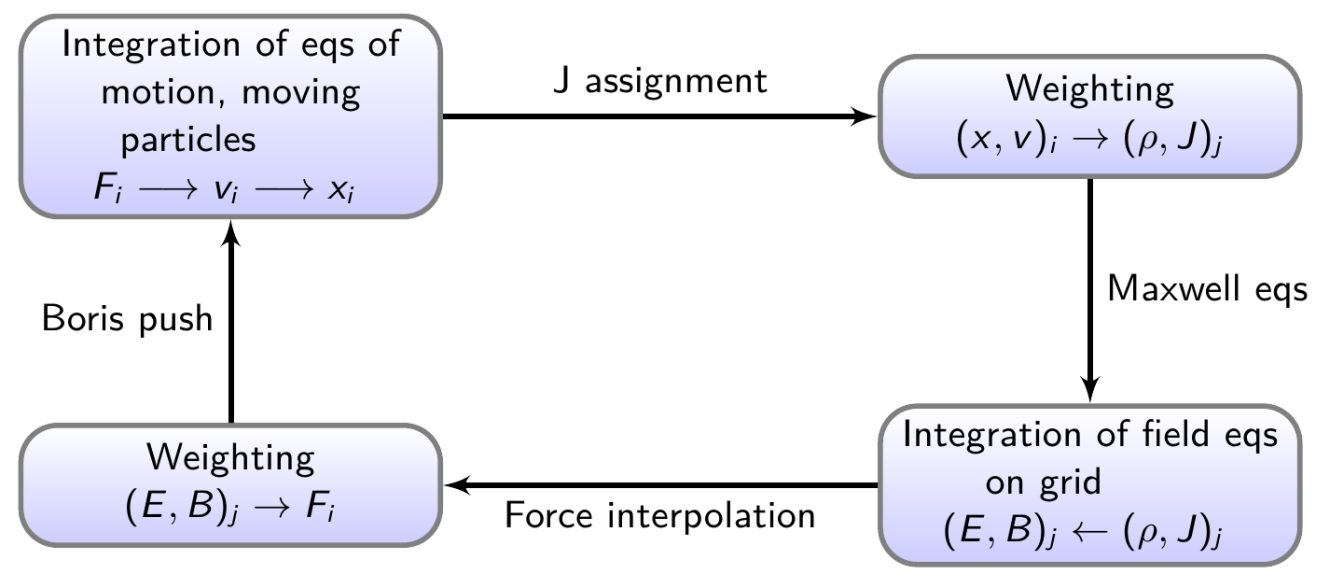

Figure 6.1: Main loop in a PIC code. See text for explanation. Adapted from Birdsall and Langdon (1991).

The reason of the introduction of a grid, instead of solving directly the equations of motion for each particle, is in order to save computational effort. The earliest attempts to simulate plasmas (Dawson 1962) used directly the explicit computation of the interparticle electric force among $N$ particles (like in N-body code simulations), which requires the huge amount of $N(N-1) / 2$ operations per time step, besides of being very noisy (Winske and Omidi 1996). On the other hand, the introduction of a grid in the PIC codes

\footnotetext{
${ }^{3}$ PIC codes are classified according to their dimensionality as "NDMV", where N and M are the dimensions of the space and velocity space, respectively (Tskhakaya et al. 2007).
} 
reduces the required operations for the force calculation to $N \log (N)$ (Pritchett 2000) 4 . In addition, this approach also reduces the fluctuations at sub-grid scales and automatically introduces the time retardation effects implicit in the Maxwell equations.

\subsection{Coarse-graining of phase space and shape function}

The PIC method represents many physical particles 5 close enough in a given point of phase space $(\vec{x}, \vec{v}) 6$, by one single computational "macro-particle" with a $\operatorname{VDF} f_{p}(\vec{x}, \vec{v})$. In the Vlasov formulation (Sec.2.1.4), this is equivalent to a discretization (sampling, coarsegraining) of the phase space. Then, a macroparticle in the PIC method is not simply a set of physical (point) particles, is more precise to say that is a fluid element in a $6 \mathrm{D}$ phase space. This can also be thought as solving the Vlasov equation Eq. (2.19) 7 with the method of characteristics (Winske and Omidi 1996) (see Sec. 2.1.4 for further details). Therefore, the total (physical) VDF $f$ is represented as the following superposition of computational phase-space elements, represented with the subscript " $p$ "(Lapenta 2012):

$$
f(\vec{x}, \vec{v}, t)=\sum_{p} M_{p} S_{x}\left(\vec{x}-\vec{x}_{p}\right) S_{v}\left(\vec{v}-\vec{v}_{p}(t)\right),=\sum_{p} f_{p}(\vec{x}, \vec{v}, t)
$$

The idea of the PIC method is assigning to the macroparticle VDF $f_{p}$ this form:

$$
f_{p}(\vec{x}, \vec{v}, t)=M_{p} S_{x}\left(\vec{x}-\vec{x}_{p}(t)\right) S_{v}\left(\vec{v}-\vec{v}_{p}(t)\right),
$$

where $\vec{x}_{p}$ and $\vec{v}_{p}$ are the phase-space coordinates around which the macro-particle is located, $M_{p}$ represent the macrofactor: the number of physical particles to a computational

\footnotetext{
${ }^{4}$ It is interesting to mention a different approach to this problem without the introduction of a grid. Indeed, there are also "mesh-free" plasma codes, based on the N-body Barnes-Hut hierarchical tree alg orithm. This is a method used to model astrophysics systems where gravitation is the inter-particle force, but with a significant speed-up over the standard N-body algorithms. The force over a particle is calculated using a multipole expansion of the net force due to the other particles located away, resulting also in a scaling as $N \log (N)$ operations. However, this approach neglects some long-range collective effects present in fullykinetic PIC plasma codes, in addition to big complexities for its parallel implementation. For further details, see the review by Gibbon et al. (2010) and references therein.

${ }^{5}$ It is not always necessary or practical to represent all the species in a plasma via this PIC method. It is often the case that is more important to model kinetically the heaviest species (ion) in the plasma, but for electrons can be enough a fluid approach. This kind of codes are called "hybrid-PIC" and are useful to model low frequency phenomena $\omega \sim \Omega_{c i}$. In order to couple the electron fluid with ions, it is necessary to use a closure in the Ohm's law to calculate the total electric field due to them (see Eq. 2.25)). This means considering a specific form for the electron pressure tensor and, frequently, neglecting their inertia. See Pritchett (2000), the chapter in Büchner et al. (2003, pp. 136-165) and the textbook Lipatov (2002) for further details. It is interesting to mention that it is also possible to have physical scenarios where the opposite approach is convenient: kinetic electrons and an ion fluid, as explained in Lyster (1992).

${ }^{6}$ In a fully relativistic formulation, it is more convenient to use as coordinate the spatial components of the 4-velocity $\vec{u}=\gamma \vec{v}$ (proper velocity), where $\gamma=1 / \sqrt{1-v^{2} / c^{2}}$ is the relativistic gamma factor. See details in Haugbølle et al. (2013).

${ }^{7}$ It is also possible to resolve directly that equation via Vlasov plasma codes. These are Eulerian codes and therefore free of the intrinsic PIC shot noise, besides of allowing the relaxation of the small grid cell size Eq. (6.37) required by the latter ones. But, on the other hand, they also require a grid in both configuration and velocity space. This make them computationally very expensive, especially for full 3D cases (with a corresponding 6D phase space and associated grid). For details, see e.g., Umeda et al. (2009) and Umeda (2012).
} 
macro-particle and $S_{x}$ and $S_{v}$ the shape functions in space and velocity, respectively. They represent the extension of the macro-particle in the phase space (they are no point particles). The choice of $M_{p}$ is a trade-off between physical fidelity (higher values, closer to nature) and computational effort (lower, simulating less particles). The standard PIC method usually considers (almost universally) the velocity shape function $S_{v}$ as a tensor product of Dirac's deltas (Lapenta 2012):

$$
S_{v}\left(\vec{v}-\vec{v}_{p}\right)=\delta\left(\vec{v}-\vec{v}_{p}\right)=\delta\left(v_{x}-v_{x, p}\right) \delta\left(v_{y}-v_{y, p}\right) \delta\left(v_{z}-v_{z, p}\right)
$$

This means no spread in the velocity space for all physical particles represented by the macroparticle: since their speed is the same, their evolution will be similar (Lapenta 2012). On the other hand, there are many choices for the shape function in the real space $S_{x}$. They are usually chosen to satisfy the following properties for requirement and convenience:

1. Compact support: $S_{x}=0$ outside a small domain $\Omega=\vec{\Delta}_{x}$ (not necessarily equal to the cell volume: it is usually chosen to be larger) in the phase space

2. Normalization: $\int_{\Omega} S_{x}\left(\vec{x}-\vec{x}_{p}\right) d^{3} \vec{x}=1$

3. Symmetric: $S_{\eta}(x-\eta)=S_{\eta}(\eta-x)$

4. Separable: $S\left(\vec{x}-\vec{x}_{p}\right)=S\left(x-x_{p}\right) S\left(y-y_{p}\right) S\left(z-z_{p}\right)$

Note that the two first properties imply that the integration of $f_{p}$ over all phase space gives the macroparticle number: $M_{p}=\int_{\Omega} f_{p} d^{3} \vec{x} d^{3} \vec{v}$. From all the functions satisfying these properties, it typically used only a small subset, the so-called b-splines. They are functions of increasingly order constructed from an iterative integration starting from the lowest order ones. The first one is defined as:

$$
b_{0}(\xi)=\left\{\begin{array}{cc}
1 & \text { if } \xi<1 / 2 \\
0 & \text { otherwise }
\end{array}\right.
$$

where $\xi=x, y, z$. The higher order b-splines of order $n$ are obtained via the generating formula (Lapenta 2012):

$$
b_{n}(\xi)=\int_{-\infty}^{\infty} b_{0}\left(\xi-\xi^{\prime}\right) b_{n-1}\left(\xi^{\prime}\right) d \xi^{\prime}
$$

If $\Delta \xi_{p}$ are the macroparticles sizes in each direction $\xi=x, y, z$, the shape function using b-splines of order $n$ is defined thus:

$$
S\left(\vec{x}-\vec{x}_{p}\right)=\frac{1}{\Delta x_{p} \Delta y_{p} \Delta z_{p}} b_{n}\left(\frac{x-x_{p}}{\Delta x_{p}}\right) b_{n}\left(\frac{y-y_{p}}{\Delta y_{p}}\right) b_{n}\left(\frac{z-z_{p}}{\Delta z_{p}}\right)
$$

Since we mentioned the macroparticle size, it is convenient to introduce the weighting function $W$ by integrating the shape function defined in Eq. (6.2) over all the domain $\Omega$, measuring thus the spatial extent of the macroparticle:

$$
W\left(\vec{x}_{c}-\vec{x}_{p}\right)=\int_{\vec{x}_{c}-\overrightarrow{\Delta x} / 2}^{\overrightarrow{x_{c}+\Delta x} / 2} S\left(\vec{x}^{\prime}-\vec{x}_{p}\right) d^{3} \vec{x}^{\prime} .
$$

where $\vec{x}_{c}$ represents a cell vertex. Note that $\overrightarrow{\Delta x}$ is usually larger than the grid cell size.

The choice of higher order shape functions has a number of consequences: 
1. The width of $W(x)$ by $\Delta x$ is increased.

2. The continuity class of these functions is increased: from $C^{n}$ to $C^{n+1}$, with $n$ the order of the interpolation scheme. In other words, the shape functions becomes smoother (Cormier-Michel et al. 2008), and so distribution of the charge density and the electromagnetic fields.

3. The quantities calculated with them become more accurate, since the aliasing effects associated with the undersampling by the interpolation is reduced (Haugbølle et al. 2013).

4. Associated with the previous point is the fact that the non-physical forces between the macroparticles smeared over the grid are also reduced, with the corresponding PIC simulated plasma behaving more similar to a real collisionless plasma (Eastwood and Hockney 1974).

5. The simulations become computationally more expensive, since their expressions require more calculations per interpolation step.

6. The numerical collisions between the macroparticles are reduced (Matsuda and Okuda 1975). This is the most important consequence for the goal of this thesis.

\subsubsection{Most common shape functions}

Due to the previously mentioned reasons, the choice of the most appropriate shape functions is a trade-off between accuracy and computation time. The ones that are proven to be more efficient and commonly used are listed below. We use $\xi=\left|x-x_{n}\right| / \Delta x$ as the relative distance with respect to the center of the macroparticle.

1. NGP (Nearest Grid Point), zero order weighting scheme. The 1D weight function is:

$$
W(x)=\left\{\begin{array}{cc}
1 & \text { if } \xi<1 / 2, \\
0 & \text { otherwise }
\end{array}\right.
$$

This $W(x)$ assigns all the charge of a macroparticle to its nearest grid point. The corresponding $S(x)$ is a Dirac delta function: each macroparticle is concentrated at one location. Although is very fast due to its simplicity, this shape function is rarely used nowadays because has very noisy properties (enhanced numerical scattering).

2. CIC (Cloud in Cell), first order (linear) weighting scheme. The 1D weight function is:

$$
W(x)= \begin{cases}1-\xi & \text { if } \xi<1, \\ 0 & \text { otherwise }\end{cases}
$$

This $W(x)$ assigns the charge of a macroparticle between the two nearest grid points by means of a linear interpolation. As explained in the previous subsection, it is obtained by convolving NGP $W(x)$ with itself. This is the most commonly used and standard shape function scheme in PIC codes since many years ago, because it provides a good (empirical) compromise between the smoothness of the interparticle force and computational speed. 
3. TSC (Triangular Shaped Cloud), second order (quadratic) weighting scheme. The $1 \mathrm{D}$ weight function is:

$$
W(x)= \begin{cases}\frac{3}{4}-\xi^{2} & \text { if } \xi<1 / 2 \\ \frac{1}{2}\left(\frac{3}{2}-\xi\right)^{2} & \text { if } 1 / 2<\xi<3 / 2 \\ 0 & \text { otherwise }\end{cases}
$$

The change from linear to quadratic interpolation makes the quantities calculated with the TSC $W(x)$ much smoother than the obtained with the CIC scheme. However, it not so often used in PIC codes, since it is also computationally more expensive.

4. PQS (Piecewise Quadratic Spline), third order (cubic) weighting scheme. The 1D weight function is:

$$
W(x)= \begin{cases}\frac{1}{6}\left(4-6 \xi^{2}+3 \xi^{3}\right) & \text { if } 0<\xi<1 \\ \frac{1}{6}\left(2-\xi^{3}\right) & \text { if } 1<\xi<2 \\ 0 & \text { otherwise }\end{cases}
$$

These different shape functions and their associated weight functions are illustrated in Fig. 6.2 for 1D and in Fig. 6.3 for 2D.
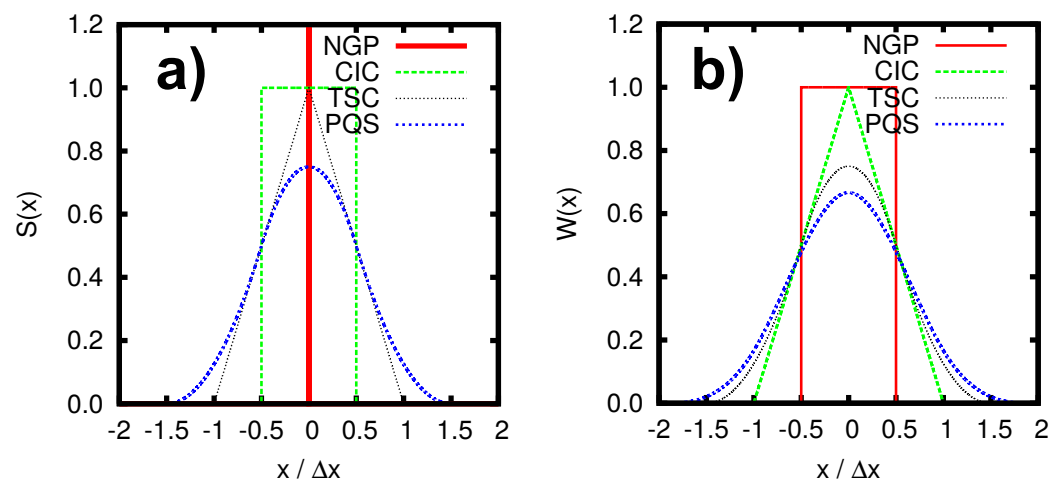

Figure 6.2: Left panel $a)$ : $1 D$ shape functions $S(x)$. Right panel $b$ ): $1 D$ weight functions $W(x)$. Those figures are for a macroparticle located at the origin $\vec{x}_{n}=0$. Adapted from Muñoz.et al. (2014). 

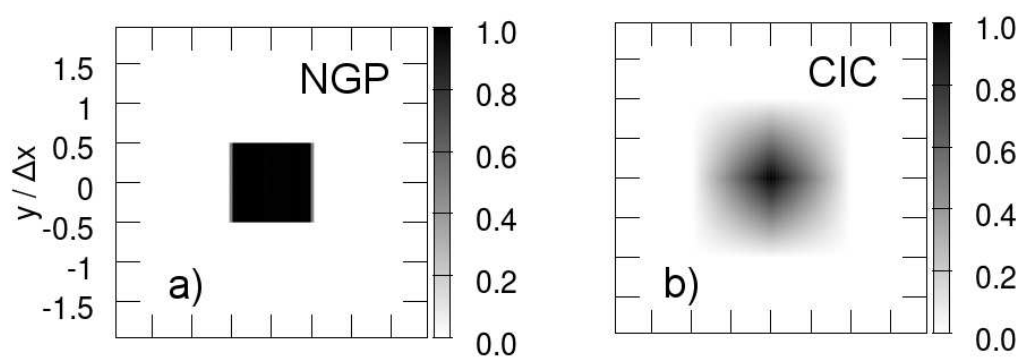

$-1.5-1-0.500 .511 .5$
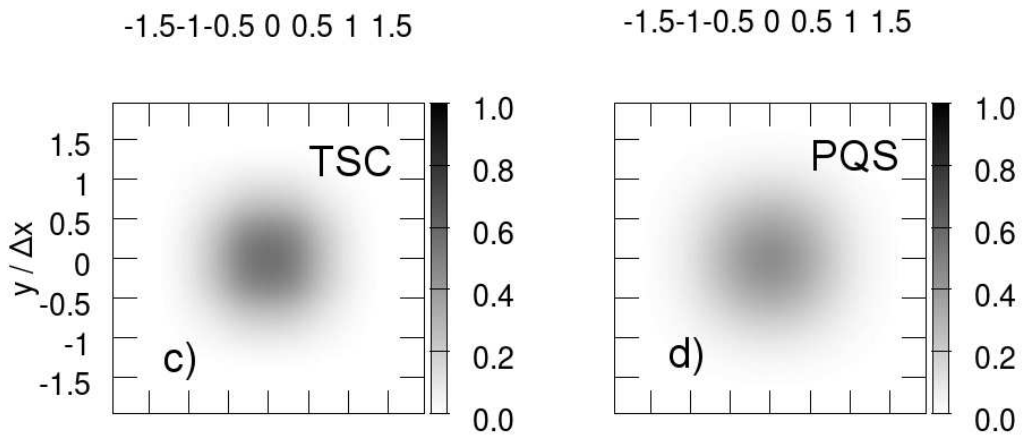

$-1.5-1-0.500 .511 .5$

$\mathrm{x} / \Delta \mathrm{x}$

$-1.5-1-0.500 .511 .5$

$\mathrm{x} / \Delta \mathrm{x}$

Figure 6.3: $2 D$ weight functions $W(x) W(y)$ for different weighting schemes. a) NGP (Nearest Grid Point). b) CIC (Cloud in Cell). c) TSC (Triangular Shaped Cloud). d) PQS (Piecewise Quadratic Spline). Adapted from Muñoz et al. (2014).

\subsection{Discrete Vlasov-Maxwell system for particles}

The introduction of a grid and the macroparticle will necessarily change the VlasovMaxwell system to be solved by the PIC code. This can be seen by replacing the definition of the macroparticle distribution function $f_{p}$ given by Eq. (6.2) in the (full, continuous) Vlasov equation Eq. (2.19) (possible due to its linearity in $f$ ) and taking the appropriate moments. This procedure will give us the equation of motion for the macroparticles.

Taking the moment of order zero, i.e.: integrating over the full phase space (symbolically: $\int f_{p} d^{3} \vec{x} d^{3} \vec{v}$ Eq. (2.19)), it is possible to prove (Lapenta 2012):

$$
\frac{d M_{p}}{d t}=0
$$

i.e.: conservation of the macrofactor (or macroparticles) in the phase space (analogous to the continuous version: the conservation of mass).

Taking the spatial moment of order 1, i.e.: multiplying by the coordinate $\vec{x}$ and then integrating over the full phase space (symbolically: $\int \vec{x} d^{3} \vec{x} d^{3} \vec{v}$ Eq. (2.19)), it is possible to get

$$
\frac{d \vec{x}_{p}}{d t}=\vec{v}_{p}
$$

i.e.: nothing more than the definition of the velocity. 
Taking the velocity moment of order 1, i.e.: multiplying by the coordinate $\vec{v}$ and then integrating over the full phase space (symbolically: $\int \vec{v} d^{3} \vec{x} d^{3} \vec{v}$ Eq. (2.19)), it is possible to get:

$$
\frac{d \vec{v}_{p}}{d t}=\frac{q_{p}}{m_{p}}\left[\vec{E}_{p}(\vec{x}, t)+\vec{v} \times \vec{B}_{p}(\vec{x}, t)\right]
$$

i.e.: formally equivalent to the Lorentz force Eq. (2.11), but with the electromagnetic fields defined (sampled) only at the grid points:

$$
\begin{aligned}
& \vec{E}_{p}=\vec{E}\left(\vec{x}_{p}\right)=\int \vec{E}(\vec{x}) S\left(\vec{x}-\vec{x}_{p}\right)=\sum_{\vec{x}_{c}} \vec{E}\left(\vec{x}_{c}\right) W\left(\vec{x}_{c}-\vec{x}_{p}\right) \\
& \vec{B}_{p}=\vec{B}\left(\vec{x}_{p}\right)=\int \vec{B}(\vec{x}) S\left(\vec{x}-\vec{x}_{p}\right)=\sum_{\vec{x}_{c}} \vec{B}\left(\vec{x}_{c}\right) W\left(\vec{x}_{c}-\vec{x}_{p}\right)
\end{aligned}
$$

where the last equality is obtained assuming constant the electromagnetic fields inside of the macroparticle volume and using the definition of the weighting function Eq. 6.7) (Haugbølle et al. 2013). Note that $q_{p}$ and $m_{p}$ corresponds to the charge and mass of each macroparticle (not the physical particle). The sum runs over all the cell vertices (subscript $\vec{c}$ ) at the boundaries of each cell volume (with the exact locations to be discussed in Sec. 6.5). Thus, the full system of equations to solve via the PIC method is given by the equations of motion Eq. (6.12)-Eq. (6.13)-Eq. (6.14), with the electromagnetic fields Eq. (6.15), in addition to the Maxwell equations and their correspondent sources to be discussed in Sec.6.7. The most important advantage of the PIC method is that the equations of motion are formally the same as the Newton equations, but with the force sampled at the grid points, even though they were derived as a discretization of the Vlasov equation. It is important to remark again that all the previous equations and quantities are calculated at the particle positions (hence the subscript $p$ ), and not on the grid points.

\subsection{Particle mover}

Due to the large quantity of simulated macroparticles, one of the most critical and time consuming parts in any PIC code is the solution of equations of motion Eq. (6.12)Eq. (6.13)-Eq. (6.14). Then, the algorithms to solve them have to be both fast and accurate. As in many other numerical codes, finite differences algorithms provide a reliable compromise between both factors, with the leap-frog (velocity-Verlet) algorithm one of the preferred choices. This is an explicit time-centered second order accurate algorithm $\left(O\left(\Delta^{2}\right)\right)$, based in the time discrete version of the aforementioned equation of motion for the macroparticles. If $\Delta t$ is the time step, each time in the code can be defined as $t_{k}=k \Delta t$, with $k$ an integer number. The leap frog algorithm updates position at these integer times but velocities at half integer times $t_{k+1 / 2}=(k+1 / 2) \Delta t$, in the following way:

$$
\begin{aligned}
\vec{x}_{p}^{k+1} & =\vec{x}_{p}^{k}+\Delta t \vec{v}_{p}^{k+1 / 2} \\
\vec{v}_{p}^{k+1 / 2} & =\vec{v}_{p}^{k-1 / 2}+\Delta t \frac{q_{p}}{m_{p}}\left(\vec{E}^{k}+\frac{\vec{v}_{p}^{k+1 / 2}+\vec{v}_{p}^{k-1 / 2}}{2} \times \vec{B}^{k}\right)
\end{aligned}
$$

\footnotetext{
${ }^{8}$ In order to get second order accuracy. Otherwise, it would be reduced to the explicit Euler algorithm which is only first order accurate (Lapenta 2012).
} 
where $v_{p}^{k}=v_{p}\left(t_{k}\right)$ and similarly for all the other physical quantities from now on. Instead of solving separately the three components of Eq. (6.18), there are other approaches involving a single solution in several steps (see Birdsall and Langdon 1991, Secs. 4.3 and 15.4). One of the most used algorithms is the so called Boris push (Boris 1970), because it is simple, accurate and fast: it requires a single evaluation of the electromagnetic fields per time step (different from Runge-Kutta algorithm requiring at least two evaluation. See Fuchs and Gunn (2006)). First, it solves separately Eq. (6.18) for the velocities at half-times applying only half of the total electric field in the right hand side (and so half of the total momentum). Second, it applies only the magnetic force $\propto \vec{v} \times \vec{B}$ (second term in the right hand side), equivalent to a rotation of $\vec{v}$ (and so energy-conserving). And third, it is applied the second half of the total electric field in the right hand side of Eq. (6.18) (and so the other half of the total momentum). This sequence of operations (depicted in Fig. 6.4), makes the algorithm more efficient due to its symmetry and time reversal or energy-conserving properties 9 . This last property, and in general the leap frog time scheme, are only possible because the second step: the rotation due to the magnetic field, since it is calculated with an implicit evaluation of the velocity (Haugbølle et al. 2013).
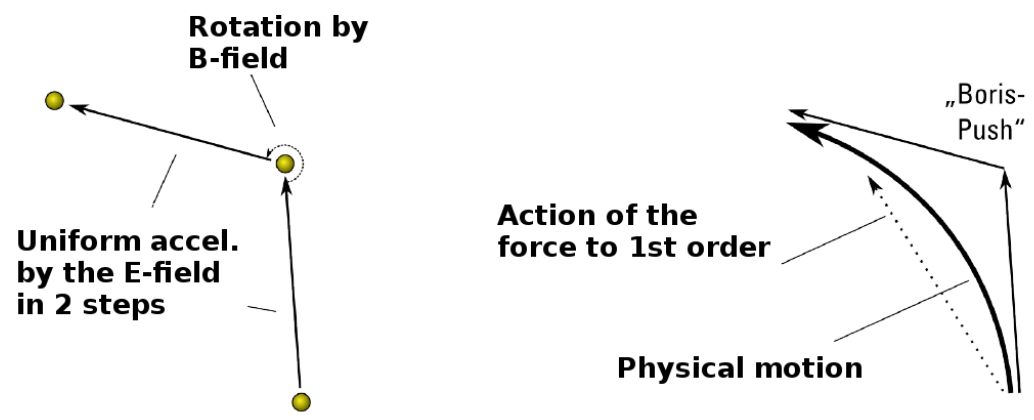

Figure 6.4: Schematics of the Boris pusher. Left: the total force over the particle is calculated in 3 steps. First, half of the uniform acceleration due to $\vec{E}$. Second, a rotation due to $\vec{B}$ (energy conserving). And third, the other half of the acceleration due to $\vec{E}$. Right: The action of the force generated by the Boris push on the particle motion is a piecewise function between the first and second application of the electric field, an straight line approximation to the real curved motion of the particle on the magnetic field. Adapted from Schreiner (2013).

ACRONYM implements the Boris pusher (in the notation of Birdsall and Langdon

\footnotetext{
${ }^{9}$ It was recently proposed by Webb (2014) that the Boris algorithm is also symplectic, i.e.: derived from a Hamiltonian. This is not common due to the intrinsic difficulty on deriving Hamiltonians from systems involving magnetic fields (they used an indirect technique based on a discrete Lagrangian). The implication is that the algorithm describe the exact particle orbit in the limit $\Delta t \rightarrow 0$. However, later another work by Zhang et al. (2015) demonstrated that the previous statement was not precise: the Boris algorithm is not symplectic: only preserves the discrete phase-space volume.
} 
(1991)) in the following way, assuming $\gamma \sim 1$ and so $\vec{u}=\gamma \vec{v} \approx \vec{v}$ (Schreiner 2013):

$$
\begin{aligned}
\vec{v} & =\vec{v}_{p}^{k-1 / 2}+\frac{q_{p} \Delta t}{2 m_{p}} \vec{E} \\
\vec{t} & =\frac{q_{p} \Delta t}{2 m_{p}} \vec{B} \\
\vec{v} & =\vec{v}^{-}+\vec{v}^{-} \times \vec{t} \\
\vec{s} & =\frac{2 \vec{t}}{1+t^{2}} \\
\vec{v}^{+} & =\vec{v}^{-}+\vec{v}^{\prime} \times \vec{s} \\
\vec{v}_{p}^{k+1 / 2} & =\vec{v}^{+}+\frac{q_{p} \Delta t}{2 m_{p}} \vec{E}
\end{aligned}
$$

where $\vec{t}$ and $\vec{s}$ are two auxiliary vectors related with the rotation angle $(t=-\tan \theta / 2$ and $s=-\sin \theta$ ), avoiding the evaluation of transcendental functions. This algorithm is relativistically correct as long as the $\gamma$ factor is not too high $(\gamma \ll 1000)$, since it is not Lorentz invariant. Indeed, Vay (2008) showed this drawback of the Boris pusher, proposing a new Lorentz invariant algorithm, called after him Vay pusher. It is also second order accurate and only a little bit slower than Boris.

\subsection{Discrete Vlasov-Maxwell system for fields: FDTD schemes and Yee lattice}

Now, the next step is choosing a convenient discretization of both time and space, in order to calculate the fields, derivatives and integrate the discrete Vlasov-Maxwell system. This is done via some scheme based in the Finite-Difference-Time-Domain (FDTD) method (see, e.g., the classical tex tbook Taflove and Hagness (2005), whose author coined this term), widely used in all areas of science involving computational electrodynamics 10 . As its name indicates, these are methods to solve numerically the fields (no potential) in the Maxwell equation using finite differences 11 for both time and space (with the requirement of resolving wavelength and frequency of the typical wavelengths found in a given system), usually with leapfrog schemes (and so in an explicit fashion), on an staggered grids. In PIC codes, the most popular and de facto standard choice for arranging these quantities is the Yee staggered lattice (Yee 1966) (see Birdsall and Langdon (1991, Sec. 15) or Taflove and Hagness (2005, Sec 3.6) for further details). The idea is that the electromagnetic fields and sources are calculated, by using the symmetries of the Maxwell equations, at different points inside of each grid cell, in order to get second order accuracy in both space and time via central differences. It uses an uniform cartesian grid. There

\footnotetext{
${ }^{10}$ for several practical and historical reasons, among them the fact of not having to use linear algebra techniques. For other reasons, see Taflove and Hagness (2005, Sec. 1.4)

${ }^{11}$ Another very different approach is solving the Maxwell equations in the Fourier space, the so called "spectral solvers", especially used when considered approx imations of the Maxwell equations such as Darwin or electrostatic (see Sec. 6.7.1). However, since FFTs require global communication on the entire simulation domain, they show bad parallel scaling performance, being one of the reasons for its not so widespread use.
} 
are slightly different conventions depending on the code. ACRONYM uses the scheme depicted in Fig. 6.5.

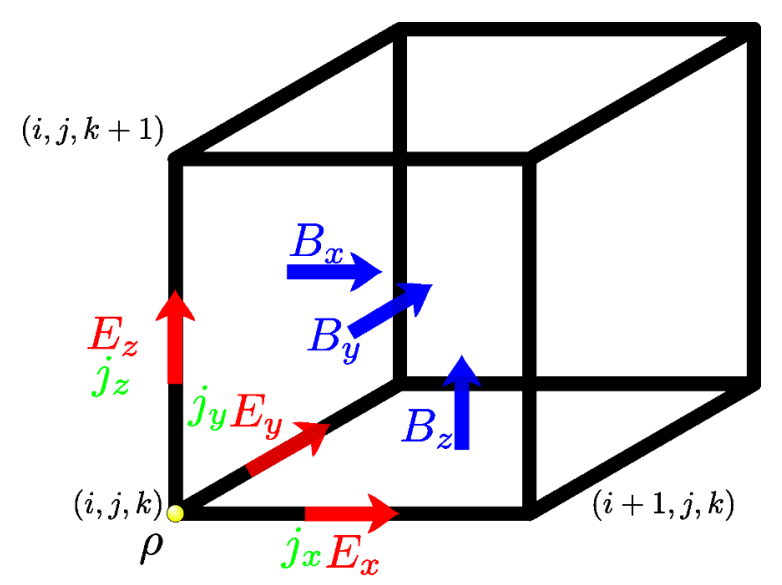

Figure 6.5: Yee lattice used by ACRONYM, showing the position in a cell where it is calculated the electromagnetic fields $\vec{E}$ and $\vec{B}$ and their sources, $\rho$ and $\vec{J}$. See main text for the explanation. From Kilian (2013).

This arrangement has the advantage of calculating the derivatives (gradient and curl) of the Maxwell equations in a consistent way, without further rearrangement, allowing at the same time an easy implementation of boundary conditions. Note that the components of $\vec{J}$ and $\vec{E}$ are calculated at the same position at the edges of the cell, as required by the right hand side of the Ampère's law Eq. (2.10). Also note that the each magnetic field component $\vec{B}$ is calculated at the faces of each cell, surrounded by the 4 components of $\vec{E}$ in the perpendicular direction as required for the curl in Faraday equation Eq. (2.9) (e.g.: $B_{z}$ at the bottom face is surrounded by the components $E_{y}$ and $E_{x}$ ). Something similar happens in the other way around: each electric field component $\vec{E}$ is surrounded by the 4 components of $\vec{B}$ in the perpendicular direction, as required by the curl in the Ampère's law.

Regarding the time integration, the Yee scheme uses a leapfrog al gorithm, storing and calculating $\vec{E}$ and $\vec{B}$ alternatively. This is an explicit scheme, avoiding numerical issues related with matrix inversion that implicit codes have, besides of being non-dissipative (Taflove and Hagness 2005). The downside is the requirement of a small time step. Different from other methods such as Runge Kutta where all the variables are calculated at the same time (time-centered), there is no necessity of storage for intermediate steps (Haugbølle et al. 2013) besides of being compatible with energy-conserving (symplectic) particle movers (see Sec.6.4).

\subsection{Coupling particles with fields: current assignment}

Now that we have prescribed the location of the fields on the cells, we have to couple the particle information with the electromagnetic fields in the Maxwell equations. For that it is necessary to calculate the sources $\rho_{c}$ and $\vec{J}$ at the cell vertices (indicated with subscript $c$ ), where the Maxwell equations are solved. Therefore, using $f_{p}$ given by Eq. (6.2) in the 
continuous definitions Eq. (2.20) and Eq. (2.21), we get (Haugbølle et al. 2013):

$$
\rho_{c}\left(\vec{x}_{c}, t\right)=\sum_{p} q_{p} M_{p} W_{x}\left(\vec{x}_{p}-\vec{x}_{c}\right)
$$

where we have assumed an average over each cell volume and used the weighting function Eq. (6.7). Note that the sum in $p$ is over all the particles of all species. This gives a charge density using the same interpolation as the fields Eq. (6.15) via the function $W_{x}$. This is not mathematically necessary, but it is natural and a very convenient choice for three different reasons (see Birdsall and Langdon (1991, Secs. 8.5 and 8.6) and Tskhakaya et al. (2007)): the absence of self-forces on the particles, the inter-particle force is symmetric (3rd Newton's law) and the conservation of momentum (as calculated from the derivative of the discrete Lorentz force Eq. (6.14)).

However, note that the explicit PIC codes as ACRONYM do not use $\rho_{c}$ for the solution of the Maxwell equations. Instead, the current density is required, for which we might use the same approach

$$
\vec{J}\left(\vec{x}_{c}\right)=\sum_{p} q_{p} \vec{v}_{p} M_{p} W_{x}\left(\vec{x}_{p}-\vec{x}_{c}\right)
$$

However, this way of calculation brings an inconvenient side effect. Indeed, the charge continuity equation (derived from Gauss and Ampère equations):

$$
\frac{\partial \rho_{c}}{\partial t}+\nabla \cdot \vec{J}=0
$$

may not be satisfied, leading to a non-physical evolution of the electromagnetic fields, since the Gauss equation for $\vec{E}$ will not be satisfied). Although Eq. (6.27) is satisfied strictly inside of each cell, the particles moving across cells (crossing boundaries) in one time step will violate charge conservation (Haugbølle et al. 2013).

One solution to this issue is making a correction $\delta \vec{E}$ to enforce the Poisson equation Eq. (2.7) at each time step, a technique called "divergence or Gauss cleaning" (Marder 1987) (option implemented in ACRONYM). Basically, it is necessary to solve the Poisson equation for the electric potential: $\nabla^{2} \delta \phi=\left(\nabla \cdot \vec{E}-\rho_{c} \epsilon_{0}\right)$, since thus we can calculate the corrected electric field $\vec{E}^{\prime}=\vec{E}-\nabla \phi=\vec{E}-\delta \vec{E}(\vec{E}$ is the original electric field as calculated from the Ampère's law). Note that this would be the only case requiring the calculation of the charge $\rho_{c}$. This additional step is computationally expensive (due to the elliptic, non local, character of the Poisson equation, requiring spectral solvers) and not friendly for parallel PIC codes (Pritchett 2000) 12 . Moreover, it has been reported by Haugbølle et al. (2013) that this method shows a worse performance in conserving energy and higher level of numerical heating than the method to be described next.

A more convenient approach to enforce Eq. (6.27) is calculating $\vec{J}$ directly from Eq. (6.27) instead of Eq. (6.26), thus preserving rigorously the condition of local charge conservation. This approach does not require to solve elliptic equations, and therefore is

\footnotetext{
${ }^{12}$ Indeed, any speedup in this step of the PIC method leads to a significant improvement in the overall performance. Due to the large amount of particles necessary to keep numerical noise at low levels, the current assignment and then the particle mover are the more time consuming steps all PIC codes (Decyk 1995).
} 
fast and easily parallelized. But there are two important disadvantages (Lapenta 2012): the conservation of momentum is lost with a consequent enhanced noise level, requiring thus a larger number of macroparticles per cell to reduce this undesired side effect.

ACRONYM uses the second approach. There are several methods implementing this "smart" way of assigning the current (Eastwood 1991, Villasenor and Buneman 1992, Esirkepov 2001, Umeda et al. 2003), but note that all of them (in the unmodified published form) are only compatible with the second order field solver (Haugbølle et al. 2013). The method used by ACRONYM is the Esirkepov scheme (see Fig. 6.6). He proposed a decomposition of the discretized version of Eq. (6.27) in a set of differential equations, decoupling the different components of $\vec{J}$. This is valid for any shape function, and it also has the advantage of allow generalizations to higher order Maxwell solvers functions (Haugbølle et al. 2013).
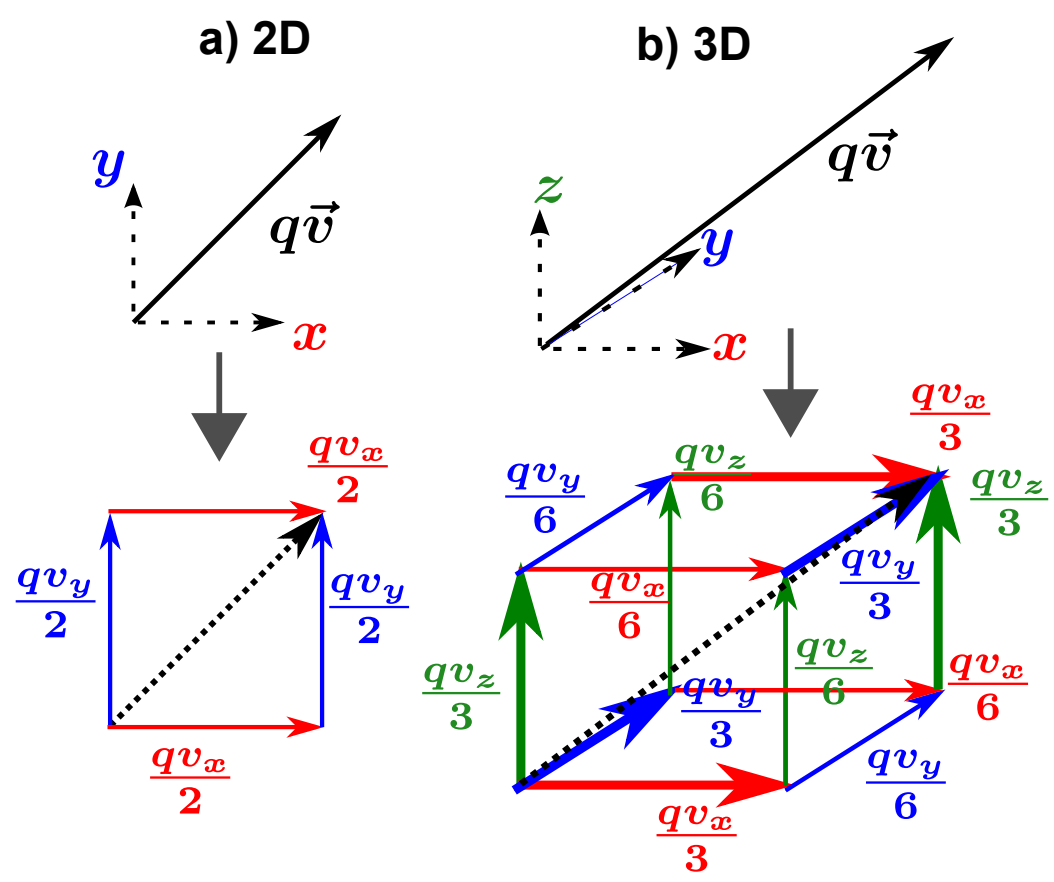

Figure 6.6: Scheme showing the Esirkepov charge assignment in $2 D(a)$ and $3 D(b)$. The flux of charge representing the current is represented by the arrows carrying charge qand velocity $\vec{v}: \vec{J}=q \vec{v}$. In $2 D($ a), the charge flux is decomposed in 2 components per spatial direction (in total 4) that are added step by step to reconstruct the total current. In $3 D(b)$, the charge flux is decomposed 12 components, each one associated with the edges of the grid cell and with a different weight. From Umeda et al. (2003).

\subsubsection{Current smoothing}

As we will see in Sec.6.8.6, the numerical noise depends mostly on the number of macroparticles per cell. But it can be numerically reduced by applying a spatial filtering to the current density after this is accumulated on the grid. One of most used filters in PIC simulations is the binomial filter. See Appendix B.1.2.2 for further details. 


\subsubsection{Energy conserving PIC codes}

It it is important to note that all the PIC codes using these conserving momentum schemes (same interpolation for particles and fields) do not conserve energy. Intuitively, this can be understood due to the "degeneration" of degrees of freedom due to the introduction of the grid and associated discretization: there are many possible microscopic configurations (position and velocities of macroparticle) leading to the same values of macroscopic quantities $\rho_{c}$ and $\vec{J}$ weighted on the grid. This was already pointed out by Abe et al. (1975) (see also Birdsall and Langdon 1991, Sec. 10.2). The deviations, however, are small, being caused by the non-conservative forces that the particles experience through the interactions between themselves though the grid (see Hockney and Eastwood 1988, Sec. 7.6).

On the other hand, there are also energy conserving PIC codes, derived from a variational formulation of the discrete Vlasov-Maxwell system, not experiencing numerical grid heating. They were first proposed by Lewis (1970), conserving energy only in the limit $\Delta t \rightarrow 0$, and recently extended to finite time steps by Markidis and Lapenta (2011) and Evstatiev and Shadwick (2013). Although at a first sight the conservation of energy might make these codes highly attractive, they are much less used in practice since they lack conservation of momentum (the Lagrangian is not invariant under displacements). This approach also produces self-forces in the particles (a highly undesired nonphysical effect), besides of having to invert large matrix systems and allowing a reduced choice of shape functions (with the consequent enhanced numerical noise). For further details, see Langdon (1973), Birdsall and Langdon (1991, Sec. 10) and (Hockney and Eastwood 1988, Sec. 5).

\subsection{Maxwell solver}

Finally, with the sources of the electromagnetic field $\vec{J}$ calculated from the previous step, we have to solve (the discrete version of) the Maxwell equations that includes the curl (Faraday and Ampère), representing a coupled system for the fields $\vec{E}$ and $\vec{B}$. For consistency reasons, it is also necessary to use a leap frog algorithm . In practice, this means that the magnetic field and density current has to be calculated at half time steps: $\vec{B}^{k \pm 1 / 2}$ and $\vec{J}^{k \pm 1 / 2}$, while the electric field at integer time steps $\vec{E}^{k}$. Thus, if these fields are calculated in the locations indicated by the Yee lattice and denoted, e.g.: $(i, j, k)$, the magnetic field will be advanced with the Faraday equation Eq. (2.9) in the following way:

$$
\vec{B}_{(i, j, k)}^{k+1 / 2}=\vec{B}_{(i, j, k)}^{k-1 / 2}-\Delta t(\nabla \times \vec{E})_{(i, j, k)}^{k}
$$

while the electric field is computed with the Ampère's law Eq. 2.10):

$$
\vec{E}_{(i, j, k)}^{k+1}=\vec{E}_{(i, j, k)}^{k}+\frac{\Delta t}{\mu_{0} \epsilon_{0}}\left[(\nabla \times \vec{B})_{(i, j, k)}^{k+1 / 2}-\mu_{0} \vec{J}_{(i, j, k)}^{k+1 / 2}\right]
$$

where, for example, the $x$ component of the finite difference operator $\nabla \times$ can be expressed explicitly in the following way (Schreiner 2013):

$$
(\nabla \times \vec{B})_{x,(i, j, k)}^{k+1 / 2} \approx \frac{1}{\Delta x}\left(B_{z,(i, j, k)}^{k+1 / 2}-B_{z,(i, j-1, k)}^{k+1 / 2}+B_{y,(i, j, k-1)}^{k+1 / 2}-B_{y,(i, j, k)}^{k+1 / 2}\right)+O\left(\Delta x^{3}\right)
$$


For details about the explicit form of these six equation on the Yee lattice, see Taflove and Hagness (2005, Sec. 3.6.3) or Birdsall and Langdon (1991).

An important feature of this Maxwell solver using a Yee lattice is that satisfies automatically the Maxwell equations containing divergence, the Gauss (Poisson) equation for the electric Eq. (2.7) and magnetic field Eq. (2.8), in each cell. This means that the initial values of $\nabla \cdot \vec{E}$ and $\nabla \cdot \vec{B}$ are preserved 13 during the evolution of the system (note that it is not equivalent to the fulfillment of the continuity equation Eq. 6.27), for which is required additional techniques, as explained in Sec.6.6). In contrast, it is interesting to mention that the enforcing of $\nabla \cdot \vec{B}$ is an important issue in MHD codes (Lapenta 2012).
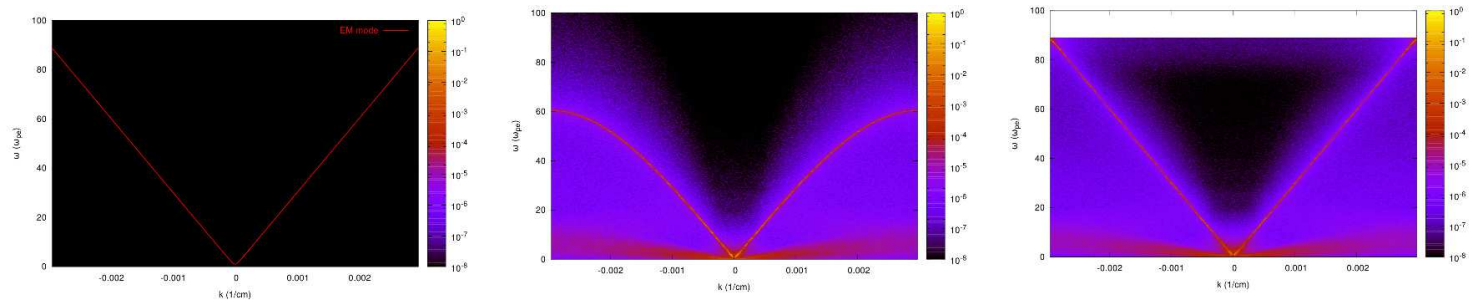

Figure 6.7: Comparison of electromagnetic dispersion relations. Left: Real theoretical Eq. (6.31) (just a couple or straight lines). Center: numerical with Yee + TSC shape function. Note the deviation for short wavelengths (high $k$ ), signature of numerically induced dispersion on the electromagnetic waves. Right: numerical with CK + TSC shape function. The dispersion of the electromagnetic waves suffers less dispersion than with Yee grid, resembling more the ideal electromagnetic waves (straight lines). From Kilian (2013).

All the Maxwell solvers, and in particular the ones based on a Yee lattice, introduce changes in the dispersion relations $(\omega, k)$ of the plasma wave modes due to discretization effects (compare Eq. 6.31) with Eq. 6.32), to be discussed in Sec. 6.8.114. Several approaches have been proposed to have more accurate dispersion relations for some parameter ranges, especially when applied to high frequency phenomena like laser wakefield accelerators (see, e.g., Vay et al. 2011). This is because the numerical dispersion relation of the Yee lattice makes electromagnetic waves travel slower than $c$ close to the cut-off frequency. One of these, implemented in ACRONYM, is the Cole-Kärkkäinen (CK) algorithm. It was formulated by Kärkkäinen and Gjonaj (2006) (extending a previous algorithm by Cole 1997) for the Maxwell equations without sources and adapted for its use to PIC codes, including source terms, by Vay et al. (2011). It is based on non-standard finite differences (NSFD) 15 , showing higher accuracy than the standard Yee lattice, without sig-

\footnotetext{
13 i.e.: they are assumed as an initial condition. For a proof, see Taflove and Hagness (2005, Sec. 3.6.9) or Birdsall and Langdon (1991, Sec. 15.6).

${ }^{14}$ Indeed, most of the linear kinetic plasma theory and stability analysis (e.g.,dispersion relations) can be redone by taking into account the grid effects through the shape function $S(x)$ via the relations Eq. 6.25) and Eq. 6.26). In Fourier space, the first relation is equivalent to replace, the charge $q \rightarrow q S(\vec{k})$, with the consequent change of the PIC plasma frequency $\omega \omega_{p e}^{2} \rightarrow \omega_{p e}^{2} S\left(\vec{k}_{n}\right)$. This means that now it is dependent on the grid wavenumber. For further details, see a brief description in Birdsall and Fellow (1991) and the extended discussion in Birdsall and Fellow (1991, Chapters 8-9).
}

\footnotetext{
${ }^{15}$ These methods improve the usual finite-difference ones by adding a correction to the derivative operators. This correction is calculated based on the analytical solutions of the equations to be solved
} 
nificant slow down in the computation (Cole 2002). It features no numerical dispersion relation along the main axis of a cartesian mesh, as long as it has cubic cells. See the simulated dispersion relations comparing with the Yee Maxwell solver in Fig. 6.7. However, it is possible to tune some coefficients in the algorithm to have accurate dispersion relations along any desired axis. This is in contrast to the Yee algorithm that has always a large numerical dispersion at high frequency along the main axis, although no numerical dispersion along the main diagonal of the cell cube (Vay et al. 2011).

It is interesting to mention that the numerical deviations from the electromagnetic wave dispersion relation at high frequencies become especially critical when there are also highly relativistic particles present in the plasma. This is because they will emit numerical Cherenkov radiation when their speed is faster than the (artificially low) speed of light in the plasma. It is due to a coupling between electromagnetic and beam modes generated by an "incompatibility" or "mismatch" between the Lagrangian treatment of particles and Eulerian treatment of electromagnetic fields (Godfrey 1975). This numerical radiation can also affect lower frequency ranges, affecting severely the evolution of the simulated plasmas (Godfrey 1974, Greenwood et al. 2004). Many approaches have been proposed to specifically eliminate this undesired effect. One of them was discussed in the previous paragraph, and even another is based in a high order version of the ordinary Yee Maxwell solver, called M24 since it is 2th order accurate in time and 4th order accurate in space (Hadi and Piket-May 1997, Hadi 2007). It has been recently implemented in ACRONYM.

\subsubsection{Approximation of the Maxwell equations}

So far, we have discussed the solution of the full set of the Maxwell equations. These kind of PIC codes are called electromagnetic, and they are especially useful to model high frequency phenomena. On the other hand, there are also electrostatic PIC codes if they solve only the Poisson or Gauss equation Eq. (2.7) for the (self-consistent) $\vec{E}$. In the latter case, a magnetic field can be included but it does not evolve: it is considered external and fixed. Its main advantage is the elimination of the CFL stability constraint (see discussion of Eq. 6.34)) by eliminating light waves (Pritchett 2000). This kind of codes usually solve these equations via spectral solvers.

Another approach are the so called Darwin (also known as magnetoinductive or nonradiative) PIC codes (first discussed in this context by Kaufman (1971). See also the early review by Hewett (1994)), which neglect the displacement current in the Ampère's law Eq. (2.10) by assuming low frequency waves or $(v / c) \ll 116$. As a result, the character of the Maxwell equations changes from hyperbolic to elliptic (Pritchett 2000). This approximation introduces a big complexity in the algorithm (elliptic equations are nonlocal), especially in the 3D case. All these categories of plasma models are included in ACRONYM, although only the full electromagnetic version will be used for the results to be shown in this thesis.

\footnotetext{
${ }^{16}$ More rigorously, that simplification in the Maxwell equations can be obtained via two very different approaches. In the first one, the formal solution to the full set of Maxwell equations in terms of scalar and vector potentials is found in the Coulomb gauge. Then, the vector potential is approximated by neglecting retardation effect. In the second approach, the same set of equations by themselves is approximated by neglecting the time derivatives in the vector potential. See Krause et al. (2007) for further details.
} 
There is even another more hybrid approach, consisting in not only an approximation of the Maxwell equations, but also on the underlying Vlasov equation, by eliminating the gyromotion of charged particles in strong magnetic fields. This gyrokinetic theory and its associated simulation codes will be discussed in Sec. 9.1.

\subsection{Numerical consequences of the coarse-graining, grid and time step}

\subsubsection{Stability conditions}

The use of explicit schemes in a PIC code involves very stringent conditions on the choice of the grid cell size and time step. Moreover, there are some other stability condition 17 that may trigger numerical instabilities if they are not taken into account. There are mainly three reasons and associated conditions (Lapenta et al. 2006):

1. Discretization of fields (Maxwell solver, Sec. 6.7). $\Delta x$ and $\Delta t$ have to be chosen in such a way that allow the propagation of the fastest wave mode. In the case of the full set of Maxwell equations, this has to be the speed or light, with the physical dispersion relation

$$
\frac{\omega^{2}}{c^{2}}=k_{x}^{2}+k_{y}^{2}+k_{z}^{2}
$$

The condition is then given by finding the (approximated) numerical dispersion relation of the electromagnetic waves propagating on the grid (obtained assuming harmonic solution in the discrete version of Faraday and Ampère's law). This depends on the Maxwell solver and so on the arrangement of fields in the grid. For the Yee lattice it is (Greenwood et al. 2004, Vay et al. 2011):

$$
\left(\frac{\sin (\omega \Delta t / 2)}{c \Delta t}\right)^{2}=\left(\frac{\sin \left(k_{x} \Delta x / 2\right)}{\Delta x}\right)^{2}+\left(\frac{\sin \left(k_{y} \Delta y / 2\right)}{\Delta y}\right)^{2}+\left(\frac{\sin \left(k_{z} \Delta z / 2\right)}{\Delta z}\right)^{2}
$$

In order to avoid numerical instability, we require to have real solutions for $\omega$, equivalent to:

$$
\left(\sin \left(\frac{\omega \Delta t}{2}\right)\right)^{2} \leq 1
$$

This allows to obtain the Courant-Friedrichs-Lewy condition (CFL, first discussed by Courant et al. (1928)), assuming that the most unstable modes should propagate at the cut-off (Nyquist) wavenumber along the main axis $k_{i, \max }=\pi / \Delta i$, the $2 \mathrm{D}$ faces diagonals and 3D main diagonal of the cell. In case of a cubic lattice: $\Delta x=\Delta y=\Delta z$, this becomes:

$$
c \Delta t_{c} \leq \min \left\{\Delta x, \frac{1}{\sqrt{2 /(\Delta x)^{2}}}, \frac{1}{\sqrt{3 /(\Delta x)^{2}}}\right\}=\frac{\Delta x}{\sqrt{3}}
$$

\footnotetext{
${ }^{17}$ See Hockney and Eastwood $(1988$, Sec. 4.4$)$ for an introduction to the stability conditions applied to the numerical schemes used in PIC codes.
} 
It is interesting to notice that this requirement becomes less restrictive in lower dimensionality: in 2D the right hand side of Eq. (6.34) is $\Delta x / \sqrt{2}$, while in $1 \mathrm{D}$ only $\Delta x$. Eq. (6.34) can be intuitively understood as the requirement that an electromagnetic wave should not propagate in a single time step $\left(c \Delta t_{c}\right)$ a distance larger $(<)$ than the grid cell size $(\Delta x \text { in } 1 D)^{18}$.

2. Discretization of equations of motion for particles (Sec. 6.3). The standard (Von Neumann) stability analysis establish that the minimum required time step to avoid instability can be calculated from the linearization with the subsequent Fourier analysis of the equation of motion, and then finding the conditions for which the maximum frequency in the system, $\omega$, has real values (otherwise, the system turns out to be unstable). In an unmagnetized plasma, this is equivalent to resolve the fastest response of the lightest particle, the plasma frequency $\omega_{p e}$ (because it is the harmonic oscillation of the electrons in presence uniquely of an electric field), giving the condition also known as Langmuir wave propagation (Tskhakaya et al. 2007, Lapenta 2012):

$$
\omega_{p e} \Delta t<2
$$

In practice, to avoid additional effects related with electron heating, a time step one order of magnitude smaller is usually chosen (found empirically): $\omega_{p e} \Delta t \lesssim 0.1$ (see Hockney and Eastwood 1988, Sec. 9.2.3.).

The condition Eq. (6.35) should also be valid for any other frequency in the plasma (replacing $\omega_{p e} \rightarrow \omega$ ). In principle, the electron gyromotion should also be resolved. However, the use of the Boris algorithm (involving a energy conserving rotation in the magnetic field, see Sec. 6.4) does not impose any additional stability requirement: the algorithm is always stable for any value of $\Omega_{c e} \Delta t$ (Bowers et al. 2008). Instead, the choice of the timestep is constrained for accuracy reasons. In order to get $1 \%$ of accuracy, the timestep has to resolve the electron Larmor gyration by (Patacchini and Hutchinson 2009):

$$
\Omega_{c e} \Delta t \lesssim 0.3
$$

This requirement becomes difficult to fulfill for strong enough magnetic fields, imposing a practical limit for simulations of strongly magnetized plasmas (at least, with explicit schemes). On the other hand, it is also interesting to mention that the electron gyromotion does not impose a constraint on the size of the electron Larmor radius $\rho_{e}$ compared to the grid cell size $\rho_{e}$. As pointed out by Melzani et al. (2013), this has an intuitive interpretation: the interpolation of the fields to the particles gives always the same particle orbit, completely independent on the grid size, providing a constant and homogeneous magnetic field.

3. Interpolation scheme (Sec. 6.2). The introduction of the shape function coupling grid and particles adds an additional stability requirement. This arises as a consequence of the loss of information from the continuum particle shape to discrete

\footnotetext{
${ }^{18}$ It is interesting to mention that $\Delta t$ obtained by this condition is usually much smaller than the required for the stability of the particle mover. As pointed out first by Boris (1970), it is possible to choose different $\Delta t$ for particles and fields, by a method known as sub-cycling, advancing the fields several times per a single particle update. See Tskhakaya et al. (2007) and Birdsall and Langdon (1991, Sec. 9.7) for further details.
} 
points, producing an aliasing from higher to lower wavenumbers. This "finite grid" instability imposes the following criterion (Lapenta 2012):

$$
\Delta x<C \lambda_{D e}
$$

where $C$ is a constant of order 1 , with the specific value depending on the method used. The derivation of Eq. 6.37) is much more involved than the previous conditions, and it can be found in Birdsall and Langdon (1991) and Hockney and Eastwood (1988). If this condition is violated, the consequence is an artificial numerical heating, to be discussed in Sec.6.8.2.

\subsubsection{Practical consequences of the stability conditions}

The three stability conditions Eq. (6.34), Eq. (6.35) and Eq. (6.37) for explicit PIC codes are very difficult to fulfill. They make the numerical modeling of kinetic plasmas computationally very expensive in many cases of interest. This is because these conditions constrain the grid cell size and time step to electron scales, but usually the phenomena to be analyzed takes place at ion (or even larger) spatial and time scales (e.g.: in magnetic reconnection 19 . For this reason it has become traditional to use reduced values of the mass ratio $m_{i} / m_{e}$ (to make closer electron and ion scales) and the inverse of the electron thermal speed $c / v_{t h, e}$ (since, due to the relations in Appendix A.3.1, this makes smaller $d_{e} / \lambda_{D e}$, and correspondingly $d_{i} / \lambda_{D e}$, the typical ion length scale). As pointed out by Pritchett (2000), for a given time measured in ion scales, the number of time steps should scale as $m_{i} / m_{e}$ in explicit PIC codes due to the stability conditions. And the number of grid points per dimension should scale as $\sqrt{m_{i} / m_{e}}$. Putting together both scalings, the computational cost of a simulation scales as $\left(m_{i} / m_{e}\right)^{(d+2) / 2}$, with $d$ the spatial dimensions. In 3D, this results in $\left(m_{i} / m_{e}\right)^{5 / 2}$, making prohibitive the use of realistic mass ratios given the current computational resources.

\subsubsection{Implicit codes}

It is interesting to mention other approaches to avoid the stringent stability conditions Eq. 6.34) and/or Eq. 6.35). Among them, one of the most important are the implicit PIC

\footnotetext{
${ }^{19}$ This kind of phenomena where many different scales are coupled are a real challenge for any kind of simulation code: there is always a trade-off between physical fidelity and the scale that it is possible to resolve (PIC codes for small scales vs MHD codes for large scales). It is interesting to mention that fullykinetic explicit PIC codes (with the more accurate physics) can be modified to deal, partially, with different length scales and at the same time satisfying the condition Eq. 6.37). The approach is known as AMR: Adaptive Mesh Refinement, consisting in adapting dynamically the grid cell size depending on the required resolution. For example, in the case of magnetic reconnection, it is possible to have high grid resolution in the diffusion region, while low resolution for the uninteresting region away from the CS. This can save a lot of computational resources, although the algorithms can be very complex (see, e.g., Fujimoto and Sydora 2008). On the other hand, it is interesting to mention that there have been also some attempts to bridge the gap between the different physics and scales used in different codes by coupling them directly. The full kinetic physics is modeled in a small region via PIC codes, and its output is used as boundary condition and input for fluid simulations. This approach was first proposed by Sugivama and Kusano (2007) (see also Usami et al. 2014), although in cases where the simulation domains of both codes have comparable sizes. In any case, their future is very promising, since it is starting to be used for an accurate modeling of space weather ( see Tóth et al. 2012, Lapenta et al. 2013, and references therein).
} 
codes, developed in the early 80s (see Mason 1983, Denavit 1981, Brackbill and Forslund 1982). All the previously described algorithms for the particle mover and Maxwell solver are explicit schemes, in the sense that the new values of the physical quantities only require the stored previous values. On the other hand, in implicit schemes the new values of the quantities appear only in implicit form in both sides of the respective equations, requiring thus the solution of a system of coupled non-linear equation (Pritchett 2000). The methods for solving these equations can be very involved. One approach to solve the implicit equations is the "kinematic", based in the solution of the linearized particle and Maxwell equations to estimate their new value (see Pritchett 2000, and references therein). But the most used so far is the so called "implicit moment" technique. They use the moment or fluid equations as an approximation for the estimations of fields, which are used in the particle mover (see Lapenta 2012, and references therein). One recent example of these kind of codes, from the same developers of ACRONYM, is PICPANTHER: "Parallel Implicit Concise PiC Allowing Non-Thermal Electromagnetic Relativity" (Kempf et al. 2014).

The advantage of implicit PIC codes is that they can be stable with longer timesteps than these required by Eq. (6.34). In principle, the stability condition on the time step is completely eliminated, and its choice is determined instead by the desired physical time scale and the accuracy of the used method. For example, under the conditions described in Pritchett (2000), the accuracy of an standard implicit method requires:

$$
0.1 \leq v_{t h, e} \Delta t / \Delta x \leq 1.0
$$

This reduces the computational cost for reaching a given time in ion scales from $m_{i} / m_{e}$ in explicit code to $\sqrt{m_{i} / m_{e}}$. But not everything is favorable for the implicit codes. In addition to the intrinsic complexity in the solution to the equations, these codes distort the physics of the unresolved length and time scales (see Lapenta 2012, and references therein).

\subsubsection{Numerical heating}

As discussed extensively in Birdsall and Langdon (1991, Secs. 8 and 13) and Hockney (1971, Sec. 9.2), since the very early times of PIC plasma simulations an artificial numerical heating of these systems was observed. This numerical heating may mimic real physical processes, making the triggering of physical instabilities driven by heating possible, besides of affecting the development of the natural instabilities of the real plasma being simulated. There are several sources of this phenomena, mostly due to two different mechanisms (Cormier-Michel et al. 2008):

\subsubsection{Grid heating and "aliasing" instability}

The first source of the self-heating was attributed the introduction of a grid: the appearance of a non physical force $F_{g}$ acting on the particles. In a real plasma, invariant under spatial translations, the inter-particle interaction force will only depend on the distance between them. But the spatial grid make the system spatially non-uniform (although periodic), losing the invariance under translations. Then, this implies that the inter-particle force will depend not only of the separation between particles, but also on their position 
relative to the grid (see Birdsall and Langdon 1991, Sec. 8.2). The difference between the interparticle force in a real plasma and the one simulated with the grid is defined as the nonphysical grid force $F_{g}$.

The most important effect of this nonphysical force is due to the aliasing between grid modes (first noticed by Langdon 1970). Indeed, by means of a Fourier analysis, the periodicity introduced by the grid can be quantified by $k_{\Delta x}=2 \pi \Delta x$. Following Melzani et al. (2013) (see also Birdsall and Langdon 1991, Sec. 8.7), any physical plasma wave mode with wavenumber $k=2 \pi / \lambda$ will generate aliases at $k_{n}= \pm k+n k_{\Delta x}$ (n: an integer). These grid aliases (in both density perturbations and grid forces $F_{g}$ ) with wavenumbers not resolved by the grid can couple resonantly with physical plasma modes, triggering parametric instabilities via non-linear wave-wave interactions. The spatial grid operates as a "pump wave". An example of such numerical instability is given in Birdsall and Langdon (1991, Sec. 8.13) based in the original work by Langdon (1970), through a linear analysis of the discrete electrostatic Vlasov equation. They found unstable wave modes arising from the aforementioned coupling. Their growth rates $\gamma$ were greatly enhanced when the Debye length was not well resolved by the grid: $\Delta x>\lambda_{D e}$, while they were heavily damped in the opposite case. This can be explained because plasma modes with large wavelengths will have $\lambda_{D e}>2 \Delta x$ (Nyquist criterion), equivalent to the known noncoupling condition from parametric instabilities: $k<-k+k_{0}$. In addition, these growth rates were found to be

$$
\gamma \propto S^{2}\left(k_{n}\right)
$$

where $S$ is shape function in Fourier space, depending on the aliases wave number $k_{n}$. Therefore, the undesired effects due to these aliases can be avoided by choosing macroparticles with an extended shape (higher order), equivalent to a sharp decaying Fourier transform. Note that these kinetic instabilities are totally independent on the number of macroparticles per cell.

The non-linear saturation of the aforementioned (linear) kinetic instability was observed to be around $k_{n} \lambda_{D e} \sim 1$ in several simulations studies, some of them compiled in Birdsall and Langdon (1991, Sec. 8.12). Therefore, when the condition Eq. 6.37) $\Delta x>C \lambda_{D e}$ was violated, the plasma experienced a very fast electron heating. This lead to an increase of $\lambda_{D e}$ until it becomes of order $\Delta x$ (more precisely $\Delta_{x} / \pi$ ), restoring the system to the marginal stability condition.

\subsubsection{2 "Scattering" heating}

But in a "real plasma" there are many more numerical effects acting on the particles that just $F_{g}$ and the aliases that produces. One can group them all together in a stochastic force $F(t)$ acting on the particles, with contributions also from computational rounding, time step, finite difference algorithm of the field equations and the use of shape functions for the macro-particles. This force, changing at each time step and different for each particle, can be modeled as due to only fluctuations in the electric field (since magnetic field fluctuations do not change energy) in a way $\delta F(t)=m \delta v / \Delta t=q \delta E$. It was found that the net result of this force is a random walk (revealing thus its stochastic origin) of the particles in velocity space. The average change in kinetic energy $\left\langle\left|(1 / 2) m \Delta v^{2}\right|\right\rangle$ of the particles due to this random walk will increase linearly with time (see Hockney and Eastwood 
1988, Eq. 9.18)

$$
\left\langle\left|(1 / 2) \Delta v^{2}\right|\right\rangle=\frac{q^{2}}{m} \Delta t|\delta \vec{E}|^{2} t_{n}
$$

where $t_{n}$ is the time after $\mathrm{n}$ time steps: $t_{n}=n \Delta t$. It can be noticed that this "stochastic heating" (further details can be found in Hockney and Eastwood (1988, Sec. 9.2) and Birdsall and Langdon (1991, Sec 13.4)) will affect most the lighter particles: electrons. Its effect on the evolution of the simulated plasma can be characterized via the "heating time" $\tau_{h}$, the time necessary to increase twice its thermal energy. By means of $2 \mathrm{D}$ electrostatic PIC simulations, Hockney (1971) and Abe et al. (1975) found the following empirical formula ${ }^{20}$ :

$$
\tau_{H} \omega_{p e} \propto \eta\left(\frac{\lambda_{D e}}{\Delta x}\right)^{2} N_{C}
$$

where $N_{c}$ is a correction to the $\Lambda$ parameter (the number of particles on a Debye sphere) due to the introduction of the grid:

$$
N_{c}=n\left(\lambda_{D e}^{2}+(W \Delta x)^{2}\right)
$$

with $W$ is a fitting parameter of order unity depending on the shape function. $\eta$ is also a fitting constant, but strongly dependent on the shape functions (in their simulations, $\eta=3$ for NGP, $\eta=600$ for CIC and $\eta=4000$ for TSC). Other simulations mentioned in the previous references also showed a dependence proportional to the length of the spatial smoothing used for the current. From this we can conclude that the numerical heating can be controlled (i.e.: longer $\tau_{h}$ ) by choosing a larger number of macroparticles per cell (through $N_{c}$ ) but especially for higher order shape functions (and only weakly on $\Delta x \lesssim \lambda_{D e}$, the critical parameter for the grid instability).

This "stochastic heating" at the end is due to the coarse-graining of phase-space. The effective scattering can be considered as a form of numerical collisions (see Sec. 6.8.3) between the macro-particles and also due to the grid. As explained by Cormier-Michel et al. (2008), whenever a macroparticle moves across the boundary of cells, it produces random electric fluctuations. These spurious electric fields, non-existent in real collisionless plasmas, are accumulated on each grid point with an associated error depending on the interpolation scheme, affecting the motion of the neighboring macroparticles when the fields are interpolated back to the macroparticles position. The net effect is analogous to the Brownian motion or random walks (a diffusive process). Finally, this leads to artificially high levels of the standard deviations in the particles' velocities, equivalent macroscopically to a numerically increased temperature.

It is interesting to mention that most of the studies about numerical heating were based on the one due to the grid instability, and to a less extent in the one due to the scattering heating. In addition, practically all of them were in reduced geometries with electrostatic PIC codes, in order to avoid additional complications to the already complex problem of determining the sources of heating. And most of the attempts to mitigate the heating were based in increasing the number of macroparticles per cell, according to Eq. (6.41), since

\footnotetext{
${ }^{20}$ There are very few reliable analytical expressions for this numerical heating, considering its many different sources.
} 
this will also reduce the PIC shot noise (see Sec.6.8.6). But note that the latter, according to Eq. (6.48), it is only weakly dependent on the shape function. For this reason, in addition to the lack of studies about the shape function, that a choice of $S$ was not considered relevant to solve this issue. Only recently there have been some studies studying these effects in more realistic plasmas: laser wakefield accelerators (Cormier-Michel et al. 2008). They found that the choice of higher order shape functions proved to be more (computationally) efficient in reducing the heating due to scattering that an equivalent increase in the number of macroparticles per cell. They also observed spurious numerical instabilities driven by numerical heating, hiding the real physical processes to be modelled. Our results to be shown in Chapter 7 agree with this conclusion for a very different setup: current sheets.

\subsubsection{Consequence of coarse-graining: numerical collisions}

Strictly speaking, PIC codes do not model a real collisionless plasma governed by the Vlasov-Maxwell equations. This is due to the inherent numerical collisions (for an extended discussion about this issue, see Melzani et al. 2013). They are generated due to the calculations on a spatial grid, a finite time step and the coarse-graining (representation of many physical particles by a single macroparticle). Thus, in practice, PIC codes solve a kinetic Boltzmann equation for the distribution function instead of the (collisionless) Vlasov equation, with an effective numerical collision operator depending on the Fourier transform of the shape function $\tilde{S}(\vec{k})$, the grid size $\Delta x$ and the time step $\Delta t$. More precisely, it is estimated being proportional to (see Birdsall and Langdon 1991, Appendix E):

$$
\left(\frac{\partial f}{\partial t}\right)_{c} \propto \int \overrightarrow{d k} \frac{\vec{k} \vec{K}}{K^{4}} \frac{\tilde{S}^{2}(\vec{k})}{|\epsilon(\vec{k}, \vec{k} \cdot \vec{v})|^{2}} \sum_{p=-\infty}^{\infty} \tilde{S}^{2}\left(\vec{k}_{p}\right) \times \int d \vec{v}^{\prime} \delta\left(\vec{k} \cdot \vec{v}-\vec{k}_{p} \cdot \vec{v}^{\prime}, \omega_{g}\right)
$$

where $\vec{\kappa}$ and $K$ are the finite difference gradient and Laplacian operators, respectively (associated with $\vec{k}$ and $k^{2}$, respectively). $\vec{k}_{p}=\vec{k}-\vec{p} \cdot \vec{k}_{g}, \vec{k}_{g}=2 \pi / \Delta\left(\vec{x}^{-1}\right)^{T}$ is the grid wave number, $\omega_{g}=2 \pi / \Delta t$ is the characteristic frequency of the time stepping, $\delta\left(\omega, \omega_{g}\right)=$ $\sum_{q=-\infty}^{\infty} \delta\left(\omega-q \omega_{g}\right)$ is a periodic delta-function comb. $\epsilon$ is the plasma dielectric function. Different from the usual dependence on the physical scenario/problem, in this case it also depends on the time integration scheme, the conservation properties of the algorithm, the shape function as well as other numerical algorithms/details of a PIC code.

We can have a more physical insight on the collision operator $(\partial f / \partial t)_{c}$ by rearranging it in the form of a Fokker-Plank collision term:

$$
\left(\frac{\partial f}{\partial t}\right)_{c}=\frac{\partial}{\partial v_{i}} D_{i j} \frac{\partial f}{\partial v_{j}}+\frac{\partial}{\partial v_{i}} A_{i} f
$$

with effective diffusion $D_{i j}$ and drag $A_{i}$ coefficients. The values of these coefficients are reduced (and with them the numerical collisions) by choosing higher order shape functions, since $\tilde{S}(\vec{k})$ decays faster. Moreover, it has been found empirically (see Birdsall and Langdon (1991, Chap. 12), Hockney and Eastwood (1988, Sec. 7.5) and Okuda (1970)) that the values of these coefficients depend mostly on the width of the macroparticle shape function compared to $\lambda_{D e}$, and to a lesser extent on the macroparticle number per cell. This is 
different from real plasmas with individual point particles where they depend mostly on the number of particles in a Debye sphere.

Note that previous investigations (Okuda 1970) have shown that the difference between the ideal Vlasov and Boltzmann equation, given by the numerical collision operator, is especially important for $2 \mathrm{D} 3 \mathrm{~V}$ plasma models in comparison with the $3 \mathrm{D}$ cases. This was confirmed by Matsuda and Okuda (1975), who showed that numerical collisions in electromagnetic PIC code simulations are reduced in regions with stronger magnetic field, in addition to be highly anisotropic in a 2D configuration with an externally applied magnetic field. This is because the electron-ion collisions, measured through the temperature relaxation time, drag and diffusion coefficients, depend on the relative direction of the magnetic field relative to the electric field fluctuations. They stated that is due to the neglect of the spatial variations in the $z$ direction: a 2D3V PIC code constrains the motion of the macroparticles to the $x-y$ plane, but solves for all three components of the velocities $v_{x}-v_{y}-v_{z}$. It is important to mention that the analysis by Matsuda and Okuda (1975) was carried out for a plasma embedded in a strong magnetic field.

Numerical collisions are also closely related with numerically generated pitch angle scattering. This is a process that redistributes particles velocities in the momentum space when a temperature anisotropy is present, without changing significantly their total speed ( $\sim$ to the total kinetic energy). Electromagnetic turbulence arising from the temperature anisotropy generates waves that transfer momentum via wave-particle interaction from the perpendicular to the parallel direction in velocity space. See more details in Kirk et al. (1994, Sec. 3.1). Similarly to pure numerical collisions, the pitch-angle scattering (either physical or numerical) is stronger in regions where the magnetic field is minimum (Coroniti 1980). This pitch-angle scattering, similar to the (either physical or numerical) collisions, is modeled through a diffusion operator in the right hand side of Boltzmann equation

Finally, it is worth to remark that the (numerical) collisional behaviour of a plasma can be measured via the collision time $\tau_{c}$ : larger values imply a more "collisionless behaviour" of the plasma. By means of 2D electrostatic PIC simulations, Hockney (1971) (see al so Hockney and Eastwood 1988) found the following empirical formula:

$$
t_{c} \omega_{p e}=n\left(\lambda_{D e}^{2}+W^{2}\right)
$$

where $W$ is the effective size of the macroparticle (as calculated from their shape function). We can see that this quantity is mostly controlled by the density $n$ and not so much on the particle shape function (different from the heating time, Eq. (6.41)).

\subsubsection{Side effect of collisions (and coarse-graining): Entropy in Vlasov plasmas}

A collisionless Vlasov plasma by definition describes reversible processes and thus keeps the entropy $S(t)$ constant for a closed system (for a discussion about non-equilibrium processes and the validity of the Vlasov approximation in a real plasma, see Klimontovich 1997). The definition of this quantity that we will be using is

$$
S(t)=-\int f(\vec{v}, t) \ln f(\vec{v}, t) d^{3} v
$$


This quantity measures the "uncertainty" in the information contained in the distribution $f$, and for that reason is sometimes called "information entropy" (Shannon 1948) 21, is different from the Gibbs entropy only by the Boltzmann's factor $k_{B} 22$. And this is almost equivalent to the thermodynamic definition of entropy (at least for a system in a local thermodynamic equilibrium) in the sense that shares the properties of (see Zubarev et al. 1996, Sec. 1.3.1): 1) additivity (extensive quantity), 2) $T d S=d U+P d V-\mu d N$ holds for reversible processes, where $U$ is the internal energy and $\mu$ the chemical potential. However, $S(t)$ as defined by Eq. 6.46) differs from the thermodynamic definition of entropy in the sense that does not monotonically increase in time until reaching maximum value at thermal equilibrium. Instead, as we mentioned, the Gibbs or information entropy $S(t)$ of a closed system cannot increase. It is only when the entropy Eq. (6.46) is calculated for any coarse-grained distribution function $f$ that $S(t)$ can increase in time, recovering the desired thermodynamic behaviour. Since the coarse-graining can be in both phase space and time, this is precisely the behaviour expected in PIC codes (corresponding to the introduction of grid and time stepping, respectively), being noticed very early in the history of these plasma model simulations (Buneman 1964). This is true even for a system in thermodynamic equilibrium which should have maximum entropy for some given constraints (see Birdsall and Langdon 1991, Sec. 12.6). Only in the differential limit, when $f$ is the fine-grained distribution function defined with respect to the infinitesimal phase space volume $d x^{3} d v^{3}, S(t)$ remains constant (it is a Casimir invariant). Choices of larger finite phase space elements will lead to higher increases in the entropy. For a proof of all these claims, see Zubarev et al. (1996, pp. 51-52) or Levin et al. (2014).

Now, the problem with the calculation of entropy gets complicated when considering dissipation, as produced eventually by numerical collisions in a PIC code. First of all, and thermodinamically speaking, the entropy should increase in presence of dissipation. The latter is characterized by the diffusion coefficient $D_{i j}$ that appears in the effective collision operator of the Boltzmann equation Eq. (6.44), depending on the shape function as well as other numerical parameters of the PIC algorithm. Then, any increase in entropy in a PIC code is not only due to the coarse-graining of phase space, but also due to the relative strength of numerical collisions. A very simplified model allow us to relate the total rate of change of entropy due to the last effect with the effective collision operator in the Boltzmann equation given by Eq. 6.43). Following Birdsall and Langdon (1991, Sec. 12.6), the latter is proportional to the increase of kinetic energy according to:

$$
\frac{d}{d t} \frac{1}{2} \overline{(\vec{v}-\overline{\vec{v}})^{2}}=-v_{t h, e}^{2} \frac{d S}{d t}=-\frac{1}{2 v_{t h, e}^{2}} \int d \vec{v} d \vec{v}\left(v_{i}-v_{i}^{\prime}\right) Q_{i j}\left(v_{j}-v_{j}^{\prime}\right) f(\vec{v}) f(\vec{v}) .
$$

This simple expression is valid for an instantaneous Maxwellian distribution function. Here, $Q_{i j}$ is a tensor proportional to the right hand side of the numerical collision operator, therefore having the same dependence as Eq. (6.43). And to the order and approximations used in the kinetic equation, the rate of change of kinetic energy is equal to the rate of

\footnotetext{
21 There are also alternatives definitions, for example the relative entropy calculated with respect to a reference distribution function $f_{0}$ (replacing the argument of the natural logarithm in Eq. 6.46) from $f(\vec{v}, t)$ by $f(\vec{v}, t) / f_{0}(\vec{v}, t)$ ), sometimes called Kullback-Leibler divergence (Kullback and Leibler 1951).

${ }^{22}$ Strictly speaking, the definition of Gibbs entropy used in statistical mechanics is a special case of the more information entropy, although we will not discuss these differences here. For further information, see Zubarev et al. (1996, Sec. 1.3).
} 
change of total energy. This correlation has already been noticed in early uniform and electrostatic simulation studies (see, e.g., Montgomery and Nielson 1970). Years later, Struckmeier (1996) and Struckmeier (2000) improved the analytical expression for the rate of change of entropy by considering other additional effects (e.g., lifting the approximation of Maxwellian distribution and allowing anisotropies), particularly suitable to be measured in PIC simulations of laser plasma beams. The model developed by them is based on the second order moment of the Vlasov-Fokker-Planck equation, relating specifically the noise and collisional effects with the numerically generated temperature anisotropies (the latter is necessary for the increase in entropy). Recent PIC simulations studies have applied and extended these models to similar simulations of laser beams, being able to distinguish the collisional effects due to the grid and the noise via measurements of the entropy and other related quantities (Hofmann and Boine-Frankenheim 2014, Boine-Frankenheim et al. 2015).

\subsubsection{Side effect of collisions: Enhanced stopping of high energy par- ticles}

Due to the collisions, a particle that travels through a plasma with speed much higher than the electron thermal speed, will experience a drag force due to the induced electrostatic field. This was already noticed by Hockney (1971) (see also Birdsall and Langdon 1991, Sec. 13.5), finding analogous empirical relations to these shown for the heating and collisions times of the previous sections. But only recently there have been some analytical progress regarding this topic. Kato (2013) and May et al. (2014) found that the rate of kinetic energy loss depends mostly on the number of macroparticles per cell and the shape function, with a very different behavior on dependence of the dimensionality. This stopping power of energetic particles can relax very quickly non thermal populations, scaling in general as $q^{2} / m$, being therefore reduced for a macroparticle with small charge. In addition, it can be avoided by using larger grid cells, higher order shape functions and current smoothing.

\subsubsection{Consequences of coarse-graining: numerical noise}

The stochastic force arising from the grid and other effects (see Sec.6.8.2) produces enhanced fluctuations levels in a PIC simulation compared to a real plasma (both are correlated). Indeed, early (2D electrostatic) PIC simulations by Hockney (1971) showed that the electric field fluctuations, a measurement of the numerical PIC noise, scale as (see also Birdsall and Langdon (1991, Sec. 13.5) and Dawson (1983)):

$$
\frac{\left\langle\epsilon_{0} E^{2} / 2\right\rangle}{(3 / 2) n_{e} k_{B} T}=\frac{K}{N_{c}}=\frac{K}{n\left(\lambda_{D e}^{2}+(W \Delta x)^{2}\right)}
$$

where $N_{c}$, defined in Eq. 6.42), is roughly the macroparticle number and $K$ is a fitting parameter. This is consistent with the general theory of fluctuations in a thermal plasma 23 ,

\footnotetext{
${ }^{23}$ It is interesting to mention that these results regarding fluctuations are beyond the scope of the kinetic Vlasov theory, because they involve the intrinsic discrete nature of a real plasma, and therefore higher order correlations in $1 / \Lambda=1 /\left(n \lambda_{D e}^{3}\right)$. See further details in Krall and Trivelpiece (1973, Sec. 11) or the classical textbooks Sitenko (1967) and Sitenko (1982).
} 
which predicts the following expression for a Maxwellian plasma with equal $T_{i}=T_{e}$ (see Krall and Trivelpiece 1973, Sec. 11.2.3).

$$
\frac{\left\langle\epsilon_{0} E^{2} / 2\right\rangle}{(3 / 2) n_{e} k_{B} T}=\int \frac{d^{3} \vec{k}}{(2 \pi)^{3}} \frac{1}{1+\left(k \lambda_{D e}\right)^{2}}=\frac{1}{(2 \pi)^{3}} \log \left(\frac{1+\left(k_{\max } \lambda_{D e}\right)^{2}}{1+\left(k_{\min } \lambda_{D e}\right)^{2}}\right) \frac{1}{n \lambda_{D e}^{3}} \approx \frac{1}{n \lambda_{D e}^{3}}=\frac{1}{\Lambda}
$$

where the last approximation is obtained neglecting the contribution from short wavelengths $k \lambda_{D e}>1$ (strongly damped). Then, we can identify the number of particles in a Debye sphere $\Lambda$ by $N_{c}$, since the latter is approximately equal to the number of macroparticles in one Debye length and also per cell $p p c$ (due to the condition $\Delta x \sim \lambda_{D e}$ to avoid grid heating). Consequently, the noise in the electric field, $\sqrt{\left\langle\epsilon_{0} E^{2} / 2\right\rangle}$, will scale as $\propto \sqrt{1 / N}$. This scaling has been measured with ACRONYM code by P. Kilian (see Fig. 6.8), showing a very good agreement with the expected theoretical behavior.

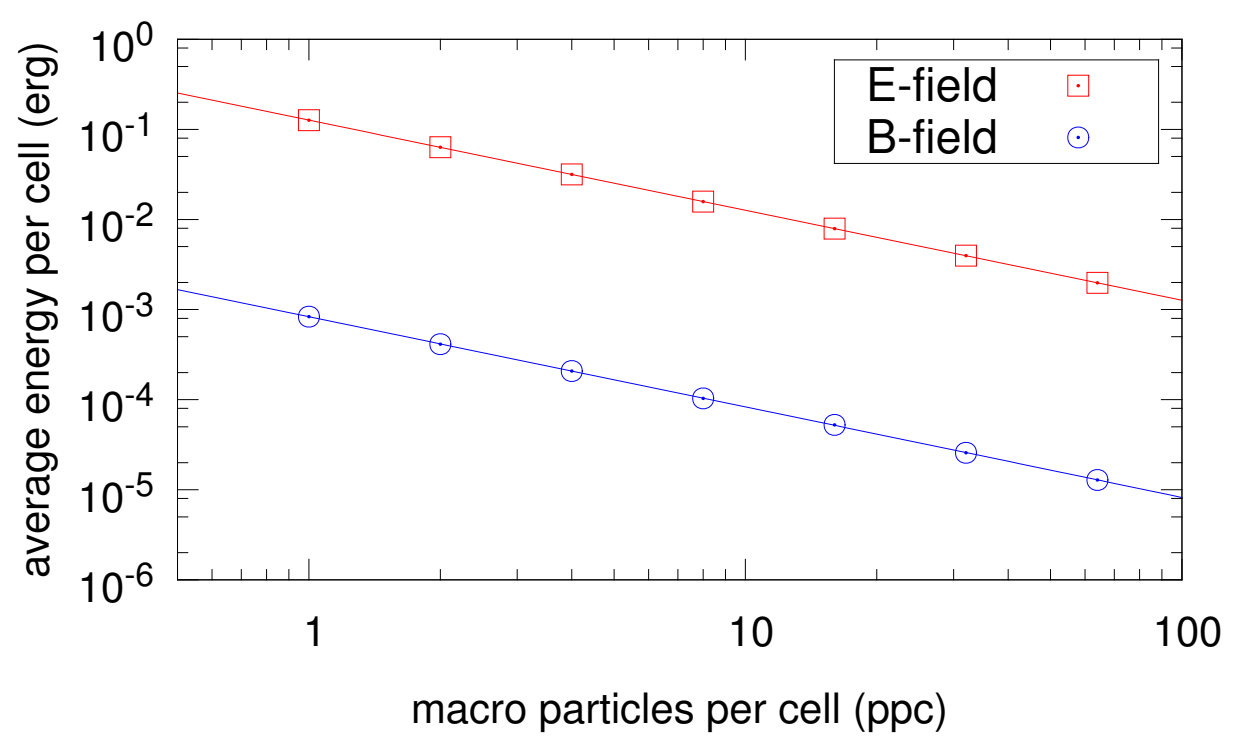

Figure 6.8: Scaling of electric and magnetic field energy with the number of macroparticles per cell, showing good agreement with Eq. (6.48). After Schreiner (2013), based in the results by $P$. Kilian.

\subsection{Parallelization}

In order to reduce the huge computational burden of solving the equation of motion for millions of macroparticles, the parallelization of PIC codes have become one important issue since some decades ago. This is especially critical nowadays, with the availability of petascale computational resources capable to use hundreds of thousands of CPUs/GPUs, requiring an optimum and efficient design of massively parallel algorithms (see, e.g., Bowers et al. 2009, Daughton et al. 2014, Burau et al. 2010, Germaschewski et al. 2013). 
The most popular strategy is the "domain decomposition'24: to divide the computational box in several smaller domains, and assigning each one of them to a single CPU (more correctly, a process or thread). This CPU has to solve all the relevant equations inside of its domain, in particular the most expensive part related with the current assignment (inter-communication is avoided in this step), and it should also be able to transfer particles to the neighboring computational domains if their motion require that. Besides of that, if the algorithm is local, like for the explicit PIC codes (but not for implicit or Darwin ones), it should also solve individually the Maxwell equations and exchange their values at their boundaries with the neighboring computational domains. Due to these reasons, the parallelization using MPI (Message Passing Interface) libraries have become a very convenient choice in most of the PIC codes used nowadays, since it allows a efficient communication between the domains (for an introduction to MPI, see Pacheco 2011, Sec. 3 ). The exchange of information between them is carried out in "ghost (or guard) cells": additional cells at the domain boundaries that are designed to exclusively transfer data and to make the interpolation of current assignment strictly local, and not representing any physical domain in the plasma.

The transfer of information between computational domains have a side effect when trying to solve the same physical problem with an increasing number of them (each one associated to one process or thread). Indeed, the increasing number of boundaries between computational domain with their associated data exchange of particles and field data may produce the opposite effect: it may slow down the total computational time of a simulation if a too high number of computational domains are used and the code parallelization is not done efficiently. This parallel performance of a PIC code (or any kind of parallel code in general) can be evaluated through scaling studies. In the computer science terminology (see Pacheco 2011, Sec. 2.6), the ideal behaviour of a parallel PIC code is called "weakly scalable" if the efficiency 25 is kept fixed when both simulation size and number of processes are increased at the same rate. On the other hand, a code/program is called "strongly scalable" if the efficiency is kept fixed for a given simulation size.

As can be seen in Fig. 6.9, the ACRONYM PIC code (to be discussed next) shows a very good weak scaling up to $65 \mathrm{k}$ processes in several supercomputers in Germany and other European countries.

\footnotetext{
${ }^{24}$ In PIC codes, this method is sometimes called GCPIC algorithm: general concurrent particle-in-cell. They were first developed for electrostatic PIC codes with spectral solvers by Liewer and Decyk (1989) and Decyk (1995). See also Pritchett (2000) for a brief description and early attempts.)

${ }^{25}$ This is defined as $E=\frac{T_{\text {seral }}}{n T_{\text {parallel }}}$ where $T_{\text {parallel }}$ is the parallel execution time of the code/program, $T_{\text {serial }}$ the serial execution time of the same (for one single process) and $n$ the number of used processes. Ideally, we would like to have $T_{\text {parallel }}=T_{\text {serial }} / n$ and so $E=1$ (a "linear speedup"), but in the real world there are many factors that makes $E$ much lower.
} 


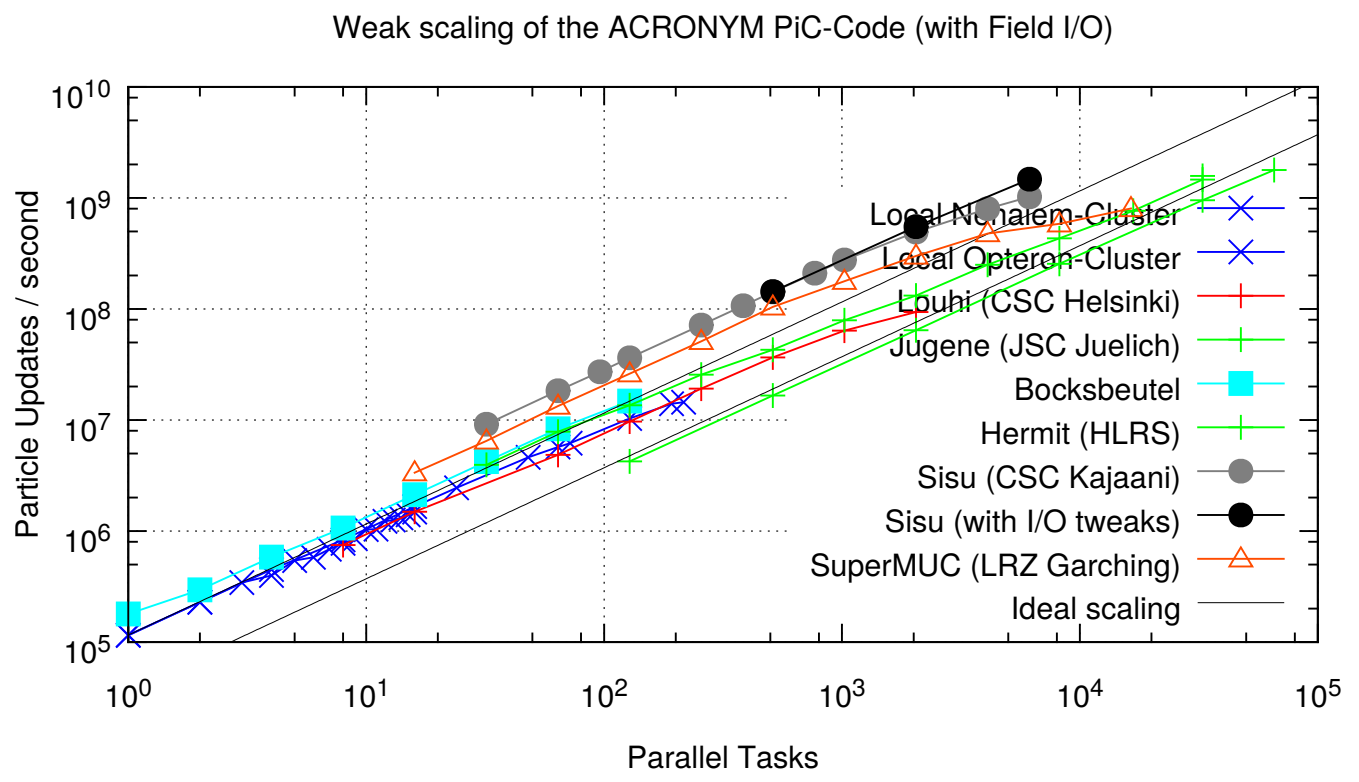

Figure 6.9: Weak scaling of ACRONYM in several supercomputers in Germany and other European countries. The $x$ axis show the number of parallel tasks or MPI processes. The $y$ axis is the "code speed" in particles updates per second. After Kilian (2013).

\subsection{The ACRONYM code}

The fully-kinetic PIC code ACRONYM ("Another Code for moving Relativistic Objects, Now with Yee lattice and Macroparticles") has been developed and improved since 2007 in the University of Würzburg, mainly during the theses written in the graduate studies of T. Burkart (Diploma and PhD: Burkart 2007, 2010), U. Ganse (Diploma and PhD: Ganse 2009, 2012), P. Kilian (Diploma and PhD: Kilian 2010, 2015), and C. Schreiner (Bachelor and Master: Schreiner 2011, 2013). Nowadays, it features26:

- Explicit scheme

- $1 \mathrm{D} 3 \mathrm{~V}, 2 \mathrm{D} 3 \mathrm{~V}$ and 3D3V cartesian geometries

- Maxwell solvers (in CGS units)

- Full electromagnetic Maxwell equations: FDTD (finite domain time difference) standard Yee lattice (2nd order accurate in space and time), FDTD 4th order solver, NSFD (Non-Standard Finite Differences) Cole-Kärkkäinen solvers CK-CK5 (Kärkkäinen and Gjonai 2006, Vay et al. 2011), and, in development stage, FDTD M24 solver with 2 th/4th order accurate in space/time, respectively (Hadi and Piket-May 1997, Greenwood et al. 2004, Hadi 2007).

- Electrostatic, using a spectral solver (with parallel FFT libraries by S. Plimpton 27 , based on the FFTW libraries 28 )

\footnotetext{
${ }^{26}$ There is also a hybrid PIC code version of ACRONYM in development stage. It features fully-kinetic ions and an electron fluid with finite inertia.

${ }^{27}$ at Sandia National Laboratories, http://www. sandia.gov/ sjplimp/docs/fft/README.html

28 "Fastest Fourier Transform in the West": http://fftw.org/
} 
- Darwin (low frequencies), using a spectral solver.

- Particle mover: (relativistic) standard Boris pusher and (ultra-relativistic) Vay pusher (Vay 2008).

- Available shape functions: NGP, CIC, TSC, PQS (piecewise quadratic shape), PCS (piecewise cubic shape) and SUDS (subtracted dipole scheme, see Kruer et al. (1973)). Option of half-order shape functions, i.e.: a given order $W^{n}$ for the even grid points and other $W^{n+1}$ at the odd grid points (Sokolov 2012). Option of fix-point particle.

- Current deposition: Charge conserving Esirkepov scheme (Esirkepov 2001) and, in development stage, Umeda scheme (Umeda et al. 2003).

- Post-compilation option of Gauss cleaning (Marder 1987) to enforce quasineutrality, by using an spectral solver in the Poisson equation

- Post-compilation option of current smoothing using a binomial filter, with optional compensation pass (Vay et al. 2011).

- Post-compilation selection of boundary conditions: Periodic, reflecting (PEC: Periodic Electric Conductor), absorbing (ABC: Absorbing Boundary Conditions, with the CFS-CMPL method: Complex Shifted Coefficient - Convolutionary Perfectly Matched Layer. See Taflove and Hagness (2005, Sec. 7.9)).

- Default species particles: electron, ion and positrons.

- Initial loading of particles: by default (non-relativistic) drifting Maxwellian distribution, with normal random numbers generated via a modified version of the Kinderman/Monahan algorithm (Leva 1992). Option to change initial random seed. Enforce of initial charge quasineutrality by loading each pair of ion/electron at the same position.

- Option for particle injection and wave launching.

- Vector operations using Blitz 29 library.

- Massively (domain) parallelized with MPI (Message Passing Interface) libraries. Automatic domain decomposition specifying number of MPI tasks.

- Post-compilation option of load balancing to dynamically resize MPI domains and thus to distribute equally the computational load.

- Output in HDF5 30 or native MPI binary format.

- Running time diagnostics for particle/electromagnetic field energy, global particle momenta per specie up to second order (density, bulk velocities and pressure tensor), local particle momenta per specie up to fourth order (for heat flux and kurtosis), temporal averages of electromagnetic fields and particle momenta, derivatives of particle momenta, particle tracking.

${ }^{29}$ http://blitz.sourceforge.net/

${ }^{30}$ Hierarchical Data Format v5 https://hdfgroup. org/HDF5/ 
- Post-processing diagnostics for power spectrum and dispersion relations, phase space and particle distribution functions, particle pitch angle.

This code have been extensively tested and applied to a wide variety of numerical and physical problems, such as investigations about the shape function (Kilian et al. 2013), particle acceleration by plasma instabilities (Burkart 2007, 2010, Burkart et al.2010, Kilian et al. 2012), particle acceleration in laser plasmas (Kilian 2010), wave-wave interaction in type II Radio Bursts (Ganse et al. 2010, 2012b,c., a, Ganse 2012, Ganse et al. 2014)), CME driven shocks in the solar wind (Kilian 2015), resonant wave-particle interaction (Schreiner 2011, 2013, Schreiner and Spanier 2014) and part of the present thesis work: current sheets instabilities (Muñoz et al. 2014, 2015). 


\section{Disclaimer for Chapter 7}

The following chapter is based on and follows very closely the line of argumentation of the work published in: P. A. Muñoz, P. Kilian, and J. Büchner, Physics of Plasmas 21, 112106, (2014) "Instabilities of collisionless current sheets revisited: the role of anisotropic heating" (Muñoz et al. 2014), also available as arXiv eprint:1501.06022, To the best of my knowledge, all the text have been rewritten and mostly expanded and/or moved to the Introductory chapters or Appendix, although some parts can still be traced to the original source. Moreover, since the line of argumentation is similar, the titles of some sections are still identical. In particular,

- 26 of 30 figures are "Reproduced with permission from P. A. Muñoz, P. Kilian, and J. Büchner, Physics of Plasmas 21, 112106, (2014), Copyright 2014, AIP Publishing LLC". Each one is identified in its corresponding caption.

- The first paragraph in Sec. 7.1.3 correspond to the last paragraph of section II in Muñoz et al. (2014).

- Sec. 7.1.4 is based on Section VI-A of Muñoz et al. (2014).

- Sec. 7.2.1 is based on Section III-A of Muñoz et al. (2014).

- Sec. 7.2.2 is based on Section III-B of Muñoz et al. (2014).

- Sec. 7.2.3 is based on Section III-C of Muñoz et al. (2014).

- Sec. 7.2.4 is based is based on Section III-D of Muñoz et al. (2014).

- Sec. 7.2.5 is based on Section VI-C of Muñoz et al. (2014).

- Sec. 7.3.1 is based on Section IV-A and IV-B of Muñoz et al. (2014).

- Sec. 7.3.2 is based on Section IV-B of Muñoz et al. (2014).

- Sec. 7.3.3 is based on Section IV-C of Muñoz et al. (2014).

- Sec. 7.3.4 is based on Section IV-D of Muñoz et al. (2014).

- Sec. 7.3.5 is based on Section IV-E of Muñoz et al. (2014).

- Sec. 7.3.6 is based on Section IV-F of Muñoz et al. (2014).

- Sec. 7.3.7 is based on Section IV-G of Muñoz et al. (2014).

- Sec. 7.3.1.1 is based on Section IV-H of Muñoz et al. (2014).

- Sec. 7.4.1 is based on Section V-A of Muñoz et al. (2014).

- Sec. 7.4.1.3 is based on Section V-B of Muñoz et al. (2014).

- Sec. 7.4.2 is based on Section V-C of Muñoz et al. (2014).

- Sec. 7.4.3 is based on Section VI-B of Muñoz et al. (2014). 


\section{Instabilities of antiparallel Harris CS and anisotropic heating}

In order to simulate current sheet instabilities and magnetic reconnection with PIC codes, it is necessary to carefully consider several numeric constraints, like stability conditions (see Sec. 6.8.1). They impose restrictions on how small the grid size $\Delta x$ and time step $\Delta t$ should be with respect to the characteristic plasma parameters $\lambda_{D e}$ and $\omega_{p e}$. In addition to that, it is also necessary to keep the numerical noise controlled due to the necessary use of a reduced number of macro-particles per cell. The direct approach for that is just increasing the number of macroparticles per cell. But this simplistic approach becomes computationally prohibitive if more realistic parameters (like mass ratio) are desired. Another, much less studied, approach is using higher order shape functions for the interpolation of PIC quantities to the grid nodes (see Sec. 6.2). As mentioned in Sec. 6.8.2, in the past there have been very few studies about the role of the numerical heating caused by this simulation parameter, mostly related with laser wake-field acceleration (Cormier-Michel et al. 2008). But the influence of the use of shape functions on the numerical heating and stability properties of collisionless current sheets has not been analyzed so far. This is the purpose of this chapter. As we are going to demonstrate, if these numerical considerations are not taken into account, they may lead to non-physical results, hiding completely the development of the physical phenomenon trying to be studied.

This chapter is divided as follows. We describe the setup of the Harris sheet simulation in Sec. 7.1. Next, we discuss the consequences of the numerically-induced anisotropic heating on the evolution of these Harris sheets in Sec.7.2, and how it can be suppressed by the use of higher order shape functions. The consequences of the numerical heating are mostly manifested in suppression of tearing mode, the instability associated with reconnection, and the appearance of an instability driven by numerically generated anisotropic heating: bifurcation. In order to prove the causation between the last two processes, we initialize CS with initially imposed temperature anisotropy in Sec. 7.3 but keeping controlled the numerical heating, seeing the natural development of bifurcation. We also observed an initially very dynamic state characterized by other secondary temperature driven micro-instabilities. Next, in Sec. 7.4 we confirmed the theoretical predictions of the tearing mode instability if all these numerical considerations are taken properly into account. Finally, we summarize our findings in the conclusion, Sec.7.5. 


\subsection{Simulation setup}

\subsubsection{General considerations}

For all the simulations to be shown in this chapter, we use a single Harris CS equilibrium (Harris 1962). It is described by a magnetic field $B_{y}$ that rotates around a neutral line, sustained by a current $J_{z}$ carried out by counterstreaming electrons and ions (see details in Sec. 3.2.1 with their respective profiles displayed in Fig. 3.1). There is no initial guide field (see Sec.7.2.5 for their effects on the simulations) nor initial background. The latter is in order to minimize additional effects due to that population and, in addition, to reduce the number of free parameters. Nevertheless, we will discuss the effects of a background plasma in Sec. 7.2.4.

The CS evolves only from the initial macroparticle numerical noise, there is no other initial perturbation. This is because we focus on the onset and growth of the spontaneous instabilities (Daughton 2005, Pritchett 2005a), the slow build-up phase of reconnection (see Chapter 4) characterized by the so called tearing mode (see Sec. 5.1). This kind of simulations require more computational time to reach the fully developed fast reconnection. Simulations with an initial perturbation allow to bypass this slow build-up phase and to reach directly the explosive reconnection phase (Birn et al. 2001), more convenient to analyze that kind of phenomena.

\subsubsection{Parameters}

As mentioned in Appendix A.3.3, there are four parameter ratios necessary to fully specify a Harris sheet in absence of background and guide field. These are chosen according to Lee and Büchner (2012).

$$
\frac{L}{d_{i}}=0.57, \quad \frac{\omega_{p e}}{\Omega_{c e}}=2.87, \quad \frac{m_{i}}{m_{e}}=180, \quad \frac{T_{i}}{T_{e}}=1,
$$

$\Omega_{c e}$ (and so $\rho_{i}$ ) is calculated with the asymptotic Harris magnetic field $B_{\infty y}$, while $\omega_{p i}$ with the central peak density $n_{0}=n_{e}=n_{i}$. Now, let us justify the choice the physical parameters. The chosen mass ratio is a compromise between computational speed and the possibility to separate the effects of ion and electron motion. The width of the CS is of the order of the ion inertial length, prone to a faster growth of the tearing mode than thicker CS, besides of the (supposed) natural limit of the thinning of CS. The ratio of frequencies $\omega_{p e} / \Omega_{c e}$ is chosen to be relatively small to save computation time. Although this parameter may affect the nonlinear saturation of the tearing mode and the relative electron to ion contribution to that stage (Coroniti 1977), the linear growth rate was shown to be independent of it (Daughton 2005).

For our discussions, it will be helpful to specify the remaining parameters necessary for the simulation setup, as well as some other few derived parameters and the relations between them. 


\begin{tabular}{|c|c|}
\hline Parameter & Standard Case \\
\hline$m_{i} / m_{e}$ & 180 \\
\hline$T_{i} / T_{e}$ & 1 \\
\hline$\omega_{p e} / \Omega_{c e}$ & 2.8688 \\
\hline$\sqrt{k_{B} T_{e} / m_{e}} / c$ & 0.1743 \\
\hline$L / d_{i}$ & 0.57735 \\
\hline$L / \rho_{i}$ & 1.1547 \\
\hline$L_{x} / d_{i}$ & 13.3 \\
\hline$L_{y} / d_{i}$ & 26.6 \\
\hline$V_{D e 0} / c$ & 0.0225 \\
\hline$V_{D e 0} / v_{t h, e}$ & 0.129 \\
\hline$\sqrt{k_{B} T_{i} / m_{i}} / c$ & 0.01299 \\
\hline$\rho_{i} / d_{i}$ & 0.5 \\
\hline$\rho_{i} / d_{e}$ & 6.7 \\
\hline$\rho_{i} / \lambda_{D e}$ & 38.48979 \\
\hline$d_{i} / \lambda_{D e}$ & 76.9795 \\
\hline$L / \lambda_{D e}$ & 44.444 \\
\hline$\omega_{p i}^{-1} / \omega_{p e}^{-1}$ & 13.416 \\
\hline$\Omega_{c i}^{-1} / \omega_{p e}^{-1}$ & 516.39 \\
\hline $0.5 L_{x} / L$ & 11.52 \\
\hline $2 \pi L / L_{y}$ & 0.136 \\
\hline$\omega_{p e}[H z]$ & $5.0459 * 10^{9}$ \\
\hline$\lambda_{D e} / \Delta x$ & 1.0 \\
\hline$\rho_{e} / \Delta x$ & 2.86886 \\
\hline$\rho_{i} / \Delta x$ & 38.48979 \\
\hline$L / \Delta x$ & 44.444 \\
\hline$\omega_{p e}^{-1} / \Delta t$ & 11.4754 \\
\hline$\Omega_{c e}^{-1} / \Delta t$ & 32.92143 \\
\hline$\Omega_{c i}^{-1} / \Delta t$ & 5925.8575 \\
\hline$N_{x} / N_{y}$ & $1024 / 2048$ \\
\hline$B_{\infty y}[G]$ & 100.000 \\
\hline
\end{tabular}

Table 7.1: Constant parameters for a Harris CS used in this chapter. The relations between them were obtained through the expressions given in Appendix A.3.1 and Appendix A.3.3.

Other simulation runs in this chapter will vary only a very few parameters with respect to the specified in Table 7.1. Their names and different parameters are in the following table. 


\begin{tabular}{ccccccc}
\hline Name/Parameter & Shape & $p p c$ & $n_{b} / n_{0}$ & total ppc per specie & $v_{t h, e y} / c$ & $T_{e, y} / T_{e, \perp}$ \\
\hline$C I C-40 p p c$ & $C I C$ & 40 & 0 & $7.28 \cdot 10^{6}$ & 0.1743 & 1 \\
$C I C-160 p p c$ & $C I C$ & 160 & 0 & $2.91 \cdot 10^{7}$ & 0.1743 & 1 \\
$C I C-360 p p c$ & $C I C$ & 360 & 0 & $1.16 \cdot 10^{8}$ & 0.1743 & 1 \\
$T S C-40 p p c$ & $T S C$ & 40 & 0 & $7.28 \cdot 10^{6}$ & 0.1743 & 1 \\
$T S C-160 p p c$ & $T S C$ & 160 & 0 & $2.91 \cdot 10^{7}$ & 0.1743 & 1 \\
\hline$C I C-40 p p c-b a c k 01$ & $C I C$ & 40 & 0.1 & $1.56 \cdot 10^{7}$ & 0.1743 & 1 \\
$C I C-40 p p c-b a c k 02$ & $C I C$ & 40 & 0.2 & $2.40 \cdot 10^{7}$ & 0.1743 & 1 \\
$C I C-360 p p c-b a c k 01$ & $C I C$ & 360 & 0.1 & $1.91 \cdot 10^{8}$ & 0.1743 & 1 \\
$T S C-40 p p c-b a c k 01$ & $T S C$ & 40 & 0.1 & $1.56 \cdot 10^{7}$ & 0.1743 & 1 \\
$T S C-160 p p c-b a c k 01$ & $T S C$ & 160 & 0.1 & $6.26 \cdot 10^{7}$ & 0.1743 & 1 \\
\hline$T S C-40 p p c-A 0.64$ & $T S C$ & 40 & 0. & $7.28 \cdot 10^{6}$ & 0.1394 & 0.64 \\
$T S C-40 p p c-A 1.21$ & $T S C$ & 40 & 0. & $7.28 \cdot 10^{6}$ & 0.1917 & 1.21 \\
$T S C-40 p p c-A 1.44$ & $T S C$ & 40 & 0. & $7.28 \cdot 10^{6}$ & 0.2092 & 1.44 \\
$T S C-40 p p c-A 1.69$ & $T S C$ & 40 & 0. & $7.28 \cdot 10^{6}$ & 0.2266 & 1.69 \\
$T S C-40 p p c-A 1.96$ & $T S C$ & 40 & 0. & $7.28 \cdot 10^{6}$ & 0.2440 & 1.96 \\
$T S C-360 p p c-A 1.96$ & $T S C$ & 360 & 0. & $1.16 \cdot 10^{8}$ & 0.2440 & 1.96 \\
$T S C-1000 p p c-A 1.96$ & $T S C$ & 1000 & 0. & $1.82 \cdot 10^{8}$ & 0.2440 & 1.96 \\
\hline$T S C-360 p p c-A 1.3$ & $T S C$ & 360 & 0. & $1.16 \cdot 10^{8}$ & 0.1987 & 1.3 \\
$T S C-360 p p c-A 1.4$ & $T S C$ & 360 & 0. & $1.16 \cdot 10^{8}$ & 0.2062 & 1.4 \\
$T S C-360 p p c-A 1.6$ & $T S C$ & 360 & 0. & $1.16 \cdot 10^{8}$ & 0.2205 & 1.6 \\
$T S C-360 p p c-A 1.8$ & $T S C$ & 360 & 0. & $1.16 \cdot 10^{8}$ & 0.2338 & 1.8 \\
\hline
\end{tabular}

Table 7.2: Parameters for the runs used in this chapter. The rest of parameters are identical to these in Table 7.1.

For the simulations, we use the PIC code ACRONYM (see Sec. 6.10 or Kilian et al. (2012) for a description) in 2.5D mode, not allowing variations in the translational invariant $\hat{z}$ direction (parallel to the current). The boundary conditions for particles and electromagnetic fields are reflecting in the $\pm x$ direction and periodic in the $\pm y$ direction (see Sec.7.1.4 for their effects on the simulations). The time step is chosen small enough to solve the electron motion and to fulfill the Courant condition $c \Delta t / \Delta x=0.5<1$ (see Eq. (6.34)). Finally, in the results to be shown, lengths are normalized to the ion inertial length $d_{i}=c / \omega_{p i}$ and times to $\Omega_{c i}^{-1}$.

The specific order in which the input parameters of this Harris CS are initialized in the ACRONYM code is described in Appendix A.3.3.

\subsubsection{Justification for the size along $y$}

The simulation box size in the $y$ direction was chosen to allow, according to the linear theory (Coppi et al. 1966), the development of up to seven unstable tearing modes with wavelength $\lambda=L_{y} / M$, with $M$ an integer satisfying $2 \pi M L / L_{y}=k_{y} L \leq 1$ (according to Table 7.1, this value is 0.136 for our parameters). Modes with shorter wavelengths, 
$k_{y} L \geq 1$, are evanescent. Thus, we can investigate the interaction and exchange of energy between magnetic islands of different size, i.e.: multimode tearing.

Note that for a simulation box size with $L_{y}=2 \pi d_{i}$, the linear theory predicts only one unstable mode with $k_{y} L=0.5$ or, equivalently, only one tearing island. Because of this, there is no interaction processes between different tearing islands and therefore no $\mathrm{X}$ point. Consistently, in the simulations (not shown here) it is possible to see a steady increase of the reconnected flux $\Psi$ until a saturated stage, but not a sudden increase in this quantity, which is a characteristic signature of the explosive phase of the reconnection process. This stabilization and saturation takes place at very small amplitudes, unimportant for space plasmas (Karimabadi 2005). The minimum size for which $\Psi$ have a sudden increase, which is the final stage of the coalescence process, is for a box size with $L_{y}=4 \pi d_{i}$, that allows three unstable modes with $k_{y} L=0.25,0.5$ and 0.75 . We checked that the time in which the explosive phase of reconnection happens and the maximum value of reconnected flux at saturated stage do not vary too much with respect to our choice of 7 unstable modes. And finally, we do not choose larger boxes in order to minimize the complications of the merging of even more magnetic islands.

\subsubsection{Justification for the size along $x$ and influence of the boundary conditions}

The choice of reflecting (also called conducting) boundary conditions in the $x$ might not be a realistic choice, especially considering applications to space plasmas. In the results to be shown, we detected waves launched from the center of the CS arising from the numerical noise. These waves propagates outwards being reflected many times between these reflecting boundaries and the center of the CS. This process leads to a periodic exchange of magnetic and kinetic energy, at time scales shorter than $\Omega_{c i}^{-1}$. By changing (extending) the simulation box size across $x$, we did not notice any significant difference in any of the processes to be described in this chapter (results not shown here). This is in agreement with previous studies (Ambrosiano et al. 1983, Melzani et al. 2013), who identified these waves as of magnetosonic nature. Therefore, we can safely ignore the influence of these boundaries in our results, as long as they are far away from the center of the CS. For our parameters, they are located 11.5L from the center (see Table 7.1).

\subsection{Origin and consequences of the numerically gener- ated anisotropic heating}

In this section, we show the results of five simulation runs with different combinations of shape functions (CIC or TSC schemes) and macroparticle per cell (ppc), specified in the first part of Table 7.1 .

\subsubsection{Numerical heating and shape function}

As we discussed in Sec. 6.6.2, the momentum preserving PIC codes (the vast majority, including ACRONYM), do not preserve the total energy exactly. This is due to 
the effect of the discretization (reduced number of macroparticles) and the introduction of the grid (via the shape function), which produces unphysical forces over the particles (Hockney and Eastwood 1988). In order to see to what extent these parameters affect the conservation of the total energy, we compare its time evolution for the aforementioned five PIC runs of Harris CS in Fig. 7.1(a).

For later times, by comparing the curves with equal number of macroparticles but different shape function, we can see that the conservation of energy is improved by two orders of magnitude just by changing the shape function. For example, for $t \gtrsim 40 \Omega_{c i}^{-1}$, the total energy for TSC-40ppc increases above its initial value by about $0.25 \%$, while for CIC-40ppc by about $25 \%$. The same is true for $160 \mathrm{ppc}$ at the same point in time: the total energy for TSC-160ppc increases above its initial value by about $0.05 \%$, while for CIC-160ppc by about $8 \%$. On the other hand, by comparing curves with equal shape function but different macroparticle number, we can infer that the pure increase of the latter does not have such significant effect on the improvement of the conservation of energy. That is true even though the numerical noise (scaling as $1 / \sqrt{p p c}$, according to the discussion in Sec. 6.8.6) is reduced in 50\% between the cases, e.g., CIC-40ppc compared to CIC-160ppc. But the change of the macroparticle number has a big impact of the computational effort, since it scales linearly with it. Thus, the run CIC-160ppc is $300 \%$ slower than CIC-40ppc, while the change of shape functions is computationally cheaper: TSC-40ppc is only 32\% slower than CIC-40ppc. 

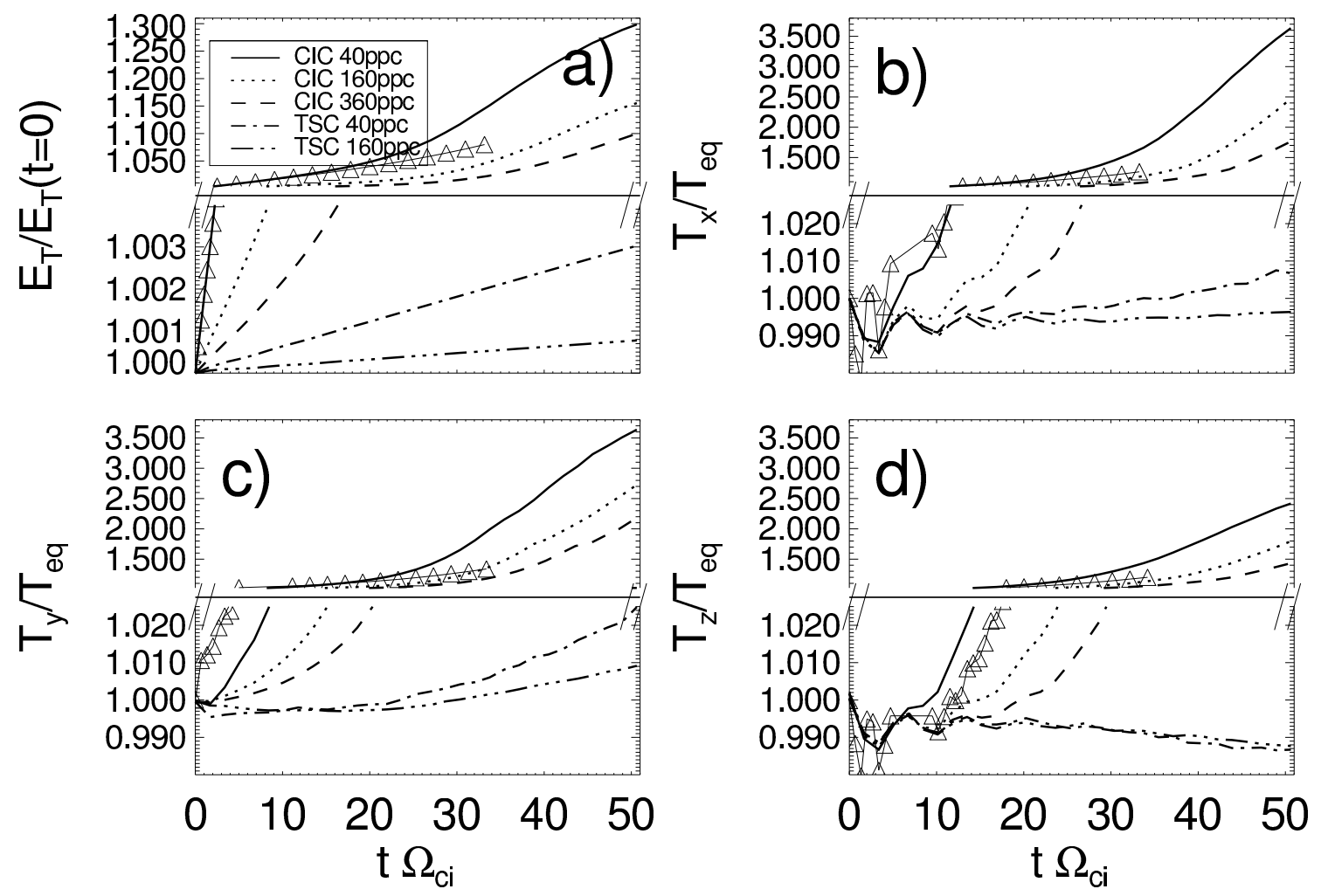

Figure 7.1: Time history of some quantities for the runs with different combinations of shape functions (CIC and TSC) and number of macroparticles per cell (40-360 ppc). It also includes a run with the XOOPIC code (using CIC shape function and 40ppc) for validation purposes (denoted by $\triangle$ ). The plots show: a) total energy (normalized to its initial value), $b$ ) average electron temperature $\left.T_{e, x}, c\right)$ average electron temperature $T_{e, y}$ and $d$ ) average electron temperature $T_{e, z}$. The temperatures are normalized to their equilibrium (initial) values. Note that we break the plots in the vertical direction in two parts, with larger scales in the upper parts. This is in order to visualize more easily the small variations of the quantities obtained using the TSC shape function, compared with the large variations in those obtained using the CIC shape function. Reproduced with permission from P. A. Muñoz, P. Kilian, and J. Büchner, Physics of Plasmas 21, 112106, (2014) Copyright 2014, AIP Publishing LLC.

In order to prove the validity of these results obtained with ACRONYM, we benchmarked it against an independent (and standard) PIC code: XOOPIC (Verboncoeur et al. 1995). Different from ACRONYM, this is an old serial code and only provides linear weighting (CIC). The results for a run with identical parameters to these of CIC-40ppc are also shown in Fig. 7.1. Note that we have not obtained results with the XOOPIC code for times greater than $t \Omega_{c i} \sim 33$, since we were using the free serial version of the code, which is much slower than ACRONYM ( $~ 58$ days (!) to reach that point in time). Although the total energy is similar in the beginning between both ACRONYM and XOOPIC runs, they start to diverge after $t \Omega_{c i} \sim 16$. From that time onwards, XOOPIC has a slightly better performance (increase of total energy $\lesssim 8 \%$ for the last time depicted $t \Omega_{c i} \sim 33$ ) than CIC-40ppc with ACRONYM (increase of total energy $\lesssim 16 \%$ for the last time depicted $t \Omega_{c i} \sim 33$ ). This difference does not affect any of the physical and numerical 
processes to be described in the next sections: they develop almost identically between both codes. Therefore, we can conclude that ACRONYM does not behave numerically different from other standard PIC codes, demonstrating the validity of our results.

It is important to mention the effects of using even higher order shape functions. For this sake, we ran another simulation test (not shown here) with the PQS shape function, but otherwise identical parameter to these of CIC-40ppc. This run was slower by $\sim 71 \%$ compared to CIC-40ppc, but it did not improve significantly the conservation of energy over TSC-40ppc. From this (and other similar evidence) we conclude that is more efficient the change of TSC over CIC than PQS over TSC shape functions, in agreement with another recent study (Cormier-Michel et al. 2008). They found that some non-physical processes related with numeric scattering do not vary significantly between cubic and quadratic interpolations rather than between quadratic and linear ones.

Now, what is the origin of this insufficient energy conservation in the CIC runs? As we can see in the components of the electron temperature averaged over the whole simulation box shown in Fig. 7.1(b)-(c)-(d), the runs that experience this effect also show enhanced numerical electron heating. They follow practically the same trend as the corresponding curves for the total energy Fig. 7.1(a): TSC runs keep this artificial heating more controlled than the CIC ones. We could check that the ion heating is negligible (not shown). From this correlation, we deduce that a higher order shape function allows a better resolution of the electron motion than just a simply increase in the macroparticle number.

By comparing Fig. 7.1(b)-(c)-(d) we can al so note that this numerical electron heating is anisotropic: $T_{e, x}$ and $T_{e, y}$ are heated more than $T_{e, z}$. We will explore the origin of this behaviour in the next subsections.

Thus, the main finding of this section is that the choice of a higher order interpolation scheme, in particular TSC over CIC, allows a better conservation of energy and reduction of numerical heating than just using more macroparticles per cell in PIC simulations of current sheets. It is "better" in the sense of being computationally cheaper. Note that early studies of electrostatic and homogeneous PIC simulations also showed this effect by comparing NGP and CIC schemes (see Hockney 1971). Our finding also agree with a more recent study by Cormier-Michel et al. (2008), who demonstrated the efficiency of TSC over CIC shape function in PIC simulations of laser wakefield accelerators.

\subsubsection{Consequences of the anisotropic numerical heating on the sta- bility of CS}

Here we are going to discuss the physical consequences of the anisotropic numerical heating on the stability of CS. As it is widely know, the natural evolution of a thin CS should lead to a development of the tearing mode: a merging of magnetic islands. This can be reproduced in the TSC-40ppc run, as indicated in the out-of-plane $J_{z}$ displayed in Fig. 7.2(b). On the other hand, we found that instead of that behaviour, CIC-40ppc (as well as the other runs with the same CIC shape function) develops a double peak structure in $J_{z}$, with a reduction of the current along the center of the CS. This phenomenon is called bifurcation. As we mentioned in Sec. 5.1.4.1, bifurcated CS sheets are associated to temperature anisotropies (Sitnov 2003, Zelenvi et al. 2004, Daughton et al. 2004, Matsui and Daughton 2008). One study (Lee and Büchner 2012) also observed a bifur- 
cated CS by using 2D PIC simulations with CIC shape functions, in agreement with our results. Since we measured strong anisotropic numerical heating for all CIC runs (see Fig. 7.1(b)-(c)-(d)), it is possible to infer that both effects are related.

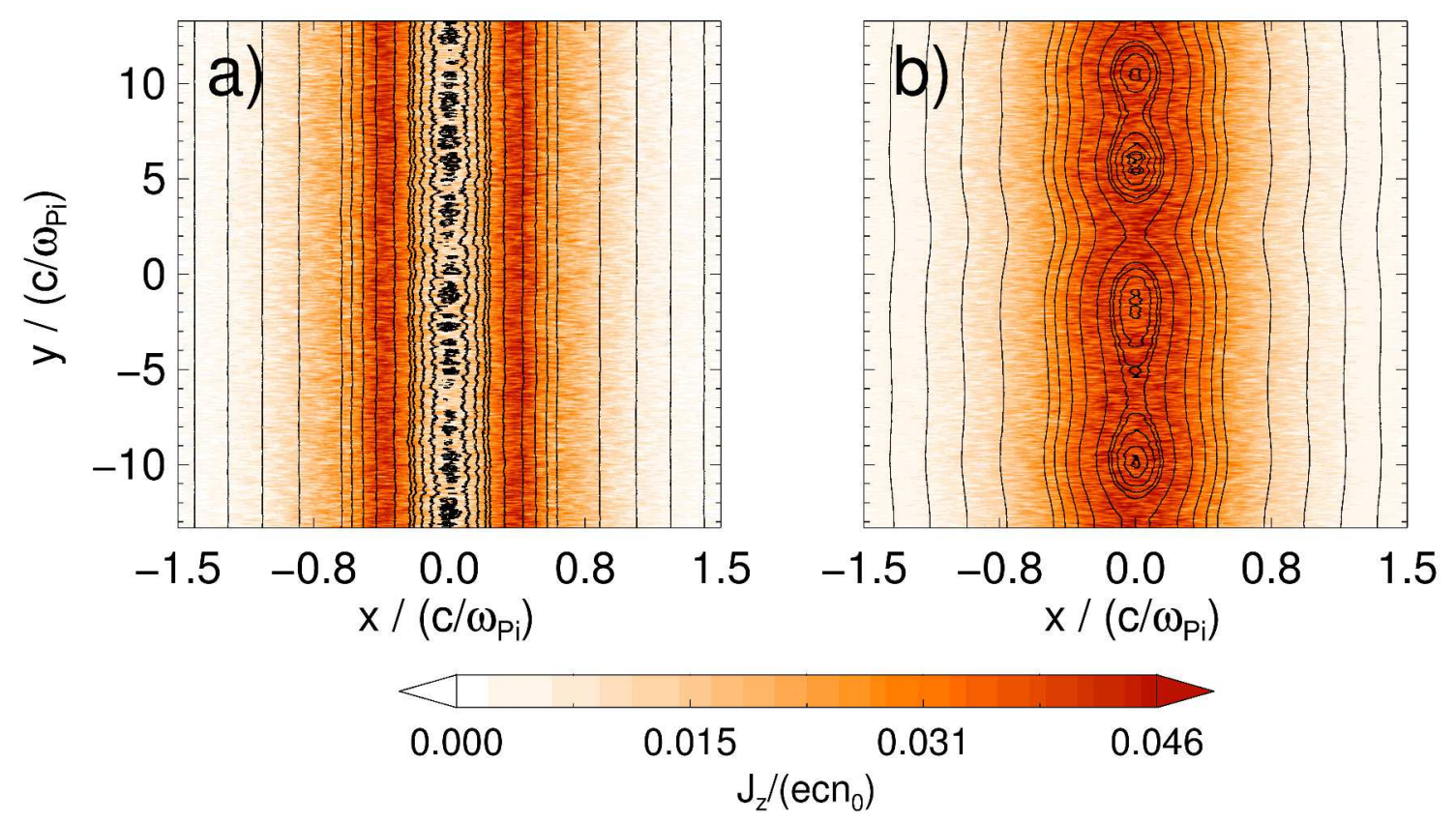

Figure 7.2: Contours of the out-of-plane component $J_{z}$ of the total current density, at the time $t=33 \Omega_{c i}^{-1}$, for two runs a) CIC-40ppc and b) TSC-40ppc. Black lines are the magnetic field lines. The run CIC-40ppc shows a bifurcated structure while TSC-40ppc shows only a single peak structure with large tearing mode islands. Reproduced with permission from P. A. Muñoz, P. Kilian, and J. Büchner, Physics of Plasmas 21, 112106, (2014) Copyright 2014, AIP Publishing LLC.

The growth of tearing mode can be characterized by the time history of the reconnected flux $\psi$, as defined by the vector potential $A_{z}$ according to Eq. (4.1). A special consideration about that definition that considers $\mathrm{X}$ and $\mathrm{O}$ points should be taken in our case of multimode tearing starting from numerical noise. This is because the position of magnetic islands is completely random and varies with time, and so are the $\mathrm{X}$ and $\mathrm{O}$ points. Therefore, and following some previous works, we chose to compute this quantity by taking the difference between the maximum and minimum value of $A_{z}$ along the line $x=0$. The evolution of a CS leads to a merging and reduction of the number of magnetic islands, and thus this definition is a representative quantity for later times. It does not represent accurately the evolution of tearing mode at the beginning, due to the large number of magnetic islands arising from noise, but that stage is not important for our purposes since is dominated by numerics. The results of this calculation are displayed in Fig. 7.3 . 

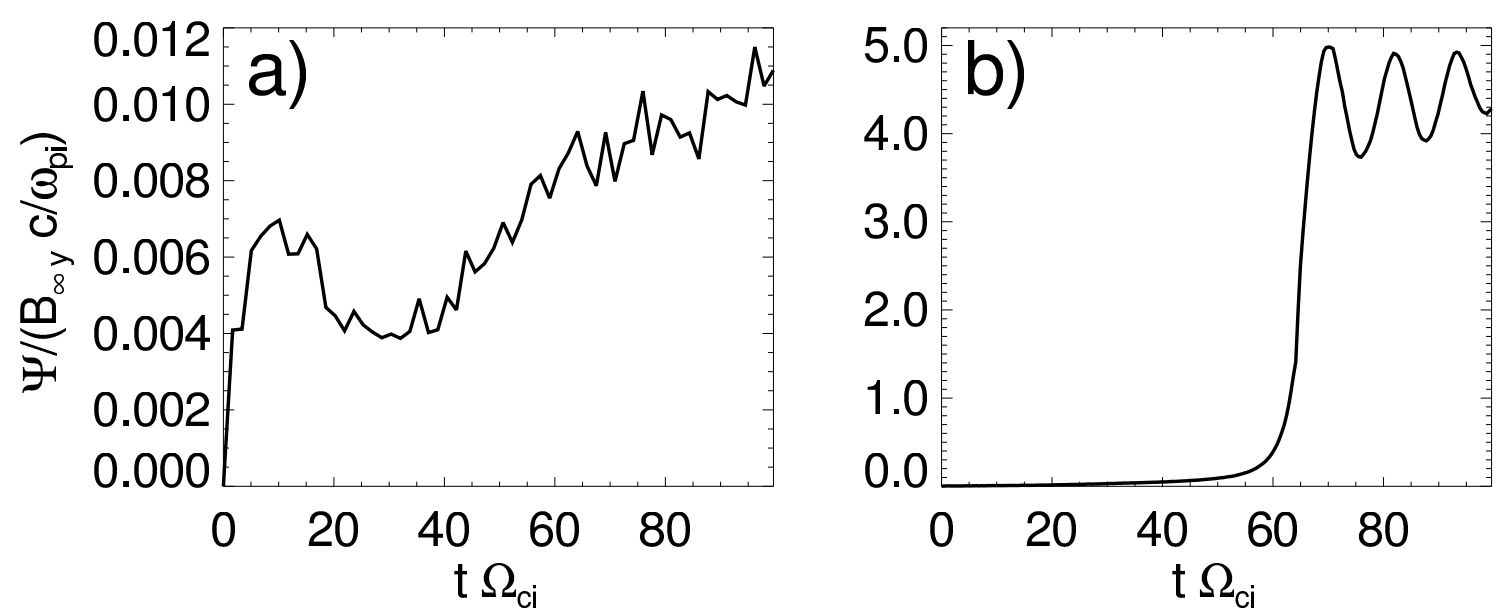

Figure 7.3: Comparison of reconnected flux $\Psi$ for the runs a) CIC-40ppc and b) TSC-4Oppc. The left panel a) (with CIC shape function) correspond to the case with anisotropic heating and CS bifurcation, while the right panel b) (with TSC shape function) correspond to a tearing instability without significant anisotropic heating nor bifurcation. Reproduced with permission from P. A. Muñoz, P. Kilian, and J. Büchner, Physics of Plasmas 21, 112106, (2014) Copyright 2014, AIP Publishing LLC.

In Fig. 7.3(b), we can see the monotonous growing of $\psi$ due to tearing mode until the explosive phase of magnetic reconnection in $t \sim 60 \Omega_{c i}^{-1}$, when there is only one remaining magnetic island. The maximum values of $\Psi$ in this stage are typical from this kind of studies of magnetic reconnection (see, e.g., Pritchett 2005a). After that time, the entire structure of the current sheet is disrupted due to outflows from the single $\mathrm{X}$ point in opposite directions, colliding due to the periodic boundary conditions. This boundary effect 1 can be avoided by choosing larger simulation boxes (Karimabadi et al. 1999) or open boundary conditions (see, e.g., Daughton et al. 2006, Ohtani and Horiuchi 2009). On the other hand, Fig. 7.3 (a) demonstrate a complete different behaviour: no growing of reconnected flux for the run CIC-40ppc, and therefore no tearing mode.

Now, let us analyze how the bifurcated CS develops for the CIC run. In Fig. 7.4 we show the integrated profile of $J_{z}$ across the inhomogeneous $x$ direction. This allows to detect that the depletion of current starts around $t \sim 15 \Omega_{c i}^{-1}$. It is driven by a reduction of $J_{e, z}$. The ion contribution $J_{i, z}$ to the total current $\left(J_{z}=J_{i, z}+J_{e, z}\right)$ is negligible. This is turn involves a reduction in the out-of-plane electron bulk velocity $V_{e, z}$, while the electron number density is practically unchanged $\left(J_{e, z}=-e n_{0 e} V_{e, z}\right)$. This agrees with the finding of a previous work (Lee and Büchner 2012).

\footnotetext{
${ }^{1}$ Although it mostly numerical in our case, its physical consequences shares some features to dipolarization fronts. See Sitnov and Swisdak (2011) for further details.
} 


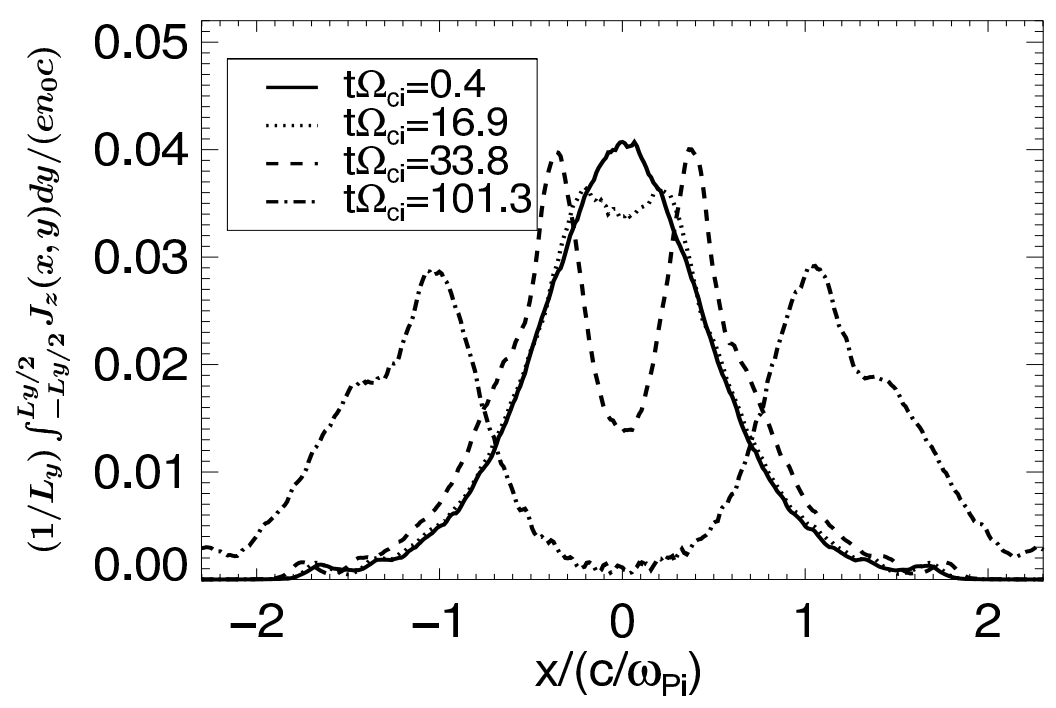

Figure 7.4: Evolution of the total current density profile $J_{z}$ showing bifurcation $(C I C$ 40ppc run). The profiles are obtained by integrating the current density along the CS: $J_{z}(x)=\left(1 / L_{y}\right) \int_{-L y / 2}^{L y / 2} J_{z}(x, y) d y$. Reproduced with permission from P. A. Muñoz, P. Kilian, and J. Büchner, Physics of Plasmas 21, 112106, (2014), Copyright 2014, AIP Publishing $L L C$.

This reduction of $V_{e, z}$ for CIC-40ppc is strongly related with the temperature anisotropy. Indeed, it can be shown from Eq. 2.24) (see Schindler and Hesse 2008, or discussion following Eq. (4.22)) that both quantities are related by the following expression

$$
\frac{P_{x x, e}-P_{z z, e}}{P_{x x, e}} \approx \frac{1}{B_{y}} \frac{d V_{e, z}}{d x} \propto \frac{T_{e, x}-T_{e, z}}{T_{e, x}} .
$$

This is valid neglecting contribution due to heat flux, assuming a gyrotropic tensor pressure (a justified assumption due to the dominant collisions in our system) and under the presence of an out-of-plane magnetic field (not shown here, but developed in these bifurcated cases in a way analogous to the be shown in Sec.7.3.2). Since there is no significant temperature anisotropy developed for TSC-40ppc run, it follows that it will not develop any reduction in $V_{e, z}$ and thus no bifurcation, in agreement with our results. Instead, it just displays the physically expected merging of magnetic tearing islands and reconnection at the end.

Now, let us describe the theoretical predictions relating temperature anisotropy and stability of the CS. For that, we need to define this quantity in our geometry. It will be given by $A_{e}=T_{e, \|} / T_{e, \perp}$, with $T_{e, \|}=T_{e, y}$ and $T_{e, \perp}=\left(T_{e, x}+T_{e, z}\right) / 2$ the temperatures in the directions parallel and perpendicular to the asymptotic Harris magnetic field (in $y$ direction). This choice is because the Harris equilibrium (Eq. (3.19)) involves only the perpendicular component of the temperature, $T_{e, \perp}$, while $T_{e, y}$ is not involved in this initial CS equilibrium. Thus, the time evolution of $A_{e}$ for the five first runs described in Table 7.2 (isotropic, with initially $A_{e}=1$ ) is displayed in Fig. 7.5. First, let us note that all the runs, in particular the ones with TSC shape function, show a monotonous steady increase in the anisotropy. This preferential electron heating in the parallel $(y)$ direction is known from the linear theory of tearing mode. This develops a parallel electric field (Chen et al. 1984), 
and therefore electrons can be more easily heated in that direction (Karimabadi et al. 2004, Vainshtein and Mazur 1982). On the other hand, the runs with CIC shape function al so develops parallel electron heating (and much more than the tearing-driven heating in the TSC runs), but it cannot be due to the tearing mode as we already demonstrated: it is only numerical.

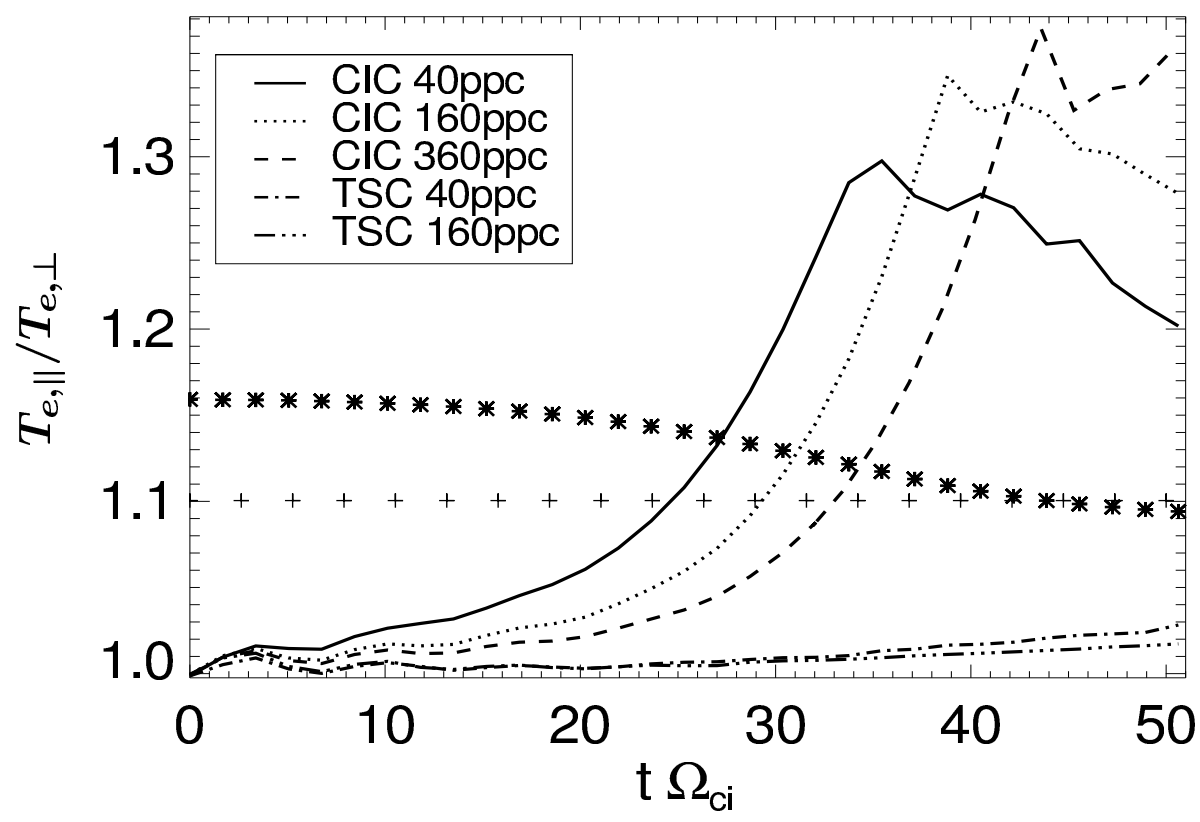

Figure 7.5: Time history of electron temperatures anisotropies $A_{e}=T_{e, \|} / T_{e, \perp}$ for the five (initially isotropic) runs with different shape functions and number of macroparticles per cell. The theoretical thresholds for tearing mode stabilization are depicted by *for Eq. (7.3) and + assuming $T_{i, \perp}=T_{e, \perp}$. The curve $*$ is calculated with the instantaneous value of $T_{i, \perp} / T_{e, \perp}$ for the run CIC-40ppc. Reproduced with permission from $P$. A. Muñoz, P. Kilian, and J. Büchner, Physics of Plasmas 21, 112106, (2014), Copyright 2014, AIP Publishing LLC.

As we mentioned in Sec.5.1.4, Chen et al. (1984) found that the tearing mode can be stabilized if the electron temperature anisotropy is higher than the threshold Eq. (5.48) (or the simplified version Eq. (5.41) found before by Laval and Pellat (1967) and Forslund (1968)). For our parameters, it reads:

$$
A_{e}=\frac{T_{e, \|}}{T_{e, \perp}}>1.159\left[2\left(1+\frac{T_{i, \perp}}{T_{e, \perp}}\right)^{-1}\right],
$$

The factor in square brackets is 1 for $T_{i, \perp}=T_{e, \perp}$, but deviates considerably from it during the course of the CS evolution for the CIC runs due to the numerical electron heating. For that reason we also plotted the full threshold in 7.5 using the maximum variation of the factor in square brackets, corresponding to the run CIC-40ppc. Thus, all the other possible cases will fall in between these curves.

Fig. 7.5 demonstrate that all the simulations that reached electron temperature anisotropies higher than the previous threshold (all the CIC runs), developed bifurcation, suppressing the tearing mode. As discussed in 5.1.4 the stabilization of tearing mode is because 
(Karimabadi et al. 2004, Karimabadi 2005) the appearance of another instability driven by temperature anisotropy: the Weibel instability. This instability propagates perpendicular to the "warmer" temperature (its source of free energy), $T_{e, x}$ in our case (since not wavevectors are allowed in the $z$ direction). In the other direction, $T_{e, y}$ for our geometry, the Weibel mode is damped. But this turns out to be the same propagation direction as the tearing mode $\left(k_{y}\right)$. Since both instabilities are driven by Landau resonance, they couple resulting in a damping or suppression of tearing mode.

The effects of the temperature anisotropy are especially important for our simulation parameters. As pointed out by Chen et al. (1984), these are enhanced for small Larmor radius (according to Table 7.1, $\rho_{e} / \lambda_{D e}=2.86$ ) and more realistic (large) mass ratios. The latter can be understood since the electron temperature anisotropy build ups on electron time scales $\left(\omega_{p e}^{-1}, \Omega_{c e}^{-1}\right)$, and therefore it will be more "accumulated" on ion time scales $\Omega_{c i}^{-1}$ if the separation of these time scales, proportional to the ratio $m_{i} / m_{e}$, is large enough. Moreover, it is known (see discussion in Sec.5.2.1), that the threshold of Weibel is mostly dominated by the electron temperature anisotropy, the ion contributions are usually negligible. An ion temperature anisotropy can only drive Weibel if its temperature anisotropy is very large and, at the same time, the electron temperature anisotropy is negligible small (Baumiohann et al. 2010). Therefore, since this scenario does not take place in any of our simulations, all the effects related with ion temperature anisotropy can be completely neglected in this work.

\subsubsection{Numerical CS bifurcation and entropy}

In the previous section we analyzed the effects of the heating developed by the CIC runs on the stability of the $\mathrm{CS}$, showing that it may lead to a non-physical evolution of the system if it is not taken properly into account. But that is not a proof that the heating arise only due to numeric considerations. In order to have an additional argument to distinguish that the numerical heating developed by the CIC shape functions is different from the real physical heating, one can look at the entropy of the system. As discussed in Sec. 6.8.4, the entropy in a collisionless Vlasov plasma should be kept strictly to zero. But the discretization of the phase space due to the introduction of a grid (via the shape function) and finite time stepping in PIC codes can generate entropy (Buneman 1964). This entropy production due to coarse graining of phase space can be interpreted as dissipation, being thus manifested through a diffusion coefficient $D_{i j}$ appearing in the effective collision operator in the right hand side of the Boltzmann equation Eq. (6.44). This coefficient $D_{i j}$ depends explicitly on numerical factors such as the shape function (see Sec. 6.8.3 or Birdsall and Langdon (1991, Sec. 12.6) for further details). As a result, the conservation of density in the phase space (Liouville's theorem) is violated, leading to a diffusion of the distribution function, manifested macroscopically as an effective (numerical or non-physical) heating (the second numerical heating mechanism mentioned in Sec. 6.8.2). Note that both numerical heating and entropy, under some assumptions, are related via Eq. (6.47).

Therefore, an increase of entropy in momentum-conserving PIC codes indicates strong numerical heating, dissipation or collisions (since all these quantities are directly related). This can be shown in the time evolution of this quantity for our usual five runs in Fig. 7.6. We use the information entropy $S$ by Shannon (1948) defined by Eq. (6.46) (equal, ex- 
cept by a constant factor, to the thermodynamic Gibbs entropy). Since $S$ is a continuous variable, we approximate it by means of the histogram estimator (Moddemeijer 1989) defined by Eq. (B.87), discretizing the phase space in bins $\Delta v^{3}$. The specific value changes with the choice of $\Delta v$ and it is defined up to a constant offset depending on the relative units of the velocity (see further details in Sec.6.8.4). For this reason, we carried out a convergence study (not shown here) to be sure that $S$ is more or less invariant through a wide range of choices of $\Delta v$ around an optimum value $\Delta v=c / 40$
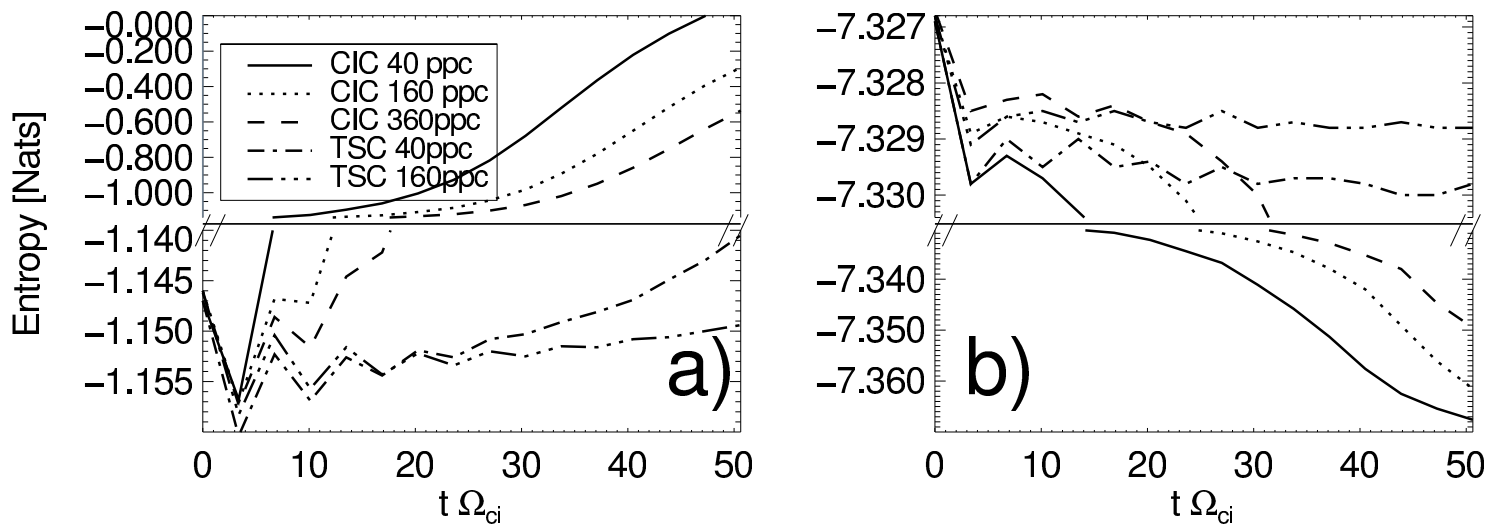

Figure 7.6: Time histories of electron $(a)$ and ion $(b)$ entropies $S(t)$ calculated according to Eq. (B.87) for the five isotropic runs. This entropy can quantify the numerical collisions. The electron entropy curves follow the same trend as the curves for the total energy and electron temperatures shown in Fig. 7.1. And for the same reasons explained in that Fig. 7.1, we break the y axis in two halves with different scales. Reproduced with permission from P. A. Muñoz, P. Kilian, and J. Büchner, Physics of Plasmas 21, 112106, (2014) Copyright 2014, AIP Publishing LLC.

In Fig. 7.6, we see that the respective curves for the electron entropy in the CIC runs show the same trend as the corresponding to the energies shown in Fig. 7.1. (a): TSC schemes preserve it better than CIC. The increase of macroparticle have the same effect, but to a much less extent. This is to be expected according to our previous discussion and Eq. 6.47). Then, this fact is a strong indication of the numerical heating dominating the CIC runs. On the other hand, the ion entropy does not vary significantly (a range of variation two order of magnitude smaller) for different shape functions and number of macroparticles, since the ion heating time scales are much larger than those of the electrons. Thus, the ion contribution to the total entropy is negligible.

Our results for CIC-40ppc case agree with a previous work by Lee and Büchner (2012). By using the XOOPIC code, that only provides the CIC shape function, they observed bifurcation of the CS. It was associated with the physical process of electrons experiencing chaotic scattering when they cross center of the CS (Büchner and Zelenyi 1989, Zelenyi et al. 2003). They also observed a growing entropy (more precisely, the relative entropy, also called Kullback-Leibler divergence according to Kullback and Leibler (1951)) since the beginning, similar to our result, associating it with a state thermodynamically more probable (less free energy). As we argued, this can also be produced only due to numerical heating. 


\subsubsection{Collisions and location of bifurcation}

According to the discussions in Sec.6.8.3 based in the original work by Matsuda and Okuda (1975), the numerical collisions in electromagnetic PIC codes weaken in presence of a magnetic field. They are stronger in unmagnetized regions, like the center of a Harris CS (even though later it becomes weakly magnetized, but it still much less than in the edges of the CS, for example). This explains that the physical consequence of these collisions seen in our CIC runs, bifurcation, starts at the center CS $x=0$ and not in other locations (like at the edges of the CS, even though these locations have less macroparticles per cell).

\subsubsection{Collisions in 2D geometry}

Previous works (Okuda 1970) have shown that the numerical diffusion coefficient $D_{i j}$ behaves very differently in 2D3V plasma models in comparison with full 3D cases. Since $D_{i j}$ measures the differences between the collisionless Vlasov and the (collisional) Boltzmann equation, it follows that the nature of numerical collisions will be different in our reduced 2D geometry. Moreover, Matsuda and Okuda (1975) al so noticed that the numerical collisions are highly anisotropic in 2D plasmas with an externally applied magnetic field. As mentioned in Sec.6.8.3, this can be understood because the coefficients characterizing numerical collisions depend explicitly on the direction of the magnetic field relative to the electric field fluctuations. But a 2D3V PIC code constrains the motion of the macroparticles to the plane $x-y$, while solving the three components of the velocity. Then, the neglected out-of-plane direction $z$ will not be affected by collisions diffusing particles in the $v_{z}$ phase space coordinate.

However, the argument and results shown in Matsuda and Okuda (1975) were carried out for a plasma in a strong magnetic field. Therefore, it might not be useful to apply it directly to our case, especially near the center of the CS. For this reason we tested this theoretical prediction by means of two simulations tests with CIC shape functions to enhance the numerical collisions. Both use CIC shape function to enhance numerical collisions, but otherwise the physical parameters were identical to the run CIC-40ppc, but without a current sheet initialization. One of them is purely $2 \mathrm{D}$ with a simulation box $[128 \Delta x \times 128 \Delta x]$, while the other one is full 3D with size [128 $\Delta x \times 128 \Delta x \times 128 \Delta x]$. The results show than the $3 \mathrm{D}$ run developed much smaller numerical heating electron temperature anisotropy than the $2 \mathrm{D}$ case. Thus, we confirmed that for our parameters, the $2 \mathrm{D}$ runs overestimate the importance of numerical collisions compared to 3D cases, in addition to make them anisotropic and so the electron temperature. Therefore, we expect than in realistic runs of $3 \mathrm{D}$ Harris CS, these numerical effects will be much less important.

\subsubsection{Numerical heating and background plasma effects}

All the results shown in the previous sections were based in simulations of Harris CS without a background plasma. This implies that there are very few macroparticles at the edges of the CS, potentially enhancing the numerical collisions or scattering in these regions, due to the stronger electromagnetic fluctuations. So, the natural question that we will address in this subsection is: how will the inclusion of a background plasma, increasing significantly the number of macroparticles in these regions, affect all the previous conclusions? 
For this sake, we use the results of six additional runs, with parameters given in the second part of Table 7.2 (identified with the suffix "back"). In a first place we compare the conservation of energy and evolution of the temperature anisotropy for cases using CIC shape function and different background populations: $10 \%$ and $20 \%$ of the peak central density of the Harris sheet, respectively. Results are depicted in Fig. 7.7. The time evolution of the numerically generated temperature anisotropy is practically identical to the case CIC-40ppc, confirming that the numerical collisions and associated scattering are not affected too much by the inclusion of the additional population. We also observed a worse energy conservation for increasing background plasma densities, but this is not related with the numerical collisions, but to other additional factors (not investigated or shown here).
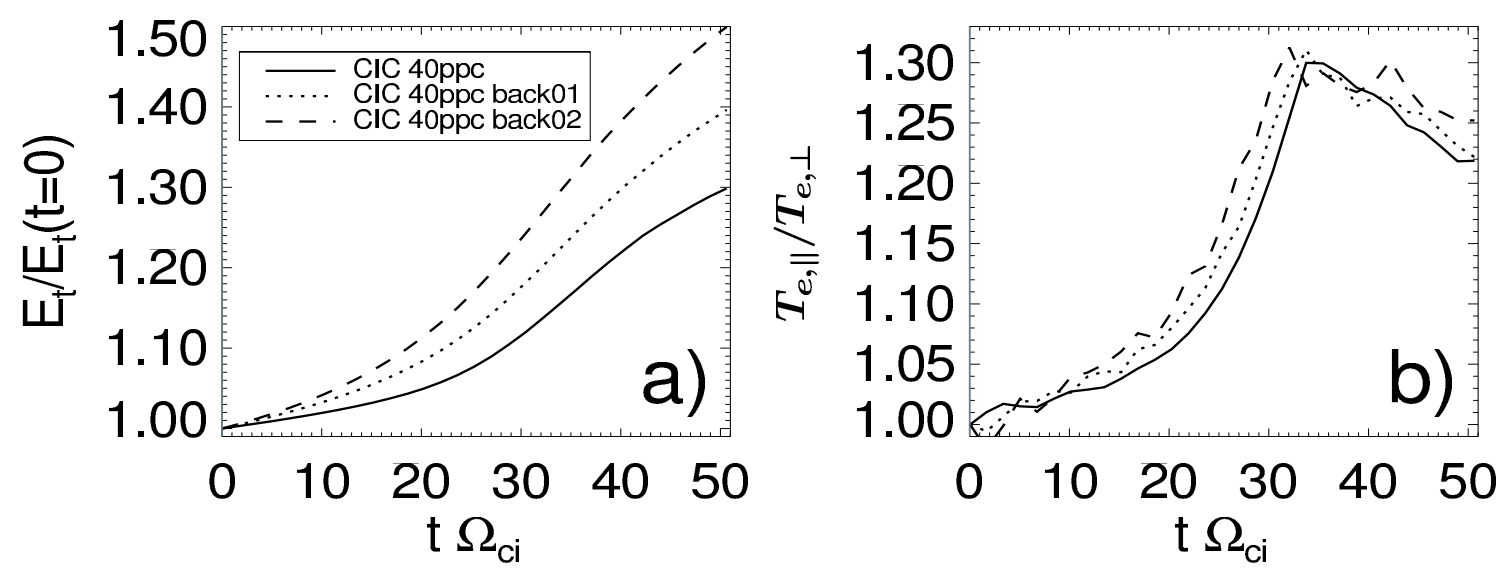

Figure 7.7: Time history of a) total energy (normalized to its initial value) and b) electron temperature anisotropy $A_{e}=T_{e, \|} / T_{e, \perp}$ for the cases of a CS without background (CIC40ppc), background population at $10 \%$ of the peak density (CIC-40ppc-back01) and at 20\% of the peak density (CIC-40ppc-back02). In all those runs the CIC scheme has been used with 40ppc. Reproduced with permission from P. A. Muñoz, P. Kilian, and J. Büchner, Physics of Plasmas 21, 112106, (2014) Copyright 2014, AIP Publishing LLC.

Next, we will prove that the previous conclusion still holds when varying the total number of macroparticles. For this sake, in Fig. 7.8 we display the time evolution of total energy and electron temperature anisotropy for runs with exactly the same parameters as the shown in Fig. 7.1, but with the inclusion of a background population of $10 \%$ of the peak central density of the Harris sheet. By comparing the total energy evolution in Figs. 7.1 (a) with Fig. 7.8 (a), we checked that it is very weakly affected by the inclusion of the additional population, for all cases with different macroparticle number and shape function. The same is valid for the evolution of temperature anisotropy for the CIC runs in Fig. 7.8(b) and Fig. 7.5. Thus, we confirmed that a background plasma does not change significantly the numerical heating and their associated effects, such as bifurcation or tearing stabilization (we checked that all these processes develop similarly). This can be understood since bifurcation takes place at the center of the CS, where numerical collisions are enhanced. A background population does not change significantly the density here, only at the edge of the CS, a region not directly involved in these numerical processes. A background population may become critical for a correct description of other 
physical processes, such as any estimation that requires the calculation of momenta of distribution function away from the center of the CS.
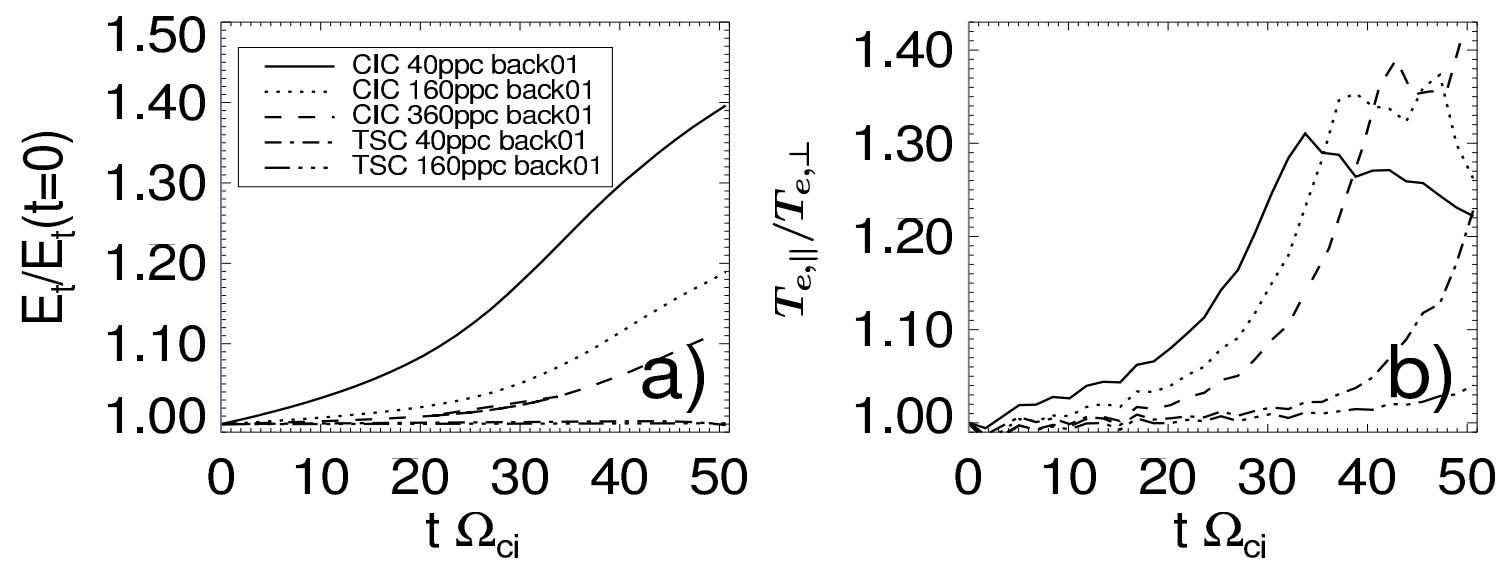

Figure 7.8: Time history of a) total energy and b) electron temperature anisotropy $A_{e}=T_{e, \|} / T_{e, \perp}$ for our standard runs described in Sec. 7.2.1, but with the addition of a background population of $10 \%$ of the peak density. Compare with the Figs. 7.1 (a) and 7.5 Reproduced with permission from P. A. Muñoz, P. Kilian, and J. Büchner, Physics of Plasmas 21, 112106, (2014) Copyright 2014, AIP Publishing LLC.

One additional observation in Fig. 7.8(b) deserves a further explanation. The TSC runs show a sudden increase in the temperature anisotropy for later times when comparing to the case without background in Fig. 7.5. This is a signature of the explosive phase of magnetic reconnection taking place (earlier than in the case without background), where significant temperature anisotropies are developed naturally in the reconnection exhaust. As reported by Karimabadi et al. (2005) via PIC simulation studies and Schmitz and Grauer (2008) via Vlasov codes, the onset of magnetic reconnection is sensitive to the initial conditions, although it does not have an overall strong dependence on the presence of a background population, and therefore some small deviations are expected (as measured by the reconnected flux). They also notices that the saturation of tearing instability have a weak dependence on the background population. On the other hand, it is known that reconnection rates are substantially reduced with increasing background plasma density (Pritchett 2001, Karimabadi et al. 2005, Schmitz and Grauen 2008). This is equivalent to an extended explosive phase of magnetic reconnection with background than without it (results not shown here). We can understand that because the quantity that determines the relative "speed" of magnetic reconnection is the Alfvén speed $V_{A}$ in the outflow region (see Sec. 4.1), with a dependence on the density $\propto 1 / \sqrt{n}$. Therefore, regions with low density in cases of CS without background will have larger $V_{A}$ (in fact, asymptotically infinite) and so the reconnection rates. As a result, the reconnection stage will be shorter than in cases with a background (with smaller values of $V_{A}$ ). This reconnection regime is sometimes called "vacuum reconnection" (Pritchett 2001), characterized by the absence of whistler waves due to the infinite Alfvén speed (see discussion in Sec. 4.2.2). 


\subsubsection{Restriction to an antiparallel magnetic field configuration}

All the results shown in the previous sections were based in simulations of Harris CS without a guide magnetic field (in the out-of-plane direction). Let us discuss a little bit about the possible effects when a finite guide field is imposed in our configuration. If it is strong enough, electron can become magnetized (gyrotropic) at the center of the current sheet, making their temperature highly isotropic on the reconnection plane $\mathrm{x}-\mathrm{y}$. This would imply that the stabilization and saturation of tearing mode will be quite insensitive to the practically negligible electron temperature anisotropy (i.e.: the tearing mode will not developed any natural significant degree of anisotropy). The saturation mechanism will have to do with other kind of processes, such as electron trapping in magnetic islands (see Karimabadi 2005, Karimabadi et al. 2005, or discussion in Sec. 5.1.3.4).

Therefore, all the processes related with numerically developed temperature anisotropy discussed in this section are critical mostly for antiparallel configuration of magnetic reconnection. In presence of a guide field, they becomes much less important. Instead, other numerical parameters play the key role for a correct modeling, such as the electron Larmor radius on the guide field. We are going to analyze the guide field effects extensively in the Chapter 8 .

\subsection{Initially imposed temperature anisotropies}

In the previous section we showed the correlation between the (numerically developed) anisotropic heating and bifurcation. The purpose of this section is to prove a causal relation between both mechanisms, by initializing a CS with a temperature anisotropy. As we will see, in these cases the CS will develop a very complex and interesting dynamics.

\subsubsection{Initial temperature anisotropy relaxation}

The results to be shown are based in seven additional simulations runs with an initial electron temperature anisotropy (called "initially anisotropic" from now on), with parameters defined in the third part of Table 7.2. All of them use TSC shape function, in order to keep the temperature anisotropy triggered by numerical heating controlled: we want to study only the consequences of the physically generated electron temperature ani sotropy $A_{e}$ (defined in Sec. 7.2.2). The temperature anisotropy is imposed by changing only $T_{e, y}=T_{e, \|}$, while keeping $T_{e, \perp}=T_{e, x}=T_{e, z}$ equal to the value necessary to keep the initial Harris equilibrium in Eq. (3.19). We chose more runs with $A_{e}>1$ rather than $A_{e}<1$, representing thus the naturally developed anisotropies during the course of the tearing mode growth evolution of the CS (see Fig.7.5). Because initially we have $T_{i}=T_{e}$, we also have an ion temperature anisotropy $A_{i}=T_{i, \|} / T_{i, \perp}$. The rest of parameters are identical to the run TSC-40ppc, with the exception of the macroparticle number in some of the runs.

The time evolution of the electron temperature related quantities $T_{e, x}, T_{e, y}$ and $A_{e}$ for the first 5 anisotropic runs with 40ppc: TSC-40ppc-A0.64, TSC-40ppc-A1.21, TSC40ppc-A1.44, TSC-40ppc-A1.69 and TSC-40ppc-A1.96 (see details in Table 7.2) is shown in Fig. 7.9. By comparing with the isotropic case TSC-40ppc also displayed in the same figure, the CS relaxes towards an isotropic state during a short transient time: $A_{e}$ goes 
down to 1 (Fig. 7.9 (c)), consequence of the decrease of the initially imposed parallel $T_{e, y}$, while at the same time the perpendicular temperature $T_{e, x}$ (similarly $T_{e, z}$, not shown here) increases above its initial equilibrium value to compensate the previous effect. Both parallel and perpendicular components of the electron temperature approaches to an asymptotic value, proportional to the initially imposed parallel $T_{e, y}$.
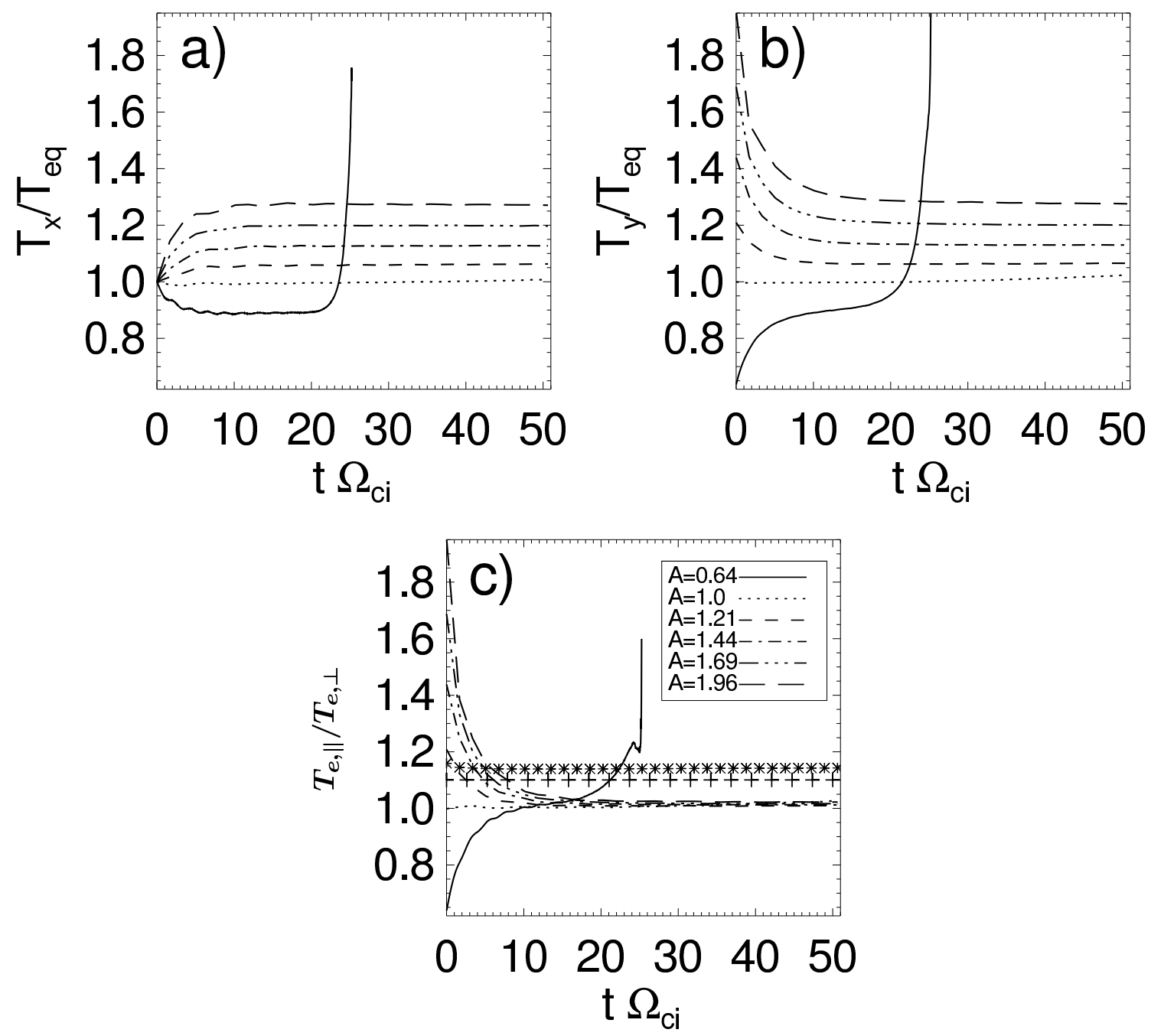

Figure 7.9: Time history of quantities characterizing the five runs with initially imposed anisotropy $A_{e}$ ("anisotropic runs"), compared to the "isotropic run" TSC-40ppc. a) average electron temperatures $T_{e, x}, b$ ) average electron temperatures $T_{e, y}=T_{e, \|}$ and c) average electron temperature anisotropy $T_{e, \|} / T_{e, \perp}$ for different initially imposed temperature anisotropies $A_{e} \in[0.6,2.0]$. The theoretical thresholds for stabilization of tearing mode are shown with lines $+E q$. (5.41) and $* E q$. (5.48). The latter one is calculated with the instantaneous value of $T_{i, \perp} / T_{e, \perp}$ for the run TSC-40ppc-A1.96. Reproduced with permission from P. A. Muñoz, P. Kilian, and J. Büchner, Physics of Plasmas 21, 112106, (2014) Copyright 2014, AIP Publishing LLC. 


\subsubsection{Enhancement of tearing mode with the opposite anisotropy}

Although not relevant for the purpose of this chapter, let us explain the seemingly strange behaviour of the run TSC-40ppc-A0.64 in Fig. 7.9. This is the only one with an opposite electron temperature anisotropy in comparison with all the rest of the simulations considered so far. We observed a faster development of tearing mode than in the isotropic case TSC-40ppc. The explosive phase of reconnection takes place already at $t \Omega_{c i} \sim 20$, explaining the sudden increase (beyond the scale) in the curves for the temperatures and anisotropy. This is consequence of the enhancement of tearing mode for anisotropies $A_{e}<1$, a well known fact since Chen et al. (1984).

\subsubsection{Influence of the number of macroparticles per cell on the anisotropy relax- ation}

Now, how can we be sure that the relaxation of the imposed anisotropy is not a numerical artifact? (like bifurcation discussed in the previous section). All the anisotropic runs are still Harris equilibria, with an anisotropy that should be preserved in an ideal collisionless Vlasov system without additional instabilities. However, as we will see, these states turn out to be unstable equilibria, prone to temperature driven microinstabilities. But first, we need to confirm that numerical collisions, tending to isotropize distribution functions in the $x-y$ plane, are not affecting our conclusions. For this sake, in Fig. 7.10 we show a convergence study on the macroparticle number of the time evolution of $A_{e}$ for anisotropic runs with initial $A_{e}=1.96$ : TSC-40ppc-A1.96, TSC-360ppc-A1.96 and TSC-1000ppc-A1.96 (see details in Table 7.2).

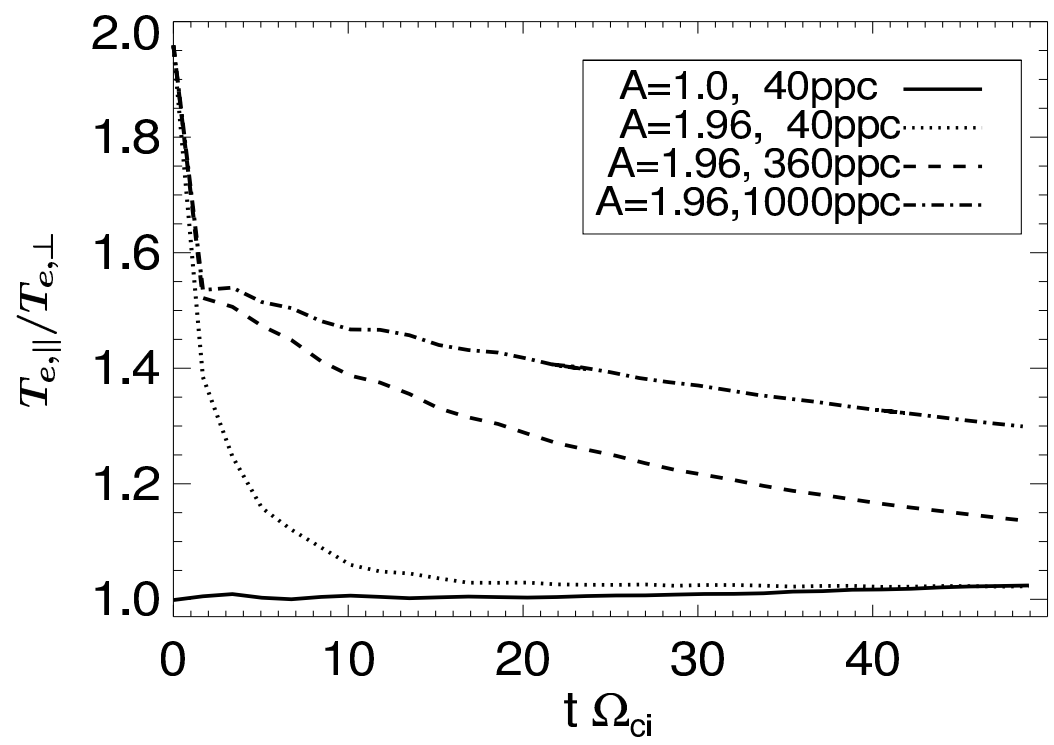

Figure 7.10: Time history of the average electron temperature anisotropy $A_{e}=T_{e, \|} / T_{e, \perp}$ for cases with an initially imposed temperature anisotropy $A_{e}=1.96$, TSC shape function and different number of macroparticles per cell (40, 360 and 1000). For comparison, the result for an isotropic initial distribution $\left(A_{e}=1.0\right)$ is also plotted with a solid line. Reproduced with permission from P. A. Muñoz, P. Kilian, and J. Büchner, Physics of Plasmas 21, 112106, (2014) Copyright 2014, AIP Publishing LLC. 
For later times, there is indeed a reduction in the decaying rate of $A_{e}$ for a higher macroparticle number, consequence of the reduction in the numerical noise (scaling as $1 / \sqrt{N}$ ) and so the (numerical) collisions and isotropization (see Fig. 7.10). This numerical isotropization, with the same dependence on $N$ as the numerical noise (Matsui 2008), is due to pitch angle scattering (see discussion at the end of Sec.6.8.3): a redistribution of the particles velocities in the momentum space, without changing significantly their total speed ( $\sim$ to the total kinetic energy) due to the good energy conservation properties of the TSC scheme. Waves are generated from the surrounding numerical electromagnetic fluctuations due to the coarse graining effects (similar to a physical turbulence), transferring momentum via wave-particle interaction from the perpendicular to the parallel direction. Since pitch angle scattering is stronger in regions with weak magnetic field, we expect stronger effects at the center of the CS, and not at their edges. Moreover, it has serious consequences for the development of the tearing mode, since it behaves analogously to numerical collisions. Indeed, it has been shown by Coroniti (1980) that (physical) pitch angle scattering can relax the anisotropy induced via tearing mode by diffusing the electrons along the magnetic field lines, and therefore cancelling the mechanism of tearing mode growth rate if it is strong enough.

\subsubsection{Temperature anisotropy relaxation via Weibel instability}

Although the long term behavior of $A_{e}$ does depend on the macroparticle number, the initial transient period showing a sudden drop does not. Indeed, Fig. 7.10 demonstrates that even the run TSC-1000ppc (with 16 times less numerical noise and pitch angle scattering as $40 \mathrm{ppc}$ ) relaxes to practically the same anisotropy level as the cases with much less macroparticles per cell. This is a strong signature of its physical origin. In order to understand the physical mechanism, let us focus in this initial short term period for one case, TSC-360ppc-A1.96. The profiles integrated along the CS $(y)$ direction of the in-plane temperature anisotropy and $B_{z}$, close to center, are shown in Fig. 7.11. 

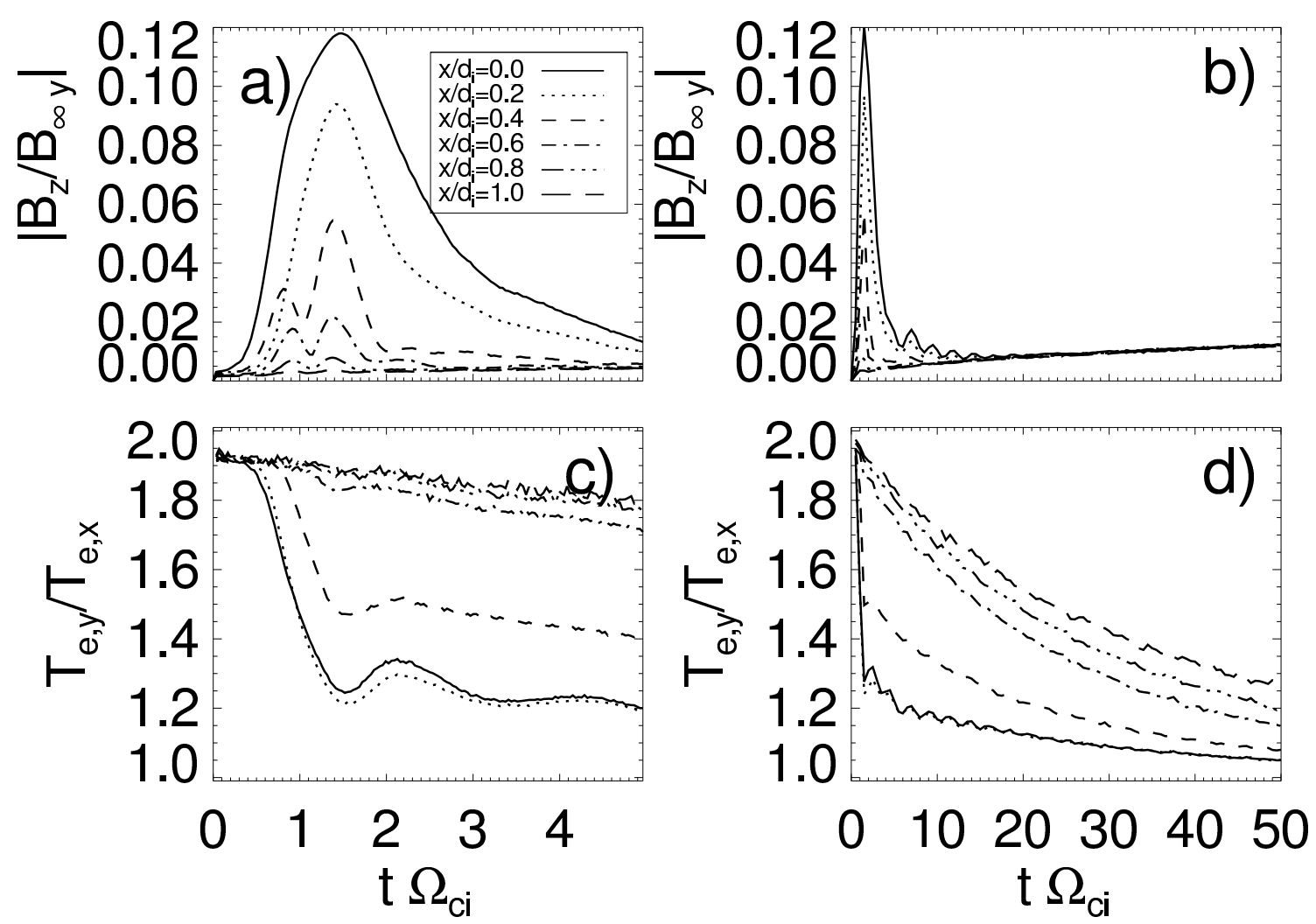

Figure 7.11: Time history of the out of plane magnetic field and temperature anisotropy averaged along $y$, at different distances $x$ from the center of the CS, for the run TSC360ppc-A1.96. a) and b) Magnetic field $\left|B_{z}\right|$. c) and d) Electron temperature anisotropy $T_{e, y} / T_{e, x}$. The left plots a) and c) are for the early stages of the system while the right plots $b)$ and d) are for the long term evolution. Reproduced with permission from P. A. Muñoz, P. Kilian, and J. Büchner, Physics of Plasmas 21, 112106, (2014) Copyright 2014, AIP Publishing LLC.

Three stages can be distinguished (1) the initial between $t \Omega_{c i}=0-0.5$ with constant anisotropy, (2) between $0.5<t \Omega_{c i}<1.5$ with a sudden anisotropy drop until a saturation value $A_{e} \sim 1.2$ and (3) $t \Omega_{c i}>1.5$, with a slow monotonously decreasing anisotropy relaxation, produced by numerical collisions and reduced with an increasing number of macroparticles per cell (see Fig. 7.10). It is interesting to note from Fig. 7.11(d) that regions away from the center of the CS will be isotropized slower. This is in agreement with the our previous discussions in 7.2.3.1; numerical collisions and pitch angle scattering are reduced in regions with stronger magnetic field.

In the initial stage (1), the conditions in the regions close to the center of the CS are favorable for the development of the (anisotropy temperature driven) Weibel instability (see Weibel 1959, or Sec. 5.2.1 for further details). This is because of the initially imposed anisotropy $T_{e, y}>T_{e, x}, T_{e, z}$ and the unmagnetized conditions there (with very high $\beta$ plasma). Growth rates and the corresponding timescales of Weibel instability are much shorter (on the order of $\omega_{p e}^{-1}$ ) than those of tearing mode $\left(\Omega_{c i}^{-1}\right)$. Therefore, the aforementioned coupling between these instabilities (leading to stabilization of tearing mode) can be completely neglected during this period $t \Omega_{c i}<0.5$. One of the main signatures of this instability is the generation of magnetic fields perpendicular to both propagation direction 
( $x$, according to the discussion of Fig. 7.5) and warmer component of the temperature ( $y$ direction), i.e.: the out-of-plane $\hat{z}$ direction, as can be seen in the time evolution of $B_{z}$ in Fig. 7.11(a). Note that regions away from the center of the CS do not show a significant increase of $B_{z}$, since they have stronger background magnetic field (and correspondingly lower $\beta$ plasma) which is known to weaken Weibel instability (cf. Sec.5.2.1). This generation of magnetic field is correlated with a corresponding decrease of electron temperature anisotropy (see Fig. 7.11(c)), the source of free energy of Weibel, and again significant only close to the center of the CS.

\subsubsection{Spatial structure of Weibel instability}

For calculation of both threshold and growth rates of Weibel mode, we need a value for the wavevector $k_{x}$. We cannot just naively use the maximum value $k_{x, \max }$ as given by Eq. (5.52), since it might predict long wavelengths, crossing to the low $\beta$ plasma regions away from the center of the CS where the unmagnetized condition for the existence of Weibel $\left(\Omega_{c e} \ll \omega_{p e}\right)$ is not satisfied any longer. Indeed, the value $\lambda_{x, \max }=2 \pi / k_{x, \max }$ gets larger for smaller anisotropies. For our parameters, an initial value $A_{e}=1.96$ corresponds to $\lambda_{x, \max }=0.8 d_{i}$, while after the initial transient period $A_{e} \sim 1.2$, corresponding to $\lambda_{x, \max }=$ $1.8 d_{i}$.

In order to solve the previous issue with the choice of $k_{x}$, we followed the approach by Lu et al. (2011). They proposed that Weibel instability in a CS will have $\lambda_{x, \max } \sim 2 L$, since the halfwidth determines the typical length scale of variation of the background magnetic field (the factor 2 is because is measured from the center to opposite directions). Therefore, we can assume $\lambda \sim d_{i}$ for our parameters at the saturation time after the initial anisotropy drop. This can be tested by measuring the length scale of the spatial structure of $B_{z}$, displayed in Fig. 7.12.: 


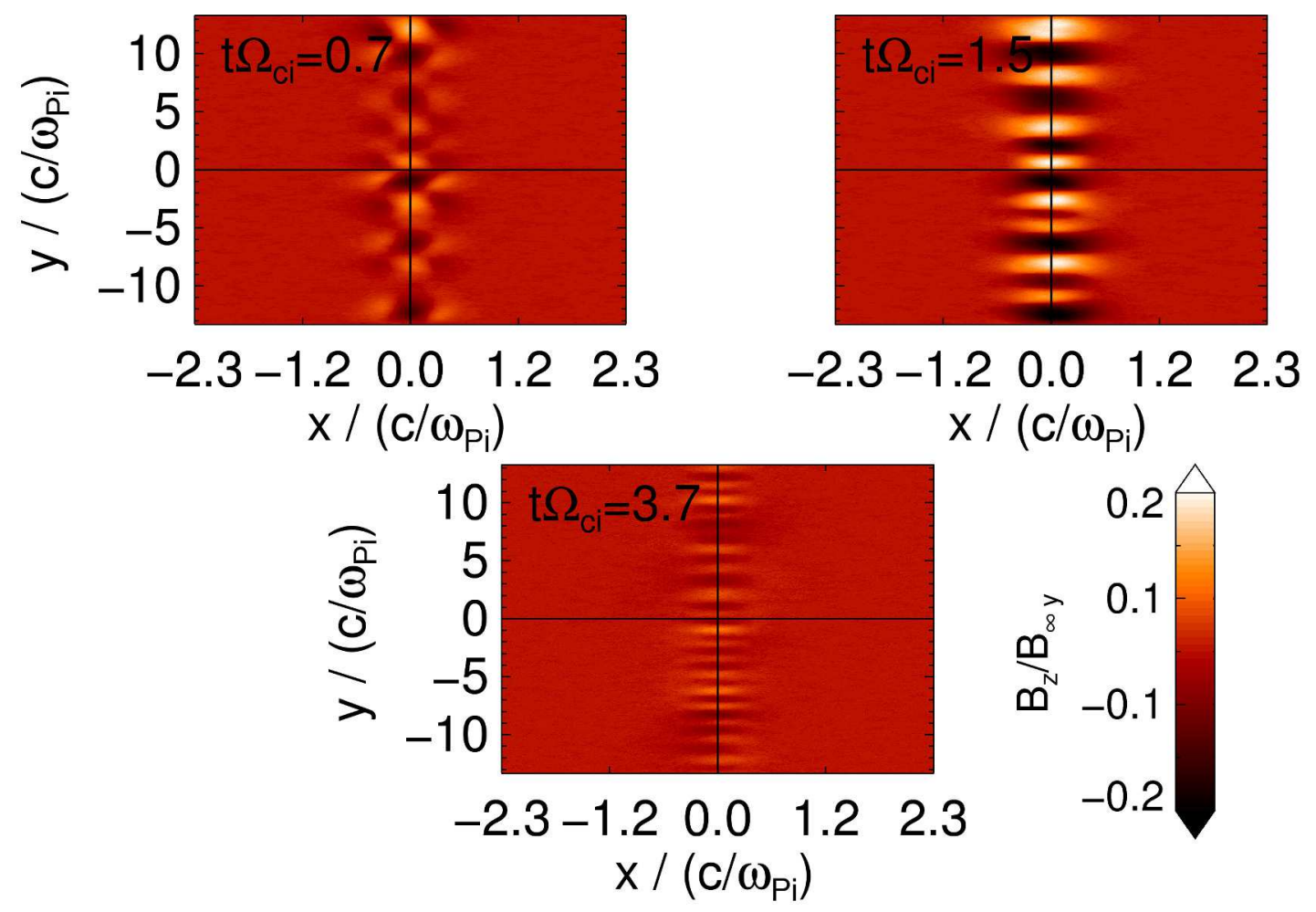

Figure 7.12: Contours of the out of plane magnetic field $B_{z}$ for the run TSC-360ppcA 1.96. The snapshots correspond to three characteristic times during the evolution of the Weibel instability. Reproduced with permission from P. A. Muñoz, P. Kilian, and J. Büchner, Physics of Plasmas 21, 112106, (2014) Copyright 2014, AIP Publishing LLC.

The "chessboard" (filamented) structure of $B_{z}$ close to the center of the CS is a characteristic signature of the Weibel instability in these scenarios, as shown in previous studies of magnetic reconnection in both electron-positron plasmas (Swisdak et al. 2008, Zenitani and Hesse 2008, Liu et al. 2009) as well as in electron-proton plasmas (Lu et al. 2011, Schoeffler et al. 2013). The temperature ani sotropy necessary for the development of Weibel instability is generated by the particles bouncing inside of the magnetic islands, also known as the Fermi mechanism (see more details in Schoeffler et al. 2013). This is not the same phenomena seen in our case, since at this initial stage there is no magnetic islands or physically generated anisotropies at all.

We can see that the size across $x$ of the $B_{z}$ structures is small at the beginning, as expected due to the high level of anisotropy. As the source of free energy (temperature energy) is depleted, they get larger, in agreement with Eq. 5.53) (see Fig. 7.12(b)). Their saturation size at $t \Omega_{c i} \sim 1.5$ is $\lambda_{\max } \sim 1 d_{i}$, in agreement with our previous estimation. The Weibel structures cannot grow further, and in particular not to the size predicted by Eq. (5.52) $\left(\lambda_{x, \max }=1.8 d_{i}\right)$, due to the constraint on the magnetic field strength.

And how about the size of the structures of $B_{z}$ along $y$ direction? The Weibel instability should not propagate in the direction of the warmer temperature $T_{e, y}$ according to linear theory. However, following Liu et al. (2009), there is mechanism for producing such $k_{y}=2 \pi / \lambda_{y}$ wavevectors when this instability is embedded in the center of a CS, like in our case. Basically, it is produced by the electrons gyrating around the Weibel generated 
magnetic field in $B_{z}$ (see also Schoeffler et al, 2013). Therefore, the wavelength $\lambda_{y}$ should have a size on the order of $2 \rho_{e, z}$, with $\rho_{e, z}$ calculated in the Weibel generated $B_{z}$ close to the center of the CS. At the peak of Weibel activity, $t \Omega_{c i}=1.5$, we measured in average $B_{z} \sim 0.1 B_{\infty y}$ inside of each cell of the "chessboard" structure. Then, the electron Larmor radius will be $\rho_{e, z} / d_{i} \sim 0.35$. This is one quarter of the measures size of Weibel cells in $y$ direction $\left(1.3 d_{i}\right)$, only a factor of two of difference with the theoretical estimate by Liu et al. (2009).

On the other hand, the previous argument also allows to explain the reduction in the size of Weibel structures along $y$ during the decaying phase $\left(t \Omega_{c i}=1.5\right)$. Because the anisotropy and so the source of free energy of Weibel gets depleted, the generated $B_{z}$ will becomes weaker, implying smaller $\rho_{e, z}$ and so $\lambda_{y}$.

\subsubsection{Weibel threshold condition}

Thus, we have validated the required $k_{x}$. So, now we can calculate the theoretical estimate for the threshold Eq. 5.54) (as derived for unmagnetized and homogeneous plasmas). It becomes

$$
A_{e}>1+\left(k_{x} c / \omega_{p e}\right)^{2} \sim 1.21
$$

This value agrees well with the observed anisotropy after $t \Omega_{c i} \gtrsim 1.5$ (see Fig.7.11 (c)), and it is higher than the required for tearing mode stabilization $\left(A_{e}=1.159\right.$ according to Eq. (7.3)). Then, we can infer that a CS initially unstable to Weibel in its center will already be stabilized the tearing mode. And in the other way around: If a CS increases monotonously its anisotropy (by means of either physical or numerical processes), first will stabilize tearing mode and only after than will become unstable to the Weibel mode. This is precisely the behaviour seen in the CIC runs (see discussion in Fig.7.5).

\subsubsection{Mirror instability produced via Weibel instability}

Now, let us analyze with more detail the behaviour of the CS in the stage (2) $0.5<$ $t \Omega_{c i}<1.5$. After $t \Omega_{c i} \sim 0.5$, we found that the weakly magnetized (high $\beta$ plasma) center of the CS (due to the Weibel generated $B_{z}$ ), but with a still high anisotropy, becomes unstable to another temperature anisotropy driven instability, known to operate under these conditions: the mirror mode (see Hasegawa 1969, Gary 1993, and discussion in Sec. 5.2.2).

\subsubsection{Field aligned temperature anisotropy}

In order to prove this claim, first we note that a necessary condition for the triggering of mirror instability is $T_{e, \perp}>T_{e, \|}$. This is the tricky part: $\|$ and $\perp$ directions are calculated with respect to the local magnetic field. This is different from Weibel instability, where these directions are measured with respect to the (initially known) imposed temperature ani sotropy. But the topology of the total $\vec{B}$ generated by Weibel instability (not only in the $z$ direction, but also in-plane) is complex (turbulent), in addition to fluctuate very quickly. Therefore, the parallel direction will have to be calculated by using $\vec{B} /|\vec{B}|$ for each location in space and time. Then, we will have to change the frame of reference (by means of a 
rotation) of the temperature tensor $T_{i j}$ to keep it locally aligned with the magnetic field and thus calculate $T_{e, \|}$. The change of reference frame give us additionally degrees of freedom in the perpendicular direction (since we have only one out three rotation axis specified), but that it is not necessary to calculate $T_{e, \perp}$ (since it only requires the diagonal components of the tensor). All the details of the calculation can be found in Appendix B.2.5. By taking into account the previous considerations, we can plot the magnetic-field-aligned temperature anisotropy $T_{e, \|} / T_{e, \perp}$ for three characteristic times in Fig.7.13. Locations with $T_{e, \perp}>T_{e, \|}$, a necessary (but not sufficient) condition for the mirror mode, are enclosed in black contour lines. They appear only after $t \Omega_{c i}>0.5$, reach maximum size around $t \Omega_{c i} \sim 1.5$, and after the anisotropy is depleted they start to disappear slowly (these times will be justified later).

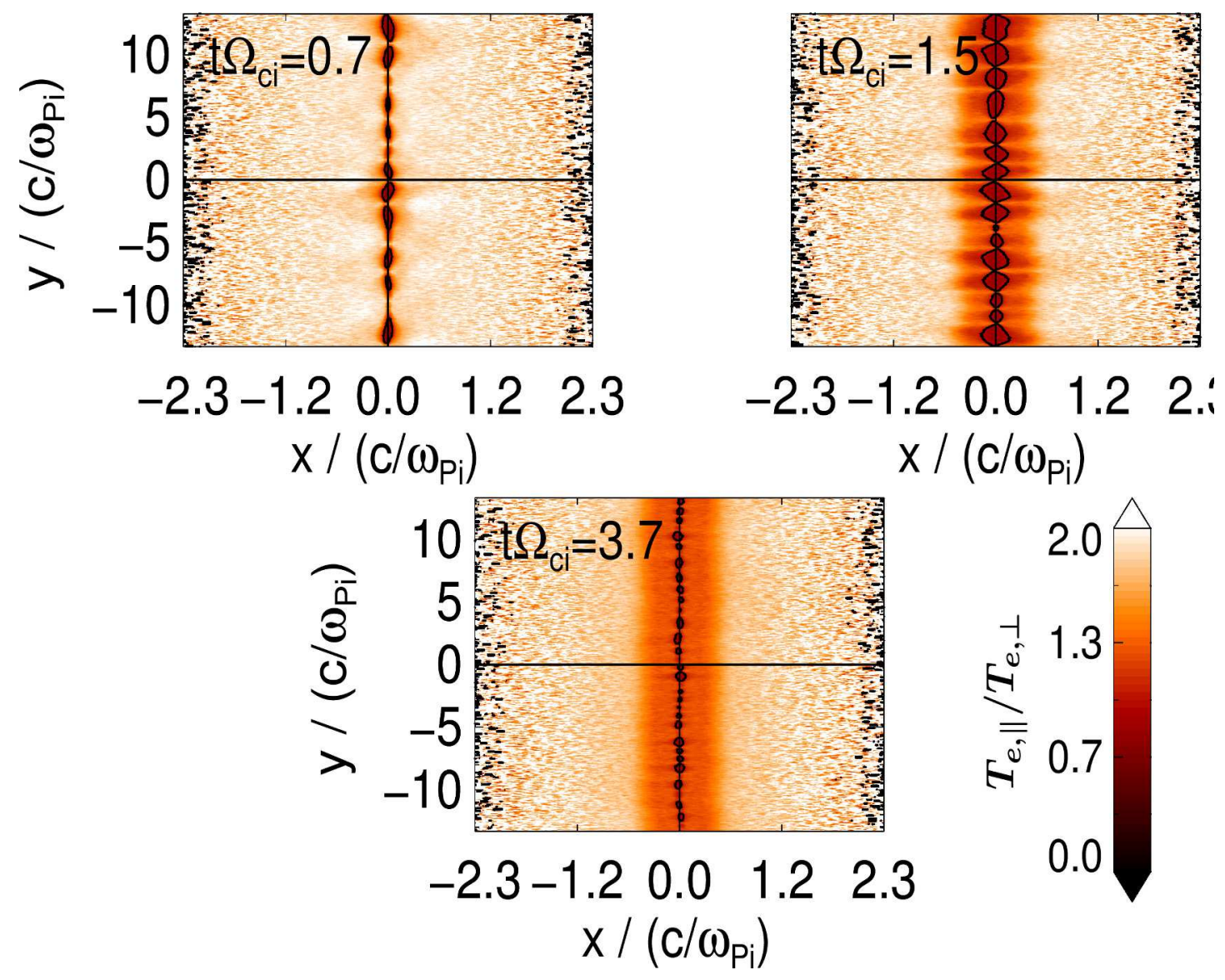

Figure 7.13: Contours of the electron temperature anisotropy $T_{e, \|} / T_{e, \perp}=P_{\|, e} / P_{\perp, e}=$ $\beta_{\|, e} / \beta_{e, \perp}$ for the run TSC-360ppc-A1.96. The snapshots correspond to the same three times shown in Fig.7.12 Note that regions with $T_{e, \perp}>T_{e, \|}$ are shown inside of the black contour lines. Reproduced with permission from P. A. Muñoz, P. Kilian, and J. Büchner, Physics of Plasmas 21, 112106, (2014) Copyright 2014, AIP Publishing LLC.

\subsubsection{Mirror threshold condition}

Let us focus now at the time of maximum activity $t \Omega_{c i} \sim 1.5$ (to be justified later) to prove the sufficient condition (threshold) for the mirror mode Eq. (5.59) $D>0$. It has higher growth rates as $D$ gets larger. First note that we can neglect the last term in 
that expression, a correction due to Hall decoupling between electrons and ions. That is because it is proportional to the difference in the electron to ion temperatures, zero in our initialization (both species are equally anisotropic), besides of not being enhanced at later times as we checked from our simulation results. Thus, a plot showing the evaluation of the full left hand side $D$ of the mirror threshold condition is displayed in Fig.7.14. Thus, we can see that the mirror condition is fulfilled inside of small scale structures very close to the center of the CS, with maximum size on electron scales $\sim d_{e}$ across the $x$ direction (much smaller than the corresponding to Weibel cells). Note that these structures are different and cannot be explained by the Weibel instability by itself.

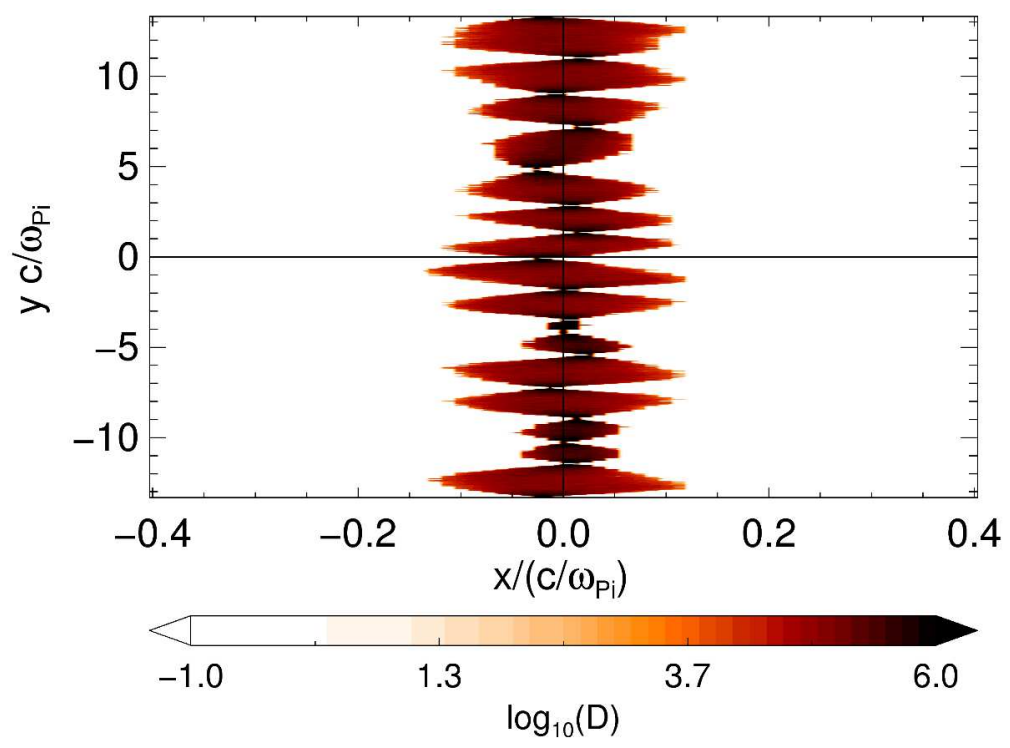

Figure 7.14: Contours of the mirror threshold value from Eq. 5.59): $\log _{10}(D)$. Only positive values are shown (corresponding to instability). This plot is for the run TSC$360 p p c-A 1.96$ at the time $t \Omega_{c i}=1.5$, corresponding to the peak of activity of both Weibel and mirror instabilities. Compare with the corresponding plots for $B_{z}$ in Fig. 7.12 and temperature anisotropy $T_{e, \|} / T_{e, \perp}$ in Fig. 7.14. Note that only a small region close to the current sheet is shown, in order to visualize the mirror structures that have a maximum typical length scale of $c / \omega_{p e}$ across the $x$ direction. Reproduced with permission from $P$. A. Muñoz, P. Kilian, and J. Büchner, Physics of Plasmas 21, 112106, (2014) Copyright 2014, AIP Publishing LLC.

In order to detect when the mirror mode is active, we display in Fig. 7.15 (top row) the time evolution of the ratio of grid points (in an area close enough to the center of the CS) satisfying the necessary condition $T_{e, \|} / T_{e, \perp}<1$ and the ones fulfilling the sufficient condition $D>0$. They are proportional to the area of the regions unstable to mirror mode. Below, in Fig. 7.15)(bottom row) we displayed the respective mean values of these quantities. By means of these diagrams, we can see that mirror mode is active $0.5<$ $t \Omega_{c i} \lesssim 5$, correlated with the times in which there is Weibel generated $B_{z}$. That is to be expected, since the mirror mode can only exist in magnetized plasmas. Furthermore, this instability reaches peak activity (both in size and strength) around $t \Omega_{c i}=1.5$, correlated with the maximum of the Weibel generated magnetic field $B_{z}$ (see Fig.7.11(a)), although with a weak temperature anisotropy. Between $1.5<t \Omega_{c i} \lesssim 5, T_{e, \|} / T_{e, \perp}<1$ (the source 
of free energy) decreases, and then the mirror instability is shutdown afterwards. It is important to remark that this temperature anisotropy is different and not related with the required anisotropy to drive the Weibel mode unstable (cf. Fig.7.11(c)).
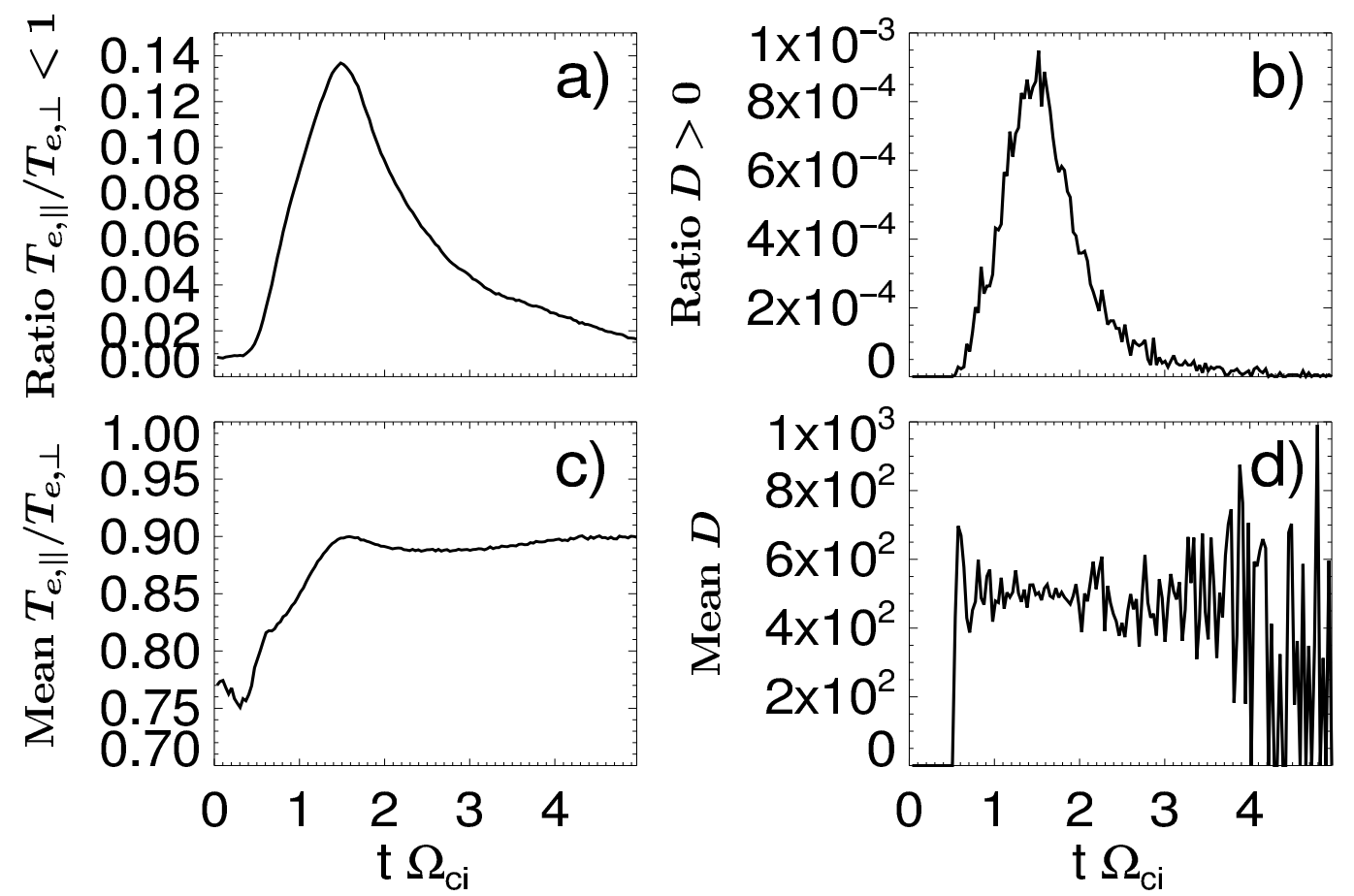

Figure 7.15: Time evolution of quantities related with the mirror instability for the run TSC-360ppc-A1.96. a) Number of grid points in $|x|<L$ that satisfy $T_{e, \|} / T_{e, \perp}<1$ divided over the total in this region. Note that $T_{e, \|} / T_{e, \perp}$ is the electron temperature anisotropy aligned with the local magnetic field, i.e.: the $\|$ direction is in the $\vec{B} /|\vec{B}|$ direction. The choice of the $|x|<L$ is basically because encompass all the area that becomes unstable to mirror instability b) Mean value of $T_{e, \|} / T_{e, \perp}$ for the grid points that satisfy the condition $\left.T_{e, \|} / T_{e, \perp}<1 . c\right)$ Number of grid points in $|x|<L$ that satisfy the mirror threshold condition Eq. (5.59), divided over the total in this region. d) Mean value of the mirror threshold condition (left hand side of Eq. (5.59)) calculated for the points that satisfy that condition. Reproduced with permission from P. A. Muñoz, P. Kilian, and J. Büchner, Physics of Plasmas 21, 112106, (2014) Copyright 2014, AIP Publishing LLC.

\subsubsection{Size of mirror mode structures and propagation direction}

The mirror unstable structures seen in Fig.7.14 are highly dynamic (at electron time scales), as a result of the turbulence generated. They deform and kink the initially antiparallel Harris magnetic field lines, since its propagation direction is perpendicular $\perp$ to the local magnetic field, mostly the $B_{z}$ generated via Weibel instability.

Even though the mirror structures change on (fast) electron time scales, it is still possible to estimate their size, not varying too much during its peak activity $\left(t \Omega_{c i} \sim 1.5\right)$. This can be then compared with the theoretical wavelengths predicted by the linear theory (see Sec. 5.2.2), in order to have another argument for the existence of mirror mode in our system. In order to do that, first we need to keep in mind that the shorter wavelengths 
allowed by mirror (non-negligible growth rates) are constrained by finite ion Larmor radius effects (Pokhotelov et al. 2000). This implies that the (perpendicular) wavelengths at maximum growth rate will have to be around $k_{\perp}^{-1} \sim \rho_{i}$. The tilted size of the measured mirror structures along $y$ direction agrees well with this prediction. This can be seen by noticing that a typical mirror island has a size $l_{x} \times l_{y}=l_{\|} \times l_{\perp}=0.2 d_{i} \times 2.0 d_{i}$, while $\lambda_{\perp, \max }=2 \pi / \rho_{i} \sim 3 d_{i}$.

According to the linear theory (see Sec. 5.2.2), mirror mode also predicts a parallel propagation, although less significant than the main perpendicular one. The Weibel generated magnetic field is mostly in $z$ but it also has a $B_{x}$ component. Then, the parallel direction will have to be along $x$ direction (since no out-of-plane propagation is allowed in our geometry), in such a way that $k_{\perp}=k_{y} \gg k_{\|}=k_{x}$. The specific propagation angle $\tan (\theta)=k_{\perp} / k_{\|}$is given then by the expression Eq. (5.62), proportional to a factor involving the electron temperature anisotropy in the mirror condition $D$ (as it appears in Eq. (5.59)). This angle $\theta$ allow us to determine the typical $\lambda_{\|, \max }$ of mirror mode. Inside of these structures it is meaningful to estimate the order of magnitude of the corresponding parameters entering into the previous expression, even though their range of variations can be very large (especially the $\beta$ plasmas). This is because $\theta$ is weakly dependent on these ones: the propagation direction does not change too much from perpendicular. Keeping in mind that consideration, we can assume typical values $\beta_{i, \|} \sim \beta_{e, \|} \sim 80$, with field aligned anisotropies $A_{e} \sim A_{i} \sim 0.99$, obtaining:

$$
\tan (\theta)=\frac{k_{\perp}}{k_{\|}}=\frac{1}{\sqrt{A}}=\sqrt{\frac{3 \beta_{i, \perp}^{-1}\left(1+\frac{\beta_{i, \perp}-\beta_{i, \|}}{2}\right)}{\left(\frac{T_{i, \perp}}{T_{i, \|}}-1\right)+\frac{T_{e, \perp}}{T_{i, \perp}}\left(\frac{T_{e, \perp}}{T_{e, \|}}-1\right)-\frac{1}{\beta_{i, \perp}}}} \sim 11^{\circ}
$$

This values matches well with the tilting angle of the mirror structure $\left(\theta=\tan ^{-1}\left(2.0 d_{i} / 0.2 d_{i}\right)\right.$ $\sim 85^{\circ}$ ), confirming thus another signature of this instability.

\subsubsection{Growth rates of the initial Weibel/mirror instabilities}

We already compared both thresholds and wavevectors of Weibel/mirror instabilities. In order to have an additional argument for their existence in our system, let us compare their growth rates with the predictions of linear theory. First we note that both instabilities operate together between $0.5<t \Omega_{c i}<1.5$, as evidenced by the increasing $\left|B_{z}\right|$ (absolute value because it alternates sign) during that period (correlated with the area of the regions $\left.T_{e, \|} / T_{e, \perp}>1\right)$. Therefore, we can measure growth rates $\gamma$ by means of a linear fitting to $\left|B_{z}(x=0)\right|$ during this period (at the center of the CS because it is most unstable region). Since our idea is to study the dependence of $\gamma$ on the imposed anisotropy, we use the results of 5 simulations runs with 360ppc and different $A_{e}$ described in the fourth part of Table 7.2: TSC-360ppc-A1.3, TSC-360ppc-A1.4, TSC-360ppc-A1.6, TSC-360ppc-A1.8 and TSC-360ppc-A1.96. The results for the growth rates are shown as black points in Fig. 7.16 (a), while the saturation peak time of $\left|B_{z}(x=0)\right|$ in Fig.7.16(b). As expected, an enhanced temperature anisotropy will increase growth rates as well as the maximum value $B_{z}$, and therefore will reach saturation sooner. The instabilities will disappear for $A_{e} \lesssim 1.2$, corresponding to Weibel threshold calculated in Eq. (7.4). This also applies to the mirror instability, since it is not allowed if there is no $B_{z}$ (generated by Weibel). 

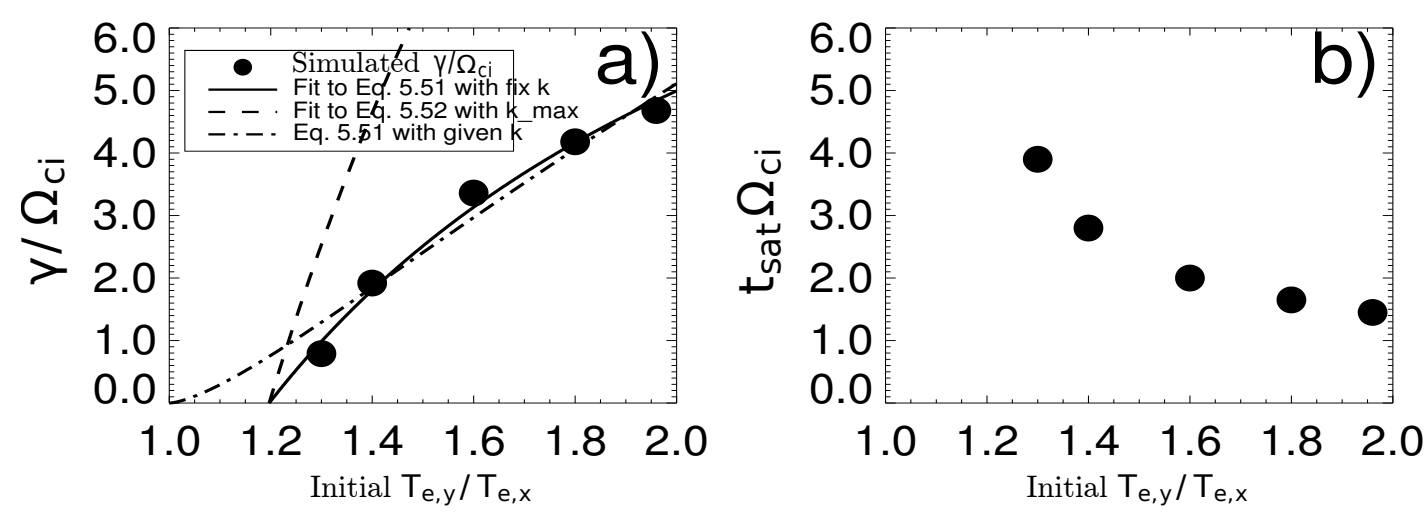

Figure 7.16: a) Comparison among simulated growth rates of the Weibel instability during the linear stage on dependence of the initially imposed electron temperature anisotropy $T_{e, y} / T_{e, x}$, a fit according to Eq. (5.51) (for fixed $k$ ), a fitting according to Eq. (5.52) (for $k_{\text {max }}$ ), and the theoretical growth rates calculated from the initial parameters in Eq. (5.51) (for fixed k). See text for details. b) Saturation time $t_{\text {sat }}$ of the Weibel instability on dependence of the initially imposed electron temperature anisotropy $T_{e, y} / T_{e, x}$. Adapted from Muñoz et al. (2014).

Now, let us compare the different ways of estimating the growth rates according to the theoretical estimation. The results in Fig. 7.16 show:

- Continuous line: Fit according to $\gamma\left(A_{e}\right) / \Omega_{c i}=\left(c_{1} / A_{e}\right) \times\left(A_{e}-1-c_{2}\right)$. This is the functional dependence given by Eq. (5.51) for the Weibel instability, but with a fixed $k_{x}$ (contained in both free parameters $c_{1}$ and $c_{2}$ ). The linear regression gives $c_{1}=$ 12.3 and $c_{2}=0.20$. This allows to calculate $k_{x}$ and then estimate the wavelength $\lambda_{x}=1.0 d_{i}$. It agrees well with the size of the Weibel structures in $B_{z}$.

- Dashed-dotted line: Fit according to $\gamma\left(A_{e}\right) / \Omega_{c i}=\left(c_{3} / A_{e}\right) \times\left(A_{e}-1\right)^{3 / 2}$. This is the functional dependence given by Eq. (5.52) assuming $k_{x}=k_{x, \max }$, being given by Eq. (5.53). The linear regression gives $c_{3}=10.22$. It reproduced the measured behaviour for larger $A_{e} \gtrsim 1.4$, while it predicts significant growth for lower anisotropies where Weibel should be stable.

- Dashed line: directly apply Eq. (5.51) with the measured maximum $k_{x}=2 \pi /(1.0 \mathrm{di})$ of the Weibel structures. It completely overestimate the measured growth rates (by a factor of 2-3). There are many possible reasons for this significant deviation from linear homogeneous theory: our system is clearly inhomogeneous, the weak magnetic field that should tend to decrease growth rates and the simultaneous presence of mirror instability

In conclusion, the linear theory for Weibel mode can only predict the dependence of growth rates on the anisotropy, knowing beforehand the allowed wavelength of the Weibel unstable mode in our geometry $\left(k_{x}\right)$. This, in turn, depends on the typical gradient scale of the magnetic field: $2 L \sim 2 \rho_{i} \sim 1.0 d_{i}$. This is the result showed by the continuous line in Fig. 7.16 (and to a lesser degree, by the dashed-dotted line in the same plot). In any case, this result is still a significant progress, even in this complex scenario where there is even another instability operating simultaneously (mirror). 
All previous estimations were for Weibel growth rates. We did not try to do something similar for the mirror mode, because their typical $\gamma$ 's, as given by Eq. 5.57) or Eq. 5.60), are very sensitive to variations in its parameters. The last is true especially for $\beta_{i}$, which varies over some orders of magnitude inside of the mirror structures and so is the range of variation of the predicted $\gamma$. Therefore, in this case comparison with the linear theory is not so meaningful to predict the behaviour of the large scale magnetic field or momenta: the growth is more dominated by the Weibel than mirror instabilities.

Summarizing the last subsections, we have found evidence, by means of the threshold condition, propagation direction and other similar signatures, that not only Weibel instability is acting in the center of the CS, but also the mirror instability. In fact, there have been some studies that have pointed out the close relationship between the mechanisms behind both (see Sec. 7.3.3)

\subsubsection{Other possible temperature driven instabilities}

In Fig. 7.13 we can also note that at the edges of the CS, outside of the mirror structures, there are regions with $T_{e, \|}>T_{e, \perp}$ prone to a temperature driven instability with this opposite condition: the firehose instability. However, we have not found signatures of its presence in our system (see Sec. 5.2.4), such as absence of resonant protons, no clear wave vector (aligned with the magnetic field) in those regions. The evidence seems to indicate that is because its predicted growth rates are about one order of magnitude lower than those of the Weibel mode. Nevertheless, their effects relating the kinking of magnetic field lines are similar to the observed in our case, as it has been reported by previous works analyzing the natural evolution of a Harris current sheet (Matteini et al. 2013).

\subsubsection{Consequence of Weibel/Mirror instabilities: bifurcation}

Here we are going to prove that the main consequence of an initially imposed anisotropy $A_{e}>1.21$, the Weibel (and therefore mirror) instability threshold according to Eq. (7.4), is the formation of a bifurcated CS. Note that this value is higher than the anisotropy threshold for tearing mode stabilization $A_{e}>1.16$ (see Eq. (7.3)), implying thus a more strict requirement for the development of bifurcation in $J_{z}$ than for suppression of tearing mode (see also Sec. 7.3.2.2). On the other hand, it is important to mention in this context that stabilization of tearing mode and mirror instability are also closely related: the force responsible for both mechanism has similar origins (Chen et al. 1984, Shi et al. 1987).

We checked the previous claim by noticing that $J_{z}$ develops a deeper dip in its center (bifurcation) when the initial $A_{e}$ is higher (plots not shown here), after the initial transient stage when Weibel instability is active. For values below the Weibel threshold, no bifurcation is visible. The evolution of this process can be exemplified in Fig. 7.17, where it is shown the profiles along $y$ of some quantities related with bifurcation, at different distances from the center of the CS. 

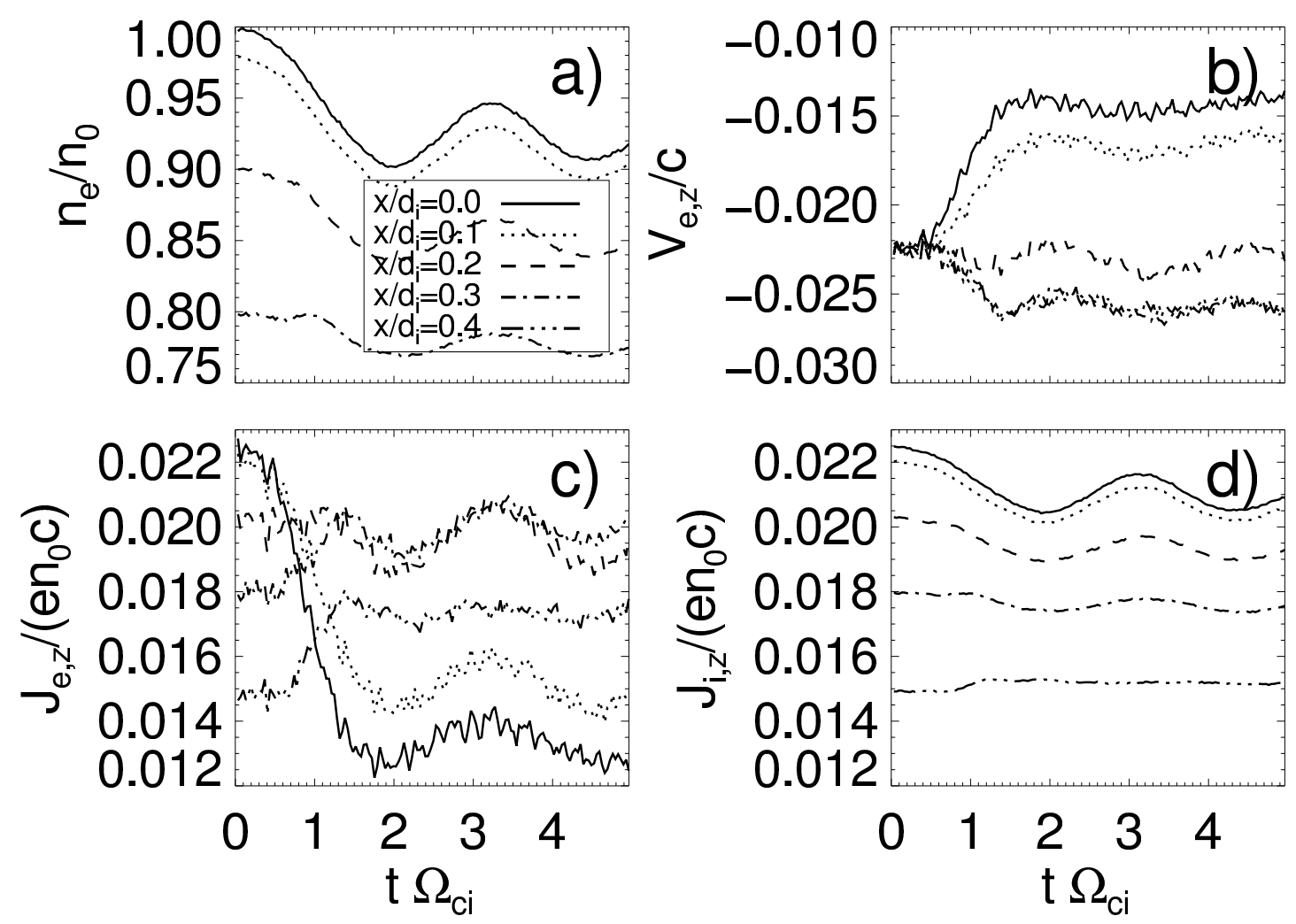

Figure 7.17: (Short) time evolution for some quantities related with bifurcation, averaged along $y$, at different distances $x$ from the center of the CS, for the run TSC-360ppc-A1.96. a) Electron density $n_{e}$. b) Electron bulk speed $\left.V_{e, z} . c\right)$ Electron current density $J_{e, z} . d$ ) Ion current density $J_{i, z}$. We do not show the ion density $n_{i}$ since its values in the course of the evolution are always very close to $n_{e}$, i.e.: the quasineutrality condition is kept everywhere to a large extent. Reproduced with permission from P. A. Muñoz, P. Kilian, and J. Büchner, Physics of Plasmas 21, 112106, (2014) Copyright 2014, AIP Publishing $L L C$.

In Fig. 7.17 (c) we can see the characteristic dip in $J_{e, z}$ at $x=0$, the signature of bifurcation (and practically no reduction in $J_{i, z}$ ). It takes place during the same period in which the initial anisotropy is exhausted (see Fig.7.11) as a result of the activity of Weibel instability. This is also correlated with the growing phase of the mirror mode (see Fig. 7.15). It does not grow beyond $t \Omega_{c i} \gtrsim 1.5$ since the numerical pitch angle scattering due to the anisotropy, responsible for this process, is stopped. So, $J_{e, z}$ at the center of the CS remains with the minimum values reached during this short period. As expected (see discussion of Fig.7.4), the reduction in $J_{e, z}$ is mostly due to $V_{e, z}$ (Fig.7.17(b)). The density $n_{e}$ (Fig. 7.17(a)) is also reduced, but to a much less extent. This can be attributed to a signature of mirror mode due to the increase of magnetic pressure: the fluctuations in both quantities should be in antiphase (as predicted by a fluid model. See Southwood and Kivelson 1993).

As we mentioned before, the highly dynamic mirror structures oscillates around the center of the CS. This process leads to a reduction in $V_{e, z}$ inside of a narrow strip with an extension equal to the maximum amplitude of these oscillations, remaining in this stage even after these structures disappear $t \Omega_{c i} \gg 1.5$. We can estimate the width of the 
bifurcated structured in $J_{z}$ as function of the initial imposed anisotropy, since it has to be on the same length scale as the component of the mirror wavevector in the parallel direction $k_{\|}$. Indeed, by assuming a fixed $k_{\perp}$ (see explanation in Sec. 7.3.3), this quantity has to be proportional to $\sqrt{A}$ by Eq. (5.62). Then, using Eq. (5.61) and Eq. (5.59), it turns out that the extension across $x$ of the mirror structures, and so the bifurcation width, is directly proportional to the instantaneous value of the anisotropy $A_{e}^{-1}-1=T_{e, \perp} / T_{e, \|}-$ 1. For values higher than 0 , this is simply the necessary condition for the triggering of mirror mode. This field aligned anisotropy never deviates too much from the marginal stability condition inside of the mirror structures, explaining bifurcation happening only close to the CS. Regions away from the center, not experiencing the mirror instability (no $T_{e, \perp} / T_{e, \|}>1$ ), will not decrease their values of $J_{z}$ and therefore no bifurcation will be observed.

\subsubsection{Influence of the number of macroparticles per cell on the Weibel/mirror instabilities}

Since the depletion of the initial anisotropy (the driver of bifurcation) depends on the macroparticle number (see Fig. 7.10), it is convenient to check for possible numerical effects on its development. This can be exemplified in Fig. 7.18 where it is shown the evolution of the profiles of $J_{z}$ across the CS (analogous to Fig. 7.4) for cases with highest anisotropy level $\left(A_{e}=1.96\right)$ and different ppc. We can see that more macroparticles per cell reduce a little bit the drop in $J_{z}$ at the center in the run TSC-360ppc-A1.96 compared to TSC-40ppc-A1.96. Nevertheless, it is still stabilizing the tearing mode. There is a trend towards the reduction, but not complete suppression, of bifurcation in the limit of a very large number of ppc. Any spontaneous instability requires some background noise/fluctuation level (either physical or numerical) in order to exist, and thus bifurcation would be suppressed only when the numerical noise is strictly zero: a non physical scenario (since there is always a thermal fluctuation level in nature). 

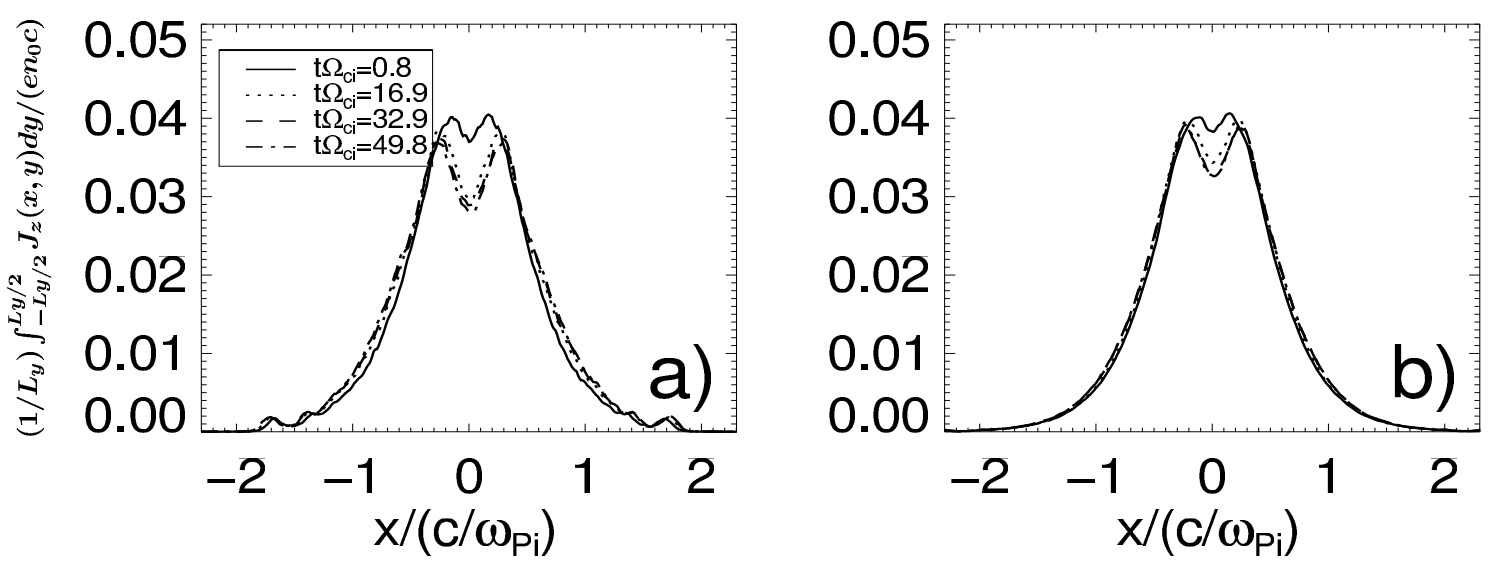

Figure 7.18: Evolution of the total current density profile $J_{z}$ showing bifurcation for runs with the highest considered initially imposed temperature anisotropy $A_{e}=1.96, T S C$ shape function and different number of macroparticles per cell: a) TSC-40ppc-A1.96 and b) TSC-360ppc-A1.96. The profiles are obtained by integrating the current density along the CS: $J_{z}(x)=\left(1 / L_{y}\right) \int_{-L y / 2}^{L y / 2} J_{z}(x, y) d y$. Reproduced with permission from $P$. A. Muñoz, P. Kilian, and J. Büchner, Physics of Plasmas 21, 112106, (2014), Copyright 2014, AIP Publishing LLC.

It is interesting to compare the different evolution of bifurcation for this case of initially imposed anisotropy Fig. 7.18 with the case of numerically generated anisotropies due to the use of CIC schemes (see Fig. 7.4). Anisotropy temperature, the driver of this process, increases in the second case but not in the first one (after the initial transient) due to the good energy conserving properties of the TSC scheme. This explains why bifurcation does not continue developing when an initially anisotropy is imposed for the TSC runs, while it does not stop to grow with the CIC runs where numerical effects continuously feed the source of free energy of this instability. Also note that in the CIC runs, the process that leads to bifurcation is not only due to pitch angle scattering like in the TSC schemes, but it has also contributions from the overall numerical heating with the associated increment in the total particle energy.

Now, let us analyze closer the initial evolution of these processes for the run with a reduced macroparticle number TSC-40ppc-A1.96, by showing the corresponding evolution the quantities showing signatures of the Weibel instability Fig.7.11. 

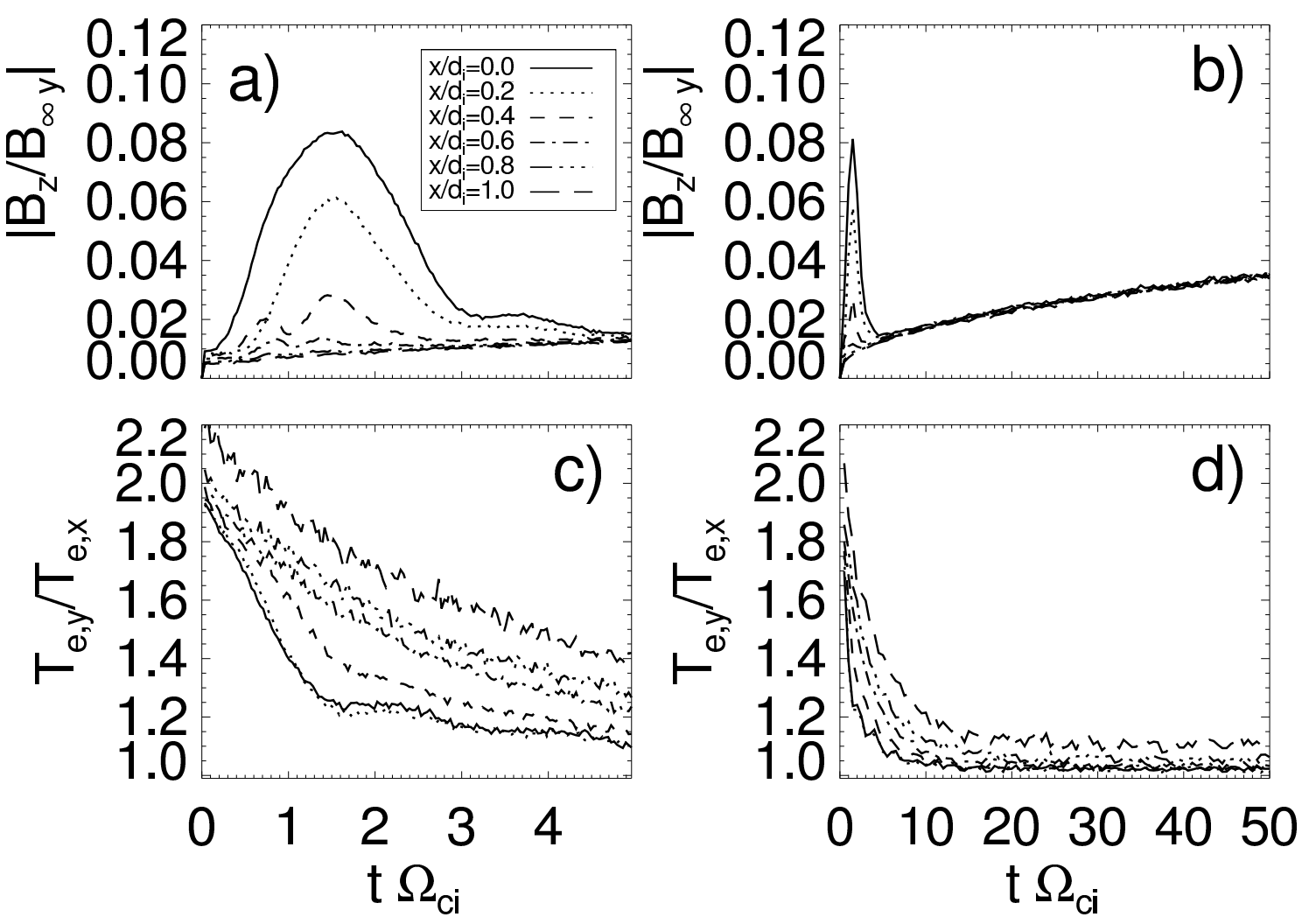

Figure 7.19: Time history of the out of plane magnetic field and temperature anisotropy averaged along $y$, at different distances $x$ from the center of the CS, for the run TSC-40ppcA 1.96. a) and b) Magnetic field $\left|B_{z}\right|$. c) and d) Electron temperature anisotropy $T_{e, y} / T_{e, x}$. The left plots $a$ ) and c) are for the early stages of the system while the right plots $b$ ) and $d$ ) are for the long term evolution. To be compared with Fig. 7.11. Reproduced with permission from P. A. Muñoz, P. Kilian, and J. Büchner, Physics of Plasmas 21, 112106, (2014) Copyright 2014, AIP Publishing LLC.

By comparing with the case with nine times more macroparticles per cell, TSC-360ppcA1.96 shown in Fig. 7.11, we note a sharp decrease in $T_{e, y} / T_{e, x}$ from the very beginning at the CS center $x=0$ : there is no period in which the anisotropy is kept to its initial value (Fig. 7.11(c)) like in the former case for $0<t \Omega_{c i}<0.5$. The consequence is that Weibel instability also operates from the beginning, as evidenced from the relaxation of anisotropy and the corresponding generation of $B_{z}$ (Fig. 7.11(a)) at a faster rate than for the case with more ppc. This can be attributed to the enhanced noise level, providing the necessary the magnetic field seed from which Weibel instability can grow. This, in turn, favors an earlier development of field aligned anisotropies $T_{e, \perp} / T_{e, \|}>1$ : the necessary condition for mirror instability. On the other hand, both Weibel and mirror instabilities saturate more or less at the same time, once this initial anisotropy is exhausted. As a result, the center of the CS will experience more time unstable to the mirror instability for $360 \mathrm{ppc}$ than for $40 \mathrm{ppc}$, explaining the stronger bifurcation in the latter case (deeper reduction in $J_{e, z}$ ), as shown in Fig.7.20 (compare with Fig.7.17).

Finally, it is interesting to note that the asymptotic value reached by $B_{z}$ for very long times is independent of the distance to the center of the CS, being only dependent on the number of macroparticles per cell (see Fig.7.11(a) and Fig.7.19). 

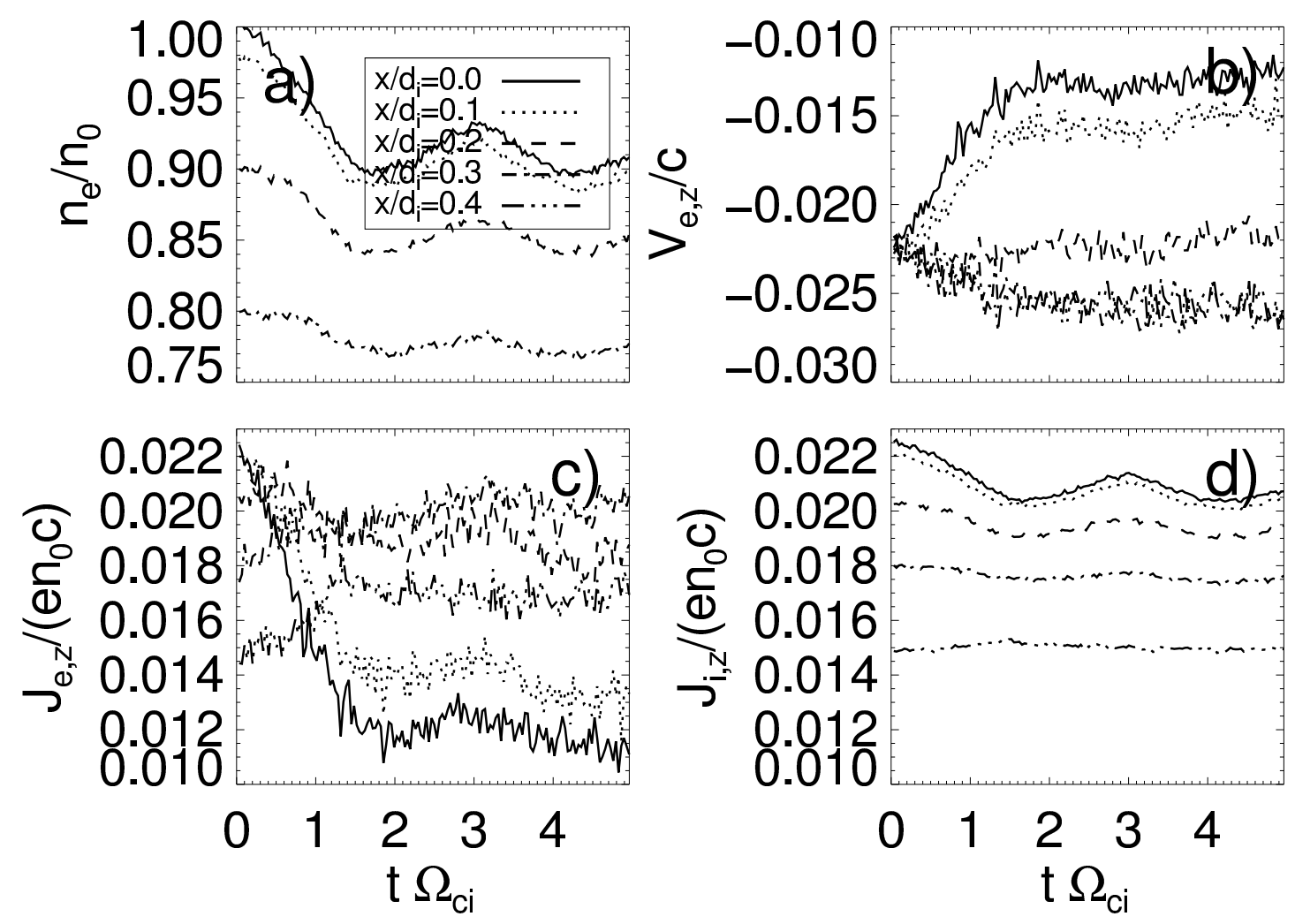

Figure 7.20: (Short) time evolution for some quantities related with bifurcation, averaged along $y$, at different distances $x$ from the center of the CS, for the run TSC-40ppc-A1.96. a) Electron density $\left.n_{e} . b\right)$ Electron bulk speed $V_{e, z} . c$ ) Electron current density $J_{e, z} . d$ ) Ion current density $J_{i, z}$. We do not show the ion density $n_{i}$ since its values in the course of the evolution are always very close to $n_{e}$, i.e.: the quasineutrality condition is kept everywhere to a large extent. To be compared with Fig. 7.17. Reproduced with permission from $P$. A. Muñoz, P. Kilian, and J. Büchner, Physics of Plasmas 21, 112106, (2014) Copyright 2014, AIP Publishing LLC.

\subsubsection{Influence of ions in the temperature anisotropy driven instabil- ities}

In this subsection we discuss the effects of an ion temperature anisotropy on the development of the aforementioned processes. For this sake, we used the results of several simulations runs not shown here for brevity. In the first set of runs, we initialize the CS with anisotropic electrons identical to the runs TSC-40ppc-AX (with $X \in[0.64,1.96]$ ) shown in Table 7.2, but enforcing isotropic ions $A_{i}=T_{i, \|} / T_{i, \perp}=1$. This allows to study the isolate the influence of anisotropic electrons. We got almost identical results to the already shown in e.g., Fig. 7.9 or Fig.7.19 (that is why it is not worthwhile to repeat them here). There are small differences, however. This can be expected because our choice of parameters implies an initial temperature ratio different from one $T_{i, \|} / T_{e, \|} \neq 1$, favoring different processes related with the Landau damping of some wave modes, as well as the thresholds and growth rates of Weibel/mirror instabilities.

In the second set of runs, we carried out simulations with an "opposite" anisotropy: the $\mathrm{CS}$ is initialized enforcing isotropic electrons $A_{e}=T_{e, \|} / T_{e, \perp}=1$ but allowing anisotropic 
ions with $A_{i} \in[0.64,1.96]$, and therefore calling them TSC-40ppc-AiX (with $X \in[0.64,1.96]$ ). Otherwise, the parameters are identical to the first set of runs. The results shown that the ion anisotropy is practically unchanged during the course of the evolution of the system (before the explosive phase of reconnection). The growth of tearing mode very similar to the isotropic run TSC-40ppc. The negligible tendency towards the isotropization of ion anisotropy can be entirely explained by numerical pitch angle scattering due to the low value of ppc. This is in agreement with the theory of anisotropic tearing by Quest et al. (2010): the tearing mode will be stabilized by an ion anisotropy only if it is much larger than any of the values of $A_{i}$ considered here. Then, this is not important for the numerical simulations of CS, since it is not likely at all such scenario due to the numerically produced heating, affecting mostly electrons. There is also no other temperature driven instabilities such as Weibel instability, since they require anisotropic electrons instead of ions (more rigorously, the ion effects are smaller by a factor $m_{i} / m_{e}$ ). The latter fact has a consequence an absence of bifurcation. Finally, it is interesting to mention than in the hypothetical case of an initially given magnetic field at the center of the CS (replacing the Weibel generated one), isotropic electron but anisotropic ions, the mirror instability can be triggered, since it also depends on ion anisotropy to more or less the same extent as for the electron anisotropy. (see Eq. (5.59)).

Therefore, we have proved that in an initially anisotropic CS, the stabilization of tearing mode together with Weibel/mirror instabilities developing in an earlier stage and producing bifurcation afterwards, are only due to the electron anisotropy, with ions playing a secondary role.

Let us close this section by summarizing the main result. We confirm that an imposed electron temperature anisotropy $A_{e}>1$ in a CS can reduce tearing mode growth rates, and even stabilizing it completely if it is strong enough. And beyond a specific threshold, it can drive temperature anisotropy instabilities such as Weibel and mirror, leading to a bifurcated structure in $J_{z}$ for cases with non-negligible numerical noise and associated pitch angle scattering (always present in PIC simulations).

\subsection{Influence of numerical parameters on the tearing in- stability}

The purpose of this section is to confirm and reproduce the theoretical predictions about tearing mode linear growth rate if (and only if) all the previous numerical considerations are taken into account. In particular, we demonstrate the influence of some numerical parameters: shape function, macroparticle number and mass ratio on these growth rates,measuring how fast is the build-up phase of magnetic reconnection.

\subsubsection{Influence of the shape function}

\subsubsection{Time evolution of power in tearing islands}

For the run TSC-40ppc, the CS develops from the beginning a long chain along $y$ direction of small magnetic islands arising from the numerical noise (mimic of the physical thermal fluctuations, but greatly enhanced due to the macrofactor). As expected 
from the linear theory (see details in Sec. 5.1), these structures start to merge generating larger magnetic islands until they saturate for a size on the order of $d_{i}$. For our parameters, and according to the discussion in Sec.7.1.3, there are be seven unstable modes satisfying $k_{y} L \leq 1$. The time evolution of these modes can be estimated, following Katanuma and Kamimura (1980), by integrating the Fourier modes of the vector potential $\left|A_{z}\left(x, k_{y}\right)\right|$ inside of the electron singular layer thickness $\pm \Delta_{N S}=\sqrt{2 \rho_{e} L}$ (see details in Appendix B.2.3). The results for the run TSC-40ppc and CIC-40ppc for comparison purposes are shown in Fig.7.21.
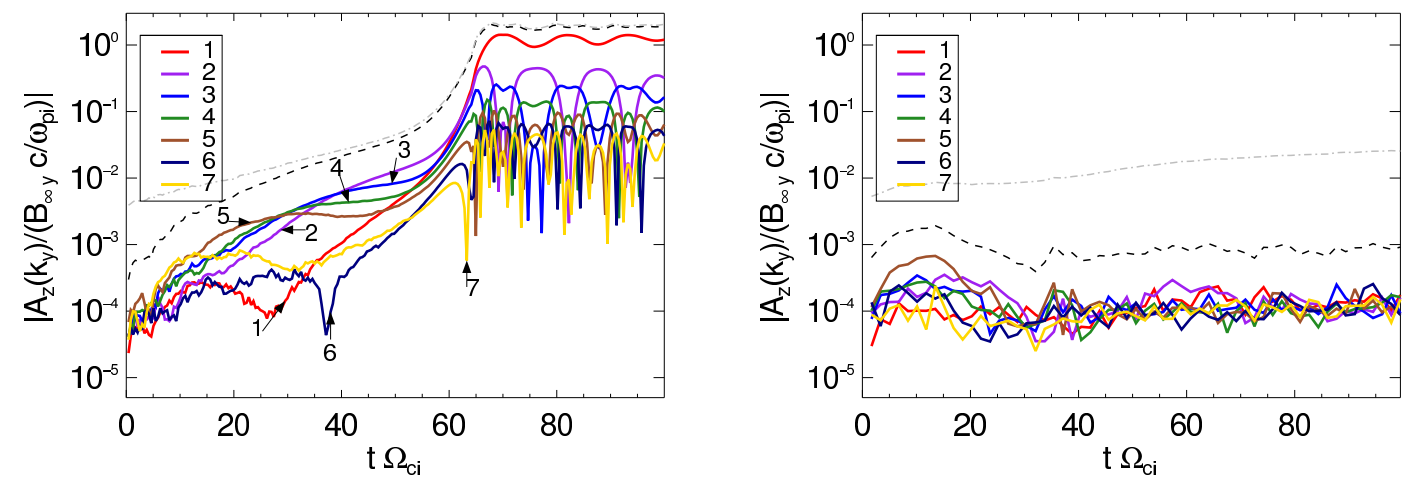

Figure 7.21: Time history of the first seven Fourier modes $\int_{x=-\Delta_{N s}}^{x=\Delta_{N s}}\left|A_{z}\left(x, k_{y}\right)\right| d x$. The dashed gray line is obtained as a sum of all Fourier modes. The dashed-dotted black line is the sum of the first seven Fourier modes shown in this plot. Left panel: TSC-4Oppc Right panel: CIC-40ppc. Reproduced with permission from P. A. Muñoz, P. Kilian, and J. Büchner, Physics of Plasmas 21, 112106, (2014) Copyright 2014, AIP Publishing LLC.

First of all, we can note in Fig. 7.21(1eft-panel) that all the seven modes reach saturated stage at the same time as the reconnected flux (see Fig. 7.3(b)). On the other hand, the suppression of tearing mode can be evidenced in Fig. 7.21(right-panel) as the non growing behaviour of the same seven modes or magnetic islands, correlated with the reconnected flux (see Fig. 7.3(a)): they are kept at the initial noise level. As we demonstrate in the previous sections, this is evidence of the stabilizing effect of bifurcation, dominating the evolution of the CS, and in agreement with a number of previous studies (Camporeale and Lapenta 2005, Matsui and Daughton 2008).

In Fig. 7.21 (a) is also plotted the sum of all Fourier modes (gray) and the sum of the first seven ones (black). The discrepancy between both curves at early times is evidence that the shortest wavelengths modes contribute to the total power only at the beginning. The global structure is then dominated by large scale magnetic islands, with a negligible contribution of the shorter scales (tearing mode is a macroscopic instability). On the other hand, for the bifurcated CS in the CIC-40ppc run shown in Fig. 7.21(b), the curves for the sum of all Fourier modes allow us to infer that the shortest wavelengths modes with $k_{y} L \gg 1$ carry a significant part of the total power throughout the evolution of the CS, in contradiction with the linear theory.

\subsubsection{Identification of linear/non linear stage}

Before the actual calculation of tearing growth rates, we need to identify the linear stage of the evolution of the different tearing modes showed in Fig. 7.21 (for the linear 
regression). Because the complex interactions of these multiple modes at different time periods, the procedure is not straightforward. We follow a criterion based on the behaviour of the shorter wavelengths modes with $k_{y} L>1$ (mode number $>7$ ), stable according to the linear theory. The linear stage is chosen up to the time in which they start to grow, $t \Omega_{c i} \sim 35$. This is the breaking point in time, in the sense that later the CS enters into the non-linear stage, not being possible a comparison with linear theory anymore. However, note that this is only a first order approximation, since some modes reach non-linear stage before than other ones. Taking into account all these details, we can nicely see a comparison in Fig. 7.22, showing the time history of the profiles of the vector potential along the center of the CS, $A_{z}(x=0)$, up to the end of the linear stage (left panel) and up to the end of the non-linear stage (right panel), corresponding to the explosive phase of reconnection, just before of the disruption of the CS.
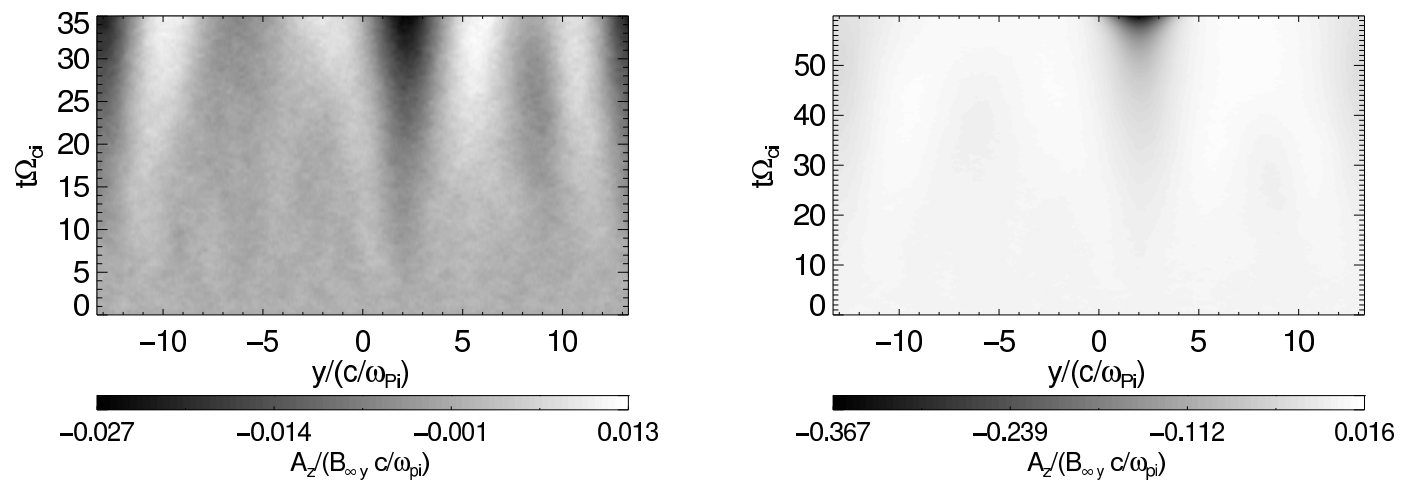

Figure 7.22: Time history of the profiles of vector potential $A_{z}(x=0, t)$ for the run TSC40ppc. Left panel: up to the limit of linear stage of tearing mode evolution $\left(t \Omega_{c i} \sim 35\right)$. Right panel: up to the explosive phase of reconnection $\left(t \Omega_{c i} \sim 60\right)$. In these stacked plots, the maxima/minima of $A_{z}$ are $O / X$ points of the magnetic islands. Reproduced with permission from P. A. Muñoz, P. Kilian, and J. Büchner, Physics of Plasmas 21, 112106, (2014) Copyright 2014, AIP Publishing LLC.

Fig.7.22(left panel) shows clearly four magnetic islands dominating the system at the end of the linear stage. This is in agreement with the linear theory (see Pritchett et al. 1991, or discussion in Sec. 5.1.2.2): the most unstable mode should have $k_{v} L \sim 0.545$ or $M=4$. This mode is shown as a green line in the left panel of Fig. 7.21. By using this value, we can estimate their size $H_{s}$ along $y: H_{s} / d_{i}=2 \pi /\left(k_{y} d_{i}\right)=6.66$. But these islands are very shallow: the difference in $A_{z}$ between their $\mathrm{O}$ and $\mathrm{X}$ points (reconnected flux) only exceeds marginally the surrounding noise level. This can be expected from this build-up phase of magnetic reconnection: the conversion of magnetic energy is not efficient.

On the other hand, Fig. 7.22(right panel) shows only one dominating magnetic island: during the non-linear stage, the power is continuously transferred towards the largest wavelength mode allowed in the system $\left(k_{y} L=0.136\right.$ or $\left.M=1\right)$, something not predicted by the linear theory. This magnetic island has a much larger reconnected flux than during the linear stage, evidence of the explosive and energetically efficient phase of magnetic reconnection. 


\subsubsection{Comparison with linear growth rates}

By applying the previous method, we can finally perform a linear regression to the seven (integrated) Fourier modes of $A_{z}$ (shown in Fig. 7.21 (left-panel)) during the linear stage of the evolution of the CS (up to $t \Omega_{c i} \sim 35$, shown in Fig. 7.22(left-panel)) and obtain the tearing mode growth rates $\gamma$ as function of the modes $k_{y} L$ shown in Fig. 7.23. We also show a few short-wavelengths modes $k_{y} L>1$ to prove that they are indeed linearly damped. The error bars are to compensate for the fact that some modes reach the linear stage earlier than other ones. We chose several time windows varying the length of the linear fitting from very early and up to $t \Omega_{c i} \sim 35$. Each one of these choices provides a slightly different $\gamma$ (for a strictly exponential growth during that period, all of them would be identical). Their mean value is plotted in Fig. 7.23, while their standard deviation are represented by the error bars lengths.

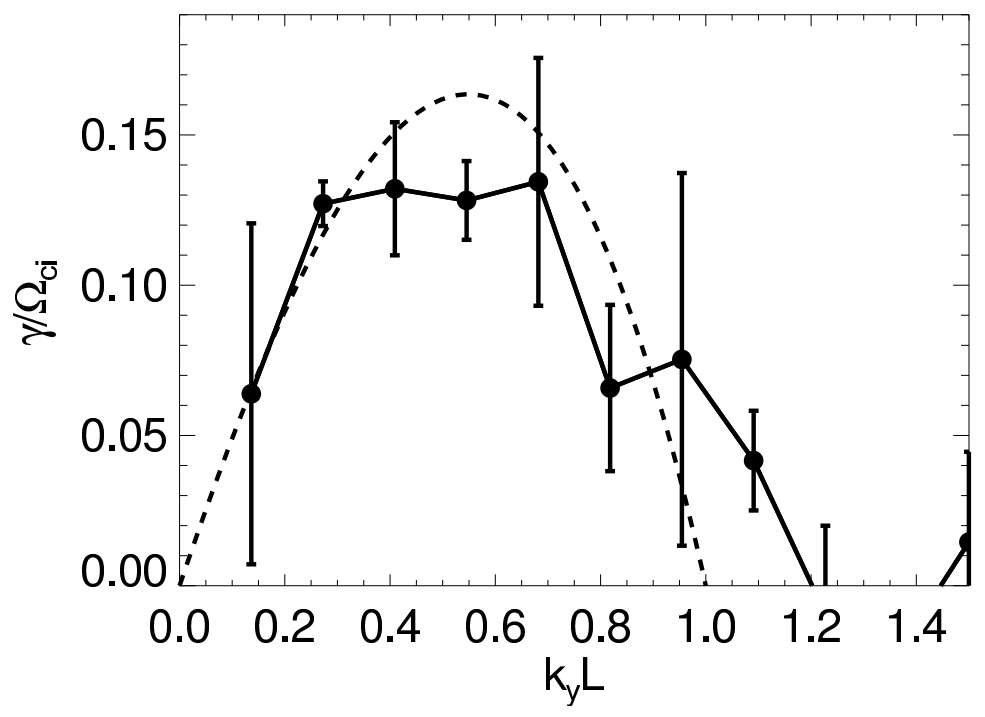

Figure 7.23: Dots connected by the solid line: simulated growth rates $\gamma$ of the Fourier modes $\int_{x=-\Delta_{N s}}^{x=\Delta_{N s}}\left|A_{z}\left(x, k_{y}\right)\right| d x$ (shown in Fig. 7.21left-panel) vs wave number $k_{y} L$ for the run TSC-4Oppc (see text for the calculation method). Dashed line: analytical estimate of the growth rate according to Eq. (7.6) for thin CS and $m_{i}=m_{e}$. Reproduced with permission from P. A. Muñoz, P. Kilian, and J. Büchner, Physics of Plasmas 21, 112106, (2014) Copyright 2014, AIP Publishing LLC.

The simulation results in Fig. 7.23 are compared to an analytical formula for the tearing mode growth rate in thin CS derived by Pritchett et al. (1991) and given in Eq. (5.14). For our parameters is:

$$
\frac{\gamma}{\Omega_{c i}}=0.2591 k_{y} L\left(2+k_{y} L\right)\left(1-k_{y} L\right)
$$

The differences between the analytical curve and our results are due to several reasons. First of all, as we already mentioned in the discussion of Eq. (5.14), the analytical curve have strong simplifying assumptions, such as equal $m_{i}=m_{e}$. More accurate numerical solutions of the Vlasov equation (Brittnacher et al. 1995, Daughton 1999, Silin et al. 2002) have shown than with realistic mass ratios the growth rates are reduced by a factor of 1.5 
to 2 (see Schindler 2007, Sec. 10.4). This might explain the discrepancy for the fastest growing mode $k_{y} L \sim 0.545$ with $\gamma / \Omega_{c i}=0.163$. Other differences can be understood since we are simulating multimode tearing, with a corresponding coupling and non-linear transfer of energy among different modes (Karimabadi et al. 2005). This is the reason because it is not worthwhile to compare our simulations results against more accurate numerical expressions (such as the previously mentioned), since the numerical uncertainties are larger than any improvement over the simple estimate Eq. (7.6).

\subsubsection{Influence of the number of macroparticles}

Here we are going to investigate the effects of the number of macroparticles per cell on the tearing mode growth rates. In order to do that, we use the simulation results of the run TSC-640ppc (see Table 7.2), with 16 times more particles than the previously analyzed TSC-40ppc, and thus reduction of numerical noise to one-quarter. The results for the time evolution of the integrated Fourier tearing modes and corresponding growth rates are shown in Fig.7.24.
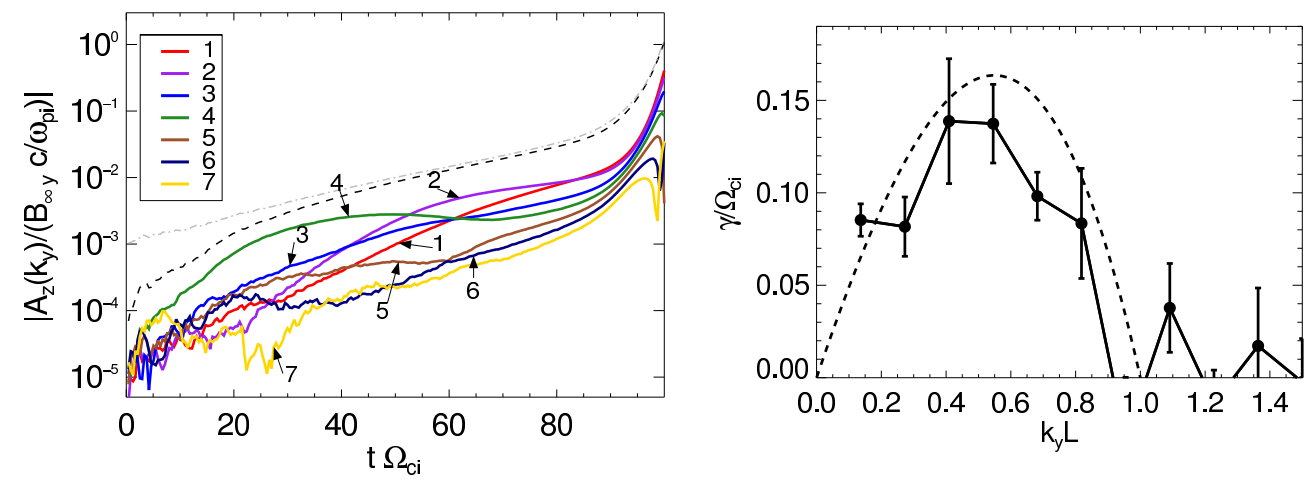

Figure 7.24: Left panel: Time history of the first seven Fourier modes $\int_{x=-\Delta_{N s}}^{x=\Delta_{N s}}\left|A_{z}\left(x, k_{y}\right)\right| d x$ for the run TSC-640ppc. To be compared with Fig. 7.21(left-panel), for the run TSC40ppc with sixteen times less macroparticles per cell. The dashed gray line is obtained as a sum of all Fourier modes. The dashed-dotted black line is the sum of the first seven Fourier modes shown in this plot.

Right panel: Dots connected by the solid line: Simulated growth rates $\gamma$ of the previous Fourier modes vs wave number $k_{y} L$ for the run TSC-64Oppc (see text for the calculation method). Dashed line: analytical estimate of the growth rate according to Eq. (7.6) for thin CS and $m_{i}=m_{e}$. Compare with the run TSC-4Oppc (sixteen times less macroparticles per cell) shown in Fig. 7.23. Reproduced with permission from P. A. Muñoz, P. Kilian, and J. Büchner, Physics of Plasmas 21, 112106, (2014) Copyright 2014, AIP Publishing $L L C$.

By comparing the corresponding Fourier modes for different number of macroparticles per cell, one can see a delay in the onset of the explosive phase of reconnection for the run TSC-640ppc. This also implies a delay in the dominance of the most unstable mode $(M=4)$, decreasing its growth rates, in agreement with previous studies (Matsui 2008). A better match with the theoretical result is expected for higher values of ppc with the corresponding lower numerical noise level. Nevertheless, the fastest growing mode is still 
the same, agreeing with the linear theory. Regarding other modes, the discrepancy can be understood since some non-linear effects are enhanced in presence of higher numerical noise level, modifying the transfer of power at different stages among the different tearing modes, with a corresponding variation in the measured growth rates (Matsui 2008), lower in general for higher ppc. In particular, note that the modes $M=1$ and $M=7$ have much smaller error bars in TSC-640ppc than TSC-40ppc. This is consequence of the fact that numerical noise mostly affects the modes with smaller growth rates, shortening the duration of their linear growth phase when it is enhanced (for TSC-40ppc).

\subsubsection{Influence of the mass ratio}

In the last part of this section, we investigate the influence of the mass ratio in all the aforementioned processes. For this purpose, we use simulations runs with very similar parameters to our standard run TSC-40ppc but with a lower mass ratio $m_{i} / m_{e}=25$. This a value typically used in many former studies of magnetic reconnection, such as the iconic "GEM challenge"(Birn et al. 2001). The results showed no significant numerical heating with the CIC scheme in comparison with our standard case CIC-40ppc. The overall evolution was very similar to these with the TSC scheme: the energy was well preserved and no significant numerical temperature anisotropies were seen, with the consequent absence of numerical artifacts leading to stabilization of tearing mode, such as bifurcation. The CS developed, with both CIC and TSC schemes, the growth of the tearing mode leading finally to the explosive phase of magnetic reconnection, with all the features predicted by the linear theory. In particular, we measured similar growth rates for both cases.

The previous behaviour can be understood recalling that the evolution of tearing mode takes place at ion scales, $\Omega_{c i}^{-1}$, while the number of iterations performed by the code has to be proportional to $\omega_{p e}^{-1}$ due to stability reasons. A smaller mass ratio will imply a shorter separation of time scales between electron and ions, since it will decrease the frequency ratio $\Omega_{c i}^{-1} / \omega_{p e}^{-1} \propto m_{i} / m_{e}$ (see relations in Appendix A.3.1). There are two consequences of this fact. First, the number of iterations before the onset of the processes depending on $\Omega_{c i}^{-1}$, such as tearing mode, will be smaller for lower values of $m_{i} / m_{e}$. And second, the accumulation of numerical errors, stochastic heating and consequently total energy will be reduced for smaller mass ratios, since it depends on the number of timesteps and thus on $\omega_{p e}^{-1}$ (see Hockney and Eastwood 1988, Sec. 9.2).

The mass ratio is also essential at shorter time scales than those of the tearing mode. Indeed, it plays a key role during the initial evolution of the runs with an initially imposed anisotropy discussed in Sec.7.3. It can be shown that both Weibel and mirror instabilities have growth rates increasing with higher values of $m_{i} / m_{e}$ (see Sec.5.2.1 and Sec. 5.2.2). Thus, PIC simulations with a too small mass ratio will underestimate the importance of these instabilities, leading to an incorrect description of the physical evolution of the CS. Moreover, the dependence of the growth rates on mass ratio is not the same for these anisotropy driven instabilities, as well as the tearing mode and many other kinetic instabilities. The change of this parameter might change the dominant instability in a CS, modifying completely the predictions given by PIC simulations (see for further details Bret 2009, Bret and Dieckmann 2010).

Thus, our conclusion for this additional run with small mass ratio is that this choice might hidden numerical instabilities (related with the anisotropic heating) that will only 
appear with more realistic (higher) mass ratios. We thought that the use of low mass ratios is the reason behind the fact that bifurcation was not seen in past PIC simulations of CS. However, PIC simulations of CS with low mass ratio might produce a completely different evolution of many physical quantities related with the reconnection process, as it has been pointed out by Karimabadi (2005).

Finally, we can conclude this section by saying that for a correct description of the time evolution of the tearing unstable modes, is essential to use at least the TSC instead of the CIC shape function, unless a very large number of macroparticles per cell are used in the simulation, something impractical from the point of view of computational savings. This requirement becomes essential in regimes with realistic (higher) mass ratios, in order to avoid instabilities associated with the numerical heating.

\subsection{Conclusions}

In this chapter, we have investigated the effects of numerical parameters on PIC simulations of collisionless Harris current sheets, in order to have a correct physical description of their evolution. Our main finding is that higher order shape functions are more efficient than increasing the number of macroparticle per cell for avoiding numerical instabilities. In particular, the use of a quadratic interpolation (TSC shape function) improves significantly the energy conservation over long times than the traditionally used linear interpolation (CIC shape function). As a result, PIC simulations with TSC shape function allow to suppress numerical collisions and anisotropic heating at computationally less cost than a larger number of macroparticles per cell. This is especially critical for larger (more realistic) mass ratios and for long PIC runs. Our results agree with a previous study of laser wakefield interactions by PIC code simulations (Cormier-Michel et al. 2008).

The previous conclusion is based in the fact that lower order shape functions (such as the often used CIC) or a insufficient number of macroparticles per cell can enhance the numerical collisions inherent to all PIC codes, leading to results with large deviations from the ideal collisionless Vlasov plasma model. This is because numerical collisions due to the coarse-graining of phase space correspond to irreversible processes, enhancing the entropy of the system due to an effective diffusion coefficient of the Boltzmann equation (a measure of the collisionality). We found that this undesirable process can be efficiently avoided by choosing higher order shape functions (see Fig.7.6), since the diffusion coefficient, and so the differences between the predictions of Vlasov and collisional plasmas, are reduced by this numerical parameter (see Eq. (6.44) and Eq. (6.43)).

Since numerical collisions can be seen as an effective scattering, they cause numerical heating, leading to an artificial (and irreversible) increase in the total energy of the system (see Fig.7.1(a)). It is important to remark than this effective-scattering heating is different from the grid heating studied since many decades ago (Langdon 1970). The latter is produced when the grid cell size is insufficiently resolved (larger than Debye length), not in our case. We also confirm that this numerical heating affects mostly electrons, being negligible for ions since it depends inversely on the mass ratio.

The previous numerically generated electron heating is anisotropic (see Fig.7.5). As pointed out by Matsuda and Okuda (1975), this is due to the anisotropic nature of numerical collisions in 2D3V PIC code simulations embedded in magnetic fields. We found 
that this anisotropic heating can lead to the stabilization of the tearing mode, the buildup phase of magnetic reconnection. We confirm that the theoretical threshold for this process(Eq. (7.3)), as derived from the theory of (physical) anisotropic tearing mode (Chen et al. 1984), can predict accurately the level of numerical anisotropy (due to either low order shape functions or too small number of macroparticles per cell) necessary for tearing suppression in our PIC simulations. This prediction also applies well for simulations that keep total energy well preserved (TSC schemes) but are initialized with an electron temperature ani sotropy.

We found that electron temperature anisotropies can drive additional instabilities in a CS. Above a certain threshold, feasible to develop in PIC simulations using low order shape functions, CS can bifurcate by reducing the out-of-plane component of the electron density current $J_{e, z}$ close to the neutral line at the center. This is, in turn, due to a reduction in the out-of-plane component of the electron bulk velocity $V_{\rho_{7}}$. Bifurcation has shown to be produced due to electron chaotic scattering (Lee and Büchner 2012), a process that can be developed if the numerical anisotropy is not controlled appropriately. Moreover, bifurcation takes place at the center of the CS, since collisions and associated scattering are stronger in regions where the magnetic field strength is minimum (Matsuda and Okuda 1975). We also confirmed that bifurcated CS inhibit tearing mode, in agreement with previous studies (Camporeale and Lapenta 2005, Matsui and Daughton 2008).

We also showed that CS can spontaneously bifurcate if an electron temperature anisotropy is imposed initially in the system, even in PIC simulations using interpolation schemes with good energy conserving properties (TSC shape function). The bifurcation strength is dependent, to a certain degree, on the noise level as evidenced by the macroparticle number. This is consequence of being generated by numerical scattering, which disappear only in the limit of negligible noise (infinite macro-particle number), a non-physical limit. We found the threshold for this process to be around $A_{e} \gtrsim 1.2$, just a little bit above the analytical estimate for tearing mode stabilization given by Eq. (7.3), a result derived from the theory of anisotropic tearing instability. It is convenient to keep in mind than this threshold should be taken just a first order approximation, since it was derived for collisionless Vlasov plasmas, a non valid assumption for the typical collisions levels usually found in PIC simulations of this physical system

We also explained the physical mechanism by which the CS bifurcates in simulations with an initially imposed temperature anisotropy. The initial state is still a Harris equilibrium, but it turns out to be unstable (besides of tearing mode) to the temperature ani sotropy driven Weibel instability close to the unmagnetized neutral line. Above a certain threshold (Eq. (7.4)), the imposed anisotropy will have to be relaxed at very fast time scales (much shorter than those of tearing mode). This process is practically independent on the macroparticle number. We found several predicted signatures of this instability, in particular the generation of filamented structures in the out-of-plane magnetic field $B_{z}$. This magnetic field provides the seed for the generation of field aligned temperature anisotropy, producing the conditions for the triggering of mirror instability. Both instabilities grow together during a short period close to the center of the CS, producing enhanced scattering due to release of the source of their free energy: the temperature anisotropy. Finally, this process leads to a bifurcation of the CS.

We also proved that only the electron temperature anisotropy is the quantity that matters for all the aforementioned numerically instabilities. In particular, by isolating the in- 
dividual contributions of ions and electrons, we confirmed that ion anisotropy do not play a relevant role in stabilization of tearing mode, at least for the levels of anisotropy feasible to be developed in PIC simulations of CS. This also agrees with previous theoretical findings (Quest et al. 2010). Furthermore, a CS with an initially imposed ion temperature anisotropy will not be relaxed before the tearing growth mode takes over. Indeed, the previous mechanism by which Weibel and mirror instabilities might be triggered in this scenario will only take place under the presence of an electron temperature anisotropy.

Finally, taking into account all these numerical considerations, in particular the choice of the TSC shape function interpolation scheme, we could calculate growth rates of the tearing mode, matching to some degree with the linear theory (see Fig. 7.23 and Fig. 7.24(right-panel)). The discrepancies can be explained due to the presence of multiple tearing modes, with the consequent non linear exchange of power among them, but also due to the enhanced noise level arising as a result of the coarse graining effect (insufficient number of macroparticle per cell). 



\section{Instabilities of Harris CS in the presence of small guide fields}

Strong magnetic guide fields along the current direction is an essential feature of current sheets in the solar corona outside magnetic null points. For this reason it is of fundamental importance to understand the stability properties of collisionless current sheets and magnetic reconnection in the presence of a relatively strong guide field. In the HallMHD model (see Sec.4.3), previous investigations have shown that a guide field affects the properties of dispersive waves allowing fast magnetic reconnection, the dissipative mechanisms responsible for the breaking of the frozen-in condition (see Sec. 4.2.3 and Sec. 4.2.4). On the other hand, kinetic studies revealed the influence of the magnetization by the guide fields of the electron orbits responsible for the tearing instability (see Sec.5.1.3). In general, guide field makes the reconnection process less efficient.

However, many unresolved questions remain, in part due to the analytical and numerical difficulties of treating the complications due to the addition of a guide field to the Harris CS equilibrium. Also, a high numerical resolution is necessary for having reliable PIC simulations addressing the onset of magnetic reconnection (tearing instability), since the numerical stability conditions and other constraints are much more severe in the case of strong guide fields rather than in the antiparallel configuration discussed in Chapter 7 . As a result, there is no agreement yet in the main contribution to the reconnected electric field in Harris CS equilibria with guide fields. In particular, the generalized Ohm's law, which has to be analyzed using a mean field approach, was not considered carefully enough in the past. Also, the properties of secondary instabilities resulting from the reconnection process under the guide field have not been completely understood yet. For these reasons, in this chapter we address these questions, starting with CS with relatively small guide fields $\left(b_{g} \lesssim 7\right)$, taking into account the computational constraints to be discussed later.

This chapter is organized as follows. First, in Sec. 8.1, we describe the simulation setup of single and double Harris CS with guide field . In Sec. 8.2 we analyze the numerical considerations to be taken into account for a proper modeling of guide field reconnection. Once this point is settled, we analyze the evolution of tearing instability and the onset of magnetic reconnection. In Sec. 8.3 we analyze the mechanism of guide field reconnection by calculating the terms of the generalized Ohm's law which break the frozen-in condition and balance the reconnection electric field. Next, in Sec. 8.4 we discuss the mean field generalized Ohm's law in CS with guide fields as a result of the interaction of particles with electromagnetic fluctuations and the micro-instabilities causing them. Finally, we summarize our findings in Sec. 8.5. 


\subsection{Simulation setup}

\subsubsection{Double Harris CS}

In this chapter from Sec. 8.2 onward 1 , we simulate a double Harris CS equilibrium adding a guide field (see Eq. (3.23)) and a constant background plasma population of electrons and ions (see Eq. (3.24)). This allows to use (double) periodic boundary conditions in the reconnection plane $x-y$ and thus to avoid any artificial numerical effect related with reflecting boundaries, in particular in the background population. The model locates the center of the two CS at $x=L_{x} / 4$ and $x=3 L_{x} / 4$, respectively. The magnetic field is implemented by repeating the single magnetic field profile Eq. (3.23) twice along $x$, with opposite sign in their shear:

$$
\vec{B}(x)=B_{\infty y}\left[\tanh \left(\frac{x-L_{x} / 4}{L}\right)-\tanh \left(\frac{x-3 L_{x} / 4}{L}\right)-1\right] \hat{y}+B_{z} \hat{z}
$$

Consistently (from Ampère's law), each CS is formed by two oppositely directed current density $J_{z}$ :

$$
J_{z}=\frac{1}{\mu_{0}} \frac{\partial B_{y}}{\partial x}=\frac{B_{\infty y}}{\mu_{0} L}\left[\cosh ^{-2}\left(\frac{x-L_{x} / 4}{L}\right)-\cosh ^{-2}\left(\frac{x-3 L_{x} / 4}{L}\right)\right]
$$

For such profile, the Harris populations (for $T_{i}=T_{e}$ ) are formed by the density profile:

$$
n_{\{e, i\}}=\frac{B_{\infty y}}{e \mu_{0} L}\left[\cosh ^{-2}\left(\frac{x-L_{x} / 4}{L}\right)+\cosh ^{-2}\left(\frac{x-3 L_{x} / 4}{L}\right)\right]
$$

The constant particle drift velocity of the single Harris sheets are inverted for each half of the $x$ domain.

$$
V_{\{i, e\}, z}(x)= \begin{cases} \pm U_{z} & \text { if } 0<x<L_{x} / 2 \\ \mp U_{z} & \text { if } L_{x} / 2<x<L_{x}\end{cases}
$$

where the signs \pm correspond to ions and electrons, respectively. Note that at the center the velocity changes discontinuously in $2 U_{z}$. But this strong shear is not a problem at all, since the Harris population vanishes (zero density) at that place if the CS are well separated (so, no particle experiences that effect). All these profiles can be seen in Fig. 8.1

\footnotetext{
${ }^{1}$ Only in the first section about onset of magnetic reconnection and tearing mode, Sec.8.1, a single CS without background will be used.
} 


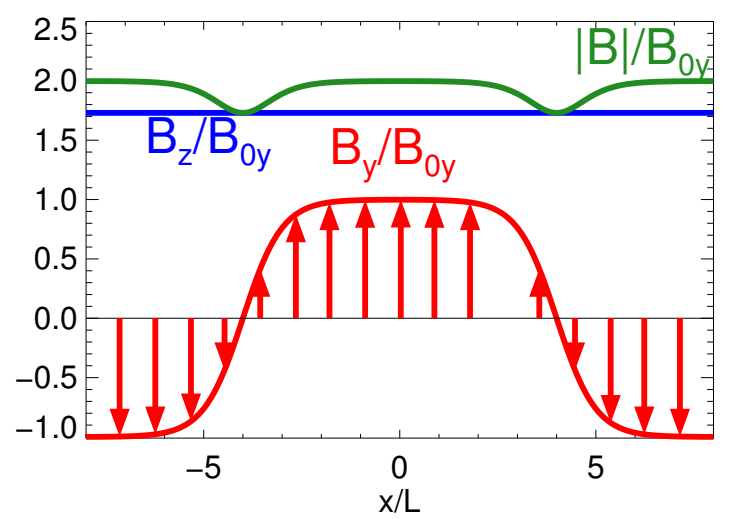

(a) $\vec{B}$

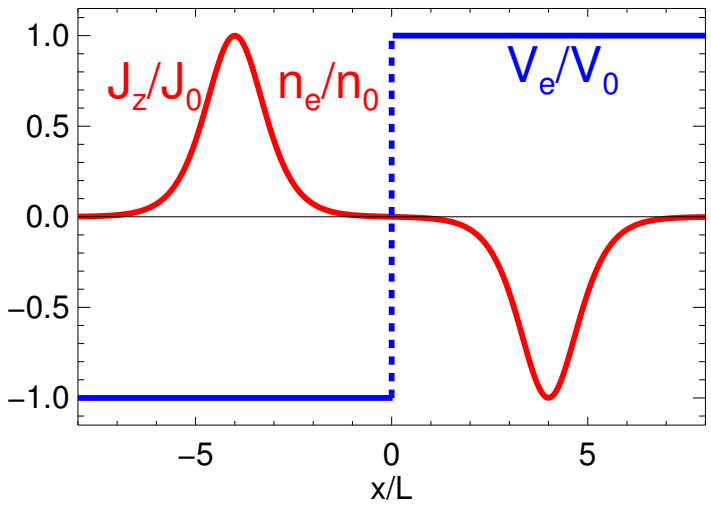

(b) $\vec{J}$

Figure 8.1: Components of the magnetic field $\vec{B}$ and current density $\vec{J}$ profiles for a Harris $C S$ with guide field $b_{g}=1.73$ given by Eq. (8.1) and Eq. (8.2), respectively. . It is assumed that the left $C S$ is at $x / L=-4$ and the right $C S$ at $x / L=4$. Compare with the corresponding force free profiles shown in Fig. 9.1.

Similar to the previous chapter, we do not apply any initial perturbation, allowing thus the study of both onset of spontaneous instabilities (tearing) and later the fully-developed nonlinear stage of magnetic reconnection. Since the evolution of the left and right CS are similar, we focus on the diagnostics of the left CS at $x=L_{x} / 4$ in the rest of this chapter, unless stated otherwise.

\subsubsection{Parameters}

As mentioned in Appendix A.3.3, in addition to the four basic parameters necessary to fully specify a Harris sheet, now we also have to specify the density of the background population $n_{b}$ and the strength of the magnetic guide field.

$$
\begin{aligned}
& \frac{L}{d_{i}}=0.5, \quad \frac{\omega_{p e}}{\Omega_{c e}}=4.16, \quad \frac{m_{i}}{m_{e}}=100, \quad \frac{T_{i}}{T_{e}}=1, \\
& \frac{n_{b}}{n_{0}}=0.2, \quad b_{g}=\frac{B_{z}}{B}{ }_{\infty y} \in[0,7]
\end{aligned}
$$

We use the same conventions for the calculation as in the previous chapter. Note that guide fields up to seven times the Harris asymptotic magnetic field are chosen (although many of the results will be shown only up to a guide field of $b_{g}=3$ ). Numerically we use the TSC shape function to minimize effects related with artificial heating (see Chapter 7 ). Now, let us specify the remaining parameters chosen for the simulation setup, as well as other derived parameters and the relations between them: 


\begin{tabular}{|c|c|}
\hline Parameter & Standard Case \\
\hline$m_{i} / m_{e}$ & 100 \\
\hline$T_{i} / T_{e}$ & 1 \\
\hline$\omega_{p e} / \Omega_{c e}$ & 4.1666 \\
\hline$\sqrt{k_{B} T_{e} / m_{e}} / c$ & 0.12 \\
\hline$L / d_{i}$ & 0.5 \\
\hline$L / \rho_{i}$ & 1.0 \\
\hline$L_{x} / d_{i}$ & $25.13=8 \pi$ \\
\hline$L_{y} / d_{i}$ & $12.56=4 \pi$ \\
\hline$V_{D e 0} / c$ & 0.024 \\
\hline$V_{D e 0} / v_{t h, e}$ & 0.2 \\
\hline$\sqrt{k_{B} T_{i} / m_{i}} / c$ & 0.012 \\
\hline$\rho_{i} / d_{i}$ & 0.5 \\
\hline$\rho_{i} / d_{e}$ & 5.0 \\
\hline$\rho_{i} / \lambda_{D e}$ & 41.667 \\
\hline$d_{i} / \lambda_{D e}$ & 83.333 \\
\hline$L / \lambda_{D e}$ & 41.667 \\
\hline$\omega_{p i}^{-1} / \omega_{p e}^{-1}$ & 10. \\
\hline$\Omega_{c i}^{-1} / \omega_{p e}^{-1}$ & 416.667 \\
\hline $0.5 L_{x} / L$ & 25.13 \\
\hline $2 \pi L / L_{y}$ & 0.25 \\
\hline$\omega_{p e}[H z]$ & $5.0 * 10^{9}$ \\
\hline$\lambda_{D e} / \Delta x$ & 1.432 \\
\hline$\rho_{e} / \Delta x$ & 5.9683 \\
\hline$\rho_{i} / \Delta x$ & 59.683 \\
\hline$L / \Delta x$ & 59.683 \\
\hline$\omega_{p e}^{-1} / \Delta t$ & 23.87324 \\
\hline$\Omega_{c e}^{-1} / \Delta t$ & 99.47183943 \\
\hline$\Omega_{c i}^{-1} / \Delta t$ & 9947.184 \\
\hline$N_{x} / N_{y}$ & $3000 / 1500$ \\
\hline$B_{\infty y}[G]$ & 68.2275 \\
\hline$n_{b} / n_{0}$ & 0.2 \\
\hline ppc Harris & $300 / 250$ \\
\hline ppc background & $60 / 50$ \\
\hline total ppc per specie & $3.77429 \cdot 10^{8}$ \\
\hline
\end{tabular}

Table 8.1: Parameters chosen for the double Harris CS simulations discussed in this chapter. The relations between them were obtained through the expressions given in Appendix A.3.1 and Appendix A.3.3.

According to the discussion in Sec. 7.1.3, the choice of the simulation box along $y$ was chosen to allow the three eigenmodes known to be the most unstable: $M=1,2,3$ 
with $2 \pi M L / L_{y}=k_{y} L=0.25,0.5,0.75$. The corresponding wavelengths are $\lambda=L_{y} / M=$ $1.0 L_{y}, 0.5 L_{y}, 0.25 L y$. This allow us to study multimode tearing but without the additional complications due to possible shorter wavelengths, less energetic eigenmodes, as these considered for non-guide field reconnection in Chapter 7 .

When thi s double CS is used, we discuss the CS instabilities by showing plots centered only in the left CS at $x=L_{x} / 4$. The periodicity in the boundary conditions guarantees us that the evolution of the right half-plane will be similar (but not totally identical in singular details).

The specific order in which the input parameters of this Harris CS are initialized in the ACRONYM code is described in Appendix A.3.3.

\subsection{Onset of guide field reconnection: Tearing instability}

The final purpose of this chapter is studying the physical effects of fully-developed magnetic reconnection. But first we analyze the onset of the tearing mode, since we are not using any definite initial perturbation. In this section we discuss simulations with parameters specified in Table 8.1 but for a single CS without background, $n_{b} / n_{0}=0$. Consistently, we use reflecting boundary conditions, similar to these used in Chapter 7 . The reason is for computational efficiency: this approach allows to reach stronger guide fields by using fewer particles due to not considering a plasma background and a second CS. The trade-off are noisier momenta of the distribution function at the edge of the CS, something not practical for the analysis of the terms in the Ohm's law.

\subsubsection{Numerical considerations with guide field}

In order to simulate correctly the tearing instability, in this subsection we briefly discuss an important numeric issue that deserves to be considered not only for that kind of study, but also for any kinetic investigation of guide field reconnection in general.

As we discussed in Sec. 7.2.5, the addition of a strong guide field to a Harris configuration magnetizes and isotropizes the electrons throughout the CS in the reconnection plane. Therefore, a stabilization of tearing mode instability related with a temperature anisotropy does not play an important role as in the non-guide field case (see Chapter 7). In addition, as predicted by Matsuda and Okuda (1975), the in-plane numerical collisions are reduced in presence of a magnetic field and so any effect related with an increasing of entropy. In our simulations, we measured this quantity, finding decreasing values for higher guide fields, in agreement with this prediction (these results are not shown here).

\subsubsection{Effects of guide field on the accuracy of particle mover}

On the other hand, the gyro-frequency of electrons in the guide field become large and and the Larmor radius smaller if $b_{g}$ is high enough. As we discussed in Sec. 6.8.1, this does not cause any problem for the stability of the Boris pusher used by ACRONYM. For the accuracy, however, the electron gyrofrequency in the guide field $\Omega_{c e}$ has to be well resolved. Hence, the timestep has to be chosen small enough according to Eq. (6.36). This condition is easily satisfied for guide fields up to $b_{g}=7$, according to the parameters 
given in Table 8.1 and the relations given in Eq. A.7):

$$
\Omega_{c e, b g} \Delta t=b_{g} \sqrt{2\left(1+\frac{T_{i}}{T_{e}}\right)} \frac{v_{t h, e}}{c} \omega_{p e} \Delta t \approx 0.01 b_{g} \ll 0.3
$$

where the subscript $b_{g}$ denotes quantities calculated on the guide field, e.g., $\rho_{e, b g}=\rho_{e} / b_{g}$. Here, 0.3 is taken to achieve a $1 \%$ of precision loss for the Boris pusher. We recovered this effect in the increasing in-plane electron temperatures and total energies for large guide field strengths, as we can see in Fig. 8.2. The out-of-plane electron temperature $T_{e, z}$ is also affected by this slow heating, but to a much less extent due to the 2D nature of our setup.
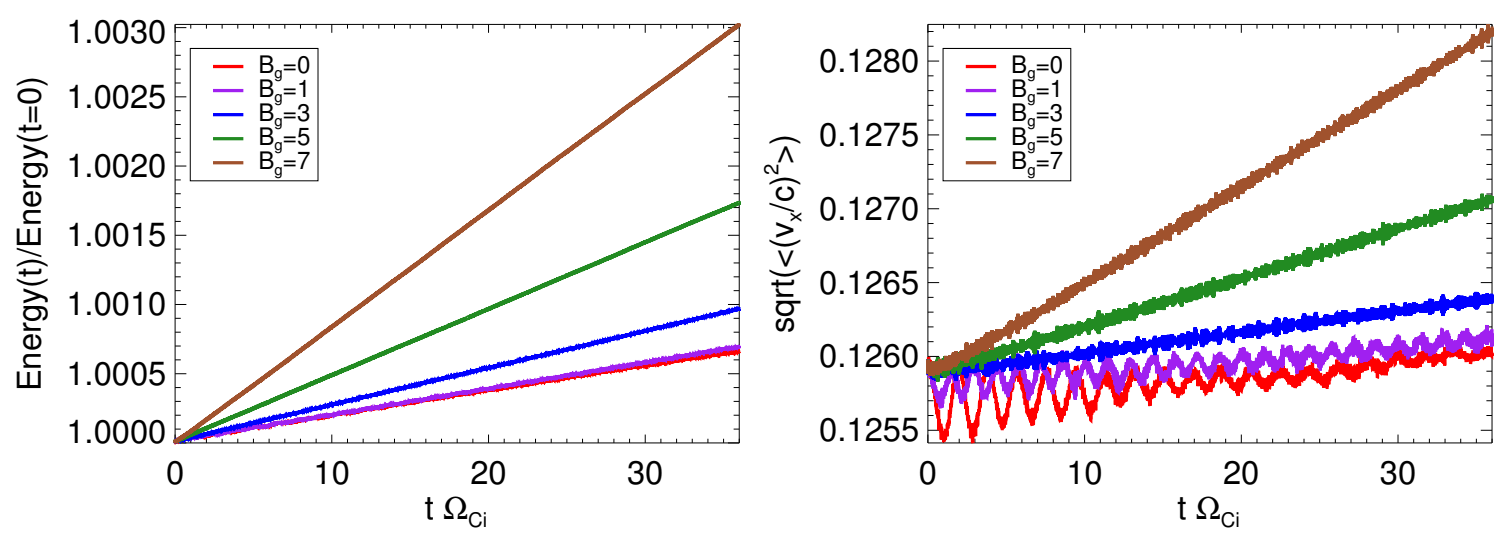

Figure 8.2: Time history of some global quantities for different guide fields. Left: Total energy. Right: Average electron temperature $T_{e, x}$

In closing this issue, it might be interesting to mention that the "wiggles" in the initial stage of both total energy and electron temperature shown in Fig. 8.2 are related with a slight lack of initial equilibrium in our setup. The initialization with the Harris equilibrium as given by Eq. (3.19) is not relativistically self-consistent, in the sense that does not distinguish between $\vec{v}$ and $\vec{u}=\gamma \vec{v}$ entering into the expressions for the temperature that sustains the magnetic pressure (Eq. (3.19)). In particular, the relativistic covariant definition of temperature (related with the stress-energy tensor) involves the covariance between $\vec{u}$ and $\vec{v}$, introducing differences with its non-relativistic definition when the drift speed and/or electron thermal speeds are in the mildly relativistic regime, as in our case. An interesting fact is that the pressure tensor loses its symmetry with the relativistic definition (it is related with the fact that the momentum flux $m \vec{u}$ may have different direction compared to the number density flux $n \vec{v}$ ). We carried out some simulation tests changing the definition of temperature in the Harris equilibrium by its relativistic counterpart Eq. (3.19). The results showed that the initial wiggles practically disappeared (plots not shown here), and that is why we thought that this behaviour is very likely due to this reason. For further details about the relativistic correct definition of temperature, see the articles by Melzani et al. (2014), Sarbach and Zannias (2013), Hesse and Zenitani (2007), Davidson (1988, p. 7) or Davidson (1974, Sec. 1.3.3). 


\subsubsection{Resolution of electron gyration and bifurcation}

However, we noticed an unexpected side-effect related with the electron gyromotion on the guide field when its associated Larmor radius $\rho_{e, b g}$ was not well resolved by the grid, something not directly related with any stability or accuracy condition of the algorithm. For this specific purpose, we analyze a different simulation to the ones specified in Table 8.1: a run initialized with a single Harris CS, no background, a relatively high guide field and other parameter similar to the ones used in Chapter 7 (note especially the higher value of mass ratio).

$$
\begin{aligned}
& \frac{L}{d_{i}}=0.5, \quad \frac{\omega_{p e}}{\Omega_{c e}}=3, \quad \frac{m_{i}}{m_{e}}=180, \quad \frac{T_{i}}{T_{e}}=1, \\
& \frac{n_{b}}{n_{0}}=0.0, \quad b_{g}=7, \quad L_{x} / L_{y}=2 \pi d_{i} / 4 \pi d_{i}, \quad \Delta x=1 \lambda_{D e}, \quad c \Delta t / \Delta x=0.5 .
\end{aligned}
$$

For this case, the ratio between electron gyromotion on the guide field and the grid cell size (given by the relation in Appendix A.3.3):

$$
\begin{aligned}
\frac{\rho_{e, b g}}{\Delta x} & =\frac{c / v_{t h, e}}{b_{g} \sqrt{2\left(1+\frac{T_{i}}{T_{e}}\right)}} \frac{1}{\Delta x / \lambda_{D e}} \approx \frac{3.03}{b_{g}} \\
\Omega_{c e, b g} \Delta t & \approx 0.027 b_{g} \ll 0.3
\end{aligned}
$$

where we have also calculated the time resolution Eq. (8.7) for this case, Now, for a guide field of $b_{g}=7$, 2 the ratio $\rho_{e, b g} / \Delta x \approx 0.43$, i. e. the Larmor gyroradius is under-resolved by the grid by a factor of two. After an initial short transient time, we could notice a drop in $J_{z}$ at the center of the CS, as can be seen in Fig. 8.3. This is analogous to the bifurcation seen in the previous chapter (in particular, see Sec.7.2). However, it is not directly related with an overall anisotropic heating such as in that case, but it is very localized. Moreover, the drop on $J_{z}$ it does not become deeper as the time goes on. This structure remains in a quasi-steady state for very long times. It stabilizes the tearing mode, inhibiting the formation and merging of magnetic islands. In this sense, it is more related with the bifurcated structure developed in Harris sheets with initial temperature anisotropy (see Sec. 7.3), although the physical process does not have to do with enhanced collisions at the center of the CS, since (as we already mentioned) they are strongly reduced in presence of a magnetic field.

In order to test the origin of this bifurcated structure of the CS, we carried out two additional simulations (subscripts " 2 " and "3") with the same physical parameters but changing the grid cell size and time step. In the run " 2 ", we use a double grid resolution: $\Delta x_{2}=0.5 \Delta x$, in addition to a necessary half time step $\Delta t_{2}=0.5 \Delta t$ to fulfill the CFL condition Eq. (6.34) with the same degree of accuracy as the original run: $c \Delta t_{2} / \Delta x_{2}=0.5$.

\footnotetext{
${ }^{2}$ In our standard set of simulations given in Table 8.1, the ratio $\rho_{e, b g} / \Delta x=5.96$, which would require much larger guide fields to see the effect to be shown, and thus much longer simulation times and spatial resolution. In addition, the reduced mass $m_{i} / m_{e}=100$ for the standard parameter set used in this chapter makes faster the development of reconnection process, hiding any numerical effect related with the accumulation of numerical heating and/or errors in the interpolation scheme. A higher mass ratio $m_{i} / m_{e}=180$ used in this special run is more prone to this kind of effect, as we demonstrated in the Chapter 7 . This justifies, in part, the choice of this different setup.
} 
Note that we keep the same physical simulation box, and therefore this simulation run have double of grid points along each direction, in addition to 4 times more total particles and it requires double number of timestep to reach the same physical time. In the run "3" we only change the time step to one half: $\Delta t_{3}=0.5 \Delta t$, while keeping the grid cell size to its original value $\Delta x_{3}=\Delta x$. In this way, the CFL condition is over-resolved $c \Delta t_{3} / \Delta x_{3}=0.25$ (so, it is even better).
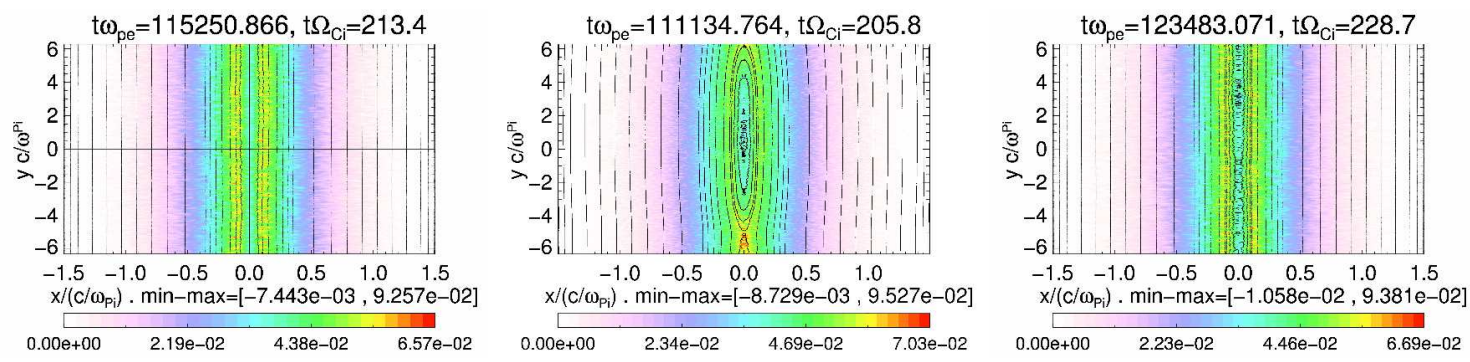

Figure 8.3: Out-of-plane current density for the strong guide field, very late in its evolution, with parameters specified in Eq. (8.8) and different time steps and grid cell sizes. Left: Run "1" with original values of $\Delta x$ and $\Delta t$. Center: Run " 2 " with $\Delta x_{2}=0.5 \Delta x$ and $\Delta t_{2}=0.5 \Delta t$. Right. Run "3" with $\Delta t_{3}=0.5 \Delta t$ and $\Delta x_{3}=\Delta x$

Comparing Fig. 8.3 (left) with Fig. 8.3(right), we can see a practical identical behavior when changing only $\Delta t$, an indication that bifurcation it is not related with the accuracy of the algorithm. On the other hand, there is a striking difference between Fig. 8.3 (left) and Fig. 8.3 (center), obtained by changing only the spatial resolution (grid cell size). We can see in the higher resolution simulation the formation of a magnetic island (with its $\mathrm{X}$ point close to the bottom of the simulation box), a signature of the tearing mode growth, the "right" physical behaviour as we explained in Chapter7: bifurcation does not appear and there is no stabilization of tearing mode. This is associated with an enhanced anisotropy $T_{x}>T_{z}$ very localized at the center of the CS with a spatial extent on the order of $\rho_{e, b g}$ (not shown here). In contrast to the expectations mentioned at the beginning of this section, it seems that collisions with the associated enhanced scattering do play a role even in this case of strong guide field, but different from the antiparallel case, they are anisotropic when comparing in-plane $(x-y)$ with the preferential out-of-plane direction $z$ (and not between $x$ and $y$ ). A higher grid resolution implies that the numerical inter-particle force due to the grid is smoothed out, and so any numerical effect related with collisions that may lead to bifurcation.

As we briefly mentioned in the discussion of Eq. $(6.36$ ), in principle there is no constraint in the relative ratio $\rho_{e, b g} / \Delta x$ due to the Boris algorithm, because it always gives the same particle orbit when the fields are interpolated back to the particles, independent on the previous ratio. However, this fact is only valid for homogeneous magnetic fields. Indeed, Melzani et al. (2013) noticed a numerical instability in cases where $\rho_{e, b g}$ is not well resolved by the grid cell size, manifested in a sudden increase of the total energy in their simulations of uniform and cold magnetized plasmas. They attributed that instability to the coupling between the aliases of the wavenumbers associated with the electron gyration $k_{n}=k \pm 2 \pi n /\left(2 \rho_{e, b g}\right)$ (with $n$ integer) and the local perturbations in the magnetic field, a mechanism analogous to the finite grid or aliasing instability when the Debye length 
is not well resolved (see Sec. 6.8.2.1). Melzani et al. (2013) also pointed out that, since this instability develops slowly, particles that experience short time in regions with underresolved Larmor radii should not affect too much to the onset of it. Our simulation of CS exhibits an inhomogeneity in the magnetic field with maximum at the center, where the Harris $B_{y}$ component reverses sign. Therefore, that inhomogeneous region may be more prone to this kind of numerical kinetic instabilities that regions away from the center of the CS, explaining thus the location of bifurcation. Moreover, due to the strong out-ofplane $b_{g}$, the particles are confined to be close to that position for longer times (cross-field motions are severely restricted), providing thus the ingredient necessary to the onset of instabilities that require heating for their triggering. To the best of our knowledge, we could not find any other report about this kind of instabilities for parameter regimes of underresolved Larmor radii. That is the reason why we thought it is important to report the possible origin of bifurcation in CS with strong guide field due to this process. However, more work is needed to reveal the details of the physical mechanism behind bifurcation in our setup.

\subsubsection{Evolution of tearing instability on dependence on guide field}

If we take properly into account the previous numeric issue, i.e., resolving spatially the electron gyration on the guide field by the grid size well enough, one can indeed see the slow growth of tearing mode manifested in the formation and merging of magnetic islands. This can be obtained by using the parameters given in Table 8.1 (in particular, note that $\rho_{e} / \Delta x=5.96$ ).

First, let use compare the evolution of tearing mode for different guide fields by showing the evolution of the reconnected flux $\Psi$ (defined in 4.1) and the integrated Fourier power of the vector potential $\left|A_{z}\left(x, k_{y}\right)\right|$ for the most unstable mode $k_{y} L=0.5$ (following the method used in Sec.7.4.1.1).
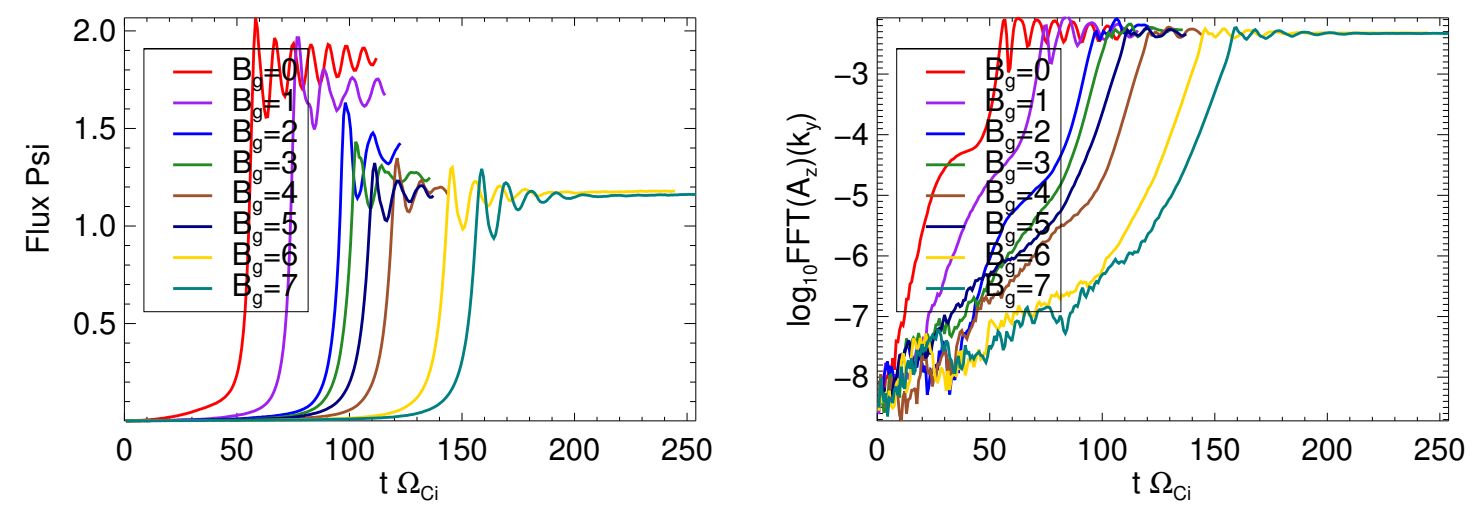

Figure 8.4: Time histories of quantities showing the evolution of tearing mode on dependence on the guide field. Left: Reconnected flux $\Psi$. Right: Integrated Fourier power of the vector potential $\int_{x=-\Delta_{N s}}^{x=\Delta_{N s}}\left|A_{z}\left(x, k_{y}\right)\right| d x$ for the most unstable mode $m=2$ or $k_{y} L=0.5$.

In Fig. 8.4 (left), the peak in the reconnected flux $\Psi$ indicates the explosive phase of reconnection, after the CS is disrupted due to the counterstreaming flows in $y$ direction resulting from the periodic boundary condition (recall discussion of Fig. 7.3 ). Since this 
peak is reached for later times for stronger guide fields, we infer that the entire reconnection onset is delayed for high $b_{g}$. This time dependence for different guide fields is summarized in Fig. 8.5. Note also that the maximum peak value of the reconnected flux is reduced for stronger guide fields, indication of the reduced efficiency of magnetic reconnection in converting the available magnetic energy stored in the system under the influence of the guide field. The large guide field behavior, however, seems to converge towards an asymptotic value for the largest $b_{g}$ considered in this study.
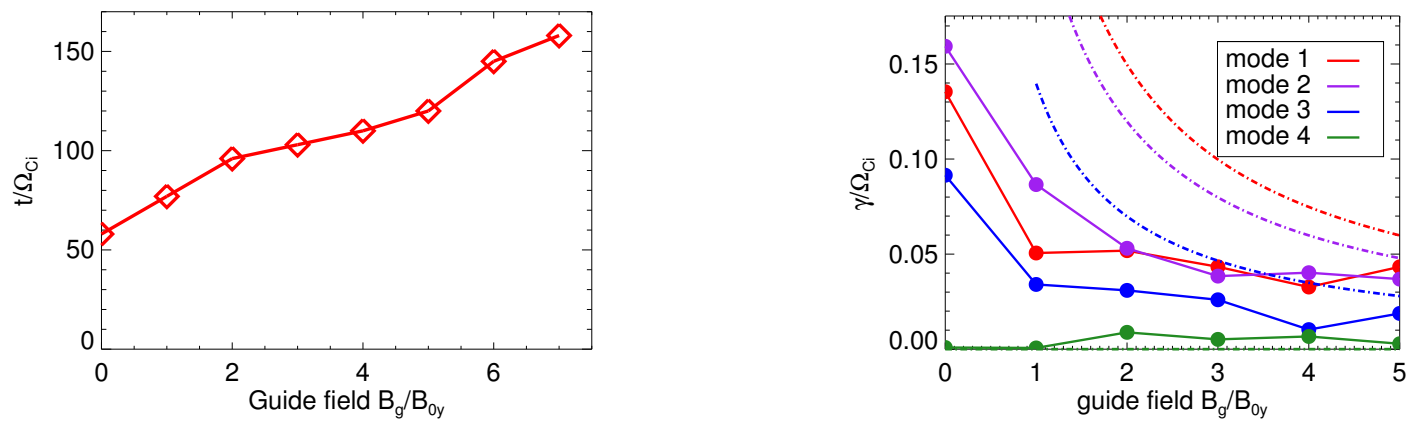

Figure 8.5: Left: Reconnection onset time (explosive phase) vs guide field strength $b_{g}$, estimated from the time history of reconnected flux shown in Fig. 8.4(left). Right: Growth rates $\gamma$ of the first modes of $A_{z}\left(k_{y}\right)$ vs guide field strength $b_{g}$. The dashed lines represent the analytical expression Eq. (8.12).

The delay in the magnetic reconnection onset (see Fig. 8.5 (left)) is also related with a slow down of the merging of magnetic tearing islands for higher $b_{g}$. This process takes place during the initial linear stage of the evolution of the CS (the build-up phase), where magnetic reconnection is not energetically efficient. This behavior can be seen in Fig. 8.4(right), representing the time evolution of the most unstable tearing mode $k_{y} L=0.5(M=2)$ on dependence on $b_{g}$ : the slope is reduced for higher guide fields. The dependence of this slope (growth rate $\gamma$ ) on $b_{g}$, along with the other 2 unstable tearing modes of this CS $(M=1,3)$, is shown in Fig. 8.5(right). The calculation method for $\gamma$ is identical to the already explained in Sec. 7.4.1.3. The dashed lines are from the analytical expression of kinetic tearing growth rates for thick CS Eq. (5.31), which for our parameters it reads:

$$
\frac{\gamma_{b g}}{\Omega_{c i}}=0.155\left(1-\left(k_{y} L\right)^{2}\right) \frac{1}{b_{g}}
$$

Note that the latter expression in valid in the limit of strong guide field, given by the expression Eq. (5.26). For our parameters, this is $b_{g}=0.213$, so all the values shown for finite guide field are in the strong regime. As we discussed in Sec.5.1.3, the previous analytical expression should not be taken as an accurate expression in our case, since it involves approximations valid beyond our parameter range (especially thick CS $L \gg \rho_{i}$ ). More accurate relations are only available as numerical solutions of the linearized Vlasov equation (see, e.g. Daughton 1999, Karimabadi et al. 2005). The main point here is just to emphasize the inverse proportionality of $\gamma \propto 1 / b_{g}$.

The previous two observations are in agreement with many previous studies. In fact, it has been known since some time ago that magnetic reconnection (in any plasma model 
involving at least the Hall term) is less efficient in presence of a guide field, in both build-up (or tearing) and explosive phase. We just discussed the reasons related with the tearing in the last paragraphs. The efficiency of the explosive phase (or fully-developed, steady state magnetic reconnection) is related with the peak values of the reconnected flux $\psi$, or equivalently, with its derivative the reconnection rates $d \psi / d t$ (proportional to the first one assuming an exponential growth, as it is often the case). Reconnection rates are reduced with increasing guide field since the dynamics of the waves allowing the fast magnetic reconnection mechanism is reduced towards smaller scales, fundamentally dominated by kinetic Alfvén waves (see Sec.4.2.2). Their dispersive properties make this process possible even when the responsible whistler waves in an antiparallel configuration are not allowed, but their phase speed and their ability to open the separatrices decreases with $b_{g}$. For a extended discussion and previous works in agreement with our results, see Sec. 4.3.3.

Before closing this section, it is necessary to make a technical remark. The fact that we are using periodic boundary conditions along $y$ makes the time period of fullydeveloped or steady state reconnection very short. It reaches their peak when the flux (see Fig. 8.4(left)) does, and then drops suddenly (not shown here). This is because the counterstreaming outflows destroy that configuration very quickly. In principle, this can be avoided by choosing longer simulation boxes or open boundary conditions, as it has been pointed out by Karimabadi et al. (2005). In the latter case, however, the definition of reconnected flux becomes more involved.

\subsection{Mechanism of guide field reconnection}

In this section, our purpose is to identify the physical mechanism that allow fast magnetic reconnection during the short phase where it reaches steady state in out setup. In other words, we will be analyzing the fully-developed stage of magnetic reconnection. As we stated in Sec. 8.1, now we switch to a double CS adding a background population as well, in order to have less noisy momenta of the distribution function (better statistics by avoiding close to vacuum regions with few particles per cell), necessary for the calculation of the term in the Ohm's law.

As we discussed in Sec. 2.2.1, there are several mechanisms for breaking the frozenin condition Eq. (2.49), each one identified with the non-ideal terms in the Ohm's law Eq. (2.27). Recall that this expression is derived from the second moment of the collisionless Vlasov equation specialized for electrons. In this subsection we are going to show the dependence of these terms on the guide field. But before, we need to address (another) numerical issue, with physical measurable consequences.

\subsubsection{Calculation methods for averages and derivatives}

Special care has to be taken when calculating both spatial and time derivatives in the right hand side of Eq. (2.27) from the raw data given by a PIC code. This is because the high PIC shot noise leads to unreliable results if these derivatives are not properly done. There is also another more important issue with physical consequences. The generalized Ohm's law in the form Eq. (2.27) can only be applied for collisionless plasmas. As we 
saw in previous chapter, the PIC noise mimic a collisional system. That is why it is convenient to get rid of noise by averaging that expression in both time and space, resembling an ensemble average. This can reduce to a large extent any numeric collision operator in the right hand side of the Boltzmann equation, thus recovering a system more similar to a Vlasov plasma. However, this procedure has a trade-off: the electromagnetic fields and quantities involving the distribution function in the left hand side of the Vlasov equation Eq. (2.19) are now ensemble averaged quantities. Then, when deriving the momenta of the Vlasov equation, there will appear additional terms due to correlations in the fluctuating parts of these quantities, as it has been shown in Sec. 4.2.5. The final result is that the mean field generalized Ohm's law Eq. (4.28) includes an additional term (in comparison with the instantaneous Ohm's law Eq. (2.25)) due to this correlated electromagnetic fluctuations Eq. (4.29), acting as an effective or "anomalous" resistivity.

\subsubsection{Time average and derivatives}

In order to decrease noise due to high frequency waves at electron time scales $\left(\omega_{p e}^{-1}\right)$, we choose to average the electromagnetic fields $\vec{E}, \vec{B}$, the current $\vec{J}$ and all the momenta of the distribution function up to order fourth (density $n$, bulk velocity $\vec{V}$, pressure tensor $P_{i j}$, heat flux $\vec{q}$ and kurtosis $K$ ) over an extended time period. By means of extensive tests (not shown here), we found that the optimum choice for the length of this average is about $\Delta T=0.5 \Omega_{c i}^{-1}$, half of the typical reconnection time scales. We are going to denote these time averages with the symbol:

$$
\langle A\rangle_{\triangle T, M A}, \quad \text { used }
$$

where " $M A$ " indicates a moving average, also known as boxcar filter or simply mean value of all the values of $A$ in the time series of length $\Delta T$ (see Appendix B.1.2.1). It is also possible to use more advanced filters, such as approximations via least square methods to detect some special features such as peak values or local maxima or minima in between the time series (see a general discussion in Appendix B.1).

It is also necessary a time derivative of the bulk flow velocity (Eulerian acceleration, calculated in a given position) in the inertia term in the generalized Ohm's law in Eq. (4.25). This is calculated via a Savitzky-Golay filter ("SG". See Appendix B.1.2.5 for further details) with a length equal to the same time period $\Delta T$ used for the time average of the quantities discussed in the last paragraph. It will be denoted as:

$$
\frac{\partial V_{e, z}}{\partial t} \approx\left\langle\frac{\partial V_{e, z}}{\partial t}\right\rangle_{\Delta T, S G} \quad \text { (used) }
$$

The choice of the same time period is in order to be self-consistent: both Eq. (8.13) and Eq. (8.14) will diminish noise in the same amount (because their cut-off frequencies will be similar). The choice of a Savitzky-Golay filter is in order to have smoother derivatives (see Appendix B.1.3.2), better detection of local maxima or minima in between the time period chosen for the average and also because it is a single pass algorithm on the time series: it does not require to store previous values for the calculation of derivatives.

In contrast, the straightforward way of calculating numerical derivatives is via finite 
differences ( $F D$, see Appendix B.1.3.1 for details) applied to the time series $\Delta T$

$$
\frac{\partial V_{e, z}}{\partial t} \approx\left\langle\frac{\partial V_{e, z}}{\partial t}\right\rangle_{\Delta T, F D} \quad \text { (not used) }
$$

but they turns out to be unreliable when applied to noisy data, especially over extended time windows like in this case (Ahnert and Abel 2007). As we explained in Appendix B.1.3.1, this can be understood in short because they are intrinsically prone to the phenomena known as "catastrophic cancellation": when two quantities with similar magnitude are subtracted, the result might have a high relative error (loss of significant digits) due to the limitations of floating point arithmetic. A time derivative calculated locally (i.e.: a short time window $\Delta T$ ) is also not convenient due to the errors introduced by the highly fluctuating PIC quantities, especially close to the reconnection peak time, the more interesting one. Our purpose in this section is to obtain the behaviour of magnetic reconnection over its typical ion time scales $\left(\Omega_{c i}^{-1}\right)$, and that is why we chose this extended average.

In principle, it is also possible to calculate derivatives via finite differences first smoothing out the time series of timesteps contained in $\Delta T$, with a final result at least as smooth as the calculated via SG filters Eq. (8.14). But that approach is not practical when applied to the in-time running diagnostics in a PIC code. It would be necessary to store all the intermediate timesteps for the calculation of derivatives via finite differences afterwards, an unnecessary memory consuming operation in two-passes. If the spatial average is de-

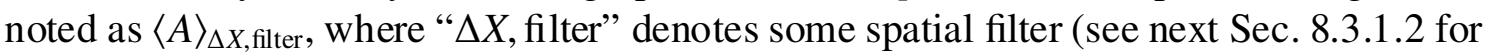
further details) over a typical length scale $\Delta X$, the two steps in this method are:

$$
\frac{\partial V_{e, z}}{\partial t} \approx\left\langle V_{e, z}\right\rangle_{\Delta x, \text { filter }} \rightarrow\left\langle\frac{\partial\left\langle V_{e, z}\right\rangle_{\Delta x, \text { filter }}}{\partial t}\right\rangle_{\Delta T, F D} \quad \text { (not used) }
$$

We will also be using the same approach Eq. 8.14) for calculating the rate of changes (again, Eulerian derivatives calculated in a fixed position) of the momenta of the distribution function up to order two (density $\partial n_{\alpha} / \partial t$, bulk velocity $\partial V_{\alpha, i} / \partial t$ and diagonal elements of the pressure tensor $\partial P_{\alpha, i i} / \partial t$ ). They are directly related with transport coefficients. All these running time diagnostics are implemented in ACRONYM.

\subsubsection{Spatial average and smoothing}

There are several more or less equivalent approaches for the calculation of spatial derivatives (necessary, e.g. for the pressure term in Ohm's law $\partial P_{e, x z} / \partial x$ ), also prone to the short wavelength PIC numerical shot noise. Following the same observations pointed out in the last subsection, it is not convenient to apply finite differences to the raw data. One can try to apply the same approach as for time derivatives and using in one-pass a S-G filter:

$$
\frac{\partial P_{e, x z}}{\partial x} \approx\left\langle\frac{\partial P_{e, x z}}{\partial x}\right\rangle_{\Delta X, S G} \quad \text { (not used) }
$$

over a typical length scale $\Delta X$. However, in this case this kind of filter lose some of its advantage in comparison with its use for time series, since a two passes algorithm on spatial data does not increase at all the computational cost (it is a post-processing diagnostic operation). In addition, a S-G filter for derivatives, although good to denoising 
small scale structures, it introduces noise to large scales, something unwanted in our case (note that we do not have that problem for the time derivatives). The other approach is smoothing out the data first and then apply finite differences:

$$
\frac{\partial P_{e, x z}}{\partial x} \approx\left\langle P_{e, x z}\right\rangle_{\Delta X_{1}, \text { filter }} \rightarrow\left\langle\frac{\partial\left\langle P_{e, x z}\right\rangle_{\Delta X_{1}, \text { filter }}}{\partial x}\right\rangle_{\Delta X_{2}, F D} \quad \text { (not used) }
$$

The filters have to be low-pass such as moving average, binomial (commonly used in current smoothing routines in PIC codes, see Appendix B.1.2.2) or Gaussian (converging to binomial for large $\Delta x$. See Appendix B.1.2.3). Each one differs in their frequency response and the choice depends on the problem to be analyzed (see Appendix B.1 for a general discussion). Note that the filter lengths $\Delta X_{1}$ and $\Delta X_{2}$ do not have to be the same . But our approach is, in order to be consistent with Eq. (8.14), applying a combination of the previous methods: first a smooth derivative using a S-G derivative filter (to preserve small scale features) and then a Gaussian filter (to smooth out noise introduced by the S-G filter to large scales):

$$
\frac{\partial P_{e, x z}}{\partial x} \approx\left\langle\frac{\partial P_{e, x z}}{\partial x}\right\rangle_{\Delta X, S G} \rightarrow\left\langle\left\langle\frac{\partial P_{e, x z}}{\partial x}\right\rangle_{\Delta X, S G}\right\rangle_{\Delta X, \text { gauss }} \quad \text { (used) }
$$

Note that we have chose the same filter length for both smoothing and derivatives $\Delta X=$ $\Delta X_{1}=\Delta X_{2}$. This is done by choosing an equivalent cutoff wavelength in the frequency response of both filters (see discussion of Eq. (B.21) for further details).

\subsubsection{Mean field generalized Ohm's law}

Now, after the previous numerical consideration, we can finally calculate each term in the generalized Ohm's law. From now on, let us omit the subscript related with the running-time average for all the quantities according to Eq. 8.13 ), in such a way that we will be denoting, e.g.,

$$
E_{z} \rightarrow\left\langle E_{z}\right\rangle_{\Delta T, M A}
$$

Thus, and using the previous filters for both spatial and time derivatives, the explicit form for calculating all terms in the generalized Ohm's law Eq. (2.25), without taking into account the contribution by correlated electromagnetic fluctuations Eq. (4.29), is given by:

$$
\begin{aligned}
& \left\langle E_{z}\right\rangle_{\Delta X, \mathrm{gauss}}+\left\langle V_{e, x} B_{y}-V_{e, y} B_{x}\right\rangle_{\Delta X, \mathrm{gauss}}= \\
& -\left\langle\frac{1}{e n_{e}}\left(\left\langle\frac{\partial P_{e, x z}}{\partial x}\right\rangle_{\Delta X, \mathrm{SG}}-\left\langle\frac{\partial P_{e, y z}}{\partial y}\right\rangle_{\Delta X, \mathrm{SG}}\right)\right\rangle_{\Delta X, \mathrm{gauss}} \\
& -\left\langle\frac{m_{e}}{e}\left(\left\langle\frac{\partial V_{e, z}}{\partial t}\right\rangle_{\Delta T, \mathrm{SG}}+V_{e, x}\left\langle\frac{\partial V_{e, z}}{\partial x}\right\rangle_{\Delta X, \mathrm{SG}}+V_{e, y}\left\langle\frac{\partial V_{e, z}}{\partial y}\right\rangle_{\Delta X, \mathrm{SG}}\right)\right\rangle_{\Delta X, \text { gauss }}
\end{aligned}
$$

In all the calculations to be shown in this chapter, the time averages and time derivatives with SG-filter have used a filter width of $\Delta T / \Delta t$ time steps. Both time and spatial derivative with SG-filter $\{\Delta T, \mathrm{SG}\}$ and $\{\Delta X, \mathrm{SG}\}$ use a polynomial order $P=2$. The spatial derivative with $\mathrm{SG}$ filter uses a width of $N=5$ (or $M=2$ ), corresponding to a cutoff 
wavelength of the corresponding smoothing filter of $\lambda_{x, \mathrm{SG}-\mathrm{cutoff}}=3.53 \Delta x=2.46 \lambda_{D e}$. The final Gaussian filter is chosen to have $\sigma=1.59$, corresponding to the same cutoff wavelength: $\lambda_{x, \text { GAUSS-cutoff }}=3.53 \Delta x=2.46 \lambda_{D e}$. The specific values of the coefficients for all these filters can be calculated with the formulas given in Appendix B.1.

As we explained at the beginning of this Sec. 8.3.1, any mismatch between the left and right hand side of this mean field Ohm's law Eq. (8.21) can be attributed to the effects of anomalous correlations between the instantaneous fluctuating quantities Eq. (4.29). This avoids the direct calculation of that term, which would require a complete information of the full time series of the instantaneous fluctuating quantities. However, the specific values of this term will depend on the chosen filter width in both time and space. This is equivalent to introduce a different separation of scales between the mean and fluctuating quantities in the Vlasov equation, which will affect any transport coefficient based on this (see discussion in Sec.4.2.5). Therefore, a rigorous calculation would have to taken into account all these effects, by means of an extensive parametric study of convergence on dependence on the chosen filter, in both space and time. The latter is particularly computationally demanding since it is done in running-time in the PIC code. This work has not been done in this thesis. Only a few examples for some specific cases were carried out, which will be presented next. Thus, our results regarding the anomalous effects in the mean field Ohm's law should be considered only as a first approximation.

\subsubsection{Dissipative mechanism in the antiparallel limit}

In order to compare how the dissipative mechanism changes with the guide field, let us display the contribution of each one of these Ohm's law terms for the limit case of $b_{g}=0$ as well as the total left and hand side, respectively. The results of the calculation by the methods explained in the previous subsection are shown in Fig. 8.6. 


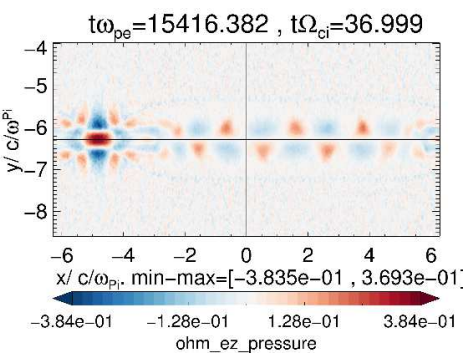

(a) $E_{z \text {,pressure }}$

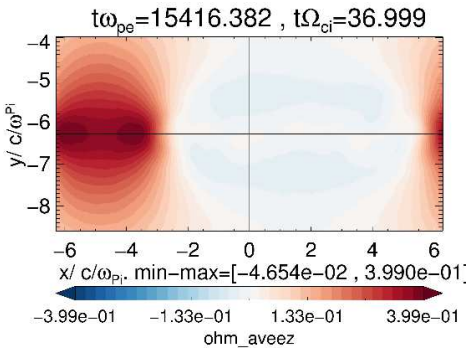

(d) (Total) $E_{z}$

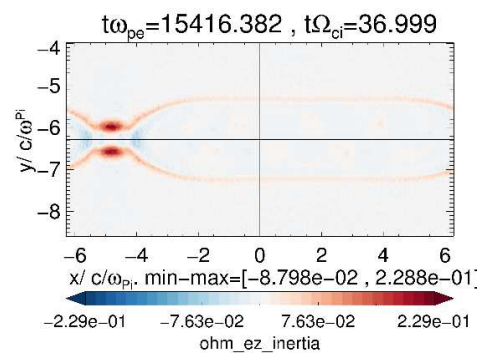

(b) $E_{z, \text { inertia }}$

$t \omega_{\mathrm{pe}}=15416.382, \mathrm{t} \Omega_{\mathrm{ci}}=36.999$

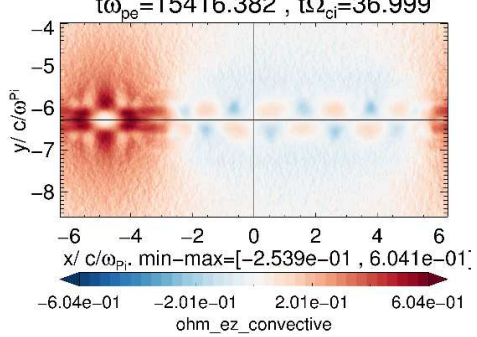

(e) $E_{z, \text { convective }}$

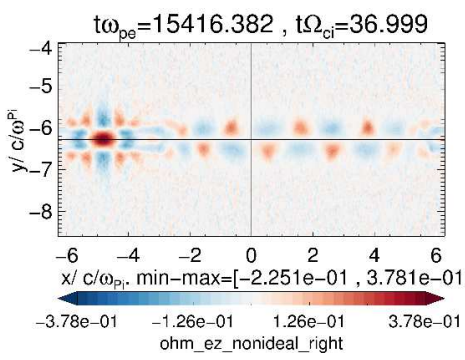

(c) $E_{R H S}=E_{z \text {,pressure }}+E_{z, \text { inertia }}$

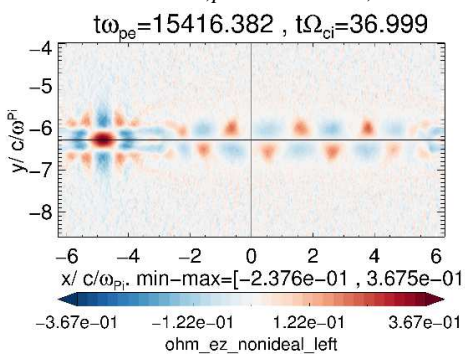

(f) $E_{L H S}=E_{z}+E_{z \text {, convective }}$

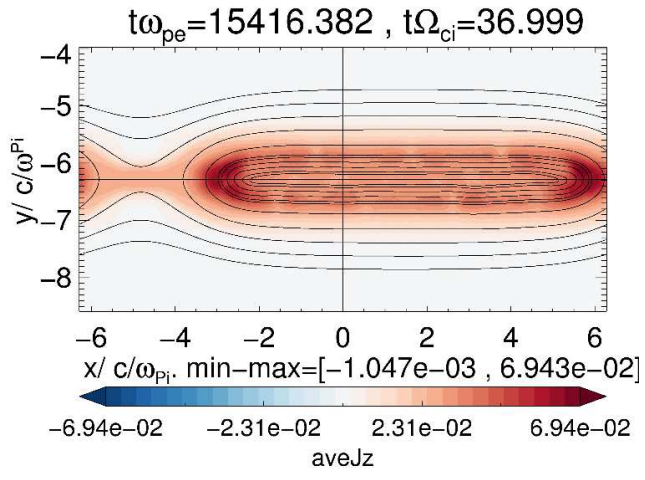

(g) $J_{z}$

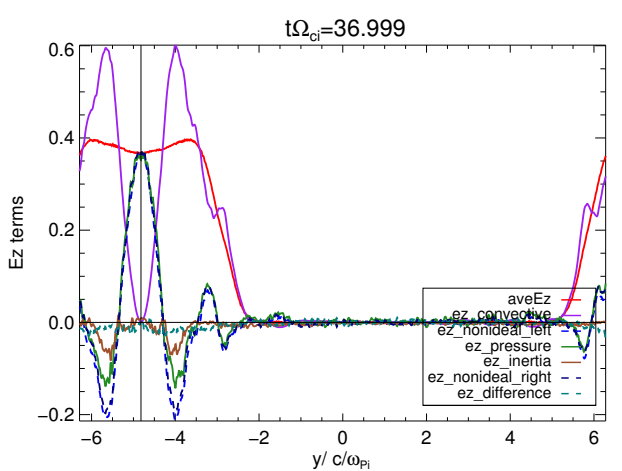

(i) Balance ofterms. Profiles along y (center)

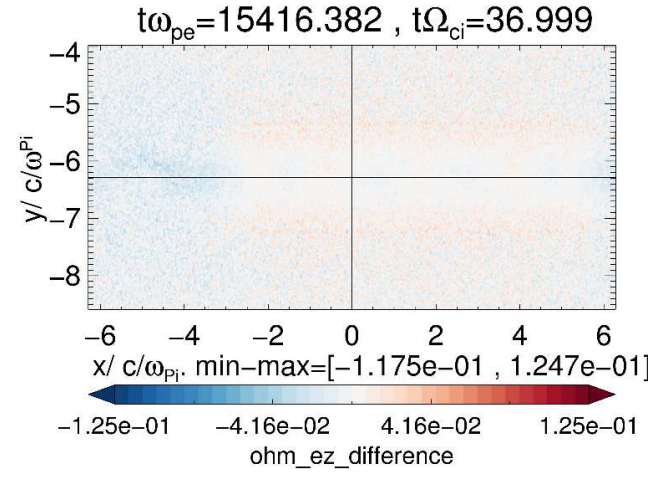

(h) $E_{R H S}-E_{L H S}$

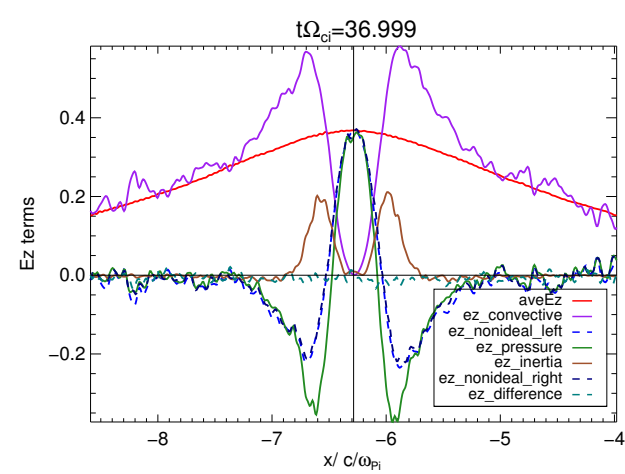

(j) Balance of terms. Profiles along $x$ (at X point)

Figure 8.6: Terms of the generalized Ohm's law for $\boldsymbol{b}_{\boldsymbol{g}}=\mathbf{0}$ at reconnection peak time. Each term was calculated with a proper time and spatial averaging according to the description and parameters given in Sec. 8.3.1.3. All the terms are in dimensionless units normalized to $B_{0} V_{A}$ (same as the reconnection rate).

First of all, in general the net difference between all the terms in the Ohm's law, as can be seen in Fig. 8.6(h), it is fulfilled to a large extent everywhere. The small differences, 
shown in the profiles Fig. 8.6(i)-(j) as teal color dashed line, can be attributed to the noise, since it seems to be random, not significantly localized and smaller than any the other terms.

Next, note that "chess-pattern" structure inside of the main magnetic island for the electron pressure term $E_{\text {pressure }}$ and, to a lesser degree, for the electron inertia term $E_{\text {inertia }}$. As we found in the previous chapter Sec.7.3.2.1, these structures are very similar to these formed due to Weibel instability (see Sec. 5.2.1). Indeed, we found a temperature anisotropy $T_{e, y}>T_{e, x}$ in all the inner region of the main magnetic island (see Fig. 8.7 (a)) above the theoretical threshold Eq. (5.54). This is due to the preferential electron parallel heating resulting of the development of tearing mode. In a very similar way, the outof-plane magnetic field $B_{z}$ generated by the Weibel instability generates a temperature aligned anisotropy $T_{e, \|}<T_{e, \perp}$ (calculated with the methods explained in Sec.7.3.3.1 and Appendix B.2.5) mostly close to the center of the magnetic island (see Fig. 8.7(b)). Their magnitude has the right threshold for the destabilization of the mirror mode (Eq. (5.59)). This is a complete analogous process to the interplay between Weibel and mirror instabilities seen in the previous chapter (see Sec.7.3.3), but in this case is completely generated by the natural anisotropies developed by the system and not by any numerical effect. The effect of this instability can be seen (see Fig. 8.7(c)), where it is shown the non-gyrotropy $D_{n g}$ of the distribution function calculated by the method explained in Appendix B.2.6. It measures relative importance of the off-diagonal terms of the pressure tensor, being recently proposed by Hesse et al. (2014). Strong deviations from a gyrotropic pressure tensor, and therefore deviations from the thermal equilibrium, are correlated with the locations where $T_{e, \|}<T_{e, \perp}$.

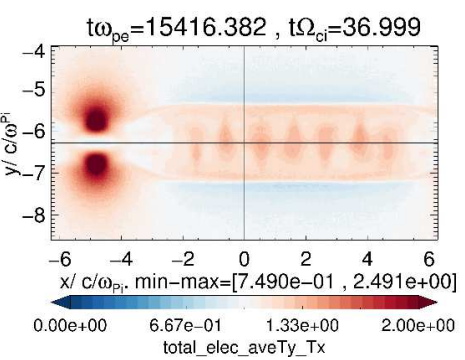

(a) $T_{e, y} / T_{e, x}$

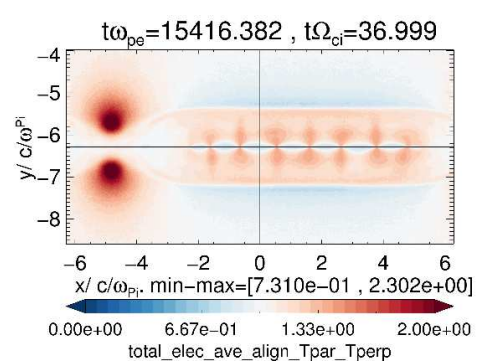

(b) $T_{e, \|} / T_{e, \perp}$

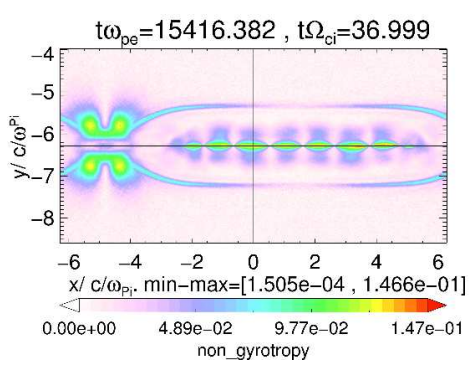

(c) Non-gyrotropy $D_{N G}$

Figure 8.7: Contour plots with quantities showing signatures of the temperature driven Weibel instability for the limit case $\boldsymbol{b}_{g}=\mathbf{0}$ at reconnection peak time

Only very few (and relatively recent) works have noticed these Weibel structures (see, e.g., Lu et al. 2011, Schoeffler et al. 2013), but it has not been recognized as a ubiquitous feature of magnetic reconnection. This might be related with the spatial resolution necessary to resolve well these structures and the low noise level associated with the use of TSC shape functions in our simulations. From the previous Chapter 7 , if these considerations are not taken into account, numerical collisions dominate the system tending to the isotropization, making disappear any effect related with anisotropy. In simulations with similar parameters but lower spatial resolution and lower number of macroparticles per cell (not shown here), the Weibel signatures related with anisotropy are completely absent. 
As can be seen in Fig. 8.6(i)-(j), close to the X point, the reconnected electric field is mostly balanced by a combinations of the pressure and inertia term, in agreement with previous studies (see Sec.4.2.3 and Sec.4.2.4). However, and different from those works, we can also notice an important contribution from the electron inertia $E_{z \text {,inertia }}>0$ at the boundaries of the main magnetic island Fig. 8.6)(b). Since there is practically no reconnected electric field in that regions, the electron pressure has to compensate this additional electric field by showing $E_{z \text {,inertia }}<0$ (see Fig. 8.6(a)).

But, what is the physical mechanism of these relative large contribution to the electric field $E_{z}$ far away from the X point? The usual suspect for the pressure term, the electron meandering close to the neutral line, cannot be responsible, since in the borders of the secondary magnetic islands the asymptotic Harris $B_{\infty y}$ is dominant, confining the electron to gyrate in small confined regions. Unexpectedly, we found that in these regions the electron bulk velocity $V_{e, z}$ is decreasing very quickly (deceleration $\partial V_{e, z} / \partial t<0$ ), even faster than in the diffusion region close to the $X$ point (Fig. $8.10(\mathrm{~g})$ ). This is the component carrying the current $J_{z}$ that sustain the current sheet. Therefore, this slow down is a signature of a diffusive process taking place at these locations (related with "anomalous" resistivity). Its effects seems to be a conversion of the bulk flow velocity into in-plane electron heating $\partial T_{e, y} / \partial t>0$, mostly along along the boundary of the magnetic island (see Fig. 8.13(d)). The other components of the temperature/pressure tensor are not heated in these locations over other places inside of the magnetic island or in the diffusion region. In addition there is also generation of a non-thermal tail of the electron in-plane 1D VDF $f\left(v_{y}\right)$ throughout the border of the magnetic island, as evidenced by the kurtosis $K_{e, y}$ (see Fig. 8.14(a)). As we mention in Appendix B.2.1, a non-thermal population can be characterized via this 4 th order moment of the distribution function, since it indicated the relative importance of the "tails" of a distribution in comparison with a Maxwellian VDF. In any case, the fact of a non-negligible $K_{e, y}$ and absence of this kind of deviations in the other components of the rate of increase of temperature or kurtosis, indicate that the electron inertia contribution is a good proxy for detecting places where this kind of behaviour are expected.

\subsubsection{Guide field dependence}

The addition of a guide field at least as high as the asymptotic Harris magnetic field $\left(b_{g}=1\right)$ changes radically the structure of all the terms in the Ohm's law, in both diffusion region as well as inside of the magnetic island (compare Fig. 8.6 with Fig. 8.8). The first observation is that the characteristic "chess board" structure in the pressure term $E_{\text {pressure }}$, signature of the Weibel instability, disappears completely inside of the main magnetic island. This is due to the magnetization of electrons on the guide field, reducing to a large extent any in-plane temperature ani sotropy inside of that region, the source of free energy of the Weibel instability. Therefore, there is no similar structures to the ones seen in Fig. 8.7 for all the guide field strengths considered in this study. 


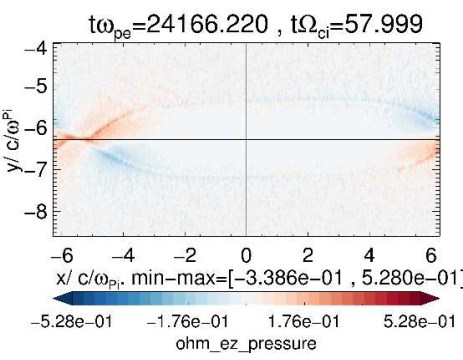

(a) $E_{z \text {,pressure }}$

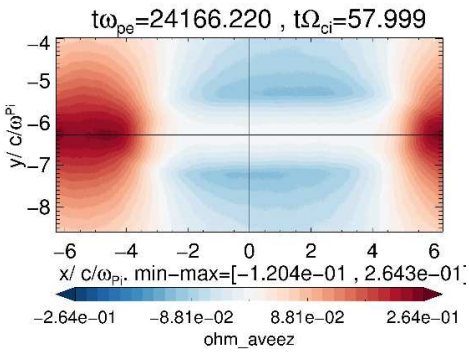

(d) (Total) $E_{z}$

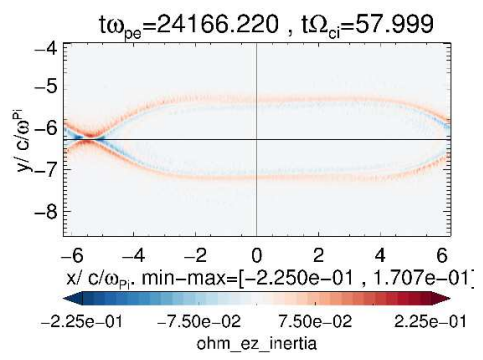

(b) $E_{\text {z,inertia }}$

$t \omega_{\mathrm{pe}}=24166.220, \mathrm{t} \Omega_{\mathrm{ci}}=57.999$

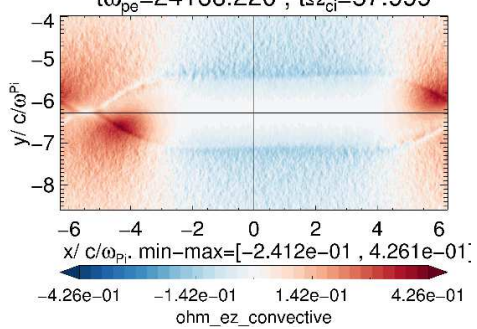

(e) $E_{z, \text { convective }}$

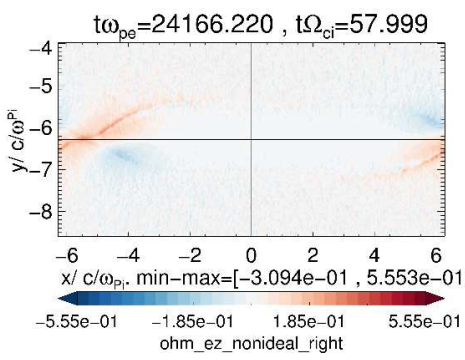

(c) $E_{R H S}=E_{z \text {,pressure }}+E_{z, \text { inertia }}$

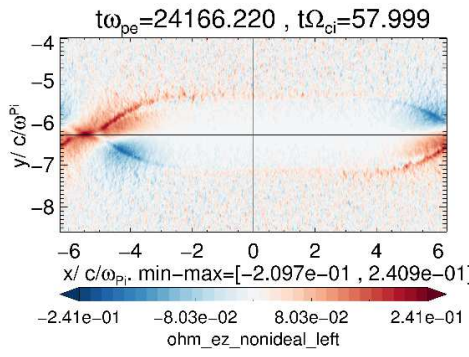

(f) $E_{L H S}=E_{z}+E_{z \text {, convective }}$

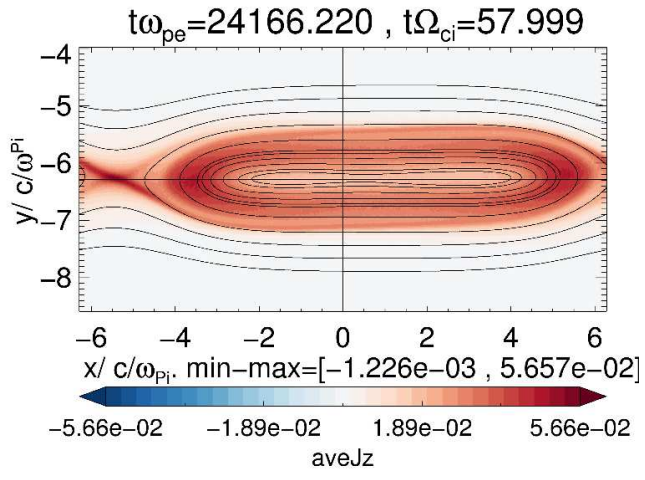

(g) $J_{z}$

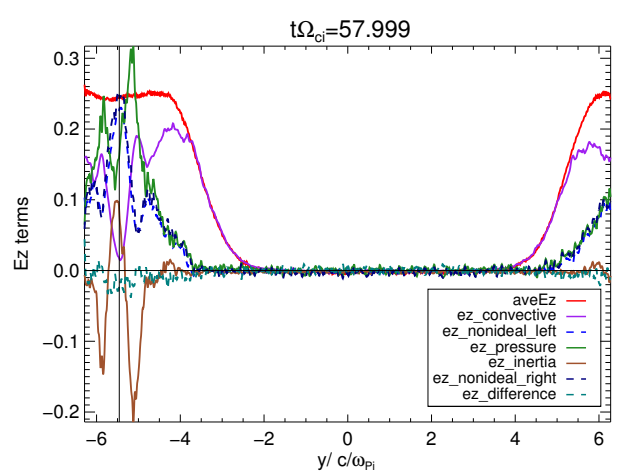

(i) Balance ofterms. Profiles along y (center)

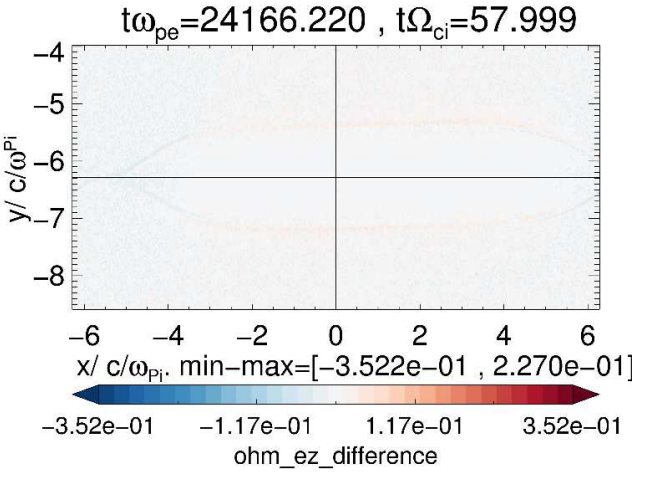

(h) $E_{R H S}-E_{L H S}$

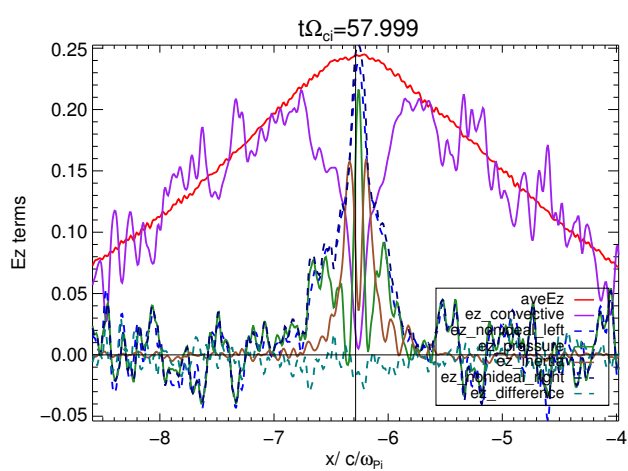

(j) Balance of terms. Profiles along $x$ (at X point)

Figure 8.8: Terms of the generalized Ohm's law for $\boldsymbol{b}_{g}=\mathbf{1}$ at reconnection peak time. Each term was calculated with a proper time and spatial averaging according to the description and parameters given in Sec. 8.3.1.3. All the terms are in dimensionless units normalized to $B_{0} V_{A}$ (same as the reconnection rate). 


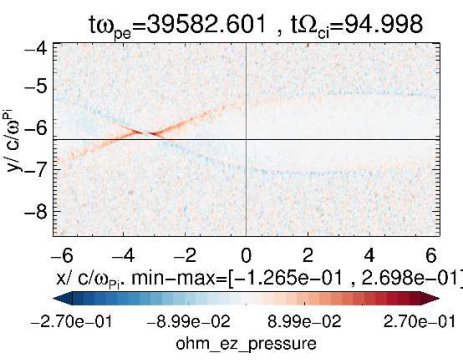

(a) $E_{z, \text { pressure }}$

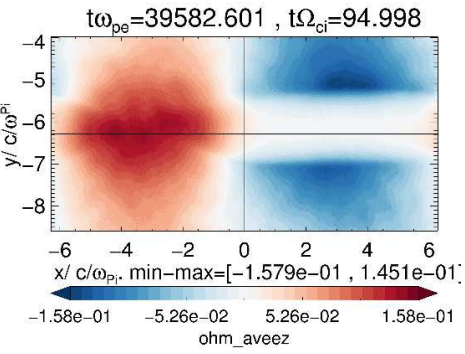

(d) (Total) $E_{z}$

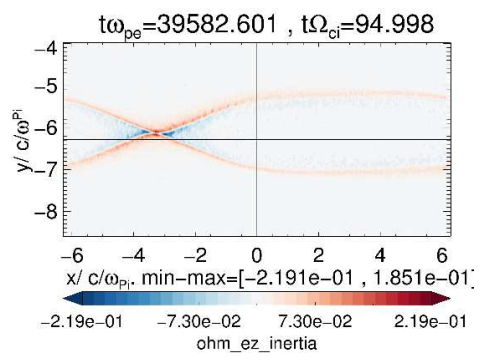

(b) $E_{z, \text { inertia }}$

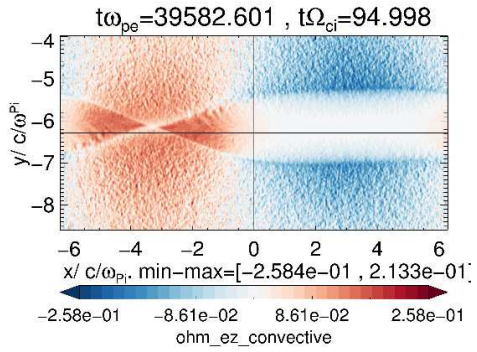

(e) $E_{z, \text { convective }}$

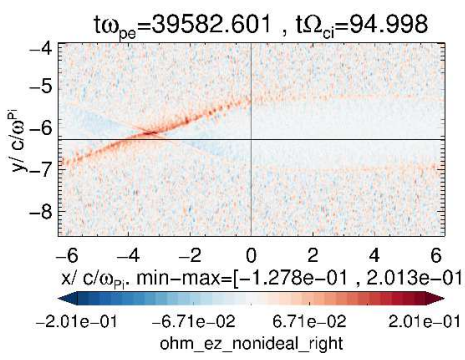

(c) $E_{R H S}=E_{z, \text { pressure }}+E_{z \text {,inertia }}$

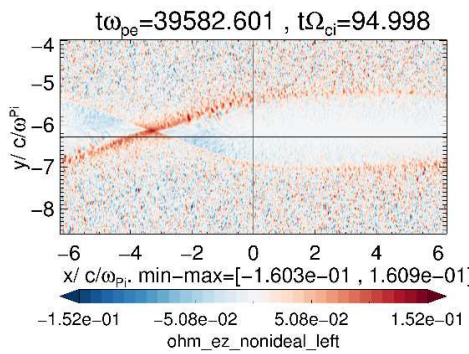

(f) $E_{L H S}=E_{z}+E_{z, \text { convective }}$

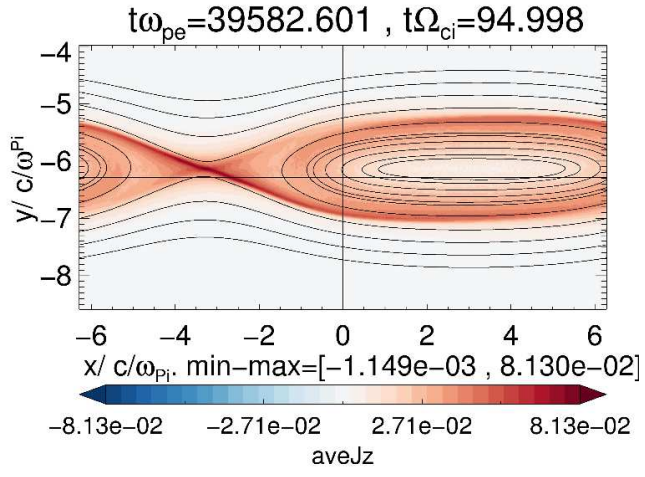

(g) $J_{z}$

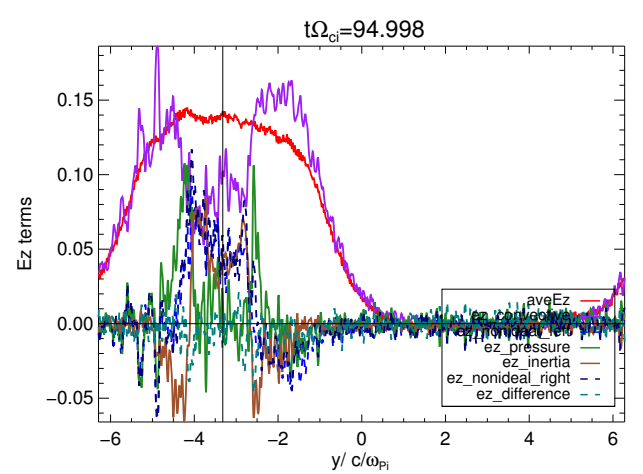

(i) Balance of terms. Profiles along y (center)

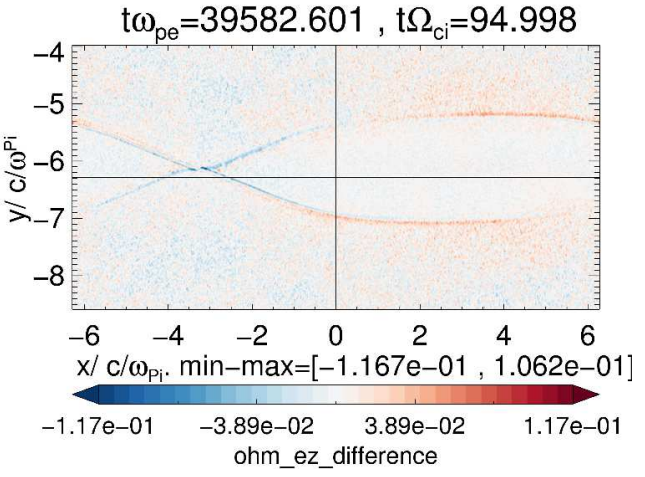

(h) $E_{R H S}-E_{L H S}$

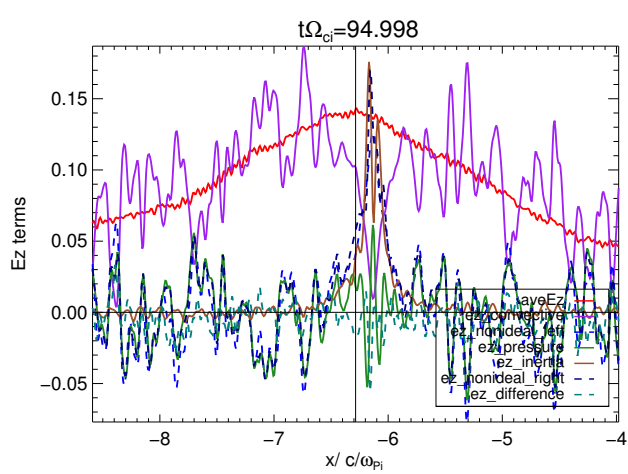

(j) Balance of terms. Profiles along $x$ (at $X$ point)

Figure 8.9: Terms of the generalized Ohm's law for $\boldsymbol{b}_{g}=\mathbf{3}$ at reconnection peak time. Each term was calculated with a proper time and spatial averaging according to the description and parameters given in Sec. 8.3.1.3. All the terms are in dimensionless units normalized to $B_{0} V_{A}$ (same as the reconnection rate). 
The balance of the terms sustaining the reconnected electric field also changes drastically with the guide field. The asymmetry introduced by the reduced shear in magnetic field and the typical length scale $\rho_{e, b g}$ reduce any large scale feature (as present in the limit $b_{g}=0$ ) of the pressure $E_{z \text {,pressure }}$ or electron inertia $E_{z \text {,inertia }}$ contributions to the reconnected electric field. They are now very concentrated in small regions in the close neighborhood of the $\mathrm{X}$ point and along the boundaries of the secondary magnetic island (see, e.g., Fig. 8.8(a)-(b)). Only in a subset of these areas the frozen in condition is violated, allowing the rearrangement of magnetic field lines leading to magnetic reconnection (see Fig. 8.8(c)). These regions becomes smaller for higher guide fields (see, e.g., Fig. 8.9 (a)-(b)-(c)), consequence of the reduced $\rho_{e, b g}$. This is even another reason for resolving properly the electron Larmor radius on the guide field by the grid cell size $\Delta x$. Although it is possible to have a stable scheme under-resolving this quantity according to the discussion in Sec. 8.2.1.2, it will not be possible to make reliable estimations about the origin of the reconnected electric field when the scales of the pressure or inertia term fall below $\Delta x$. The importance of the inertia term increases with the guide field in comparison with the pressure term, in agreement with previous simulations and theoretical studies (see Sec.4.2.4). For our next discussions note also that the inertia term is always symmetric with respect to the central line of the CS, while the pressure contribution to the reconnected electric field becomes asymmetric under the influence of the guide field. It is positive in the pair of separatrices with low density.

\subsection{Deviations in the generalized Ohm's law and cross- field streaming instabilities}

An important observation in the previous plots is that the balance of terms in the Ohm's law loses accuracy with increasing guide field (compare Fig. 8.8(h) with Fig. 8.9(h)). This is not only due to the enhanced noise, but also an indication of enhanced correlated electromagnetic fluctuations levels, appearing as additional terms $E_{z, \text { anomalous }}$ in the mean field Ohm's law (recall Sec. 4.2.5 and the definition Eq. (4.29)). This is different from the antiparallel case (see Fig. 8.6(h), because the guide field localizes the deviations in the Ohm's law at some specific locations. Although we are going to present some arguments in favor of these kind of processes taking place in our system, a more extensive parametric study of the effects of guide field and filters on noise are required to fully confirm this claim. A detailed analysis of the full time series from the simulation is required to rigorously separate the steady state from the fluctuating parts, and thus to have a more reliable calculation method for the ensemble averages (and not only in time, but also in space).

The additional fluctuating term $E_{z \text {,anomalous }}$ not modeled by the simple two-fluid Ohm's law is very localized along the separatrices and in the outer boundary of the main magnetic island. Their length scales, increasingly smaller for higher guide fields due to the reduced electron Larmor radius $\rho_{e, b g}$, can only be resolved by a good enough spatial resolution (simulations with guide fields but smaller resolution, not shown here, do not display this behaviour). The effect of this $E_{z \text {,anomalous }}$ term is correlated with a slow down of the current carriers, as can be seen in the deceleration of the out-of-plane bulk velocity $\partial V_{e, z} / \partial t$ (see Fig. 8.10 (bottom-row)). Therefore, their effects are closely related with the electron inertia contribution to the reconnected electric field $E_{z, \text { inertia }}$. In particular, $\partial V_{e, z} / \partial t$ in- 
creases a little bit for larger guide fields (e.g.: $\left.b_{g}=3\right)$, but what it is more important: their peak values become localized at the edges of the magnetic island, where is the main term responsible for sustaining the out-of-plane electric field in the opposite direction to the reconnected one: $E_{z}<0$. This electric field does not contribute to reconnection, only taking place close to the $\mathrm{X}$ point, but additional secondary processes related with turbulence. Note that the relative importance of this electric field with opposite polarity outside of the edge of the main magnetic island increases with the guide field (compare, e.g., Fig. 8.6(d) with Fig. 8.8(d) and Fig. 8.9(d)).

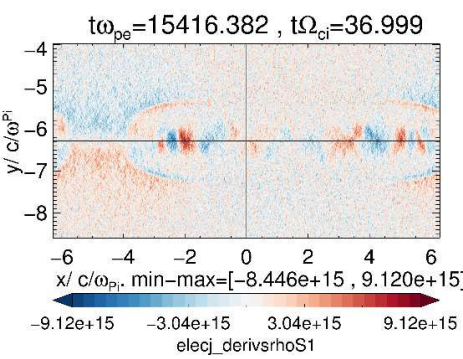

(a) $b_{g}=0$

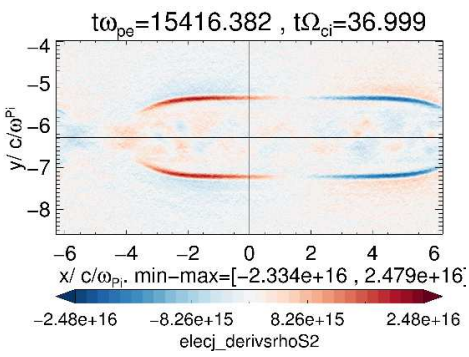

(d) $b_{g}=0$

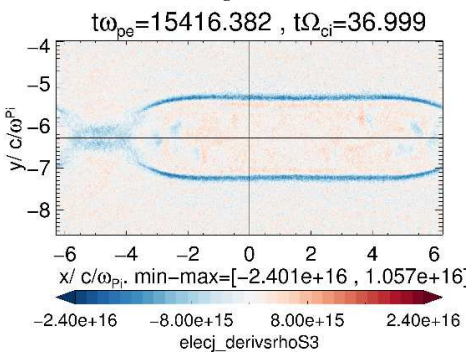

(g) $b_{g}=0$

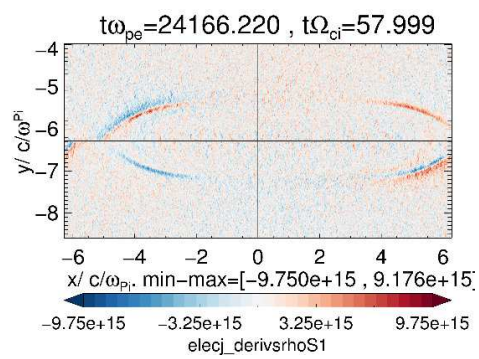

(b) $b_{g}=1$

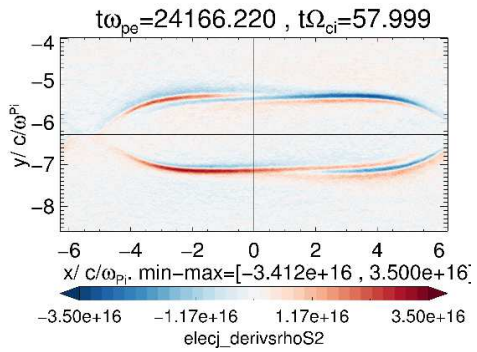

(e) $b_{g}=1$

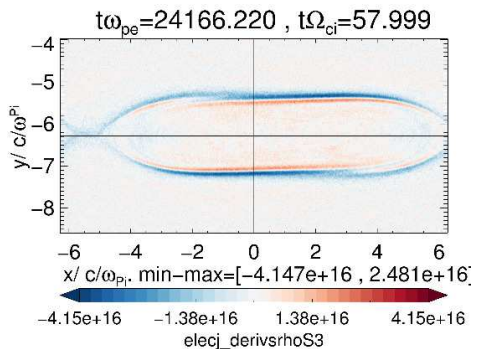

(h) $b_{g}=1$

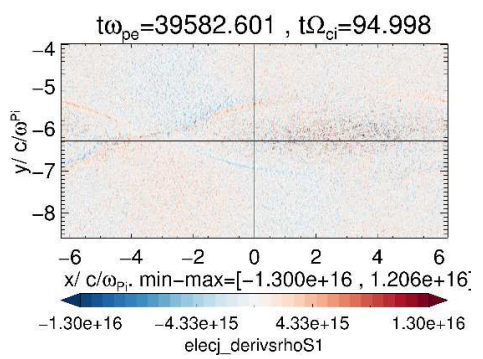

(c) $b_{g}=3$

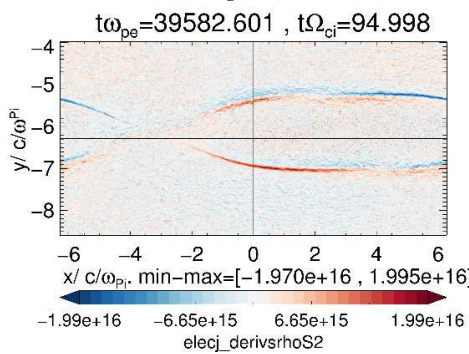

(f) $b_{g}=3$

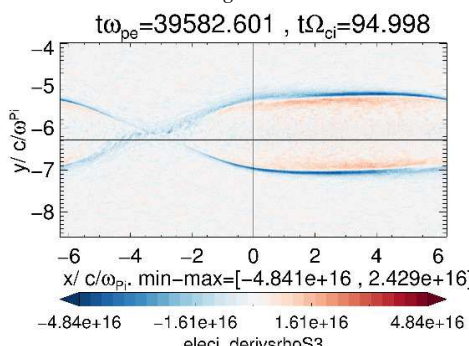

(i) $b_{g}=3$

Figure 8.10: Contour plots of different components of the electron bulk (Eulerian) acceleration $\partial \vec{V}_{e} / \partial t$ at reconnection peak time for different guide fields (in C.G.S. units). These derivatives were calculated in running-time with the methods explained in Sec. 8.3.1.1. Top row: In-plane $\partial V_{e, x} / \partial t$. Middle row: In-plane $\partial V_{e, y} / \partial t$. Bottom row: Out-of-plane $\partial V_{e, z} / \partial t$.

\subsubsection{Electron bulk acceleration and cross-field instabilities}

On the other hand, and different from the usual case where the anomalous electric field $E_{\text {anomalous }}$ arises from current aligned streaming instabilities, in this case that contribution can also arise from cross-field streaming instabilities such as the modified two-stream instability MTSI (see Sec. 5.4.2.2 and al so Yoon and Lui (2006)), allowed to grow in our 
2D geometry because it has practically perpendicular propagation (in-plane) to the local magnetic field (mostly along $\hat{z}$ for larger guide field such as $b_{g}=3$ ). If operating, the component $E_{z \text {,anomalous }}$ should have contributions from the electromagnetic term Eq. (4.29), proportional to $\left[\delta\left(n_{e} \vec{V}_{e}\right) \times \delta \vec{B}\right]_{z}$. The MTSI instability gains importance only in a guide field configuration, because their source of free energy are the always present in-plane streaming flows, with typically Alfvénic speeds in the outflow region. In this case, due to the effects of guide field, these particles are deflected from the separatrices toward the edges of the secondary magnetic islands. They concentrate in concentric thin layers where these particles experience an opposite directed in-plane acceleration and deceleration (see Fig. 8.10 (middle-row)). This might lead to instabilities with a shear flow as their source of free energy. In particular, MTSI, since it grows faster in the perpendicular direction to the magnetic field. One signature of this cross-field streaming instability taking place is that after the time shown in this plot, these regions become turbulent, dissipating the free energy stored in the counter-propagating electron flows. This can be seen in Fig. 8.11.

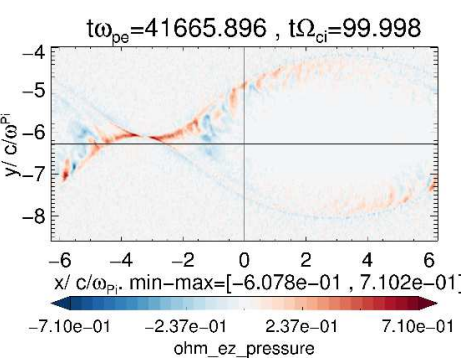

(a) $E_{z \text {,pressure }}$

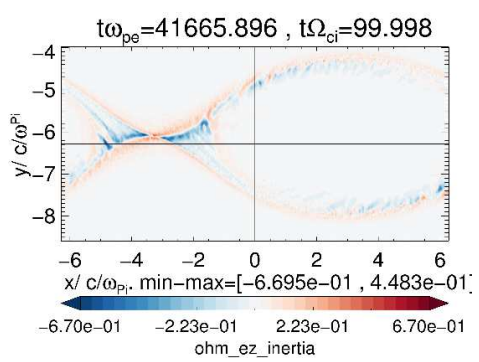

(b) $E_{z, \text { inertia }}$

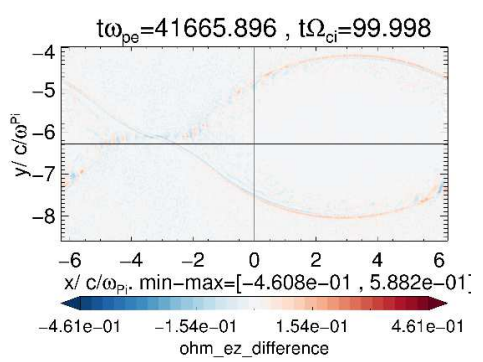

(c) $E_{z, R H S}-E_{z, L H S}$

Figure 8.11: Some terms of the generalized Ohm's law for $\boldsymbol{b}_{\boldsymbol{g}}=\mathbf{3}$ for a time after the reconnection peak time (compare with Fig. 8.9). Each term was calculated with a proper time and spatial averaging according to the description and parameters given in Sec. 8.3.1.3. All the terms are in dimensionless units normalized to $B_{0} V_{A}$ (same as the reconnection rate).

The same regions (boundary of magnetic island) where this wave activity is taking place also show enhanced increase in density $\partial n_{e} / \partial t$ in very thin layers (see Fig. $8.12(\mathrm{c}) 3^{3}$ . This implies a very large gradient in density, potentially prone to LHDI (see Sec. 5.4.2.4). This instability has oblique propagation, being also allowed in our 2D geometry with an out-of-plane guide field. And their typical time and length scales are similar to these of MTSI. Note that this kind of instabilities develop away from the $\mathrm{X}$ point and diffusion region, since in that regions the flows have very low speeds, being accelerated only in the outflow region and along the separatrices (see Fig. 8.10(middle-row)).

\footnotetext{
${ }^{3}$ Note the fast increase of density $\partial n_{e} / \partial t$ for the antiparallel case $b_{g}=0$ is concentrated throughout the magnetic island, although more in the outflow region next to the X point (Fig. 8.12(a)). But already with a guide field $b_{g}=1$ (Fig. 8.12 (b)) the increase in density shifts to the boundary of the magnetic island, due to the change of electron trajectories in the outflows produced by the guide field. A stronger guide field makes this effect even more notorious and localized in very thin layers, with the corresponding generation of steep density gradients prone to LHDI (Fig. 8.12(c)).
} 


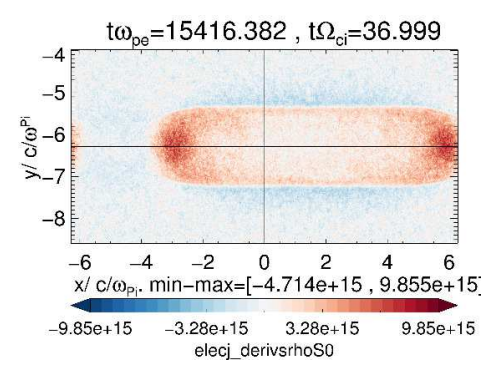

(a) $b_{g}=0$

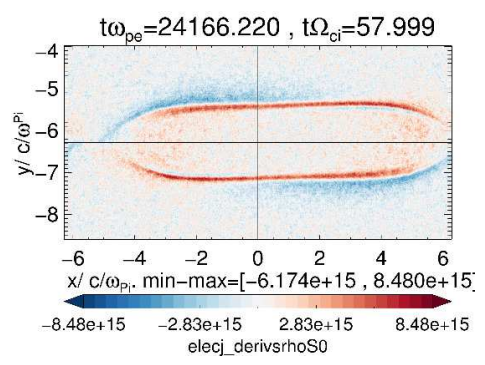

(b) $b_{g}=1$

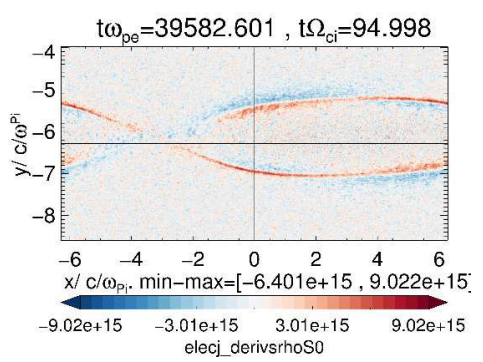

(c) $b_{g}=3$

Figure 8.12: Contour plots of the rate of change (Eulerian derivative) of electron number density $\partial n_{e} / \partial t$ (in C.G.S units) at reconnection peak time for different guide fields. This derivative was calculated in running-time with the methods explained in Sec. 8.3.1.1.

There is even another reason why these kind of cross-field instabilities can develop only under the presence of a guide field. This is because higher guide field Harris CS simulations delay the onset of the fully-developed stage of magnetic reconnection (recall Fig. 8.4). Therefore, the previous instabilities with typical time scales on the order of the lower hybrid frequency $\Omega_{l h}^{-1}$, will have more time to develop and to reach saturated state in comparison in cases with lower guide fields.

All the previous observations are arguments in favor of the identification of Fig. 8.11 as signature of instabilities driven by streamings and gradients at the boundaries of the magnetic islands. And this seems to be always correlated with the presence of an important contribution of the electron inertia $E_{z \text {,inertia }}$ to the negative electric field $\left(E_{z}<0\right)$, a deceleration of the out-of-plane electron bulk flow $\partial V_{e, z} / \partial t<0$ and the appearance of non-negligible $E_{z \text {,anomalous }}$ in the mean field Ohm's law. All these processes become increasingly more important for higher guide fields. The fact that guide field reconnection can give origin to higher levels of anomalous resistivity (due to the higher saturation levels of MTSI) is in agreement with previous studies (Yoon and Lui 2006).

\subsubsection{Thermal and non-thermal effects of the instabilities and devia- tions in Ohm's law}

Another consequence of the previous instabilities, as well as the deviations in the Ohm's law, is in the rate of electron heating. Indeed, it is remarkably different between the antiparallel cases and the guide field ones where the deviations and streaming instabilities are taking place. In the antiparallel case, the tearing mode induces a preferential heating along its wavenumber vector $\vec{k}_{y}$ over both $x$ and $z$ directions. The temperature increases faster in this direction $\partial T_{e, y} / \partial t>\partial T_{e, x} / \partial t \sim \partial T_{e, z} / \partial t$, mostly in the outer boundary of the main magnetic island in comparison with its inside. In the perpendicular direction the heating takes place mostly uniformly everywhere inside of the island. The addition of a guide field changes gradually this picture. For $b_{g}=1$, the heating ratios along the tearing direction $y$ and $z$ becomes similar, while $x$ decreases its importance: $\partial T_{e, z} / \partial t \sim \partial T_{e, y} / \partial t>\partial T_{e, x} / \partial t$. What it is more important is that there is no significant increase of temperatures inside of the magnetic islands anymore. All the heating activity moves to the separatrices and the outer boundary of the secondary magnetic islands, where the anomalous momentum transfer due to $E_{z \text {,anomalous }}$ as well the cross-field 
streaming instabilities seen in Fig. 8.11 are active. A stronger guide field reduce further the importance of the in-plane heating (more isotropic now), becoming dominant only in the out-of-plane direction (due to the free streaming of electrons along that direction): $\partial T_{e, z} / \partial t \gg \partial T_{e, y} / \partial t \sim \partial T_{e, x} / \partial t$.

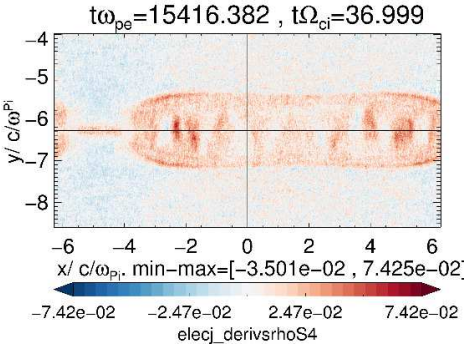

(a) $b_{g}=0$

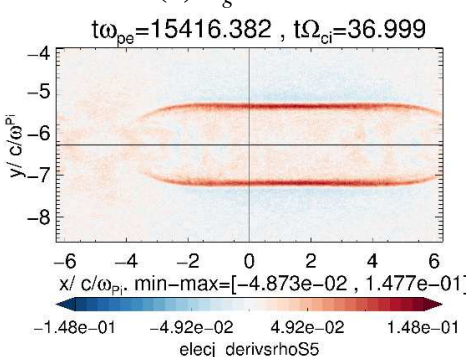

(d) $b_{g}=0$

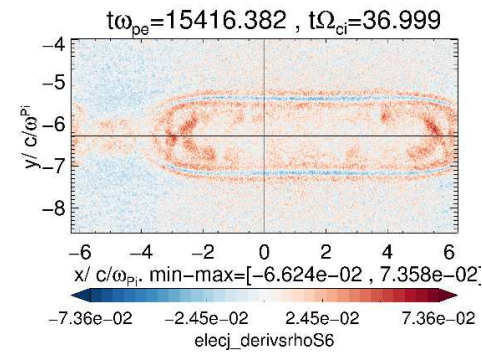

(g) $b_{g}=0$

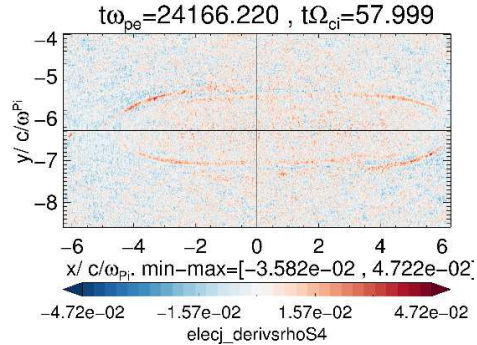

(b) $b_{g}=1$

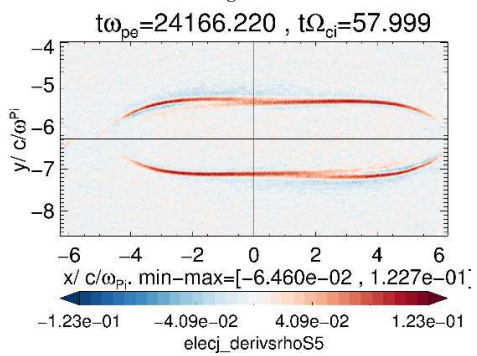

(e) $b_{g}=1$

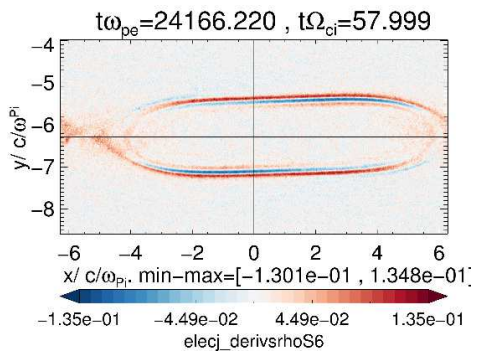

(h) $b_{g}=1$

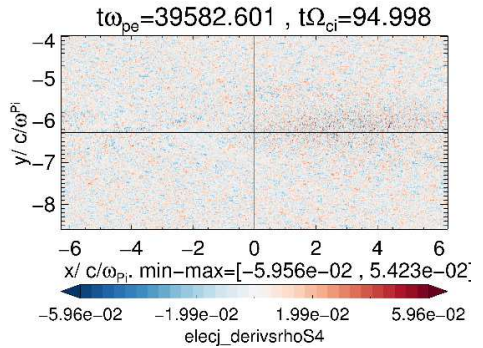

(c) $b_{g}=3$

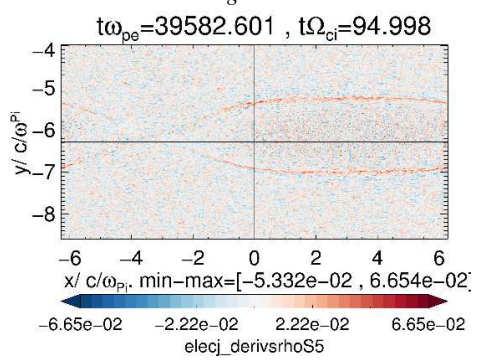

(f) $b_{g}=3$

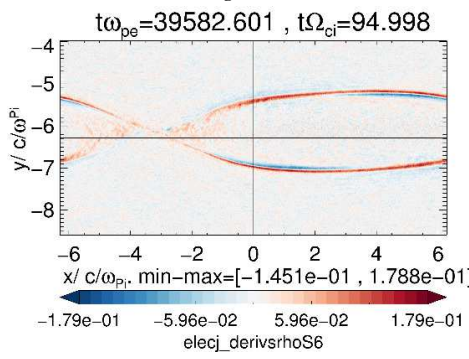

(i) $b_{g}=3$

Figure 8.13: Contour plots of different components of the electron heating rates (Eulerian derivatives) $\partial T_{e} / \partial t$ at reconnection peak time (in C.G.S. units) for different guide fields. These derivatives were calculated in running-time with the methods explained in Sec. 8.3.1.1. Top row: In-plane $\partial T_{e, x} / \partial t$. Middle row: In-plane $\partial T_{e, y} / \partial t$. Bottom row: Out-of-plane $\partial T_{e, z} / \partial t$.

There are also non-thermal consequences of the previous deviations in the Ohm's law and instabilities. As can be seen in Fig. 8.14, the location of non-thermal electrons changes radically between the antiparallel and guide field cases. In the antiparallel case, there is a dominant $K_{e, z}>0$ in the immediate neighborhood of the diffusion region, indicating non-Maxwellian tails in the 1D VDF $f\left(v_{z}\right)$. As we mentioned previously, there is non-negligible in-plane kurtosis $K_{e, y}$ in a extended region close to the diffusion region as well as in the border of the magnetic island, with opposite signs (see Fig. 8.14(leftcolumn)). The absolute values of these deviation are much smaller than those in $K_{e, z}$. On the other hand, a guide field makes all the deviations from the Maxwellian VDF very localized in the diffusion region and the border of the magnetic island. For $b_{g}=1$ (see 
Fig. $8.14\left(\right.$ middle-column)),, both $K_{e, y}$ and $K_{e, z}$ indicate similar deviations from equilibrium in both $y$ and $z$ direction. But for larger guide fields, $b_{g}=3$ (see Fig. 8.14)(rightcolumn)), the $z$ component becomes dominant over extended regions along the border of the magnetic island, signature of enhanced non-thermal processes taking place there. Therefore, this indicates an additional consequence and correlation with the places where the cross-field streaming instabilities and deviations $E_{z \text {,anomalous }}$ are expected to operate

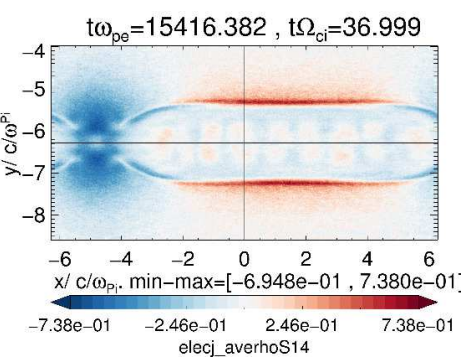

(a) $b_{g}=0$

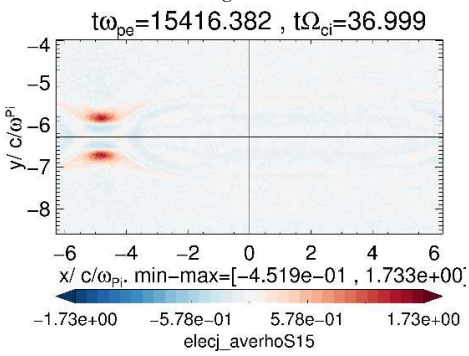

(d) $b_{g}=0$

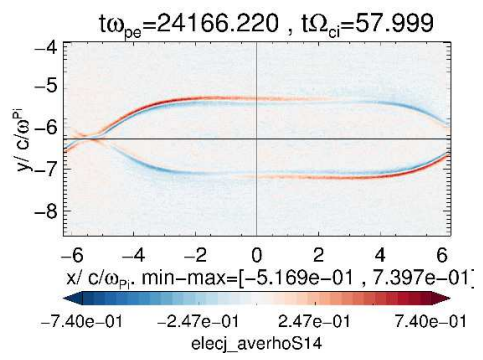

(b) $b_{g}=1$

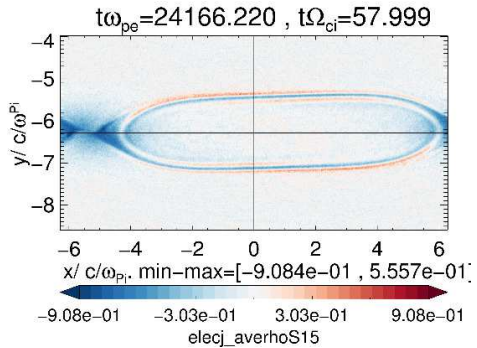

(e) $b_{g}=1$

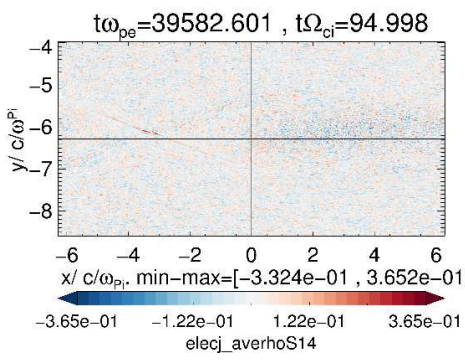

(c) $b_{g}=3$

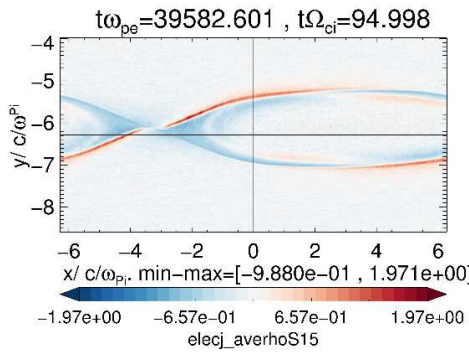

(f) $b_{g}=3$

Figure 8.14: Contour plots of electron excess kurtosis for different guide fields at reconnection peak time (in C.G.S. units). This 4th order momenta of the distribution function is explained in Appendix B.2.1. Top row: In-plane $K_{e, y}$. Bottom row $K_{e, z}$. The x component is not shown since it is negligible in comparison with the other components of the kurtosis.

\subsection{Conclusions}

In this chapter, we analyzed instabilities and reconnection in Harris CS with small guide fields $b_{g}<7$ (focusing especially in the range $0<b_{g}<3$ ). First, and during the course of a calibration of numerical parameters suitable for PIC simulations of guide field reconnection, we found that tearing mode can be stabilized and the CS can develop a bifurcated structure if the electron Larmor radius on the guide field $\rho_{e, b g}$ is not well resolved by the grid cell size $\Delta x$. It is necessary at least $\rho_{e, b g}>0.5 \Delta x$ to avoid this artificial effect. That condition becomes more difficult to fulfill for more realistic parameters: higher mass ratios $\left(m_{i} / m_{e}\right)$ and lower electron thermal speeds $v_{t h, e}$. It is important to remark that this effect has nothing to do with stability or accuracy conditions of the algorithm used by the PIC code (Boris pusher to advance the particles), and it has not been reported before in this kind of setup. Furthermore, it is of a different nature that the bifurcation of CS seen in the previous Chapter 7 driven by temperature anisotropies: it does not become deeper with time and it is not associated with an increase in the total energy.

Once the previous numerical consideration was properly taken into account, we could recover the expected delay in the reconnection onset for increasing guide fields, with the 
associated reduction of tearing growth rates for guide fields as high as $b_{g}=7$ in agreement with theoretical estimations. Note that the requirement of resolving well enough the electron Larmor radius on the guide field makes the PIC simulations for even higher guide fields computationally more demanding, and that is why in the next Chapter 9 we are going to change the approach for reaching the large guide field limit.

Then, we developed calculation methods for time and spatial averages (in both running time and post-processing) required for a proper calculation of the terms in the generalized Ohm's law, as well as numerical derivatives of some momenta of the distribution function. By means of that, we explored the dissipative mechanism responsible for the breaking of the frozen-in condition. In the limit case of an antiparallel configuration, the reconnected electric field is sustained mostly by the non-gyrotropy of the pressure tensor, and to a lesser extent by the electron inertia, in agreement with the theoretical expectations and many previous works.

In the limit case of zero guide field we also found signatures of Weibel instability inside of the magnetic island. We noticed that this process, driven by temperature anisotropy, it is only possible with a good enough spatial resolution and relatively high number of macroparticles per cell. Otherwise, numerical collisions dominate the system tending to the isotropization of the distribution functions and suppressing any temperature driven instability.

Next, under the influence of a guide field, we confirmed that the reconnected electric field is sustained by the effects of both non-gyrotropy of the pressure tensor as well as the electron inertia, but now confined to smaller scales. The latter term becomes increasingly more important for larger $b_{g}$, as the length scales of the electron gyroradius $\rho_{e, b g}$ on the guide field decrease. This emphasizes even more the requirement of a good resolution of the grid cell size to be able to distinguish these effects.

However, we noticed that the balance of terms in the generalized Ohm's law loses accuracy for higher guide fields. This is not only due to the enhanced noise level, but it seems to be additional processes responsible for this behaviour. Indeed, we found signatures that the appearance of an unbalanced term in the mean field Ohm's law is possibly due to anomalous electromagnetic fluctuations $E_{z \text {,anomalous }}$, especially relevant for the magnetic island, away from the diffusion region and $\mathrm{X}$ point. The argument for this claim are: 1) a very localized region where this unbalance holds: mostly in the boundaries of the magnetic island 2) a spatial correlation with the deceleration of the electron bulk flow speed $\partial V_{e, z} / \partial t<0$, always related with anomalous resistivity, 3) some signatures of additional streaming and gradient driven instabilities that might result as consequence of this effect. In addition, we also noticed that the electron inertia term $E_{z \text {,inertia }}$ is a good proxy for the locations where this anomalous term should be operating.

Finally, we found that the aforementioned instabilities associated with the locations where $E_{z, \text { anomalous }}$ is non-negligible have basically two different origins. The first one is possibly the cross-field streaming instability MTSI, due to counterstreaming electron flows in the boundaries of the secondary magnetic islands. The second one is LHDI, due to the strong density gradient quickly developed in the same regions due to pile-up of electrons deflected by the guide field. Both instabilities, with similar time and length scales, only appear under the influence of a guide field, and they become stronger for larger $b_{g}$. Their effects are an enhanced electron heating along the $z$ direction, the generation of non-thermal tails of the electron distribution function (mostly in the same $z$ direction) and 
turbulent wave activity in these regions after their saturation time. 


\section{Disclaimer for Chapter 9}

The work to be shown in the following chapter was result of a code benchmark collaboration with D. Told and F. Jenko (at Max-Planck-Institut für Plasmaphysik and Department of Physics and Astronomy, University of California, Los Angeles, USA). The gyrokinetic simulations with the GENE code were carried out by D. Told. However, the PIC simulations, analysis and comparison of results was done by the author of this thesis.

Roughly part of the first half of this chapter is based on and follows very closely the line of argumentation of the article: P. A. Muñoz, D. Told, P. Kilian, J. Büchner and F. Jenko "Gyrokinetic and kinetic particle-in-cell simulations of guide-field reconnection. I: Macroscopic effects of the electron flows", Physics of Plasmas 22, 082110, (2015) (Muñoz et al. 2015), also available as arXiv eprint:1504.01351. Most of the text has been rewritten and mostly expanded and/or moved to the Introductory chapters or Appendix, although some parts can still be traced to the original source. In particular, some sentences or sections of paragraphs can be similar or identical to sections of the previous (non-referred) versions available on the arXiv eprint.

In particular, comparing with the published version:

- 11 from 13 figures of Muñoz et al. (2015) have been used in this chapter, explicitly indicated in the caption (from a total of 33 figures).

- Sec. 9.3.2 is based on Section III-B of Muñoz et al. (2015).

- Sec. 9.4.1 is based on Section IV-A of Muñoz et al. (2015).

- Sec. 9.4.2 is based on Section IV-B of Muñoz et al. (2015).

- Sec. 9.5.1 is based on Section V-A and Appendix A of Muñoz et al. (2015).

- Sec. 9.5.2 is based on parts of Section V-B of Muñoz et al. (2015).

- Sec. 9.5.3 is based on parts of Section V-B of Muñoz et al. (2015).

- Sec. 9.5.4 is based on parts of Section V-C of Muñoz et al. (2015).

- Sec. 9.5.5 is based on Section V-D of Muñoz et al. (2015)

- Sec. 9.5.6 is based on Section V-E of Muñoz et al. (2015).

- Sec. 9.9.1 (few paragraphs) are based on parts of Section VI of Muñoz et al. (2015).

- Sec. 9.9.2 (few paragraphs) are based on parts of Section VI of Muñoz et al. (2015). 



\section{Instabilities of force free CS in moderate and large guide fields: comparison with gyrokinetic simulations}

Since in laboratory and fusion environments the plasma $\beta$ is even smaller than in the solar corona (equivalent to very strong guide magnetic fields $b_{g} \rightarrow \infty$ ), it is often used an approximation of the Vlasov-Maxwell system, called gyrokinetic theory. This approach, to be explained in Sec. 9.1, eliminates the particle fast gyromotion on strong magnetic fields. However, for moderate guide fields strengths, more typical in the solar corona, it is not clear to what extent GK can be applied.

There is an additional numerical interest in this kind of approach/codes. As we already showed in previous chapters, PIC simulations of magnetic reconnection can be computationally very demanding if more realistic parameters are used. This is because the stability conditions require resolve electron time $\left(\omega_{p e}^{-1}, \Omega_{c e}^{-1}\right)$ and length $\left(\Lambda_{D e}\right)$ scales (Sec. 6.8.1), although the global evolution is on time $\left(\Omega_{c i}^{-1}\right)$ and length $\left(L \gg d_{i}\right)$ scales orders of magnitude larger. Besides of that, it is necessary to keep controlled the numerical noise, which requires the computationally demanding requirement of a higher large number of particles per cell and higher order shape functions. Otherwise, numerical instabilities can be triggered that can completely hidden the development of the physical phenomenon wanted to be modeled (see Sec. 6.8.1). There have been proposed several approaches to tackle the previous problem since several decades ago, some of them explained in Sec. 6.7.1 and Sec. 6.8.1.2. One of the most successful and popular in modeling laboratory and fusion plasmas (not mentioned in the general discussion about PIC codes in Chapter 6) is precisely the aforementioned gyrokinetic theory, showing huge speeding ups over the computationally demanding PIC simulations. Only recently it has started to be applied for applications to magnetic reconnection (see Sec. 9.1.2), but in the limit of very large guide fields. This approach might also be incredible useful for solar coronal simulations of magnetic reconnection, but again, it is uncertain how reliable can their results be trusted in the more realistic limit of moderate guide fields.

For these reasons, in this chapter we aim towards the identification of the parameter regimes, i.e.: guide field strength, for where the gyrokinetic approach can give an accurate description of magnetic reconnection in the solar corona. We investigate the limits of its applicability by means of a detailed comparison benchmark with the fully-kinetic PIC code ACRONYM, which does not have any approximation regarding guide field 
strengths.

Only recently there was a benchmark study of magnetic reconnection with both plasma models carried out by TenBarge et al. (2014), showing exciting results (see details in Sec. 9.1.2). This motivated us to follow their approach and extend their work, by answering some of the unsolved questions that remained in the original paper. In this chapter, we are going to investigate the differences in the development of magnetic reconnection between both codes, especially in the PIC low guide field regime. In other words, we want to establish the limits of applicability of the GK simulations: how low can the PIC guide field be in order to have a similar description of this physical process. As we mentioned, this is of particular relevance for the range of guide fields typically found in the Solar corona (large, but far from being as dominant as in fusion plasmas). We are going to show that the answer depends of several numerical and physical parameters, and it is also dependent on the spatial location (some areas show better convergence between both codes for lower guide field than other ones). There are numerical reasons for that, related with a necessary different initialization of both codes and the way in which the pressure equilibrium condition is considered. These numerical constraints lead to a different physical evolution of the system in the PIC low guide field regime, breaking the comparison with the GK results, mostly due to the effects of a shear flow and secondary magnetic islands.

This chapter is divided as follows. First we introduce the gyrokinetic theory and the recent progress in gyrokinetic simulations of magnetic reconnection in Sec. 9.1.2. We describe the setup of the simulations in Sec. 9.2. Then, in Sec. 9.3, we show our reproduction of the previous comparison study by TenBarge et al. (2014) in which our work is based. This is done in order to identify precisely to what extent the linear scaling reported by them is satisfied in our case, as well as to identify the open problems and differences. Those are manifested in both magnetic and thermal pressures. In Sec. 9.4, it is discussed how a core magnetic field appears in the PIC low guide field regime as a result of the different treatment of the pressure equilibrium condition between both codes, in the framework of a two fluid model. Next, in Sec. 9.5, we discuss the physical mechanism behind that process, due to an initial shear flow in the PIC low guide field regime. The differences in the behaviour and location of the different components of the diagonal terms of the pressure tensor (thermal fluctuations) are discussed in Sec.9.6. Afterwards, in Sec. 9.7, we focus in the non thermal features given by the off-diagonal terms of the pressure tensor and dissipative mechanisms, in addition to non-thermal features revealed via the analysis of the electrons VDFs and energy spectra. In Sec. 9.8 we studied how cross-streaming instabilities develop in the PIC low guide field regime than cannot be captured by the gyrokinetic approach. The effects of a high $\beta$ plasma are analyzed in Sec. 9.9. In Sec. 9.10 we make some remarks about the reliability of the PIC results in the high guide field regime due to the role of the numerical noise. And finally we summarize our findings in the conclusion, Sec.9.11. 


\subsection{Gyrokinetic approach}

\subsubsection{Gyrokinetic theory}

When a plasma is strongly magnetized, the particles mostly perform a gyromotion around the guiding center, in the perpendicular direction to the magnetic field. If all the perpendicular motions different to this gyration are considered small enough, we have the so called gyrokinetic approximation (see the original approach described in Frieman and Chen (1982), or the more recent review in Brizard and Hahm (2007)). This approach reduces the dimensionality of solving the Vlasov-Maxwell the system from a phase space of 6 to 5 components (Howes et al. 2006), by eliminating the electron gyromotion from the dynamics of the system.

The gyrokinetic equations can be applied when the following conditions are satisfied (Howes et al. 2006):

1. Strong magnetized conditions:

$$
\rho_{i}=\frac{v_{t h, i}}{\Omega_{c i}} \ll L
$$

where $L$ is a typical length scale of the gradients in a system. It is important to note that this condition do not require low beta plasmas. High beta plasmas, such those of the solar wind, also satisfies this condition even though they have weak magnetic field, because the spatial variations of the gradient are extended over large $L$. This also defines the so called ordering parameter $\epsilon$ :

$$
\epsilon=\frac{\rho_{i}}{l_{0}} \ll 1
$$

where $l_{0}$ is a typical length scale, smaller than the parallel wavelengths of the fluctuations. $\epsilon$ is used for the formal expansion of all the equations used in the gyrokinetic approach.

The definition Eq. (9.2) has an important consequence for the study of thin current sheets. From Eq. (A.4), these structures have to be sustained by out-of-plane drifts speeds $U_{d} / v_{t h, i}$, inversely proportional to the halfwidth measured in units of $\rho_{i}$. This means that the drift speed, and thus the current, is on order $\epsilon$. Thinner current sheets, of order $L \lesssim \rho_{i}$, will break the GK ordering.

2. Low frequency phenomena

$$
\omega \sim \frac{v_{t h, i}}{l_{0}} \sim O\left(\epsilon \Omega_{c i}\right) \ll \Omega_{c i}
$$

where $\omega$ is the typical frequency of the fluctuations of the distribution function $\delta f_{1}$, magnetic $\delta \vec{B}$, and electric fields $\delta \vec{E}$, respectively. The collision frequency is also assumed to have this frequency: $v \sim \omega$ (gyrokinetic cannot treat properly systems with collisional frequencies much higher than $\omega$ ). This allows to average over the Larmor radius of the particles, eliminating the dimension perpendicular to the magnetic field. 
3. Small fluctuations with respect to the equilibrium. Let $F_{0}, B_{0}$ and $E_{0}=v_{t h, i} B_{0}$ the equilibrium distribution function, magnetic and electric fields, respectively. Then, we have.

$$
\frac{\delta f_{1}}{F_{0}} \sim \frac{\delta \vec{B}}{B_{0}} \sim \frac{\delta \vec{E}}{v_{t h, i} B_{0}} \sim O(\epsilon)
$$

The equilibrium distribution function is allowed to vary slow enough:

$$
\frac{1}{F_{0}} \frac{\partial F_{0}}{\partial t} \sim O\left(\frac{1}{t_{\text {heat }}}\right)
$$

where $t_{\text {heat }}$ is the so called heating time-scale:

$$
t_{\text {heat }}=\frac{1}{\epsilon^{2}} \frac{l_{0}}{v_{t h, i}} \sim O\left(\frac{1}{\epsilon^{3} \Omega_{c i}}\right)
$$

4. Small/large spatial variations across/along the magnetic field.

$$
\begin{aligned}
& k_{\perp} \sim \frac{b \times \nabla \delta f_{1}}{\delta f} \sim \frac{b \times \nabla \delta \vec{B}}{|\delta \vec{B}|} \sim \frac{b \times \nabla \delta \vec{E}}{|\delta \vec{E}|} \sim O\left(\frac{1}{\rho_{i}}\right) \quad \Leftrightarrow \quad k_{\perp} \rho_{i} \sim 1 \\
& k_{\|} \sim \frac{b \cdot \nabla \delta f_{1}}{\delta f} \sim \frac{b \cdot \nabla \delta \vec{B}}{|\delta \vec{B}|} \sim \frac{b \cdot \nabla \delta \vec{E}}{|\delta \vec{E}|} \sim O\left(\frac{1}{l_{0}}\right)
\end{aligned}
$$

where $b$ is the direction of the equilibrium magnetic field. The two previous conditions imply that the anisotropy of the fluctuations in the gyrokinetic approach is also of order epsilon:

$$
\frac{k_{\|}}{k_{\perp}}=\frac{\rho_{i}}{l_{0}} \sim O(\epsilon)
$$

This equation reproduces a key prediction/conjecture of the theory of MHD turbulence: the energy cascade of the magnetic field have at its very end parallel wavelength much larger than the perpendicular ones: $k_{\|} \ll k_{\perp}$. This prediction is also supported by a large number of observations in the solar wind and in MHD simulations (see, e.g.: Howes et al. 2006, Schekochihin et al. 2009, and references therein).

Another important consequence of Eq. (9.9) is that imposes an additional constraint on the perpendicular bulk speed $\vec{u}_{\perp}$. It is known than in strongly magnetized plasmas, the motion of particles perpendicular to the magnetic field is dominated by drifts. Assuming no strong gradients, the dominant drift will be the $\vec{E} \times \vec{B}$, which implies, by using Eq. (9.4),

$$
\vec{u}_{\perp} \sim \frac{\delta \vec{E} \times \vec{B}_{0}}{B_{0}^{2}} \sim O\left(\epsilon v_{t h, i}\right)
$$

Thus, the maximum spatial fluctuations perpendicular to the magnetic field are of the order

$$
l_{\perp} \sim \frac{\vec{u}_{\perp}}{\omega} \sim \frac{1}{k_{\perp}} \sim O\left(\rho_{i}\right)
$$


As a consequence, the GK ordering breaks down for perpendicular speeds on the order of the ion thermal speed. Thus, the usual GK approach is valid for subsonic speeds. However, recently there have been extensions of the theory that do allow supersonic speeds under some circumstances (see Sharma et al. 2015, and references therein).

Under the previous assumptions is possible to derive the gyrokinetic equations. See the detailed derivation in, e.g., Brizard and Hahm (2007) or the theses by Merz (2008), Told (2012). The gyrokinetic model have been widely used for studies of turbulence in fusion research (Jenko et al. 2000, Brizard and Hahm 2007) and astrophysical turbulence (Howes et al. 2006, Schekochihin et al. 2009).

It is important to mention that the gyrokinetic equations order out fast MHD waves, Langmuir oscillations and cyclotron resonances. On the other hand, they retain finiteLarmor effects, parallel Landau damping, transit time damping and slow MHD waves (as long as they are anisotropic $\left.k_{\|} \sim \epsilon k_{\perp}\right)$.

Now, codes based in the gyrokinetic approach have proved to be useful to model strongly magnetized plasmas, where that plasma model holds. Their main numerical advantage is that they can reduce the computationally cost of the simulations in some order of magnitude in comparison with PIC codes. This is because they allow to overcome the limitation in grid size and time stepping from PIC codes: $\Delta x<\lambda_{D e}$ and $\Delta t \omega_{p e}<1$, respectively, to the more relaxed conditions:

$$
\Delta x<\rho_{i}, \quad \Delta t \Omega_{c i}<1
$$

In this way, GK simulations can allow the use of larger ion to electron mass ratios, larger simulations boxes and longer time scales than those used by PIC codes. One consequence of the GK ordering is the fact that the background magnetic field $B$ varies on timescales much larger than the particle gyromotion. As a consequence, the magnetic moment $\mu$ Eq. (2.60) is an adiabatic invariant. For this reason, it is used in GK codes to define the perpendicular velocity grid, usually normalized with respect to a factor depending on the thermal particle energy $\mu_{\text {norm }}=k_{B} T_{i} / B$. The parallel velocity grid is defined independently, being normalized to a factor depending on both thermal and ion sound speeds (not involving the background magnetic field).

The results of the gyrokinetic simulations to be shown in this thesis were performed by GENE code1 ("Gyrokinetic Electromagnetic Numerical Experiment"). It is a Eulerian gyrokinetic code (fluid-like, no intrinsic noise), using explicit finite-difference methods of the predictor-corrector type with a dissipative scheme. The code is second-order accurate in phase space and time (see more details in Merz 2008, Told 2012).

\subsubsection{Gyrokinetic simulations of tearing instability/magnetic recon- nection}

Simulations based on the gyrokinetic approach have proved to be successful to model very accurately fusion and laboratory plasmas since some decades ago. However, only recently has started to be applied to study magnetic reconnection, with different purposes

\footnotetext{
${ }^{1}$ See Jenko et al. (2000). Freely available at http://genecode . org/
} 
and results. There are several drawbacks of this approach, however. The gyrokinetic model has to assume an initial equilibrium with a very strong guide field very $B_{g} / B_{\infty y} \gg 1$. In addition, several wave modes that might play a key role in reconnection but have high frequencies $\omega \lesssim \Omega_{c e}$, are ordered out. The first gyrokinetic simulations of tearing mode/magnetic reconnection were performed by Sydora (2001), Wan et al. (2005a) and Wan et al. (2005b), using a initial Gaussian current distribution that gives a magnetic field with an error function profile. These studies allowed the use of realistic mass ratios, something prohibitively expensive for PIC simulation at that time. Later, gyrokinetic simulations by Rogers et al. (2007) used an initial sinusoidal magnetic field equal to Eq. (3.25) for the reconnecting component, while the out-of-plane component is taken to be constant and high enough. Their analysis was restricted for cases with the ion sound Larmor radius less than the electron skin depth $\rho_{s}=\left(v_{t h, i}+v_{t h, e} / \sqrt{m_{i} / m_{e}}\right) / \Omega_{c i}<d_{e}$. They studied linear and non-linear tearing growth rates and reconnection rates, finding good agreement with previous theoretical estimations based in two fluid and kinetic models (in the two asymptotic regimes of large and small tearing stability parameter $\Delta^{\prime}$ ) for low beta plasma regimes. They applied linear theory obtained with fluid models (Porcelli 1991) valid in the range $2\left(m_{e} / m_{i}\right) \ll \beta_{\text {total }} \ll\left(m_{e} / m_{i}\right)^{1 / 4}$. For high beta plasma regimes, however, they reported large deviation from the existing analytical estimations, possible due to the role played by finite Larmor radius. These deviations from fluid theories were confirmed by Numata et al. (2011), which expanded the previous work by studying a wider parameter range of plasma beta, but using a different magnetic field profile and small collisional effects.

Wang et al. $(2008,2011)$ performed simulations of magnetic reconnection with a hybrid model in between the traditional gyrokinetic and PIC approaches called GeFi: "Gyrokinetic electrons plus Fully kinetic ions" (developed in Lin et al. 2005, 2011). With this code, their simulation model could access to frequency ranges $\omega<\Omega_{c e}$, allowing whistler and lower-hybrid waves, besides of including finite electron Larmor radius effects (off-diagonal components of the pressure tensor, of critical importance in studies of magnetic reconnection). They used this code to study drift instabilities in a Harris current sheet with finite guide field, something not allowed by the traditional gyrokinetic approach. Consistently, they compared their simulations results with the predictions of a gyrokinetic eigenmode theory extended to include finite Larmor radius effects. Wang et al. (2011) also benchmarked their results with those obtained with a Darwin PIC code simulation. They found large deviations between the linear tearing growth rates calculated with both codes for thinner CS $L \ll \rho_{i}$ and stronger guide fields $B_{g} \gtrsim B_{\infty y}$. Later, this model was applied to study other instabilities in the reconnection process, such as LHDI (Tummel et al. 2014).

Pueschel et al. (2011) performed simulations of magnetic reconnection with the same code used in our study (GENE), covering a much wider range of parameters than those used in previous studies. They initialized a current sheet with several periodic sinusoidal magnetic field profiles (one of them similar to the one used in Rogers et al. 2007). Their results still showed good agreement with the same fluid theory used by Rogers et al. (2007) during the linear stage

Another studies by Numata and Loureiro (2014, 2015) studied tearing instability with an initial magnetic field profile based on a modified Harris current sheet, multiplied by a special function to enforce periodicity (in addition to a strong guide field). They focused 
mostly in the effects of a small but non negligible collisionality. The main finding of this study is that gyrokinetic simulations of reconnection in low beta plasma show strong electron heating via Landau damping, in agreement with previous studies using fluidkinetic codes (Loureiro et al. 2013b). Ion heating is much smaller, being relevant only in high beta environments (Numata and Loureiro 2014).

It is important to remark that gyrokinetic simulations of magnetic reconnection can be truly collisionless, not being affected by numerical collisions like in PIC simulations. A consequence of this fact is that a completely saturated state of magnetic reconnection can be run backwards in time, in such a way that particle energy can be given back to the magnetic field lines (Ishizawa and Watanabe 2013).

Zacharias et al. (2012) and Kobayashi et al. (2014) applied gyrokinetic model to study reconnection in a system with strong gradients in both density and temperature, finding suitable range of parameters where reconnection can be stabilized or destabilized by them.

Hornsby et al. (2014b,a c) studied interaction of unstable tearing modes with (3D) gyrokinetic turbulence. In a similar way, Pueschel et al. (2014) applied gyrokinetic codes to study secondary magnetic reconnection events in fully developed 2D turbulence, arising from a reconnection driven by linear tearing mode. They used the same initial sinusoidal current sheet as in Rogers et al. (2007) and Pueschel et al. (2011), but repeated many times inside of the simulation box. They found a strong temperature anisotropy arising from the linear tearing mode (in contradiction with previous studies), a result important for our future discussions.

It is important to remark that most of the previous studies make comparisons of simulation results with fluid theories, because gyrokinetic theory of magnetic reconnection is far from being complete, and is much less developed than the full kinetic collisionless theory. Although gyrokinetic theory is a radical simplification from the Vlasov theory, its intrinsic mathematical complexity is not less than considering the full Vlasov-Maxwell system (Zocco and Schekochihin 2011). In this sense, gyrokinetic simulations of magnetic reconnection have also been benchmarked against gyrofluid codes (which retain some of the kinetic effects present in gyrokinetic models) to study tearing modes (Zacharias et al. 2014).

The main question that arises from the gyrokinetic simulations of magnetic reconnection is to what extent they can model accurately this process, in the sense of an agreement with the results given by the fully-kinetic PIC simulations. This is a question of central importance in order to have reliable results, without resorting to expensive PIC simulations. As mentioned previously, only very recently the first attempt for solving this question was started by TenBarge et al. (2014). They carried out and compared PIC with GK simulations of magnetic reconnection in the large guide field limit. The global evolution was similar in both cases, with a quadrupolar structure of thermal and magnetic pressures around the X point. The PIC runs displayed a convergence to the GK morphology of these structures for increasingly larger guide fields. They showed that most of the reconnection related quantities, such as the reconnection rate, outflow speeds, out-of-plane magnetic field, scale linearly with the guide field in the PIC case, converging to the GK result in the large $b_{g}$ limit. The good agreement among these values is obtained after a proper normalization and matching between the $\epsilon$ parameter of GK with $b_{g}$ in PIC. This is under the assumption of a total plasma $\beta$ constant for different PIC guide fields, which implies a reduction in the reconnected magnetic field $B_{\infty y}$ to obtain the effect of a stronger guide 
field. They also noticed that the morphological convergence between the results given by both codes required lower guide fields for high beta plasmas $\left(\beta_{i}=1\right)$. The work to be shown in this chapter is an extension of theirs.

\subsection{Simulation setup}

\subsubsection{Force free CS}

\subsubsection{Justification for the use of a force free CS}

Different from the previous chapters, the initialization of the CS cannot be a Harris equilibrium in gyrokinetic simulations. This is because the equilibrium distribution function for the background in GK is always a non-drifting Maxwellian $f_{0}=\left(n_{0} /\left(\sqrt{2 \pi} v_{t h}\right)^{3}\right)$ $\exp \left[\frac{1}{2 v_{t h}^{2}}\left(v_{\perp}^{2}+v_{z}^{2}\right)\right]$ (see, e.g., the theses by Merz (2008, Sec. 2.5) and Told (2012, Sec. 2.4.1)). Then, the particles that carry the initial current in the Harris case (spatially varying drifting Maxwellians) are considered to be a perturbation on this background. This means that both density and drift speed are perturbed quantities in the electron VDF Eq. 3.11):

$$
\begin{aligned}
f=f_{0}+\delta f_{\text {harris }} & =f_{0}+\delta\left\{\frac{n(x)}{\left(\sqrt{2 \pi} v_{t h, e}\right)^{3}} \exp \left[\frac{-1}{2 v_{t h, e}^{2}}\left(v_{\perp}^{2}+\left(v_{z}-U_{d}\right)^{2}\right)\right]\right\} \\
& =f_{0}+\left\{\frac{\delta n(x)}{\left(\sqrt{2 \pi} v_{t h, e}\right)^{3}} \exp \left[\frac{-1}{2 v_{t h, e}^{2}}\left(v_{\perp}^{2}+\left(v_{z}-\delta U_{d}\right)^{2}\right)\right]\right\} \\
& \approx f_{0}+\frac{\delta n(x)}{\left(\sqrt{2 \pi} v_{t h, e}\right)^{3}} \exp \left[\frac{-1}{2 v_{t h, e}^{2}}\left(v_{\perp}^{2}+v_{z}^{2}\right)\right]\left\{1+\frac{2 v_{z} \delta U_{d}+\ldots}{2 v_{t h, e}^{2}}\right\} \\
& \approx f_{0}+\frac{\delta n(x)}{n_{0}} \underbrace{\frac{n_{0}}{\left(\sqrt{2 \pi} v_{t h, e}\right)^{3}} \exp \left[\frac{-1}{2 v_{t h e}^{2}}\left(v_{\perp}^{2}+v_{z}^{2}\right)\right]}_{=f_{0}}+O\left(\delta^{2}\right) \\
& =f_{0}\left(1+\frac{\delta n(x)}{n_{0}}\right)+O\left(\delta^{2}\right)
\end{aligned}
$$

where we can identify $\delta f_{\text {harris }}=f_{0} \frac{\delta n(x)}{n_{m}}$. But according to the gyrokinetic equations (see, e.g., Merz (2008, Sec. 2.5.2) or Told (2012, Sec. 2.5.2)), the perturbed parallel current density $J_{\|}=J_{z}$ is proportional to:

$$
\delta J_{e, \|} \propto e \int v_{z} \delta f d v_{\|} d v_{\perp}=e \int v_{z} f_{0} \frac{\delta n(x)}{n_{0}} d v_{\|} d v_{\perp}=0 .
$$

Therefore, there is no initial current that can sustain the sheared Harris magnetic field to first order in GK. For this reason we use instead a force free CS $\vec{J} \times \vec{B}=0$. We discussed and compared it to the Harris equilibrium in Sec. 3.2.2. Since there is no known kinetic equilibrium of a force free configuration with a guide field (see Sec. 3.2.2.3), we chose the magnetic field profile given by Eq. (3.42), corresponding a one with constant magnitude that rotates around the center of the CS by an angle given by Eq. (3.44). The sheared magnetic field is sustained by the current given by Eq. (3.45), with a $J_{z}$ component with 
the same form as in the Harris case. This current is chosen to be carried only for electrons (and stationary ions), with the VDF Eq. (3.47) featuring a spatially varying drift speed $\vec{V}_{e, y / z}(\vec{x})$ and constant density $n_{0}$. Because the equilibrium VDF in GK has the same last property of constant density, the full GK VDF is equal to the perturbation of the force free VDF:

$$
\begin{aligned}
f=\delta f_{\mathrm{ff}} & =\delta\left\{\frac{n_{0}}{\left(\sqrt{2 \pi} v_{t h, e}\right)^{3}} \exp \left[\frac{-1}{2 v_{t h, e}^{2}}\left(v_{x}^{2}+\left(v_{y}-V_{e, y}(\vec{x})\right)^{2}+\left(v_{z}-V_{e, z}(\vec{x})\right)^{2}\right)\right]\right\} \\
& =\frac{n_{0}}{\left(\sqrt{2 \pi} v_{t h, e}\right)^{3}} \exp \left[\frac{-1}{2 v_{t h, e}^{2}}\left(v_{x}^{2}+\left(v_{y}-\delta V_{e, y}(\vec{x})\right)^{2}+\left(v_{z}-\delta V_{e, z}(\vec{x})\right)^{2}\right)\right] \\
& \approx \frac{n_{0}}{\left(\sqrt{2 \pi} v_{t h, e}\right)^{3}} \exp \left[\frac{-1}{2 v_{t h, e}^{2}}\left(v_{\perp}^{2}+v_{z}^{2}\right)\right]\left\{1+\frac{2 v_{y} \delta V_{e, y}(\vec{x})+\ldots}{2 v_{t h, e}^{2}}\right\}\left\{1+\frac{2 v_{z} \delta V_{e, z}(\vec{x})+\ldots}{2 v_{t h, e}^{2}}\right\}+O\left(\delta^{2}\right) \\
& =f_{0}\left(1+\frac{v_{y} \delta V_{e, y}(\vec{x})}{v_{t h, e}^{2}}+\frac{v_{z} \delta V_{e, z}(\vec{x})}{v_{t h, e}^{2}}\right)+O\left(\delta^{2}\right)
\end{aligned}
$$

In this way, now we have a non-zero initial perturbed current in the parallel direction

$$
\begin{aligned}
\delta J_{e, \|} & \propto e \int v_{z} \delta f d v_{\|} d v_{\perp}=e \int v_{z} f_{0}\left(1+\frac{v_{y} \delta V_{e, y}(\vec{x})}{v_{t h, e}^{2}}+\frac{v_{z} \delta V_{e, z}(\vec{x})}{v_{t h, e}^{2}}\right) d v_{\|} d v_{\perp} \\
& =e \frac{\delta V_{e, z}(\vec{x})}{v_{t h, e}^{2}} \int v_{z}^{2} f_{0} d v_{\|} d v_{\perp} \neq 0
\end{aligned}
$$

while in the perpendicular direction is still zero:

$$
\delta J_{e, \perp} \propto e \int v_{\perp} \delta f d v_{\|} d v_{\perp}=e \frac{\delta V_{e, y}(\vec{x})}{v_{t h, e}^{2}} \int v_{y} v_{\perp} f_{0} d v_{\|} d v_{\perp}=0
$$

by using the symmetry properties of $f_{0}$. Then, the force free VDF Eq. (3.47) can provide an initial current in the out-of-plane direction capable to sustain the reconnected magnetic field, and this is the reason because we are going to use it instead of the Harris equilibrium for a comparison with GK. On the other hand, Eq. (9.25) indicates that there is no initial perpendicular current in the GK initialization (to a first order), different from the Harris case. This will be a source of differences in the results to be shown.

\subsubsection{Double force free CS to be used}

Now, in order to avoid numerical issues related with the boundary conditions for a single force free CS, and following the approach by TenBarge et al. (2014), we use instead a double force free CS with periodic boundary conditions. The left one is centered in $x=L_{x} / 4$ and the right one in $x=3 L_{x} / 4$. In order to have a zero asymptotic magnetic field, this has to rotate in opposite directions for each CS, being given by:

$$
\begin{aligned}
& B_{y}=B_{\infty y}\left[\tanh \left(\frac{x-L_{x} / 4}{L}\right)-\tanh \left(\frac{x-3 L_{x} / 4}{L}\right)-1\right] \\
& B_{z}=B_{\infty y} \sqrt{b_{g}^{2}+\cosh ^{-2}\left(\frac{x-L_{x} / 4}{L}\right)+\cosh ^{-2}\left(\frac{x-3 L_{x} / 4}{L}\right)}
\end{aligned}
$$


with $b_{g}=\frac{B_{g}}{B_{\infty y}}$ the guide field. Consistently, we have the corresponding current density profile:

$$
\begin{aligned}
& J_{e, z}=\frac{1}{\mu_{0}} \frac{\partial B_{y}}{\partial x}=\frac{B_{\infty y}}{\mu_{0} L}\left[\cosh ^{-2}\left(\frac{x-L_{x} / 4}{L}\right)-\cosh ^{-2}\left(\frac{x-3 L_{x} / 4}{L}\right)\right] \\
& J_{e, y}=-\frac{1}{\mu_{0}} \frac{\partial B_{z}}{\partial x}=\frac{B_{\infty y}}{\mu_{0} L}\left[\frac{\tanh \left(\frac{x-L_{x} / 4}{L}\right) \cosh ^{-2}\left(\frac{x-L_{x} / 4}{L}\right)+\tanh \left(\frac{x-3 L_{x} / 4}{L}\right) \cosh ^{-2}\left(\frac{x-3 L_{x} / 4}{L}\right)}{\sqrt{b_{g}^{2}+\cosh ^{-2}\left(\frac{x-L_{x} / 4}{L}\right)+\cosh ^{-2}\left(\frac{x-3 L_{x} / 4}{L}\right)}}\right]
\end{aligned}
$$

Both profiles can be seen in Fig. 9.1

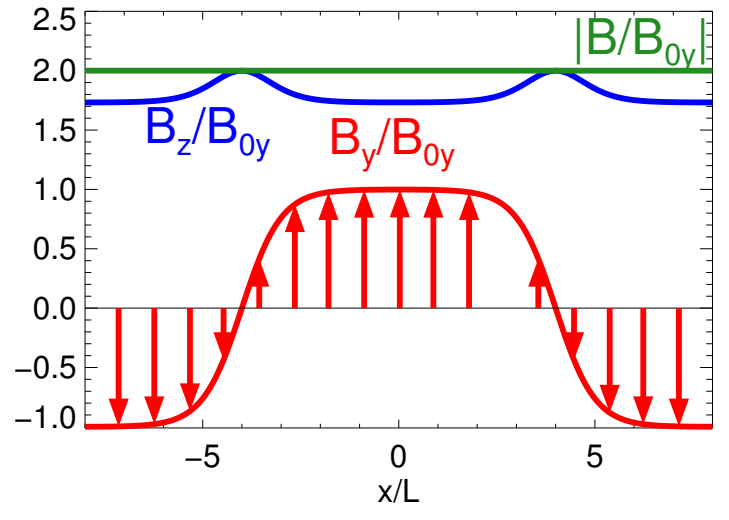

(a) $\vec{B}$

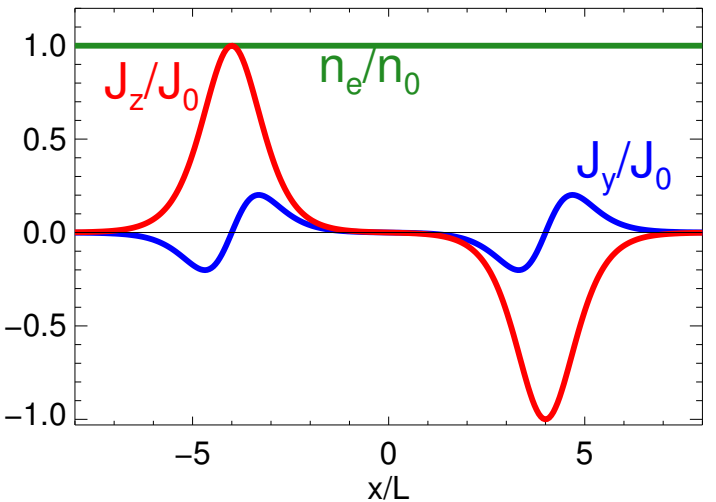

(b) $\vec{J}$ and $n_{e}$

Figure 9.1: Components of the magnetic field $\vec{B}$ and current density $\vec{J}$ profiles for a double force free CS with total magnetic field $B_{T}=2$ and $b_{g}=1.73$, given by Eq. (9.27) and Eq. (9.29), respectively. It is assumed that the left CS is at $x / L=-4$ and the right $C S$ at $x / L=4$. Compare with the corresponding Harris CS profiles shown in Fig. 8.1.

The initial in-plane current given by Eq. (9.29) represents a counterstreaming electron shear flow with magnitude $V_{e, y}=-J_{e, y} /\left(e n_{0}\right)$, parallel to the asymptotic magnetic field.

Note that we are going to assume initially the same constant density for both ion and electrons, $n_{e}=n_{i}=n_{0}$, besides of a spatially constant and equal temperature for both species $T_{i}=T_{e}$.

Because the evolution of the left and right CS are very similar, we are going to focus only in the left one in the rest of this chapter, unless stated otherwise.

\subsubsection{Perturbation}

Since in this chapter we are not interested in the reconnection onset, we applied an initial large scale perturbation to trigger this process. We chose a perturbation used in some other recent works of magnetic reconnection (see, e.g., Liu et al. 2014), given by the vector potential $\delta A_{z}$ :

$$
\delta A_{z}=\delta P \frac{L_{y}}{2 \pi} \sin \left(\frac{2 \pi\left(y+L_{y} / 4\right)}{L_{y}}\right) \sin ^{2}\left(\frac{2 \pi x}{L_{x}}\right),
$$


which will generate and $\mathrm{X}$ point (local minimum of $A_{z}$ ) in the center of the left CS [ $\left.L_{x} / 4, L y / 2\right]$ and a $\mathrm{O}$ point (local maximum of $A_{z}$ ) in the center of the right CS [ $\left.3 L_{x} / 4, L y / 2\right]$. Since the PIC code ACRONYM use the field formulation, we need the explicit perturbation for the magnetic field:

$$
\begin{aligned}
& \delta B_{x}=\frac{\partial \delta A_{z}}{\partial y}=\delta P \cos \left(\frac{2 \pi\left(y+L_{y} / 4\right)}{L_{y}}\right) \sin ^{2}\left(\frac{2 \pi x}{L_{x}}\right) \\
& \delta B_{y}=-\frac{\partial \delta A_{z}}{\partial x}=-\delta P \frac{L_{y}}{L_{x}} \sin \left(\frac{2 \pi\left(y+L_{y} / 4\right)}{L_{y}}\right) \sin \left(\frac{4 \pi x)}{L_{x}}\right)
\end{aligned}
$$

The shift in $-L_{y} / 4$ is to have the $\mathrm{X}$ and $\mathrm{O}$ points at $L_{y} / 2$ for the left and right CS, respectively (for a domain $\left[0, L_{y}\right]$ ). The perturbation can be seen in Fig. 9.2].

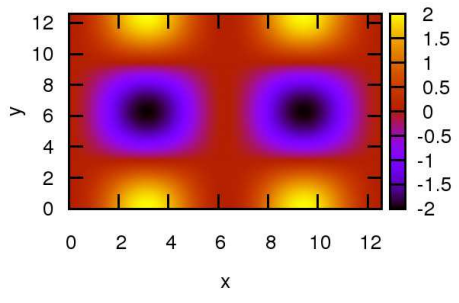

(a) $\delta A_{z}$

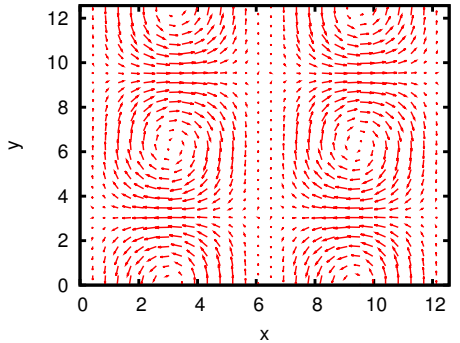

(b) $\delta \vec{B}$

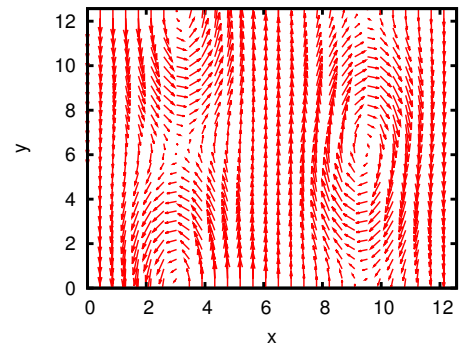

(c) $\vec{B}+\delta \vec{B}$

Figure 9.2: Initial perturbation of the double force free CS. Left. Contour plot of the vector potential Eq. (9.30). Center: Vector field of the perturbation $\delta \vec{B}$ Eq. (9.31). Right: Vector field of the total initial magnetic field including the perturbation $\vec{B}+\delta \vec{B}$. $\delta P$ has be chosen large enough to see the initial X point at the center of the domain for the left CS and the $O$ point at the right $C S$.

\subsubsection{Parameters}

Following the original work by TenBarge et al. (2014), we use two sets of parameters called "low" and "high" beta cases, where the main distinction is that the first one has $\beta_{i}=0.01$, while the second one $\beta_{i}=1.0$. This ion plasma beta and all the other parameters whose definition includes the magnetic field are calculated with respect to the total one $B_{T}$. The only exception will be the Alfvén speed, calculated with respect to the asymptotic magnetic field $B_{\infty y}$. The latter also implies that the Alfvén time $\tau_{A}=L / V_{A}$ will be calculated with respect to this quantity. The total magnetic field $B_{T}$ will be kept constant for different PIC guide field, implying that the effect of a higher guide field $b_{g}$ will be reached by reducing the asymptotic magnetic field $B_{\infty y}$. This choice is different from TenBarge et al. (2014), where the guide field $B_{g}$ is kept constant, with a consequent change of the total magnetic field $B_{T}$ and total plasma beta. This approach allow us to reach the very low guide field regime with the PIC code, and thus to be able to distinguish some phenomena that would not appear otherwise (with the original choice of keeping $B_{g}$ constant). Obviously, this choice does not affect significantly the comparison with the GK simulations in the large guide field limit, where convergence is expected. A critical 
consequence of this choice for the performance of the PIC runs is that the Alfvén time, measured in units of $\omega_{p e}^{-1}$, depend on the following parameters (obtained via the relations given in Appendix A.3.2)

$$
\tau_{A} \omega_{p e}=\frac{\sqrt{T_{i} / T_{e}} m_{i} / m_{e}}{v_{t h, e} / c} \beta_{i} \frac{L}{\rho_{i}} \sqrt{1+b_{g}^{2}}
$$

In the PIC runs, because the time step has to be proportional to $\omega_{p e}^{-1}$ for stability reasons, the latter expression will also be proportional to the number of time steps used to reach a given time measured in $\tau_{A}$, and thus to the computational effort. This implies that PIC high guide field runs are more expensive to run than the low beta ones. We can also see that high $\beta$ plasmas have the same negative effect on the PIC runs (slower runs)

On the other hand, different from previous chapters, we are going to use the thermal speed defined with the factor of two $v_{\{e, i\}}=\sqrt{2 k_{B} T_{\{e, i\}} / m_{\{e, i\}}}$. With these considerations, all the definitions of the parameters and relation between them are given in the appendix Appendix A.3, where special care has been taken to distinguish those ones which are common between the Harris and force free cases and the ones that are only valid in some of these configurations.

In the plots and discussions to be shown in this chapter, the lengths will be normalized to $\rho_{i}$ and the times to the Alfvén time $\tau_{A}=L / V_{A}$. The reconnection rate will be normalized to $\dot{\psi}_{N}=B_{\infty y} V_{A}$ and current density: $J_{N}=e n_{0} V_{A} \sqrt{\beta_{i}}=e n_{0} v_{t h, i} / \sqrt{1+b_{g}^{2}}$. This implies that the normalized peak central value of the initial out-of-plane current density $J_{z}$ in Eq. (9.28) is given by:

$$
J_{z N}:=\frac{J_{z}(t=0)}{J_{N}}=\frac{e n_{0} U_{e}}{J_{N}}=\frac{U_{e}}{v_{t h, i}} \sqrt{1+b_{g}^{2}}=\frac{U_{e}}{V_{A} \sqrt{\beta_{i}}}=\frac{1}{\left(L / d_{i}\right) \sqrt{\beta_{i}}},
$$

which is independent on the guide field strength.

For the low beta set of parameters, we use the results of 5 PIC guide field runs, while 4 runs were used in the high beta case. All the basic parameters independent on the guide fields in each low/beta case, as well as the most important derived parameters to be used in this chapter, are given in Table 9.1 


\begin{tabular}{|c|c|c|}
\hline Parameter & Low beta & High beta \\
\hline$\beta_{i}$ & 0.01 & 1.0 \\
\hline$m_{i} / m_{e}$ & 25 & 25 \\
\hline$T_{i} / T_{e}$ & 1 & 1 \\
\hline$\omega_{p e} / \Omega_{c e}$ & 0.8 & 4.0 \\
\hline$\sqrt{2 k_{B} T_{e} / m_{e}} / c$ & 0.125 & 0.25 \\
\hline$L / \rho_{i}$ & 2 & 1 \\
\hline$L_{x} / \rho_{i}\left(=L_{y} / \rho_{i}\right)$ & $40 \pi$ & $20 \pi$ \\
\hline$V_{A T} / c$ & 0.25 & 0.05 \\
\hline$V_{D e 0} / V_{A}$ & 5 & 1 \\
\hline$\sqrt{2 k_{B} T_{i} / m_{i}} / c$ & 0.025 & 0.05 \\
\hline$J_{z}(t=0) / J_{N}$ & 50 & 1 \\
\hline$\rho_{i} / d_{i}$ & 0.1 & 1.0 \\
\hline$\rho_{i} / d_{e}$ & 0.5 & 5.0 \\
\hline$\rho_{i} / \lambda_{D e}$ & 5.656 & 28.284 \\
\hline$\rho_{e} / \lambda_{D e}$ & 1.131 & 5.656 \\
\hline$L / d_{e}$ & 2 & 5 \\
\hline$L / \lambda_{D e}$ & 11.314 & 28.284 \\
\hline$\omega_{p i}^{-1} / \omega_{p e}^{-1}$ & 5 & 5 \\
\hline$\Omega_{l h}^{-1} / \omega_{p e}^{-1}$ & 6.4031 & 20.615 \\
\hline$\Omega_{c i}^{-1} / \omega_{p e}^{-1}$ & 20 & 100 \\
\hline $0.5 L_{x} / L$ & 31.416 & 31.416 \\
\hline $2 \pi L / L_{x}$ & 0.1 & 0.1 \\
\hline$\omega_{p e}[H z]$ & $5 * 10^{9}$ & $5 * 10^{9}$ \\
\hline$\lambda_{D e} / \Delta x$ & 1.440 & 0.7202 \\
\hline$\rho_{e} / \Delta x$ & 1.629 & 4.0743 \\
\hline$\rho_{i} / \Delta x$ & 8.148 & 20.372 \\
\hline$L / \Delta x$ & 16.297 & 20.378 \\
\hline$\omega_{p e}^{-1} / \Delta t$ & 32.594 & 8.1487 \\
\hline$\Omega_{c e}^{-1} / \Delta t$ & 26.076 & 32.595 \\
\hline$\Omega_{c i}^{-1} / \Delta t$ & 651.89 & 814.87 \\
\hline$N_{x}\left(=N_{y}\right)$ & 1024 & 1280 \\
\hline ppc & 1000 & 1000 \\
\hline total ppc per specie & $2.097 * 10^{9}$ & $3.277 * 10^{9}$ \\
\hline$B_{\text {total }}[G]$ & 355.35 & 71.07 \\
\hline
\end{tabular}

Table 9.1: Parameters independent on the guide field. The derived parameters and relations were obtained using the expressions given in Appendix A.3.1 and Appendix A.3.2. Note the simultaneous modification in $\omega_{p e} / \Omega_{c e}$ and $v_{t h, e}$ when changing from the low to high beta regime, since their product is constant and proportional to $\beta_{i}$.

Because the choice of keeping $B_{T}$ constant and varying only the relative guide field $b_{g}$ in the PIC runs, some parameters will change for different $b_{g}$. These ones are given in 
Table 9.2 and Table 9.3 for low/high beta cases, respectively.

\begin{tabular}{cccccc}
\hline Parameter & $b_{g}=5$ & $b_{g}=10$ & $b_{g}=20$ & $b_{g}=30$ & $b_{g}=50$ \\
\hline$\tau_{A} / \omega_{p e}^{-1}$ & 20.396 & 40.199 & 80.099 & 120.06 & 200.04 \\
$\Omega_{c i}^{-1} / \tau_{A}$ & 0.9806 & 0.4975 & 0.2497 & 0.1666 & 0.0999 \\
$\omega_{p e} / \Omega_{c e, \infty}$ & 4.079 & 8.039 & 16.02 & 24.013 & 40.008 \\
\hline$V_{A} / c$ & 0.04903 & 0.02487 & 0.01248 & 0.008329 & 0.004999 \\
$V_{A} / v_{t h, i}$ & 1.96116 & 0.9950 & 0.49937 & 0.3331 & 0.1999 \\
$V_{A} / v_{t h, e}$ & 0.39223 & 0.19900 & 0.09987 & 0.0666 & 0.03999 \\
$V_{D e 0} / c$ & 0.24514 & 0.12438 & 0.06242 & 0.041643 & 0.02499 \\
$V_{D e 0} / v_{t h, i}$ & 9.8058 & 4.9751 & 2.4968 & 1.6657 & 0.9998 \\
$V_{D e 0} / v_{t h, e}$ & 1.9611 & 0.99503 & 0.49937 & 0.3331 & 0.19996 \\
\hline$\beta_{k} / 2$ & 0.26 & 1.01 & 4.01 & 9.01 & 25.01 \\
$\mu_{k}$ & 5.252 & 20.402 & 81.002 & 182.002 & 505.202 \\
\hline$\tau_{A} / \Delta t$ & 664.8 & 1310.3 & 2610.85 & 3913.56 & 6520.3 \\
$B_{\infty y}[G]$ & 69.690 & 35.358 & 17.7454 & 11.838 & 7.1056 \\
$B_{G}[G]$ & 348.451 & 353.588 & 354.908 & 355.15 & 355.28 \\
$\phi=\tan ^{-1}\left(b_{g}\right)\left[{ }^{\circ}\right]$ & 78.690 & 84.289 & 87.1376 & 88.090 & 88.854 \\
$\theta=\cos ^{-1}\left[\left(b_{g}^{2}-1\right) /\left(1+b_{g}^{2}\right)\right]\left[{ }^{\circ}\right]$ & 22.619 & 11.421 & 5.7248 & 3.8183 & 2.2915
\end{tabular}

Table 9.2: Parameters dependent on the guide field for the high beta $\beta_{i}=0.01$ case. The derived parameters and relations were obtained using the expression given in Appendix A.3.1 and Appendix A.3.2. 


\begin{tabular}{ccccc}
\hline Parameter & $b_{g}=1$ & $b_{g}=3$ & $b_{g}=5$ & $b_{g}=10$ \\
\hline$\tau_{A} / \omega_{p e}^{-1}$ & 141.421 & 316.227 & 509.90 & 1004.98 \\
$\Omega_{c i}^{-1} / \tau_{A}$ & 0.7071 & 0.3162 & 0.19611 & 0.09950 \\
$\omega_{p e} / \Omega_{c e, \infty}$ & 5.6568 & 12.649 & 20.396 & 40.199 \\
\hline$V_{A} / c$ & 0.03535 & 0.01581 & 0.009805 & 0.0049751 \\
$V_{A} / v_{t h, i}$ & 0.7071 & 0.3162 & 0.19611 & 0.09950 \\
$V_{A} / v_{t h, e}$ & 0.1414 & 0.0632 & 0.03922 & 0.01990 \\
$V_{D e 0} / c$ & 0.03535 & 0.01581 & 0.009805 & 0.004975 \\
$V_{D e 0} / v_{t h, i}$ & 0.70710 & 0.31622 & 0.1961 & 0.0995 \\
$V_{D e 0} / v_{t h, e}$ & 0.14142 & 0.06324 & 0.03922 & 0.01990 \\
\hline$\beta_{k} / 2$ & 2. & 10. & 26. & 101. \\
$\mu_{k}$ & 0.8 & 4. & 10.4 & 40.4 \\
\hline$\tau_{A} / \Delta t$ & 1152.40 & 2576.85 & 4155.05 & 8189.37 \\
$B_{\infty y}[G]$ & 50.2543 & 22.474 & 13.938 & 7.0717 \\
$B_{G}[G]$ & 50.2543 & 67.423 & 69.690 & 70.717 \\
$\phi=\tan ^{-1}\left(b_{g}\right)\left[{ }^{\circ}\right]$ & 45 & 71.565 & 78.690 & 84.289 \\
$\theta=\cos ^{-1}\left[\left(b_{g}^{2}-1\right) /\left(1+b_{g}^{2}\right)\right]\left[{ }^{\circ}\right]$ & 90 & 36.869 & 22.619 & 11.421
\end{tabular}

Table 9.3: Parameters dependent on the guide field for the high beta $\beta_{i}=1.0$ case.

The specific order in which the input parameters of this force free CS are initialized in the ACRONYM code is described in Appendix A.3.2.

Now, a last clarification about other remaining numerical parameters. Both codes use double periodic boundary conditions ( $x$ and $y$ directions). The initial perturbation strength in Eq. (9.30) is $\delta P=0.01$. For the PIC runs, we use a grid size $\Delta x$ of $N_{x}=N_{y}=1024$ cells in the low beta case (with $\rho_{e} / \Delta x=1.69$ ), while for the high beta case is $N_{x}=N_{y}=1280$ cells (with $\rho_{e} / \Delta x=4.07$ ). The time step is chosen to be $\Delta t \omega_{p e}=0.03 / 0.12$ in the low/high beta case, respectively, to fulfill the CFL condition with $(c \Delta t) / \Delta x=0.5<1$. 1000 particles per cell are used in both cases for each species. Finally, a TSC shape function and no current smoothing were used. For the GK runs, the spatial grid is $N_{x}=$ $N_{y}=1024$ for both cases, while the parallel/perpendicular velocity grid is chosen to be $L_{v}=3 v_{t h, i}, L_{\mu}=9 k_{B} T_{i} / B_{T}$, with $32 \times 20$ points in the space $\left(v_{\|}, \mu\right) . \mu$ is the (adiabatic invariant) magnetic moment. In the GK simulations, the initial noise level and spectrum were chosen to match with the corresponding one in the PIC runs. Then, this noise acts as an additional perturbation on top of the one described by Eq. (9.30).

A fundamental parameter that is necessary to specify in the GENE GK runs is the ordering parameter $\epsilon$, connecting the perturbed quantities with the reality. It is defined as follows:

$$
\epsilon=\frac{1}{b_{\infty y, \text { norm }} b_{g, r e f}}
$$

where $b_{\infty y, n o r m}=B_{\infty y} / B_{\infty y, r e f}$ is the normalized asymptotic magnetic field with respect to a reference value $B_{\infty y, r e f}$ expressed in code units. The initialization in the GK runs gives $b_{\infty y, \text { norm }}=0.05 / 2.5$ for the low/high beta cases, respectively. $b_{g, r e f}$ is a reference 
guide field to match with the results provided by the PIC runs. It will be specified in the discussion of next Sec. 9.3.2.

Finally, some comments about the performance of both codes. Due to the constraint given by Eq. (9.33), the range of computing time for the ACRONYM PIC runs varies in a factor of ten between the lowest and highest considered $b_{g}$. In fact, for the low beta case the PIC case $b_{g}=5$ uses $3.38 \cdot 10^{4} \mathrm{CPU}$ core-hours, while $3.33 \cdot 10^{5} \mathrm{CPU}$ core-hours for the case $b_{g}=50$. On the other hand, the single GENE GK code simulation with which those runs were compared used only $350 \mathrm{CPU}$ core hours, representing a speed-up by a factor of $10^{2}-10^{3}$ when comparing to the low/high guide field regimes. This huge computing saving by the GK runs, especially in the strong guide field regime, motivates us further to investigate what properties of magnetic reconnection can be accurately modelled by comparing to the respective PIC simulations.

\subsection{Global evolution: reproduction of previous work}

\subsubsection{Reconnection rates and fast magnetic reconnection}

In this section, we first reproduce the results of the previous comparison work by TenBarge et al. (2014) in order to identify the key open problems that will be addressed in this chapter. The results of our runs with the ACRONYM PIC and GK GENE code simulations can be summarized in the time history of the reconnection rates $(d \Psi / d t) / \dot{\Psi}_{N}$, shown in Fig. 9.3 for both low and high beta cases.
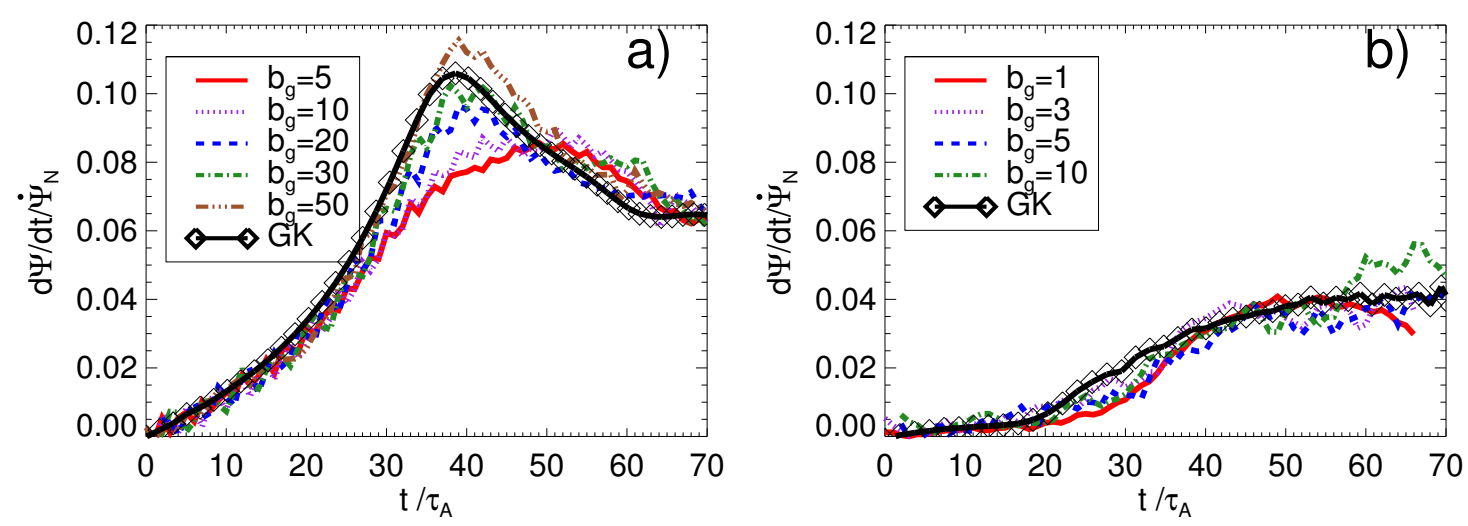

Figure 9.3: Comparison of reconnection rates $d \psi / d t$ for the left CS among different PIC guide field cases and the corresponding GK result. This quantity is calculated as the difference in the out-of-plane vector potential $A_{z}$ between the $X$ and $O$ points according to Eq. (B.82). Left a): Low beta $\beta_{i}=0.01$ case. Right b): High beta $\beta_{i}=1.0$ case. Reproduced with permission from P. A. Muñoz, D. Told, P. Kilian, J. Büchner and F. Jenko, Physics of Plasmas 22, 082110, (2015) Copyright 2015, AIP Publishing LLC.

The low beta case reaches reconnection peak time around $t=40 \tau_{A}$ for all the PIC cases and the GK runs, decreasing later on. This is due to the formation of secondary magnetic islands, as can be seen in the Fig. 9.4 for a guide field of $b_{g}=10$ at different times. These and all the other figures from the PIC runs shown in this chapter, unless 
stated otherwise, have been averaged over $t=0.5 \tau_{A}$ to reduce the effects of the numerical noise.
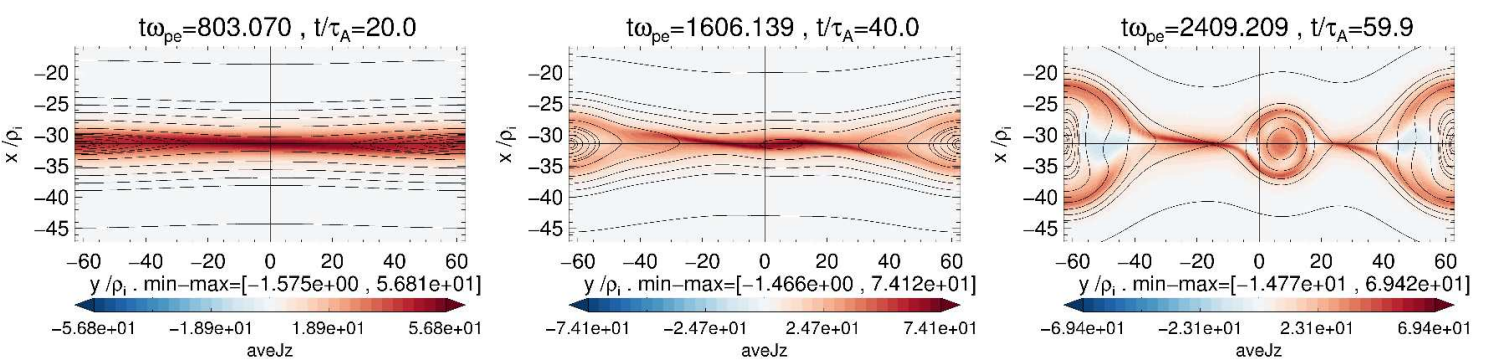

Figure 9.4: Contour of the out-of-plane current density $J_{z}$ for the PIC run $b_{g}=10$ (low beta case) at different times. All PIC quantities are averaged over $t=0.5 \tau_{A}$

The high beta case shows a similar behavior, although the peak the values are smaller by a factor of 2, not showing a drop in reconnection rates later on. Both facts are translated in less open separatrices and secondary magnetic islands affecting less the reconnection at the main X point (less "deep" magnetic islands), as can be seen in Fig.Fig. 9.5.
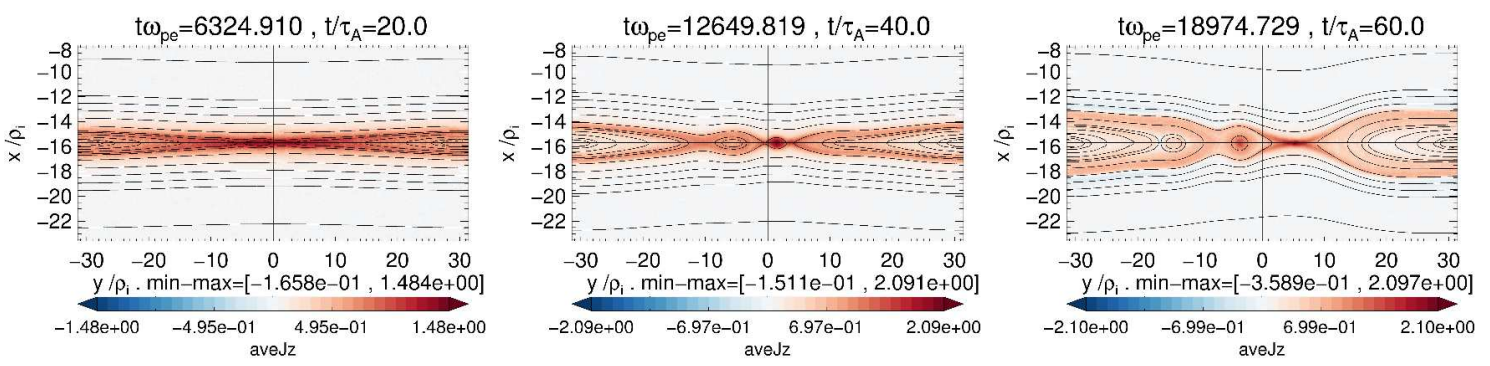

Figure 9.5: Contour of the out-of-plane current density $J_{z}$ for the PIC run $b_{g}=3$ (high beta case) at different times. All PIC quantities are averaged over $t=0.5 \tau_{A}$

Although TenBarge et al. (2014) obtained a similar behaviour, there are some minor differences. They observed formation of secondary magnetic islands for later times than in our case: $t \gtrsim 75 \tau_{A}$. Since these structures are sensitive to the initial noise in our simulation, we think that a reason for this difference might be the higher level of numerical noise in ACRONYM compared to the VPIC code used by them, but we cannot prove this assertion unless more specific details about the VPIC initialization are given. Also, note that the formation of secondary magnetic islands is completely dependent on the initial noise. Even changing the random seed used for the particle initialization will change the location of these structures. Thus, a direct comparison between different PIC guide field and GK runs cannot be done directly.

Let us discuss the magnetic reconnection regimes of these cases. As explained in Sec. 4.2.2, the standard theory for explaining under which regimes fast magnetic reconnection is expected in based in the presence of dispersive waves. According to the two fluid model first developed by Rogers et al. (2001) (see also Ricci et al. 2004), there are two parameters: $\mu_{k}$ (Eq. (4.10)) and $\beta_{k} / 2$ (Eq. (4.13)) which determine if the dispersive whistler or kinetic Alfvén (KAWs) waves can be present in a given magnetic reconnection regime. If any of them are allowed, magnetic reconnection is fast $(d \Psi / d t) / \dot{\Psi}_{N} \sim 0.1$. 
Our low beta case, according to the Table 9.2 have these parameters between the range $\left(\mu_{k}, \beta_{k} / 2\right)=(5.25,0.26)$ for $b_{g}=5$, to $\left(\mu_{k}, \beta_{k} / 2\right)=(505,25)$ for $b_{g}=50$. This will not allow whistler waves which requires $\mu_{k} \ll 1$ nor KAWs that require $\beta_{k} / 2 \gg \max \left\{\mu_{k}, 1\right\}$ and therefore reconnection should be slow. This is in contradiction to our result that show fast reconnection rates in this regime, but in agreement with the original work by TenBarge et al. (2014). As discussed extensively in Sec. 4.2.2.2 the explanation for this contradiction has been a very active topic of research during the last year, with several possible explanations. Although there is no a full definitive answer yet, all these evidences seem to indicate that fast dispersive waves, such as whistler and kinetic Alfvén waves, seems not to play the essential role to explain fast magnetic reconnection in collisionless plasmas. At least in our case, in basis to the results to be shown in Sec. 9.6, it seems that processes like the temperature anisotropy might be the responsible of fast reconnection, as it has been pointed out recently by Cassak et al. (2015). However, more work is needed to clarify this issue in our simulations. Since the purpose of this chapter is different, we are not going to analyze this issue in this work anymore.

On the other hand, according to the Table 9.3, the high beta case have the dispersive parameters between the range $\left(\mu_{k}, \beta_{k} / 2\right)=(0.8,2.0)$ for $b_{g}=1$, to $\left(\mu_{k}, \beta_{k} / 2\right)=(40.4,101)$ for $b_{g}=10$. Both extreme cases, and therefore all the guide field between them, satisfies the condition $\beta_{k} / 2 \gg \max \left\{\mu_{k}, 1\right\}$ for fast magnetic reconnection mediated via KAWs. This regime, although is still fast $(d \psi / d t$ on the order of 0.1$)$, is predicted to have smaller reconnection rates than the on mediated via whistler waves, in agreement with our results $(d \psi / d t \sim 0.04)$.

All the results and plots to be shown from now on are based in the low beta case. The slightly different conclusions for the high beta case will be analyzed only in the Sec.9.9.

\subsubsection{Parity/symmetry of magnetic reconnection quantities and lin- ear scaling}

Here we use the definitions and theoretical framework explained in Sec. 4.3.1. There, we justified, by using the two fluid theory developed by Rogers et al. (2003), that in the strong guide field regime limit $b_{g} \gg 1$ both thermal $\delta P_{t h} / P_{t h, 0}$ and magnetic $\delta B_{z} / B_{g}$ pressures fluctuations show display an antisymmetric structure in the separatrices around the $\mathrm{X}$ point, with opposite polarity due to the pressure equilibrium condition. Note that in the strong guide field limit, the magnetic pressure is dominated by the out-of-plane component, and that is why we use only that component. The theoretical estimates for the strength of these quantities in Eq. (4.33) and Eq. (4.36), for location in the separatrices away from both $\mathrm{X}$ and $\mathrm{O}$ points, predict a linear dependence of both of them on $\left(d_{i} / l_{x}\right)\left(1 / b_{g}\right) \sim 1 /\left(\sqrt{\beta_{i}} b_{g}\right)$. The first factor $\left(d_{i} / l_{x}\right)$ is constant for each low/high beta set of parameters. Therefore, the fluctuations $\delta P_{t h} \ll P_{t h, 0}$ are predicted to be inversely proportional to the guide field $b_{g}$ providing that $b_{g} \gg 1$, in such a way that the following quantity is constant for different PIC guide fields.

$$
\Gamma \delta P_{t h}=\frac{\sqrt{1+b_{g}^{2}}}{b_{g, r e f}} \delta P_{t h}=\frac{\sqrt{1+b_{g}^{2}}}{10} \delta P_{t h}
$$


where the scaling factor $\Gamma$ is defined as follows:

$$
\Gamma=\frac{\sqrt{1+b_{g}^{2}}}{b_{g, r e f}}
$$

where $b_{g, r e f}$ is a reference guide field, chosen to be $b_{g}=10 / 5$ in the low/high beta case, respectively. The same is valid for $\delta B_{z} / B_{g}$. Then, by choosing the same reference guide field for the GK ordering parameter Eq. (9.35), we have $\epsilon=2 / 0.04$ for the low/high beta case, respectively. This specification has a consequence that the GK thermal pressure fluctuations $\delta P_{t h}$ should have the same value as the PIC Eq. (9.36). The same is valid for $\delta B_{z}$. This linear scaling with the guide field is only valid assuming $\beta_{i}$ constant, which implies reducing $B_{\infty y}$ to have the effect of a stronger guide field. If we had chosen to keep $B_{\infty y}$ constant and increase $B_{g}$ to have a higher $b_{g}$ effect, the previous consideration would not be valid (we would be changing $\beta_{i}$ ) and a direct comparison between the different PIC guide field and GK runs would not be possible.

The results of this linear scaling prediction, not shown by the previous work (TenBarge et al. 2014), are displayed in the Fig. 9.6 and Fig. 9.7 for different PIC guide fields runs and the corresponding GK result, at a time shortly after the reconnection peak, where there are presence of secondary magnetic islands.
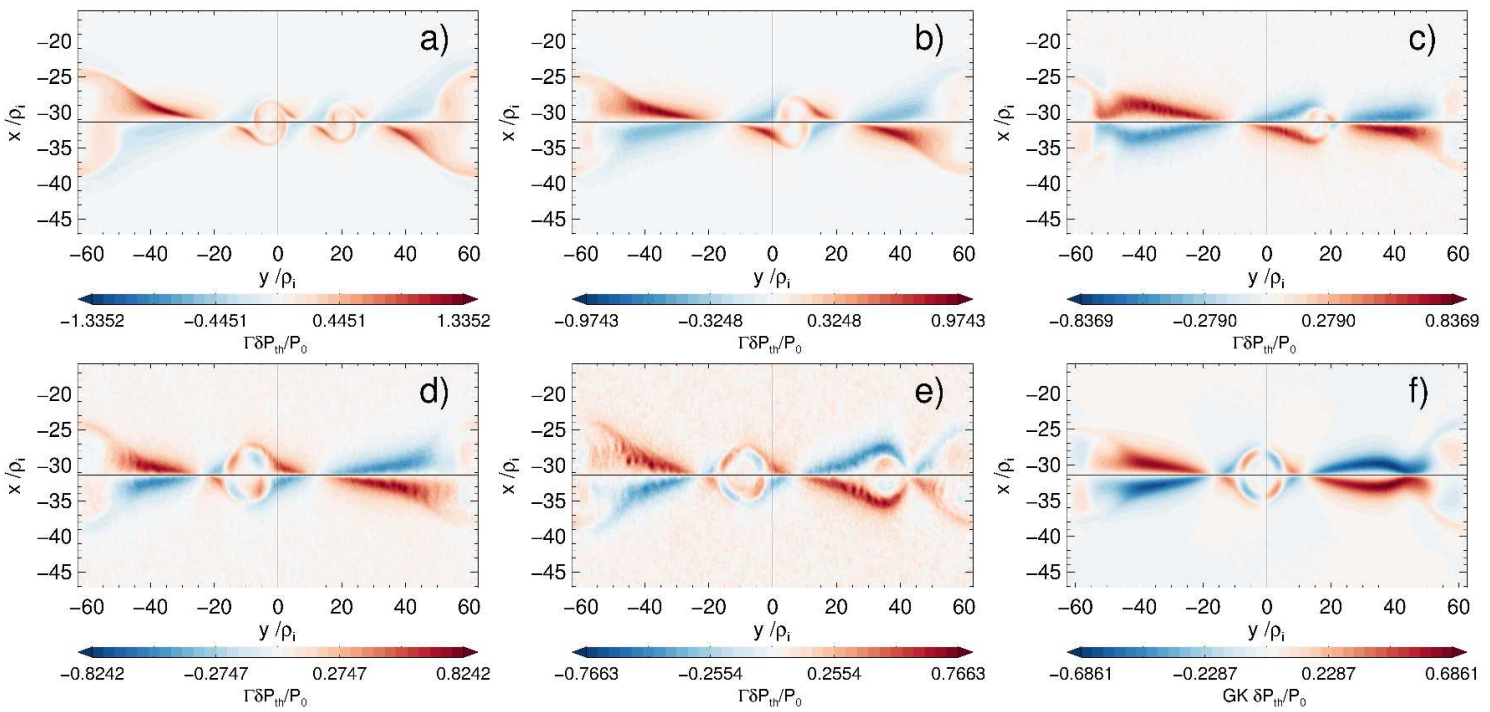

Figure 9.6: Contour plots of the scaled fluctuations in the (perpendicular) thermal pressure $\Gamma \delta P_{t h} / P_{t h, 0}$ for different guide fields in the PIC runs and the corresponding GK result, at a time $t / \tau_{A}=50$ shortly after the reconnection peak time. a) PIC $b_{g}=5, b$ ) PIC $\left.b_{g}=10, c\right)$ PIC $\left.b_{g}=20, d\right)$ PIC $\left.\left.b_{g}=30, e\right) P I C b_{g}=50, f\right)$ GK. In the PIC runs, $\delta P_{\text {th }}=P_{\text {th }}(t)-2 n_{0} k_{B} T_{i}$. The scaling factor $\Gamma$ (Eq. (9.37)) for the PIC runs was calculated using a reference guide field $b_{g, r e f}=10$. The color scheme is scaled between \pm the mean plus 3.5 standard deviations of the plotted quantity, a representative maximum as explained in the discussion of Fig. 9.9. All PIC quantities are averaged over $t=0.5 \tau_{A}$. Reproduced with permission from P. A. Muñoz, D. Told, P. Kilian, J. Büchner and F. Jenko, Physics of Plasmas 22, 082110, (2015) Copyright 2015, AIP Publishing LLC. 

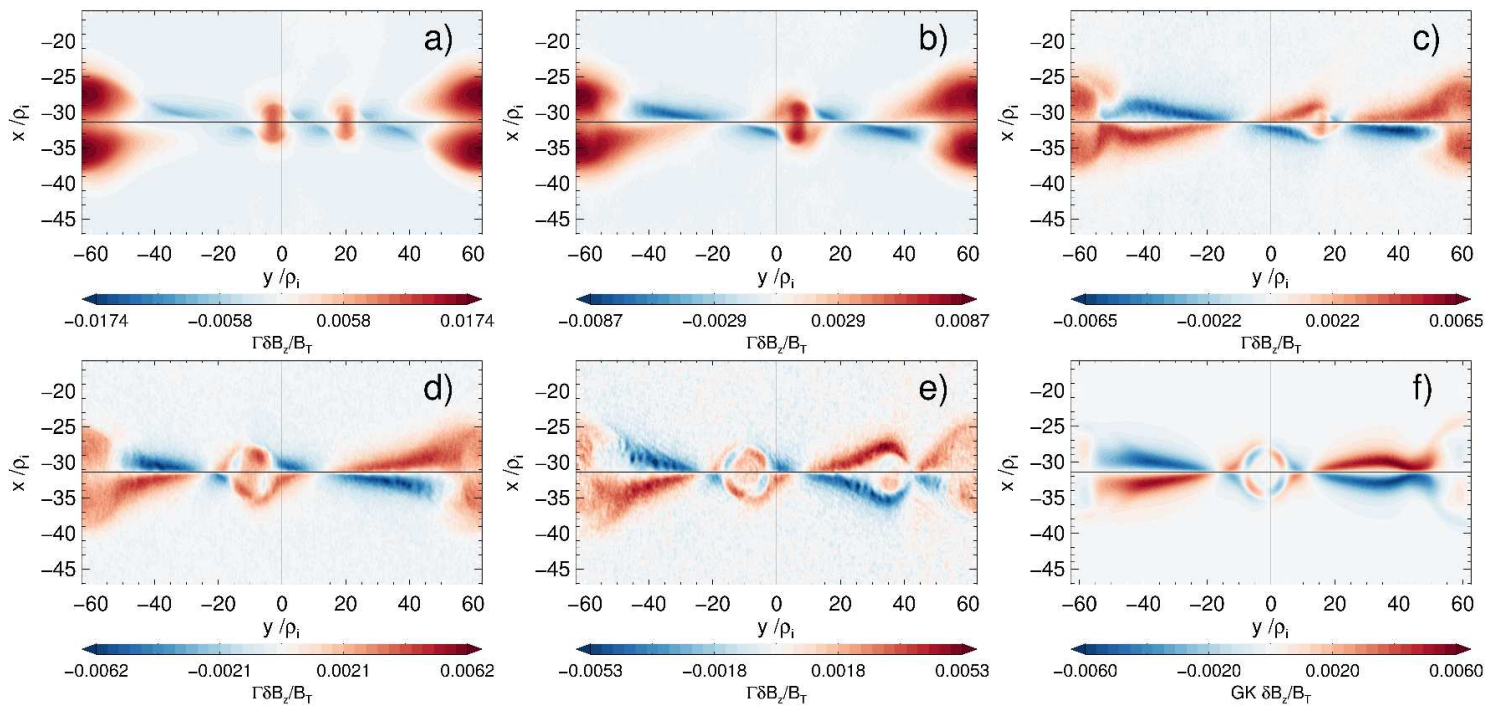

Figure 9.7: Contours of the scaled fluctuations in the out-of-plane magnetic field $\Gamma \delta B_{z} / B_{T}$ for different guide fields in the PIC runs and the corresponding GK result, at the same time $t=50 \tau_{A}$ as shown in Fig. 9.0. a) PIC $\left.b_{g}=5, b\right)$ PIC $b_{g}=10$, c) PIC $\left.b_{g}=20, d\right)$ PIC $b_{g}=30$, e) PIC $\left.b_{g}=50, f\right)$ GK. In the PIC runs, $\delta B_{z}=B_{z}(t)-B_{z}(t=0)$, where the initial $B_{z}(t=0) \approx B_{g}$ for large enough guide field according to Eq. 9.27). The scaling factor $\Gamma$ is the same used in Fig. 9.6. The color scheme is calculated using the same method described in Fig. 9.6. Reproduced with permission from P. A. Muñoz, D. Told, P. Kilian, J. Büchner and F. Jenko, Physics of Plasmas 22, 082110, (2015) Copyright 2015, AIP Publishing LLC.

From Fig. 9.6 and Fig. 9.7 we can infer the convergence of the PIC results towards the GK ones in the limit of strong guide field, not only in the linear scaling to the guide field, but also in the converging symmetry. This observation was already confirmed by TenBarge et al. (2014) in the region close to the separatrices, where that model holds. However, we can immediately notice that not only the symmetry between both separatrices is broken in the low guide field regime, but also the appearance of an additional magnetic field in the secondary magnetic islands (see Fig. 9.7(a)-(b))not visible in GK or the strong PIC guide field regimes.

\subsection{Core magnetic field and pressure equilibrium condi- tion}

Since this section is devoted to the phenomena taking place at the secondary magnetic islands, it is convenient to clarify this term. They are structures appearing as a result of the tearing mode, with a different (smaller) wavelength than the one imposed by the large scale initial perturbation. They start at electron length scales, growing up to ion scales. In this sense, our definition does not exactly match with some previous studies (Chen et al. 2012, Zhou et al. 2012, Huang et al. 2014), which limit secondary islands to electron length scales. Other works have stated that secondary islands have opposite outof-plane current density to those of the primary islands (Huang et al. 2013). Again, the 
structures in our simulations do not match these features. In any case, we expect the formation of these structures in our setup, since it is known that guide field reconnection produce a much burstier reconnection forming many secondary magnetic islands, in comparison with the antiparallel case (Drake et al. 2006).

\subsubsection{Numerical reason for core magnetic field and pressure equilib- rium condition}

In this section we are going to explain the numerical origin of the core-magnetic field seen in the secondary magnetic islands, as well as in the $y$ boundaries, for the PIC low guide field regime as shown in Fig. 9.7(a)-(b).

The previous observation has to do with deviation from the pressure equilibrium condition, since there is no corresponding drop in the (perpendicular) thermal pressure in the same previous locations (see Fig. 9.6(a)-(b)). In the GK code, the sum of magnetic field pressure and perpendicular thermal pressure is kept identically equal to zero (to machine precision). This is because the GK model keeps the perpendicular force balance to order $\epsilon$, as a consequence of the perpendicular GK Ampère's law:

$$
(\nabla \times \delta \vec{B})_{\perp}=\nabla_{\perp} \delta B_{z}=\mu_{0} \delta \vec{J}_{\perp}
$$

than can be rewritten as (see, e.g., Roach et al. 2005, Schekochihin et al. 2009, Abel et al. 2013):

$$
\nabla_{\perp} \cdot \delta \overline{\overline{P_{\perp}}}+\frac{B \nabla_{\perp} \delta B_{\|}}{2 \mu_{0}}=0
$$

where the quantities denoted as $\delta$ are of order $\epsilon . \delta \overline{\overline{P_{\perp}}}$ is the (total) perturbed perpendicular pressure tensor, $\delta B_{\|}$the perturbed parallel magnetic field and $\delta J_{\perp}$ is the perturbed perpendicular current density. This expression is equivalent to the one obtained in the framework of the two-fluid theory previously discussed, as can be seen by combining the thermal (Eq. (4.33)) and magnetic (Eq. (4.36)) pressure fluctuations.

$$
\frac{\delta B_{z}}{B_{g}}=-\beta_{i} \frac{\delta P_{t h}}{P_{t h, 0}}
$$

The difference between Eq. (9.39) and Eq. (9.40) is that in the first one, $\delta \overline{\overline{P_{\perp}}}$ may possible include off-diagonal terms contributing to finite ion Larmor effects, while in the second equation, $\delta P_{t h}$ has to be a scalar quantity. On the other hand, different from the GK model, PIC codes may allow large deviations from the pressure equilibrium conditions (they solve the full system of Vlasov-Maxwell equations), especially in the low guide field regime, since the previous expressions are valid only for $b_{g} \gg 1$, where results converge.

The respective 2D (frequency) histograms with the correlation of the left and right hand side of Eq. 9.40) are shown in Fig. 9.8(middle row) for different guide fields, at the same point in time that is shown in Fig. 9.6 and Fig. 9.7. An ideal pressure equilibrium in this limit, given by Eq. (9.40), should follow a straight line with slope $-\beta_{i}$ (black diagonal line in the middle row of Fig. 9.8 ). These plots were generated by selecting the interesting 
region close enough to the center of the $\mathrm{CS}$, in order to eliminate as much as possible any fluctuation in the almost unperturbed region away from the CS (not involved in the reconnection process). The chosen region contains all the points with a current density $J_{z}$ above $10 \%$ of its initial peak value (see Fig. 9.8(top row)). In order to show the locations of deviations in the pressure equilibrium condition, we also plot in Fig. 9.8(bottom row) the corresponding fluctuations in the total pressure,

$$
\frac{\delta P_{\text {total }}}{P_{t h, 0}}=\frac{P_{t h}+P_{m a g}}{P_{t h, 0}}-1-\frac{1}{2 \beta_{i}}
$$

with $P_{m a g}=B^{2} /\left(2 \mu_{0}\right)$. 


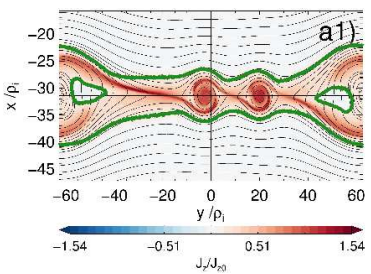

(a) $J_{z} / J_{0} b_{g}=5$

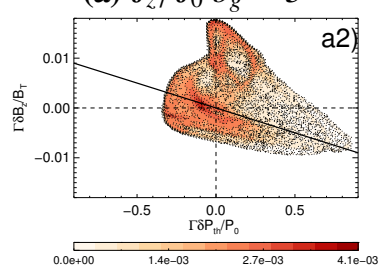

(e) $b_{g}=5$

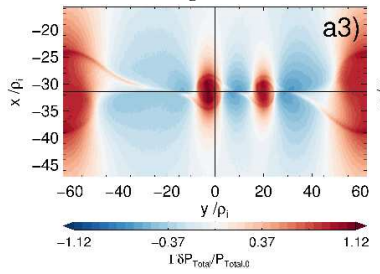

(i) $\Gamma \delta P_{\text {total }} / P_{t h, 0} b_{g}=5$

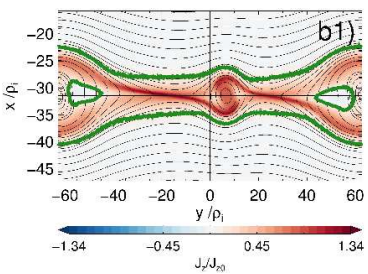

(b) $J_{z} / J_{0} b_{g}=10$

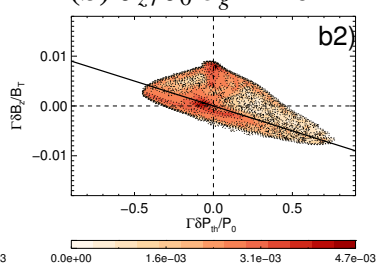

(f) $b_{g}=10$

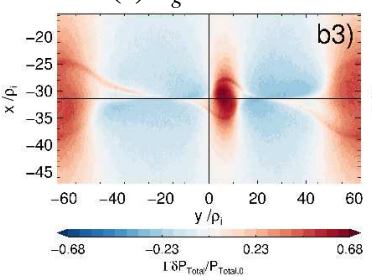

(j) $\Gamma \delta P_{\text {total }} / P_{t h, 0} b_{g}=10$

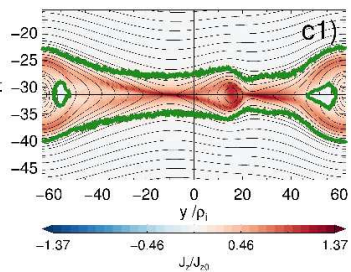

(c) $J_{z} / J_{0} b_{g}=20$

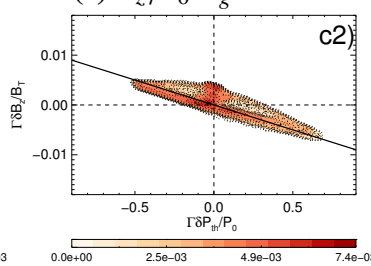

(g) $b_{g}=20$

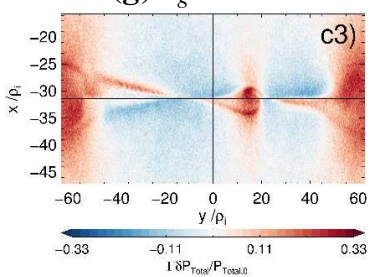

(k) $\Gamma \delta P_{\text {total }} / P_{t h, 0} b_{g}=20$

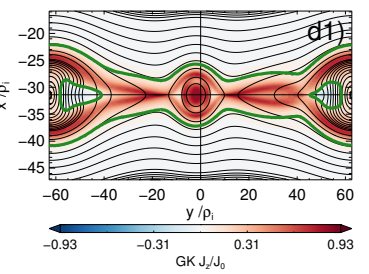

(d) $G K$

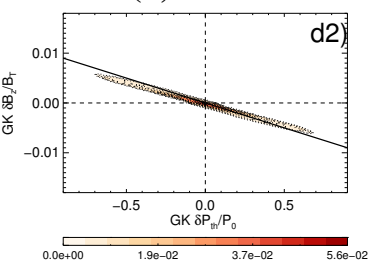

(h) $G K$

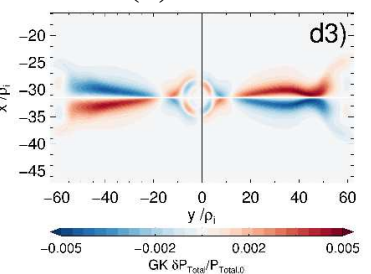

(l) $G K \Gamma \delta P_{\text {total }} / P_{\text {th }, 0}$

Figure 9.8: Top row. Contour plots of the out-of-plane current density $J_{z} / J_{z}(t=0)$ for different PIC guide fields and the corresponding GK result, at a time $t / \tau_{A}=50$. al) PIC $\left.b_{g}=5, b 1\right)$ PIC $\left.b_{g}=10, c 1\right)$ PIC $\left.b_{g}=20, d 1\right)$ GK. Magnetic field lines are shown in black contour lines. Note that because Eq. (9.34), $J_{z}(t=0)=J_{z N} \cdot J_{N}$ varies with the PIC guide field (through $J_{N}$ ). The region inside the green contour corresponds to values of $J_{z}$ higher than $10 \%$ of the initial value $J_{z}(t=0)$.

Middle row: Respective $2 D$ (frequency) histograms with the correlation between the magnetic and thermal fluctuations $\Gamma \delta B_{z} / B_{T}$ and $\Gamma \delta P_{t h} / P_{t h, 0}$ for the same runs and time as above. a2) PIC $\left.b_{g}=5, b 2\right)$ PIC $\left.b_{g}=10, c 2\right)$ PIC $\left.b_{g}=20, d 2\right) G K$. The points used to generate these plots correspond to those located inside of the green contour in the plots for $J_{z}$ in the first row. The diagonal black straight line with slope $-\beta_{i}$ represents the pressure equilibrium condition in the limit of strong guide field Eq. (9.40).

Bottom row: Respective contour plots for the scaled total pressure $\Gamma \delta P_{\text {total }} / P_{\text {th }, 0}$. a3) PIC $b_{g}=5$, b3) PIC $\left.b_{g}=10, c 3\right)$ PIC $b_{g}=20$. d3) GK. The latter is identically zero to machine precision. Reproduced with permission from P. A. Muñoz, D. Told, P. Kilian, J. Büchner and F. Jenko, Physics of Plasmas 22, 082110, (2015) Copyright 2015, AIP Publishing LLC.

In Fig. 9.8 (a2-a3) we can see clearly large deviations from the diagonal straight line of pressure equilibrium for PIC guide field runs $b_{g}=5$ and $b_{g}=10$, with a big spread that becomes increasingly negligible for a guide field $b_{g}=20$. The latter seems to converge to the GK result (all the points exactly on the straight line). Note a distinctive "bump" in the region $\delta P_{t h} \sim 0$ with $\delta B_{z} \gtrsim 0$ (upper part), which is very noticeably for a PIC guide field $b_{g}=5$. There, the strong magnetic fluctuations are not compensated by a drop in the thermal pressure. We can see in the corresponding plots of the fluctuations in the total pressure $\delta P_{\text {total }}$ (Fig. 9.8 (bottom row)), that these regions are mostly in the secondary mag- 
netic islands and in the $y$ boundaries. This excess of total pressure generates a net force towards the exterior of the magnetic islands, leading to an expansion in reconnection time scales $\left(\sim \tau_{A}\right)$. Note that the $\delta P_{\text {total }}$ increases going to the lower guide field regime, even though this quantity has been scaled linearly to the guide field. That is to be expected, since the maximum level of fluctuations for this time are of the order of the initial equilibrium values, and the assumption on which Eq. (9.40) is based (small fluctuations, the same one used in GK) breaks down. For times after the reconnection peak, when secondary islands are active, we could observe that $\delta P_{\text {total }}$ actually scales quadratically with the guide field. This means that $\Gamma^{2} \delta P_{\text {total }} / P_{t h, 0}$ shows the same fluctuation level for different guide fields.

In the 2D histograms of Fig. 9.8(top row) it is also possible to discern an asymmetric distribution along the straight line of the pressure equilibrium condition that is more important in the PIC low guide field regime: there are more points located in the right bottom quadrant $\left(\delta P_{t h}>0\right.$ and $\left.\delta B_{z}<0\right)$ than in the left upper quadrant $\left(\delta P_{t h}<0\right.$ and $\left.\delta B_{z}>0\right)$. The corresponding GK results show a symmetric behaviour (see also Fig. 9.6 ). This is an indication of the asymmetry in the separatrices for the PIC low guide field regime: the maximum values of $\delta P_{t h}$ are larger than the absolute value of the minimum ones in each pair of separatrices. $\delta B_{z}$ displays similar behaviour but to a much smaller extent. We will explain the reasons in Sec. 9.5.1.

Another important observation in the middle row of Fig. 9.8 is the presence of a set of points in a straight line parallel to the pressure equilibrium line, but shifted upwards. We checked that they are located in the regions with enhanced current density: both the pair of separatrices with enhanced number density (especially far away from the X point) and the "S" shape inside of the secondary magnetic islands. These regions contributes to further deviations in the pressure equilibrium condition in both $\delta P_{t h}$ and $\delta B_{z}$.

Therefore, although the magnetic field fluctuations $\delta B_{z}$ predicted by the GK simulations can be similar to the PIC ones for low guide fields $\left(b_{g}=5,10\right)$ close to the separatrices, this becomes invalid in regions inside of the secondary magnetic islands or the periodic $y$ boundaries. That phenomenon can be understood because of the larger deviations from the pressure equilibrium condition generated by an uncompensated additional magnetic pressure, significant only in the PIC low guide field regime.

\subsubsection{Time evolution of deviations in the pressure equilibrium condi- tion}

A convergence study focusing in one single time, like the one shown in the previous Fig. 9.6 and Fig. 9.7 may be misleading. Furthermore, as we already showed, even though the reconnection rates may take similar values for a given time, the evolution of the physical quantities associated with the reconnection process is very different. That is why in this section we analyze the time evolution of the quantities related to the pressure equilibrium between both codes. Thus, we can detect from what time the previously described core magnetic field, and the associated deviations in the pressure equilibrium condition, start to appear. This also complements the work of TenBarge et al. (2014), since in that comparison work the time evolution of the quantities related with reconnection was not investigated.

Now, in order to make quantitative estimations, we chose to track the maximum of 
$\delta P_{t h}$ and $\delta B_{z}$ in a region centered in the left CS (same area shown in Fig. 9.6 and Fig. 9.7). Since the PIC simulations are very prone to numerical noise, we chose the maximum value of the previous quantities as equal to the mean plus 3.5 standard deviations. We do not consider the absolute maximum because is very prone to outlier values, especially in the PIC high guide field regime. In addition, the initial value of the respective fluctuating quantity is subtracted, since it mostly measures the numerical PIC noise, being enhanced for higher guide fields. Moreover, the scaling given by the estimates Eq. (4.33) and Eq. (4.36) requires an initial zero offset in order to have a proper comparison with the corresponding GK results. The results are shown in Fig. 9.9.

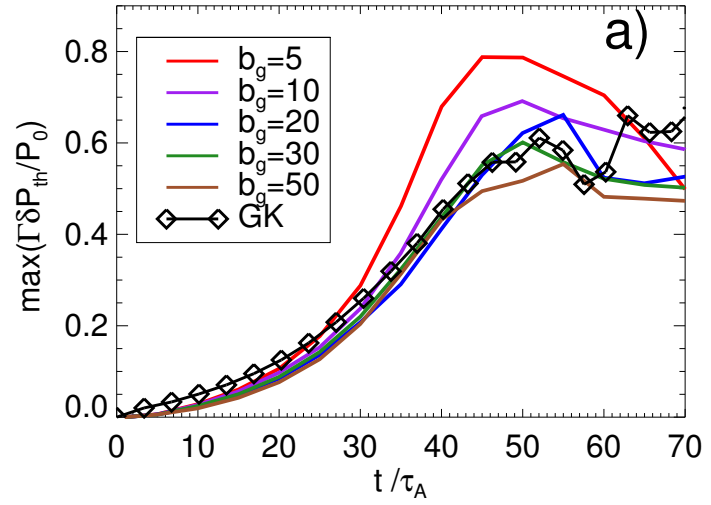

(a) $\Gamma \delta P_{t h} / P_{0}$

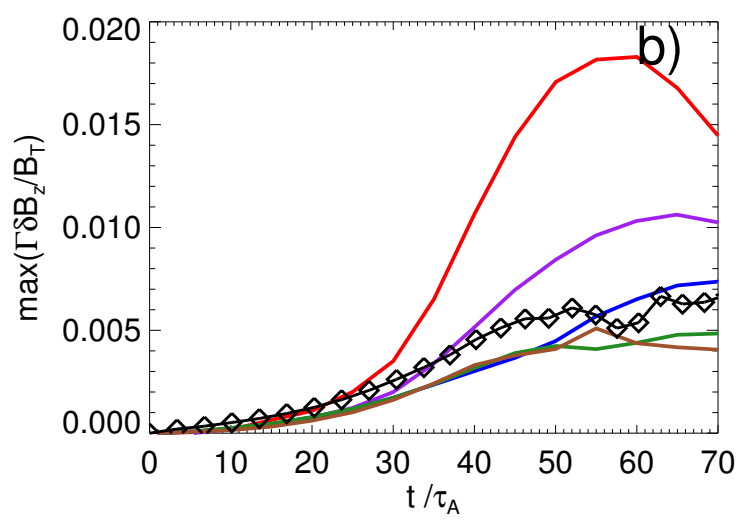

(b) $\Gamma \delta B_{z} / B_{T}$

Figure 9.9: Time history of the maximum value of the scaled thermal pressure $\Gamma \delta P_{t h} / P_{t h, 0}$ (a) and magnetic fluctuations $\Gamma \delta B_{z} / B_{T}$ (b) for different PIC guide field cases and the corresponding GK result. In the PIC runs, the "maximum" is defined as equal to the mean plus 3.5 standard deviations of the respective quantities, consistent with the color scheme used in Fig. 9.6 and Fig. 9.7. In addition, the initial value of the respective fluctuating quantities is subtracted in the PIC results. As explained in the main text, these methods are for reducing the effects of the numerical PIC noise as much as possible. Reproduced with permission from P. A. Muñoz, D. Told, P. Kilian, J. Büchner and F. Jenko, Physics of Plasmas 22, 082110, (2015) Copyright 2015, AIP Publishing LLC.

First of all, we can note in Fig. 9.9 that the reconnection peak time is around $t \sim 40 \tau_{A}$, correlated with the onset time for the formation of secondary magnetic islands. Later, around $t \sim 50 \tau_{A}$, the thermal fluctuations $\delta P_{t h}$ reach their maximum. These times are the same for all guide fields. The magnetic fluctuations reach maximum values even later. This suggests that the process generating $\delta B_{z}$ for these times is different from the Hall term due to the reconnection process itself (close to the $\mathrm{X}$ point, in the separatrices). It is driven by physical processes deeply in the non-linear phase of magnetic reconnection, not being straightforward to describe them as an steady process. This is also the justification for showing these quantities at the time $t=50 \tau_{A}$ in the previous and most of the following figures of this chapter.

In Fig. 9.9(a)-(b) we can see that the time evolution of both maximum $\Gamma \delta P_{\text {th }}$ and $\Gamma \delta B_{z}$ shows a convergence among the PIC runs in the strong guide field limit, i.e.: the respective curves get closer and closer for all guide fields $b_{g} \gtrsim 20$, following the same trend as the corresponding GK curve and the strongest PIC guide field $b_{g}=50$. As it was pointed out 
by TenBarge et al. (2014), this agreement extends to many other particle related quantities (densities, temperatures, outflow speeds). However, significant discrepancies are visible for the PIC low guide field regime. These deviations start to be noticeably for earlier times for lower values of $b_{g}$. For example, the curves of $\Gamma \delta B_{z}$ between the PIC run $b_{g}=5$ start to diverge from the GK one already in $t \gtrsim 20 \tau_{A}$, while the curve for the PIC run $b_{g}=30$ only after $t \gtrsim 45 \tau_{A}$. Note that deviations among $\Gamma \delta P_{t h}$ curves are smaller than the corresponding to the $\Gamma \delta B_{z}$ curves.

The physical reasons of the differences in the PIC runs for $\Gamma \delta P_{t h}$ as shown in Fig. 9.9)(a) will be addressed in the Sec. 9.6. They have to do with the different contributions of electrons and ions to the specific components of the pressure tensor, as well as non-thermal features of the electron distribution function. In the rest of this section we explain and analyze with much more detail the differences regarding the magnetic fluctuations $\Gamma \delta B_{z}$ shown in Fig. 9.9(b).

In order to complement the information that Fig. 9.9 provide us, we need to correlate the behavior shown there with the pressure equilibrium condition (i.e.: the location in the space of thermal $\delta P_{t h}$ and magnetic $\delta B_{z}$ fluctuations). For that purpose, in Fig. 9.10 we show the 2D histograms relating the fluctuations of thermal and magnetic pressures, for three characteristic times in the evolution of the lowest PIC guide field run $b_{g}=5$.

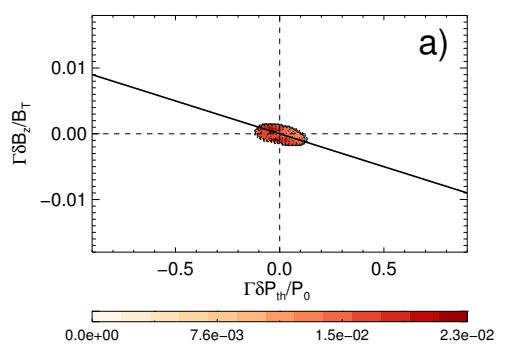

(a) $t / \tau_{A}=20$

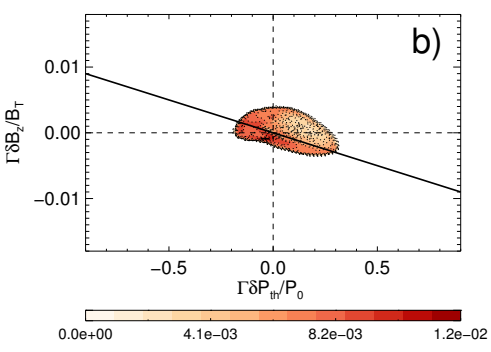

(b) $t / \tau_{A}=30$

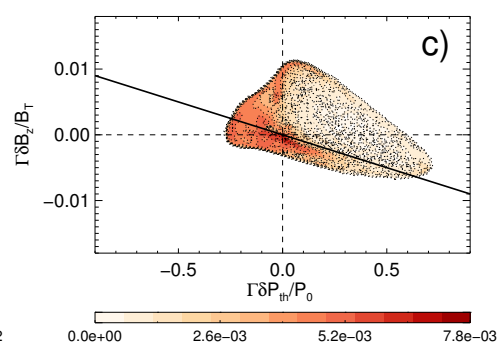

(c) $t / \tau_{A}=40$

Figure 9.10: $2 D$ (frequency) histograms wit the correlation between the scaled thermal and magnetic fluctuations $\Gamma \delta B_{z} / B_{T}$ and $\Gamma \delta P_{t h} / P_{t h, 0}$, for three characteristic times in the evolution of a low enough PIC guide field run $b_{g}=5$. a) $\left.t=20 \tau_{A}, b\right) t=30 \tau_{A}, c$ ) $t=40 \tau_{A}$. Similarly to Fig. 9.8, the points used to generate these plots correspond to the regions with the current density $J_{z}$ above the $10 \%$ of its initial value $J_{z}(t=0)$. The diagonal black line represents the pressure equilibrium condition in the limit of strong guide field Eq. (9.40). Reproduced with permission from P. A. Muñoz, D. Told, P. Kilian, J. Büchner and F. Jenko, Physics of Plasmas 22, 082110,(2015) Copyright 2015, AIP Publishing LLC.

We can see in Fig.9.10 the presence of an asymmetric distribution of points in the right bottom quadrant $\left(\delta P_{t h}>0\right.$ and $\left.\delta B_{z}<0\right)$, with respect to he pressure equilibrium line. We saw that this behavior starts from the very beginning $\left(t=20 \tau_{A}\right)$, and therefore is due to a process always operative in the system. For these earlier times (shown in Fig.9.10(a)), the points tracked are mostly located in this region corresponding to the separatrices. Later, before of the reconnection peak time $\left(t \sim 30 \tau_{A}\right.$, Fig. 9.10 (b) $)$, a "bump" $\delta P_{t h} \sim 0$ with $\delta B_{z} \gtrsim 0$ (signature of violation of the pressure equilibrium condition) starts to develop inside of the secondary magnetic islands or the $y$ boundaries. Therefore, this has to be 
caused by a process associated with the evolution reconnection. Finally, in Fig. 9.10(c) for $t \sim 40 \tau_{A}$, the "bump region" becomes larger, indicating that that the locations where the pressure equilibrium condition is violated shift from the separatrices to the "bump" The physical origin of this phenomenon, related with the action of the shear flow on the magnetic islands, will be explained in the following Sec. 9.5 .

Thus, in this section we have shown that the evolution for the magnetic and thermal pressures is similar in the PIC and GK simulations, especially during the linear phase of magnetic reconnection. However, the formation of secondary magnetic islands breaks this convergence, because it generates large magnetic fluctuations $\delta B_{z}$ only in the PIC low guide field regime, with maxima reached for times much later than the reconnection peak time.

\subsection{Core magnetic field and shear flow}

In this section we describe the physical mechanism that leads to the generation of core magnetic field in the PIC low guide field simulations, as a result of a necessarily slightly different initialization in comparison with the GK runs, thus complementing the previous Sec. 9.4. There have been some previous works that have found the same features, such as Karimabadi et al. (1999) (explained at the end of Sec. 9.5.1) and Zhou et al. (2014). The latter work reported a core-magnetic field inside of secondary magnetic islands when these structures coalesce, as result of a Hall effect that twists magnetic field lines, plus flux transport with the associated pile up of the out-of-plane magnetic field.

\subsubsection{Initial shear flow}

We found that the development of strong in-plane currents generates leads to the generation of core magnetic field in the PIC low guide field runs (see, e.g., Fig. 9.7(a)-(b)). The latter are not the expected ones due to the pure reconnection process, but instead they are generated because of the initial shear flow parallel to the reconnected magnetic field, important only in that parameter regime. This can be seen by rewriting the factor outside of the expression involving transcendental functions in Eq. (9.29) as:

$$
V_{e y 0}=\frac{1}{\sqrt{\beta_{i}}\left(L / \rho_{i}\right)} V_{A}=\frac{1}{\beta_{i}\left(L / \rho_{i}\right)} \frac{1}{\sqrt{1+b_{g}^{2}}} v_{t h, i}
$$

where the last equality is valid because the PIC runs will invariably have to change the following ratio for different guide fields (due to the choice of keeping the total plasma $\beta$ constant):

$$
\frac{V_{A}}{v_{t h, i}}=\frac{1}{\sqrt{1+b_{g}^{2}}} \frac{1}{\sqrt{\beta_{i}}} .
$$

Note that this ratio $V_{A} / v_{t h, i}$ increases for lower PIC guide fields: from $V_{A} / v_{t h, i}=0.2 \rightarrow$ 1.96 going from $b_{g}=50 \rightarrow 5$ in this low beta case. Thus, we can see that $V_{e y 0}$ is independent on the guide field in units of $V_{A}$, but (approx.) inversely dependent on it in 
terms of $v_{t h, i}$. We also have to take into account that the value of this shear flow depends, in addition, on the guide field through the (spatially dependent) denominator in Eq. (9.29). As a result, the maximum peak values of the shear flow, in the low beta case, have the dependence on the guide field shown in Fig. 9.11 .
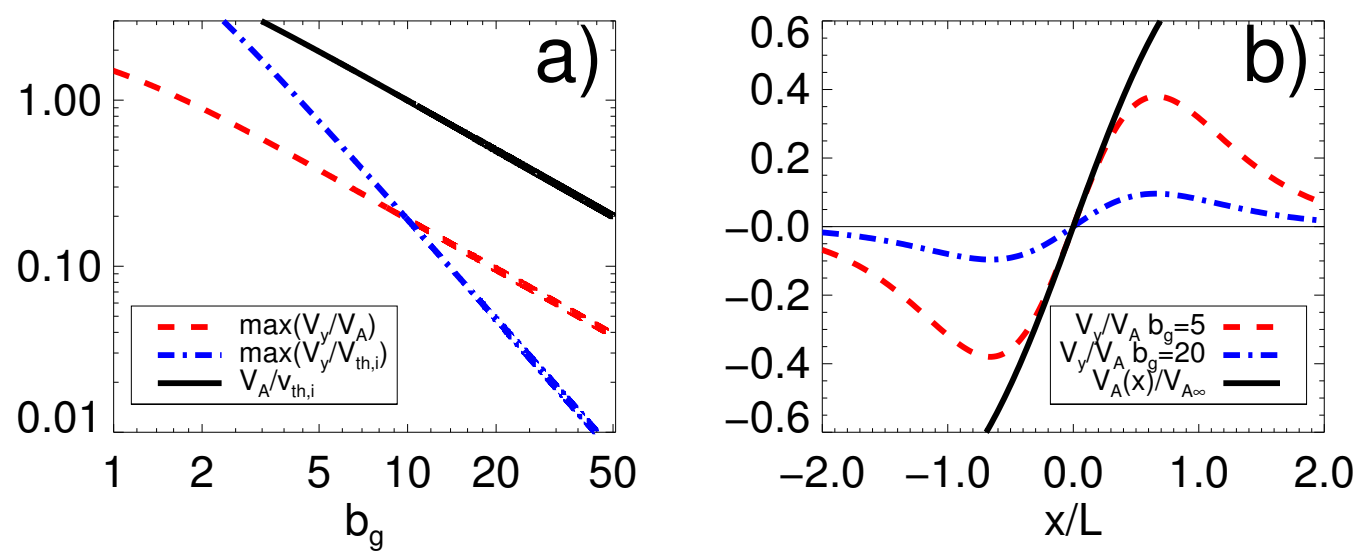

Figure 9.11: a) Maximum initial value of the in-plane electron flow speed $V_{e, y}$ on dependence on the guide field $b_{g}$, normalized to the in-plane Alfvén speed $V_{A}$ and to the constant $v_{t h, i}$. The ratio of both normalization factors, $V_{A} / v_{t h, i}$, is shown in black continuous line. Note that both axis are in logarithmic scale.

b) Initial profiles of in-plane electron flow speed $V_{e, y}(x) / V_{A}$ across a CS centered in $x=0$ for two values of guide field. The initial in-plane local Alfvén speed $V_{A}(x)$ is shown in black continuous line. Reproduced with permission from P. A. Muñoz, D. Told, P. Kilian, J. Büchner and F. Jenko, Physics of Plasmas 22, 082110, (2015) Copyright 2015, AIP Publishing LLC.

This dependence implies that the PIC initialization will have an additional source of free energy due to this shear flow, with a total kinetic bulk energy (in absolute units or in terms of $v_{t h, i}$ ) strongly dependent on the guide field (approximately, $\propto 1 / b_{g}^{4}$ ), becoming negligible in the large guide field limit or the GK initialization. In the latter, it is enforced to be exactly zero, as we already mentioned in Eq. (9.25). Otherwise, a non vanishing $\delta J_{e, \perp}$ would imply, by means of Eq. (9.38), a $\delta B_{z}$ of second order, ruled out from the standard GK equations.

On the other hand, the ratio $V_{A} / v_{t h, i}$ also plays an essential role in the GK theory, because of the perpendicular drift approximation as discussed in Sec. 9.1 (specifically in the discussion of Eq. (9.10)). It basically states that the range of validity of GK assumes $\vec{V}_{\perp} \sim O\left(\epsilon v_{t h, i}\right)$. Then, in-plane speeds that approach to $v_{t h, i}$ will make GK invalid. But this is precisely the case when $V_{A} / V_{t h, i}$ is of order 1 , since it is known that reconnection outflows will develop speeds on the order of $V_{A}$ for ions (and even larger for electrons), as discussed in Sec.4.2.1. Therefore, it is predicted that the GK results will deviate from the real physical behaviour of a Vlasov plasma modeled via PIC simulations especially for the cases $b_{g}=5$ or $b_{g}=10$. These guide fields, as seen in Fig. 9.11, have $V_{A} / v_{t h, i} \lesssim 1$ and also maximum peak values of the shear flow within a significant fraction of $v_{t h, i}$.

The asymmetric separatrices seen in Fig. 9.8, especially in the sense that positive 
pressure fluctuations are larger than the negative ones $\left(\delta P_{t h}>0\right.$ over $\left.\delta P_{t h}<0\right)$ are also due to the initial shear flow. The model developed by Cassak (2011a) already featured a similar behaviour, being attributed to the dynamic pressure of the shear flow, which can tilt the outflow in its incoming direction (see discussion in Sec.5.3.4). As a result, more electrons are piled up preferentially in one pair of the separatrices over the other one for sufficiently low PIC guide fields, increasing density, temperature and thermal pressure (see Fig. 9.6). This is a behaviour not predicted by the two fluid model sketched in Sec. 4.3.1, since it was developed without these initial flows.

It is important to mention that the " $\mathrm{S}$ " shape of $\delta B_{z}$ in the secondary magnetic islands is not directly related to the asymmetry induced by the shear flow. This has been observed in previous hybrid simulations such as in Karimabadi et al. (1999), where it was attributed to an ubiquitous feature of guide field reconnection (such as Harris sheets without initial shear flow), due to the asymmetric shift of the outflows from the $\mathrm{X}$ points in comparison with anti-parallel reconnection. However, that study was carried out in the regime of guide fields even smaller than the reconnected magnetic field $\left(b_{g}<1\right)$, and therefore the conclusion might not be applied directly to our case.

\subsubsection{Current/flows in secondary magnetic islands}

For the cases of PIC runs with low enough guide field, the (stronger) initial shear flow induces a net vortical current during the development of the reconnection process. This takes place in the magnetic islands, in both secondary (close to $\mathrm{X}$ point) and primary ones (close the periodic $y$ boundaries), as can be seen in Fig. 9.12. That current is generated only after the formation of these islands, since the magnetic field lines are wrapped up around them, deflecting the initial electron shear flow into that direction. The result is the generation of a net out-of-plane magnetic field (see Fig. 9.7) in the out-of-plane direction $(-z$ direction), same as the curl of $\vec{J}$. This process was already seen in previous 2D MHD and Hall-MHD simulations (Shi et al. 2005) for shear flows with sub-Alfvénic speeds, in agreement with our parameter range (see discussion in Sec. 5.3.5). 

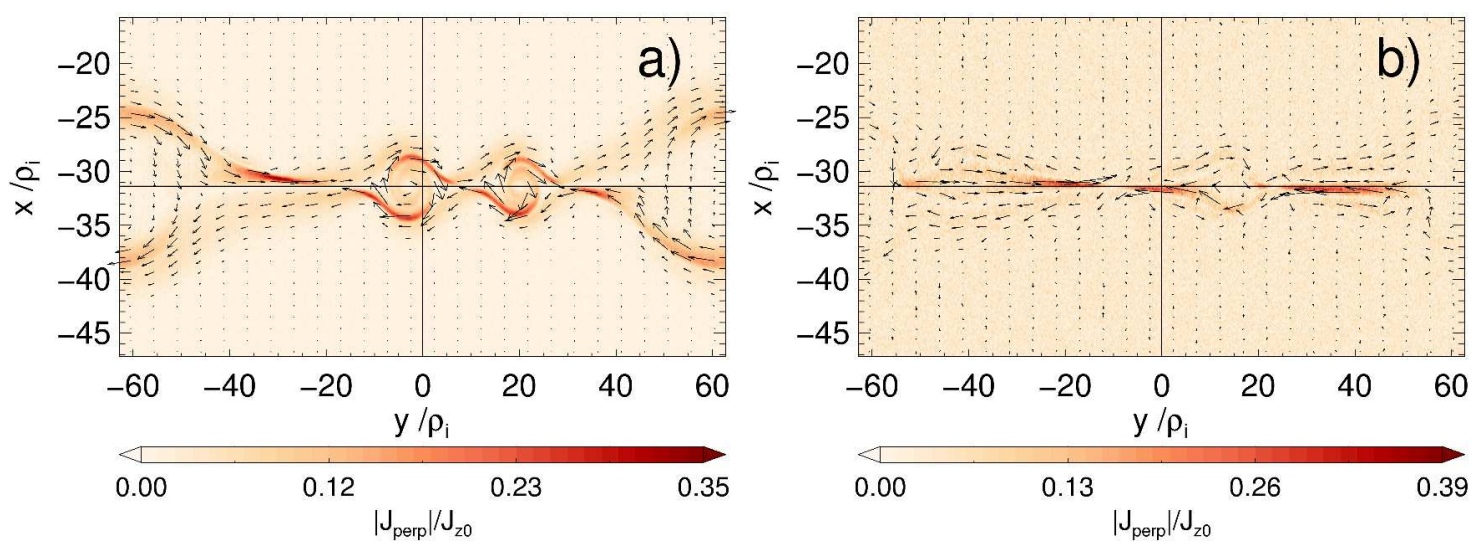

Figure 9.12: Vector plot of the in-plane current $\vec{J}_{\perp}=J_{x} \hat{x}+J_{y} \hat{y}$ for two cases of PIC guide fields $a$ ) $b_{g}=5$ and $b$ ) $b_{g}=20$, at a time $t=50 \tau_{A}$. Color coded is the magnitude of this inplane current $\left|\vec{J}_{\perp}\right| / J_{z}(t=0)$. Note that because Eq. (9.34), $J_{z}(t=0)=J_{z N} \cdot J_{N}$ varies with the PIC guide field (through $J_{N}$ ). Reproduced with permission from P. A. Muñoz, D. Told, P. Kilian, J. Büchner and F. Jenko, Physics of Plasmas 22, 082110, (2015), Copyright 2015, AIP Publishing LLC.

Note that the structure of the core magnetic field becomes more symmetric inside of the secondary magnetic islands in the PIC low guide field regime than the GK or PIC high guide field regime (see Fig. 9.7). This is in agreement with Hosseinpour and Mohammadi (2013) (see more details in Sec.5.3.5), who predicted that the Hall magnetic field due to tearing mode under influence of a shear flow can exhibit a significant symmetric structure for low $\beta_{y}<1$ (in our case, for PIC $b_{g}=5, \beta_{y} \approx 0.52$ ), while it should be symmetric (same prediction as in Rogers et al. 2001) in the limit $\beta_{y} \gg 1$ (in our case, for PIC $b_{g}=50$, $\beta_{y} \approx 50$ ).

The decoupling of motions between electrons and ions (Hall effect) is the physical two-fluid process generating the intra-island current, as shown in Fig. 9.13 for two PIC guide field cases and the corresponding GK result. Especifically, in Fig. 9.13(b1), we can see that for the case of PIC $b_{g}=5$ (the lowest guide field considered), the ions always follow the reconnection outflow from the $\mathrm{X}$ point. On the other hand, the electrons are not affected too much by that outflow, keeping their initial (sheared) bulk velocity flow pattern (Fig. 9.13 (a1)) but deformed due to the presence of the magnetic islands, in such a way that a vortical flow is produced. This characteristic flow pattern is barely visible for higher guide fields $\left(b_{g} \gtrsim 20\right.$, as seen in Fig. 9.13(a2)-(b2)) and totally absent for the GK run (see Fig. 9.13(a3)-(b3)), where it mostly follows the reconnection outflow.

Finally, it is interesting to mention that the current in the magnetic islands is associated with a dynamo process, i.e.: $\vec{J} \cdot \vec{E}<0$. This means a a transfer of energy from the bulk electron motion to the magnetic field (see Fig. 9.23). This effect does not take place for the cases of PIC high guide field or GK runs. The only locations where $\vec{J} \cdot \vec{E}<0$ are close to the outflows, due to the bulk motion of the plasma. We are going to discuss more extensively this issue in Sec. 9.7 .3 , by comparing with the dissipation $\vec{J} \cdot \vec{E}>0$ close to the $\mathrm{X}$ points. 


\subsubsection{Electron/ion flow in later times}
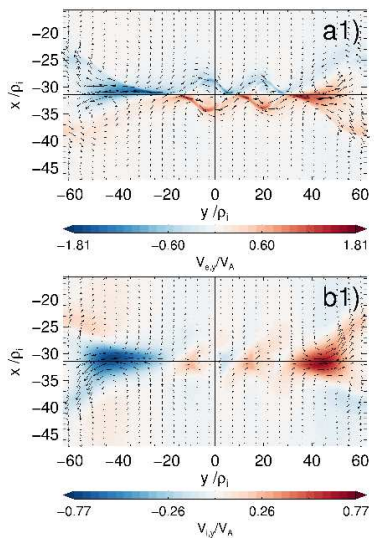
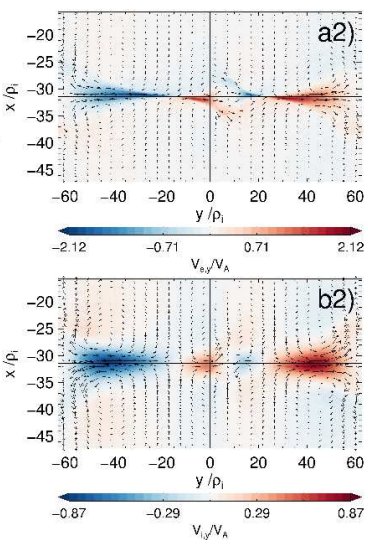
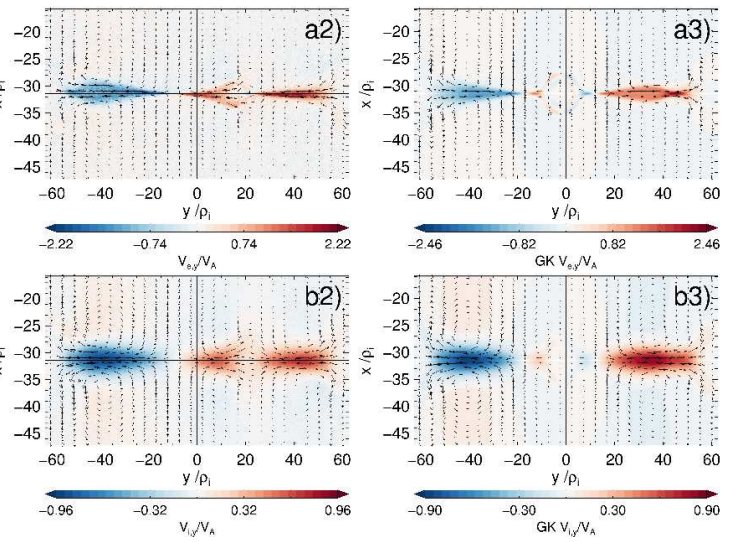

Figure 9.13: Top row: Vector plot of the in-plane electron bulk velocity for different PIC guide fields and the corresponding GK result, at a time $t=50 \tau_{A}$. Color coded is the $V_{e, y} / V_{A}$ component. a1) PIC $b_{g}=5$, a2) PIC $\left.b_{g}=20, a 3\right) G K$.

Bottom row: Same as above but for the in-plane ion bulk velocity $\left.V_{i, y} / V_{A} . b 1\right) P I C b_{g}=5$, b2) $\left.P I C b_{g}=20, b 3\right)$ GK. Reproduced with permission from P. A. Muñoz, D. Told, $P$. Kilian, J. Büchner and F. Jenko, Physics of Plasmas 22, 082110, (2015) Copyright 2015, AIP Publishing LLC.

In good agreement with the standard two fluid theory of magnetic reconnection by Shay et al. (2001) (discussed in Sec.4.2.1), we could observe that the speed of the electron outflows from the main $\mathrm{X}$ point due to reconnection is about the double of the asymptotic ion Alfvén speed $V_{e, y} \sim 2.2 V_{A}$, with some small variation among different PIC runs (see Fig. 9.13(a)-(b)). On the other hand, the ion outflow speeds are practically constant among different PIC guide fields and the GK runs (see Fig. 9.13(d)-(f)), and reaching sub-Alfvénic values $V_{e, y} \sim 0.8 V_{A}$. Thus, the critical PIC guide field for which the shear flow can generate the currents building up the core magnetic field $\left(b_{g} \lesssim 20\right)$ can be determined, in principle, by measuring how comparable are the values of the initial shear flow (strongly dependent on the guide field according to Fig. 9.11) with the constancy of the reconnection outflow speeds.

It is important to mention that because the generation of magnetic field is due to a Hall effect, their effects will be stronger when the CS is much thinner than the ion sound Larmor radius $\rho_{s}$ (Nakamura et al. 2008), which applies very well to our case ( $L=2 \rho_{i}=$ $\left.0.2 d_{i}\right)$. From this we can also predict that this kind of effects will be strongly reduced in thicker CS, and thus the results of PIC and GK simulations will be more similar.

In Fig. 9.13 we can also note another important difference between the secondary magnetic islands for different PIC guide field regimes. For $b_{g}=5$, the electron outflow from the secondary $\mathrm{X}$ points close to these islands is strong enough to be noticed over the dominant outflow from the main X point (see Fig. 9.13(a1)). On the other hand, for higher guide field such as $b_{g}=20$, the electron outflow speed from the secondary magnetic islands is reduced to such extent that cannot overcome the reconnection outflow from the main X point and it is simply carried away from this (Fig. 9.13(a2)). As it can be expected, this behaviour is also seen in the GK simulations (Fig. 9.13(a3)). Therefore, 
although the secondary magnetic islands are formed for all guide field regimes in the PIC runs and also in GK simulations, displaying a similar morphology, they have essentially different properties. The dominance of the main $\mathrm{X}$ point is much more significant for the PIC low guide field regime than in the high guide field one or the GK runs, a behaviour that can only be captured by PIC simulations.

\subsubsection{Time evolution of electron/ion flows: influence of initial shear flow}

Now, let us investigate how these currents are build up from the initial one in the PIC runs with sufficiently low guide field. First, in order to distinguish the relative contributions of the initial shear flow and the reconnection outflows, it is convenient to analyze the system for earlier times, during the linear phase of tearing mode growth, when there are no secondary magnetic islands. This can be seen in Fig. 9.14, where we compare the full electron outflow and the one obtained by subtracting the initial shear flow.
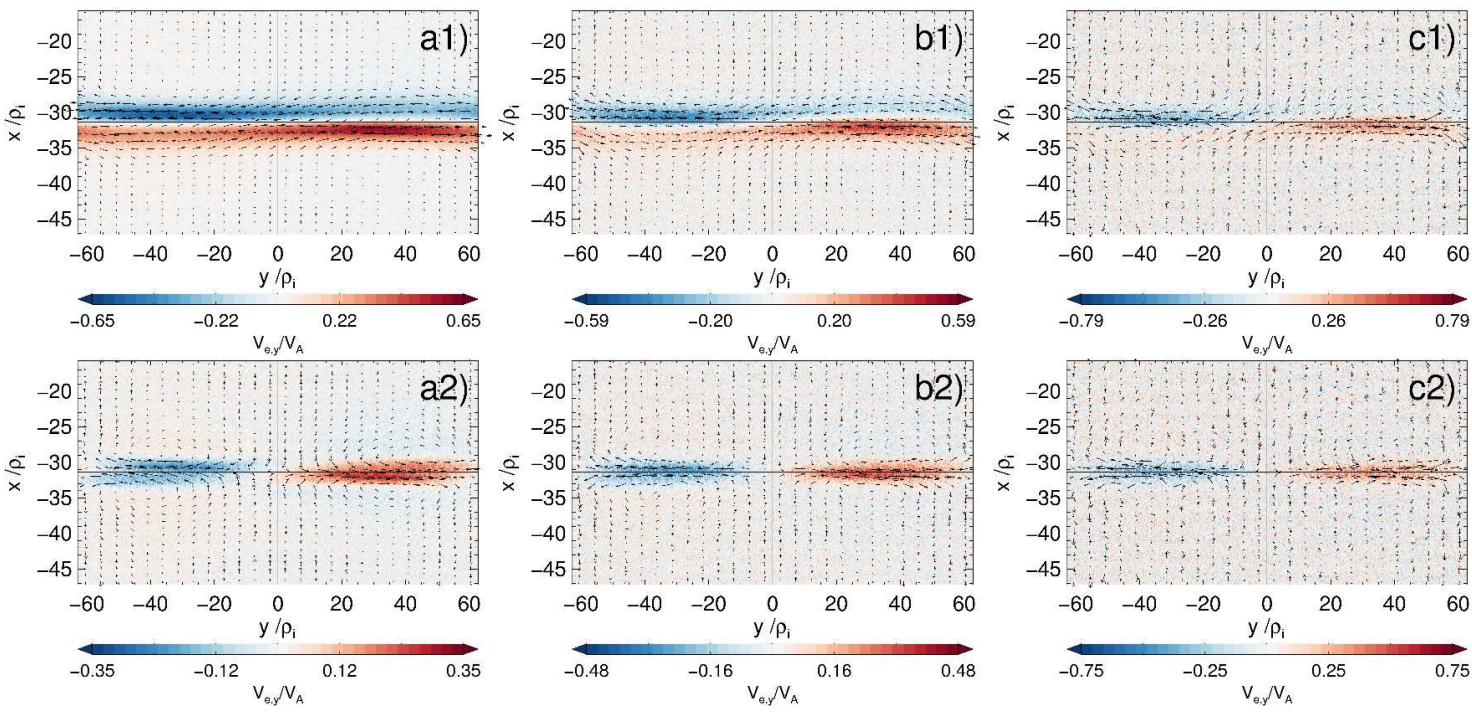

Figure 9.14: Top row: Vector plots of the in-plane electron bulk velocity for different PIC guide fields, at a time $t=20 \tau_{A}$, early in the linear phase of reconnection. Color coded is the $V_{e, y} / V_{A}$ component. al) PIC $\left.b_{g}=5, b 1\right)$ PIC $\left.b_{g}=10, c 1\right)$ PIC $b_{g}=20$.

Bottom row: Same vector plots as above, but with the initial shear flow subtracted: $\left(V_{e, y}-\right.$ $\left.V_{e, y}(t=0)\right) / V_{A}$. This gives the outflow due to reconnection. a2) PIC $\left.b_{g}=5, b 2\right)$ PIC $\left.b_{g}=10, c 2\right) P I C b_{g}=20$.

In Fig. 9.14(a1) and Fig. 9.14(a2), the electron flow is dominated by the initial shear flow for this case of smallest guide field $b_{g}=5$ : the reconnection outflow is very weak to produce any appreciable effect. This can be measured through the maximum values: the total flow speed reaches $V_{y} \sim 0.65 V_{A}$, while the reconnection outflow speed reaches $V_{y} \sim 0.35 V_{A}$. On the opposite case, for a stronger guide field of $b_{g}=20$ (Fig. 9.14)(c1) and Fig. 9.14(c2)), the shear flow is weak enough that the overall electron flow mostly follows the reconnection one, being only weakly deflected (total flow speed reaches $V_{y} \sim 0.79 V_{A}$, while the reconnection outflow speed reaches $\left.V_{y} \sim 0.75 V_{A}\right)$. For later times, the dynamics 
is dominated by the reconnection outflow in both cases, in such a way that $V_{e, y} \gg V_{e, y}(t=$ 0 ) (see Fig. 9.13). Note that the reconnection outflows are always symmetric (even), while the odd parity of the shear flow with respect to the center of the CS breaks this symmetry, as we already explained in Sec.9.5.1.

We checked that the electron and ion inflow/outflow speeds have similar values among different PIC guide field and GK simulations when measured in units of the Alfvén speed for different times (see, e.g., Fig. 9.13). The agreement, as can be expected, is better in the linear phase of reconnection. As a result, the in-plane current $\vec{J}_{\perp}$ (proportional to $\vec{V}_{i, \perp}-\vec{V}_{e, \perp}$ ) will also display similar values among different PIC guide fields when the same normalization is used (see Fig. 9.12). But the generation of the magnetic field $B_{z}$ depends on $J_{\perp}$ measured in absolute units (without normalization to $J_{N}$ ). The initial value of the unnormalized current does change with the guide field due to the relative ratio of Alfvén to thermal speeds Eq. (9.43). Furthermore, the generation of magnetic field $\delta B_{z}$ can be estimated as follows, by using the Ampère's law, neglecting displacement current and approximating the $\operatorname{curl} \nabla \times \vec{B}$ by the gradient scale length $1 / \Delta L$ :

$$
\frac{\delta B_{z}}{B_{g}} \approx \frac{\Delta L}{\rho_{i}}\left(\frac{\mu_{0} \rho_{i}}{B_{g}}\right) J_{\perp}
$$

Defining the constant $\Lambda=\mu_{0} \rho_{i} / B_{g}$, we infer that $\delta B_{z} / B_{g}$ in absolute units ( $B_{g}$ is constant for different PIC guide fields) is on the order of $\Lambda J_{\perp}$ when the length scale of the magnetic field is on the order of $\rho_{i}$, not dependent on the guide field. We checked that $\rho_{i}$ does not change too much for all the shown times, equivalent to a weakly varying ion temperature and thus a proportionality between $\delta B_{z}$ and $J_{\perp}$ (force free condition). Now, in the simulations, a good estimate of $\Delta L$ is the size across the $x$ direction of the secondary magnetic islands close to the $X$ point: $\sim 10 \rho_{i}$, or the magnetic island at the $y$ boundaries $\left(\sim 18 \rho_{i}\right)$. In this way, we can calculate the evolution of the terms in the right hand side of Eq. (9.44) that only depends on the current $\Lambda J_{\perp}$, with results shown in Fig. 9.15. 


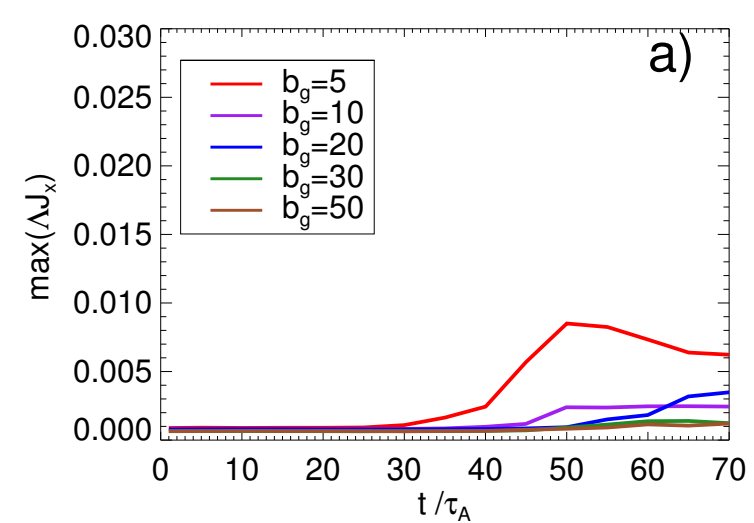

(a) $J_{x}$

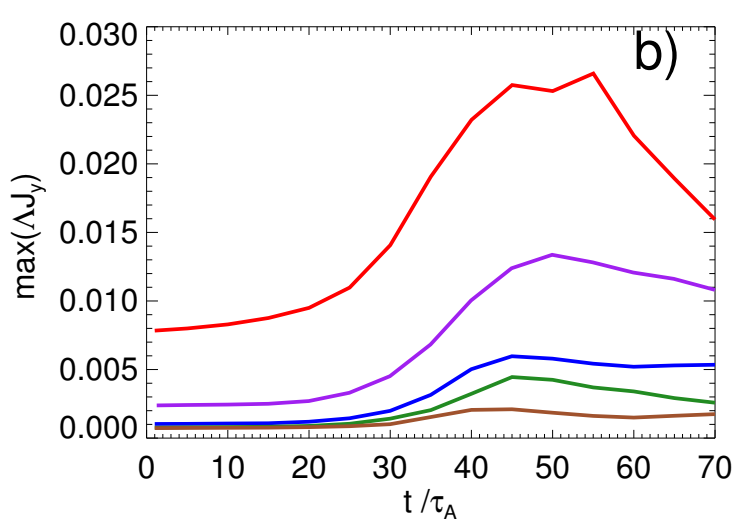

(b) $J_{x}$

Figure 9.15: Time history of the maximum value of the fluctuations in the in-plane current for different PIC guide fields cases. a) $\Lambda J_{x}$. b) $\Lambda J_{y}$. Note that this quantity does not have a normalization factor dependent on the guide field as Fig. 9.12 (it is measured in absolute units). These values have to be compared with the ones for $\delta B_{z} / B_{g}$ shown in Fig. 9.9. Reproduced with permission from P. A. Muñoz, D. Told, P. Kilian, J. Büchner and F. Jenko, Physics of Plasmas 22, 082110, (2015). Copyright 2015, AIP Publishing $L L C$.

Fig. 9.15(b) shows that the maxima of $J_{y}$, and so $\delta B_{z}$, are important only in the PIC low guide field regime, when measured without normalization. Or in other words, magnetic field generation is only effective in the lower PIC guide field regime. More specifically, in Fig. 9.15(b) we can see that both in-plane components $J_{x}$ and $J_{y}$ start to grow after the reconnection peak time and the associated formation of secondary magnetic islands. Nevertheless, most of the contributions to the total in-plane current come from the $J_{y}$ component (compare with Fig. 9.15(a)). By comparing the previous plot of $\Lambda J_{y}$ with the time history of the maximum values of $\delta B_{z}$ (see Fig. 9.9(b)), taking off the factor $\Gamma$, we can confirm a good agreement (in the order of magnitude) of both quantities (e.g., for $b_{g}=5$, the maximum is $\left.\delta B_{z} / B_{g} \sim 0.034\right)$. Note that these maxima of $J_{y}$ are reached only at the borders of the regions where are located the associated maxima of $\delta B_{z}$ : in the boundaries of the magnetic islands (the contribution from the separatrices can be neglected since its curl is practically zero).

Summarizing, all the previous evidence indicates that the core magnetic field $\delta B_{z}$ is generated by the combined effect of the initial shear flow and formation of secondary magnetic islands start to form. It has more relative importance in the PIC low guide field regime, and it is one of the main features different between PIC and GK simulations of magnetic reconnection of force free current sheets.

\subsubsection{Boundary effects in core magnetic field generation}

Not only a core magnetic field is generated in the secondary magnetic islands in the PIC low guide field regime, but also at the $y$ boundaries. The dominant numerical reason is due to the colliding outflows from reconnection in the main $\mathrm{X}$ point and the periodic boundary conditions (equivalent to a configuration with multiple $\mathrm{X}$ points). Reconnection in the PIC low guide field regime produces faster electron outflows in absolute units, since 
they have approximate similar values in units of $V_{A}$, but this scales as $\propto 1 / b_{g}$. As discussed in Sec.7.2.2, this might be avoided by using other numeric techniques. Note that the main secondary island in the $\mathrm{O}$ point of this CS (due to the initial perturbation), is located in this same place ( $y$ boundaries), generating a magnetic field via the same process described before for the secondary magnetic islands. Thus, the total magnetic field in these locations has contributions of both physical and boundary effects.

\subsubsection{Influence of shear flow in reconnection}

Now, let us focus on the effects of the shear flow and associated magnetic field generation in reconnection. The general trend is towards a reduction of reconnection rates in the very low end of analyzed guide fields range, $b_{g}=5,10$, compared to the high guide field regime or the GK runs (see Fig. 9.3). There are at least 3 different reasons:

- The additional magnetic pressure due to to the core magnetic field in the magnetic islands inhibits the CS thinning because it makes the reconnection outflows slower, and thus a reduction in reconnection rates should be observed. And it is precisely in the regime of PIC low guide field where the relative importance of this additional out-of-plane magnetic field is greater (see, e.g., Fig.9.9).

- We already explained in Sec.5.3.5 that, following the argument by Cassak (2011a), the outflow speed should also be smaller (and thus reconnection rates) due to the reduction in the magnetic tension of the reconnected magnetic field lines. This release of tension is produced due to the initial shear flow, and was confirmed by a kinetic dispersion relation and 2D PIC simulations for thin CS (Roytershteyn and Daughton 2008).

- Finally, the formation of core magnetic field in the magnetic islands by the Hall currents implies a higher magnetic energy in the system compared to the case without them. Therefore, the amount of magnetic energy converted into particle energy via magnetic reconnection is reduced when the core magnetic field is present.

Overall, if the total plasma $\beta$ is kept constant, it is clear that reconnection rates should be reduced in the PIC low guide field regime. We can have a first order estimate to what extent the reconnection rates should be reduced in presence of a shear flow by calculating the factor $\left(1-V_{0}^{2} / V_{A}^{2}\right)$ according to Eq. (5.77). This expression, derived by Cassak (2011a), is valid under a Hall-MHD model (without guide field). As we can see in Fig. 9.11, the previous reduction factor is non-negligible in the lower PIC guide field regime $b_{g}=5,10$ (with factors 0.57 and 0.89 , respectively). For the corresponding PIC runs, the measured reduction in reconnection rates is on the order of $20 \%$ compared to the GK results (see Fig. 9.3), equivalent to a factor of 0.8. Note that although the estimate should not be applied directly to our case because of the strong guide field regime, the qualitative conclusion still holds. 


\subsubsection{Other related studies about magnetic field generation by shear flows}

In closing this section, it is important to mention that previous studies have reported the generation of large scale and long duration magnetic fields as a result of the electron scale kinetic Kelvin-Helmholtz (K-H) instability (Alves et al. 2012, Grismayer et al. 2013a, Alves et al. 2014). As discussed extensively in Sec.5.3.3, this instability is closely related to the vortical flow pattern seen in our low PIC guide field runs inside of the secondary magnetic islands. However, these studies were for cold $\left(v_{\text {the }} \ll V_{0}\right)$ shear flows in unmagnetized plasmas, and therefore do not apply directly to our case. Nevertheless, the physical mechanism behind this, associated with a current imbalance resulting from the mixing of electrons crossing the deformed shear interface during the non-linear evolution, seems to be quite general. This can be tested by the theoretical estimate for the generated magnetic field Eq. (5.74).

\section{6 (Thermal) heating mechanisms}

In this section we will address the physical origin of the larger thermal pressure perturbations $\delta P_{t h}$ in the PIC low guide field regime, comparing to the respective limiting GK results. Nevertheless, we focus mostly in the PIC runs in the low guide field regime, since they show very different to the ones obtained via the GK simulations. First of all, in the time evolution of the maximum values of this quantity show in Fig. 9.9(a) and all the subsequent analysis, we considered only perpendicular thermal pressure fluctuations $\delta P_{t h, \perp}$, due to the pressure equilibrium condition. When considering the full scalar pressure including parallel perturbation contributions: $\delta P_{t h, t o t a l}=\delta P_{t h, \perp}+\delta P_{t h, \|}$, the differences are even larger. These maxima of these quantities, in the PIC low guide field regime, are located in the boundary of the secondary magnetic islands, as well as in one pair of separatrices. The origin of these differences in this regime are related the heating of electrons and ions in different directions with respect to the magnetic field, as well as other dissipative processes and non thermal mechanisms.

First we are going to analyze how different are heated the electrons and ions with respect to the magnetic field in the PIC runs on dependence on $b_{g}$, by looking at the locations where these processes develops. The purpose is distinguish which heating mechanisms are generated by the reconnection process itself and which ones are due to other instabilities. The respective contour plots of these quantities for some PIC guide fields are shown in Fig. 9.16, at the same usual time $t=50 \tau_{A}$. 

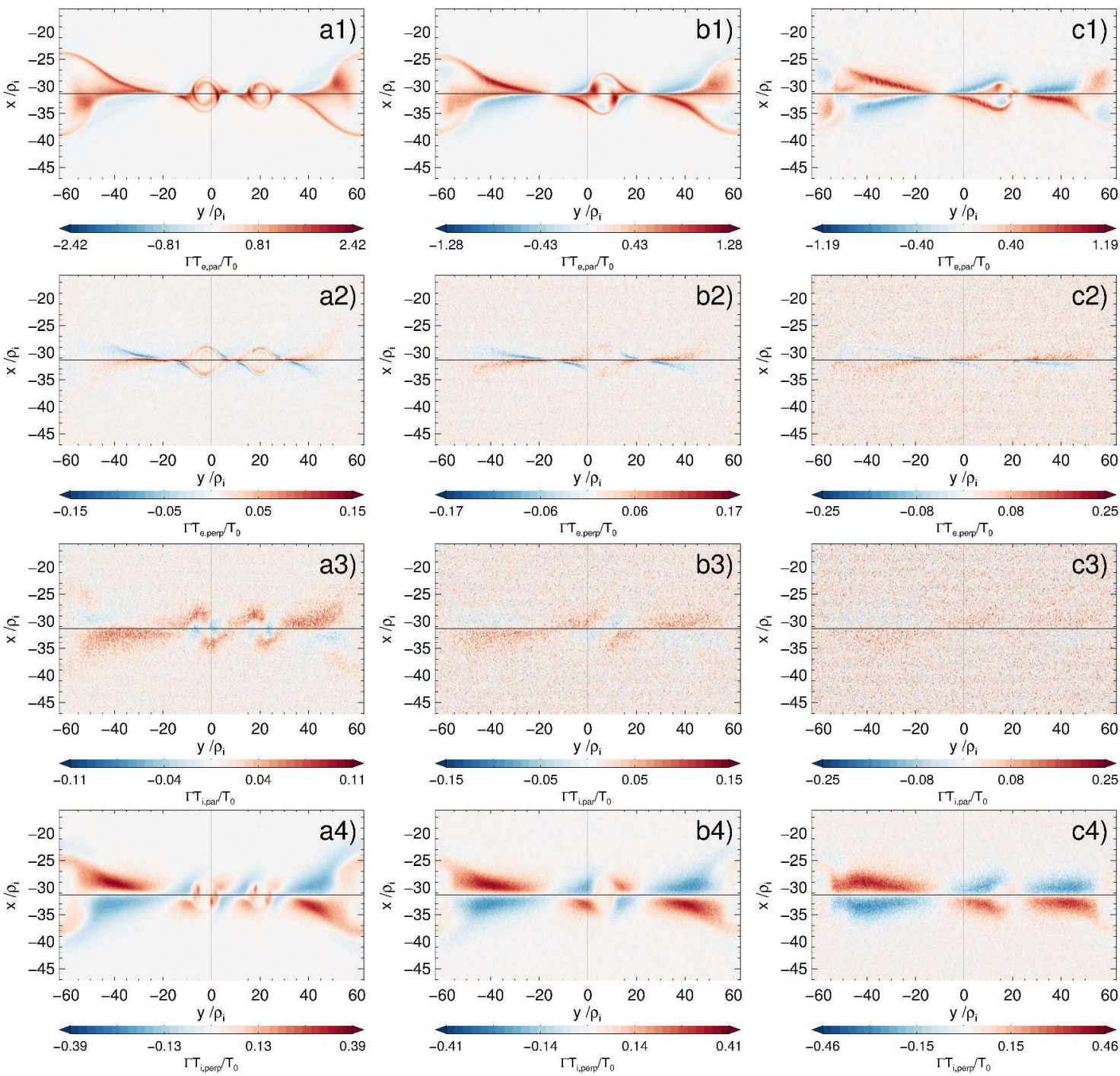

Figure 9.16: Electrons and ion temperatures are reconnection peak time $t=50 \tau_{A}$ for different guide fields, scaled with the factor $\Gamma$. Top row 1: $\delta \Gamma T_{e, \|} / T_{0}$. Row 2: $\delta \Gamma T_{e, \perp} / T_{0}$. Row 3: $\delta \Gamma T_{i, \|} / T_{0}$. Row 4: $\delta \Gamma T_{i, \perp} / T_{0}$. Increasing guide field from left to right column: $a$ ) $\left.\left.b_{g}=5, b\right) b_{g}=10, c\right) b_{g}=20$.

In Fig. 9.16, it can be seen that $T_{e, \|}$ and $T_{i, \perp}$ take maximum and minimum values in the same pair of separatrices, correlated with the polarity of enhanced density $n_{e} / n_{0}>1$ and thermal pressure (see Fig. 9.6). These quantities have more or less same values when scaled on the guide field, except in the case $T_{e, \|}$ for $b_{g}=5$ showing large deviations. On the other hand, both $T_{e, \perp}$ and $T_{i, \|}$ have opposite polarity. This means that the perpendicular ion temperature is heated more than the parallel one $T_{i, \perp}>T_{i, \|}$ in the same pair of separatrices where the parallel electron temperature is enhanced. Moreover their temperature is increased only in a fraction of the electron one (is reduced in a factor of $\sqrt{m_{i} / m_{e}}$ ). But because the ion density has more or less the same distribution as the electron density, the overall contributions of the ions to the thermal pressure $P_{i, t h}=n_{i} k_{b} T_{i}$ has the same sign as those of electrons (i.e.: correlated with the sign of the density fluctuations).

An important numerical issue to take into account is the strong numerical heating 
observed in the PIC high guide field cases (see, e.g., Fig. 9.16 (3rd column) for $b_{g}=20$ ). The maximum of the $T_{e, \perp}$ and $T_{i, \|}$ are just a little bit above of the surrounding background temperatures, enhanced at the noise level. Therefore, the quantitative conclusions in this regime may be misleading. That is the reason because we are not going to analyze in detail the behavior for guide fields in the range $20<b_{g}<50$ in the remainder of this section. More about this issue will be discussed in Sec. 9.10 .

We also need to prove that the previous behaviour of temperatures is characteristic during all the reconnection process and not only during the specific time shown there $\left(t=50 \tau_{A}\right)$. With this purpose, and similar to the method used for the magnetic and thermal pressures in Fig. 9.9, we plot the maximum of the parallel and perpendicular electron/ion temperature in an area close to the left CS. The results are shown in Fig. 9.17. In general, we can see a fast convergence for all the PIC runs with guide field $b_{g} \gtrsim 20$ towards the gyrokinetic result, and larger deviation for $b_{g}=5,10$.
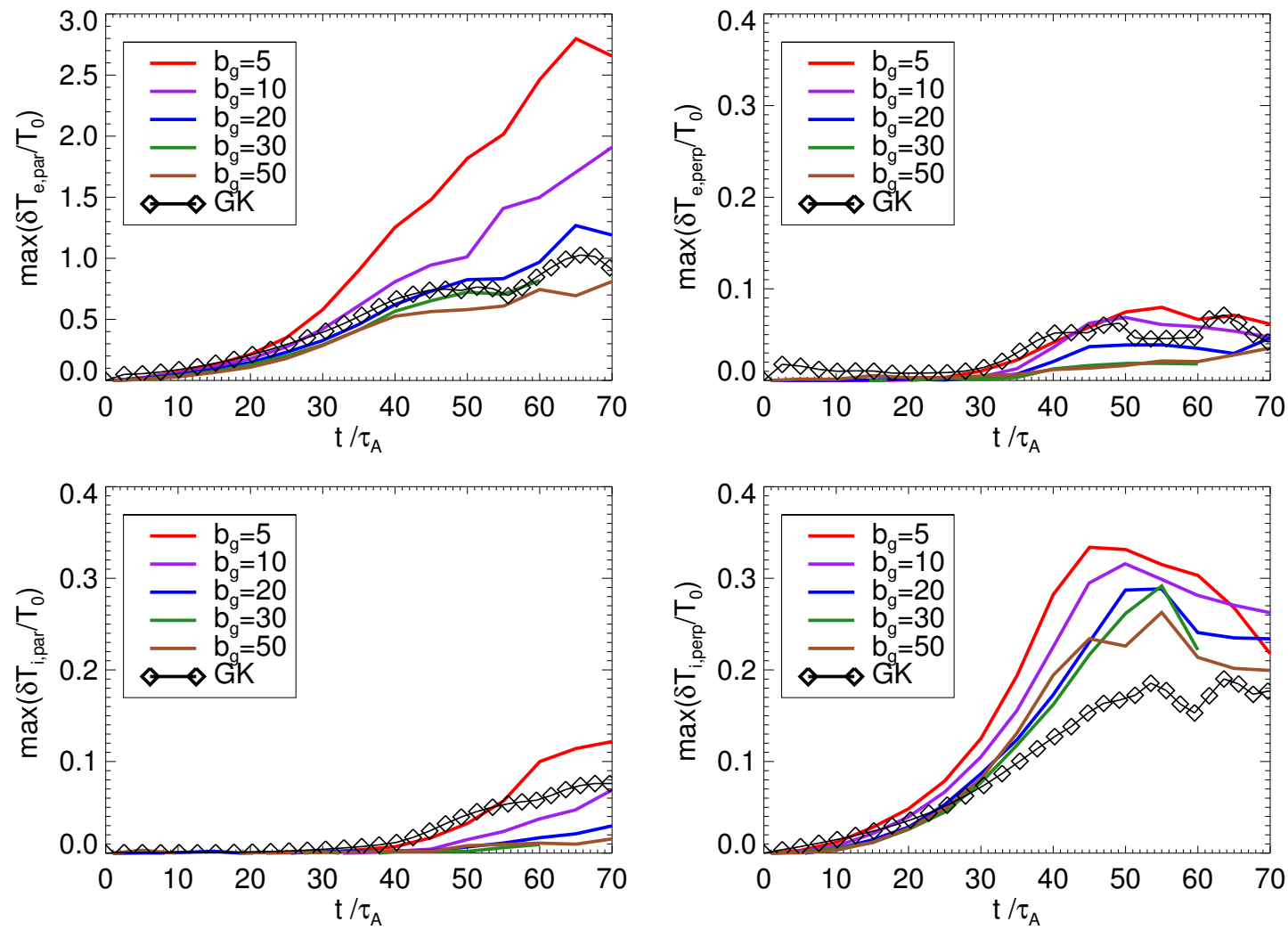

Figure 9.17: Time evolution of maximum of $\|$ and $\perp$ temperatures (normalized to the initial $\left.T_{0}\right)$. Top: electron $\Gamma \delta T_{e, \|}$ and $\Gamma \delta T_{e, \perp}$. Bottom: ion $\Gamma \delta T_{i,,}, \Gamma \delta T_{i, \perp}$. Note the different $y$ range for $\Gamma \delta T_{e, \|}$ in comparison with the other ones.

In Fig. 9.17 a-b), we can note that the parallel electron temperature $T_{e, \|}$ increases from the very beginning, as a result of the pile-up of them as the separatrices are slowly open due to reconnection. This is also due to the fact that they can be easily accelerated due to parallel electric fields $E_{\|}$generated during this process, mostly in the out-of-plane direction. On the other hand, the perpendicular component of the electron temperature $T_{e, \perp}$ only start to suddenly increase after the reconnection peak, as a result of processes related 
with the formation of secondary magnetic islands. Both mechanisms are well reproduced by both PIC and GK runs. On the other hand, in Fig. 9.17c-d), we can see that ions develop the opposite behaviour to the electrons: their perpendicular component is heated from the beginning, while their parallel component is heated only after the reconnection peak

This behaviour can be explained on basis to the CGL approximation discussed in Sec. 2.6. This plasma approximation is specially suitable for strongly magnetized systems, like this case of low $\beta$ plasma and predicts the invariance of two constants relating the two components of the parallel and perpendicular of the thermal pressure with the magnetic field. In particular $T_{e, \|} \propto n^{2} / B^{2}$ and $T_{e, \perp} \propto n B$. In basis of this plasma model, Egedal et al. (2013) showed that the conditions in the magnetic reconnection regions are favorable for the development of strong electron ani sotropies $T_{e, \|}>T_{e, \perp}$, especially in low $\beta$ plasmas according to a closure relations and scaling law that applies well to our case (see also Hirabayashi and Hoshino 2013). This process physically originates from electron trapping, and it can be modeled well enough for both PIC and GK plasma models, at least during the linear phase of magnetic reconnection. But this relies on the assumption of negligible heat flux. We checked that this quantity starts to become important after the formation of secondary magnetic islands, especially in the PIC low guide field regime (results not shown here), and this might explain the differences between our results and the GK plasma model that underestimates the parallel electron heating. Note also that these strong electron anisotropies might explain the fast reconnection rates developed in this low $\beta$ plasma (Cassak et al. 2015) where it was traditionally thought that reconnection should be slow. However, more work is needed to reveal the physical mechanism behind this process in our case (see also discussion of Fig. 9.3).

\subsection{Non-thermal and dissipative mechanisms}

\subsubsection{Deviations from thermal equilibrium}

A characterization of the differences between PIC/GK would not be complete without an analysis of the momenta of the distribution function that gives information about out-of-equilibrium processes. The previous section about heating processes only gives information about the diagonal component of the thermal pressure. This analysis is interesting because most of the out-of-equilibrium processes cannot be modeled via fluid models, in addition to the GK restriction concerning the small fluctuations from the (equilibrium) Maxwellian distribution function. Moreover, there are many instabilities, like the ones to be shown in Sec. 9.8, that can generate strong non-thermal features especially in the electron VDF, due to the non-linear wave-particle interactions at kinetic scales.

These processes can be divided in the ones that affect to the off-diagonal terms of the pressure tensor (2nd order momenta) and the ones affecting to other high order momenta of the VDF

The relative importance of the off-diagonal terms of the pressure tensor can be quantified through the non-gyrotropy $D_{n g}$ the distribution function, by using the definition Eq. (B.93) (proposed by Hesse et al. 2014), as explained in Appendix B.2.6. A comparison of this quantity for different guide fields is shown in Fig. 9.18. 

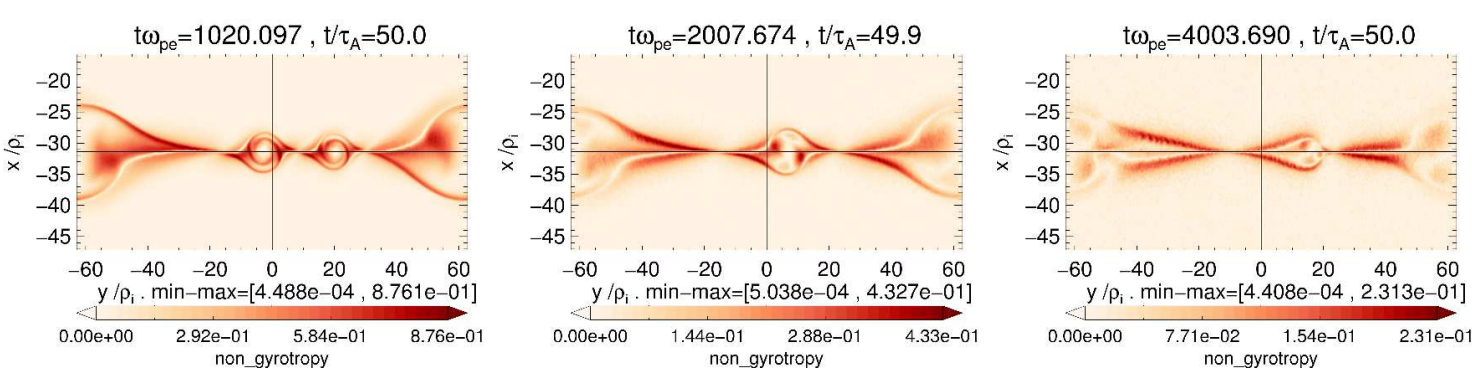

Figure 9.18: Contour plots of the non-gyrotropy $D_{N g}$ of the electron VDF using the definition Eq. (B.93) for different guide fields at reconnection peak time. Left: $b_{g}=5$. Center: $b_{g}=10$, Right: $b_{g}=20$. Note that the non gyrotropy is bounded between $0<D_{N g}<1$.

The off-diagonal terms in the electron pressure tensor, contributing to high values of $D_{n g}$ as shown in Fig. 9.18, are related with a fluid pseudo-viscosity and the dynamics of kinetic Alfvén waves, being generically classified as finite electron Larmor radius effects. As we are going to discuss in Sec. 9.8, they are very related with steep local pressure gradients, a source of free energy for micro-instabilities. Note that reconnection also produces off-diagonal terms of the pressure tensor, especially in the component $P_{y z}$, whose gradient can sustain the reconnected electric field. But this only takes place very close to the X points. Indeed, we can see the highest values of $D_{n g}$ are reached in the pair of separatrices with stronger density (same as current density), as well as around the secondary magnetic islands, away from the $\mathrm{X}$ point and therefore not directly related with reconnection.

Now, let us switch to the analysis of processes related with higher order momenta of the distribution function and, therefore, strong deviations from a Maxwellian distribution function. This is particularly interesting for the PIC simulations, since the GK approach by definition cannot model processes deviating too much from the thermal equilibrium.

As we explain in Appendix B.2.1, a non-thermal population can be characterized very efficiently (in the sense of not having to use the full information of the VDF) via the kurtosis, the 4th order moment of the VDF, defined by Eq. (B.72). The results are shown in Fig. 9.19 for the (electron) kurtosis $K_{y}$ and $K_{z}$ at reconnection peak time for a guide field of $b_{g}=5$. We can see signatures of a non-thermal electron population in the component $v_{y}$ (high values of $K_{y}>0$ ) very localized in one separatrix, but especially in the component $v_{z}$ along an extended region in both separatrices, as well as in the outflow region. These regions are both spatially and temporally correlated with the locations and time where the instabilities to be described in Sec. 9.8 are active, being therefore a consequence of them. This non-thermal population vanishes for the PIC runs with higher guide field. Also note that the highest values of kurtosis are much more localized than those of the non-gyrotropy (see Fig. 9.18(a)).

The highest values of kurtosis $K_{z}>0$ are in the low density pair of separatrices, while the high density pair has $K_{z}<0$. The behaviour of $K_{y}$ is the opposite. Now, let us check how well these values correlate with the locations where electron are being accelerated. For this purpose, we identified the most energetic electrons in an area close to both the separatrices and secondary magnetic islands. The results are shown in Fig. 9.20. 

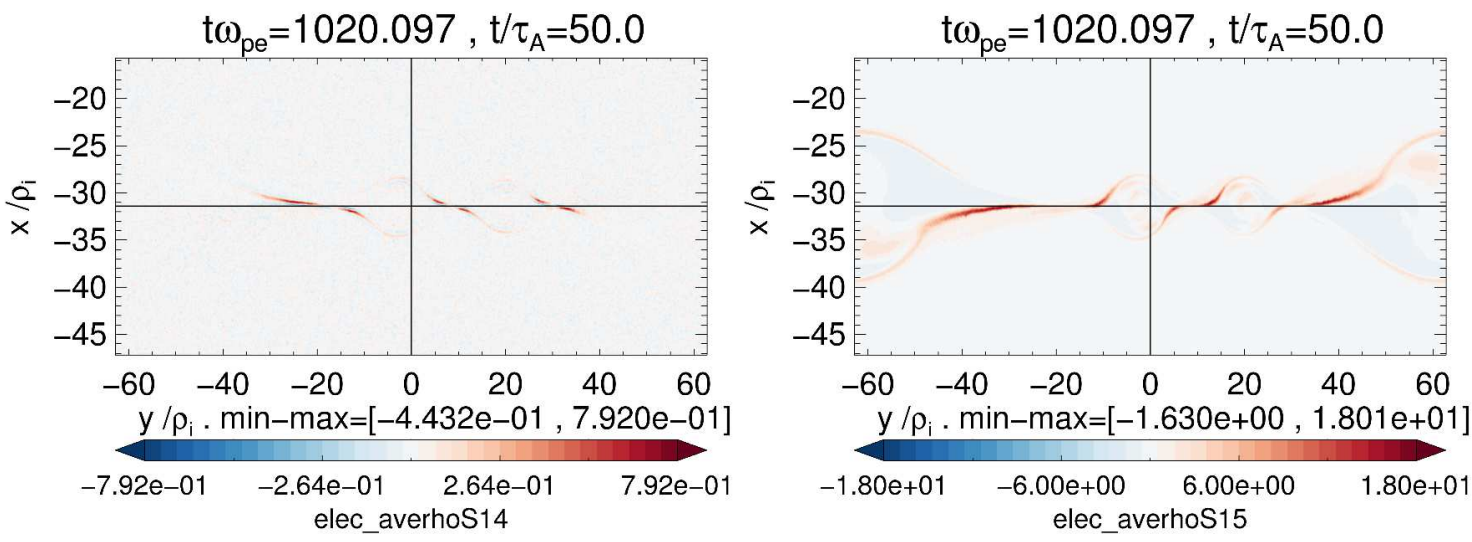

Figure 9.19: Left a): Electron Kurtosis $K_{y}$. Right b): Electron Kurtosis $K_{z}$. The kurtosis in $x$ direction is negligible (practically Maxwellian distribution function).
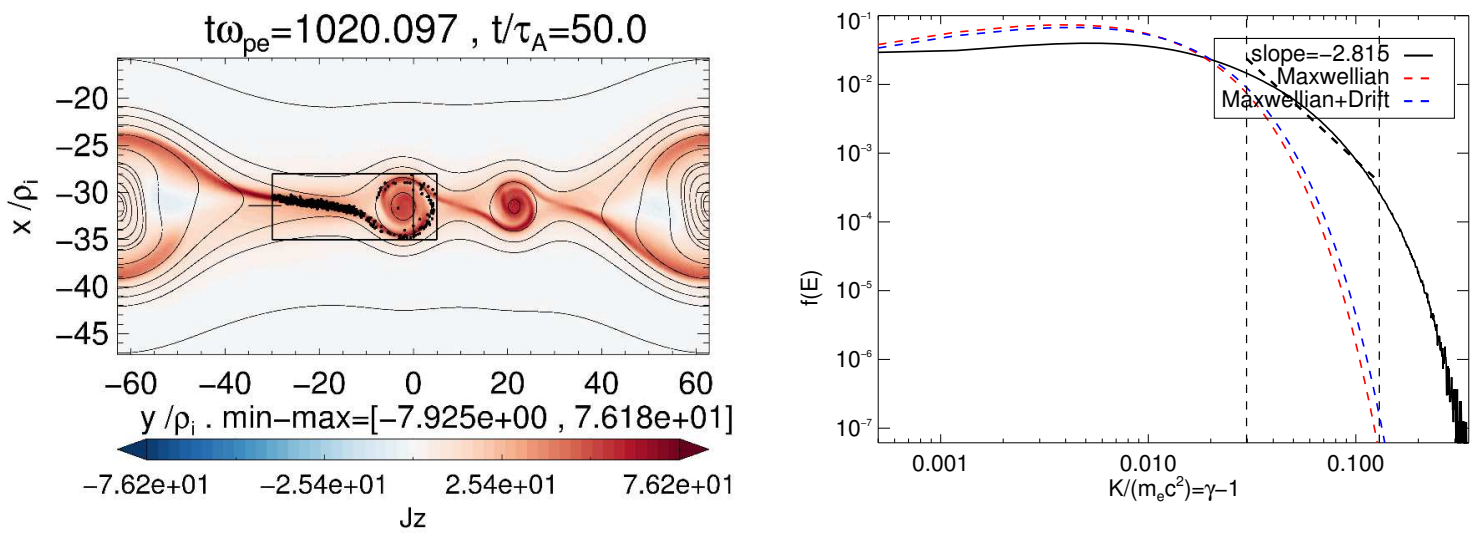

Figure 9.20: Left panel: Location of the 1000 most energetic electrons inside of the black region. Right panel: Electron energy spectra $K / m_{e} c^{2}=\gamma-1$, with $K$ the relativistic kinetic energy and $\gamma$ the relativistic factor (log-log scale), for the same region shown above. The slope indicates the power-law index fitted to the spectrum of non-thermal particles with energy inside of the region shown in vertical dashed bars. The red curve is a (theoretical) Maxwellian with the same initial electron thermal speed.

Most of the accelerated particles are located in the enhanced density separatrix, and fewer in the outer boundary of the secondary magnetic island (almost no energetic particles are inside of the latter). The energy spectra exhibits a relatively hard tail (power law index of -2.8). But in summary, positive values of the kurtosis $K_{z}>0$ do not correlate with these acceleration regions. Instead, they exhibit mostly the opposite behavior: $K_{z}>0$. In order to understand why, we need to resort to the full information contained in the electron VDF. This is shown in Fig. 9.21 for three small characteristic regions in one separatrix, at the edge of one secondary magnetic island, and in the outflow region. 

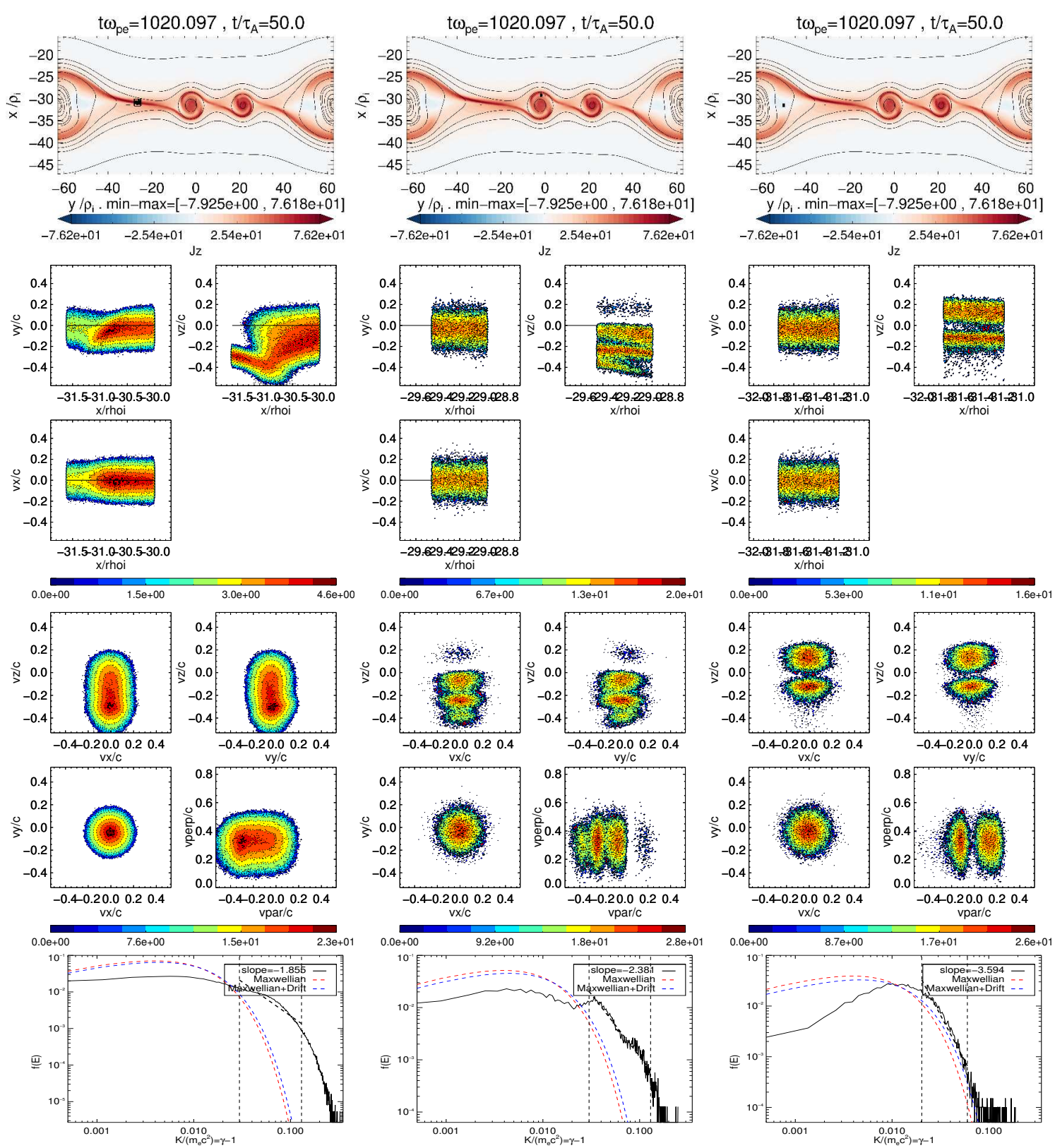

Figure 9.21: Signatures of electron acceleration and beam formation for the PIC run $b_{g}=5$ at reconnection peak time $t=50 \tau_{A}$.

Top row: Out-of-plane current density $J_{z}$ showing in a black square three selected regions. Middle top row: Phase spaces ( $x$ vs $\left.v_{x}\right),\left(x v s v_{y}\right)$ and $\left(x v s v_{z}\right)$ of the electron distribution function for the regions shown above. Black dots indicate the position of the 1000 most energetic particles inside of the chosen region.

Middle bottom row: Electron velocity distribution functions $\left(v_{x} v s v_{y}\right),\left(v_{x} v s v_{z}\right),\left(v_{y} v s v_{z}\right)$ and $\left(v_{\|} v s v_{\perp}\right)$ for the same regions shown above.

Bottom row: Electron energy spectra $K / m_{e} c^{2}=\gamma-1$, with $K$ the relativistic kinetic energy and $\gamma$ the relativistic factor (log-log scale), for the same regions shown above. The slope indicates the power-law index fitted to the spectrum of non-thermal particles with energy inside of the region shown in vertical dashed bars. The red curve is a (theoretical) Maxwellian with the same initial electron thermal speed 
In Fig. 9.21, we finally disclose the reason of the anomalous behaviour of the kurtosis. The VDF shown in the middle bottom row exhibits double peak structures (beams) along $v_{z}$ in all the regions indicated. In particular it can be seen a highly structured electron velocity distribution function inside of the magnetic islands, with several beams in the $v_{z}$ direction. Unfortunately, this streaming free energy cannot be released so easily since no wave can propagate along $z$ direction. This obviously bias the result of $K_{z}$ based on single peak structures. Nevertheless, even for $K_{z}<0$ it is still a good indicator of the locations where particles are being accelerated.

On the other hand, in Fig. 9.21 (bottom row) we can confirm, through the energy spectra, that the accelerated particles are indeed in one separatrix and, to a lesser degree, in the secondary magnetic island, but not in the outflow region. Although it is also shows a beam formation in this region, its energy spectra does not deviate too much from a Maxwellian one. Therefore, we can conclude that the process leading to beam formation is different from the one accelerating particles. However, more work is needed to disclose its mechanism.

Before closing this section, three important observations are worth to mention for possible future work extending the results shown here. First, the phase space plots in Fig. 9.21 (middle top row) show signature of electron trapping (elliptical structures) in the phase-space $x-v_{z}$ for earlier times such as $t=37 \tau_{A}$ (plots not shown here). These elliptical trapping structures saturates afterwards, and for the time shown here $t=50 \tau_{A}$, they lead to regions in the phase space with absence of particles, generating thus the double peak structures (beams). The second remark is about how misleading can be the macroscopic moment $J_{z}$ to describe the structure of the reconnection outflow. Indeed, in Fig. 9.21)(3rd column) demonstrates that there are two beams drifting in opposite directions along $z$, but their mean is practically zero, and so $V_{z}$ and $J_{z}$. This microscopic behaviour cannot be captured with only the analysis of this first order momenta. And finally, the third remark is that we carried out a similar analysis for higher guide field $\left(b_{g}=20\right)$. All these processes are diminished: less accelerated particles and practically no double peak structure in the electron VDF for $v_{z}$.

\subsubsection{Charge separation and in-plane electric fields}

Another important difference between the GK and PIC runs with low enough guide field are the deviations from quasineutrality in the latter ones, something not allowed in the GK approach. This can be seen by comparing Fig. 9.22 a 1 for $b_{g}=5$ with Fig. 9.22 a2) for $b_{g}=30$, showing the charge separation $\rho_{c}=\left(n_{i}-n_{e}\right) / n_{i}$. This charge separation is produced due to the decoupling of ion and electron motions due to their different inertia. The low guide field regime in the PIC runs favors this kind of processes, since the overall dynamics of the system is faster in absolute terms (higher ratio of $V_{A} / v_{t h, i}$, as discussed in Sec. 9.5.1). Note that the locations where the charge separation is higher are correlated with the regions where the relative perpendicular electron-ion drift speed take higher values, as can be seen by comparing Fig. 9.22 a1 with Fig. 9.24a1). These are in the asymmetric separatrix arm and in the outer boundary of secondary magnetic islands. Therefore, it can be expected that those regions will experience additionally a host of kinetic effects typically found below Debye lengths. Because the GK runs used a zero Debye length, more differences are expected in these regions compared to the PIC runs. 
Even more: the linear theory of all previously described micro-instabilities relies heavily on the quasineutrality assumption. Then, one can infer that in these regions with nonneutral plasmas the dynamics of the system might be very different from the explained previously (Davidson 1988), and thus all the previous estimations should be handled with caution and just as a first order estimation.

It is important to remark that the charge separation in the PIC low guide field regime is unnaturally high, because it is dependent on the frequency ratio $\omega_{p e} / \Omega_{c e}$ that is kept much lower in PIC simulations than in real physical environments (like planetary magnetospheres), due to computational constraints (Chen et al. 2012). This artificial effect is additionally enhanced for the low mass ratios used in our runs, since this parameter brings much closer the electron and ion dynamics: the electrons cannot screen effectively any charge separation driven by ion motion because they are heavier than they should be in reality.
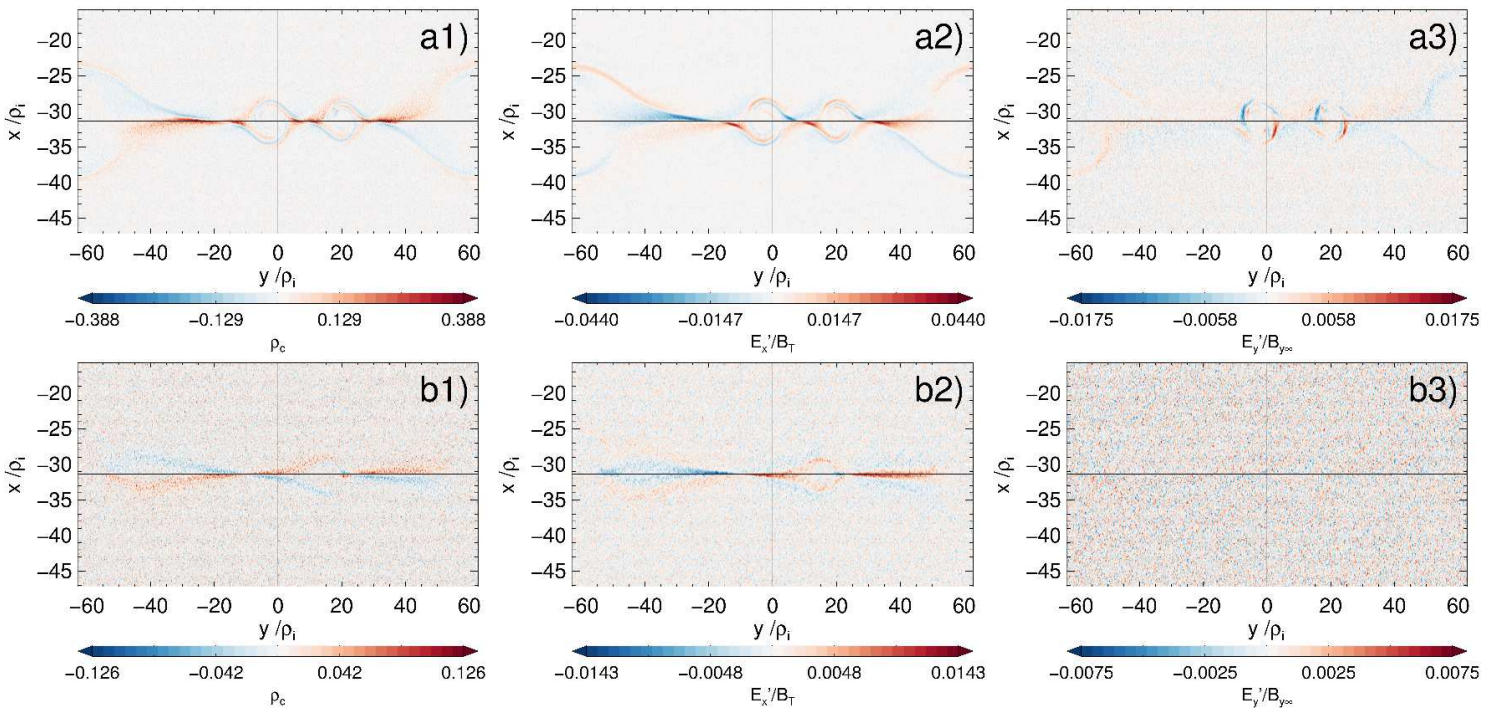

Figure 9.22: Top row al-3): guide field $b_{g}=5$. Bottom row b1-3): guide field $b_{g}=30$. Left column a1-b1: charge separation $\rho_{c}=\left(n_{i}-n_{e}\right) / n_{i}$. Middle column a2-b2: Non ideal electric field $E_{x}^{\prime} / B_{T}$. Right column a3-b3: Non ideal electric field $E_{y}^{\prime} / B_{T}$. The non-ideal in-plane electric fields have been calculated subtracting the convective electric field due to electron motion (i.e.: in its own reference frame): $E_{x, y}^{\prime}=E_{x, y}-\left(\vec{V}_{e} \times \vec{B}\right)_{x, y}$. All the quantities are calculated at reconnection peak time $t=50 \tau_{A}$. Note that all the color scales have been scaled between the minimum and maximum values in the plotted region.

The charge separation produce local steady in-plane electric fields in the region around the separatrices and in the outer boundaries of the secondary magnetic islands. Same as in the previous sections, those electric field are computed in the electron frame of reference $\vec{E}^{\prime}$ as defined by Eq. (2.26). The strongest electric fields are in the same direction of the gradients generated via the shear flow and/or reconnection: across the current sheet in the $x$ direction Fig. 9.22 a2). The $E_{y}$ component in Fig. 9.22a3) is much weaker since the gradients in that direction arise as a result of secondary instabilities, not driven directly by the reconnection process. Note that although in the regime between $b_{g}=5-20$ the charge separation and $E_{x}^{\prime}$ component scale linearly with the guide field, for higher guide 
fields they are of comparable magnitude to the surrounding noise level, which is kept more or less constant (compare the top row a1-3) for $b_{g}=5$ with the bottom row b1-b3) for $b_{g}=20$ in Fig. 9.22. There is even no visible large scale structures for $E_{y}^{\prime}$ in the case of $b_{g}=20$. This can be seen more clearly in Fig. 9.32 in Sec. 9.10 , where we discuss the dependence of these and other quantities on the numerical PIC noise.

\subsubsection{Dissipation measures}

Dissipation of electromagnetic to particle energy can happen as consequence of the reconnection process (always close to the diffusion region in the $\mathrm{X}$ point) or due to secondary instabilities. Therefore, it is important to investigate the differences in the dissipation between the PIC runs for different guide fields and the corresponding GK results. However, there are many different possible measurements of the dissipation. The first one is just directly to use $\vec{J} \cdot \vec{E}$. But there are several potential drawbacks of using this definition, specially because it has contributions from the convective electric fields due to electron motion, which are important in some parameter regimes. Therefore, it is more convenient to use the electric fields calculated in the electron frame of reference according to Eq. (2.26), changing the resulting dissipation since it is not a Lorentz invariant. In addition, we already saw that charge separation effects contribute to additional in-plane electric field in the PIC low guide field regime, adding an unwanted effect not related with a "true" dissipation (see Fig. 9.22). A self-consistent definition that takes into account all these effects (being therefore relativistically covariant) was proposed recently by Zenitani et al. (2011). It is claimed that allows to identify the location and size of the dissipation region more precisely. In the non-relativistic regime (for relativistic factors $\gamma \sim 1$ ), it is given by:

$$
D_{e}=\vec{J}^{\prime} \cdot \vec{E}^{\prime}=\vec{J} \cdot\left(\vec{E}+\vec{V}_{e} \times \vec{B}\right)-\rho_{c} \vec{V}_{e} \cdot \vec{E}
$$

where $\rho_{c}$ is the net charge separation, a correction due to the work done by moving the charge against the electric field . The results of this calculation for differences guide fields are shown in Fig. 9.23 (bottom row).

First of all, in Fig. 9.23 we can see the previously mentioned dynamo effect $\vec{J} \cdot \vec{E}<0$ inside of the secondary magnetic islands, due to the generation of core-magnetic field resulting from the shear flow, becoming stronger in the low guide field regime (e.g., $b_{g}=5$ ). Second, note the asymmetry in the dissipation region with $\vec{J} \cdot \vec{E}>0$ along the separatrices. By comparing with the symmetric dissipation regions using the definition $D_{e}=\overrightarrow{J^{\prime}} \cdot \overrightarrow{E^{\prime}}$, we can conclude that it is mostly due to bulk electron motions, a side effect of the shear flow and not contributing with a true dissipation of energy. This is confirmed by the corresponding GK results: they also show a symmetric diffusion region around the $\mathrm{X}$ point (plot not shown here). And third, note that indeed $D_{e}$ localizes very well the X points: its maximum positives values are reached in these regions. This is in contrast to the usual dissipation $\vec{J} \cdot \vec{E}$, exhibiting elongated regions with $\vec{J} \cdot \vec{E}>0$ along one of the separatrices. But the region close to the $\mathrm{X}$ point do not show signatures of enhanced thermal, non thermal or wave activity: the pressure tensor is not particularly non-gyrotropic here (see Fig. 9.18) and although there are indications of the presence of particle acceleration (see Fig. 9.19), other regions (separatrix) seems to be much more effective in producing this 


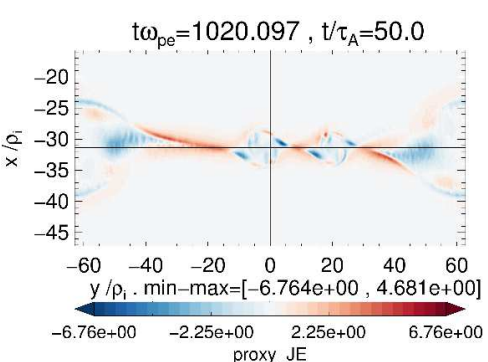

(a) $b_{g}=5$

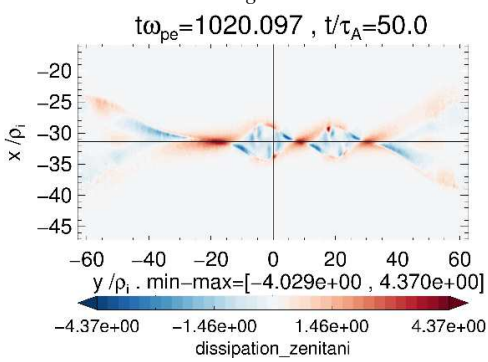

(d) $b_{g}=5$

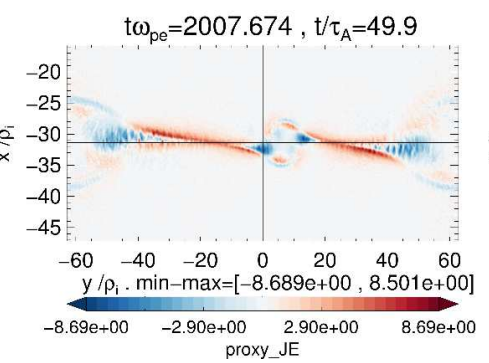

(b) $b_{g}=10$

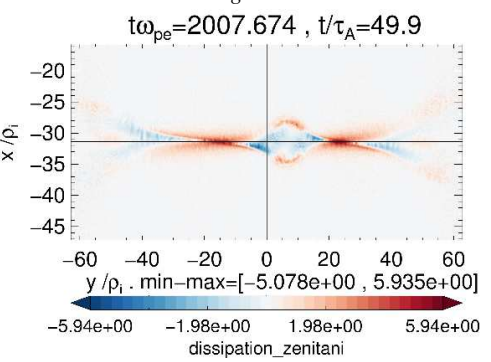

(e) $b_{g}=10$

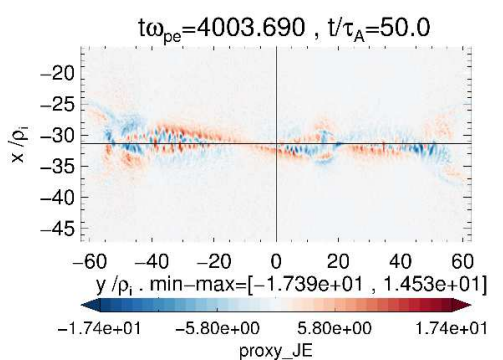

(c) $b_{g}=20$

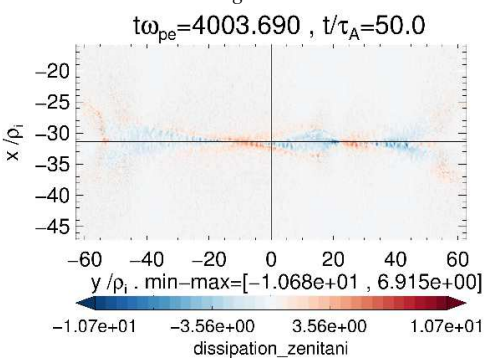

(f) $b_{g}=20$

Figure 9.23: Contour plots of two dissipation measures for different guide fields, at reconnection peak time, normalized to $J_{0} B_{\infty y} V_{A}$. Top: $\vec{J} \cdot \vec{E}$. Bottom: Covariant dissipation in the electron frame of reference $D_{e}=\vec{J}^{\prime} \cdot \vec{E}^{\prime}$, following Eq. (9.45).

kind of high energy phenomena. There is only a weak correlation with the locations of deviations from quasineutrality, but nothing more important than that (see Fig. 9.22).

\subsection{Evidence for cross-field streaming instabilities}

There are a host of micro-instabilities that may be triggered by flows as their source of free energy. Their threshold depends critically on the ratio of the flow speed $V$ with respect to the electron/ion thermal speeds $V / v_{t h, i}$. On the other hand, reconnection dynamics is governed by the value of the in-plane Alfvén speed $V_{A}$. Therefore, the relative value of the Alfvén to ion thermal speeds play a critical role in determining if a system would be unstable to some of these instabilities. But the ratio $V_{A} / v_{t h, i}$ in Eq. (9.43) do changes for different PIC guide field runs, while for GK depends that may depend on the choice of $\beta$. Therefore, it is natural to expect that a PIC run with low guide field would be potentially more prone to some of these instabilities. Furthermore, the characteristic frequency of some of them may be higher than the ones allowed by the GK approach, making a difference when comparing with the corresponding PIC results. For these reasons, it is interesting to analyze the evidences for the possible instabilities in this regime case by case.

\subsubsection{Criteria for instabilities ruled out}

Note that we are interested in the micro-instabilities driven by a relative electron-ion drift mostly perpendicular to the magnetic field $\vec{V}_{e-i} \perp \vec{B}$. This is because the dominant guide magnetic field is in the out-of-plane $\hat{z}$ direction, perpendicular to the reconnection 
plane $\hat{x}-\hat{y}$ where the distinctive flows should develop, and due to our $2 \mathrm{D}$ geometry that do not allow instabilities propagating along the $\hat{z}$ direction. The different characteristic of this family of instabilities were discussed in Sec. 5.4.2. Thus, we ruled out streaming instabilities that propagates along the magnetic field direction, such as the well-known ionacoustic or Buneman instabilities . There are also instabilities that propagates obliquely to the magnetic field but with a parallel streaming as their source of free energy (see Sec.5.4.1), such as the ion cyclotron harmonic wave instability (ICHWI). We also rule out these ones, since their growth rates are on the order of $\Omega_{c i}$, corresponding to typical time scales of magnetic reconnection, and thus being unlikely to be of importance.

But not all is lost. Other category of instabilities are the so called cross-field streaming, propagating almost perpendicular to the magnetic field direction (in this case, mostly along $\hat{z}$ ), being fed by streamings also perpendicular (in this case, in the reconnection plane). Now, one of the most important cross-field streaming instabilities in principle allowed in our system is the electron cyclotron drift instability (ECDI) (see Sec. 5.4.2.1 and the pionner works by Forslund et al. 1970, Lampe et al. 1971, Forslund et al. 1972). This instability operates at frequencies given by the harmonics of $\Omega_{c e}$, and with very high threshold speeds: $\frac{V_{0}}{v_{t h, e}} \gtrsim \frac{\Omega_{c e}}{\omega_{p e}}>1$. This is a value higher than the obtained in our runs for the low beta case, and that is why we also ruled out the existence of this instability in our system. Note that with more realistic frequencies ratios $\left(\omega_{p e} / \Omega_{c e} \gg 1\right)$, this condition can be more easily satisfied.

Thus, finally we have three remaining options of cross-field instabilities, to be discussed next:

\subsubsection{Modified two stream (MTSI)/ Kinetic cross-field streaming (KCSI) instabilities}

As discussed in Sec.5.4.2.2, this non-resonant or reactive (fluid-like) instability has a weak temperature ratio dependence and a threshold of the order of $v_{t h, i}$, with typical frequencies in the lower-hybrid range (intermediate between $\Omega_{c e}<\omega<\Omega_{c i}$ ). In this point it is interesting to mention the work by Fujimoto and Machida (2003), proposing an interplay between both KCSI and ECDI taking place during magnetic reconnection, as discussed in Sec.5.4.2.3. That study was applied in a high beta regime, for applications to Harris current sheet without guide field. We propose that a similar mechanism is operative in our simulations, although with only MTSI since ECDI is already ruled out due to its high threshold speed. This may explain in part of the different behaviour seen in the low guide field PIC runs in comparison with the high guide field cases or the gyrokinetic limit. We will focus in the region close to the separatrices (not in the secondary magnetic islands). In our case, the character of these instabilities will change somewhat from the analysis by Fujimoto and Machida (2003) in the low beta regime.

Let us make some quantitative estimations to test the possible existence of MTSI in our scenario. This instability is possibly triggered during the early, linear stage of the reconnection before its peak $(t \gtrsim 40)$. First, recall that the instability domain of allowed drift speeds of MTSI is bounded in both directions. The bottom threshold is obtained by the requirement of overcoming ion Landau damping, while the upper threshold is given by the stabilizing effects of electromagnetic terms in the otherwise electrostatic instability, resulting thus the expression Eq. (5.101) written for our parameters (in the low beta case) 
as:

$$
1<\frac{V}{v_{t h, i}} \lesssim \sqrt{2 \frac{1+\beta_{e}}{\beta_{i}}} \sim 20
$$
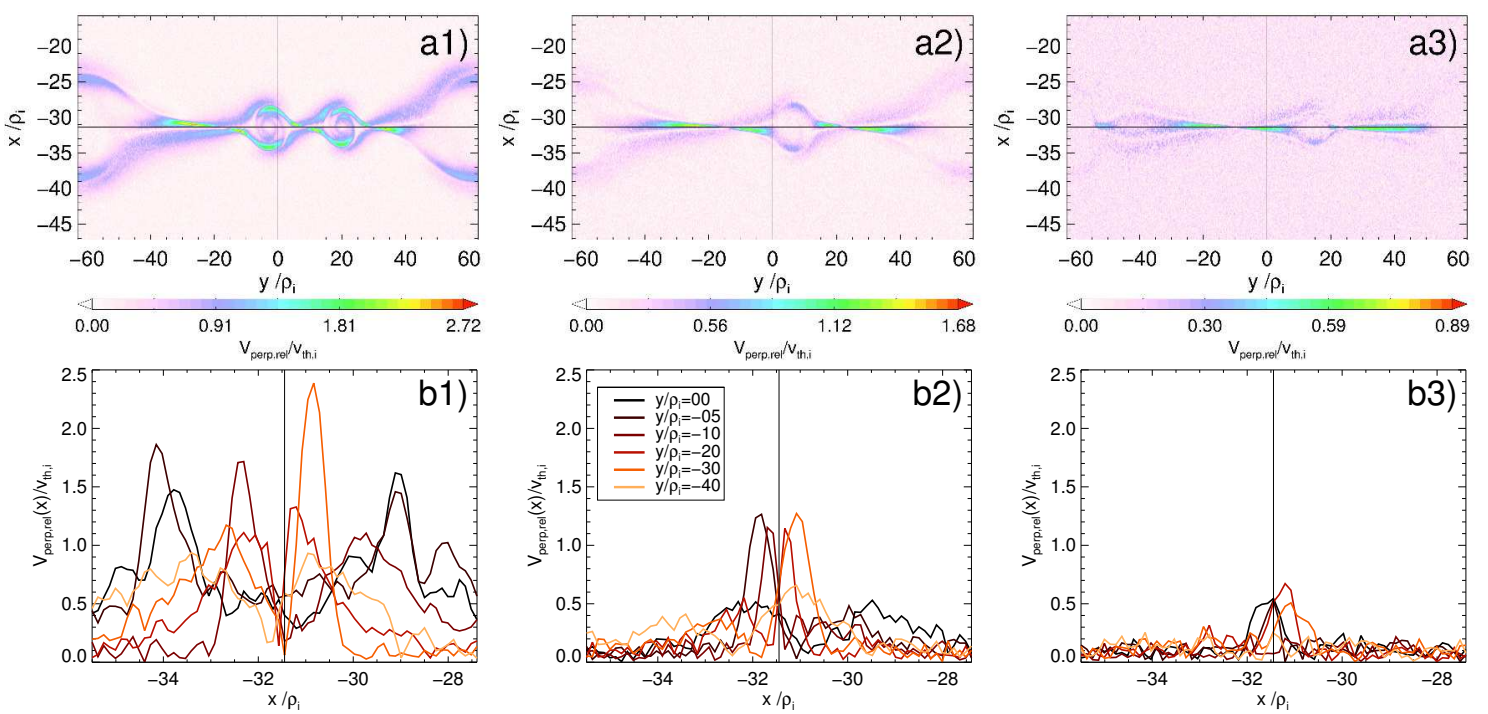

Figure 9.24: Relative perpendicular streaming electron-ion drift speed $V_{\perp, \text { rel }} / v_{\text {th,i }}=$ $\left|\vec{V}_{e, \perp}-\vec{V}_{i, \perp}\right| / v_{t h, i}$ across the left CS, for three different values of guide field at reconnection peak time $t=50 \tau_{A}$. Top row al-3) Contour plots (scaled between zero and the maximum in each case). Bottom row b1-3): Profiles across $x$ at different distance $y$. Left column a1)-b1) $b_{g}=5$. Middle column $a 2-b 2 b_{g}=10$. Right column a3-b3 $b_{g}=20$. All the quantities have been normalized to the instantaneous and local value of $v_{t h, i}(\vec{x}, t)$. The threshold for MTSI is 1 in these units.

We can see in Fig. 9.24 that only in the low guide field regime $b_{g}=5$ and $b_{g}=$ 10 the instability condition is reached in some localized regions in the separatrices. It never reaches values higher than $\left|\vec{V}_{e, \perp}-\vec{V}_{i, \perp}\right| / v_{t h, i}>1$ for guide field strengths $b_{g} \gtrsim$ 20. For later times the relative drift speed does not increase further much. Note the asymmetry in the separatrices: in one pair the relative drift speed is higher than in the other, and correspondingly the growth rate and non-linear effects of the MTSI instability are expected to be more important. This is due to the influence of the initial shear flow, and therefore is natural to see that the locations with higher values of relative electronion streaming become more symmetric and close to the outflows for increasing guide fields. None of these processes are captured for high guide field cases or the gyrokinetic simulations.

Second, MTSI has a both maximum growth rates and real frequencies on the order of the lower hybrid frequency $\Omega_{l h}$ (see discussion of Eq. (5.92)). This is in a frequency range outside of the allowed by the gyrokinetic approach, and it should be observable only with PIC simulations. Note that this quantity is guide field independent on the PIC runs and in between the ion and electron cyclotron frequencies, $\Omega_{c e}^{-1} / \omega_{p e}^{-1}=0.8<\Omega_{l h}^{-1} / \omega_{p e}^{-1} \sim 6.4<$ $\Omega_{c i}^{-1} / \omega_{p e}^{-1}=20$, for the low beta case. Compare this frequency with the Alfvén time, for 
a case with low guide field $b_{g}=5: \tau_{A} / \omega_{p e}^{-1} \sim 20$ and a high guide field case $b_{g}=50$ $\tau_{A} / \omega_{p e}^{-1} \sim 200$. On the other hand, we mentioned that for low beta plasmas, the levels of electrostatic and magnetic fluctuations will be similar (Wu et al. 1983). And indeed, we saw some signatures of high frequency fluctuations in both magnetic fields and the electric fields measured in the electron frame of reference (Eq. (9.45)), although the plots are not shown here

Third, the linear theory (McBride 1972) predicts an almost perpendicular propagation, with an angle (between $\vec{k}$ and $\vec{B}$ ) at maximum growth rate $\tan \theta=k_{\perp} / k_{\|}=\sqrt{m_{e} / m_{i}}$. For our parameters is $\theta \sim 78.7$. Note that $k_{\|}$will shift from being strictly in the $z$ direction in the infinite guide field limit (not observable due to the $2 \mathrm{D}$ constraint) to have a more significant in-plane component for low guide fields, because the ratio $B_{\infty y} / B_{g} \sim 1 / \hat{b}_{g}$ is reduced. Therefore, the large but required $k_{\|}$component of MTSI (there is no instability for strictly perpendicular propagation) will become more significant, and so its effects, in the low guide field regime. Regarding the wavelength of the most unstable modes, the linear theory predicts a perpendicular wavenumber at maximum growth rate of the order $k_{\perp, \max } \sim \frac{\Omega_{l h}}{2 V_{0}}($ see Eq. $(5.92)$ ), which for our parameters implies a wavelength

$$
\frac{\lambda_{\perp, \max }}{\rho_{i}} \sim 4.023 \frac{V_{0}}{v_{t h, i}}
$$

We have estimated this wavelength in the contours plots of the electrostatic fluctuations in FigFig. 9.22. Note that this quantity imply shorter wavelengths for higher guide fields or earlier times, because the ratio $V_{0} / v_{t h, i}$ is reduced.

Fifth, and finally, kinetic and electromagnetic effects are known to reduce substantially the consequences of this instability in the high beta range of parameters (Wu et al. 1983, Winske et al. 1985). This is because electrons become more resonant producing enhanced Landau damping. Numerical solutions of the linearized dispersion relation show that the growth rates are significantly reduced as well as the ion/electron heating. In addition, the heating becomes more isotropic for ions, while more anisotropic for electrons (more field aligned). And the relative heating ratio between electrons and ions changes from one to higher values (more energy goes to electrons than ions). Another difference in the high beta range of parameters is that range of unstable wavenumbers is increased and the propagation angle at maximum growth rate is even more oblique. This implies that the in-plane component of the unstable waves decreases in comparison with the low beta case, and therefore the observable wavelengths should be reduced.

Now, some words about the influence of these instabilities in reconnection. We observed that in the low guide field regime where this instability operates, the reconnection rate decrease (see Fig.9.3). In addition to all the reasons given in Sec.9.5.6 if these microinstabilities operates, its effect will also tend to reduce reconnection rate as well. This is because in our setup this kind of instabilities are of secondary importance in comparison with magnetic reconnection, and the electrostatic fluctuations can absorb part of the magnetic energy released via reconnection, decreasing its rate (see some other examples from previous works in Sec. 5.4.2.3)

Summarizing this section, there are strong indications that a cross-field streaming driven micro-instability operates for PIC runs with low enough guide field. Its main effect seems to be the enhanced electrostatic fluctuations taking place in one pair of the 
separatrices, heating the electrons in these regions and contributing to the asymmetry between thermal and magnetic pressures there.

\subsubsection{Gradients driven instabilities}

\subsubsection{Lower hybrid drift instability (LHDI)}

As discussed extensively in Sec. 5.4.2.4, LHDI is a instability driven by pressure gradients: either in density or temperature. And similarly to MTSI, it also has growth rates and typical frequencies are al so on the order of $\Omega_{l h}$. The mechanism is indeed very similar: the diamagnetic drifts generated by these gradients produce cross-field currents that behaves analogously to the ones required for the triggering of MTSI, as can be seen from the Eq. (5.104) or Eq. (5.105). It states that cross-drift speeds in units of $v_{t h, i}$ are proportional to gradients in length scales of $\rho_{i}$. Thus, if present, their effects are added up (destabilization becomes easier). Since the threshold is given by Eq. (5.107), we infer that gradients steeper than $\rho_{i}$ or diamagnetic drift speeds higher than $v_{t h, i}$ will drive unstable LHDI. But that expression was derived assuming unmagnetized ions $\omega \gg \Omega_{c i}$ and neglecting finite ion Larmor radius effects $k_{\perp} \rho_{i} \ll 1$. But for gradients on the order of (ironically) $\rho_{i}$ or diamagnetic drift speeds on the order of $v_{t h, i}$, especially the second assumption breaks down. A more accurate threshold condition was derived by Davidson et al. (1977) taking into account these finite ion Larmor effects and given in Eq. (5.112). Note that these thresholds are not absolute, in the sense that LHDI can still operative with gradient lengths below $\rho_{i}$, but with reduced growth rates (Davidson and Gladd 1975). In this sense, LHDI is a quite ubiquitous instability, not being shut-down completely so easily. Another important remark provided by full numerical solutions is that the typical wavelengths of LHDI for our parameter regime $T_{i} \sim T_{e}$ (where many analytical approximations breaks down) is $k_{\perp} \rho_{e} \sim 1$ over a wide range of values of diamagnetic drifts speeds around $V_{D, \perp} \sim v_{t h, i}$ (Davidson and Gladd 1975). These are wavelengths shorter than those of MTSI, implying that electron finite Larmor radius effects are essential to describe properly this instability.

Now, let us compare the respective LHDI thresholds in the PIC runs for the gradient strengths. The maximum values reached by these perpendicular electron pressure gradient across the CS at different distances $y$ from the center are shown in Fig. 9.25 at a reconnection peak time. The threshold values Eq. (5.107) or Eq. (5.112) are only marginally reached in some localized regions for the cases with lowest guide field $b_{g}=5$ and $b_{g}=10$, especially across the separatrices and in the outer boundary of the secondary magnetic islands. Then, in these places enhanced wave activity due to this instability is expected. 

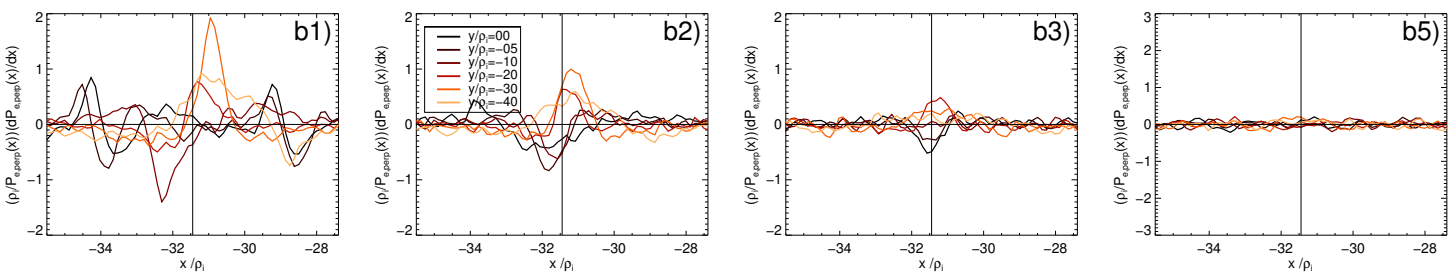

Figure 9.25: Profiles across the left CS (at different distances y) of the gradient length scale of the perpendicular electron pressure $\left(\rho_{i} / P_{e, \perp}\right) d P_{e, \perp} / d x$ in units of $\rho_{i}$, for different guide fields. $\left.\left.\left.b 1): b_{g}=5, b 2\right): b_{g}=10, b 3\right) b_{g}=20, b 4\right) b_{g}=50$. All of them are for $a$ time $t=50 \tau_{A}$

\subsubsection{Comparison of ExB and diamagnetic drifts (MTSI vs LHDI)}

As we already mentioned, those steps gradients produce cross-field currents that behaves similarly to those of MTSI. A natural question is to determine their relative contribution to the total net current, or which one is dominant: either the diamagnetic drift driven by gradients that feeds LHDI, or the $V_{E x B}$ drift source of free energy of MTSI (since $V_{E x B}$ is approximately the electron frame of reference, implying that the ions will also have a relative drift of $-V_{E x B}$ with respect to them). By combining both expressions and assuming some other approximations, it is possible to establish that a system will become unstable to MTSI and LHDI when both previously mentioned drifts are present whenever the condition Eq. (5.106) holds. in other words, each drift individually should be higher than $v_{t h, i}$ assuming $T_{i}=T_{e}$, or otherwise, one of them should compensate the another weaker one by having proportionally much higher values than $v_{t h, i}$. A comparison of these drifts can be seen in Fig. 9.26.
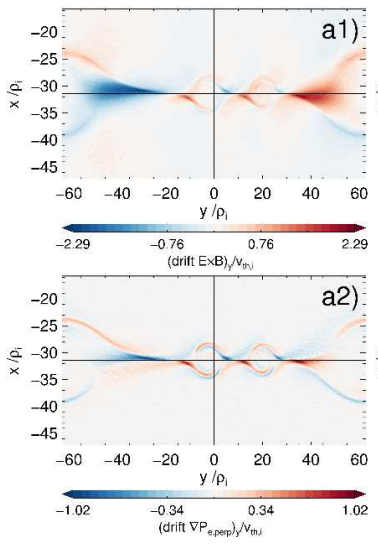
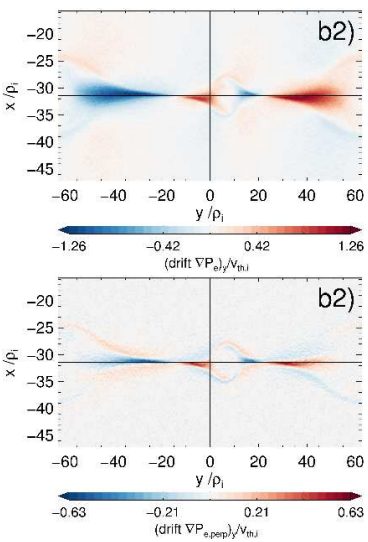
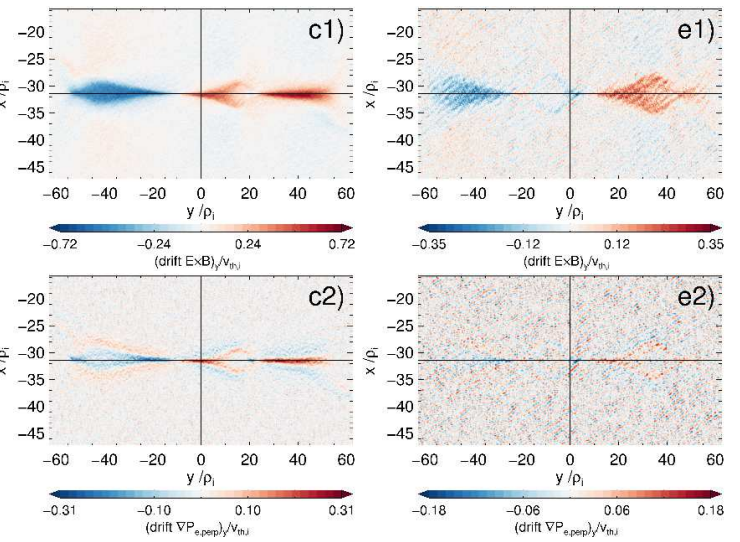

Figure 9.26: Contour plots of characteristic drifts along y direction (with color tables scaled between \pm the maximum value in each case, in order to improve visualization). Top row: drift $V_{E x B, y}$. Bottom row: diamagnetic drift $V_{D, y}$. Columns more to the right indicate higher guide fields. a1-2) $b_{g}=5$. b1-2) $b_{g}=10$. cl-2) $\left.b_{g}=20 . d 1-2\right) b_{g}=50$. The normalization is with respect to the initial value of $v_{t h, i}$.

The first point to note in Fig. 9.26 is that the steep gradients regions (proportional to the diamagnetic speed by Eq. (5.104)) prone to LHDI are much more localized than those where MTSI is expected (compare also with Fig. 9.24). These locations also change 
from being in one of the separatrices (and secondary magnetic islands) to the outflows by increasing guide field, due to the weakening asymmetric effect of the initial shear flow.

Second, the relative ratio of the diamagnetic to $E \times B$ drift is roughly 0.5 (taking into account the peak maximum values), being more or less constant among different guide field strengths. Note that both drifts scale inversely with the guide field in units of $v_{t h, i}$, or equivalently, they have the same values in units of the Alfvén speed $V_{A}$ for different guide fields. The diamagnetic drift reach supersonic speeds only for guide fields $b_{g} \lesssim 5$, while the $E x B$ drift for guide fields $b_{g} \lesssim 10$. Therefore, LHDI is expected to play a less important but it is still significant role in comparison with MTSI for the low guide field regime, because it always adds-up free energy available for instability, enhancing growth rates. Another consequence of the relative ordering of these drift speeds is that the GK perpendicular drift approximation should break down precisely for these guide field values where $V_{E x B}$ or $V_{D}$ reach supersonic speeds (see Sec. 9.5.1).

Finally, in both Fig. 9.26 and Fig. 9.25 we can see that the gradient effects are completely negligible in the large guide field limit, since these are reduced below the level of numerical noise. On the other hand, the $\vec{E} \times \vec{B}$ drift is still significant even at $b_{g}=50$, since after all these are the motions that drive reconnection process. Therefore, this is even another reason where convergence with the GK runs is expected in this regime.

An important point is that the small wavelengths of LHDI instabilities make necessary the inclusion of finite electron Larmor radius effects. Whenever there are steep gradients at length scales smaller than $\rho_{i}$, the inclusion of these effects become essential. We already showed that a signature of the importance of these effects is given by the non-gyrotropy of the electron VDF $D_{N g}$. And indeed, the locations where this quantity takes higher values, as shown in Fig. 9.18, are correlated with the regions where the diamagnetic drift peaks (see Fig. 9.26(bottom)).

\subsubsection{Electron-ion hybrid (EIH)/Kinetic Kelvin-Helmholtz instabilities}

Not only gradients in pressure can trigger instabilities. The reconnection process can generate, in the PIC low guide field regime, very steep gradients in the flow as well. In these cases the free energy source comes from these strong sheared flows producing the instabilities discussed in Sec. 5.3.2.1, in particular the so called electron-ion hybrid (EIH) instability. This has also frequencies in the lower hybrid-range $\Omega_{l h}$ when the gradients in velocity (with frequency units) are steeper than that value, according to Eq. (5.72). As in the discussion of MTSI and LHDI, instabilities with frequencies in this intermediate range between $\Omega_{c e}<\omega<\Omega_{c i}$ can drive particles out of their $\vec{E} \times \vec{B}$ drifts, thus breaking one of the GK assumptions and making differences with the PIC runs in the low guide field regime. Because we already saw the effects of instabilities in this frequency range in the previous section, it is worth and interesting to check the conditions for the triggering of EIH in our case. Furthermore, the source of free energy of these instabilities have effects that always add up (never compensates): relative streaming/ExB drift drives MTSI, pressure gradients/diamagnetic drifts drives LHDI, and velocity shear drives EIH. Other related source of free energy, the gradient in magnetic field strength, is ruled out because it is negligible for the low beta case.

Therefore, since the flow that can drive this instability has to be in the $y$ direction, we can estimate the conditions for its existence by showing the gradients in the flow speed 
$\frac{d V_{e, y}}{d x}$ measured in units of $\Omega_{l h}$, as shown in Fig.Fig. 9.27 .
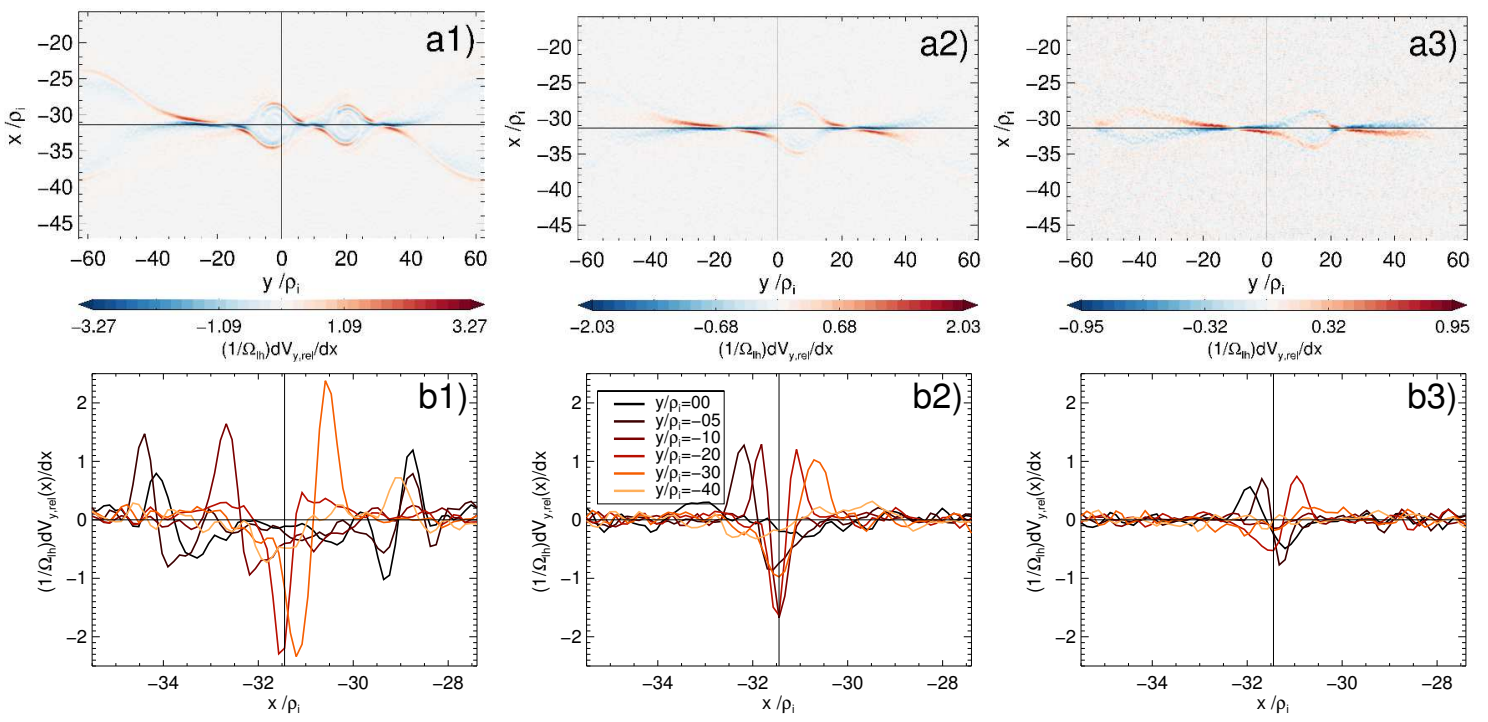

Figure 9.27: Top row al-3)/bottom row b1-3): Contour plots/profiles across $X$ of the normalized shear flow strength $\left(1 / \Omega_{l h}\right) d V_{e, y} / d x$ for different guide fields. al-bl: $b_{g}=5$, $a 2-b 2: b_{g}=10, a 3-b 3 b_{g}=20$. All the plots are for a time $t=50 \tau_{A}$. The threshold of EIH is 1 in these units.

Two important consequences can be extracted from Fig.Fig. 9.27. First of all, the shear flow gradients are steeper than the threshold condition Eq. (5.72) in some localized regions around the asymmetric separatrix arm and the outer boundary of the secondary islands, similarly to the discussion of the previous instabilities. Then, enhanced wave activity and ion heating is expected in the same locations due to EIH. Second, the peak value has an offset with respect to the one from the relative electron-ion drift speed (see Fig. 9.24) or (see Fig. 9.25) pressure gradients. The shear flow gradient peaks at the edges of the thin channels with enhanced relative streaming speed, and therefore it might extend the instability region by the main MTSI instability on the order of $\rho_{i}$.

\subsubsection{Thermal effects due to secondary instabilities}

Both linear and quasilinear theories of the previous three described instabilities related with cross-streaming and gradients make relevant predictions related with the ion/electron heating. We verified that the parallel electron and perpendicular ion heating take place exactly for the locations and times where the relative-streaming and pressure/velocity gradients crosses their respective thresholds. All those processes goes down for increasing guide field, since the source of free energy of the instabilities is reduced, converging to the GK results.

Quasilinear estimations at saturation time of MTSI (in the low beta regime) predicts perpendicular ion heating comparable to a parallel electron heating (same behaviour seen in our runs), with a magnitude given by Eq. (5.98) proportional to the cross-streaming speed magnitude $V_{0}$. This means that the additional heating due to this instability scale inversely with the guide field, because of the dependence of $V_{0}$, which is a good estimate 
for the behaviour seen in Fig. 9.17a-c. On the other hand, we could not observe that the streaming free energy is equipartitioned between the thermal energies of ions and electrons, due to possibly other assumptions not taking into account there. The heating mechanism due to MTSI can be understood as follows (McBride 1972, Fujimoto and Machida 2003). As we mentioned, this instability has almost perpendicular propagation, but nevertheless requires a finite $k_{\|}$in order to exist. It is in this direction parallel to the magnetic field where the oblique MTSI waves produce potential troughs where the electrons can be trapped, due to their free streaming along this direction. For higher guide fields and GK runs, $k_{\|}$is completely negligible because the $2 \mathrm{D}$ geometry of the runs (it is almost completely in the $\hat{z}$ direction). But in the lower guide field regime the parallel direction has a small but significant in-plane component, since the ratio with the in-plane magnetic field is reduced. Therefore, only for reduced guide fields potential troughs can be formed along the projection of $k_{\|}$on the reconnection plane, and thus the efficiency of parallel electron heating is enhanced.

On the other hand, since LHDI has a completely analogous mechanism (see Sec. 9.8.3), which a contribution to the source of free energy coming from the diamagnetic drift, a similar prediction to MTSI related to the heating is expected for this instability.

Finally, EIH instability also predicts a perpendicular ion heating (Romero and Ganguli 1993, Ganguli et al. 1994), basically due to resonant interaction of the lower hybrid waves generated by them with the ion VDF. Therefore, its contribution to the overall additional ion heating adds up with the ones due to the previous instabilities.

Some of the theoretical disagreements with the results of our simulations can be understood since it is necessary to keep in mind that different from the original studies of instabilities in homogeneous plasmas, a saturated or stable state is not reached due to the highly dynamic conditions of the evolving current sheet: the source of free energy is continuously fed by the development of magnetic reconnection.

\subsection{Finite plasma beta effects}

In this section we address the effects of the plasma beta in all the previous results, using the "high beta" case runs with $\beta_{i}=1.0$. In general, the basic phenomena to be described are similar to the previously discussed low beta case $\left(\beta_{i}=0.01\right)$. But when comparing the different PIC guide fields in the range $b_{g}=1 \rightarrow 10$ with GK runs, we notice that the agreement is better, as seen in the reconnection rates of Fig. 9.3 (b), as well as in the time evolution of the scaled magnetic fluctuations $\Gamma \delta B_{z}$, shown in Fig. 9.28(b).

\subsubsection{Differences in the pressure equilibrium condition}

There are at least two related reasons for the better agreement in the high beta case.

The first one is because the absolute magnitude of the fluctuating quantities $\delta P_{\text {th }}$ and $\delta B_{z}$ are proportional to $\propto 1 / \sqrt{\beta}$ (see Eq. (4.33) and Eq. (4.36)), and thus smaller by around one order of magnitude compared to the low beta case. In addition, the GK ordering parameter $\epsilon$ will also be reduced (see discussion of Eq. (9.35)), improving the validity of the model in this parameter regime. The physical consequences in that the largest deviations from the pressure equilibrium condition will be reduced by the same amount, 


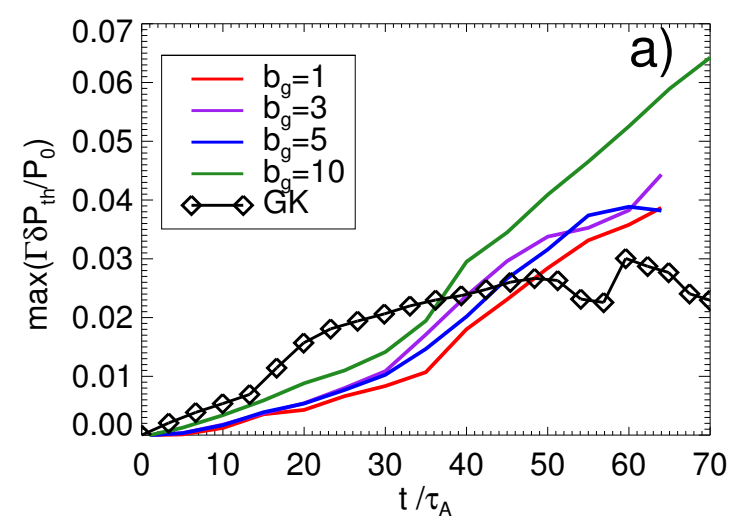

(a) $\Gamma \delta P_{t h}$

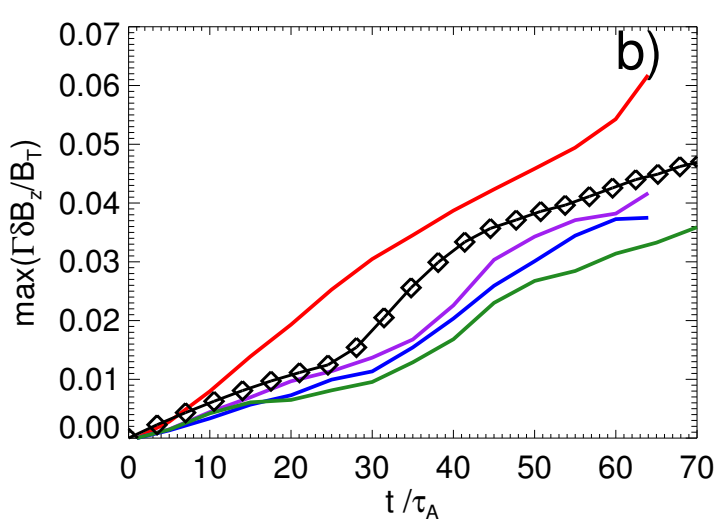

(b) $\Gamma \delta B_{z} / B_{T}$

Figure 9.28: Time history of the maximum value of the scaled thermal $\Gamma \delta P_{t h} / P_{t h, 0}(a)$ and magnetic fluctuations $\Gamma \delta B_{z} / B_{T}(b)$ for different PIC guide fields and the corresponding $G K$ result, in the high beta case $\beta=1.0$. To be compared with Fig. 9.9. The scaling factor $\Gamma$ (Eq. (9.37)) for the PIC runs was calculated using a reference guide field $b_{g, r e f}=5$. Reproduced with permission from P. A. Muñoz, D. Told, P. Kilian, J. Büchner and F. Jenko, Physics of Plasmas 22, 082110, (2015) Copyright 2015, AIP Publishing LLC.

making more accurate the predictions of the GK approach compared to a Vlasov plasma (and the respective GK/PIC simulations). This can be seen in the plots comparing the thermal $\delta P_{t h}$, magnetic $\delta B_{z}$ and total pressure $\delta P_{\text {total }}$ fluctuations in Fig. 9.29 for different PIC guide fields and the corresponding GK result. Specifically, from looking at the values of Fig. 9.29 (4th column) we can infer that the maximum net force due to the total pressure imbalance in the PIC low guide field regime will be much smaller than in the low beta case. The time evolution of the thermal pressure fluctuations $\Gamma \delta P_{t h}$ in Fig. 9.28(a) displays a different behaviour between the results given by both codes. 


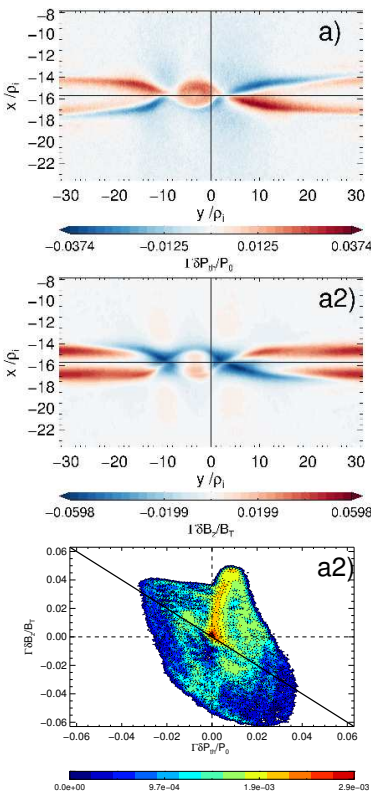

(a) $B g=1$

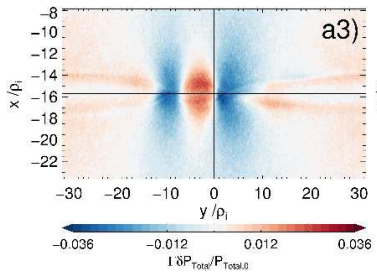

(e) $\Gamma \delta P_{\text {total }} / P_{t h, 0} b g=1$
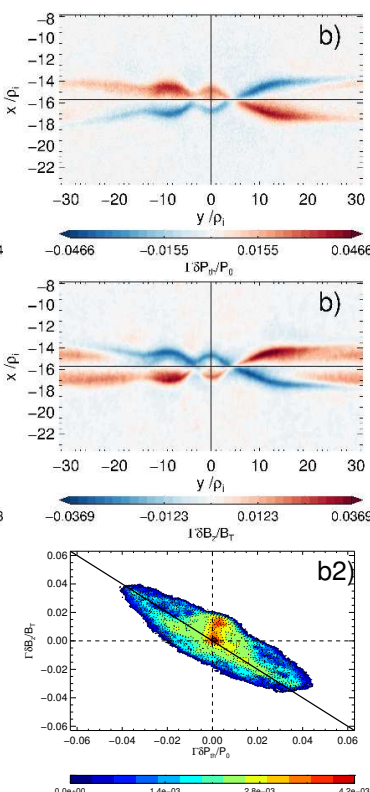

(b) $B g=3$

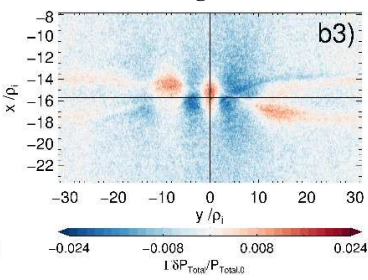

(f) $\Gamma \delta P_{\text {total }} / P_{t h, 0} b g=3$
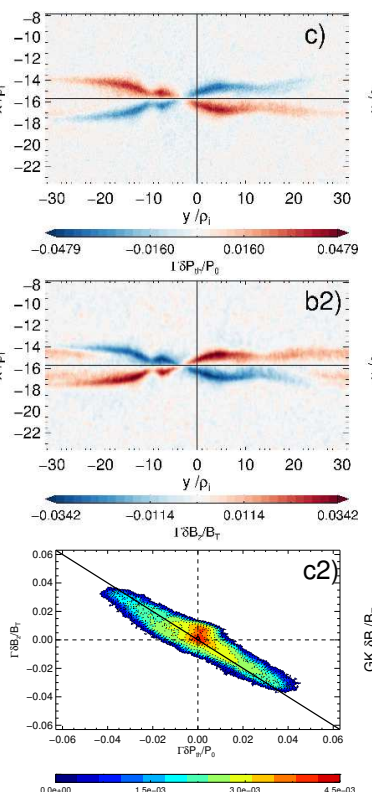

(c) $b_{g}=5$

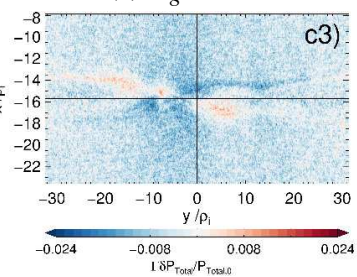

(g) $\Gamma \delta P_{\text {total }} / P_{t h, 0} b_{g}=5$
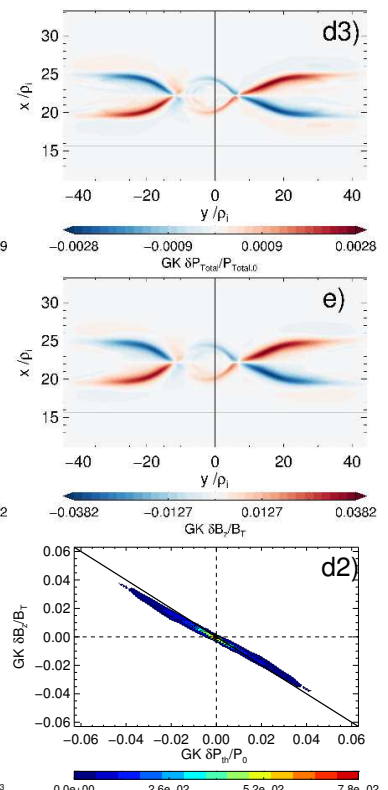

(d) $G K$

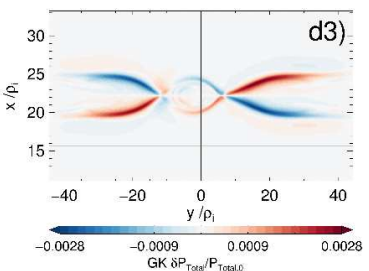

(h) $G K \Gamma \delta P_{\text {total }} / P_{\text {th }, 0}$

Figure 9.29: High beta case. Contour plots of several scaled fluctuations for different PIC guide fields and the corresponding GK result, at a time $t=50 \tau_{A}$.

First row: $\left.\left.\left.\left.\Gamma \delta P_{t h} / P_{0} a 1\right) b_{g}=1, b 1\right) b_{g}=3, c 1\right) b_{g}=5, d l\right) G K$. (to be compared with Fig. (9.6).

Second row $\left.\left.\left.\left.\Gamma \delta B_{z} / B_{T} a 2\right) b_{g}=1, b 2\right) b_{g}=3, c 2\right) b_{g}=5, d 2\right) G K$. (to be compared with Fig. 9.7).

Third row: Respective $2 D$ (frequency) histograms with the correlation between the magnetic and thermal fluctuations $\Gamma \delta B_{z} / B_{T}$ and $\Gamma \delta P_{t h} / P_{t h, 0}$. a3) $\left.b_{g}=1, b 3\right) b_{g}=3, c 3$ ) $\left.b_{g}=5, d 3\right) G K$. The points used to generate these plots correspond to the regions with the current density $J_{z}$ above the $10 \%$ of its initial value $J_{z}(t=0)$. The diagonal black straight line with slope $-\beta_{\text {in }}$ represents the pressure equilibrium condition in the limit of strong guide field Eq. (9.40).

Fourth row: Respective contour plots for the scaled total pressure $\Gamma \delta P_{\text {total }} / P_{\text {th }, 0}$ a4) $\left.\left.\left.b_{g}=1, b 4\right) b_{g}=3, c 4\right) b_{g}=5, d 4\right) G K$. The latter is identically zero to machine precision. (to be compared with Fig. Q.8).

However, compared to the low beta case, we can immediately notice a big difference inside of the secondary magnetic islands in the PIC low guide field regime (Fig. 9.29)(first columnn)). Besides of the expected core-magnetic field, there is also a thermal pressure contribution negligible in the PIC high guide field regime or the GK simulations. This makes the "bump" in the histograms $\delta P_{t h}-\delta B_{z}$ (Fig. 9.29(3rd columnn)) shifts from being in the $y$ axis (as in the low beta case) to the first quadrant. 


\subsubsection{Flow pattern structure in magnetic islands and separatrices}

The second reason for the better agreement in this high beta case between both simulations models for the PIC guide field runs with $b_{g} \gtrsim 3$, compared to the low beta ones, is also due to another fact related with the magnitude of the in-plane currents. The range of variation of the Alfvén to ion thermal speed (Eq. (9.43)), is decreased by a small amount in comparison to the low beta case: $V_{A} / v_{t h, i}=0.1 \rightarrow 0.7$ going from $b_{g}=10 \rightarrow 1$. This means that the Alfvén velocities are always subsonic (even in the extreme case of $b_{g}=0$, where a comparison between PIC and GK simulations would not make sense). As a result, the maximum values of the initial shear flow will al so be subsonic. For the lowest PIC guide field considered, $b_{g}=1$, the value is $\max \left(V_{y 0}\right) \sim 0.3 V_{A} \sim 0.2 v_{t h, i}$. Moreover, due to the fact that the maximum electron/ion outflows speeds in units of $V_{A}$ are proportional to the reconnection rates, they will be reduced at least by a factor of two according to Fig. 9.3(b). Therefore, these speeds will become negligible when measured in units of $v_{t h, i}$, compared to the low beta case. For example, the measured maximum outflows speeds are on the order of $0.75 V_{A}$ for electrons (see Fig. 9.30 (top row)) and $0.3 V_{A}$ for ions (see Fig. 9.30(bottom row)). This reduction is approximately by a factor of 3 compared with the corresponding values in the low beta case.
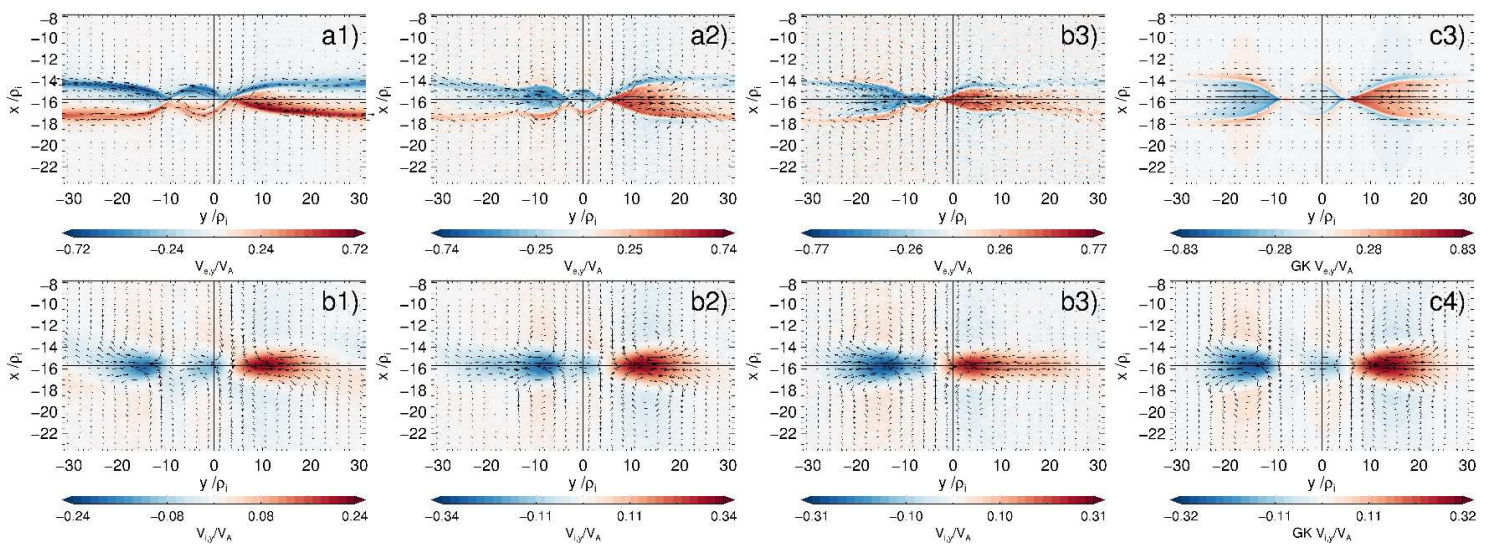

Figure 9.30: High beta case. Top row: Vector plot of the in-plane electron bulk velocity for different PIC guide fields and the corresponding GK result, at a time $t=50 \tau_{A}$. Color coded is the $V_{e, y} / V_{A}$ component. a1) PIC $b_{g}=1$, a2) PIC $b_{g}=3$, a3) PIC $b_{g}=5$, a4) GK.

Bottom row: Same as above but for the ion in-plane bulk velocity $V_{i, y} / V_{A}$. b1) PIC $b_{g}=1$, b2) $\left.\left.P I C b_{g}=3, b 2\right) P I C b_{g}=5, b 4\right) G K$.

The previous observation has two physical consequences. For the GK code, the maximum deviations from the drift approximation where this approach holds, proportional to the maximum speeds in units of $v_{t h, i}$, will be smaller than in the low beta case (see discussion in Sec. 9.5.1). Consequently, a comparison with the PIC runs will be more reliable. For the PIC code, smaller reconnection rates implies less open separatrices and smaller secondary magnetic islands. In the small guide field regime $\left(b_{g} \approx 1\right)$ there is still a strong effect of the shear flow around these structures, producing currents (see, e.g., Fig. 9.30(a1)-(a1)) which become negligible for $b_{g} \gtrsim 3$ (see, e.g., Fig. 9.30(b3)-(b3) for $\left.b_{g}=5\right)$. In fact, the Hall term and the corresponding decoupling of electrons and ions 
is facilitated in high plasma beta environments. That is why we observed in-plane current densities twice as high as the values seen in the low beta case (Fig. 9.12), as can be calculated from the electron/ion flows in Fig. 9.30. But the generation of core magnetic field, according to the estimate Eq. (9.44), is proportional to the length scale $\Delta L$, which is roughly five times smaller in this case compared to the low beta case (secondary magnetic islands close to the $X$ point have size around $\sim 2 \rho_{i}$ across the $x$ direction, while the magnetic island at $y$ boundaries has size around $\sim 4 \rho_{i}$ ). Furthermore, the magnetic field generation due to the colliding outflows at the $y$ boundaries is decreased, since the maximum outflows speeds are smaller than in the low beta case. All these predicted significant deviations for the lowest PIC guide field case, $b_{g}=1$, can be seen in in the time evolution of $\Gamma \delta B_{z}$ in Fig. 9.28 (b). Note that according to the previous discussion, they are much smaller in absolute terms compared with the low beta case: convergence with the GK results (overlap of curves) is already reached for values $b_{g} \gtrsim 3$ (see, e.g., the second column of Fig. 9.29 for $b_{g}=5$ ). It is important to mention that this reduction of core magnetic field strength in high $\beta$ plasmas was already found in hybrid simulations by Karimabadi et al. (1999) (although for very low guide fields $b_{g}<1$ ).

There is another critical difference in the in-plane flow pattern in this high beta case outside of the secondary magnetic islands. Both PIC high guide field simulations and the GK runs show electrons returning in the outer boundary of the separatrices with respect to the direction of the main reconnection outflow (see Fig. 2.29(c1)-(d1)). Thus, the $V_{e, y}$ component has an octupolar structure in these regions. This sheared velocity field generates enhanced density currents and associated magnetic field in these regions (although with the classical quadrupolar structure, not like the octupolar one seen in the work by Graf von der Pahlen and Tsiklauri 2013). The first effect can be seen in Fig. 9.31(c). Indeed, the maximum values of the magnetic field as seen in the time history of Fig. 9.28(a) are reached in these separatrices instead of the secondary magnetic islands. In the low guide field regime, however, the initial shear flow is so dominant than the returning electron pattern is destroyed. Instead, in this regime, the magnetic field is generated only due to the ion-electron decoupling.
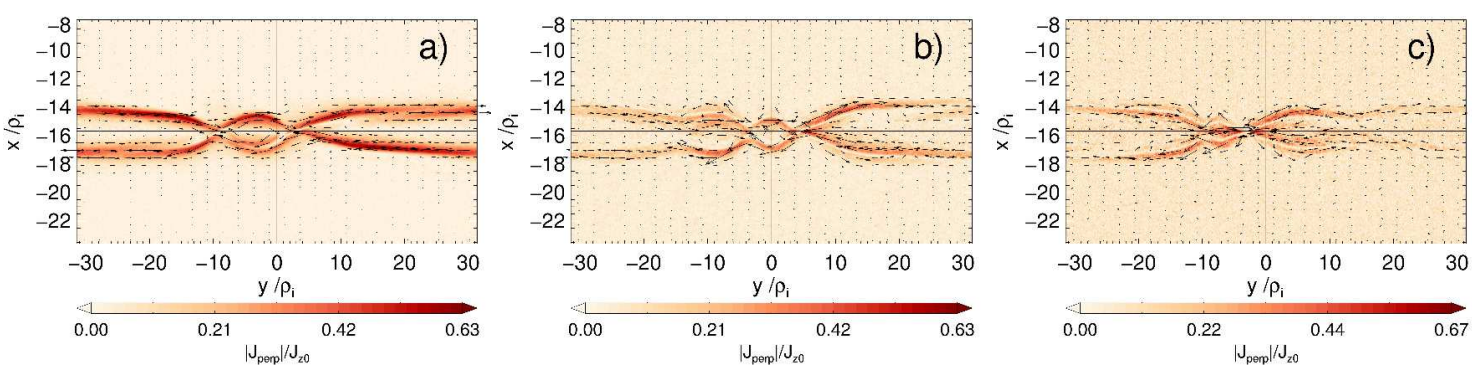

Figure 9.31: Vector plot of the in-plane current $\vec{J}_{\perp}=J_{x} \hat{x}+J_{y} \hat{y}$ for two cases of PIC guide fields $a$ ) $\left.\left.b_{g}=1, b\right) b_{g}=3, b\right) b_{g}=5$, at a time $t=50 \tau_{A}$. Color coded is the magnitude of this in-plane current $\left|\vec{J}_{\perp}\right| / J_{z}(t=0)$. Note that because Eq. 9.34), $J_{z}(t=0)=J_{z N} * J_{N}$ varies with the PIC guide field (through $J_{N}$ ). 


\subsubsection{Summary: critical parameters for reliable PIC/GK compari- son}

Therefore, by analyzing a different set of runs in a high $\beta$ plasma regime, we could distinguish between the different effects of violations of the gyrokinetic ordering in the GK runs and the initial shear flow always present in the PIC force free runs. The first effect is encoded in the parameter $\epsilon$ (Eq. $(9.35)$ ), which can be as high as 4 in the low beta case for the lowest guide field $b_{g}=5$. Then, strong deviations from the PIC simulation results are be expected in that regime. On the other hand, $\epsilon=0.2$ for the lowest guide field $b_{g}=1$ in the high beta case, and a better agreement is observed. However, we still could find generation of core magnetic field and other phenomena associated with the initial shear flow, although with reduced strength. This also breaks the comparison between PIC to GK, but it does not only rely on the relative value of $V_{A} / v_{t h, i}$ and other parameter associated to the initial shear flow, but also in the fact that reconnection rates are smaller (less open separatrices and smaller magnetic islands). Thus, we can conclude that a reliable comparison between PIC-GK simulations of magnetic reconnection can be obtained with parameters that provides smaller fluctuations levels (high $\beta$ plasmas), lower ratios $V_{A} / v_{t h, i}$ and also relatively small reconnection rates $d \Psi / d t$ (proportional to $V_{e, \text { out }} / V_{A}$, with $V_{e, \text { out }}$ the maximum electron outflow speeds). The balance of this three parameters guarantee a good convergence. However, PIC codes shows an enhanced numerical heating in high $\beta$ plasmas, making a comparison with GK not so reliable in this regime.

\subsection{Role of numerical noise}

It is important to mention at this point that the PIC runs in the high guide field regime are much more affected by numerical noise than in the low guide field regime. This is because, in our setup, $B_{\infty y}$ decreases for increasing $b_{g}$. Then, for large guide fields the signal-to-noise ratio can be very low, as can be seen in the extreme case $b_{g}=50$ in Fig. 9.6 (e) and Fig. 9.7(e) (even with the extended temporal average). Therefore, although in principle there is no limitation due to numerical constrains on the electron gyromotion for even higher guide fields (in our setup, $\rho_{e}$ and $\Omega_{c e}$ are constant for different guide fields), the PIC results in this regime will not be reliable due to this unfortunate fact.

The numerical noise affects more some quantities over other ones, with different physical effects. One of the most notorious is the in-plane electric field in the electron frame of reference, and therefore the charge separation, an non-ideal effect absent in the GK approach (see Sec. 9.7.2). In order to prove this, in Fig. 9.32 we display some "typical" maximum values of the charge separation and in-plane electric fields on dependence on the guide field. We also plotted 3 times the standard deviation of these fields away from the CS in order to have an estimation on the noise level. 

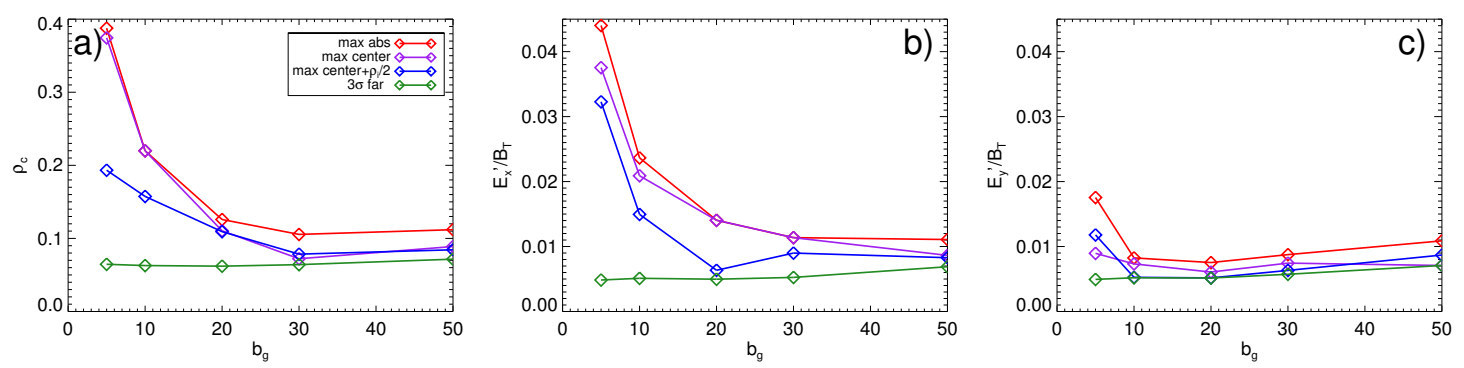

Figure 9.32: Typical maximum values vs guide field $b_{g}$ of the different fields associated with charge separation, at reconnection peak time $t=50 \tau_{A}$. a) $\rho_{c} b$ ) $E_{x}^{\prime} / B_{T}$ (in the electron frame of reference), c) $E_{y}^{\prime} / B_{T}$ (in the electron frame of reference). Red curve "max abs" display the absolute maximum around the plotted region. Purple curve max center" is the maximum along the center. Blue curve "max center $+\rho_{i} / 2$ " is the maximum of a line shifted in $\rho_{i} / 2$ with respect to the center. and 3 times the standard deviation $\sigma$ of a line along $y$ away from the $C S$.

In Fig. 9.32 we can see that the noise level for $\rho_{c}$ and $E_{x}^{\prime}$ is reached approximately for $b_{g} \gtrsim 30$. Since $E_{y}$ is weaker initially, it reaches noise level for weaker guide fields $b_{g} \gtrsim 10,20$. Therefore, we can establish that quasineutrality is reached for guide fields $b_{g} \gtrsim 30$, and therefore a comparison with gyrokinetic should be more reliable in this regime.

The numerical noise is manifested through numerical heating, therefore affecting directly the measured temperatures. Since those are a second order momenta of the distribution function, they are also more affected due to fluctuations resulting from the reduced macroparticle number. Similar as for the electric field, we show the influence of the guide field on Fig. 9.33. We can see that any attempt to extract meaningful information from $T_{i, \|}$ or $\delta T_{e, \perp}$ is not reliably at all for guide fields $b_{g}>20$, since the noise level is more or less at the same level as the global maxim of these quantities. This requirement is less restrictive for the other components of the temperature $T_{e, \|}$ or $\delta T_{i, \perp}$, they can still be measured reliable even for higher guide fields: their fluctuating values are higher an so the signal to noise ratio 

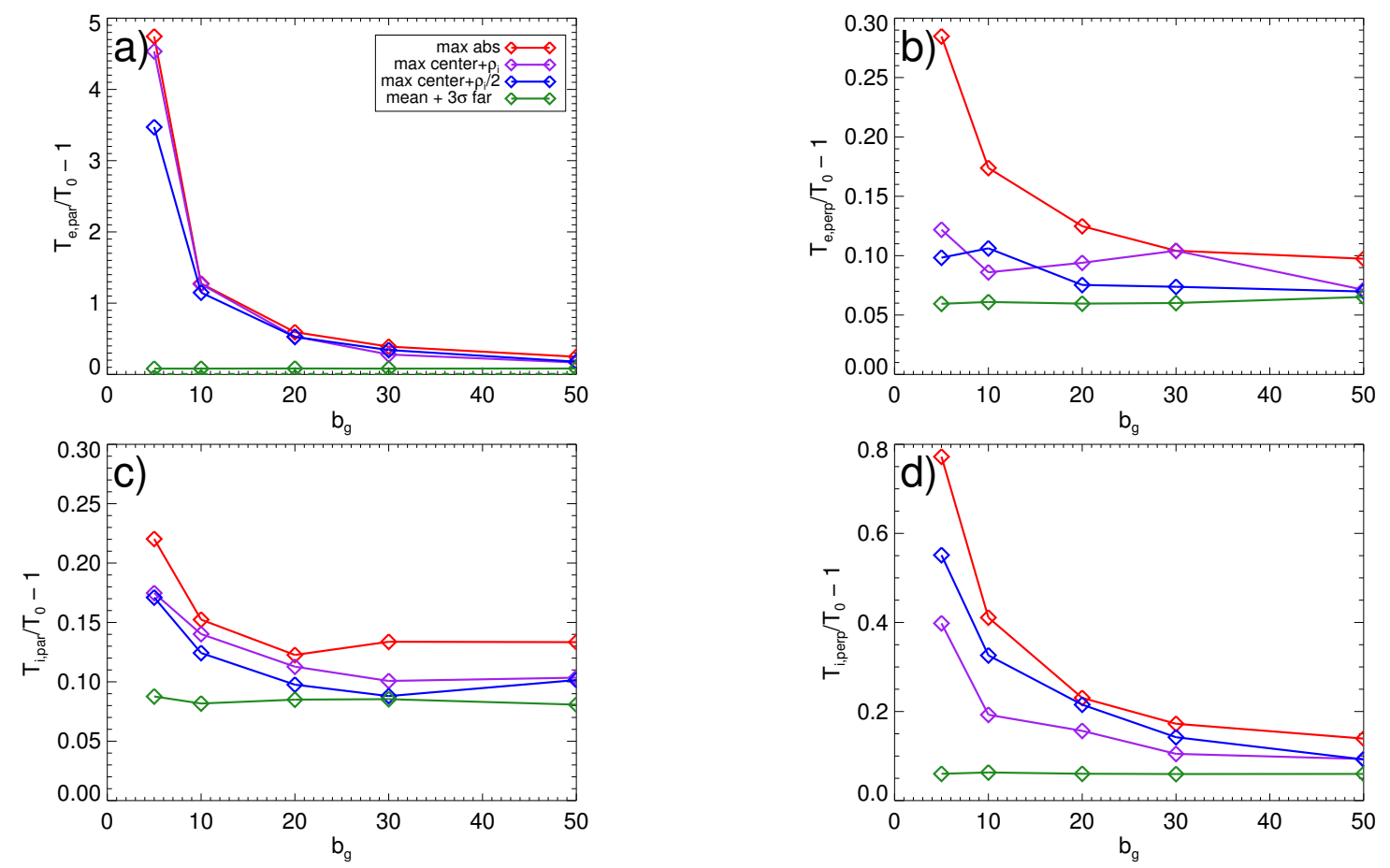

Figure 9.33: Typical maximum values $v$ s guide field $b_{g}$ of the components of the temperatures, at reconnection peak time $t=50 \tau_{A}$. a) $\left.\left.\left.\Gamma \delta T_{e, \|}, b\right) \Gamma \delta T_{e, \perp}, c\right) \Gamma \delta T_{i, \|}, d\right) \Gamma \delta T_{i, \perp}$. Red curve "max abs" display the absolute maximum around the plotted region. Purple curve max center" is the maximum along the center. Blue curve "max center $+\rho_{i} / 2$ " is the maximum of a line shifted in $\rho_{i} / 2$ with respect to the center. and 3 times the standard deviation $\sigma$ of a line along y away from the CS.

On the other hand, there is in general a higher level of numerical noise, and associated numerical heating, in the high beta case compared to the low beta one for the PIC runs. It becomes increasingly important for higher guide fields, reducing even faster the signal-tonoise ratio. This is, in part, responsible for the monotonically increasing thermal pressure fluctuations in Fig. 9.28(a) for later times, especially in the case $b_{g}=10$. This observation was already pointed out in the original work by TenBarge et al. (2014), being a well known consequence of the enhanced numerical collisions in weakly magnetized environments simulated by PIC codes, or equivalently, in high beta plasmas (Matsuda and Okuda 1975).

\subsection{Conclusions}

By means of PIC and gyrokinetic simulations, we have extended a previous study of magnetic reconnection in the limit of strong guide field (TenBarge et al. 2014) using an independent set of PIC and gyrokinetic codes. We established the limits of applicability of gyrokinetic simulations compared to PIC ones in the low guide field regime, and the physical reasons behind these differences. This is of critical importance for application to the solar coronal plasmas where the guide field is large but not too much. It is important to mention that most of the following conclusions are based in set of runs that keep the 
ion plasma beta constant in $\beta_{i}=0.01$ (a value also suitable for the Solar corona).

First, we identified the limits regarding the linear scaling on the guide field of both thermal $\left(\delta P_{t h}\right)$ and magnetic $\left(\delta B_{z}\right)$ fluctuations in the PIC runs. The linear scaling is based on a standard two-fluid theory (Rogers et al. 2001) strictly satisfied by the GK runs and asymptotically by the PIC plasma model in the limit $b_{g} \rightarrow \infty$. We confirmed that the linear scaling and therefore convergence between PIC and GK models works well for guide field as low as $b_{g}=5$ or $b_{g}=10$, but different from the previous work by TenBarge et al. (2014), we found that is only valid in a very limited region close to the X point. Large deviations between both plasma models take place in the secondary magnetic islands, formed around the reconnection peak time. This implies that the agreement between PIC and GK simulations of magnetic reconnection is better during the linear phase but not reliable afterwards. This fact also limits the validity of the GK approach when comparing to PIC simulations to much larger guide fields $b_{g} \gtrsim 30$ in locations close to or inside of the secondary magnetic islands.

The main reason of that discrepancy is the generation of a core-magnetic field (out-ofplane component) inside of the magnetic islands in the PIC low guide field regime $b_{g} \lesssim 30$. This not compensated by a corresponding drop in the thermal pressure, being therefore a deviation from the pressure equilibrium condition allowed only in the PIC plasma model but not in GK (that keeps that condition to machine precision). The physical mechanism that generates this core-magnetic field is due to the in-plane shear flow present in the PIC force free CS initialization, although negligible in the high guide field regime. Correspondingly, this shear flow is absent in the GK force free CS initialization. The effects of that shear flow, stronger in the PIC low guide field regime are: 1) asymmetric separatrices, 2 ) reduction in reconnection rates 3 ) different flow pattern inside of the secondary magnetic islands and 4) generation of vortical in-plane Hall currents due to the wrapping of magnetic field lines around the secondary magnetic islands. The latter effect produces the core-magnetic field, evidenced also due to a transfer of energy between particles to the fields (dynamo effect $\vec{J} \cdot \vec{E}<0$ ). We could also notice the generation of an out-of-plane magnetic field in the periodic $y$ boundaries, due to the colliding outflows coming from the main reconnection $\mathrm{X}$ point, non negligible even for stronger guide fields.

In the second part of this chapter, from Sec. 9.6 onward, we analyzed mostly how microscopic processes and instabilities develop in the large guide field limit in the fullykinetic model via PIC codes. Most of these processes are beyond the scope of the gyrokinetic approach because they involve high frequency waves and strong deviations from Maxwellian distributions function. And it is precisely in this kind of mechanisms where the conversion of energy that affects large scale phenomena related with magnetic reconnection, such as those observed in the Solar corona, matters the most.

When analyzing the thermal behaviour of the simulations, we found a very strong parallel electron heating $T_{e, \|}$ acting from the very beginning, and to a lesser degree, perpendicular ion heating $T_{i, \perp}$. This behaviour is reproduced qualitatively well by the GK code, but the PIC results in the low guide field regime reveal a more active heating in the pair of separatrices with enhanced density than the GK results, even after they are scaled on the guide field. Other components are affected only after the reconnection peak time, where secondary magnetic islands start to appear. We could relate qualitatively this behavior with the double adiabatic or CGL equations, indicating a conservation of the magnetic moment $\mu$ to a first approximation in PIC codes. On the other hand, this quantity is strictly 
conserved in the GK approach.

The strong differences in the thermal behavior for the PIC codes in the low guide field regime gave us a hint to investigate the non thermal features. First, we identified all the regions where the tensor pressure is non-gyrotropic on dependence on the guide field, finding that for low $b_{g}$, there is an extended region around both separatrices and outflow region where these quasi-viscosity is important, and not only to sustain the reconnected electric field as in the standard two-fluid theory of Hall reconnection. Then, by means of an analysis of the kurtosis of the distribution function (4th order momenta), we could identify very precisely that the points with the largest deviations between PIC and GK results are where there are more presence of accelerated particles along the out-of-plane direction, as evidenced for the hard tails in their energy spectra. We could validate the previous results given by the kurtosis via a full analysis of the particle distribution function. This al so allowed us to find strong beam formation in the low guide field regime along the $z$ direction, not only close to the diffusion region and separatrices as it is known from previous works, but also in the secondary magnetic islands and the outflow region. However, the free energy in these beams cannot be (directly) released due to $2 \mathrm{D}$ dimensionality constraints.

Then, we compared two different measures of dissipation. We found than for positive values $(\vec{J} \cdot \vec{E}>0$, implying transfer of energy from fields to particles) they do not necessarily correlate with the expected locations where there should be more energetic phenomena: beam formation or accelerated particles. Instead, locations where these measures predict transfer of energy from the particles to the fields $(\vec{J} \cdot \vec{E}>0)$ is where we can see the largest deviations from the thermal equilibrium.

An additional evidence for the differences between PIC and GK results in the low guide field regime is due to the charge separation. The GK runs shown here are strictly quasineutral, while the PIC codes allow arbitrary deviations even slightly below the Debye length, if sufficiently resolved (as in our case). In the PIC low guide field regime, and due to the small mass ratio and frequency ratio $\omega_{p e} / \Omega_{c e}$, there are strong deviations from quasineutrality leading to in-plane electric fields in the electron frame of reference (i.e.: non ideal electric fields). Although there is some correlation with some of the other already mentioned phenomena, more work is needed in order to distinguish its physical consequences from the numerical ones and thus to establish a causal relation.

We also proved that an important source of differences between the predicted behavior of magnetic reconnection is the ratio of electron outflow to ion thermal speed, proportional to $V_{A} / v_{t h, i}$. This ratio becomes increasingly higher in the PIC low guide field regime due to generation of supersonic outflows $\left(V_{A} / v_{t h, i} \sim 1\right.$ for $\left.b_{g} \lesssim 10\right)$, but this is not allowed in the GK approach. Instead, it is constrained to values $V_{A} / v_{t h, i} \lesssim 1$ : otherwise it will break the GK assumption of perpendicular drift approximation: it allows particles move out of their orbits as given by the cross-field drifts. Since it is known that relative electron-ion streaming is a important source of free energy for microinstabilities, it is natural to expect that when the aforementioned ratio takes higher values in the PIC runs, the CS can become unstable for this plasma model but not in the GK simulations. Indeed, we found evidence (mostly via an analysis of thresholds) of several cross-field instabilities allowed to grow in our reduced 2D geometry due to the presence of guide field. The first one is the modified two streaming (MTSI, also known as kinetic cross-field streaming KCSI) instability, driven by the pure electron-ion relative streaming or, equivalently, the $\vec{E} \times \vec{B}$ drift. 
Second, the lower hybrid drift instability (LHDI), driven by pressure gradients or crossfields diamagnetic drifts. And third, an electron-ion hybrid (EIH) instability, a version of the kinetic Kelvin-Helmholtz instability driven by gradients in the shear flow. All these instabilities are operating in the same spatial locations (enhanced density separatrix arm), have typical frequencies and wavelengths beyond the allowed ones by GK $\left(\Omega_{l h}<\omega<\Omega_{c e}\right.$ and $\rho_{e}<\lambda<\rho_{i}$ ), in such a way that their effects are always added.

Finally, two important remarks about the consequences of the aforementioned crossfield streaming instabilities. First, note that a non-linear saturation of these instabilities leading to turbulence would require times longer than the allowed in our simulations due to periodic boundary conditions and small simulation box. And second, note that the same setup in a full 3D geometry would allow additional decay channels for these instabilities, producing more turbulence and many other interesting effects worth to be investigated for applications to realistic scenarios, such as Solar coronal plasmas.

On the other hand, we also analyzed briefly the effects of a high plasma beta $\beta_{i}=1.0$. Although not directly applied for the Solar corona, it is interesting to know the effects that this parameter has on the aforementioned phenomena. The general characteristics of most of the analyzed phenomena remain similar, but the convergence between GK and PIC plasma models is less restrictive. Even in the regions close to the secondary magnetic islands, where additional effects take place only in the PIC runs (core-magnetic field generation), the agreement in thermal and magnetic fluctuations only require guide fields as low as $b_{g} \gtrsim 3$. There are several related reasons for that, allowing us to determine the optimum parameter range for a reliable comparison of PIC and GK simulations of magnetic reconnection. First, the fluctuation level of both $\delta P_{t h}$ and $\delta B_{z}$ scales as $1 / \sqrt{\beta_{i}}$ and thus the small $\epsilon$ approximation of GK is better satisfied for higher $\beta$ plasmas and a better agreement with PIC is expected. The drawback in the latter is that the PIC runs suffer of enhanced numerical heating in this parameter range. Second, a better comparison is obtained for parameters with $V_{A} / v_{t h, i} \ll 1$ (related with the $\epsilon$ in the GK code). In the PIC runs this is for 1) making smaller the shear flow strength that generates the core-magnetic field and 2) to quench cross-field micro-instabilities such as MTSI, as well as the gradient driven LHDI and shear flow driven EIH, generating heating and non-thermal features only captured by the PIC approach. And third, the agreement between both plasma model work better in high $\beta$ plasmas since the reconnection rates are reduced. In the GK model this implies less energetic outflows that might potentially break the perpendicular drift approximation. And in the PIC plasma model, this leads to less open separatrices and thus more elongated magnetic islands where the generation of core-magnetic field is less efficient, allowing a more reliable comparison with the GK approach. Thus, it is necessary a careful choice of these parameters in order to guarantee an accurate physical description of magnetic reconnection in the GK approach when compared to the kinetic plasma description as modeled with PIC codes.

Finally, and even with all these limitations of the GK model that we found, it it important to remark their computational advantages. GK simulations of magnetic reconnection perform remarkably well and much faster in the high guide field regime in comparison with the PIC ones. For the codes and the low beta range of parameters showed in this study, the GK GENE code required $10^{3}$ less CPU-core hours than the corresponding run with the PIC ACRONYM code for a guide field $b_{g}=50$. And due to the all previous considerations, for these cases there are very few differences in the phenomenology of 
magnetic reconnection in both plasma models, and therefore the GK approach can accurately reproduce all the physics involved in this process (in both X points and secondary magnetic islands). Moreover, for such high guide fields the PIC runs also have necessarily very small noise to signal ratio, making the extraction of useful physical information somehow unreliable. 



\section{Conclusions and Outlook}

\subsection{Conclusions}

The goal of this investigation was to analyze instabilities of collisionless current sheets (CS) leading to and also formed by magnetic reconnection, as it can be expected in the Solar corona. Since the Solar coronal plasma is largely collisionless, it must be analyzed kinetically. We used fully kinetic Particle-in-Cell (PIC) codes to simulate the nonlinear dynamics of the coronal plasma phenomena. In addition to collisionless CS properties and magnetic reconnection in other plasmas, for Solar conditions it is important to take into account the presence of finite magnetic guide fields in the current direction. While the cases of small and infinitely large guide field are more or less well understood, the cases of moderately large guide fields, typical of the collisionless plasma conditions of the Solar corona, are not well understood yet. In order to validate our simulation approach, we analyzed the limiting cases of zero (antiparallel configuration) and infinitely large guide fields (gyrokinetic approximation).

In order to reach our goals, we first gradually introduced the concepts and methods to put our work in the context of existing theories in the introductory chapters 1-6. In Chapter 1, we discussed the importance of current sheets, their instabilities and magnetic reconnection in the solar corona. In addition, we gave an overview of our aims and introduced the structure of this thesis work. In Chapter 2, we briefly reviewed the necessary plasma physics background. In Chapter 3, we described the most important kinetic CS equilibria forming the basis of all the following discussions. Then, in Chapter 4, we reviewed steady state models of fully developed magnetic reconnection. In Chapter 5, we briefly described and analyzed the most important instabilities of collisionless CS that we found in our work. The last introductory Chapter 6 is devoted to the main numerical simulation method used in this thesis: fully kinetic PiC codes, with an emphasis on the methods used by the ACRONYM code.

In Chapter 7 , describing the first part of our results, we analyzed spontaneous instabilities of Harris CS in an antiparallel configuration with no guide field taken into account, with an emphasis on numerically-induced effects. The purpose was testing the numerical requirements of the code to correctly simulate collisionless plasmas (with higher, more realistic mass ratios). We showed that simulations with these more realistic parameters are often prone to pitfalls of the underlying numerical algorithms.

Numerically-induced instabilities detected in Chapter 7 are due to an anisotropic heating and an often hardly conserved total plasma energy. This may lead, e.g., to a bifurcation of the CS (reduction in the out-of-plane current density $J_{z}$ ), inhibiting the tearing instability. We found that this artificial process, generated at micro-scales by the enhanced PIC 
shot noise mimic of collisional physical phenomena, can be avoided by choosing a higher order interpolation scheme (shape function) in the code. In particular, the advantage of a second order scheme (TSC) over the commonly used linear scheme (CIC) turned out to be essential and computationally much more efficient than just increasing the number of macroparticles per cell as usually done. This is especially important for simulations with larger (more realistic) mass ratios and for PIC runs over long times, probably being a key factor in the design of the upcoming next-generation large scale PIC simulations.

In Chapter 7, we also found that for high enough anisotropically distributed particles the Harris-type CS bifurcates. This is due to the numerical scattering close to the center of the CS. Bifurcation weakens with reduced scattering generated, e.g, by numerical noise. It is not negligible for macroparticle numbers commonly used in numerical simulations. We found good agreement between the levels of electron temperature anisotropy required for the appearance of bifurcation and the predictions for tearing stabilization, comparing them with the predictions of the anisotropic tearing instability.

In Chapter 7 we also investigated the relation between bifurcation and other temperature driven instabilities in cases with initially imposed anisotropy. The region close to the unmagnetized center of a non guide field Harris CS can be unstable, e.g., to the Weibel and mirror instabilities. We found evidence of a Weibel instability in the generation of filamented structures in the generated out-of-plane magnetic field, the correct growth rates and threshold conditions. The magnetic fields generated by the Weibel instability provided the seed that can activate the (magnetized) mirror instability, releasing very quickly the initially imposed anisotropy in the form of waves, turbulence and anisotropic electron heating, leading finally to a bifurcation of the Harris CS.

In Chapter 8 and Chapter 9 , we reported our results about the investigation of the influence of a magnetic guide field in the current direction on the CS stability and magnetic reconnection. This is important for understanding the Solar corona, since magnetic fields generated the photosphere permeates the corona, causing the formation of current sheets in complex topologies prone to instabilities and energy conversion by magnetic reconnection.

In particular, in the second part of our results in Chapter 8 , we investigated the spontaneous tearing instability of and reconnection through Harris CS with moderately large guide fields $\left(b_{g} \lesssim 7\right)$. We found numerically-induced bifurcation due to scattering if the electron Larmor radius is not well resolved by the grid cell size. This imposes an additional constraint on the simulations parameters above the usual stability conditions of PIC codes. This has not been analyzed before and it will become critical when in the future more realistic parameters will become used. Only when this effect is taken into account, we found a decrease of the tearing mode growth rates and also a delay of the onset of reconnection with increasing guide field strengths.

In Chapter 8 we further analyzed the mechanisms supporting the reconnected electric field via the two-fluid Ohm's law. For that sake we first derived a proper treatment of spatial and temporal derivatives and averages. We implemented them in both running code and in the post-processing diagnostics. As a result, we confirmed previous theoretical predictions regarding the relative importance of the non-gyrotropic pressure and electron inertia contributions to the violations of the frozen-in condition. However, we additionally found indications of the presence of additional unbalanced terms in the mean-fieldOhm's law. We conjectured that they are due to the action of electromagnetic fluctuations 
(turbulence), which becomes increasingly important for higher guide fields (or smaller $\beta$ plasmas). They are more influential in the outer boundary of magnetic islands, away from the diffusion region of reconnection. They are strongly correlated with the deceleration of the electron bulk drift speed $\left(\partial V_{e, z} / \partial t<0\right)$, usually associated with anomalous resistivity. Moreover, in these same locations we found signatures of 1) cross-field streaming instabilities due to counterstreaming electron flows and 2) pressure gradient driven instabilities, which heat the electrons in the out-of-plane (guide field) direction, generate non-thermal tails of the electron distribution function also in the out-of-plane direction and turbulent wave activity in these regions beyond their saturation time.

In the third part of our results Chapter 9 , we reported the results of our investigations of collisionless CS in the large guide field limit $\left(5 \lesssim b_{g} \lesssim 50\right)$. We compared our PIC simulation results with those obtained by a gyrokinetic (GK) code (GENE). We investigated the properties of the fully-developed stage of magnetic reconnection, bypassing the slow linear growth of the tearing mode instability by applying an initial perturbation. The large guide field limit is important for the analysis of the low $\beta$ plasmas in the solar corona. Also, for better applicability to the Solar corona, we considered as initial equilibrium a force free CS. We established the limits of applicability of the gyrokinetic approach, derived for the strong guide field limit $b_{g} \rightarrow \infty$, in describing the realistic regime of finite (low) guide fields, well described by PIC codes. The study benchmarks that GK code simulations can help to save computational resources by using them instead of the more expensive PIC ones in the parameter regime that we identified.

We found that both plasma theories: fully kinetic and GK, develop magnetic reconnection with similar features inside of secondary magnetic islands, only if strong enough guide fields are imposed in the PIC code $\left(b_{g}>30\right.$ for $\left.\beta_{i}=0.01\right)$. In the non-ideal region close to the $\mathrm{X}$ point, the convergence between both models work better in the sense that lower PIC guide fields $\left(b_{g}>5\right.$ for $\left.\beta_{i}=0.01\right)$ are required, in agreement with previous studies. PIC code low guide field simulations reveal a larger magnetic pressure inside of secondary magnetic islands. This is not compensated by a corresponding decrease in thermal pressure taking into account the ideal pressure equilibrium condition. GK simulations keep this condition to machine precision by construction, while fully-kinetic PIC code simulations allow arbitrary deviations. This turned out to be the source of differences between the results of the two plasma models: GK and the more accurate PIC. The physical mechanism generating additional core-magnetic field in the secondary magnetic islands is a shear flow instability. It was discovered in the PIC code simulation for the low guide field regime. It is negligible in the high $b_{g}$ limit and absent by construction in the GK initialization. Due to a mechanism similar to the Kelvin-Helmholtz instability, this shear flow instability decouples electrons from ions via a Hall effect, generating in-plane currents and wrapping up the magnetic field in vortical patterns around the secondary magnetic islands. All this is related with a dynamo effect $(\vec{J} \cdot \vec{E}<0)$, decreasing the efficiency of magnetic reconnection.

In Chapter 9 we also detected another caveat of the GK plasma description. The ratio of the electron outflow speed to the ion thermal speed, proportional to $V_{A} / v_{t h, i}$, is higher for the PIC code low guide field regime, reaching values close to 1 for $b_{g} \lesssim 10$. This violates the GK ordering, by allowing particles moving out of their orbits given by their cross-field drifts. This behaviour was disclosed by our PIC code kinetic investigations. In addition, the initial shear flow also produces asymmetric separatrices and other related effects when 
the ratio $V_{A} / v_{t h, i}$ is higher. Moreover, PIC simulation in the low guide field regime allowed us to find evidence of a cross-field streaming instability due to the high relative drift speed between electron and ions when $V_{A} / v_{t h, i}>1$. This instability is associated with shear flows and pressure gradients. It produces electron heating, non thermal features such as beam formation and electron acceleration especially in the asymmetric pair of the separatrices and at the edge of the magnetic islands. And due to the (short) wavelengths and (high) frequencies involved, this instability is ordered out in the GK approach and can only be captured with a fully-kinetic PIC plasma model.

Finally, in Chapter 9 we determined the plasma $\beta$ dependence of GK simulations toward the more correct PIC results. Higher $\beta$ require lower guide fields to obtain reduced reconnection rates. In this limit, it is more convenient to use gyrokinetic instead of PIC codes simulations and still having reliable results.

\subsection{Outlook}

There are many interesting possible extensions of the work developed in this thesis. The logical next step is extending the CS PIC simulations to full 3D geometries. Although the computations are necessarily more expensive, luckily many of the undesired numerical effects, such as numerical collisions, noise and heating, in addition to braking of accelerated particles, are reduced in 3D geometries. Preliminary results, not reproduced here, already showed exciting new physical phenomena taking place in these configurations, due to the possibility of current aligned microinstabilities such as Buneman instability. They are especially important in guide field configurations and for thinner CS, and we already detected microturbulence and strongly non-thermal features in the particle distribution function. In larger 3D simulations is expected that the tearing magnetic island become flux ropes, coupling with other instabilities and generating very thin electron scale current layers where turbulence is the dominant dissipation mechanism (see Treumann and Baumjohann (2013b) and references therein). In order to observe such processes, we have to extend the simulation box domain and/or changing the boundary conditions from periodic to open ones in the downstream direction, in order to allow a regime of fully developed magnetic reconnection for longer times. This will also allow us to observe efficient mechanisms of particle acceleration in these configurations.

The methods developed for the time-spatial averaging in Chapter 8 will also allow us to calculate properly the instability and transport properties of CS and reconnection in fully 3D geometry of collisionless plasmas with finite guide fields, typically found in the Solar corona. In addition, extensions of the aforementioned statistical averaging methods, possibly implemented in running time during a PIC simulation, can help us to identify more precisely and effectively the different instabilities in magnetic reconnection via the analysis of the wave activity. All these methods will improve the calculation of transport properties, which influence not only the microscale processes, but also the macroscopic dynamics of the solar corona and other collisionless plasmas. Thus, we will be bridging the gap between the microphysics of the diffusion region and the large scales effects characteristics of the release of energy in magnetic reconnection. 


\section{A Terminology, definitions and relations}

\section{A.1 Plasma parameters and abbreviations}

\section{A.1.1 Abbreviations}

1. CS: current sheet

2. PIC: Particle-in-cell (code)

3. GK: gyrokinetic

4. ppc: macroparticles per cell

\section{A.1.2 List of symbols and definitions}

We will be using MKS units everywhere (although the ACRONYM PIC code still uses CGS units).

Unless stated otherwise, we use the Einstein convention (implicit sum over repeated indices) in all the equations involving tensors, e.g.: $V_{j} P_{i j}=\sum_{j} V_{j} P_{i j}$.

- I, field aligned directions are with respect to the magnetic field $\vec{B}$ (unless in the context of temperature anisotropies in unmagnetized scenarios: Sec.(7.3.2).

- $\perp$, transverse, cross-field aligned directions are with respect to the magnetic field $\vec{B}$ (unless in the context of temperature anisotropies in unmagnetized scenarios: Sec. 7.3.2).

- ()$_{\alpha}$ : specie $\alpha=e, i$ of the plasma. Omitted when the meaning is clear.

- $\varepsilon_{i j k}$. Levi-Civita symbol (for representing vector cross product $\times$ )

- $C=\sqrt{C_{x}^{2}+C_{y}^{2}+C_{z}^{2}}$ for any vector quantity $\vec{C}=C_{x} \hat{x}+C_{y} \hat{y}+C_{z} \hat{z}$, unless stated otherwise.

- $\vec{B}$ : Magnetic field

- $\vec{E}$ : Electric field 
- $\overrightarrow{A:}$ Vector potential

- $\psi$ : Reconnected flux

- $d \psi / d t \propto E_{z}$ : Reconnection rate

- L: CS Halfwidth

- $\Delta_{N S}=\sqrt{2 \rho_{e} L}$ : Electron singular layer thickness

- $L_{x / y / z}$ : Simulation box size in $x / y / z$ direction

- $N_{x / y / z}$. Grid points in $x / y / z$ direction

- $\omega_{p e}=\sqrt{n_{e} e^{2} /\left(\epsilon_{0} m_{e}\right)}$ : (electron) plasma frequency

- $\omega_{p i}=\sqrt{n_{i} e^{2} /\left(\epsilon_{0} m_{i}\right)}$ : ion plasma frequency

- $\lambda_{D e}=v_{t h, e} / \omega_{p e}=\sqrt{\epsilon_{0} k_{B} T_{e} /\left(n_{0} e^{2}\right)}$ : Debye length

- $\Lambda=n \lambda_{D e}^{3}$ : Plasma parameter.

- $M_{p}=N_{\text {physical }} / N_{\text {numerical }}$ : Macrofactor.

- $v_{t h,\{e, i\}}=\sqrt{k_{B} T_{\{e, i\}} / m_{\{e, i\}}}:$ Electron/ion thermal speed

- $c_{s}=\sqrt{\gamma P / \rho}=\sqrt{\gamma k_{B} T / m_{i}}$ : sound speed

- $c$ : speed of light

- $T_{\{e, i\}}$ : Electron/ion temperature

- $d_{\{e, i\}}=c / \omega_{\{p e, p i\}}:$ Electron/ion skin depth or inertial length

- $\Omega_{c\{e, i\}}=e B / m_{e, i}$ : Electron/ion Larmor frequency or gyrofrequency

- $\rho_{\{e, i\}}=v_{t h,\{e, i\}} / \Omega_{c\{e, i\}}=m_{\{e, i\}} v_{t h,\{e, i\}} /\left(q_{\{e, i\}} B\right)$ : Electron/ion Larmor radius

- $\Omega_{l h}=\omega_{p i} / \sqrt{1+\omega_{p e}^{2} / \Omega_{c e}^{2}}$ Lower hybrid frequency

- $V_{A(e)}=B / \sqrt{\mu_{0} n_{i(e)} m_{i(e)}}$ : (electron) Alfvén speed

- $\tau_{A}=L / V_{A}:$ Alfvén time

- $v_{c}=v_{t h} / \lambda_{c}$ : collision frequency, where $\lambda_{c}$ is the collisional mean free path.

- $\eta=m_{e} v_{c} /\left(n e^{2}\right)$ : resistivity

- $R_{M}=\left(\mu_{0} / \eta\right) L_{B} V$ Magnetic Reynolds number (ratio between convective to diffusive term in the induction equation Eq. (2.45)).$L_{B}$ is the length scale of variation of $\vec{B}$ and $V$ a characteristic fluid velocity.

- $R_{M}=\left(\mu_{0} / \eta\right) L_{B} V_{A}$ : Lundquist number (same as $R_{M}$ with $V=V_{A}$ ) 
- $K=\lambda / L$ : Knudsen number. $L$ is a characteristic length scale.

- $\mu=m v_{\perp}^{2} / 2 B$ Magnetic moment

- $\gamma$ : Growth rates

- $f(\vec{x}, \vec{v})$ distribution function

- $(\vec{x}, \vec{v}):$ spatial/velocity coordinates (6D phase space)

- $n_{\{e, i\}}$ : Electron/ion number density

- $m_{\{e, i\}}:$ Electron/ion mass

- $q_{\{e, i\}}=\mp:$ Electron/ion charge

- $\rho_{c,\{e, i\}}=q_{\{e, i\}} n_{\{e, i\}}:$ Electron/ion charge density

- $\vec{V}_{\{e, i\}}$ : Electron/ion bulk flow velocity

- $\vec{J}_{\{e, i\}}:$ Electron/ion current density

- $P_{i j}$ : Pressure tensor

- $p=P_{j j} / 3$, given $P_{i j}=p \delta_{i j}$ : Scalar pressure

- $Q_{i j k}$ : Heat tensor

- $q_{i}=Q_{i j j}$ Heat flux vector

- $K_{i}: 1 \mathrm{D}$ (excess) Kurtosis along $i$ direction

- $\beta_{\{e, i\}}=2 \mu_{0} n_{\{e, i\}} k_{B} T_{\{e, i\}} / B^{2}:$ electron/ion plasma beta

- $B_{\infty y}$ : asymptotic reconnected magnetic field

- $B_{G}$ : magnetic guide field strength (along $\hat{z}$ direction)

- $b_{g}=B_{G} / B_{\infty y}$ : relative guide field

- $N_{\text {total }}$ : Total number of macroparticles

\section{A.2 Geometry}

Unless stated otherwise, the geometry of our setup for both single and double CS simulations is shown in Fig. A.1. 


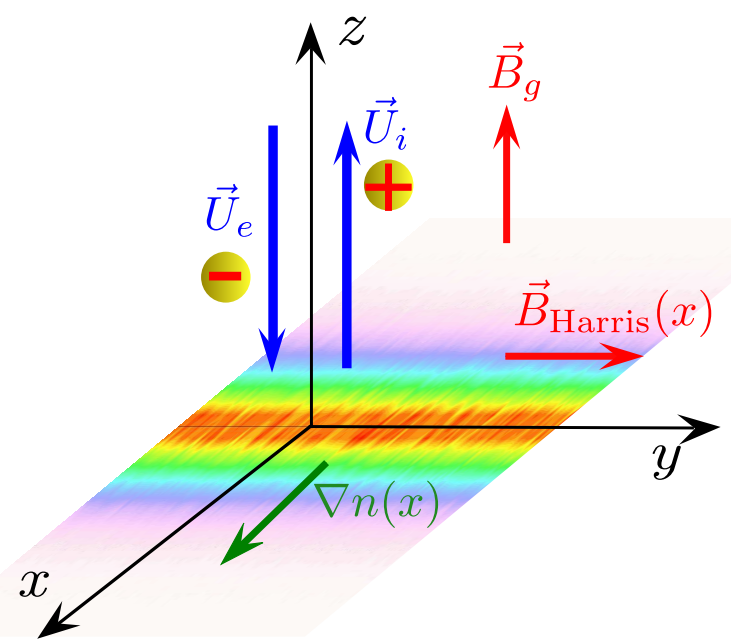

Figure A.1: Schematics of the geometry of the simulation setup for single Harris CS. The magnetic field $\vec{B}$ is in the y direction on dependence on the $x$ coordinate. Correspondingly, the density $n$ and current density $\vec{J}$ vary along the $x$ direction. The $2 D$ simulations are carried out in the $x-y$ plane (the reconnection plane). The CS is sustained by counterstreaming electrons and ions that produce a current density $\vec{J}$ in the out-of-plane direction z. The magnetic guide field is also in the out-of-plane direction $z$. For force cases the setup is practically identical, although the ions are not drifting $U_{i}=0$, there is no density gradient $\nabla n=0$ and the out of plane guide field is dependent on $x: B_{g}=B_{G}(x)$.

Guide field reconnection is also called component reconnection. When there is no guide field, it is also called antiparallel reconnection.

\section{A.3 Relation between quantities and parameter setup}

For the tuning and setup of the parameters in the initial equilibria in our simulations, it is very convenient express them in terms of a few characteristic dimensionless ratios. They are not independent, however. Several relations exist among them, some of them general and other ones specific of the initial equilibria. They show that these parameters cannot be chosen independently. We are going to list the most used ones in the following very handy list:

\section{A.3.1 General relations between plasma parameters}

$\frac{d_{i}}{\lambda_{D e}}=\sqrt{\frac{m_{i}}{m_{e}}} \frac{c}{v_{t h, e}}, \quad \frac{d_{e}}{\lambda_{D e}}=\frac{c}{v_{t h, e}}=\frac{\omega_{p e}^{-1}}{\lambda_{D e} / c}, \quad \frac{\rho_{e}}{\lambda_{d e}}=\frac{\omega_{p e}}{\Omega_{c e}}, \quad d_{i}=\frac{V_{A}}{\Omega_{c i}}, \quad d_{e}=\frac{V_{A e}}{\Omega_{c e}}$

$\frac{v_{t h, i}}{V_{A}}=\frac{\rho_{i}}{d_{i}}, \quad \frac{\Omega_{c i}^{-1}}{\omega_{p e}^{-1}}=\frac{\omega_{p e}}{\Omega_{c e}} \frac{m_{i}}{m_{e}} \quad \frac{V_{A}}{c}=\sqrt{\frac{m_{e}}{m_{i}}} \frac{\Omega_{c e}}{\omega_{p e}}=\frac{\Omega_{c i}}{\omega_{p i}} \quad \frac{V_{A e}}{c}=\frac{\Omega_{c e}}{\omega_{p e}}$

$\sqrt{\beta_{i}}=\sqrt{2} \frac{v_{t h, i}}{V_{A}}=\sqrt{2} \frac{\rho_{i}}{d_{i}}=\frac{\rho_{e}}{d_{e}} \sqrt{\frac{2 T_{i}}{T_{e}}}=\frac{\omega_{p e}}{\Omega_{c e}} \frac{v_{t h, e}}{c} \sqrt{\frac{2 T_{i}}{T_{e}}}$ 
General relations for any CS equilibria (from Ampère's law):

$$
\left|U_{e}-U_{i}\right|=\frac{B_{\infty y}}{\mu_{0} e n_{0} L}=\frac{c}{\frac{L}{d_{i}}\left(\omega_{p e} / \Omega_{c e}\right) \sqrt{m_{i} / m_{e}}} \quad \Leftrightarrow \quad \frac{\left|U_{e}-U_{i}\right|}{v_{t h, i}}=\frac{\rho_{i}}{L} \frac{\left(c / v_{t h, e}\right)^{2}}{\left(\omega_{p e} / \Omega_{c e}\right)^{2}} \frac{T_{e}}{T_{i}}
$$

\section{A.3.2 Independent input parameters for a Force Free CS}

In this subsection we are going to show explicitly the order in which the independent input parameters of a force free CS (see Sec. 3.2.2.3) are used for the simulation setup in the ACRONYM code, in addition to give some other useful relations valid for this initialization.

All the previous and the following relations where it is involved implicitly the magnetic field, e.g.: $\Omega_{c e}$ and $V_{A}$, are calculated with respect to the in-plane component $B_{\infty y}$. This is different from the choice in Chapter 9 , where $\Omega_{c e}$ (but not $V_{A}$ ), is calculated with respect to the total magnetic field $\sqrt{B_{\infty y}^{2}+B_{g}^{2}}$. Then, in order to apply that convention to the following and previous list of relations, it is necessary to add a conversion factor in this way: $\Omega_{c e}=\Omega_{c e, \text { Btotal }} / \sqrt{1+b_{g}^{2}}$.

1. Mass ratio $m_{i} / m_{e}$

2. Temperature ratio. $T_{i} / T_{e}$

3. Electron thermal speed to light speed $v_{t h, e} / c$. It defines $T_{e}$. With 1) and 2), it defines $T_{i}$.

4. Physical scale of the system is fixed by specifying $\omega_{p e}$ or $n_{e}$.

5. Frequency ratio $\omega_{p e} / \Omega_{c e}$. The asymptotic magnetic field strength $B_{\infty y}$ is then calculated from this ratio and the physical value of $\omega_{p e}$ from step 4)

$$
B_{\infty y}=\frac{\Omega_{c e}}{\omega_{p e}} \frac{m_{e} \omega_{p e}}{e}=\frac{\Omega_{c e}}{\omega_{p e}} \sqrt{\frac{\epsilon_{0}}{n_{0} m_{e}}}
$$

6. Ratio halfwidth to ion skin depth $L / d_{i}$ (or $L / \rho_{i}$ ). The initial drift speed $U_{d} / c$ (or $\left.U_{d} / v_{t h, i}\right)$ is then calculated via Eq. A.4). Note that in this case $U_{D}=U_{e}$ and $U_{i}=0$ (stationary ions). Note also that requires the computation of $B_{\infty y}$ from step 5).

7. Relative guide field $b_{g}=B_{G} / B_{\infty}$. It requires the computation of $B_{\infty y}$ from step 5).

Additional relations:

$$
\tau_{A} \omega_{p e}=\frac{L \omega_{p e}}{V_{A}}=\frac{\omega_{p e}}{\Omega_{c e}} \frac{m_{i}}{m_{e}} \frac{L}{d_{i}}=\frac{\sqrt{T_{i} / T_{e}} m_{i} / m_{e}}{v_{t h, e} / c} \beta_{i} \frac{L}{\rho_{i}}, \quad \frac{\Omega_{c i}^{-1}}{\tau_{A}}=\frac{c / \omega_{p i}}{L}
$$




\section{A.3.3 Independent input parameters for a Harris CS}

In this subsection we are going to show explicitly the order in which the independent input parameters of a Harris CS (see Sec. 3.2.1) are used for the simulation setup in the ACRONYM code, in addition to give some other useful relations valid for this initialization.

Here, all the quantities involving density $n_{e}$ are calculated with the peak central value, while the quantities involving the magnetic field are calculated with respect to the asymptotic value $B_{\infty y}$

The pressure equilibrium condition Eq. (3.19) imposes a constrain between the electron thermal speed (related with thermal pressure) and the frequency ratio (related with magnetic pressure);

$$
\frac{\omega_{p e}}{\Omega_{c e}}=\frac{c}{v_{t h, e}} \sqrt{\frac{1}{2\left(1+\frac{T_{i}}{T_{e}}\right)}}
$$

Then, there is one less free parameter in comparison with the force free case in Appendix A.3.2 ( 3 and 5 gets combined), although we also have to specify additionally the background population: We also have the kinetic condition for absence of initial electric fields Eq. (3.22), relating electron $U_{e}$ and ion $U_{i}$ drifts.

1. Mass ratio $m_{i} / m_{e}$

2. Temperature ratio. $T_{i} / T_{e}$

3. Physical scale of the system is fixed by specifying $\omega_{p e}$ or $n_{e}$.

4. Electron thermal speed to light speed $v_{t h, e} / c$. It defines $T_{e}$. With 1) and 2), it defines $T_{i}$. From Eq. (A.7), it defines the frequency ratio $\omega_{p e} / \Omega_{c e}$. And from step 3), we can get the asymptotic magnetic field strength

$$
B_{\infty y}=\omega_{p e} \frac{m_{e}}{e} \frac{v_{t h, e}}{c} \sqrt{2\left(1+\frac{T_{i}}{T_{e}}\right)}
$$

5. Ratio halfwidth to ion skin depth $L / d_{i}$ (or $L / \rho_{i}$ ). The initial drift speed $U_{\{i, e\}} / c$ (or $\left.U_{\{i, e\}} / v_{t h, i}\right)$ is then calculated via Eq. A.4), expressing the frequency ratio in terms of the thermal speed via step 4). Finally, the electron drift speed can be calculated by eliminating $U_{i}$ in via Eq. (3.22).

$$
\begin{aligned}
\left|U_{i}-U_{e}\right| & =\frac{B_{\infty y}}{\mu_{0} e n_{0} L}=\frac{v_{t h, e}}{c} \sqrt{\frac{m_{e}}{m_{i}}} \sqrt{2\left(1+\frac{T_{i}}{T_{e}}\right)} \frac{d_{i}}{L} \\
U_{e} & =-\frac{v_{t h, e}}{c} \sqrt{\frac{m_{e}}{m_{i}}} \sqrt{\frac{2}{\left(1+\frac{T_{i}}{T_{e}}\right)}} \frac{d_{i}}{L}
\end{aligned}
$$

6. Relative guide field $b_{g}=B_{G} / B_{\infty}$. It requires the computation of $B_{\infty y}$ from step 5).

7. Ratio background population to peak density: $n_{b} / n_{e}$ 
Additional relations/constrains for Harris cases

$$
\begin{aligned}
& \frac{V_{A}}{c}=\frac{v_{t h, e}}{c} \sqrt{2 \frac{m_{e}}{m_{i}}\left(1+\frac{T_{i}}{T_{e}}\right)}=\frac{\Omega_{c e}}{\omega_{p e}} \sqrt{\frac{m_{e}}{m_{i}}}, \quad \beta_{i}=\frac{T_{i} / T_{e}}{1+\frac{T_{i}}{T_{e}}}, \quad \beta_{e}=\frac{1}{1+\frac{T_{i}}{T_{e}}}, \quad \beta=1 \\
& \frac{\rho_{i}}{d_{i}}=\frac{\sqrt{\frac{T_{i}}{T_{e}}}}{\sqrt{2\left(1+\frac{T_{i}}{T_{e}}\right)}}=\frac{v_{t h, i}}{V_{A}}, \quad \frac{\rho_{e}}{\lambda_{D e}}=\frac{c}{v_{t h, e}} \frac{1}{\sqrt{2\left(1+\frac{T_{i}}{T_{e}}\right)}}, \quad \frac{\Omega_{c i}^{-1}}{\omega_{p e}^{-1}}=\frac{c}{v_{t h, e}} \frac{m_{i} / m_{e}}{\sqrt{2\left(1+\frac{T_{i}}{T_{e}}\right)}}
\end{aligned}
$$

Note that $\beta_{(i, e)}$ and $V_{A}$ are not calculated with the thermal and magnetic pressure measured at the same place 1 . One numerical parameter useful to estimate computational cost is the number of macroparticles per cell in the whole simulation box for a single Harris CS:

$$
N_{\text {total }}=\operatorname{ppc} \cdot N_{x} N_{y} \frac{2 L}{L_{x}} \tanh \left(\frac{L_{x}}{2 L}\right)
$$

\footnotetext{
${ }^{1}$ the density is measured at the center, while the magnetic field at the infinity. If we want to calculate this quantities at either the center or asymptotically away, they are either infinity or zero
} 



\section{B Numerical tools}

\section{B.1 Digital filters}

Digital filters are discrete mathematical operators whose aim is smoothing out a signal in the real space. Let $\left\{A_{k}\right\}=\left\{A_{0}, A_{1}, \ldots, A_{n-1}\right\}$ a (1D) set of $n-1$ data points (the input signal). The smooth data series (output signal) is the linear combination defined by

$$
\left\{\left\langle A_{k}\right\rangle\right\}=\sum_{j=-M}^{M} g_{j} A_{k+j}, \quad \text { for } \quad k=0,1,2, \ldots n-1
$$

where $\left\{g_{j}\right\}$ are the $N=2 M+1$ filter coefficients (or weights, or impulse response1) with $M=(N-1) / 2$ an integer. $N$ is also known as the filter width. This indicates that the filters coefficients are usually chosen to be odd. Note that the number of data points has to be larger than the filter width: $n>2 N+1$. Each one of the $n$ data points requires a sliding window of $N$ neighbor points and $N$ associated filter coefficients (known ones, calculated only once), implying that only a subset set of $n-2 M$ of the total data set of $n$ points can be filtered: $M$ points at the beginning and at the end cannot be computed with Eq. B.1) (although this kind of end effects are usually solved by extrapolating the original data set). In addition, any set of coefficients $\left\{g_{i}\right\}$ is a smoothing filter if satisfies the following conditions(Marchand 1983):

- Symmetric: $g_{-k}=g_{k}$. This is required to have a real valued frequency response (see below). When applied for smoothing current or density in the spatial grid of a PIC codes, this also guarantees momentum conservation (Birdsall and Langdon 1991).

- Normalized: $\sum_{i=-m}^{m} g_{i}=1$. This implies that the mean of the original and filtered data set are the same.

- Bounded: $\sum_{i=-m}^{m}\left|g_{i}\right|<\infty$

- Bounded frequency response function: $\left|G\left(\omega_{j}\right)\right|=\sum_{l=-M}^{M}\left|g_{l} \cos (l \theta)\right|<1$, with $0<$ $\theta<2 \pi$ (see Eq. (B.20)).

The last two conditions guarantee that the (discrete) Fourier transform of the filter coefficients are always real numbers (Marchand 1983). This fact is of central importance for the design of these filters, as we will see.

\footnotetext{
1 Since $N$ is finite, this kind of filters belong to the category of Finite Impulse Response (FIR). For the basics of all the concepts to be introduced in this section about the field of Digital Signal Processing (DSP), see the textbook Orfanidis (2010) freely available at http://www .ece.rutgers.edu/ orfanidi/intro2sp/, in particular the Chapter 3.
} 
For comparison purposes, it also important to give the continuous version of the filter Eq. (B.1) (Sagaut 2006):

$$
\langle A(t)\rangle=\int_{-\infty}^{\infty} A(\xi) G(t-\xi) d^{3} \xi
$$

where $G(t-\xi)$ is known as the kernel of the filter.

\section{B.1.1 Frequency response}

For our discussions, it is convenient to give the explicit expression of the discrete Fourier transform (recall the continuous definition in Eq. (1.1)) of the smoothed (filtered) signal:

$$
\begin{aligned}
\widetilde{\left\langle A_{j}\right\rangle} & =\frac{1}{n} \sum_{k=0}^{n-1}\left\langle A_{k}\right\rangle \exp \left(-\mathrm{i} \frac{2 \pi j}{n} k\right) \\
\Leftrightarrow \quad\left\langle\widetilde{A\left(\omega_{j}\right)}\right\rangle & =\frac{1}{n} \sum_{k=0}^{N-1}\left\langle A_{k}\right\rangle \exp \left(-\mathrm{i} \omega_{j} t_{k}\right)
\end{aligned}
$$

where $j=0,1, \ldots, n-1$ are the indices of the $n$ Fourier coefficients associated with the original input signal, $\Delta$ is the (uniform) time interval between each data point, $\omega_{j}=$ $2 \pi j /(n \Delta)$ is the discrete angular frequency (in physical units!), $t_{k}=k \Delta$ is the discrete time in which each data point $A_{i}$ was measured 2 . The sampling frequency (in physical units) is $f_{s}=1 / \Delta$ and the Nyquist (or folding) frequency is $f_{N}=f_{s} / 2=1 / 2 \Delta$ according to the sampling theorem: the sampling rate should be at least twice the maximum frequency allowed in the system (Orfanidis 2010).

Now, let us find a relation between the filter coefficients in Fourier space and the output signal by replacing the general form of the filter Eq. (B.1) in the definition of the discrete Fourier transform of the filter Eq. (B.4)

\footnotetext{
${ }^{2}$ or the discrete position $x_{k}=k \Delta$ in which each data point is located in a spatial grid. Note that in all this section we are going to talk in term of a time series signal. The discussion is analogous for a spatially defined signal (on a grid), changing the frequency $\omega_{j}$ by the wave number $k_{j}$.
} 


$$
\begin{aligned}
\left\langle\widetilde{A\left(\omega_{j}\right)}\right\rangle & =\frac{1}{n} \sum_{k=0}^{n-1}\left\langle A_{k}\right\rangle e^{-\mathrm{i} \omega_{j} t_{k}} \\
& =\frac{1}{n} \sum_{k=0}^{n-1}\left(\sum_{l=-M}^{M} g_{l} A_{k-l}\right) e^{-\mathrm{i} \omega_{j} t_{k}} \\
& =\frac{1}{n} \sum_{k=0}^{n-1}\left(g_{-M} A_{k-M}+g_{-M+1} A_{k-M+1}+\cdots+g_{-1} A_{k-1}+g_{0} A_{k}+g_{1} A_{k+1}\right. \\
& \left.+\cdots+g_{M-1} A_{k+M-1}+g_{M} A_{k+M}\right) e^{-\mathrm{i} \omega_{j} t_{k}} \\
& =\frac{1}{n} \sum_{k=-M}^{n-1-M} g_{M} A_{k} e^{-\mathrm{i} \omega_{j} t_{k+M}}+\sum_{k=-M+1}^{n-1-M+1} g_{M-1} A_{k} e^{-\mathrm{i} \omega_{j} t_{k+M-1}}+\ldots \\
& +\sum_{k=-1}^{n-1-1} g_{1} A_{k} e^{-\mathrm{i} \omega_{j} t_{k+1}}+\sum_{k=0}^{n-1} g_{0} A_{k} e^{-\mathrm{i} \omega_{j} t_{k}}+\sum_{k=1}^{n-1+1} g_{1} A_{k} e^{-\mathrm{i} \omega_{j} t_{k-1}+\ldots}{ }^{n-1+M} \\
& \left.+\sum_{k=M-1}^{n-1-M-1} g_{M-1} A_{k} e^{-\mathrm{i} \omega_{j} t_{k-M+1}}+\sum_{k=M}^{n} g_{M} A_{k} e^{-\mathrm{i} \omega_{j} t_{k-M}}\right) \\
& =\frac{1}{n} \sum_{k=0}^{n-1} A_{k} e^{-\mathrm{i} \omega_{j} t_{k}}\left(g_{M}\left(e^{-\mathrm{i} M \omega_{j} \Delta}+e^{+\mathrm{i} M \omega_{j} \Delta}\right)+g_{M-1}\left(e^{-\mathrm{i}(M-1) \omega_{j} \Delta}+e^{+\mathrm{i}(M+1) \omega_{j} \Delta}\right)\right. \\
& =\frac{1}{n} \sum_{k=0}^{n-1} A_{k} e^{-\mathrm{i} \omega_{j} t_{k}}\left[g_{0}+2 \sum_{l=0}^{M} g_{l} \cos \left(l \omega_{j} \Delta\right)\right] \\
& \left.+\cdots+g_{2}\left(e^{-\mathrm{i} 2 \omega_{j} \Delta}+e^{+\mathrm{i} 2 \omega_{j} \Delta}\right)+g_{1}\left(e^{-\mathrm{i} \omega_{j} \Delta}+e^{+\mathrm{i} \omega_{j} \Delta}\right)+1\right) \\
& \ldots+g_{M=0}^{n-1} A_{k} e^{-\mathrm{i} \omega_{j} t_{k}}\left(\frac{g_{0}}{2}+g_{1} \cos \left(\omega_{j} \Delta\right)+g_{2} \cos \left(2 \omega_{j} \Delta\right)\right.
\end{aligned}
$$

where we have used the symmetry properties of the filter coefficients and the definition of $t_{k}$. Note that the argument of the transcendental function in the right hand side is (as expected) dimensionless: $\omega_{j} \Delta=2 \pi j / n$. The terms in the last sum are all reals, consequence of the symmetry of the filter coefficients. The last relation can be rearranged by using the definition of the discrete Fourier transform of the input (not filtered) signal

$$
\begin{aligned}
\left\langle\widetilde{A\left(\omega_{j}\right)}\right\rangle & =\widetilde{A\left(\omega_{j}\right)}\left[g_{0}+2 \sum_{l=1}^{M} g_{l} \cos \left(l \omega_{j} \Delta\right)\right] \\
& =\widetilde{G\left(\omega_{j}\right)} \widetilde{A\left(\omega_{j}\right)}
\end{aligned}
$$

Where we have introduced $\widetilde{G\left(\omega_{j}\right)}$ known as frequency response (or transfer function for the continuous case) of the filter. Thus, Eq. (B.18) states that that the action of a filter in the Fourier space is nothing else that the multiplication of the frequency response and the input signal (it does not apply only to filters, it is actually a much more general result). 
And obviously, the frequency response is nothing else than the discrete Fourier transform of the filter coefficients $g_{k}$ :

$$
\widetilde{G}\left(\omega_{j}\right)=\frac{1}{n} \sum_{k=0}^{n-1} g_{k} \exp \left(-\mathrm{i} \omega_{j} t_{k}\right)
$$

In our case, the explicit form of the frequency response can be expressed as:

$$
\widetilde{G\left(\omega_{j}\right)}:=g_{0}+2 \sum_{l=1}^{M} g_{l} \cos \left(l \omega_{j} \Delta\right)=\sum_{l=-M}^{M} g_{l} \cos \left(l \omega_{j} \Delta\right)
$$

Note that (obviously) all the $N=2 M+1$ coefficients of the frequency response $g_{l}$ are also normalized to 1 . Intuitively, the frequency response gives a measure of the range of frequencies modified by the filter. If $\widetilde{G\left(\omega_{j}\right)}$ does not modify low frequency and heavily smooth out high frequencies, the filter is called a "low-pass" filter. In the opposite case (if it allows high frequencies but blocks low frequencies), it is called a "high-pass" filter. The range of frequencies where the filter is (supposed) to block frequencies is called "stopband", while the range of allowed frequencies is called "passband". The range between both is called the "transition band". Because of the properties of the Fourier transform (at least for FIR filters), the length of the this range of frequencies is inversely proportional to the filter width. In between of the transition band, the frequency in which a low-pass filter attenuates to a half the original signal is called the "cutoff":

$$
\left\langle A\left(\widetilde{\left.\omega_{\text {cutoff }}\right)}\right\rangle=\frac{A\left(\widetilde{\omega_{\text {cutoff }}}\right)}{2} \quad \Rightarrow \quad G\left(\widetilde{\omega_{\text {cutoff }}}\right)=\frac{1}{2}\right.
$$

Frequencies higher than $\omega_{\text {cutoff }}$ will be (gradually) attenuated. In the digital signal processing (DSP) community, it is more common to express the effect of a filter in decibels, which for this case will be:

$$
10 \log _{10}\left(\frac{\left\langle A\left(\widetilde{\omega_{\text {cutoff }}}\right)\right\rangle}{A\left(\omega_{\text {cutoff }}\right)}\right)=10 \log _{10}\left(\frac{1}{2}\right) \approx-3 \mathrm{~dB}
$$

In any case, the frequency cutoff is associated with a typical timescale in the real space, which can be written normalized to the timestep $\Delta$ as follows:

$$
\frac{t_{\text {cutoff }}}{\Delta}=\frac{2 \pi}{\omega_{\text {cutoff }} \Delta}=\frac{n \Delta}{j_{\text {cutoff }}}
$$

where $j_{\text {cutoff }}$ is the index associated with the (discrete) frequency cutoff. Signals with temporal fluctuations shorter than $t_{\text {cutoff }}$ will be attenuated. In this sense, this quantity determines a resolved time scale. Similarly, for spatial signals, fluctuations with length scales shorter than the length cutoff $\lambda_{\text {cutoff }}=2 \pi /\left(k_{\text {cutoff }} \Delta\right)$ will be smoothed out (and lengths larger than $\lambda_{\text {cutoff }}$ will be resolved by the filter).

\section{B.1.2 Examples of commonly used filters}

Now, we are going to give the exact representation (discrete and in many cases continuous for gain insight in the physical meaning) of the most commonly used filters (in 1D). 


\section{B.1.2.1 Moving average}

Also called boxcar or top-hat filter, it is the simplest filter, assigning to each point in the interval the mean value of the $\left\{A_{n}\right\}$ on the given interval:

$$
\left\{\left\langle A_{k}\right\rangle\right\}=\frac{1}{2 M+1} \sum_{i=-M}^{M} A_{k-i}=\frac{1}{N} \sum_{i=1}^{N} A_{k-i}
$$

where all the coefficients are equal to

$$
g_{i}=\frac{1}{2 M+1}=\frac{1}{N}, \quad \text { for } \quad i=-M, \ldots,-1,0,1, \ldots, M
$$

This filter works relatively well if the data set want to be smoothed is more or less constant or changes linearly inside of the interval of width $N$. But any other feature such as local maxima or minima will be reduced, something impractical if one wants to extract physical information of such features. In these cases, one has to resort to another kind of filters (see, e.g., Appendix B.1.2.5). The reduction of noise by a moving average can be measured by the reduction in the standard deviation $\sigma$ of the original data set to $\sigma / \sqrt{M}$.

The frequency response can be calculated from Eq. (B.20) by setting all the coefficients $g_{l}=1$ and using the known expression for the sum of a geometric series

$$
\widetilde{G\left(\omega_{j}\right)}:=\frac{1}{2 M+1}\left[\frac{\sin \left(\frac{2 M+1}{2} \omega_{j} \Delta\right)}{\sin \left(\frac{\omega_{j} \Delta}{2}\right)}\right]=\frac{1}{N}\left[\frac{\sin \left(\frac{N \omega_{j} \Delta}{2}\right)}{\sin \left(\frac{\omega_{j} \Delta}{2}\right)}\right]
$$

An explicit example is the 5 points moving average with $M=2$, whose filter formula, respective frequency response and the allowed discrete frequencies are:

$$
\begin{aligned}
\left\langle A_{k}\right\rangle_{M A} & =\frac{1}{5}\left[A_{k-2}+A_{k-1}+A_{k}+A_{k+1}+A_{k+2}\right] \\
\widetilde{G_{M A}}\left(\omega_{j}\right) & =\frac{1}{5}\left[1+2 \cos \left(\omega_{j} \Delta\right)+2 \cos \left(2 \omega_{j} \Delta\right)\right]=\frac{1}{5}\left[\frac{\sin \left(\frac{5 \omega_{j} \Delta}{2}\right)}{\sin \left(\frac{\omega_{j} \Delta}{2}\right)}\right] \\
\omega_{j} \Delta & =\frac{2 \pi j}{n}=\left\{0, \frac{2 \pi}{n}, \frac{4 \pi}{n}, \frac{6 \pi}{n}, \ldots, \frac{2 \pi(n-1)}{n}\right\}
\end{aligned}
$$

We can see the frequency responses for varies filter lengths $N$ in Fig. B.1. They show a very undesirable property characteristic of this filters: for frequencies higher than $1 / N$, $\widetilde{G\left(\omega_{j}\right)}$ oscillates instead of being close to zero (the ideal behaviour). It is only zero at the multiples of $1 / N$. And there is even regions where $\widetilde{G\left(\omega_{j}\right)}<0$, implying a "phase reversal" of the original signal. Then, a large part of high frequencies will be allowed, although damped and even reversed: the original signal will be distorted, even in absence of noise. This is obviously a non wanted behaviour for a filter with the purpose of "smoothing" data (and not distorting it): a trade-off because of its simplicity. 


\section{B.1.2.2 Binomial}

The binomial filter is one of the most used ones in PIC simulations for the current smoothing (see, e.g, Birdsall and Langdon 1991, Appendix C). Its name comes from the fact that the weights in Eq. (B.1) are chosen according to the binomial coefficients (Pascal triangle):

$$
g_{k}=\frac{1}{\sum_{l=0}^{N} A_{l}}\left(\begin{array}{l}
N \\
k
\end{array}\right)=\frac{1}{\sum_{l=0}^{N} A_{l}} \frac{N !}{k !(N-k) !}
$$

Note that by definition the binomial coefficients are always symmetric, a necessary condition for being filter coefficients. The denominator is for the fulfillment of the normalization condition.

Let us now specialize for the smallest case allowed: $N=3$ and $M=1$, with binomial coefficients $\{1,2,1\}$ (numerator of the previous expression). Since we need to satisfy the normalization condition, we have to divide by their sum resulting in the filter coefficients $g_{0}=2 / 4$ and $g_{-1}=g_{1}=1 / 4$. Thus, plugging this into the general definition for $g_{i}$ in Eq. (B.1), we obtain the so called 3-point binomial filter, a very common choice in PIC codes (Vay et al. 2011). Its explicit formula and corresponding frequency response (calculated from Eq. (B.20)) are:

$$
\begin{aligned}
\left\langle A_{\mathrm{M} 1-\text { bin }, \mathrm{k}}\right\rangle & =\frac{1}{4}\left[A_{k-1}+2 A_{k}+A_{k+1}\right] \\
\widetilde{G}_{\mathrm{M} 1-\mathrm{bin}}\left(\omega_{j}\right) & =\frac{1}{2}\left[1+\cos \left(\omega_{j} \Delta\right)\right]=\cos ^{2}\left(\frac{\omega_{j} \Delta}{2}\right)
\end{aligned}
$$

The nice thing about the binomial filter is that more extended filters can obtained by a repeated convolution of the basic 3-point filter Eq. (B.31) for $M=1$ (this is more efficient that calculate all the binomial coefficients for large values of $M$ ). Thus, the frequency response for a filter of length $M=(N-1) / 2$ will be given by:

$$
\begin{aligned}
\widetilde{G_{\mathrm{M}-\text { bin }}}\left(\omega_{j}\right) & =\left(\widetilde{G_{\mathrm{M} 1-\text { bin }}}\left(\omega_{j}\right)\right)^{M} \\
& =\cos ^{2 M}\left(\frac{\omega_{j} \Delta}{2}\right)=\cos ^{N-1}\left(\frac{\omega_{j} \Delta}{2}\right)
\end{aligned}
$$

Note that this frequency response is always positive, implying no change in phase of the original signal. Furthermore, it is possible to show that, under some general assumptions, a binomial filter is "maximally" flat in the passband and with the highest slope in the transition band among all the digital filters of order $M$. This implies that it will modify very little the (low) frequencies wanted to be kept, while it will cutoff the undesired (high) frequencies efficiently, in opposition to the simpler moving average. See the proof of this and other additional properties of the binomial filter in Marchand (1983).

Following the definition in Eq. (B.21), it is possible to find a closed analytical form for the cutoff frequency by solving

$$
\begin{aligned}
\frac{1}{2} & =\cos ^{2 M}\left(\frac{\omega_{\text {cutoff }} \Delta}{2}\right) \\
\Rightarrow \omega_{\text {cutoff }} \Delta & =2 \operatorname{acos}\left(2^{-1 /(2 M)}\right)
\end{aligned}
$$


from where we can get the resolved time scale using Eq. (B.23):

$$
\frac{t_{\text {cutoff }}}{\Delta}=\frac{\pi}{\operatorname{acos}\left(2^{-1 /(2 M)}\right)}
$$

Some examples of frequencies responses of this filter are given in Fig. B.1 on dependence on the width or number of passes of the 3 point filter $M$.

\section{B.1.2.3 Gaussian filter}

This filter is obtained with weights in Eq. (B.1) following the continuous Gaussian or normal probability distribution function.

$$
\begin{aligned}
G(t-\xi) & =\frac{1}{\sqrt{2 \pi} \sigma} \exp \left(-\frac{|t-\xi|^{2}}{2 \sigma^{2}}\right) \\
\widetilde{G}(f) & =\exp \left(-\frac{f^{2}}{2 \sigma_{f}^{2}}\right)
\end{aligned}
$$

where we have also indicated their respective frequency response (Fourier transform) and $\sigma, \sigma_{f}$ are the standard deviations in real and Fourier space, respectively. The discrete version of the Gaussian filter (weights $g_{k}$ ) can be obtained by sampling the continuous version Eq. (B.38) at multiples of the inverse of the standard deviation: $\{0, \pm 1 / \sigma, \pm 2 / \sigma, \ldots\}$. Since the original is an infinite signal, these discrete values are truncated when $G(t-\xi)$ is below a small given threshold. Finally, all these values are normalized to 1 . The discrete frequency response is obtained by simply using the definition Eq. (B.20). However, for a large enough filter width $N$, it can be shown than the coefficients of the binomial filter converge to the Gaussian one (Marchand 1983), because of the central limit theorem. This is often more efficient than calculate the sampled Gaussian distribution, but only if $\sigma$ is small enough. Otherwise, the second method is more convenient.
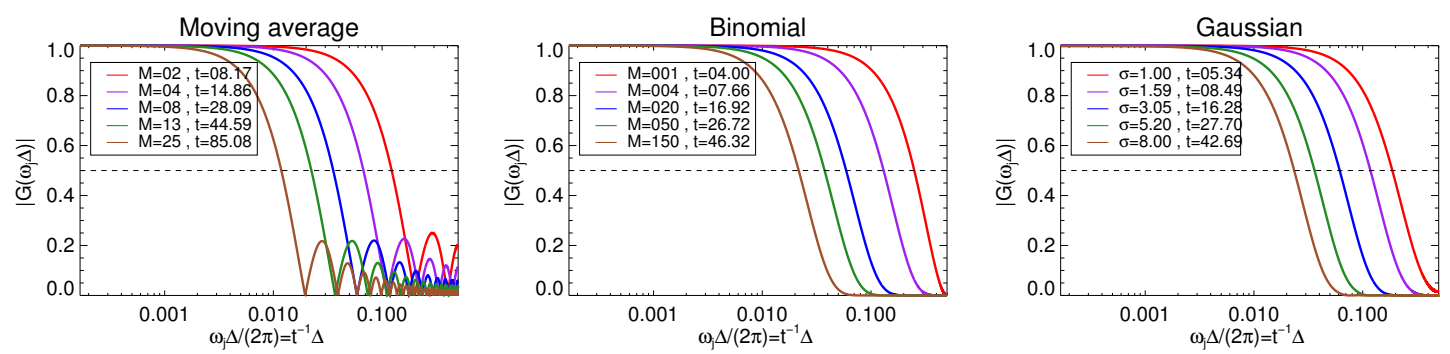

Figure B.1: Frequency responses $|G(\omega \Delta)|$ of several filters, with the cutoff time $t_{\text {cut }} / \Delta$ indicated. Left: Moving average for different (half)widths $M$ (note the ripples in the high frequency range). Center: Binomial for different halfwidth or passes of the basic 3 point filter M. Right: Gaussian for different $\sigma$. The last two filters do not show ripples in the high frequency range, attenuating efficiently any noise there. Note that the approximation for the Gaussian cut-off Eq. (B.42) works better for large values of $\sigma$.

According to the basic properties of Fourier transforms, the standard deviations of the filters in real and frequency domain are related by

$$
\sigma \sigma_{f}=\frac{1}{2 \pi}
$$


From this expression it is possible to prove than the cutoff frequency of the discrete Gaussian filter is:

$$
\omega_{\text {cutoff }} \Delta=2 \pi \sqrt{2} \log (2)\left(\sigma_{f} \Delta\right)=\frac{\sqrt{2} \log (2)}{\sigma / \Delta}
$$

(the factor $\sqrt{2} \log (2)$ comes the full width at half maximum of the continuous Gaussian distribution function) The associated time cutoff or resolved time scale is:

$$
\frac{t_{\text {cutoff }}}{\Delta}=\frac{2 \pi \sigma / \Delta}{\sqrt{2} \log (2)} \approx 6 \frac{\sigma}{\Delta}
$$

This is precisely the reason why this filter is very handy: its time cutoff can be specified in terms of its standard deviation. Note, however, that the previous formula is precise only if the filter width is large enough $(\sigma / \Delta \gg 1)$. Some examples of frequency response of this filter are given in Fig. B.1.

\section{B.1.2.4 Sharp-cutoff}

Although it is not used in this thesis, it is useful to know the specific form of the filter that has the "opposite" form of the moving average and the ideal behavior: it eliminates completely all the frequencies higher that the cutoff $\omega_{\text {cutoff }}$, while it does not modify at all the lowpass band of frequencies (Sagaut 2006):

$$
\begin{aligned}
G(x-\xi) & =\frac{\sin \left(k_{\text {cutoff }}(x-\xi)\right)}{k_{\text {cutoff }}(x-\xi)}, \quad \text { with } \quad k_{c}=\frac{\pi}{\Delta} \\
\widetilde{G}(k) & = \begin{cases}1 / \Delta & \text { if }|k|<k_{\text {cutoff }}, \\
0 & \text { otherwise. }\end{cases}
\end{aligned}
$$

This theoretical filter, known as sharp-cutoff or sinc cannot be done in practice, since it is not causal

\section{B.1.2.5 Savitzky-Golay filters}

Savitzky-Golay (lowpass) filters (Savitzky and Golay 1964) smooth data based on a (local) polynomial approximation, using a least-squares approach. They were first proposed for improving the detection of peaks in (noisy) signals from chemical spectra 3 , becoming in one of the standard techniques in that field since then. The main advantage of the Savitzky-Golay (S-G) filters is that they keep the shape of the peaks of a signal (usually appearing in spectroscopic analysis with a Gaussian form), while at the same time smoothing out the overall data. Or in other words, under the assumption that the original data can be locally well approximated by a polynomial, this filter can smooth the signal without significant loss of resolution (Press et al. 2007). This is in contrast to

\footnotetext{
${ }^{3}$ The paper by Savitzky-Golay also is one of the ten most cited $(\sim 10 k)$ in all the history of the journal Analytical Chemistry, even though "only" describes a numerical method. It is less known but it still important in the digital signal processing (DSP) field, and not too known outside of these fields (Schafer 2011).
} 
other more conventional filters that, together with smoothing out the data, also make any peak flatter and difficult to distinguish. This effect can be seen in Fig. B.2(left), where it is shown the action of a moving average and S-G filters with the same window length on synthetic noisy data (Press et al. 2007). The moving average broadens and decrease the amplitude of the "spectral" peaks to the right of the data sets. On the other hand, the S-G filter preserves both height and width of the peaks (the shape). But we already see the trade-off of that nice feature: the large scales features (the broad peak to the left of the data set) becomes noisier with the S-G filter.
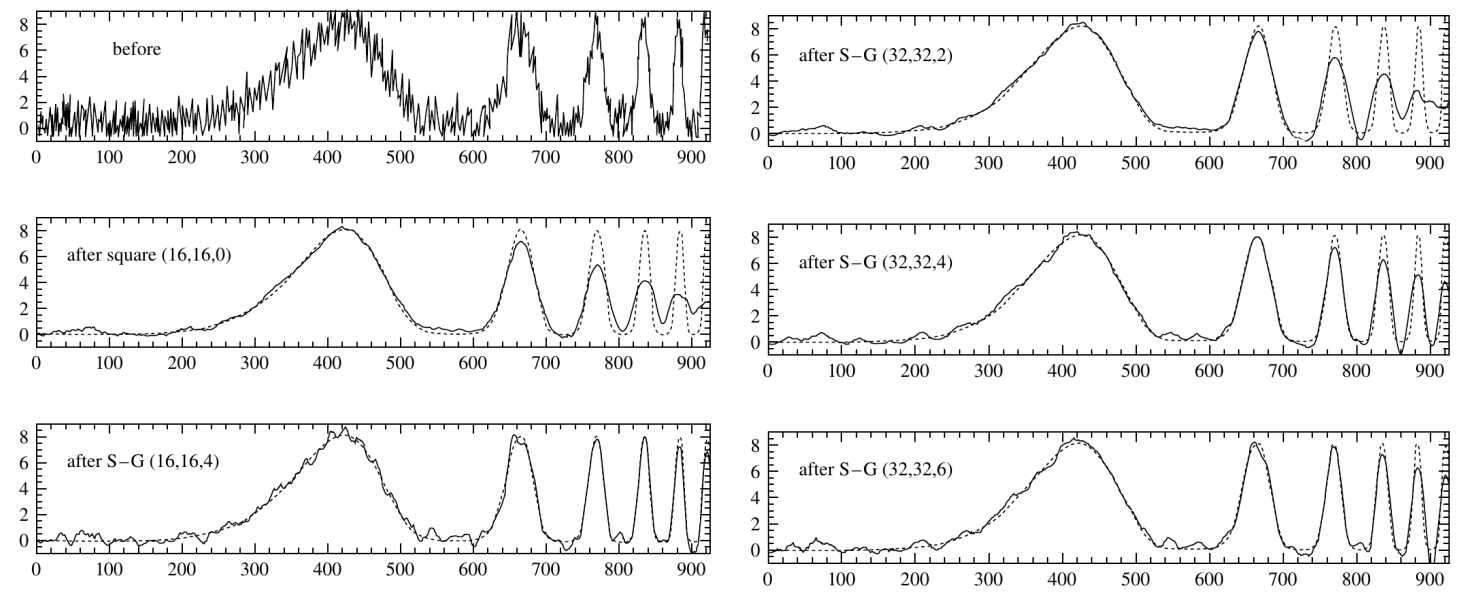

Figure B.2: Effect of the $S$-G filter on synthetic data with added Gaussian noise. Left: Superimposed the smoothed data obtained with a moving average (top) with $N=33, S-G$ filter with $M=16$ (and $N=33$ ) and polynomial order 2 (center) and same as previous but with polynomial order 4 (bottom). Adapted from Fig. 14.9.1 of Press et al. (2007). Right. Comparison of $S$ - $G$ filters with $M=32$ and different polynomial orders. Top: 2, Center: 4, Bottom: 6. Adapted from Fig. 14.9.2 of Press et al. (2007). $P$

The smoothed data via S-G filters is obtained by sampling a given polynomial of order

$$
p(n)=\sum_{k=0}^{P} a_{k} n^{k}
$$

that fits locally to the $N=2 M+1$ points of a data set $\left\{A_{n}\right\}$, equivalent to a fixed linear combination of them (Schafer 2011) with filter coefficients uniquely specified for a given polynomial of order $P$ and filter width $2 M+1$. This implies that it is not necessary to calculate them again for each interval where smoothed data is wanted. The fitting via least-squares approximation is obtained by minimizing the error function

$$
\epsilon_{N}=\sum_{n=-M}^{M}\left(p(n)-A_{n}\right)^{2}
$$

\footnotetext{
${ }^{4}$ Under some conditions, it is possible to extend this filter for an even number of data points (Luo et al. 2005a).
} 
which is equivalent to derive them with respect to each one of the polynomial coefficients $a_{k}: \partial \epsilon_{N} / \partial a_{i}$. This procedure give $P+1$ equations with $P+1$ unknowns with unique solution if and only if $P \gtrsim 2 M$ (Schafer 2011). Then, the coefficients of this system of equations can be obtained via standard inversion matrix methods 5 With this method (or any similar), the specific S-G coefficients for polynomials order $P=2$ (or 3) and $P=4$ (or 5) as function of the filter width are 6 :

$$
\begin{aligned}
& g_{\mathrm{SG}-\mathrm{P} 2 \mathrm{k}, \mathrm{k}}=3 \frac{\left(3 M^{2}+3 M-1-5 k^{2}\right)}{(2 M+3)(2 M+1)(2 M-1)} \\
& g_{\mathrm{SG}-\mathrm{P} 4 \mathrm{k}, \mathrm{K}}=15 \frac{\left(15 M^{4}+30 M^{3}-35 M^{2}-50 M+12-35\left(2 M^{2}+2 M-3\right) k^{2}+63 k^{4}\right)}{4(2 M+5)(2 M+3)(2 M+1)(2 M-1)(2 M-3)}
\end{aligned}
$$

Thus, e.g., for the case with $(P=2, M=2)$ the explicit filter formula and the respective frequency response (via Eq. $($ B.20) $)$ are:

$$
\begin{aligned}
\left\langle A_{\mathrm{SG}-\mathrm{P} 2 \mathrm{M} 2, \mathrm{k}}\right\rangle & =\frac{1}{35}\left[-3 A_{k-2}+12 A_{k-1}+17 A_{k}+12 A_{k+1}-3 A_{k+2}\right] \\
G_{\mathrm{SG}-\mathrm{P} 2 \mathrm{M} 2, \mathrm{k}}\left(\omega_{j}\right) & =\frac{1}{35}\left[17+24 \cos \left(\omega_{j} \Delta\right)-6 \cos \left(2 \omega_{j} \Delta\right)\right] .
\end{aligned}
$$

Note that, different from binomial of Gaussian filters and similar to the moving average, the frequency response can be negative, implying a phase change for high frequencies. This is one of the not so good features of these filters. See Fig. B.3 for a comparison of S-G filters for different $M$ and $P$, and Fig. B.4 for a comparison of S-G against others filters. Now, let us list some other properties (Schafer 2011):

\footnotetext{
${ }^{5}$ See details, e.g., in the textbook Orfanidis (2010, Sec. 8.3.5). There is also an alternative approach for obtaining these coefficients relying in the use of the (orthogonal) Gram polynomials (see Gorry 1990, including pseudo-code!). This approach is used in many efficient implementations of this algorithm, due to its recursive character.

${ }^{6}$ For a comprehensive compilation of formulas for various cases and combinations of $M$ and $P$, see, e.g, Madden (1978), which corrected several typos in the original formulas given in the paper by Savitzky and Golay (1964).
} 

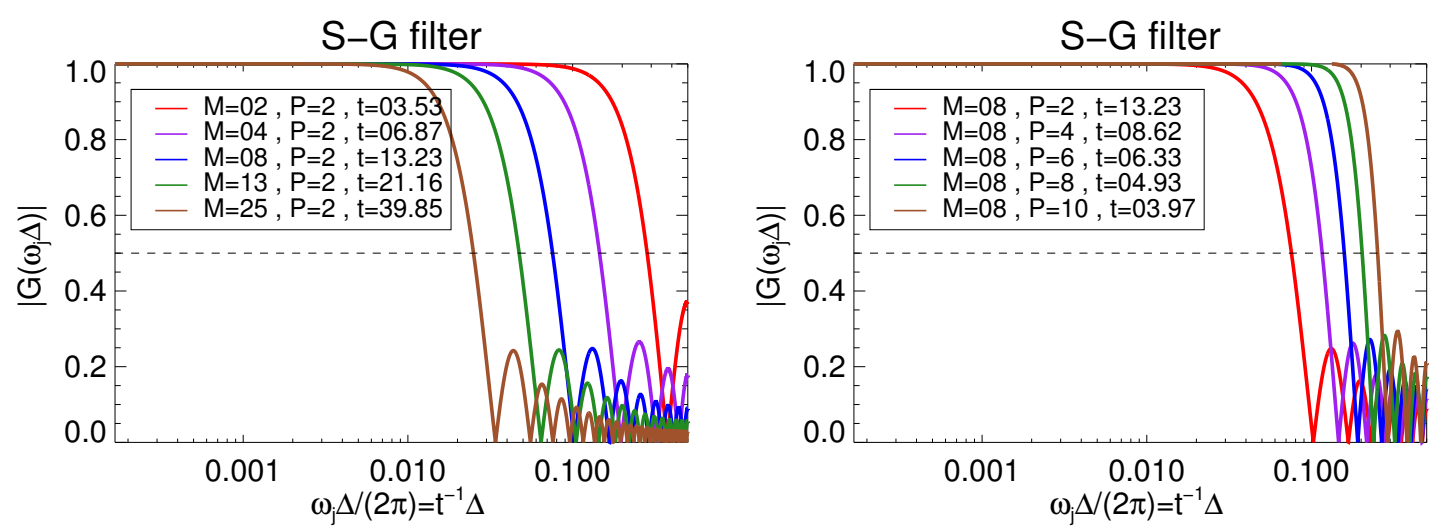

Figure B.3: Frequency responses $|G(\omega \Delta)|$ of Savitzky-Golay filters with the cutoff-time indicated. Left: dependence on the filter width $M$ for a fixed polynomial order P. Right: dependence on the polynomial order $P$ for a fixed filter width $M$. Note that the same cutoff can be obtained with a different combinations of these parameters, although with a different behavior in the transition band and in the ripples for high frequencies.

- The "peak conservation" property of the S-G filters can be derived more formally from Parseval's theorem, stating that the first $P$ momenta of the original signal $A_{k}$ are preserved by the filter $\left\langle A_{k}\right\rangle_{S G}$ :

$$
\sum_{k=-\infty}^{\infty} k^{r}\left\langle A_{k}\right\rangle_{S G}=\sum_{k=-\infty}^{\infty} k^{r} A_{k}, \quad \text { for } \quad r=1,2, \ldots, P
$$

It can also be proven that, for a fixed $\mathrm{N}$, a S-G filter is the momentum conserving filter with maximum noise reduction (Bromba and Ziegler 1981). In the context of spectroscopy, this property is physically equivalent to preserve the area under a spectral line (momenta order 0 ), its mean position in time (momenta order 1 ), its line width (momenta order 2 ) and so on (Press et al. 2007)7.

- The last property has a corollary in the frequency domain: the S-G filters have very flat passband, but on the other hand, the stopband does not attenuate signals so well compared to binomial or Gaussian filters. The peak gain in this range of frequencies increases for higher polynomial orders $P$ (given a fixed filter width $M$ ).

- The moving average (see Appendix B.1.2.1) is a particular case of S-G filter for zero order polynomials $P=0$ and filter length of 3 (or $M=1$ ). Therefore, this filter will not preserve the spectroscopic line width, since it is the momenta of order 2. In fact, this was one of the reasons because Savitzky and Golay developed the filter named after them: they wanted to improve the tendency of moving averages to flatten and widen peaks in the spectra

- In Eq. (B.1), the coefficient $g_{0}=\left\langle A_{0}\right\rangle$ (smoothed value at the center of the interval).

\footnotetext{
7 This fact is of fundamental importance in our studies of magnetic reconnection for increasing guide fields, where the length scales of the important features (e.g. border of magnetic islands) are reduced due to the smaller electron Larmor radius ( see Chapter 8 ).
} 
- In Eq. (B.1), the coefficient $g_{k}$ corresponds to the smoothed value of the $k$-th derivative of the data set $\left\{A_{k}\right\}$ evaluated at the center of the interval, divided over $k$ ! (see Madden 1978).

- S-G filters with polynomial of order $P$ and $P+1$ are identical if $P$ is an even integer (i.e.: 2 and 3, 4 and 5).

- For a fixed filter width $M$, the S-G passband increases approximately proportional to the polynomial order $P$.

- The cut-off frequency $\omega_{\text {cutoff }}$ is inversely proportional to the filter width $M$.

- An empirical formula for the cutoff frequency is $\omega_{\text {cutoff }}=(P+1) /(3.2 M-4.6)$ for large filter widths $M \gtrsim 25$ and $P<M$. Note that different combinations of $P$ and $M$ can give the same cutoff frequency.

- Since the noise amplification factor scales as the sum of the squares of the coefficient $\sum_{j=-M}^{M} g_{j}^{2}$ (Luo et al. 2005b, Orfanidis 2010), the S-G filter will attenuate better the noise for larger $M$ and/or $P$. Or in other words, the signal to noise ratio will increase for higher values of these parameters. For quantitative estimations about the amount of noise reduction and improvement of signal to noise ratio, see Bromba and Ziegler (1981).
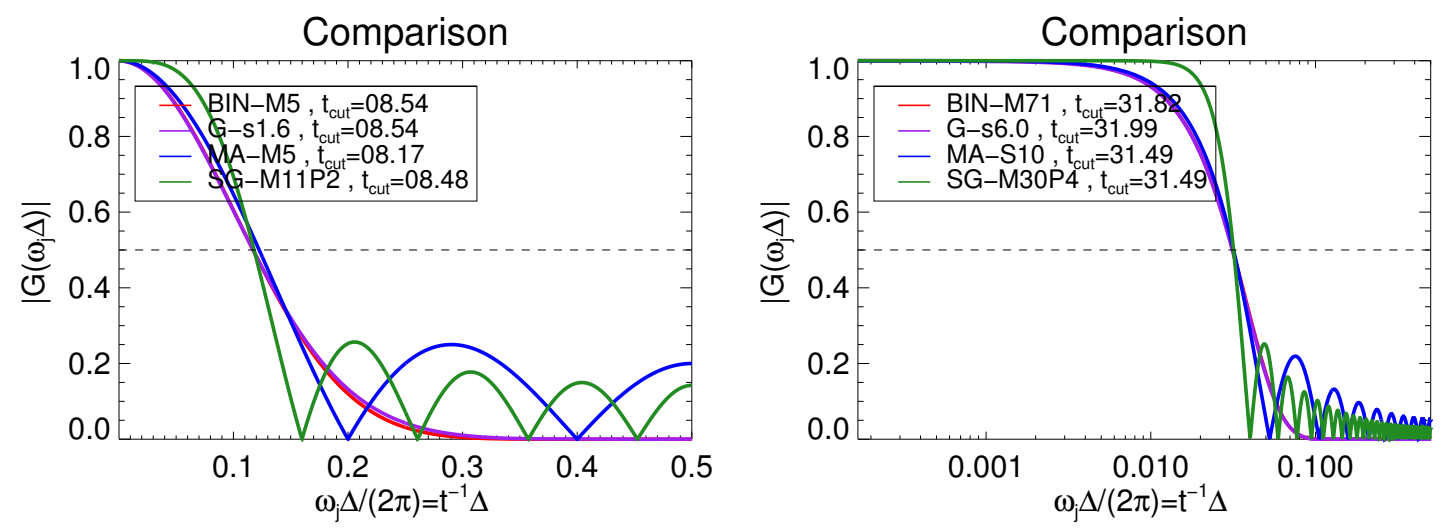

Figure B.4: Comparison of the frequency responses $|G(\omega \Delta)|$ of several filters (binomial with width $M$, Gaussian with $\sigma$, moving average with width $M$ and Savitzky-Golay for width $M$ and polynomial order $P$ ) for the same cutoff time $t_{\text {cutoff. Left: Comparison for } a}$ short $t_{\text {cutoff }} \approx 8.5$ (note the linear scale for the $x$-axis). Right: Comparison for a longer $t_{\text {cutoff }} \approx 32$ (note the logarithmic scale for the $x$-axis). Although binomial and Gaussian filters (practically indistinguishable for the values shown here) attenuate efficiently noise in the high frequency range when compared to $S$-G filters, they have shorter pass bands and less steeper transition bands than the latter ones. These are useful properties if one wants to get rid of given frequencies while reducing to the minimum the distortion introduced by the filter to large scales. Note that the moving average have the "bad" properties of both $S-G$ and binomial/Gaussian filter: short passband (distortion to large scales) and ripples in the stop band (lack of efficient reduction of noise to small scales) 


\section{B.1.3 Numerical derivatives}

Similar to Eq. (B.1), numerical derivatives can be calculated by choosing suitable coefficients $g_{j}^{q}$ and then forming a linear combination with the data points, usually centered in the same way as smoothing filters:

$$
\left(\frac{d^{q}\{\langle A\rangle\}}{d x^{q}}\right)_{k}=\sum_{j=-M}^{M} g_{j}^{(q)} A_{k+j}, \quad \text { for } \quad k=-M,-M+1, \ldots,-1,0,1, \ldots, M-1, M
$$

where $q$ indicates the order of the derivative (other symbols are identical to the ones in Eq. (B.1)). The angular brackets have been maintained in the left hand side to indicate that a numerical derivative can also (but not necessarily) smooth the result.

\section{B.1.3.1 Extended finite differences}

The standard way of calculating numerical derivatives is via finite differences (called central differences if they are in the form Eq. (B.52), providing better accuracy than asymmetric calculations). As explained in every textbook of numerical analysis, they are obtained by approximating locally the value of the function by a polynomial of order $P$, equivalent to a Taylor approximation. Higher order polynomial approximations will require more points, called in this context the "stencil" (instead of filter width as for the designed for smoothing purposes). The explicit formula for the first order derivative 8 is given by Ahnert and Abel (2007):

$$
\left(\frac{d A}{d t}\right)_{k, F D}=\sum_{j=1}^{M} \alpha_{j} \frac{A_{k+j}-A_{k-j}}{2 j \Delta}
$$

with $g_{j}^{(1)}=\alpha_{j} /(2 j \Delta)$ and

$$
\alpha_{j}=2 \cdot(-1)^{j+1} \frac{\left(\begin{array}{c}
k \\
k-j
\end{array}\right)}{\left(\begin{array}{c}
k+j \\
k
\end{array}\right)}
$$

Note the antisymmetric character of all these expressions $\left(g_{-k}^{(1)}=-g_{k}^{(1)}\right)$ in opposition to the first property of the smoothing filters (and $g_{0}=0$ ). The first three lower order ones for the first derivative (with accuracy 2, 4 and 6, respectively), are given by:

$$
\begin{aligned}
& \left(\frac{d A}{d t}\right)_{k, F D-1 M}=\frac{-A_{k-1}+A_{k+1}}{2 \Delta} \\
& \left(\frac{d A}{d t}\right)_{k, F D-2 M}=\frac{A_{k-2}-8 A_{k-1}+8 A_{k+1}-A_{k+2}}{12 \Delta} \\
& \left(\frac{d A}{d t}\right)_{k, F D-3 M}=\frac{-A_{k-3}+9 A_{k-2}-45 A_{k-1}+45 A_{k+1}-9 A_{k+2}+A_{k+3}}{60 \Delta}
\end{aligned}
$$

\footnotetext{
${ }^{8}$ The weights or coefficients of finite differences for arbitrary stencil lengths and derivative order can be computed easily via the algorithm published by Fornberg (1988).
} 
In Eq. B.53), the error between the approximation and the exact derivative scales theoretically as $(O)\left(\Delta^{M}\right)$ : larger stencils (using more points for the derivatives) will decrease the error, as well as the choice of smaller timestep $\Delta$. However, this is only valid if the data is noise-free. In realistic cases the original data set contains intrinsic errors. These errors may originate from both noise in the measurements and discretization effects. Then, the subtraction of two similar numbers in the numerator of Eq. (B.53), a necessary operation due to the use of differences in all these algorithms, will lead potentially to a loss of digits of significance (due to the limitations of floating point arithmetic). Thus, the result of the approximation for the derivative can be much less accurate than the original data set, even to the extreme of being completely meaningless (Ahnert and Abel 2007).

The frequency response of the finite differences for first order derivatives will change in comparison with the one for smoothing filters due to the aforementioned antisymmetry property: instead of adding the complex exponentials in Eq. (B.9), it will appear a minus sign in such a way that the exponentials term will be subtracted and then:

$$
\left.\widetilde{G_{F D}\left(\omega_{j}\right.}\right):=-2 i \sum_{l=1}^{M} g_{l}^{(1)} \sin \left(l \omega_{j} \Delta\right)
$$

For example, for the aforementioned first central differences formulae:

$$
\begin{aligned}
& G_{F D-1 M}\left(\omega_{j}\right)=-\frac{i}{\Delta} \sin \left(\omega_{j} \Delta\right) \\
& G_{F D-2 M}\left(\omega_{j}\right)=-\frac{i}{6 \Delta}\left[8 \sin \left(\omega_{j} \Delta\right)-\sin \left(2 \omega_{j} \Delta\right)\right] \\
& G_{F D-3 M} \widetilde{\tau}\left(\omega_{j}\right)=-\frac{i}{30 \Delta}\left[45 \sin \left(\omega_{j} \Delta\right)-9 \sin \left(2 \omega_{j} \Delta\right)+\sin \left(3 \omega_{j} \Delta\right)\right]
\end{aligned}
$$

An ideal derivative would have a frequency response $\widetilde{G_{\text {ideal }}}\left(\omega_{j}\right)=i \omega_{j}$ (the time derivative with respect to $\delta$ of the complex exponential). As we can see in Fig. B.5, the central differences approximates that behaviour in the low frequencies. For larger filter width, they get closer and closer to the asymptotic line. But this is inconvenient from the practical point of view: they do allow high frequencies without a significant damping, something unwanted if one wants to differentiate noisy (experimental) data, since most of the noise is precisely in that range. It is even worse: since the gain of the $\widetilde{G_{F D}\left(\omega_{j}\right)}$ can be significant for large $\omega_{j}$, the numerical differentiation can indeed amplify the noise. Therefore, a practical filter should not follow the linear frequency response of the ideal derivative: it should instead be a lowpass blocking high frequencies.

\section{B.1.3.2 Smooth derivatives with S-G filters}

The Savitzky-Golay filters can also be applied for calculating numerical derivatives, often in a much smoother way than finite differences 8 . It can be proven that the S-G derivative filter is equivalent to convolving a $S-G$ smoothing filter and the derivative of the input signal (Luo et al. 2005b). An intuitive way of seeing this is because they are based on a least square approximation: these filters do not use the exact values of the

\footnotetext{
${ }^{9}$ In this context, they are sometimes called Lanczos differentiators. See Usui and Amidror (1982) for further details and extended comparisons among different numerical methods for derivatives.
} 
time series to be differentiated as in the case of finite differences (since they are based on interpolation), instead: they use modified (smoothed) values of the input data.

It it interesting to mention that the S-G filters were historically first developed due to that reason, since the derivatives of the spectroscopic data carry important physical information. The method developed by Savitzky and Golay (1964) is essentially the same for both smoothing an any higher derivative order. The simplicity of this general purpose algorithm contributed to its popularity in the years after its original publication (Riordon et al. 2000).

Some properties of the S-G first order derivative filters are (Luo et al. 2005b):

- The filters with polynomials of order $P$ and $P+1$ are identical if $P$ is an odd integer (i.e.: 1 and 2, 3 and 4).

- The polynomial order and filter width should satisfy $P<M-1$ to get the smoothing effect on the derivative. Otherwise, the expressions are reduced to the simpler finite differences (the least-square approximation degenerates into an interpolation)

- The frequency response is flatter and closer to the ideal derivative $i \omega_{j}$ for higher order polynomial $P$, although it also widen the lowpass band.

- The first $P$ momenta of the derivative of the signal $(d A / d t)_{k}$ are preserved by the filter $(d A / d t)_{k, S G}$

- A corollary of the latter property is that these derivative filters minimize the noise amplification factor under that restriction.

- In general, for any derivative order: $\sum_{j=-M}^{M} g_{j}^{(q)} j^{q}=q$ ! (Madden 1978).

- In general, for any derivative order: $\sum_{j=-M}^{M} g_{j}^{(j)}=0$ for $q \neq 0$ (Madden 1978).

For example, the coefficients for the first order derivative approximated by a polynomial order $P=1$ (or 2) and arbitrary width $M$ are given by 10 :

$$
g_{\mathrm{SG} 1 \mathrm{der}-\mathrm{P} 1, \mathrm{k}}=\frac{1}{\Delta} \frac{3 k}{(2 M+3)(2 M+1)(2 M-1)}
$$

Specializing for the smallest width of $M=3(M=2=P$ would be identical to the finite difference algorithm), we can obtain the explicit formulas for the filter and their corresponding frequency response (calculated via Eq. (B.58)):

$$
\begin{aligned}
\left(\frac{d A}{d t}\right)_{k, S G 1 d e r-P 1 M 3} & =\frac{-3 A_{k-3}-2 A_{k-2}-A_{k-1}+A_{k+1}+2 A_{k+2}+3 A_{k+3}}{28 \Delta} \\
G_{k, S G 1 \text { der-P1M3 }}\left(\omega_{j}\right) & =-\frac{i}{6 \Delta}\left[\sin \left(\omega_{j} \Delta\right)+2 \sin \left(2 \omega_{j} \Delta\right)+3 \sin \left(3 \omega_{j} \Delta\right)\right]
\end{aligned}
$$

It is interesting to compare the previous expression with the equivalent finite difference algorithm for $M=3$ in Eq. (B.57) and Eq. (B.61). In opposition to the frequency response of finite differences, the ones for S-G derivatives attenuates much better the signal at

\footnotetext{
${ }^{10}$ See some other ex plicit formulas in Madden (1978).
} 
higher frequencies, although still with the characteristic ripples. Note that the gain or amplitude in that region decreases for higher values of $N$.
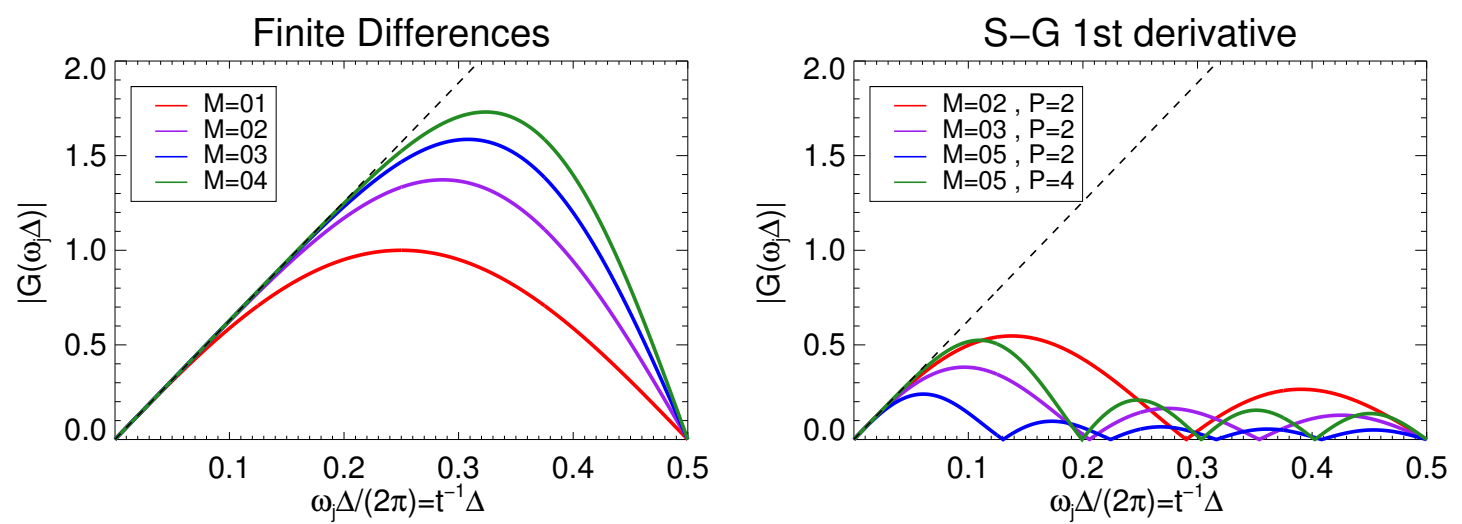

Figure B.5: Frequency responses $|G(\omega \Delta)|$ of finite differences (left) and first order derivatives via Savitzky-Golay filters (right) for given width $M$ and polynomial order $P$. The dashed line indicates the frequency response of an ideal differentiator $\left(i \omega_{j}\right)$. Note that finite differences do no attenuate noise in the high frequency range, instead: they amplify any signal with frequencies up to the Nyquist frequency. This noise amplification gets worse for wider widths (or stencils). In contrast, S-G filters attenuate partially the high frequency noise, being more efficient in that for longer widths (exactly the opposite to finite differences).

\section{B.1.3.3 Combining smoothing formulas and derivatives}

One can combine for smoothing formulas and derivatives in any desired order. Their frequency response can be obtained by a simply convolution in the Fourier space. In this way, in principle it is possible to design a combination of operations with an optimum behavior in both high and low frequency ranges. See an example in Fig. B.6. We can see that an efficient reduction of noise for high frequencies can be obtained by combining a Gaussian filter with $\sigma=1.6$ and finite differences with $M=1$ (red line). However, this reduction is very gradual over a long range of frequencies, a not convenient behaviour inherited from the wide transition band of the Gaussian filter. Wider Gaussian filters $(\sigma=3.0)$ and longer stencils for the finite differences $(M=2)$ keep the same behaviour (purple line), although with additional noise reduction and distorted derivatives in large scales. Single pass first order derivatives with S-G filters (green, brown and dark blue lines) reduce, but do not attenuate completely the high frequency noise. But in comparison with a combination of Gaussian filter and finite difference, the specific frequency in which the curve change from the ideal differentiator (in low frequencies) to block noise (in high frequencies) is much more localized (steeper slope). This is a very nice property since it allows to define more precisely the separation between small and large scales. This transition gets steeper for wider filter widths or polynomial order (compare with the brown and dark blue lines). But the best of both worlds can be obtained by combining a single pass derivative with SG filter (with $M=10$ and $P=2$ ) and Gaussian smoothing afterwards (with $\sigma=3.0$ ), as shown in light blue line. The noise is completely smoothed 
out in the high frequency range (inherited from the Gaussian filter), while the frequency determining the change from differentiator to block noise is more localized than the Gaussian one (inherited from the SG filter), in the sense of a steeper slope (although it is not so localized as for the pure $S-G$ filter).

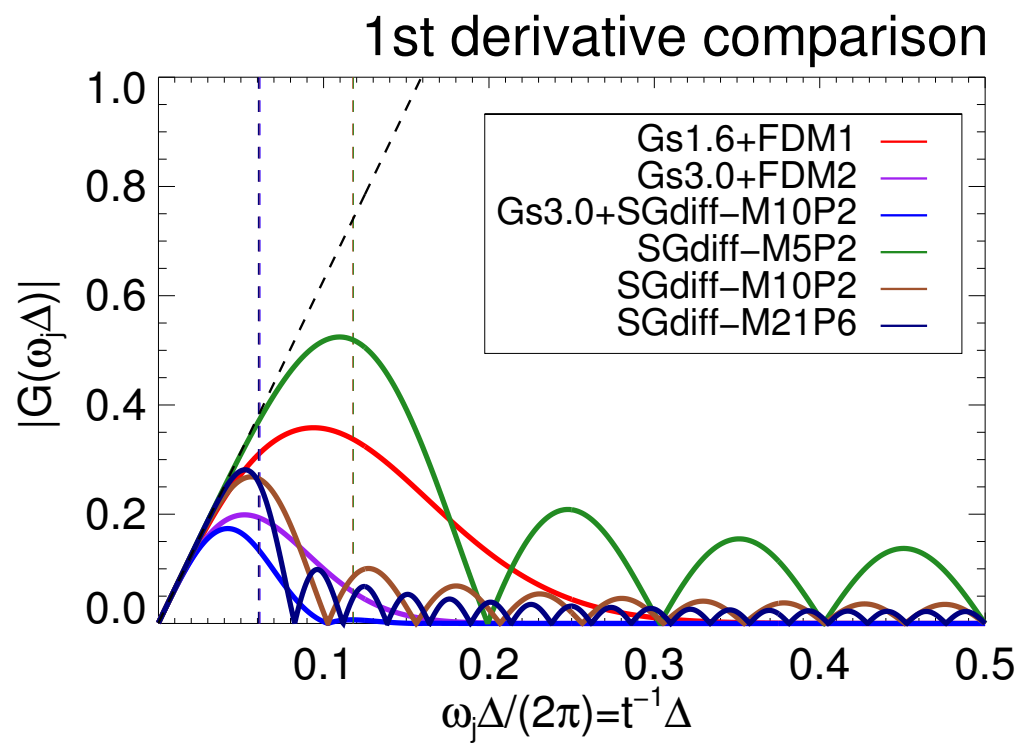

Figure B.6: Comparison of frequency responses for different combination of derivatives via convolution in the frequency domain. The dashed line indicates the frequency response of an ideal differentiator $\left(i \omega_{j}\right)$. The single pass 1st order derivatives with Savitzky-Golay filters are indicated with their corresponding width $M$ and polynomial order $P$ in green, brown and dark blue lines. For the rest of combinations, see explanation in the main text.

\section{B.2 Some calculation methods}

\section{B.2.1 Higher order momenta of the distribution function}

Although not used so often, higher order momenta of the distribution function (i.e., higher than two) can reveal important physical information in the system. But instead of using directly the VDF and associated momenta with physical dimensions, it is more convenient to work with dimensionless units and the terminology used in statistics.

In order to define the higher order momenta, we need to start with the definitions of the lower ones. The moments or order zero and one are identical to the described in Eq. (2.3) and Eq. (2.4), repeated here for the sake of clarity and to emphasize the terminology to be used:

$$
V=\langle v\rangle=\frac{1}{n} \int_{-\infty}^{\infty} v f d v, \quad n=\int_{-\infty}^{\infty} f d v
$$

where we have assumed 1D VDF $f=f(v)$. The moment of order 1 is usually called the expectation value of $f$. The moment of order 2 of this VDF is called the variance $\sigma^{2}$, 
proportional to the trace of the pressure tensor Eq. (2.5):

$$
\sigma^{2}=\left\langle(v-\langle v\rangle)^{2}\right\rangle=\left\langle v^{2}\right\rangle-\langle v\rangle^{2}:=\frac{1}{n} \int_{-\infty}^{\infty}(v-V)^{4} f d v
$$

With this we can define the normalized excess kurtosis $K$, proportional to the fourth moment of the VDF: 11

$$
K=\frac{\left\langle(v-V)^{4}\right\rangle}{\left(\sigma^{2}\right)^{2}}-3=\frac{1}{\left(\sigma^{2}\right)^{2}} \frac{1}{n} \int_{-\infty}^{\infty}(v-V)^{4} f d v-3
$$

the -3 in the definition Eq. (B.67) makes the kurtosis of a Maxwellian (Gaussian) distribution equal to 0 . Kurtosis measures the flatness of a distribution function, the relative importance of its tails. $K>0$ indicates a more sharp "peaked" distribution ("leptokurtic") with "fatter" tails, while $K<0$ the opposite: a flatter distribution ("platykurtic") with a wider peak and "thinner" tails. Kurtosis has been used as a proxy of intermittent ("bursty") signals in fully developed turbulence (see, e.g., Servidio et al. 2011, Wan et al. 2012, Leonardis 2013, Karimabadi et al. 2014), since these signals are characterized by "fatter" tails of probability density function for the electromagnetic fields $\vec{E}$ or $\vec{B}$. In a similar way, positive values of $K$ in a electron VDF might be signature of locations of particle acceleration (since it would indicate more particles at the tails).

Now, in order to extend these definitions to a full 3D geometry, we need to define the covariance $\sigma_{i j}^{2}$ as a a $3 \times 3$ matrix, with 6 independent components:

$$
\sigma_{i j}^{2}=\left\langle\left(v_{i}-\left\langle v_{j}\right\rangle\right)^{2}\right\rangle=\left\langle v_{i} v_{j}\right\rangle-\left\langle v_{i}\right\rangle\left\langle v_{j}\right\rangle:=\frac{1}{n} \int_{-\infty}^{\infty}\left(v_{i}-V_{i}\right)\left(v_{j}-V_{j}\right) f d v^{3}
$$

The pressure tensor $P_{i j}(\sim$ thermal energy density) can be written in terms of the covariance as:

$$
P_{i j}=n k_{B} T_{i j}=m n \sigma_{i j}^{2}=m \int_{-\infty}^{\infty}\left(v_{i}-V_{j}\right)\left(v_{j}-V_{j}\right) f d^{3} v
$$

where $k_{B} T_{i j}=m \sigma_{i j}^{2}$ is the temperature tensor ( average thermal energy in $i$ and $j$ direction per physical particle). Thus, the kurtosis becomes a $4 \times 4$ tensor with 15 independent components:

$$
\begin{aligned}
K_{i j k l} & =\frac{\left\langle\left(v_{i}-V_{i}\right)\left(v_{j}-V_{j}\right)\left(v_{k}-V_{k}\right)\left(v_{l}-V_{l}\right)\right\rangle}{\sigma_{i j}^{2} \sigma_{k l}^{2}}-3 \\
& =\frac{1}{\sigma_{i j}^{2} \sigma_{k l}^{2}} \frac{1}{n} \int_{-\infty}^{\infty}\left(v_{i}-V_{i}\right)\left(v_{j}-V_{j}\right)\left(v_{k}-V_{k}\right)\left(v_{l}-V_{l}\right) f d v^{3}-3
\end{aligned}
$$

\footnotetext{
${ }^{11}$ Although it is not used in this thesis, it is interesting to mention that the third order statistical moment of a VDF is called skewness, measuring the asymmetry of the distribution function. Physically, it is proportional to the heat tensor $Q_{i j k}$, whose trace is the heat flux $q_{i}=\sum_{j} Q_{i j j}$ that it is related with the transport of energy.
} 
One way of simplifying the information contained in the kurtosis tensor is looking at its "diagonal" components (in the 4D space):

$$
K_{i}=\frac{\left\langle\left(v_{i}-V_{i}\right)^{4}\right\rangle}{\left(\sigma_{i i}^{2}\right)^{2}}-3=\frac{1}{\left(\sigma_{i i}^{2}\right)^{2}} \frac{1}{n} \int_{-\infty}^{\infty}\left(v_{i}-V_{i}\right)^{4} f d v^{3}-3
$$

where $i=x, y, z$. These quantities $K_{i}$ can be interpreted as the equivalent 1D kurtosis (Eq. (B.67)) of a 1D reduced electron distribution function.

In order to calculate all these quantities with (discrete) particles in the running time diagnostics, it is convenient to use one-pass algorithms, in order to avoid to loop twice or more over all the particles in each cell (the computationally more expensive part of the calculation). Therefore, the previous momenta of the distribution function have to be rearranged in the following way:

$$
\begin{aligned}
V_{i} & =\frac{1}{N} \sum_{l=1}^{N} v_{i}^{l} \\
\sigma_{i j}^{2} & =\frac{1}{N} \sum_{l=1}^{N}\left(v_{i}^{l}-V_{i}\right)\left(v_{j}^{l}-V_{j}\right)=\frac{1}{N} \sum_{l=1}^{N} v_{i}^{l} v_{j}^{l}-V_{i} V_{j} \\
Q_{i i i} & =\frac{1}{N} \sum_{l=1}^{N}\left(v_{i}^{l}-V_{i}\right)^{3}=\frac{1}{N} \sum_{l=1}^{N}\left(v_{i}^{l}\right)^{3}-3 V_{i} \sigma_{i i}^{2}-V_{i}^{3} \\
K_{i} & =\frac{1}{\left(\sigma_{i i}^{2}\right)^{2}} \frac{1}{N} \sum_{l=1}^{N}\left(v_{i}^{l}-V_{i}\right)^{4}-3 \\
& =\frac{1}{\left(\sigma_{i i}^{2}\right)^{2}} \frac{1}{N} \sum_{l=1}^{N}\left[v_{i}^{l}-4\left(v_{i}^{l}\right)^{3} V_{i}+6\left(v_{i}^{l}\right)^{2} V_{i}^{2}-4\left(v_{i}^{l}\right) V_{i}^{3}+V_{i}^{4}\right]- \\
& =\frac{1}{\left(\sigma_{i i}^{2}\right)^{2}}\left[\frac{1}{N} \sum_{l=1}^{N}\left(v_{i}^{l}\right)^{4}-4 V_{i} Q_{i i i}-6 V_{i}^{2} \sigma_{i i}^{2}-V_{i}^{4}\right]-
\end{aligned}
$$

with the index $l$ running over each one of the total $N$ macroparticles inside and around the grid cell $\Delta x$ contributing to the weighting of the momenta (depending on the shape function, slightly above $\Delta x$. See Sec. 6.2 for further details). Note that all the previous expressions are calculated in terms of central order momenta defined by $\sum_{l=1}^{N}\left(v_{i}^{l}\right)^{n}$, with $n=0,1,2,3,4$. In addition, the previous computed lower order momenta have been used in each case by means of recurrence relations. These running time diagnostics for the 4th order momenta kurtosis (as well as for the 3rd order momenta: heat flux) are implemented in the ACRONYM code.

\section{B.2.2 Reconnection rate}

It is not too practical to calculate reconnection rates by using directly the definition Eq. (4.2), since $E_{z}$ is highly sensitive to noise in $2 \mathrm{D}$ simulations. This can be avoided by averaging over time scales $T$ such as $\omega_{p e}^{-1} \ll T \ll \Omega_{c i}$, but there is still the uncertainty about the specific temporal length of the average that might change the final result. For 
that reason, we calculate reconnection rates with the definition based in the magnetic flux Eq. (4.1). By introducing the vector potential $\vec{B}=\nabla \times \vec{A}$ in our 2D configuration (no variations in the $z$ direction):

$$
B_{x}=\frac{\partial A_{z}}{\partial y} \quad B_{y}=-\frac{\partial A_{z}}{\partial x}
$$

we can notice that the reconnected flux density is always path-independent in a 2D configuration provided that the normal to the surface $d \vec{S}$ is on-the-plane $x-y$ (see, e.g., (Somov 2013))

$$
d \psi^{\prime}=\vec{B} \cdot d \vec{S}=B_{y} d x-B_{x} d y=\frac{\partial A_{z}}{\partial x} d x+\frac{\partial A_{z}}{\partial y} d y=d A_{z}
$$

or, equivalently,

$$
\psi^{\prime}=\frac{1}{\Delta z} \oint \vec{A} \cdot d \vec{l}=\frac{1}{\Delta z}\left(-\int_{O+}^{O-} A_{z} d z-\int_{O-}^{X-} A_{y} d y+\int_{X-}^{X+} A_{z} d z+\int_{X+}^{O+} A_{y} d y\right)=A_{z}(X)-A_{z}(O)
$$

$\frac{d \psi}{d t}=\frac{d}{d t}\left(A_{z}(X)-A_{z}(O)\right)$

In other words, the reconnection rate is the rate of variation of the difference in the vector potential between the X and O point. Since the PIC code ACRONYM works with fields and not potentials, it is convenient to obtain $A_{z}$ by means of

$$
A_{z}(x, y)=\int B_{x}(x, y) d y+\operatorname{const}(x), \quad A_{z}(x, y)=-\int B_{y}(x, y) d x+\operatorname{const}(y)
$$

note that this can be applied everywhere, not only in the center of the CS.

\section{B.2.3 Tearing mode growth rates}

We compute growth rates from the vector potential following the standard definition (see, e.g., Katanuma and Kamimura 1980, Matsui 2008). The idea is, first, to obtain to Fourier transform $A_{z}(x, y, t)$ along the periodic direction $y$ for each position $x$ :

$$
\left|\tilde{A}_{z}\left(x, k_{y}\right)\right|=\left|\frac{1}{L_{y}} \int_{0}^{L_{y}} A_{z}(x, y) \exp \left(-i k_{y} y\right) d y\right|=\left|\tilde{A}_{z}(x, m)\right|, \quad \text { with } k_{y}=2 \pi m / L_{y}
$$

with $m$ the mode number, and represent how many entire wavelengths fit in the entire simulation box size in $y$ direction. It is convenient to average over some range $\Delta x$ in the $x$ direction to diminish noise effects. We chose to average over the electron singular layer $\Delta x=\Delta_{N s}=\sqrt{2 \rho_{e} L}$ (see Karimabadi 2005, Matsui and Daughton 2008). In this way, we have the $m$ Fourier modes for each CS. For example, if the center of a current sheet is located at $x_{0}$, we have:

$$
\left|\tilde{A_{z}}\left(x_{0}, m\right)\right|=\frac{1}{2 \Delta x} \int_{x_{0}-\Delta x}^{x_{0}+\Delta x}\left|\tilde{A}_{z}(x, m)\right|=\left|\frac{1}{2 \Delta x L_{y}} \int_{x_{0}-\Delta x}^{x_{0}+\Delta x} \int_{0}^{L_{y}} A_{z}(x, y) \exp \left(-\frac{i 2 \pi m y}{L_{y}}\right) d y\right|
$$


It also convenient to calculate the total power up to mode number $n$ :

$$
P(n)=\sum_{m=1}^{m=n}\left|\tilde{A}_{z}\left(x_{0}, m\right)\right|
$$

\section{B.2.4 Discrete coarse-grained entropy}

The information entropy as defined by Eq. (6.46) is calculated by means of a histogram estimator (Moddemeijer 1989):

$$
S \approx-\sum_{i, j, k}^{N_{x}, N_{y}, N_{z}} \rho_{i, j, k} \ln \rho_{i, j, k}+3 \ln (\Delta v),
$$

where $\rho_{i, j, k}=f_{i, j, k} \Delta v^{3}$ are the bin-related probabilities of the macroparticles to occupy the cell $(i, j, k)$ in the velocity space with a bin size $\Delta v^{3}$. In Eq. (B.87) $f_{i, j, k}$ is simply the discrete approximation of the continuous distribution function $f(\vec{v}, t)$ in each cell. Note that the entropy Eq. (B.87) is defined up to a constant offset depending on the relative units of $\Delta v$ (in our case, in units of the speed of light $c$ ). Since we are using natural logarithms, the units of $S$ are nats (1.44 bits). See Moddemeijer (1989) for details about the calculation of accuracy, introduced bias and variance in the relation Eq. (B.87).

\section{B.2.5 Rotation of pressure tensor}

In order to calculate consistently the temperatures anisotropies and other related quantities, it is necessary to define precisely the parallel $(\|)$ and perpendicular $(\perp)$ directions to the magnetic field for the pressure tensor. Let $P_{i j}$ be the full tensor pressure in the "simulation" reference frame $(\hat{x}, \hat{y}, \hat{z})$ and $\hat{b}=\vec{B} / B$ the unitary vector in the direction of the local magnetic field. In the simulation frame the diagonal elements of the pressure tensor can be written, without loss of generality, as $P_{i j}=\operatorname{diag}\left(P_{\perp}, P_{\perp}, P_{\|}\right)$, in such a way that $P_{\perp}=\left(P_{x x}+P_{y y}\right) / 2$ and $P_{\|}=P_{z z}$. We can choose another reference frame $\left(\hat{x}^{\prime}, \hat{y}^{\prime}, \hat{z}^{\prime}\right)$ where one of its axis ( $(\hat{z})$ is parallel to $\hat{b}$ and thus to construct the rotation matrix $R$ (with elements given by the cosines of the angles between the old and new reference frames). Note that only three out nine components of the matrix $R$ are defined by $\hat{b}$, but we do not need the remaining ones for our purposes (this is a consequence of the two additional degrees of freedom by fixing only one out three of the rotation axis). Then, the pressure tensor in the new reference frame can be calculated as

$$
P_{i j}^{\prime}=R_{i k} P_{k l} R_{l j}^{T}
$$

with components given by:

$$
\begin{aligned}
P_{x x}^{\prime} & =P_{y y}^{\prime}=\frac{1}{2}\left[\sum_{i} P_{i i}-\sum_{i} \sum_{j} b_{i} b_{j} P_{i j}\right]=P_{\perp}^{\prime} \\
P_{z z}^{\prime} & =\sum_{i} \sum_{j} b_{i} b_{j} P_{i j}=P_{\|}^{\prime}
\end{aligned}
$$


where $i=x, y, z$ and $R^{T}$ is the transpose matrix of $R$. Although there are six arbitrary terms in $R_{i j}$, they are not needed for the diagonal components of $P_{i j}^{\prime}$, since the trace is invariant under orthogonal transformations: $\operatorname{trace}\left(P_{i j}\right)=P_{x x}+P_{y y}+P_{z z}=\operatorname{trace}\left(P_{i j}^{\prime}\right)$. Therefore, all the results concerning temperatures anisotropies will be calculated according to the previous definition of $P_{\|}^{\prime}$ and $P_{\perp}^{\prime}$, since the parallel direction will always be in the direction of the local magnetic field (and we will drop off all the prime superscripts from now onwards).

\section{B.2.6 Non-Gyrotropy of the pressure tensor}

In general, distributions functions in collisionless plasmas tend to be gyrotropic, i.e., symmetric around the magnetic field direction. This implies that the tensor pressure $G_{i j}$ of these gyrotropic distributions can always be decomposed in two parts:

$$
G_{i j}=G_{\perp} \mathbb{I}_{i j}+\left(G_{\perp}-G_{\|}\right) \hat{b}_{i} \hat{b}_{j}
$$

where $G_{\|}=G_{i j} \hat{b}_{i} \hat{b}_{j}$ is the pressure parallel to the magnetic field lines and $G_{\perp}=\left(\operatorname{trace}\left(G_{i j}\right)-\right.$ $\left.G_{\|}\right) / 2$. Note that this definition coincides with the components of a general tensor pressure in a reference frame locally aligned with the magnetic field, given by Eq. (B.89), providing that $P_{i j}$ is diagonal in the simulation reference frame.

A velocity distribution function with an arbitrary pressure tensor $P_{i j}$ can always be decomposed as $P_{i j}=G_{i j}+N_{i j}$, where $G_{i j}$ is the gyrotropic part given by Eq. (B.91) and $N_{i j}$ is the nongyrotropic part. According to Hesse et al. (2014), $G_{i j}$ represents physically a pressure tensor of a gyrotropic distribution function with the same thermal energy as the full distribution $P_{i j}$. The relative importance of $N_{i j}$ with respect to $G_{i j}$ can be calculated by means of the Frobenius norm of those tensors, defined as

$$
\|A\|=\sqrt{\sum_{i=1}^{3} \lambda_{i}}=\sqrt{\operatorname{trace}\left(A^{T} A\right)}=\sqrt{\sum_{i=1}^{3} \sum_{j=1}^{3} A_{i j}}
$$

where $\lambda_{i}$ are the eigenvalues of $A_{i j}$. Due to the last equality, we can avoid the calculation of the eigenvalue problem. Then, the degree of non-gyrotropy of $P_{i j}$ (deviations from the expression Eq. (B.91)) can be estimated by means of the definition given in Hesse et al. (2014):

$$
D_{n g}=\frac{\|N\|}{\|G\|}=\frac{\sqrt{\sum_{i=1}^{3} \sum_{j=1}^{3} N_{i j}}}{\operatorname{trace}\left(G_{i j}\right)}
$$

where $D_{n g}$ is the degree of anisotropy for a velocity distribution function with an arbitrary pressure tensor. The norm of $G$ has that form since it is a diagonal matrix by construction.

Finally, it is important to mention other alternative ways of computing the non-gyrotropy of the VDF. Many of them are based in calculating first the eigenvalues of the pressure tensor in order to find their main and secondary axis and thus its proper frame of reference. But usually these matrix methods turn out to be computationally more expensive, especially if they are done during the running time of a PIC code. For further details, see Servidio et al. (2014). 


\section{Bibliography}

Abe, H., Miyamoto, J., and Itatani, R. Grid effects on the plasma simulation by the finitesized particle. J. Comput. Phys., 19, 134-149 (1975). (cited in page 132, 140)

Abel, I. G., Plunk, G. G., Wang, E., Barnes, M., Cowley, S. C., Dorland, W., and Schekochihin, A. A. Multiscale gyrokinetics for rotating tokamak plasmas: fluctuations, transport and energy flows. Reports Prog. Phys., 76,116201 (2013). (cited in page 247)

Abraham-Shrauner, B. Force-free Jacobian equilibria for Vlasov-Maxwell plasmas. Phys. Plasmas, 20, 102117,(2013). (cited in page 46)

Ahedo, E. and Ramos, J. J. Parametric analysis of the two-fluid tearing instability. Plasma Phys. Control. Fusion, 51, 055018 (2009). (cited in page 81)

Ahnert, K. and Abel, M. Numerical differentiation of experimental data: local versus global methods. Comput. Phys. Commun., 177, 764-774 (2007). (cited in page 209, 317, 318,

Alfvén, H. Existence of electromagnetic-hydrodynamic waves. Nature, 150, 405-406 (1942). (cited in page 36)

Alves, E. P., Grismayer, T., Martins, S. F., Fiúza, F., Fonseca, R. a., and Silva, L. O. Large-Scale Magnetic Field Generation Via The Kinetic Kelvin-Helmholtz Instability In Unmagnetized Scenarios. Astrophys. J., 746, L14 (2012). (cited in page 99, 262)

Alves, E. P., Grismayer, T., Fonseca, R., and O Silva, L. Electron-scale shear instabilities: magnetic field generation and particle acceleration in astrophysical jets. New J. Phys., 16,035007 (2014). (cited in page 99, 100, 112, 262)

Ambrosiano, J., Lee, L. C., and Fu, Z. F. Simulation of the collisionless tearing instability in an anisotropic neutral sheet. J. Geophys. Res., 91, 113 (1986). (cited in page 59)

Ambrosiano, J. J., Lee, L. C., and Swift, D. W. Simulation of the ion tearing instability in the presence of a background plasma. J. Geophys. Res., 88, 7860(1983). (cited in page 155)

Aschwanden, M. J. Physics of the Solar Corona. Springer Praxis Books. Springer Berlin Heidelberg (2005). (cited in page 13) 
Baalrud, S. D., Bhattacharjee, A., and Huang, Y.-M. Reduced magnetohydrodynamic theory of oblique plasmoid instabilities. Phys. Plasmas, 19,022101 (2012). (cited in page 81.

Balikhin, M. and Gedalin, M. Generalization of the Harris current sheet model for nonrelativistic, relativistic and pair plasmas. J. Plasma Phys., 74,749 (2008). (cited in page 43.

Balogh, A., Bykov, A., Cargill, P., and Dendy, R. Microphysics of Cosmic Plasmas, volume 47. Springer (2014). (cited in page 20)

Baumjohann, W. and Treumann, R. A. Basic space plasma physics. Imperial College Press ; Distributed by World Scientific Pub. (1997). (cited in page 18, 25, 26, 28, 30, 34, 42)

Baumjohann, W., Nakamura, R., and Treumann, R. A. Magnetic guide field generation in collisionless current sheets. Ann. Geophys., 28,789-793(2010). (cited in page 88, 89, 90, 163)

Bellan, P. M. Fundamentals of Plasma Physics. Cambridge University Press, Cambridge, UK ; New York (2006). (cited in page 25, 33, 42, 76)

Bernstein, I. Waves in a Plasma in a Magnetic Field. Phys. Rev.,109, 10-21(1958). (cited in page 104)

Bessho, N. and Bhattacharjee, A. Collisionless reconnection in an electron-positron plasma. Phys. Rev. Lett., 245001, 23-26 (2005). (cited in page 58)

Birdsall, C. K. and Fellow, L. Particle-in-Cell charged-particle simulations, Plus Monte Carlo collision with neutral atom, PIC-MCC. IEEE Trans. Plasma Sci., 19,65-85 (1991). (cited in page 119, 133)

Birdsall, C. and Langdon, A. B. Plasma Physics via Computer Simulation. IOP Publishing, Bristol, England (1991). (cited in page 106, 119, 120, 127, 128, 130, 132, 133, 136, 137, 138, 139, 140, 141, 143, 144, 163, 305, 310,

Birn, J. Forced magnetic reconnection. Geophys. Res. Lett.,32, L06105(2005). (cited in page 55)

Birn, J. and Hesse, M. Reconnection rates in driven magnetic reconnection. Phys. Plasmas, 14,082306 (2007). (cited in page 49)

Birn, J., Drake, J. F., Shay, M. A., Rogers, B. N., Denton, R. E., Hesse, M., Kuznetsova, M., Ma, Z. W., Bhattacharjee, A., Otto, A., and Pritchett, P. L. Geospace Environmental Modeling (GEM) Magnetic Reconnection Challenge. J. Geophys. Res., 106, 3715-3719 (2001). (cited in page 54, 68, 152, 192)

Biskamp, D. and Schwarz, E. Localization, the clue to fast magnetic reconnection. Phys. Plasmas, 8, 4729 (2001). (cited in page 60) 
Biskamp, D. Magnetic Reconnection in Plasmas. Cambridge University Press, Cambridge ; New York (2000). (cited in page 49, 53, 63, 76, 77)

Biskamp, D., Schwarz, E., and Drake, J. F. Two-fluid theory of collisionless magnetic reconnection. Phys. Plasmas, 4, 1002(1997). (cited in page 67)

Bittencourt, J. Fundamental of Plasma Physics. Springer (2004). (cited in page 25, 28)

Bobrova, N. a., Bulanov, S. V., Sakai, J. I., and Sugiyama, D. Force-free equilibria and reconnection of the magnetic field lines in collisionless plasma configurations. Phys. Plasmas, 8, 759-768,(2001). (cited in page 43, 44, 45, 80,

Bohlin, H., Von Stechow, A., Rahbarnia, K., Grulke, O., and Klinger, T. VINETA II: A linear magnetic reconnection experiment. Rev. Sci. Instrum., 85, 023501(2014). (cited in page 18)

Boine-Frankenheim, O., Hofmann, I., Struckmeier, J., and Appel, S. Artificial collisions, entropy and emittance growth in computer simulations of intense beams. Nucl. Inst. Methods Phys. Res. A, 770, 164-168 (2015). (cited in page 144)

Boris, J. P. Relativistic Plasma Simulation - Optimization of a Hybrid Code. In J. Boris, editor, Proc. Fourth Conf. Numer. Simul. Plasmas, Washingt. DC, pages 3-67. Naval Research Laboratory (1970). (cited in page 127, 136)

Bowers, K. J., Albright, B. J., Yin, L., Bergen, B., and Kwan, T. J. T. Ultrahigh performance three-dimensional electromagnetic relativistic kinetic plasma simulation. Phys. Plasmas, 15, 055703 (2008). (cited in page 136)

Bowers, K. J., Albright, B. J., Yin, L., Daughton, W., Roytershteyn, V., Bergen, B., and Kwan, T. J. T. Advances in petascale kinetic plasma simulation with VPIC and Roadrunner. J. Phys. Conf. Ser., 180,012055 (2009). (cited in page 145)

Boyd, T. and Sanderson, J. The Physics of Plasmas. Cambridge University Press (2003). (cited in page 15, 25, 28, 32, 34)

Brackbill, J. U. Particle methods. Int. J. Numer. Methods Fluids, 47, 693-705(2005). (cited in page 119)

Brackbill, J. and Forslund, D. An implicit method for electromagnetic plasma simulation in two dimensions. J. Comput. Phys., 46, 271-308 (1982). (cited in page 138)

Bret, A. Weibel, Two-Stream, Filamentation, Oblique, Bell, Buneman...Which One Grows Faster? Astrophys. J., 699, 990-1003 (2009). (cited in page 89, 192)

Bret, A. and Dieckmann, M. E. How large can the electron to proton mass ratio be in particle-in-cell simulations of unstable systems? Phys. Plasmas, 17,032109 (2010). (cited in page 192)

Bret, A., Firpo, M.-C., and Deutsch, C. Electromagnetic instabilities for relativistic beamplasma interaction in whole $\mathrm{k}$ space: Nonrelativistic beam and plasma temperature effects. Phys. Rev. E, 72,016403 (2005). (cited in page 89) 
Bret, A. Collisional behaviors of astrophysical collisionless plasmas. J. Plasma Phys., 81,1-19 (2015). (cited in page 99)

Brittnacher, M., Quest, K. B., and Karimabadi, H. A new approach to the linear theory of single-species tearing in two-dimensional quasi-neutral sheets. J. Geophys. Res., 100, 3551 (1995). (cited in page 75, 190)

Brizard, A. J. and Hahm, T. S. Foundations of nonlinear gyrokinetic theory. Rev. Mod. Phys., 79, 421-468 (2007). (cited in page 229, 231)

Bromba, M. U. a. and Ziegler, H. Application hints for Savitzky-Golay digital smoothing filters. Anal. Chem., 53, 1583-1586(1981). (cited in page 315, 316)

Brown, M. R., Browning, P. K., Dieckmann, M. E., Furno, I., and Intrator, T. P. Microphysics of Cosmic Plasmas: Hierarchies of Plasma Instabilities from MHD to Kinetic. Space Sci. Rev., pages 1-27(2013). (cited in page 104)

Büchner, J. About the third integral of charged particle motion in strongly curved magnetic fields. Astron. Nachrichten A J. all Fields Astron., 307, 191-199 (1986). (cited in page 73 (1)

Büchner, J. Locating Current Sheets in the Solar Corona. Space Sci. Rev., 122, 149-160 (2006). (cited in page 16)

Büchner, J. Astrophysical reconnection and collisionless dissipation. Plasma Phys. Control. Fusion, 49, B325-B339 (2007). (cited in page 14)

Büchner, J. and Zelenyi, L. M. Chaotization of the electron motion as the cause of an internal magnetotail instability and substorm onset. J. Geophys. Res., 92,13456(1987). (cited in page 60, 86)

Büchner, J. and Daughton, W. Basic theory of collisionless reconnection. In Birn, J. and Priest, E. R., editors, Reconnect. Magn. Fields Magnetohydrodinamics Collisionless Theory Obs., chapter 3, pages 87-166. Cambridge Univ. Press (2007). (cited in page 16)

Büchner, J. and Elkina, N. Vlasov Code Simulation of Anomalous Resistivity. Space Sci. Rev., 121, 237-252 (2006). (cited in page 63)

Büchner, J. and Nikutowski, B. Acceleration of the fast solar wind by reconnection. In Sol. Wind 11/SOHO 16, Connect. Sun Heliosph., pages 141-146 (2005). (cited in page 17)

Büchner, J. and Zelenyi, L. M. Regular and chaotic charged particle motion in magnetotaillike field reversals: 1. Basic theory of trapped motion. J. Geophys. Res., $\mathbf{9 4 , 1 1 8 2 1}$ (1989). (cited in page 164)

Büchner, J. and Zelenyi, L. M. Regular and chaotic particle motion in sheared magnetic field reversals. Adv. Sp. Res., 11, 177-182 (1991). (cited in page 73)

Büchner, J., Kuznetsova, M., and Zelenyi, L. M. Sheared field tearing mode instability and creation of flux ropes in the Earth magnetotail. Geophys. Res. Lett., 18,385-388 (1991). (cited in page 80) 
Büchner, J., Dum, C., and Scholer, M. Space Plasma Simulation. Springer-Verlag, Berlin Heidelberg (2003). (cited in page 119, 121)

Buneman, O. Dissipation of Currents in Ionized Media. Phys. Rev.,115, 503-517(1959). (cited in page 119)

Buneman, O. Instability of electrons drifting through ions across a magnetic field. J. Nucl. Energy. Part C, Plasma Physics, Accel. Thermonucl. Res., 4, 111-117(1962). (cited in page 105)

Buneman, O. Models of Collisionless Shock Fronts. Phys. Fluids, 7, S3 (1964). (cited in page 143, 163,

Burau, H., Widera, R., Honig, W., Juckeland, G., Debus, A., Kluge, T., Schramm, U., Cowan, T. E., Sauerbrey, R., and Bussmann, M. PIConGPU: A Fully Relativistic Particle-in-Cell Code for a GPU Cluster. IEEE Trans. Plasma Sci., 38, 2831-2839 (2010). (cited in page 145)

Burkart, T. Simulation von Teilchenbeschleunigung durch die Weibel-Instabilität mit Hilfe eines PiC-Codes. Diploma thesis, University of Würzburg (2007). (cited in page 147, 149.

Burkart, T. Der Einfluss des fundamentalen Massenverhältnisses auf die Teilchenbeschleunigung durch Plasmainstabilitäten. PhD thesis, University of Würzburg (2010). (cited in page 147, 149

Burkart, T., Elbracht, O., Ganse, U., and Spanier, F. The influence of the mass-ratio on the acceleration of particles by filamentation instabilities. Astrophys. J., 720, 1318-1324 (2010). (cited in page 149)

Burkhart, G. R. and Chen, J. Collisionless tearing instability of a bi-Maxwellian neutral sheet - An integrodifferential treatment with exact particle orbits. Phys. Fluids B Plasma Phys., 1, 1578(1989). (cited in page 84)

Cai, D., Storey, L. R. O., and Neubert, T. Kinetic equilibria of plasma shear layers. Phys. Fluids B Plasma Phys., 2, 75(1990). (cited in page 97, 98)

Cai, D., Storey, L. R. O., and Itoh, T. Particle simulation of the kinetic Kelvin-Helmholtz instability in a magnetoplasma. Phys. Fluids B Plasma Phys., 5, 3507(1993). (cited in page 97)

Califano, F., Prandi, R., Pegoraro, F., and Bulanov, S. V. Two-dimensional electronmagnetohydrodynamic instabilities. Phys. Plasmas, 6, 2332-2339 (1999). (cited in page 101)

Camporeale, E. and Burgess, D. Electron firehose instability: Kinetic linear theory and two-dimensional particle-in-cell simulations. J. Geophys. Res., 113, A07107 (2008). (cited in page 94) 
Camporeale, E. and Lapenta, G. Model of bifurcated current sheets in the Earth's magnetotail: Equilibrium and stability. J. Geophys. Res., 110, A07206 (2005). (cited in page $87,188,194$

Cassak, P. A. Theory and simulations of the scaling of magnetic reconnection with symmetric shear flow. Phys. Plasmas, 18,072106 (2011a). (cited in page 103, 255, 261)

Cassak, P. A. Scaling of the magnetic reconnection rate with symmetric shear flow. Phys. Plasmas, 18,074501 (2011b). (cited in page 103)

Cassak, P. A. and Shay, M. A. Magnetic Reconnection for Coronal Conditions: Reconnection Rates, Secondary Islands and Onset. Space Sci. Rev.,172, 283-302(2011). (cited in page 13, 15, 19, 49, 50, 69,

Cassak, P. A., Baylor, R. N., Fermo, R. L., Beidler, M. T., Shay, M. A., Swisdak, M., Drake, J. F., and Karimabadi, H. Fast magnetic reconnection due to anisotropic electron pressure. Phys. Plasmas, 22, 020705 (2015). (cited in page 59, 244, 265)

Cerri, S. S., Henri, P., Califano, F., Del Sarto, D., Faganello, M., and Pegoraro, F. Extended fluid models: Pressure tensor effects and equilibria. Phys. Plasmas, 20, 112112 (2013). (cited in page 98)

Cerri, S. S., Pegoraro, F., Califano, F., Del Sarto, D., and Jenko, F. Pressure tensor in the presence of velocity shear: Stationary solutions and self-consistent equilibria. Phys. Plasmas, 21, 112109 (2014). (cited in page 98)

Chacón, L., Knoll, D. a., and Finn, J. M. Hall MHD effects on the 2D KelvinHelmholtz/tearing instability. Phys. Lett. Sect. A Gen. At. Solid State Phys., 308, 187-197(2003). (cited in page 101)

Chacón, L., Simakov, A. N., Lukin, V. S., and Zocco, A. Fast reconnection in nonrelativistic 2D electron-positron plasmas. Phys. Rev. Lett., 101, 3-6 (2008). (cited in page 58

Chandrasekhar, S. Hydrodynamic and hydromagnetic stability. International Series of Monographs on Physics, Oxford (1961). (cited in page 95)

Channell, P. Exact Vlasov-Maxwell equilibria with sheared magnetic fields. Phys. Fluids, 19, 1541(1976). (cited in page 44, 45)

Che, H., Drake, J. F., and Swisdak, M. A current filamentation mechanism for breaking magnetic field lines during reconnection. Nature, 474, 184-7,(2011). (cited in page 48)

Chen, J., Palmadesso, P. J., Fedder, J. A., and Lyon, J. G. Fast collisionless tearing in an anisotropic neutral sheet. Geophys. Res. Lett.,11, 12-15(1984). (cited in page 84, 85, 93, 161, 162, 163, 170, 181, 194,

Chen, L., Daughton, W., and Bhattacharjee, A. In-plane electric fields in magnetic islands during collisionless magnetic reconnection. Phys. Plasmas, 19,112902 (2012). (cited in page 106, 246, 270) 
Chen, Q., Otto, A., and Lee, L. C. Tearing instability, Kelvin-Helmholtz instability, and magnetic reconnection. J. Geophys. Res., 102, 151 (1997). (cited in page 101)

Chen, S.-H., Le, G., and Fok, M.-C. Magnetospheric boundary perturbations on MHD and kinetic scales. J. Geophys. Res. Sp. Phys., 120, 113-137(2015). (cited in page 98 , 99, 105)

Chew, G. F., Goldberger, M. L., and Low, F. E. The Boltzmann Equation and the OneFluid Hydromagnetic Equations in the Absence of Particle Collisions. Proc. R. Soc. A Math. Phys. Eng. Sci., 236, 112-118 (1956). (cited in page 37)

Cole, J. B. A high-accuracy realization of the yee algorithm using non-standard finite differences. IEEE Trans. Microw. Theory Tech.,45, 991-996(1997). (cited in page 133)

Cole, J. B. High-accuracy yee algorithm based on nonstandard finite differences: New developments and verifications. IEEE Trans. Antennas Propag., 50, 1185-1191(2002). (cited in page 134)

Coppi, B., Laval, G., and Pellat, R. Dynamics of the Geomagnetic Tail. Phys. Rev. Lett., 16, 1207-1210 (1966). (cited in page 72, 154)

Cormier-Michel, E., Shadwick, B., Geddes, C. G. R., Esarey, E., Schroeder, C. B., and Leemans, W. Unphysical kinetic effects in particle-in-cell modeling of laser wakefield accelerators. Phys. Rev. E, 78,016404(2008). (cited in page 123, 138, 140, 141, 151, 158, 193)

Coroniti, F. V. Nonlinear Evolution of the Collisionless Tearing Mode. Phys. Rev. Lett., 38, 1355-1358 (1977). (cited in page 152)

Coroniti, F. V. On the tearing mode in quasi-neutral sheets. J. Geophys. Res., $\mathbf{8 5 , 6 7 1 9}$ (1980). (cited in page142,171)

Courant, R., Friedrichs, K., and Lewy, H. Über die partiellen Differenzengleichungen der mathematischen Physik. Math. Ann., 100, 32-74 (1928). (cited in page 135)

Cravens, T. Physics of solar system plasmas. Cambridge University Press (1997). (cited in page 25, 28, 31,

Das, A. and Kaw, P. Nonlocal sausage-like instability of current channels in electron magnetohydrodynamics. Phys. Plasmas, 8,4518 (2001). (cited in page 97)

Daughton, W., Roytershteyn, V., Karimabadi, H., Yin, L., Albright, B. J., Gary, S. P., Bowers, K. J., and Consistent, S. Secondary Island Formation in Collisional and Collisionless Kinetic Simulations of Magnetic Reconnection. In AIP Conf. Proc., volume 1320, page 144 (2011a). (cited in page 52, 58)

Daughton, W. The unstable eigenmodes of a neutral sheet. Phys. Plasmas, 6, 1329(1999). (cited in page 75, 190, 206)

Daughton, W. Electromagnetic properties of the lower-hybrid drift instability in a thin current sheet. Phys. Plasmas, 10, 3103(2003). (cited in page 75) 
Daughton, W. Kinetic theory of collisionless tearing at the magnetopause. J. Geophys. Res., 110, A03217 (2005). (cited in page 78, 79, 152)

Daughton, W. and Karimabadi, H. Collisionless magnetic reconnection in large-scale electron-positron plasmas. Phys. Plasmas, 14,072303 (2007). (cited in page 58)

Daughton, W., Lapenta, G., and Ricci, P. Nonlinear Evolution of the Lower-Hybrid Drift Instability in a Current Sheet. Phys. Rev. Lett., 93, 105004 (2004). (cited in page 84, 86, 87, 158)

Daughton, W., Scudder, J., and Karimabadi, H. Fully kinetic simulations of undriven magnetic reconnection with open boundary conditions. Phys. Plasmas, 13,072101 (2006). (cited in page 160)

Daughton, W., Roytershteyn, V., Karimabadi, H., Yin, L., Albright, B. J., Bergen, B., and Bowers, K. J. Role of electron physics in the development of turbulent magnetic reconnection in collisionless plasmas. Nat. Phys.,7, 539-542 (2011b). (cited in page 81)

Daughton, W., Nakamura, T. K. M., Karimabadi, H., Roytershteyn, V., and Loring, B. Computing the reconnection rate in turbulent kinetic layers by using electron mixing to identify topology. Phys. Plasmas, 21,052307(2014). (cited in page 145)

Davidson, R. C., Gladd, N. T., Wu, C. S., and Huba, J. D. Effects of finite plasma beta on the lower-hybrid-drift instability. Phys. Fluids, 20,301(1977). (cited in page 111 115, 116, 276,

Davidson, R. and Krall, N. Anomalous transport in high-temperature plasmas with applications to solenoidal fusion systems. Nucl. Fusion, 17, 1313-1372 (1977). (cited in page 63, 116

Davidson, R. C. Nonlinear Development of Electromagnetic Instabilities in Anisotropic Plasmas. Phys. Fluids, 15, 317(1972). (cited in page 89)

Davidson, R. C. Theory of nonneutral Plasmas. W.A. Benjamin, Inc. (1974). (cited in page 202

Davidson, R. C. Waves and instabilities in nonneutral plasmas. In AIP Conf. Proc., volume 175, pages 139-209 (1988). (cited in page 202, 270)

Davidson, R. C. and Gladd, N. T. Anomalous transport properties associated with the lower-hybrid-drift instability. Phys. Fluids, 18, 1327 (1975). (cited in page 114, 115, 276)

Dawson, J. One-Dimensional Plasma Model. Phys. Fluids, 5, 445-459 (1962). (cited in page 119, (120)

Dawson, J. M. Particle simulation of plasmas. Rev. Mod. Phys., 55, 403-447 (1983). (cited in page 119, 144)

Decyk, V. K. Skeleton PIC codes for parallel computers. Comput. Phys. Commun., 87, 87-94(1995). (cited in page 130, 146) 
Denavit, H. Time-Filtering Particle Simulations. J. Comput. Phys., 366, 337-366 (1981). (cited in page 138)

Divin, A., Khotyaintsev, Y. V., Vaivads, A., André, M., Markidis, S., and Lapenta, G. Evolution of the Lower Hybrid drift instability at reconnection jet front. J. Geophys. Res. Sp. Phys., 120, n/a-n/a (2015). (cited in page 117)

Dobrowolny, M. Instability of a neutral sheet. Nuovo Cim. B Ser. 10, 55, 427-442 (1968). (cited in page 73)

Drake, J. F. and Lee, Y. Nonlinear Evolution of Collisionless and Semicollisional Tearing Modes. Phys. Rev. Lett., 39, 453-456 (1977a). (cited in page 76, 77)

Drake, J. F. and Lee, Y. C. Kinetic theory of tearing instabilities. Phys. Fluids, 20, 1341 (1977b). (cited in page 72, 73, 74, 78, 79, 81, 82, 84)

Drake, J. F., Swisdak, M., Cattell, C., Shay, M. A., Rogers, B. N., and Zeiler, A. Formation of electron holes and particle energization during magnetic reconnection. Science, 299, 873-7 (2003). (cited in page 48, 67)

Drake, J. F., Swisdak, M., Schoeffler, K. M., Rogers, B. N., and Kobayashi, S. Formation of secondary islands during magnetic reconnection. Geophys. Res. Lett., 33, L13105 (2006). (cited in page 247)

Drummond, W. E. and Rosenbluth, M. N. Anomalous diffusion arising from microinstabilities in a plasma. Phys. Fluids, 5, 1507(1962). (cited in page 104, 105)

Dungey, J. W. Interplanetary magnetic field and the auroral zones. Phys. Rev. Lett., 6, 47-48 (1961). (cited in page 17)

Dungey, J. Conditions for the occurrence of electrical discharges in astrophysical systems. Philos. Mag. Ser. 7, 44, 725-738 (1953). (cited in page 49)

Eastwood, J. The virtual particle electromagnetic particle-mesh method. Comput. Phys. Commun., 64,252-266 (1991). (cited in page 131)

Eastwood, J. and Hockney, R. Shaping the force law in two-dimensional particle-mesh models. J. Comput. Phys., 16,342-359 (1974). (cited in page 123)

Egedal, J., Le, A., and Daughton, W. A review of pressure anisotropy caused by electron trapping in collisionless plasma, and its implications for magnetic reconnection. Phys. Plasmas, 20,061201(2013). (cited in page 53, 265)

Esirkepov, T. Exact charge conservation scheme for Particle-in-Cell simulation with an arbitrary form-factor. Comput. Phys. Commun., 135, 144-153 (2001). (cited in page131, 148)

Evstatiev, E. and Shadwick, B. Variational formulation of particle algorithms for kinetic plasma simulations. J. Comput. Phys., 245, 376-398 (2013). (cited in page 132) 
Fermo, R. L., Drake, J. F., and Swisdak, M. Secondary Magnetic Islands Generated by the Kelvin-Helmholtz Instability in a Reconnecting Current Sheet. Phys. Rev. Lett., 108, 255005(2012). (cited in page 102)

Filippychev, D. S. Hybrid Simulation Of Space Plasmas : Models With Massless Fluid Representation Of Electrons. IV. Kelvin - Helmholtz Instability. Comput. Math. Model., 13,119-142 (2002). (cited in page 98)

Fisk, L. a., Schwadron, N. a., and Zurbuchen, T. H. Acceleration of the fast solar wind by the emergence of new magnetic flux. J. Geophys. Res., 104, 19765(1999). (cited in page (17)

Fornberg, B. Generation of finite difference formulas on arbitrarily spaced grids. Math. Comput.,51, 699-706(1988). (cited in page 317)

Forslund, D., Morse, R., and Nielson, C. Electron cyclotron drift instability. Phys. Rev. Lett., 25, 1266 (1970). (cited in page 105, 106, 273)

Forslund, D., Morse, R., Nielson, C., and Fu, J. Electron Cyclotron Drift Instability and Turbulence. Phys. Fluids, 15, 1303-1318,(1972). (cited in page 106, 107, 273)

Forslund, D. W. A model of the plasma sheet in the earth's magnetosphere. PhD thesis, Princeton University (1968). (cited in page 82, 83, 84, 162)

Fried, B. D. Mechanism for Instability of Transverse Plasma Waves. Phys. Fluids, 2, 337 (1959). (cited in page 89)

Fried, B. D. and Conte, S. D. The Plasma Dispersion Function: the Hilbert transform of the Gaussian. Academic Pres (1961). (cited in page 75)

Friedberg, J. and Gerwin, R. Lower hybrid drift instability at low drift velocities. Phys. Fluids, 20, 1311-1315 (1977). (cited in page 115)

Frieman, E. A. and Chen, L. Nonlinear gyrokinetic equations for low-frequency electromagnetic waves in general plasma equilibria. Phys. Fluids, 25, 502-508(1982). (cited in page 229

Fu, W. Z. and Hau, L. N. Vlasov-Maxwell equilibrium solutions for Harris sheet magnetic field with Kappa velocity distribution. Phys. Plasmas, 12,1-4 (2005). (cited in page 43)

Fuchs, V. and Gunn, J. P. On the integration of equations of motion for particle-in-cell codes. J. Comput. Phys., 214, 299-315(2006). (cited in page 127)

Fujimoto, K. and Machida, S. An electron heating mechanism in the outflow region from the X-type neutral line. J. Geophys. Res. Sp. Phys., 108,1-11(2003). (cited in page 107, 112, 273, 280,

Fujimoto, K. and Sydora, R. D. Electromagnetic particle-in-cell simulations on magnetic reconnection with adaptive mesh refinement. Comput. Phys. Commun., 178, 915-923 (2008). (cited in page 137) 
Furth, H. P., Killeen, J., and Rosenbluth, M. N. Finite-Resistivity Instabilities of a Sheet Pinch. Phys. Fluids, 6, 459 (1963). (cited in page 69, 70, 71, 72, 73, 74, 84)

Galeev, A. Reconnection in the magnetotail. Space Sci. Rev., 23,411-425(1979). (cited in page 72 ,

Galeev, A. and Sudan, R. Basic Plasma Physics II. North-Holland Pub., Amsterdam ; New York : New York, N.Y (1984). (cited in page 16, 71, 73)

Ganguli, G., Lee, Y. C., and Palmadesso, P. J. Kinetic theory for electrostatic waves due to transverse velocity shears. Phys. Fluids, 31, 823 (1988). (cited in page 97, 98)

Ganguli, G., Keskinen, M. J., Romero, H., Heelis, R., Moore, T., and Pollock, C. Coupling of microprocesses and macroprocesses due to velocity shear: An application to the lowaltitude ionosphere. J. Geophys. Res., 99, 8873-8889 (1994). (cited in page 280)

Ganse, U., Kilian, P., Vainio, R., and Spanier, F. Emission of Type II Radio Bursts Single-Beam Versus Two-Beam Scenario. Sol. Phys., 280, 551-560 (2012a). (cited in page 149)

Ganse, U. Typ II Radiobursts bei koronalen Masseauswürfen - Simulation mit PiC-Codes. Diploma thesis, University of Würzburg (2009). (cited in page 147)

Ganse, U. Kinetische Simulationen solarer Typ II Radiobursts. PhD thesis, University of Würzburg (2012). (cited in page 147, 149)

Ganse, U., Burkart, T., Spanier, F., and Vainio, R. Kinetic simulations of solar type II radio burst emission processes. AIP Conf. Proc., 1216, 245-248 (2010). (cited in page 149)

Ganse, U., Kilian, P., Siegel, S., and Spanier, F. Numerical challenges in kinetic simulations of three-wave interactions. arXiv Prepr., 1207.6870 (2012b). (cited in page 149)

Ganse, U., Kilian, P., Spanier, F., and Vainio, R. Nonlinear Wave Interactions as Emission Process of Type II Radio Bursts. Astrophys. J.,751, 145(2012c). (cited in page 149)

Ganse, U., Kilian, P., Spanier, F., and Vainio, R. Fundamental and harmonic plasma emission in different plasma environments. Astron. Astrophys., [564, 15 (2014). (cited in page 149)

Gary, S. P. Longitudinal waves in a perpendicular collisionless plasma shock. I - Cold ions. J. Plasma Phys., 4,739-751,(1970). (cited in page 105, 113)

Gary, S. P. Theory of space plasma microinstabilities. Cambridge University Press (1993). (cited in page 19, 87, 90, 91, 94, 175,

Gary, S. P. and Cairns, I. H. Electron temperature anisotropy instabilities: Whistler, electrostatic and z mode. J. Geophys. Res., 104, 19835(1999). (cited in page 93)

Gary, S. P. and Karimabadi, H. Linear theory of electron temperature anisotropy instabilities: Whistler, mirror, and Weibel. J. Geophys. Res., 111, A11224(2006). (cited in page 92, 93) 
Gary, S. P. and Karimabadi, H. Fluctuations in electron-positron plasmas: Linear theory and implications for turbulence. Phys. Plasmas, 16,042104(2009). (cited in page 58)

Gary, S. P. and Li, H. Whistler Heat Flux Instability at High Beta. Astrophys. J., 529, 1131-1135 (2000). (cited in page 93)

Gary, S. P., Tokar, R. L., and Winske, D. A. N. Ion/ion and electron/ion cross-field instabilities near the lower hybrid frequency. J. Geophys. Res., 92, 10029(1987). (cited in page 108, 109,111,

Génot, V., Schwartz, S. J., Mazelle, C., Balikhin, M., Dunlop, M., and Bauer, T. M. Kinetic study of the mirror mode. J. Geophys. Res., 106,21611 (2001). (cited in page 90,

Germaschewski, K., Fox, W., Ahmadi, N., Wang, L., Abbott, S., Ruhl, H., and Bhattacharjee, A. The Plasma Simulation Code: A modern particle-in-cell code with loadbalancing and GPU support. arXiv Prepr., 1310.7866 (2013). (cited in page 145)

Gibbon, P., Speck, R., Karmakar, A., Arnold, L., Frings, W., Berberich, B., Reiter, D., and Mašek, M. Progress in mesh-free plasma simulation with parallel tree codes. IEEE Trans. Plasma Sci., 38, 2367-2376 (2010). (cited in page 121)

Giovanelli, R. G. A Theory of Chromospheric Flares. Nature, 158,81-82(1946). (cited in page 49.

Gladd, N. T. The lower hybrid drift instability and the modified two stream instability in high density theta pinch environments. Plasma Phys., 18, 27-40 (1976). (cited in page 114, 115, 116)

Godfrey, B. B. Numerical Cherenkov instabilities in electromagnetic particle codes. $J$. Comput. Phys., 15, 504-521(1974). (cited in page 134)

Godfrey, B. B. Canonical momenta and numerical instabilities in particle codes. J. Comput. Phys., 19, 58-76(1975). (cited in page 134)

Gorry, P. A. General least-squares smoothing and differentiation by the convolution (Savitzky-Golay) method. Anal. Chem.,62, 570-573 (1990). (cited in page 314)

Gosling, J. T. Magnetic Reconnection in the Solar Wind. Space Sci. Rev., 172, 187-200 (2011). (cited in page 17)

Graf von der Pahlen, J. and Tsiklauri, D. The effect of guide-field and boundary conditions on collisionless magnetic reconnection in a stressed X-point collapse. Phys. Plasmas, 21,012901 (2013). (cited in page 284)

Greco, A. and Taktakishvili, A. Ion dynamics in the near-Earth magnetotail: Magnetic turbulence versus normal component of the average magnetic field. J. Geophys. Res., 107, 1267 (2002). (cited in page 86) 
Greenwood, A. D., Cartwright, K. L., Luginsland, J. W., and Baca, E. a. On the elimination of numerical Cerenkov radiation in PIC simulations. J. Comput. Phys., 201, 665-684 (2004). (cited in page 134, 135, 147)

Grismayer, T., Alves, E. P., Fonseca, R. A., and Silva, L. O. Dc-magnetic-field generation in unmagnetized shear flows. Phys. Rev. Lett., 111,015005 (2013a). (cited in page 99 , 100, 262)

Grismayer, T., Alves, E. P., Fonseca, R. a., and Silva, L. O. Theory of multidimensional electron-scale instabilities in unmagnetized shear flows. Plasma Phys. Control. Fusion, 55, 124031 (2013b). (cited in page 99)

Gruzinov, A. GRB: magnetic fields, cosmic rays, and emission from first principles? arXiv Prepr., 0803.1182 (2008). (cited in page 99)

Guo, Y., Yi, L., Quan-ming, L., and Shui, W. A hybrid simulation study of magnetic reconnection in anisotropic plasmas. Chinese Astron. Astrophys., 27, 374-382 (2003). (cited in page 59)

Hadi, M. and Piket-May, M. A modified FDTD $(2,4)$ scheme for modeling electrically large structures with high-phase accuracy. IEEE Trans. Antennas Propag., 45, 254-264 (1997). (cited in page 134, 147)

Hadi, M. F. A finite volumes-based 3-D low dispersion FDTD algorithm. IEEE Trans. Antennas Propag., 55, 2287-2293 (2007). (cited in page 134 147)

Haijima, K., Tanaka, K., Fujimoto, M., and Shinohara, I. Electron temperature anisotropy effects on tearing mode in ion-scale current sheets. Adv. Sp. Res., 41, 1643-1648(2008). (cited in page 83)

Hall, A. N. Finite ion Larmor radius modifications to the firehose and mirror instabilities. J. Plasma Phys., 21, 431(1979). (cited in page 91)

Harlow, F. H. PIC and its progeny. Comput. Phys. Commun., 48,1-10.(1988). (cited in page 119)

Harris, E. G. On a plasma sheath separating regions of oppositely directed magnetic field. Nuovo Cim.,23, 115-121, (1962). (cited in page 40, 43, 152)

Harrison, M. G. and Neukirch, T. Some remarks on one-dimensional force-free VlasovMaxwell equilibria. Phys. Plasmas, 16,022106(2009a). (cited in page 45)

Harrison, M. G. and Neukirch, T. One-Dimensional Vlasov-Maxwell Equilibrium for the Force-Free Harris Sheet. Phys. Rev. Lett., 102, 135003 (2009b). (cited in page 40, 43, 44, 45, 46)

Haruki, T. and Sakai, J. I. Rapid dissipation of magnetic field energy driven by plasma flows in force-free collisionless pair plasmas. Phys. Plasmas, 8, 1538-1544 (2001). (cited in page 112) 
Hasegawa, A. Drift Mirror Instability in the Magnetosphere. Phys. Fluids, 12, 2642 (1969). (cited in page 90, 93, 175)

Hasegawa, A. Plasma Instabilities and Nonlinear Effects, volume 8 of Physics and Chemistry in Space. Springer Berlin Heidelberg, Berlin, Heidelberg (1975). (cited in page 91, 104, 105)

Haugbølle, T., Frederiksen, J. T., and Nordlund, A. photon-plasma: A modern high-order particle-in-cell code. Phys. Plasmas, 20,062904(2013). (cited in page 121, 123, 126, 127, 129, 130, 131,

Hededal, C. B. and Nishikawa, K.-I. The Influence of an Ambient Magnetic Field on Relativistic collisionless Plasma Shocks. Astrophys. J., 623, L89-L92 (2005). (cited in page 90,

Hellinger, P. Comment on the linear mirror instability near the threshold. Phys. Plasmas, 14,082105 (2007). (cited in page 91, 92)

Henri, P., Cerri, S. S., Califano, F., Pegoraro, F., Rossi, C., Faganello, M., Šebek, O., Trávníček, P. M., Hellinger, P., Frederiksen, J. T., Nordlund, A., Markidis, S., Keppens, R., and Lapenta, G. Nonlinear evolution of the magnetized Kelvin-Helmholtz instability: from fluid to kinetic modeling. Phys. Plasmas, 20,102118 (2013). (cited in page 98)

Hesse, M., Aunai, N., Birn, J., Cassak, P., Denton, R. E., Drake, J. F., Gombosi, T., Hoshino, M., Matthaeus, W., Sibeck, D., and Zenitani, S. Theory and Modeling for the Magnetospheric Multiscale Mission. Space Sci. Rev., pages 1-54(2014). (cited in page 17, 60, 61, 213, 265, 326

Hesse, M. and Zenitani, S. Dissipation in relativistic pair-plasma reconnection. Phys. Plasmas, 14, 112102(2007). (cited in page 202)

Hesse, M., Winske, D., and Birn, J. On the ion-scale structure of thin current sheets in the magnetotail. Phys. Scr., T74, 63-66(1998). (cited in page 87)

Hesse, M., Kuznetsova, M., and Birn, J. The role of electron heat flux in guide-field magnetic reconnection. Phys. Plasmas, 11, 5387(2004). (cited in page 61)

Hesse, M., Forbes, T. G., and Birn, J. On the Relation between Reconnected Magnetic Flux and Parallel Electric Fields in the Solar Corona. Astrophys. J., 631, 1227-1238 (2005a). (cited in page 60)

Hesse, M., Kuznetsova, M., Schindler, K., and Birn, J. Three-dimensional modeling of electron quasiviscous dissipation in guide-field magnetic reconnection. Phys. Plasmas, 12,100704 (2005b). (cited in page 48)

Hesse, M., Neukirch, T., Schindler, K., Kuznetsova, M., and Zenitani, S. The Diffusion Region in Collisionless Magnetic Reconnection. Space Sci. Rev., 160,3-23 (2011). (cited in page 61) 
Hewett, D. Low-frequency electromagnetic (Darwin) applications in plasma simulation. Comput. Phys. Commun., 84,243-277(1994). (cited in page 134)

Hirabayashi, K. and Hoshino, M. Magnetic reconnection under anisotropic magnetohydrodynamic approximation. Phys. Plasmas, 20,112111(2013). (cited in page 83, 265)

Hockney, R. W. and Eastwood, J. Computer Simulation Using Particles. IOP Publishing (1988). (cited in page 119, 132, 135, 136, 137, 139, 140, 141, 142, 156, 192)

Hockney, R. Measurements of collision and heating times in a two-dimensional thermal computer plasma. J. Comput. Phys., 8, 19-44,(1971). (cited in page 138, 140, 142, 144, (158)

Hofmann, I. and Boine-Frankenheim, O. Grid induced noise and entropy growth in 3d particle-in-cell simulation of high intensity beams. Phys. Rev. Spec. Top. - Accel. Beams, 17, 124201 (2014). (cited in page 144)

Hoh, F. C. Stability of Sheet Pinch. Phys. Fluids, 9,277(1966). (cited in page43)

Horiuchi, R. and Sato, T. Particle simulation study of collisionless driven reconnection in a sheared magnetic field. Phys. Plasmas, 4,277(1997). (cited in page 62, 68)

Horiuchi, R., Usami, S., and Ohtani, H. Influence of a Guide Field on Collisionless Driven Reconnection. Plasma Fusion Res., 9, 1401092 (2014). (cited in page 62, 68)

Hornsby, W., Migliano, P., and Bucholz, R. The linear tearing instability in three dimensional, toroidal gyrokinetic simulations. Phys. Plasmas, 22,022118 (2014a). (cited in page 233)

Hornsby, W., Migliano, P., Buchholz, R., Peeters, A., Zarzoso, D., Casson, F., and Poli, E. Nonlinear seed island generation by three-dimensional electromagnetic, gyrokinetic turbulence. arXiv Prepr., 1403.1520 (2014b). (cited in page233)

Hornsby, W., Migliano, P., Buchholz, R., Zarzoso, D., Casson, F., Poli, E., and Peeters, A. On seed island generation and the non-linear interaction of the tearing mode with electromagnetic gyro-kinetic turbulence. arXiv Prepr., 1409.0648 (2014c). (cited in page 233

Hoshino, M. The electrostatic effect for the collisionless tearing mode. J. Geophys. Res., 92,7368-7380 (1987). (cited in page 75)

Hoshino, M., Nishida, A., Mukai, T., Saito, Y., Yamamoto, T., and Kokubun, S. Structure of plasma sheet in magnetotail: Double-peaked electric current sheet. J. Geophys. Res., 101, 24775 (1996). (cited in page 87)

Hosseinpour, M. and Mohammadi, M. A. On the structure of guide magnetic field in the inertia-driven magnetic reconnection with the presence of shear flow. Phys. Plasmas, 20,114501 (2013). (cited in page 65, 66, 103, 256) 
Howes, G. G., Cowley, S. C., Dorland, W., Hammett, G. W., Quataert, E., and Schekochihin, A. a. Astrophysical Gyrokinetics: Basic Equations and Linear Theory. Astrophys. $J .$, 651, 590-614 (2006). (cited in page 229, 230, 231)

Huang, C., Lu, Q., Wu, M., Lu, S., and Wang, S. Out-of-plane electron currents in magnetic islands formed during collisionless magnetic reconnection. J. Geophys. Res. Sp. Phys., 118, 991-996 (2013). (cited in page 246)

Huang, S. Y., Zhou, M., Yuan, Z. G., Deng, X. H., Sahraoui, F., Pang, Y., and Fu, S. Kinetic simulations of electric field structure within magnetic island during magnetic reconnection and their applications to the satellite observations. J. Geophys. Res. Sp. Phys., 119, 7402-7412 (2014). (cited in page 246)

Huba, J. D. Hall dynamics of the Kelvin-Helmholtz instability. Phys. Rev. Lett., 72,2033-2036 (1994). (cited in page 97, 98)

Huba, J. D., Gladd, N. T., and Papadopoulos, K. The lower-hybrid-drift instability as a source of anomalous resistivity for magnetic field line reconnection. Geophys. Res. Lett.,4, 125 (1977). (cited in page 116, 117)

Huba, J. D., Gladd, N. T., and Papadopoulos, K. Lower-hybrid-drift wave turbulence in the distant magnetotail. J. Geophys. Res., 83, 5217 (1978). (cited in page 117)

Huebl, A., Pugmire, D., Schmitt, F., Pausch, R., and Bussmann, M. Visualizing the Radiation of the Kelvin-Helmholtz Instability. IEEE Trans. Plasma Sci., 42, 9-10 (2014). (cited in page 98)

Huntington, C. M., Fiuza, F., Ross, J. S., Zylstra, a. B., Drake, R. P., Froula, D. H., Gregori, G., Kugland, N. L., Kuranz, C. C., Levy, M. C., Li, C. K., Meinecke, J., Morita, T., Petrasso, R., Plechaty, C., Remington, B. a., Ryutov, D. D., Sakawa, Y., Spitkovsky, A., Takabe, H., and Park, H. S. Observation of magnetic field generation via the Weibel instability in interpenetrating plasma flows. Nat. Phys.,11,1-13 (2015). (cited in page 89)

Ishizawa, A. and Watanabe, T.-H. Reversible collisionless magnetic reconnection. Phys. Plasmas, 20, 102116 (2013). (cited in page 233)

Janaki, M. S., Dasgupta, B., and Yoon, P. H. A two-fluid model of the bifurcated current sheet. J. Geophys. Res., 117, A12201(2012). (cited in page 86)

Jenko, F., Dorland, W., Kotschenreuther, M., and Rogers, B. N. Electron temperature gradient driven turbulence. Phys. Plasmas, 7, 1904(2000). (cited in page 231)

Ji, H. and Daughton, W. Phase diagram for magnetic reconnection in heliophysical, astrophysical, and laboratory plasmas. Phys. Plasmas, 18,111207(2011). (cited in page 15)

Johnson, E. A. Gaussian-Moment Relaxation Closures for Verifiable Numerical Simulation of Fast Magnetic Reconnection in Plasma. PhD thesis, University of WisconsinMadison (2011). (cited in page 31, 33) 
Kagan, D., Sironi, L., Cerutti, B., and Giannios, D. Relativistic Magnetic Reconnection in Pair Plasmas and Its Astrophysical Applications. Space Sci. Rev., pages 1-29 (2015). (cited in page 18)

Karimabadi, H. Physics of saturation of collisionless tearing mode as a function of guide field. J. Geophys. Res., 110, A03214,(2005). (cited in page 76, 77, 78, 82, 155, 163, 168, 193, 324)

Karimabadi, H., Krauss-Varban, D., Omidi, N., and Vu, H. X. Magnetic structure of the reconnection layer and core field generation in plasmoids. J. Geophys. Res., 104, 12313 (1999). (cited in page 160, 253, 255, 284)

Karimabadi, H., Daughton, W., and Quest, K. B. Role of electron temperature anisotropy in the onset of magnetic reconnection. Geophys. Res. Lett., 31, L18801 (2004). (cited in page 58, 82, 83, 85, 162, 163,

Karimabadi, H., Daughton, W., and Quest, K. B. Antiparallel versus component merging at the magnetopause: Current bifurcation and intermittent reconnection. J. Geophys. Res., 110, A03213 (2005). (cited in page 64, 68, 74, 76, 79, 80, 82, 83, 87, 167, 168, 191, 206, 207)

Karimabadi, H., Roytershteyn, V., Daughton, W., and Liu, Y.-H. Recent Evolution in the Theory of Magnetic Reconnection and Its Connection with Turbulence. Space Sci. Rev., 178, 307-323 (2013). (cited in page 49, 50)

Karimabadi, H., Roytershteyn, V., Vu, H. X., Omelchenko, Y. A., Scudder, J., Daughton, W., Dimmock, A., Nykyri, K., Wan, M., Sibeck, D., Tatineni, M., Majumdar, A., Loring, B., and Geveci, B. The link between shocks, turbulence, and magnetic reconnection in collisionless plasmas. Phys. Plasmas, 21,062308 (2014). (cited in page 322)

Kärkkäinen, M. and Gjonaj, E. Low-dispersion wake field calculation tools. In Proc. ICAP 2006, pages 35-40 (2006). (cited in page 133, 147)

Karlický, M. Solar flares: radio and X-ray signatures of magnetic reconnection processes. Res. Astron. Astrophys., 14, 753-772 (2014). (cited in page 15)

Katanuma, I. and Kamimura, T. Simulation studies of the collisionless tearing instabilities. Phys. Fluids, 23, 2500 (1980). (cited in page 74, 188, 324)

Kato, T. N. Energy Loss of High-Energy Particles in Particle-in-Cell Simulation. arXiv Prepr., 1312.5507 (2013). (cited in page 144)

Kaufman, A. N. The Darwin Model as a Tool for Electromagnetic Plasma Simulation. Phys. Fluids, 14,446(1971). (cited in page 134)

Kemel, K., Henri, P., Lapenta, G., Califano, F., and Markidis, S. Kinetic instability plasma turbulence during the nonlinear stage of the Kelvin-Helmholtz. arXiv Prepr., 1412.1266 (2014). (cited in page 98) 
Kempf, A., Kilian, P., Ganse, U., and Schreiner, C. PICPANTHER : A simple , concise implementation of the relativistic moment implicit Particle-in-Cell method. Comput. Phys. Commun., 188, 198-207(2014). (cited in page 138)

Kilian, P., Ganse, U., and Spanier, F. Different Choices of the Form Factor in Particle-inCell Simulations. In Pogorelov, N., Audit, E., and Zank, G., editors, Numer. Model. Sp. Plasma Flows, volume 474 of Astronomical Society of the Pacific Conference Series, page 208 (2013). (cited in page 149)

Kilian, P. Teilchenbeschleunigung in Laserplasmen: Kinetische Simulationen. Diploma thesis, University of Würzburg (2010). (cited in page 147, 149)

Kilian, P. press-Particle code simulation of CME driven shocks. Technical report, MPS (2013). (cited in page 129, 133, 147)

Kilian, P. Teilchenbeschleunigung an kollisionsfreien Schockfronten. Phd thesis in preparation, University of Würzburg (2015). (cited in page 147,149)

Kilian, P., Burkart, T., and Spanier, F. The Influence of the Mass Ratio on Particle Acceleration by the Filamentation Instability. In Nagel, W. E., Kröner, D. B., and Resch, M. M., editors, High Perform. Comput. Sci. Eng. '11, pages 5-13. Springer Berlin Heidelberg, Berlin, Heidelberg (2012). (cited in page 119, 149, 154)

Kindel, J. M. and Kennel, C. F. Topside current instabilities. J. Geophys. Res., 76, 3055 (1971). (cited in page 104)

Kirk, J. G., Melrose, D. B., and Priest, E. R. Plasma Astrophysics, volume 24 of Saas-Fee Advanced Courses. Springer-Verlag, Berlin/Heidelberg (1994). (cited in page 142)

Kivelson, M. G. and Russell, C. T. Introduction to space physics. Cambridge University Press, Cambridge ; New York (1995). (cited in page 15, 17)

Klein, K. G. The kinetic plasma physics of solar wind turbulence. PhD thesis, University of Iowa (2013). (cited in page 90, 91)

Kleva, R. G., Drake, J. F., and Waelbroeck, F. L. Fast reconnection in high temperature plasmas. Phys. Plasmas, 2, 23-34(1995). (cited in page 59, 64, 65)

Klimontovich, Y. L. Physics of collisionless plasma. Physics-Uspekhi, 40, 21-51(1997). (cited in page 142)

Kobayashi, S., Rogers, B. N., and Numata, R. Gyrokinetic simulations of collisionless reconnection in turbulent non-uniform plasmas. Phys. Plasmas, 21,040704 (2014). (cited in page 233)

Koskinen, H. Physics of Space Storms: From the Solar Surface to the Earth. SpringerVerlag, Berlin Heidelberg (2011). (cited in page 16, 25, 52)

Krall, N. a. and Liewer, P. C. Low-frequency instabilities in magnetic pulses. Phys. Rev. $A, 4,2094-2103$ (1971). (cited in page 107, 113, 114) 
Krall, N. A. and Liewer, P. C. Turbulent Heating and Resistivity in Cool-Electron $\theta-$ Pinches. Phys. Fluids, 15, 1166 (1972). (cited in page 107)

Krall, N. A. and Trivelpiece, A. W. Principles of Plasma Physics. McGraw-Hill (1973). (cited in page 25, 28, 29, 30, 40, 88, 89, 90, 94, 144, 145)

Krause, T. B., Apte, A., and Morrison, P. J. A unified approach to the Darwin approximation. Phys. Plasmas, 14,102112 (2007). (cited in page 134)

Kruer, W., Dawson, J. M., and Rosen, B. The dipole expansion method for plasma simulation. J. Comput. Phys., 13, 114-129 (1973). (cited in page 148)

Kullback, S. and Leibler, R. On information and sufficiency. Ann. Math. Stat.,22, 79-86 (1951). (cited in page 143, 164)

Kuznetsov, E. a., Passot, T., and Sulem, P. L. On the mirror instability in the presence of electron temperature anisotropy. Phys. Plasmas, 19,090701 (2012). (cited in page 92)

Kuznetsova, M. M., Hesse, M., and Winske, D. Toward a transport model of collisionless magnetic reconnection. J. Geophys. Res., 105, 7601 (2000). (cited in page 60)

Lampe, M., Manheimer, W. M., McBride, J. B., Orens, J. H., Shanny, R., and Sudan, R. N. Nonlinear development of the beam-cyclotron instability. Phys. Rev. Lett., 26, 1221-1225 (1971). (cited in page 107, 273)

Landau, L. D. On the vibrations of the electronic plasma. Zh. Eksp. Teor. Fiz, 10, 25 (1946). (cited in page 18)

Langdon, A. Effects of the spatial grid in simulation plasmas. J. Comput. Phys., 6, 247-267 (1970). (cited in page 139, 193)

Langdon, A. "Energy-conserving" plasma simulation algorithms. J. Comput. Phys., 12,247-268(1973). (cited in page 132)

Lapenta, G., Markidis, S., Divin, A., Goldman, M., and Newman, D. Scales of guide field reconnection at the hydrogen mass ratio. Phys. Plasmas, 17,082106(2010). (cited in page 64, 66)

Lapenta, G. Particle simulations of space weather. J. Comput. Phys., 231, 795-821(2012). (cited in page 119, 121, 122, 125, 126, 131, 133, 136, 137, 138)

Lapenta, G., Brackbill, J. U., and Ricci, P. Kinetic approach to microscopic-macroscopic coupling in space and laboratory plasmas. Phys. Plasmas, 13, 055904(2006). (cited in page 135

Lapenta, G., Pierrard, V., Keppens, R., Markidis, S., Poedts, S., Šebek, O., Trávníček, P. M., Henri, P., Califano, F., Pegoraro, F., Faganello, M., Olshevsky, V., Restante, A. L., Nordlund, A. k., Frederiksen, J. T., Mackay, D. H., Parnell, C. E., Bemporad, A., Susino, R., and Borremans, K. SWIFF: Space weather integrated forecasting framework. J. Sp. Weather Sp. Clim., 3, A05(2013). (cited in page 137) 
Lashmore-Davies, C. and Martin, T. Electrostatic instabilities driven by an electric current perpendicular to a magnetic field. Nucl. Fusion, 13, 193-203(1973). (cited in page 105, 106, 108, 109, 110, 113, 114,

Laval, G. and Pellat, R. Stability of the plane neutral sheet for oblique propagation in anisotropic temperature Pap. 36. In Proc. ESRIN Study Gr., page 5, Frascati, Italy (1967). European Space Research Organization. (cited in page 82, 162)

Laval, G., Pellat, R., and Vuillemin, M. Instabilités électromagnétiques des plasmas sans collisions (CN-21/71). In Plasma Phys. Control. Nucl. Fusion Res. Vol. II, page 259 (1966). (cited in page73)

Lee, K.-W. and Büchner, J. Spontaneous Bifurcation of Single Peaked Current Sheets by Chaotic Electron Scattering. arXiv Prepr., 1205.1437 (2012). (cited in page 152, 158, 160, 164, 194,

Lembege, B. and Pellat, R. Stability of a thick two-dimensional quasineutral sheet. Phys. Fluids, 25, 1995 (1982). (cited in page 72)

Lemons, D. S. and Gary, S. P. Electromagnetic effects on the modified two-stream instability. J. Geophys. Res., 82, 2337-2342(1977). (cited in page 111, 114)

Lemons, D. S., Winske, D., and Gary, S. P. Electrostatic ion cyclotron velocity shear instability. J. Geophys. Res. Sp. Phys., 97, 19381-19387(1992). (cited in page 97)

Leonardis, E. Quantifying Finite Range Plasma Turbulence. PhD thesis, University of Warwick (2013). (cited in page 322)

Leva, J. L. A fast normal random number generator. ACM Trans. Math. Softw., 18, 449-453 (1992). (cited in page 148)

Levin, Y., Pakter, R., Rizzato, F. B., Teles, T. N., and Benetti, F. P. C. Nonequilibrium statistical mechanics of systems with long-range interactions. Phys. Rep., [535, 1-60 (2014). (cited in page 143)

Lewis, H. Energy-conserving numerical approximations for Vlasov plasmas. J. Comput. Phys., 6, 136-141 (1970). (cited in page 132)

Li, H., Nishimura, K., Barnes, D. C., Gary, S. P., and Colgate, S. a. Magnetic dissipation in a force-free plasma with a sheet-pinch configuration. Phys. Plasmas, 10, 2763-2771 (2003). (cited in page 45, 72, 81)

Liang, E., Boettcher, M., and Smith, I. Magnetic Field Generation and Particle Energization At Relativistic Shear Boundaries in Collisionless Electron-Positron Plasmas. Astrophys. J.,766, L19 (2013a). (cited in page 100)

Liang, E., Fu, W., Boettcher, M., Smith, I., and Roustazadeh, P. Relativistic PositronElectron-Ion Shear Flows and Application To Gamma-Ray Bursts. Astrophys. J., 779, L27 (2013b). (cited in page 100) 
Liewer, P. C. and Decyk, V. K. A general concurrent algorithm for plasma particle-in-cell simulation codes. J. Comput. Phys., 322, 302-322 (1989). (cited in page 146)

Lin, Y., Wang, X. Y., Chen, L., Lu, X., and Kong, W. An improved gyrokinetic electron and fully kinetic ion particle simulation scheme: benchmark with a linear tearing mode. Plasma Phys. Control. Fusion, 53,054013 (2011). (cited in page 80, 232)

Lin, Y., Wang, X., Lin, Z., and Chen, L. A gyrokinetic electron and fully kinetic ion plasma simulation model. Plasma Phys. Control. Fusion, 47, 657-669 (2005). (cited in page 232

Lipatov, A. S. The Hybrid Multiscale Simulation Technology. Springer-Verlag (2002). (cited in page 121)

Liu, Y.-H., Swisdak, M., and Drake, J. F. The Weibel instability inside the electronpositron Harris sheet. Phys. Plasmas, 16, 042101(2009). (cited in page 90, 174, 175)

Liu, Y.-H., Daughton, W., Karimabadi, H., Li, H., and Roytershteyn, V. Bifurcated Structure of the Electron Diffusion Region in Three-Dimensional Magnetic Reconnection. Phys. Rev. Lett., 110, 265004(2013). (cited in page 48, 81)

Liu, Y.-H., Daughton, W., Karimabadi, H., Li, H., and Peter Gary, S. Do dispersive waves play a role in collisionless magnetic reconnection? Phys. Plasmas, 21,022113(2014). (cited in page 48, 55, 59, 102, 112, 236)

Loureiro, N. F., Schekochihin, A. A., and Cowley, S. C. Instability of current sheets and formation of plasmoid chains. Phys. Plasmas, 14, 100703 (2007). (cited in page 52)

Loureiro, N. F., Schekochihin, A. A., and Uzdensky, D. A. Plasmoid and KelvinHelmholtz instabilities in Sweet-Parker current sheets. Phys. Rev. E, 87,013102 (2013a). (cited in page 52)

Loureiro, N. F., Schekochihin, A. A., and Zocco, A. Fast Collisionless Reconnection and Electron Heating in Strongly Magnetized Plasmas. Phys. Rev. Lett., 111, 025002 (2013b). (cited in page 233)

Lu, S., Lu, Q., Shao, X., Yoon, P. H., and Wang, S. Weibel instability and structures of magnetic island in anti-parallel collisionless magnetic reconnection. Phys. Plasmas, 18,072105 (2011). (cited in page 90, 173, 174, 213)

Lui, a. T. Y., Zheng, Y., Rème, H., Dunlop, M. W., Gustafsson, G., and Owen, C. J. Breakdown of the frozen-in condition in the Earth's magnetotail. J. Geophys. Res. Sp. Phys., 112, 1-15 (2007). (cited in page 63)

Luo, J., Ying, K., and Bai, J. Savitzky-Golay smoothing and differentiation filter for even number data. Signal Processing, 85, 1429-1434 (2005a). (cited in page 313)

Luo, J., Ying, K., He, P., and Bai, J. Properties of Savitzky-Golay digital differentiators. Digit. Signal Process., 15, 122-136 (2005b). (cited in page 316, 318, 319) 
Lyster, P. M. A fluid-ion and particle-electron model for low-frequency plasma instabilities. J. Comput. Phys., 102, 180-193 (1992). (cited in page 121)

Ma, X. Kelvin-Helmholtz Instability And Magnetic Reconnection At The Earth's Magnetospheric Boundary. PhD thesis, Fairbanks, Alaska (2012). (cited in page 95, 101)

Madden, H. H. Comments on the Savitzky-Golay convolution method for least-squaresfit smoothing and differentiation of digital data. Anal. Chem., 50, 1383-1386 (1978). (cited in page 314, 316, 319)

Mahajan, S. and Hazeltine, R. Sheared-flow generalization of the Harris sheet. Phys. Plasmas, 7, 1287(2000). (cited in page 98)

Malakit, K., Cassak, P. a., Shay, M. a., and Drake, J. F. The hall effect in magnetic reconnection: Hybrid versus Hall-less hybrid simulations. Geophys. Res. Lett., $\mathbf{3 6}, 1-5$ (2009). (cited in page 58)

Marchand, P. Binomial smoothing filter: A way to avoid some pitfalls of least-squares polynomial smoothing. Rev. Sci. Instrum., 54,1034 (1983). (cited in page 305, 310, 311)

Marder, B. A method for incorporating Gauss' law into electromagnetic PIC codes. $J$. Comput. Phys., 68, 48-55(1987). (cited in page 130, 148)

Markidis, S. and Lapenta, G. The energy conserving particle-in-cell method. J. Comput. Phys., 230, 7037-7052 (2011). (cited in page 132)

Mason, R. J. Implicit moment PIC-hybrid simulation of collisional plasmas. J. Comput. Phys., 51,484-501(1983). (cited in page 138)

Matsuda, Y. and Okuda, H. Collisions in multi-dimensional plasma simulations. Phys. Fluids, 18,1740 (1975). (cited in page 123, 142, 165, 193, 194, 201, 287)

Matsui, T. Kinetic theory and simulation of collisionless tearing in bifurcated current sheets. PhD thesis, University of Iowa (2008). (cited in page 87, 171, 191, 192, 324)

Matsui, T. and Daughton, W. Kinetic theory and simulation of collisionless tearing in bifurcated current sheets. Phys. Plasmas, 15,012901 (2008). (cited in page 87, 158, 188, 194, 324)

Matsumoto, H. and Omura, Y. Computer Space Plasma Physics : Simulation Techniques and Software. Terra Scientific Publishing Company (1993). (cited in page119)

Matteini, L., Landi, S., Velli, M., and Matthaeus, W. H. Proton Temperature Anisotropy and Magnetic Reconnection in the Solar Wind: Effects of Kinetic Instabilities on Current Sheet Stability. Astrophys. J., 763, 142 (2013). (cited in page 94, 181)

May, J., Tonge, J., Ellis, I., Mori, W. B., Fiuza, F., Fonseca, R. a., Silva, L. O., and Ren, C. Enhanced stopping of macro-particles in particle-in-cell simulations. Phys. Plasmas, 21, 052703 (2014). (cited in page 144) 
McBride, J. and Ott, E. Electromagnetic and finite $\beta$ e effects on the modified two stream instability. Phys. Lett. A, 39,363-364(1972). (cited in page 110)

McBride, J. B. Theory and Simulation of Turbulent Heating by the Modified Two-Stream Instability. Phys. Fluids, 15, 2367,(1972). (cited in page 107, 108, 109, 110, 111, 275, 280)

Medvedev, M. V. and Loeb, A. Generation of Magnetic Fields in the Relativistic Shock of Gamma-Ray Burst Sources. Astrophys. J., 526, 697-706(1999). (cited in page 89)

Melzani, M., Winisdoerffer, C., Walder, R., Folini, D., Favre, J. M., Krastanov, S., and Messmer, P. Apar-T: code, validation, and physical interpretation of particle-in-cell results. Astron. Astrophys., 558, A133 (2013). (cited in page 136, 139, 141, 155, 204, 205)

Melzani, M., Walder, R., Folini, D., Winisdoerffer, C., and Favre, J. M. Relativistic magnetic reconnection in collisionless ion-electron plasmas explored with particle-incell simulations. Astron. Astrophys.,570, A111 (2014). (cited in page 202)

Merz, F. Gyrokinetic Simulation of Multimode Plasma Turbulence. $\mathrm{PhD}$ thesis, University of Münster (2008). (cited in page 231, 234)

Michno, M. Temperature instabilities in the solar wind plasma. $\mathrm{PhD}$ thesis, University of Bochum (2014). (cited in page 94)

Mirnov, V. V., Hegna, C. C., and Prager, S. C. Two-fluid tearing instability in force-free magnetic configuration. Phys. Plasmas, 11,4468-4482(2004). (cited in page 81)

Miura, A. and Pritchett, P. L. Nonlocal stability analysis of the MHD Kelvin-Helmholtz instability in a compressible plasma. J. Geophys. Res., 87, 7431-7444(1982). (cited in page 95, 96)

Moddemeijer, R. On estimation of entropy and mutual information of continuous distributions. Signal Processing, 16, 233-248(1989). (cited in page 164, 325)

Modestov, M., Bychkov, V., Brodin, G., Marklund, M., and Brandenburg, A. Evolution of the magnetic field generated by the Kelvin-Helmholtz instability. Phys. Plasmas, 21, 072126 (2014). (cited in page101)

Mok, C., Ryu, C.-M., Yoon, P. H., and Lui, A. T. Y. Global two-fluid stability of bifurcated current sheets. J. Geophys. Res., 111, A03203 (2006). (cited in page 86)

Montgomery, D. and Nielson, C. Thermal Relaxation in One- and Two-Dimensional Plasma Models. Phys. Fluids, 13,1405(1970). (cited in page 144)

Muñoz, P. A., Kilian, P., and Büchner, J. Instabilities of collisionless current sheets revisited: the role of anisotropic heating. Phys. Plasmas, 21, 112106 (2014). (cited in page 124, 125, 149, 150, 180, 
Muñoz, P. A., Told, D., Kilian, P., Büchner, J., and Jenko, F. Gyrokinetic and kinetic particle-in-cell simulations of guide-field reconnection. I. Macroscopic effects of the electron flows. Phys. Plasmas, 22, 082110 (2015). (cited in page 149, 225)

Nakamura, T. K. M., Fujimoto, M., and Otto, A. Structure of an MHD-scale KelvinHelmholtz vortex: Two-dimensional two-fluid simulations including finite electron inertial effects. J. Geophys. Res. Sp. Phys., 113, A09204(2008). (cited in page 101, 102, 257)

Nakamura, T. K. M., Hasegawa, H., and Shinohara, I. Kinetic effects on the KelvinHelmholtz instability in ion-to-magnetohydrodynamic scale transverse velocity shear layers: Particle simulations. Phys. Plasmas, 17,042119 (2010). (cited in page 98)

Nakamura, T. K. M., Hasegawa, H., Shinohara, I., and Fujimoto, M. Evolution of an MHD-scale Kelvin-Helmholtz vortex accompanied by magnetic reconnection: Twodimensional particle simulations. J. Geophys. Res. Sp. Phys., 116, 1-18 (2011). (cited in page 98, 102

Nakamura, T. K. M., Daughton, W., Karimabadi, H., and Eriksson, S. Three-dimensional dynamics of vortex-induced reconnection and comparison with THEMIS observations. J. Geophys. Res. Sp. Phys., 118, 5742-5757(2013). (cited in page 102)

Neukirch, T., Wilson, F., and Harrison, M. G. A detailed investigation of the properties of a Vlasov-Maxwell equilibrium for the force-free Harris sheet. Phys. Plasmas, 16, 122102 (2009). (cited in page 44, 45, 46)

Nishikawa, K. I., Hardee, P., Zhang, B., Dutan, I., Medvedev, M., Choi, E. J., Min, K. W., Niemiec, J., Mizuno, Y., Nordlund, A., Frederiksen, J. T., Sol, H., Pohl, M., and Hartmann, D. H. Magnetic field generation in a jet-sheath plasma via the kinetic KelvinHelmholtz instability. Ann. Geophys., 31, 1535-1541 (2013). (cited in page 100)

Nishikawa, K. I., Hardee, P. E., Dutan, I., Zhang, B., Meli, A., Choi, E. J., and Min, K. Radiation from Particles Accelerated in Relativistic Jet Shocks and Shear-flows. arXiv Prepr., 1412.7064 (2014). (cited in page 100)

Nishimura, K., Liang, E., and Gary, S. P. Charge separation effects in magnetized electronion plasma expansion into a vacuum. Phys. Plasmas, 10,4559 (2003). (cited in page 45)

Numata, R. and Loureiro, N. F. Electron and Ion Heating During Magnetic Reconnection in Weakly Collisional Plasmas. In Proc. 12th Asia Pacific Phys. Conf., volume 1, page 015044. Journal of the Physical Society of Japan (2014). (cited in page 232, 233)

Numata, R. and Loureiro, N. F. Ion and electron heating during magnetic reconnection in weakly collisional plasmas. J. Plasma Phys., 81, 305810201 (2015). (cited in page 232)

Numata, R., Dorland, W., Howes, G. G., Loureiro, N. F., Rogers, B. N., and Tatsuno, T. Gyrokinetic simulations of the tearing instability. Phys. Plasmas, 18,112106 (2011). (cited in page 232) 
Ohtani, H. and Horiuchi, R. Open Boundary Condition for Particle Simulation in Magnetic Reconnection Research. Plasma Fusion Res., 4, 024-024 (2009). (cited in page 160)

Okuda, H. Collisions in a Plasma of Finite-Size Particles. Phys. Fluids, 13, 2123 (1970). (cited in page 141, 142, 165)

Orfanidis, S. J. Introduction to Signal Processing. Pearson Education, Inc. (2010). (cited in page 305, 306, 314, 316

Osman, K. T., Matthaeus, W. H., Gosling, J. T., Greco, A., Servidio, S., Hnat, B., Chapman, S. C., and Phan, T. D. Magnetic Reconnection and Intermittent Turbulence in the Solar Wind. Phys. Rev. Lett., 112,5 (2014). (cited in page 18)

Pacheco, P. S. An Introduction to Parallel Programming. Elsevier Inc. (2011). (cited in page 146)

Parker, E. N. Sweet's mechanism for merging magnetic fields in conducting fluids. $J$. Geophys. Res., 62, 509 (1957). (cited in page 49, 50)

Parker, E. N. Dynamics of the Interplanetary Gas and Magnetic Fields. Astrophys. J., 128, 664 (1958). (cited in page 94)

Parker, E. N. Magnetic neutral sheets in evolving fields. I - General theory. II - Formation of the solar corona. Astrophys. J., 264, 642(1983). (cited in page 13)

Parker, E. Spontaneous current sheets in magnetic fields: with applications to stellar $x$-rays. Oxford University Press, New York (1994). (cited in page 13, 16)

Parks, G. K. Physics of Space Plasmas. Westview Press (2004). (cited in page 25)

Patacchini, L. and Hutchinson, I. H. Explicit time-reversible orbit integration in Particle In Cell codes with static homogeneous magnetic field. J. Comput. Phys., 228, 2604-2615 (2009). (cited in page 136)

Petschek, H. E. Magnetic Field Annihilation. Phys. Sol. Flares, Proc. AAS-NASA Symp., page 425 (1964). (cited in page 52)

Pokhotelov, O. A. Linear theory of the mirror instability in non-Maxwellian space plasmas. J. Geophys. Res., 107, 1312 (2002). (cited in page 92)

Pokhotelov, O. A., Balikhin, M. A., Alleyne, H. S.-C. K., and Onishchenko, O. G. Mirror instability with finite electron temperature effects. J. Geophys. Res., 105, 2393 (2000). (cited in page 91, 92, 179)

Pokhotelov, O. A., Sagdeev, R. Z., Balikhin, M. a., Fedun, V. N., and Dudnikova, G. I. Nonlinear Mirror and Weibel modes: peculiarities of quasi-linear dynamics. Ann. Geophys., 28, 2161-2167 (2010). (cited in page 93)

Porcelli, F. Collisionless $\mathrm{m}=1$ tearing mode. Phys. Rev. Lett., 66, 425-428(1991). (cited in page 80 , 232 
Press, W. H., Teukolsky, S. a., Vetterling, W. T., and Flannery, B. P. Numerical Recipes: The Art of Scientific Computing. Third Edition. Cambridge University Press (2007). (cited in page 312,313, 315)

Priest, E. R., Longcope, D. W., and Heyvaerts, J. Coronal Heating at Separators and Separatrices. Astrophys. J.,624, 1057-1071(2005). (cited in page 13)

Priest, E. R. and Forbes, T. G. Magnetic Reconnection : MHD Theory and Applications. Cambridge University Press (2000). (cited in page 49, 69, 70, 71, 76)

Pritchett, P. L. Simulation of collisionless electrostatic velocity-shear-driven instabilities. Phys. Fluids B Plasma Phys., 5, 3770 (1993). (cited in page 97)

Pritchett, P. L. Particle-in-cell simulations of magnetosphere electrodynamics. IEEE Trans. Plasma Sci., 28, 1976-1990 (2000). (cited in page 119, 121, 130, 134, 137, 138, (146)

Pritchett, P. L. Geospace Environment Modeling magnetic reconnection challenge: Simulations with a full particle electromagnetic code. J. Geophys. Res., 106, 3783-3798 (2001). (cited in page 54, 68, 167)

Pritchett, P. L. Onset and saturation of guide-field magnetic reconnection. Phys. Plasmas, 12,062301,(2005a). (cited in page 62, 66, 78, 152, 160)

Pritchett, P. L. The "Newton Challenge": Kinetic aspects of forced magnetic reconnection. J. Geophys. Res., 110, A10213 (2005b). (cited in page 55)

Pritchett, P. L. Relativistic electron production during guide field magnetic reconnection. J. Geophys. Res., 111,1-11 (2006). (cited in page 67)

Pritchett, P. L. and Coroniti, F. V. Three-dimensional collisionless magnetic reconnection in the presence of a guide field. J. Geophys. Res., 109, A01220 (2004). (cited in page 680

Pritchett, P. L., Coroniti, F. V., Pellat, R., and Karimabadi, H. Collisionless reconnection in two-dimensional magnetotail equilibria. J. Geophys. Res., 96,11523 (1991). (cited in page 74, 80, 189, 190,

Pueschel, M. J., Jenko, F., Told, D., and Büchner, J. Gyrokinetic simulations of magnetic reconnection. Phys. Plasmas, 18, 112102 (2011). (cited in page 80, 232, 233)

Pueschel, M. J., Told, D., Terry, P. W., Jenko, F., Zweibel, E. G., Zhdankin, V., and Lesch, H. Magnetic Reconnection Turbulence in Strong Guide Fields: Basic Properties and Application to Coronal Heating. Astrophys. J. Suppl. Ser., 213,30 (2014). (cited in page 233)

Quest, K. B. and Coroniti, F. V. Tearing at the dayside magnetopause. J. Geophys. Res., 86,3289 (1981a). (cited in page 73)

Quest, K. B. and Coroniti, F. V. Linear theory of tearing in a high- $\beta$ plasma. J. Geophys. Res., 86, 3299 (1981b). (cited in page 78, 79, 82) 
Quest, K. B., Karimabadi, H., and Daughton, W. Linear theory of anisotropy driven modes in a Harris neutral sheet. Phys. Plasmas, 17,022107(2010). (cited in page 42 , 85, 86, 187, 195,

Ricci, P., Brackbill, J. U., Daughton, W., and Lapenta, G. Collisionless magnetic reconnection in the presence of a guide field. Phys. Plasmas, 11, 4102 (2004). (cited in page 56, 57, 58, 60, 62, 65, 66, 68, 84, 86, 243,

Riordon, J., Zubritsky, E., and Newman, A. Top 10 Articles. Anal. Chem., 72, 324-329 (2000). (cited in page 319)

Roach, C. M., Applegate, D. J., Connor, J. W., Cowley, S. C., Dorland, W. D., Hastie, R. J., Joiner, N., Saarelma, S., Schekochihin, A. A., Akers, R. J., Brickley, C., Field, A. R., Valovic, M., and Team, t. M. Microstability physics as illuminated in the spherical tokamak. Plasma Phys. Control. Fusion, 47, B323-B336(2005). (cited in page 247)

Rogers, B. N., Denton, R. E., Drake, J. F., and Shay, M. A. Role of Dispersive Waves in Collisionless Magnetic Reconnection. Phys. Rev. Lett., 87, 195004(2001). (cited in page 55, 57, 58, 59, 64, 67, 68, 103, 243, 256, 288)

Rogers, B. N., Denton, R. E., and Drake, J. F. Signatures of collisionless magnetic reconnection. J. Geophys. Res., 108, 1111 (2003). (cited in page 64, 65, 66, 244)

Rogers, B. N., Kobayashi, S., Ricci, P., Dorland, W., Drake, J., and Tatsuno, T. Gyrokinetic simulations of collisionless magnetic reconnection. Phys. Plasmas, 14,092110 (2007). (cited in page 80, 232, 233)

Romero, H. and Ganguli, G. Nonlinear evolution of a strongly sheared cross-field plasma flow. Phys. Fluids B, 5,3163(1993). (cited in page 280)

Romero, H., Ganguli, G., Lee, Y. C., and Palmadesso, P. J. Electron-ion hybrid instabilities driven by velocity shear in a magnetized plasma. Phys. Fluids B Plasma Phys., 4,1708 (1992). (cited in page 98)

Roytershteyn, V. and Daughton, W. Collisionless instability of thin current sheets in the presence of sheared parallel flows. Phys. Plasmas, 15,082901 (2008). (cited in page 103 , 261

Runov, A. Current sheet structure near magnetic X-line observed by Cluster. Geophys. Res. Lett., 30, 1579 (2003). (cited in page 86)

Sagaut, P. Large Eddy Simulation for Incompressible Flows. Springer Berlin Heidelberg (2006). (cited in page 306, 312

Sakai, J. I., Sugiyama, D., Haruki, T., Bobrova, N., and Bulanov, S. Magnetic field energy dissipation due to particle trapping in a force-free configuration of collisionless pair plasmas. Phys. Rev. E. Stat. Nonlin. Soft Matter Phys., 63, 046408 (2001). (cited in page 45) 
Sakai, J. I. and Matsuo, A. Three-dimensional dynamics of relativistic flows in pair plasmas with force-free magnetic configuration. Phys. Plasmas, 11, 3251-3258 (2004). (cited in page 45, 112)

Sarbach, O. and Zannias, T. Relativistic Kinetic Theory: An Introduction. In AIP Conf. Proc., volume 1548, page 134 (2013). (cited in page 202)

Savitzky, A. and Golay, M. J. E. Smoothing and Differentiation of Data by Simplified Least Squares Procedures. Anal. Chem., 36, 1627-1639 (1964). (cited in page 312 , 314, 319,

Schafer, R. What is a savitzky-golay filter? Signal Process. Mag. IEEE, pages 111-117 (2011). (cited in page 312, 313, 314)

Schekochihin, A. A., Cowley, S. C., Dorland, W., Hammett, G. W., Howes, G. G., Quataert, E., and Tatsuno, T. Astrophysical Gyrokinetics: Kinetic and Fluid Turbulent Cascades in Magnetized Weakly Collisional Plasmas. Astrophys. J. Suppl. Ser., 182, 310-377 (2009). (cited in page 230, 231, 247)

Schindler, K. A theory of the substorm mechanism. J. Geophys. Res., 79, 2803-2810 (1974). (cited in page 72)

Schindler, K. Physics of Space Plasma Activity. Cambridge University Press, Cambridge ; New York (2007). (cited in page 16, 30, 62, 69, 71, 73, 191)

Schindler, K. and Birn, J. Magnetospheric physics. Phys. Rep., 47, 109-165 (1978). (cited in page 17)

Schindler, K. and Hesse, M. Formation of thin bifurcated current sheets by quasisteady compression. Phys. Plasmas, 15, 042902 (2008). (cited in page 86, 161)

Schmitz, H. and Grauer, R. Vlasov simulations of collisionless magnetic reconnection without background density. Commun. Nonlinear Sci. Numer. Simul., 13,169-173 (2008). (cited in page 167)

Schoeffler, K. M., Drake, J. F., and Swisdak, M. The Effects of Plasma Beta and Anisotropy Instabilities on the Dynamics of Reconnecting Magnetic Fields in the Heliosheath. Astrophys. J.,743, 70 (2011). (cited in page 94)

Schoeffler, K. M., Drake, J. F., Swisdak, M., and Knizhnik, K. The Role of Pressure Anisotropy on Particle Acceleration during Magnetic Reconnection. Astrophys. J., 764, 126 (2013). (cited in page 90, 174, 175, 213)

Schreiner, C. Anregung und Analyse dispersiver, rechts-zirkularer Moden in numerischen Simulationen kinetischer Plasmen. Bachelor thesis, University of Würzburg (2011). (cited in page 147, 149)

Schreiner, C. Resonante Welle-Teilchen-Wechselwirkung in kinetischen Plasmen. Master thesis, University of Würzburg (2013). (cited in page 127, 128, 132, 145, 147, 149) 
Schreiner, C. and Spanier, F. Wave-particle-interaction in kinetic plasmas. Comput. Phys. Commun., 185, 1981-1986(2014). (cited in page 149)

Schwartz, S. J., Zweibel, E. G., and Goldman, M. Microphysics in astrophysical plasmas. Space Sci. Rev., 178, 81-99 (2013). (cited in page 20)

Servidio, S., Dmitruk, P., Greco, A., Wan, M., Donato, S., Cassak, P. a., Shay, M. A., Carbone, V., and Matthaeus, W. H. Magnetic reconnection as an element of turbulence. Nonlinear Process. Geophys., 18, 675-695(2011). (cited in page 322)

Servidio, S., Valentini, F., Perrone, D., Greco, A., Califano, F., Matthaeus, W. H., and Veltri, P. A kinetic model of plasma turbulence. J. Plasma Phys., pages 1-35 (2014). (cited in page 326)

Sestero, A. Self-Consistent Description of a Warm Stationary Plasma in a Uniformly Sheared Magnetic Field. Phys. Fluids, 10,193 (1967). (cited in page 44)

Shannon, C. A mathematical theory of communication. Bell Syst. Tech. J., 27, 379-423 (1948). (cited in page 143, 163)

Sharma, S., Sengupta, S., and Sen, A. Particle-in-cell simulation of large amplitude ionacoustic solitons. Phys. Plasmas, 22,022115(2015). (cited in page 231)

Shay, M. A., Drake, J. F., Denton, R. E., and Biskamp, D. Structure of the dissipation region during collisionless magnetic reconnection. J. Geophys. Res., 103,9165 (1998). (cited in page 53, 54, 87)

Shay, M. A., Drake, J. F., Rogers, B. N., and Denton, R. E. Alfvénic collisionless magnetic reconnection and the Hall term. J. Geophys. Res., 106, 3759-3772(2001). (cited in page 53, 54, 112, 257)

Shi, Q. Q., Pu, Z. Y., Zhang, H., Fu, S. Y., Xiao, C. J., Zong, Q. G., Fritz, T. A., and Liu, Z. X. Simulation studies of high-latitude magnetospheric boundary dynamics. Surv. Geophys., 26, 369-386 (2005). (cited in page 103, 255)

Shi, Y., Lee, L. C., and Fu, Z. F. A study of tearing instability in the presence of a pressure anisotropy. J. Geophys. Res., 92, 12171 (1987). (cited in page 93, 181)

Silin, I. Theory and Vlasov-code simulations of thin current sheet instabilities in collisionless space plasmas. $\mathrm{PhD}$ thesis, University of Braunschweig (2004). (cited in page 16)

Silin, I. and Büchner, J. Nonlinear instability of thin current sheets in antiparallel and guided magnetic fields. Phys. Plasmas, 10,3561(2003). (cited in page 16)

Silin, I., Büchner, J., and Zelenyi, L. M. Instabilities of collisionless current sheets: Theory and simulations. Phys. Plasmas, 9, 1104 (2002). (cited in page 16, 75, 190)

Sironi, L. and Narayan, R. Electron Heating By the Ion Cyclotron Instability in Collisionless Accretion Flows. I. Compression-Driven Instabilities and the Electron Heating Mechanism. Astrophys. J., 800, 88 (2015). (cited in page 87, 93) 
Sitenko, A. G. Electromagnetic Fluctuations in Plasma. Academic Press, New York (1967). (cited in page 144)

Sitenko, A. G. Fluctuations and Non-linear Wave Interactions in Plasmas. Pergamon Press Ltd (1982). (cited in page 144)

Sitnov, M. I. A model of the bifurcated current sheet. Geophys. Res. Lett., 30, 1712 (2003). (cited in page 86, 158)

Sitnov, M. I. and Swisdak, M. Onset of collisionless magnetic reconnection in twodimensional current sheets and formation of dipolarization fronts. J. Geophys. Res., 116, A12216 (2011). (cited in page 160)

Sokolov, I. V. Alternating-order interpolation in a charge-conserving scheme for particlein-cell simulations. Comput. Phys. Commun.,184,320-328(2012). (cited in page 148)

Somov, B. V. Plasma astrophysics, Part I: Fundamentals and Practice. Springer, New York ; Heidelberg, 2nd ed. edition (2013). (cited in page 25, 28, 50, 324)

Somov, B. V. Plasma Astrophysics Part II: Reconnection and Flares. Springer (2006). (cited in page 13, 14, 17)

Southwood, D. J. and Kivelson, M. G. Mirror instability: 1. Physical mechanism of linear instability. J. Geophys. Res., 98, 9181 (1993). (cited in page 91, 92, 182)

Stanier, A., Simakov, A. N., Chacón, L., and Daughton, W. Fast magnetic reconnection with large guide fields. Phys. Plasmas, 22,010701 (2015). (cited in page 55, 59)

Stark, C. R. and Neukirch, T. Collisionless distribution function for the relativistic forcefree Harris sheet. Phys. Plasmas, 19,012115(2012). (cited in page 46)

Stix, T. H. Waves in Plasmas. American Institute of Physics (1992). (cited in page 28)

Stockem, A., Lerche, I., and Schlickeiser, R. On the Physical Realization of Twodimensional Turbulence Fields in Magnetized Interplanetary Plasmas. Astrophys. J., 651, 584-589 (2006). (cited in page 89, 90)

Struckmeier, J. Stochastic effects in real and simulated ion beams. Phys. Rev. Spec. Top. Accel. Beams, 3, 034202 (2000). (cited in page 144)

Struckmeier, J. Concept of entropy in the realm of charged particle beams. Phys. Rev. E, 54, 830-837(1996). (cited in page 144)

Sugiyama, T. and Kusano, K. Multi-scale plasma simulation by the interlocking of magnetohydrodynamic model and particle-in-cell kinetic model. J. Comput. Phys., 227, 1340-1352 (2007). (cited in page 137)

Sundar, S. and Das, A. Free energy source for flow shear driven instabilities in electronmagnetohydrodynamics. Phys. Plasmas, 17,042106 (2010). (cited in page 97)

Swanson, D. G. Plasma Waves, 2nd Edition. IOP Publishing (2003). (cited in page 29) 
Sweet, P. The Neutral Point Theory of Solar Flares. In Lehnert., B., editor, Electromagn. Phenom. Cosm. Phys., page 123. Cambridge University Press (1958). (cited in page 50)

Swisdak, M., Liu, Y., and Drake, J. F. Development of a Turbulent Outflow During Electron-Positron Magnetic Reconnection. Astrophys. J., 680, 999-1008(2008). (cited in page 90, 174

Sydora, R. D. Nonlinear dynamics of small-scale magnetic islands in high temperature plasmas. Phys. Plasmas, 8, 1929-1934(2001). (cited in page 232)

Taflove, A. and Hagness, S. C. Computational Electrodynamics: The Finite-Difference Time-Domain Method. Artech House, Inc., 3rd edition (2005). (cited in page 128, 129 133,148 )

Tajiri, M. Propagation of Hydromagnetic Waves in Collisionless Plasma. II. Kinetic Approach. J. Phys. Soc. Japan, 22, 1482-1494(1967). (cited in page 91)

Tautz, R. C. and Schlickeiser, R. Counterstreaming magnetized plasmas. II. Perpendicular wave propagation. Phys. Plasmas, 13,062901 (2006). (cited in page 89)

Tautz, R. C. and Schlickeiser, R. Spontaneous emission of Weibel fluctuations by anisotropic distributions. Phys. Plasmas, 14, 102102 (2007). (cited in page 90)

Tautz, R. C. and Shalchi, A. On particle transport in Weibel generated magnetic fluctuations. Phys. Plasmas, 15, 052304 (2008). (cited in page 89, 90)

TenBarge, J. M., Daughton, W., Karimabadi, H., Howes, G. G., and Dorland, W. Collisionless reconnection in the large guide field regime: Gyrokinetic versus particle-in-cell simulations. Phys. Plasmas, 21, 020708 (2014). (cited in page 48, 55, 58, 228, 233, 235, 237, 242, 243, 244, 245, 246, 250, 252, 287, 288)

Told, D. Gyrokinetic Microturbulence in Transport Barriers. PhD thesis, University of Ülm (2012). (cited in page 231,234)

Tóth, G., van der Holst, B., Sokolov, I. V., De Zeeuw, D. L., Gombosi, T. I., Fang, F., Manchester, W. B., Meng, X., Najib, D., Powell, K. G., Stout, Q. F., Glocer, A., Ma, Y. J., and Opher, M. Adaptive numerical algorithms in space weather modeling. $J$. Comput. Phys., 231, 870-903(2012). (cited in page 137)

Treumann, R. a. and Baumjohann, W. Brief Communication: Weibel, Firehose and Mirror mode relations. Nonlinear Process. Geophys., 21, 143-148 (2014). (cited in page 89, (93)

Treumann, R. A. Origin of resistivity in reconnection. Earth, Planets Sp., 53, 453-462 (2001). (cited in page 25, 63, 106, 108, 109,

Treumann, R. A. and Baumjohann, W. Advanced Space Plasma Physics. Imperial College Press ; Distributed by World Scientific Pub. (2001). (cited in page 88, 91, 94, 108, 114)

Treumann, R. A. and Baumjohann, W. A note on the Weibel instability and thermal fluctuations. Ann. Geophys., 30, 427-431(2012). (cited in page 89) 
Treumann, R. A. and Baumjohann, W. A note on the structure and kinematics of Harris current sheets. arXiv Prepr. (2013a). (cited in page43)

Treumann, R. A. and Baumjohann, W. Collisionless magnetic reconnection in space plasmas. Front. Phys., 1, 31,(2013b). (cited in page 49, 55, 57, 60, 62, 63, 67, 72, 78, 296)

Treumann, R. A., Nakamura, R., and Baumjohann, W. Collisionless reconnection: mechanism of self-ignition in thin plane homogeneous current sheets. Ann. Geophys., 28, 1935-1943 (2010). (cited in page 89, 90)

Tskhakaya, D., Matyash, K., Schneider, R., and Taccogna, F. The Particle-In-Cell Method. Contrib. to Plasma Phys., 47, 563-594(2007). (cited in page 119, 120, 130, 136)

Tummel, K., Chen, L., Wang, Z., Wang, X. Y., and Lin, Y. Gyrokinetic theory of electrostatic lower-hybrid drift instabilities in a current sheet with guide field. Phys. Plasmas, 21, 052104 (2014). (cited in page232)

Umeda, T. Simulation of Collisionless Plasma With the Vlasov Method. In Doherty, B. S. and Molloy, A. N., editors, Comput. Phys., chapter 5, pages 315-332. Nova Science Publishers (2012). (cited in page 121)

Umeda, T., Omura, Y., Tominaga, T., and Matsumoto, H. A new charge conservation method in electromagnetic particle-in-cell simulations. Comput. Phys. Commun., 156, 73-85 (2003). (cited in page 131, 148)

Umeda, T., Togano, K., and Ogino, T. Two-dimensional full-electromagnetic Vlasov code with conservative scheme and its application to magnetic reconnection. Comput. Phys. Commun., 180, 365-374 (2009). (cited in page 121)

Umeda, T., Miwa, J.-i., Matsumoto, Y., Nakamura, T. K. M., Togano, K., Fukazawa, K., and Shinohara, I. Full electromagnetic Vlasov code simulation of the Kelvin-Helmholtz instability. Phys. Plasmas, 17, 052311(2010). (cited in page 98)

Usami, S., Horiuchi, R., Ohtani, H., and Den, M. Multi-Hierarchy Simulation of Collisionless Driven Reconnection by Real-Space Decomposition. In J. Phys. Conf. Ser., volume 561, page 012021 (2014). (cited in page 137)

Usui, S. and Amidror, I. Digital low-pass differentiation for biological signal processing. IEEE Trans. Biomed. Eng., 29, 686-693(1982). (cited in page 318)

Uzdensky, D. A. Magnetic Reconnection in Astrophysical Systems. arXiv Prepr. (2006). (cited in page 53)

Uzdensky, D. A. and Kulsrud, R. M. Physical origin of the quadrupole out-of-plane magnetic field in Hall-magnetohydrodynamic reconnection. Phys. Plasmas, 13, 062305 (2006). (cited in page 54)

Uzdensky, D. a. and Rightley, S. Plasma Physics of Extreme Astrophysical Environments. Reports Prog. Phys., 77, 036902 (2014). (cited in page 18) 
Vainshtein, S. I. and Mazur, V. A. Tearing instability in a neutral sheet with temperature anisotropy. Plasma Phys., 24,965-975(1982). (cited in page 84, 162)

Vasyliunas, V. M. Theoretical models of magnetic field line merging. Rev. Geophys., 13,303 (1975). (cited in page 60)

Vay, J. L. Simulation of beams or plasmas crossing at relativistic velocity. Phys. Plasmas, 15, 056701 (2008). (cited in page 128, 148)

Vay, J.-L., Geddes, C. G. R., Cormier-Michel, E., and Grote, D. Numerical methods for instability mitigation in the modeling of laser wakefield accelerators in a Lorentzboosted frame. J. Comput. Phys., 230, 5908-5929 (2011). (cited in page 133, 134, 135, 147, 148, 310,

Vay, J.-1. and Godfrey, B. B. Modeling of relativistic plasmas with the Particle-In-Cell method. Comptes Rendus Mécanique, 1,1-9 (2014). (cited in page 119)

Verboncoeur, J. P. Particle simulation of plasmas: review and advances. Plasma Phys. Control. Fusion, 47, A231-A260 (2005). (cited in page 119)

Verboncoeur, J. P., Langdon, A. B., and Gladd, N. T. An object-oriented electromagnetic PIC code. Comput. Phys. Commun., 87, 199-211(1995). (cited in page 157)

Villasenor, J. and Buneman, O. Rigorous charge conservation for local electromagnetic field solvers. Comput. Phys. Commun., 69,306-316(1992). (cited in page 131)

Vlasov, A. A. On vibration properties of electron gas. Zh. Eksp. Teor. Fiz, 8, 291 (1938). (cited in page 29)

Vörös, Z., Sasunov, Y. L., Semenov, V. S., Zaqarashvili, T. V., Bruno, R., and Khodachenko, M. Reconnection Outflow Generated Turbulence in the Solar Wind. Astrophys. J., 797, L10 (2014). (cited in page 18)

Walker, A. D. M. Magnetohydrodynamic Waves in Geospace. IOP Publishing Ltd. (2005). (cited in page 30)

Wan, M., Matthaeus, W. H., Karimabadi, H., Roytershteyn, V., Shay, M. A., Wu, P., Daughton, W., Loring, B., and Chapman, S. C. Intermittent Dissipation at Kinetic Scales in Collisionless Plasma Turbulence. Phys. Rev. Lett., 109, 195001(2012). (cited in page 322)

Wan, W., Chen, Y., and Parker, S. E. Gyrokinetic $\delta$ f simulation of the collisionless and semicollisional tearing mode instability. Phys. Plasmas, 12,012311,(2005a). (cited in page 232)

Wan, W., Chen, Y., and Parker, S. E. delta f Simulation of the collisionless tearing mode instability with a gyrokinetic ion response. IEEE Trans. Plasma Sci., 33,609-614 (2005b). (cited in page 232)

Wang, X. Y., Lin, Y., Chen, L., and Lin, Z. A particle simulation of current sheet instabilities under finite guide field. Phys. Plasmas, 15,072103 (2008). (cited in page 232) 
Wang, X. Y., Lin, Y., Chen, L., Lu, X., and Kong, W. Investigation of tearing instability using GeFi particle simulation model. Phys. Plasmas, 18,122102 (2011). (cited in page 80,232

Wang, Z., Pritchett, P. L., and Ashour-Abdalla, M. Kinetic effects on the velocity-sheardriven instability. Phys. Fluids B Plasma Phys.,4,1092 (1992). (cited in page 97)

Webb, S. D. Symplectic integration of magnetic systems. J. Comput. Phys., 270, 570-576 (2014). (cited in page 127)

Weibel, E. Spontaneously Growing Transverse Waves in a Plasma Due to an Anisotropic Velocity Distribution. Phys. Rev. Lett., 2, 83-84 (1959). (cited in page 88, 172)

Wilson, F. and Neukirch, T. A family of one-dimensional Vlasov-Maxwell equilibria for the force-free Harris sheet. Phys. Plasmas, 18, 082108 (2011). (cited in page 46)

Winske, D. and Omidi, N. A nonspecialist's guide to kinetic simulations of space plasmas. J. Geophys. Res., 101, 17287,(1996). (cited in page 119, 120,121)

Winske, D., Quest, K. B., Tanaka, M., and Wu, C. S. Plasma heating at collisionless shocks due to the kinetic cross-field streaming instability. J. Geophys. Res., 90, 123-136 (1985). (cited in page 111, 275)

Winske, D., Giacalone, J., Thomsen, M. F., and Mellott, M. M. A comparative study of plasma heating by ion acoustic and modified two-stream instabilities at subcritical quasi-perpendicular shocks. J. Geophys. Res. Sp. Phys., 92, 4411-4422(1987). (cited in page 106)

Wong, H. V. Electrostatic Electron-Ion Streaming Instability. Phys. Fluids, 13, 757-760 (1970). (cited in page 105)

Wu, C. S., Winske, D., Papadopoulos, K., Zhou, Y. M., Tsai, S. T., and Guo, S. C. A kinetic cross-field streaming instability. Phys. Fluids, 26, 1259 (1983). (cited in page111, 275)

Xu, X., Wang, Y., Wei, F., Feng, X., Deng, X., Zhou, M., Pang, Y., and Wong, H.-c. Direct evidence for kinetic effects associated with solar wind reconnection. Sci. Rep., 5, 1-6 (2015). (cited in page 18)

Yamada, M., Kulsrud, R. M., and Ji, H. Magnetic reconnection. Rev. Mod. Phys., 82, 603-664 (2010). (cited in page 18, 49, 60, 62, 67)

Yee, K. S. Numerical solution of initial boundary value problems involving maxwell's equations in isotropic media. IEEE Trans. Antennas Propag., 14,302-307 (1966). (cited in page 128)

Yoon, P. H. and Lui, a. T. Y. Lower-hybrid drift and Buneman instabilities in current sheets with guide field. Phys. Plasmas, 15,112105 (2008). (cited in page 114)

Yoon, P. H. and Lui, A. T. Y. Quasi-linear theory of anomalous resistivity. J. Geophys. Res., 111, A02203 (2006). (cited in page 62, 63, 218, 220), 
Yoon, P. H., Drake, J. F., and Lui, A. T. Y. Theory and simulation of Kelvin-Helmholtz instability in the geomagnetic tail. J. Geophys. Res., 101, 27327 (1996). (cited in page 97)

Yoon, P. H., Janaki, M. S., and Dasgupta, B. Kinetic model of Janaki et al.'s bifurcated current sheet. J. Geophys. Res. Sp. Phys., 119, 260-267(2014). (cited in page 86)

Zacharias, O., Kleiber, R., and Hatzky, R. Gyrokinetic simulations of collisionless tearing modes. In J. Phys. Conf. Ser., volume 401, page 012026 (2012). (cited in page 233)

Zacharias, O., Comisso, L., Grasso, D., Kleiber, R., Borchardt, M., and Hatzky, R. Numerical comparison between a gyrofluid and gyrokinetic model investigating collisionless magnetic reconnection. Phys. Plasmas, 21, 062106 (2014). (cited in page 233)

Zelenyi, L. M., Malova, H. V., and Popov, V. Y. Splitting of thin current sheets in the Earth's magnetosphere. J. Exp. Theor. Phys. Lett., 78,296-299 (2003). (cited in page 164)

Zelenyi, L. M., Malova, H. V., Popov, V. Y., Delcourt, D., and Sharma, A. S. Nonlinear equilibrium structure of thin currents sheets: influence of electron pressure anisotropy. Nonlinear Process. Geophys., 11, 579-587 (2004). (cited in page 86, 158)

Zenitani, S. and Hesse, M. The role of the Weibel instability at the reconnection jet front in relativistic pair plasma reconnection. Phys. Plasmas, 15,022101 (2008). (cited in page 90, 174)

Zenitani, S., Hesse, M., Klimas, A., and Kuznetsova, M. New Measure of the Dissipation Region in Collisionless Magnetic Reconnection. Phys. Rev. Lett., 106, 195003 (2011). (cited in page 271)

Zhang, S., Jia, Y., and Sun, Q. Comment on "Symplectic integration of magnetic systems" by Stephen D. Webb [J. Comput. Phys. 270 (2014) 570-576]. J. Comput. Phys., 282, 43-46 (2015). (cited in page 127)

Zhang, W., Lin, Z., Yoon, P. H., and Wang, X. Y. Two-Fluid Formulation of Lower-Hybrid Drift Instabilities in Current-Sheet Equilibrium with a Guide Field. Commun. Comput. Phys., 4, 719-728 (2008). (cited in page 101)

Zhou, M., Deng, X. H., and Huang, S. Y. Electric field structure inside the secondary island in the reconnection diffusion region. Phys. Plasmas, 19,042902 (2012). (cited in page 246)

Zhou, M., Pang, Y., Deng, X., Huang, S., and Lai, X. Plasma Physics of Magnetic Island Coalescence during Magnetic Reconnection. J. Geophys. Res. Sp. Phys., 119, 6177-6189 (2014). (cited in page 253)

Zhou, Y., Li, Y., and Wu, C. Stabilizing effects of a magnetic field gradient in a perpendicular shock wave on electron-cyclotron-drift instability. Phys. Fluids, 27, 2049-2054 (1984). (cited in page 107) 
Zocco, A. and Schekochihin, A. a. Reduced fluid-kinetic equations for low-frequency dynamics, magnetic reconnection, and electron heating in low-beta plasmas. Phys. Plasmas, 18, 102309 (2011). (cited in page 233)

Zubarev, D., Morozov, V., and Röpke, G. Statistical Mechanics of Nonequilibrium Processes. Akademie Verlag (1996). (cited in page 143)

Zweibel, E. G. and Yamada, M. Magnetic Reconnection in Astrophysical and Laboratory Plasmas. Annu. Rev. Astron. Astrophys., 47, 291-332 (2009). (cited in page 18, 49, 51, 52, 54, 70, 


\section{Scientific Contributions}

\section{Refereed publications}

- P. A. Muñoz, D. Told, P. Kilian, J. Büchner and F. Jenko "Gyrokinetic and kinetic particle-in-cell simulations of guide-field reconnection. I: Macroscopic effects of the electron flows", Physics of Plasmas 22, 082110, 2015.

- P. A. Muñoz, P. Kilian, and J. Büchner, "Instabilities of collisionless current sheets revisited: The role of anisotropic heating”, Physics of Plasmas 21, 112106, 2014.

\section{Conference proceedings}

- P. A. Muñoz, J. A. Araneda, "Nonlinear behavior of double ion beam distributions", Journal of Physics: Conference Series 511, 012004, 2014 (devoted to ICPPLAWPP 2010). 



\section{Acknowledgements}

First of all, I would like to express my gratitude to my supervisor Professor Jörg Büchner, for trusting and giving me the unique opportunity of working in this institute as his PhD student. I really appreciate all his advice and suggestions over these three years. I will always remember all the chances that he gave me to enjoy very interesting and fruitful discussions about science with him and other people, in conferences, in the collaborations with other groups that he organized and in our weekly group meetings. His very deep physical insights and knowledge about Plasma Physics always gave me hints to investigate more and more and thus to quench my endless thirst for knowledge.

But having started a PhD in this institute would not have been possible at all without the advice of Professor Jaime Araneda during my undergraduate studies in Chile. I am very grateful of having introduced me to the fascinating field of kinetic Plasma Physics. He was and he still is my inspiration as a scientist and person. His continuous guidance and advice when I was studying in Concepción allowing me to have the required academic preparation to face the challenge to pursue a $\mathrm{PhD}$ in Physics here in Germany.

My work in this institute made a 180 degree change around the middle of my $\mathrm{PhD}$, when in a critical point of my research I met and I started to work with Patrick Kilian. I cannot express with any words my gratitude to him for so many things. His deep knowledge about PIC codes (as well as in many other areas of Physics) changed radically the way in which I was developing my work. I learned of him all the small simulation tricks and details that can only be acquired by the experience accumulated over years of continuous work. For the first time I felt like part of a true "kinetic plasma and PIC" team when working and discussing with him. The research presented in this thesis would not have been possible at all without his continuous support, help and always interesting conversations about the more diverse topics. In practice, he became my model on how a simulation scientist should be.

I also want to thank the IMPRS for Solar System Science for giving me the opportunity to finance this $\mathrm{PhD}$, in particular to the former coordinator, the late Dieter Schmitt, who helped me a lot when I started here. And of course, I also want to say many thanks to the current coordinator Sonja Schuh for all her help, for taking care of each PhD student and in general for her very impressive organizational skills that are making this IMPRS school everyday better.

I would also like to thank to Profs. Hardi Peter and Jens Niemeyer for having accepted to be part of my TAC committee, as well as Profs. W. Glatzel, U. Motschmann, L. Gizon, A. Reiners and Dr. Wolfram Schmidt for agreeing to be part of the examination committee.

I am also thankful to all the rest of members of our TSSSP group. Jan Skála and Fabien Widmer for their always cheerful attitude that helped to improve my mood when 
facing the daily difficulties associated with the research, in addition to share some many nice moments throughout all these years. Also Neeraj Jain for his help in many different things since my first year here and Jeff Lee for his support at the beginning.

I also have to say a very big thank you to my friend and former flat-mate Rakesh Yadav for so many things, and to all my former and current office-mates for making our office a very nice place to work. And of course, to all the fellow PhD students known generically as the "coffee group" with whom I could share nice moments, that helped me to have a little bit of "human company" everyday after lunch, and in particular Kok Leng for always keeping that joyful attitude so characteristic of him.

My sincere apologies to all the people that I am forgetting to mention now.

And last but not least, tengo que agradecer a Dios y a mi familia. Ellos siempre creyeron en mis capacidades para lograr el desafío de estudiar un doctorado en Física aquí en el Viejo Continente. A pesar de la distancia y dificultades con que me fui encontrando, siempre me apoyaron enviándome ánimos todo este tiempo. Esta tesis está dedicada a ellos. 


\section{Curriculum vitae}

\section{Personal}

- Last Name: Muñoz Sepúlveda

- First and Middle Name: Patricio Alejandro

- Date of birth: 17.04.1987

- Place of birth: Concepción, Chile

- Nationality: Chilean

\section{Education}

- 03/1993 - 12/1996 : Primary School, Escuela D-557 José Hipólito Salas y Toro, Chiguayante, Chile.

- 03/1997 - 12/2000 : Primary School, Liceo Salesiano Domingo Savio, Concepción, Chile.

- 03/2001 - 12/2004 : High School, Liceo Salesiano Domingo Savio, Concepción, Chile.

- 03/2005 - 12/2008 : Bachelor in Physical Sciences, Universidad de Concepción, Concepción, Chile.

- 08/2009 - 04/2011 : Master of Science with Major in Physics, Universidad de Concepción, Concepción, Chile. Thesis title: "Kinetic Theory of Parametric Instabilities in Plasmas“.

- 02/2012 - 06/2015 : PhD candidate, Max-Planck-Institut für Sonnensystemforschung and Georg-August-Universität Göttingen, Göttingen, Germany. Thesis title: "Fully kinetic PiC simulations of current sheet instabilities for the Solar corona“. 


\section{Patricio A. Muñoz Sepúlveda: Fully kinetic PiC simulations of current sheet instabilities for the Solar corona}

In the Solar corona, magnetic energy is conjectured to be released through current sheets to be transformed to particle, plasma energy and heating by magnetic reconnection. Since the coronal plasma is collisionless, these are essentially kinetic plasma processes. Their nonlinear physics and properties can be described best by Particle-in-Cell ( $\mathrm{PiC}$ ) numerical simulations. Since the coronal plasma is magnetically dominated, and in contrast to previous kinetic simulations of current sheets, the presence of large guide magnetic fields has to be taken into account. We aim at finding and describing the resulting dominating kinetic instabilities, turbulent processes and anomalous (collisionless) transport effects.

In order to validate our methods, first we analyze the limit case of zero guide field (antiparallel configuration). We find several instabilities driven by temperature anisotropy inhibiting the tearing mode. They might be numerically induced when more realistic parameters (high mass ratios) are used in $\mathrm{PiC}$ simulations. This numerical temperature anisotropy can be efficiently reduced by using higher order shape functions. For current sheets in the presence of small guide fields, we show evidence of non collisional resistivity in the generalized Ohm's law. And in the limit of infinite guide fields, we compare our kinetic simulation results with gyrokinetic theory. Although there is agreement in some quantities such as reconnection rates between both plasma models, we find a magnetic field generation only in $\mathrm{PiC}$ simulations with finite guide fields, due to an initial shear flow in the force free current sheet initialization. In addition, we also find signatures of cross-streaming instabilities producing anisotropic electron heating and acceleration. 